
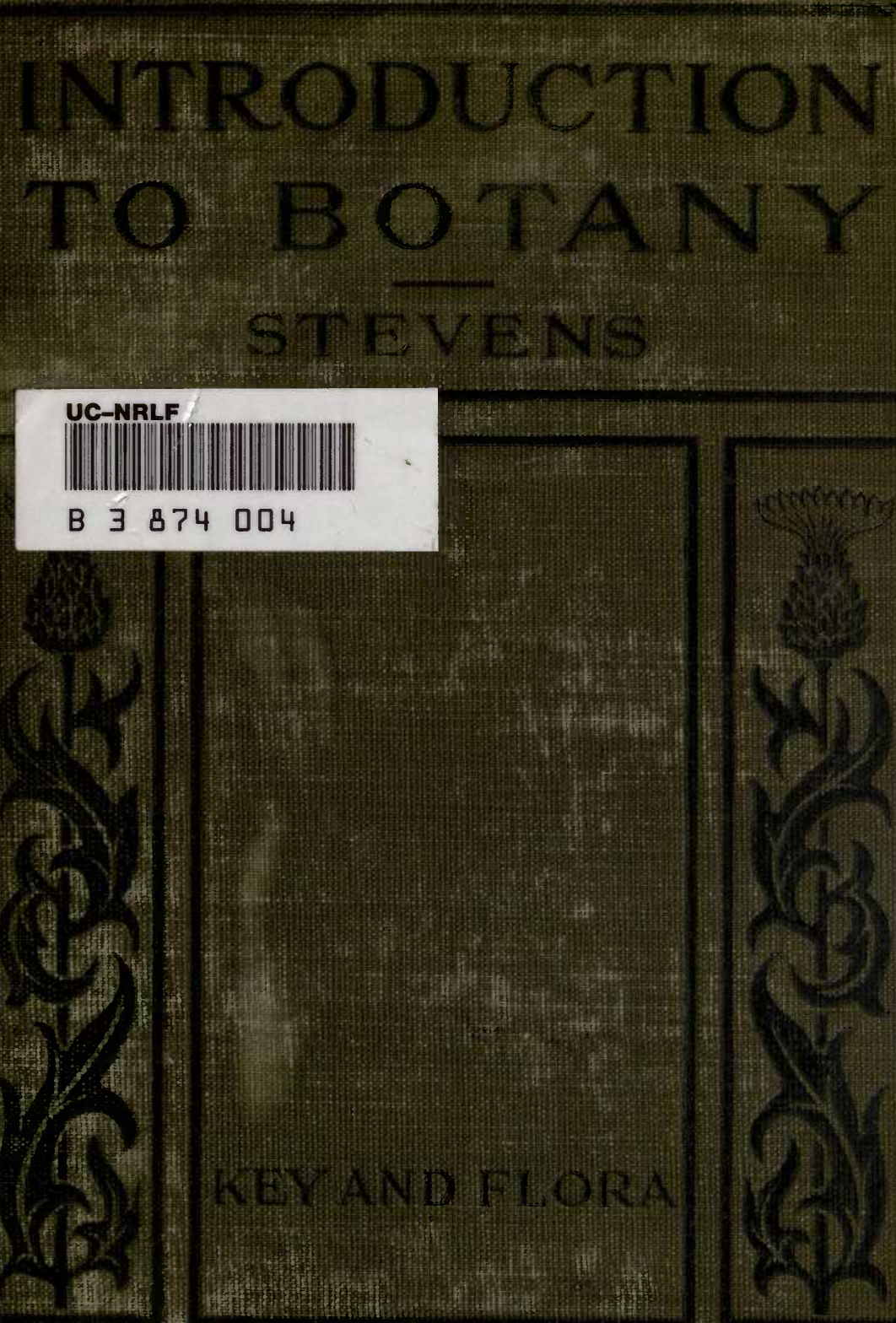

H:

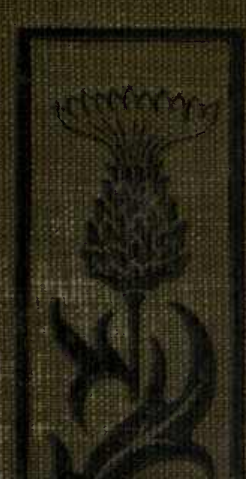


UNIVERSITY OF CALIFORNIA

\section{DEPARTMENT OF EDUCATION}

GIFT OF THE PUBLISHER
No. 392 .
Received 1903

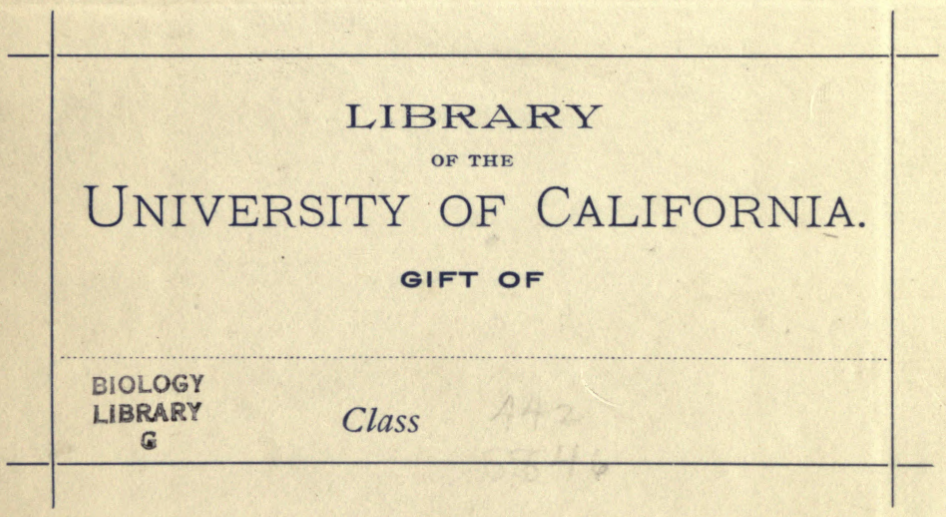






\title{
INTRODUCTION TO BOTANY
}

\author{
BY \\ WILLIAM CHASE STEVENS \\ PROFESSOR OF BOTANY IN THE UNIVERSTTY OF KANSAS
}

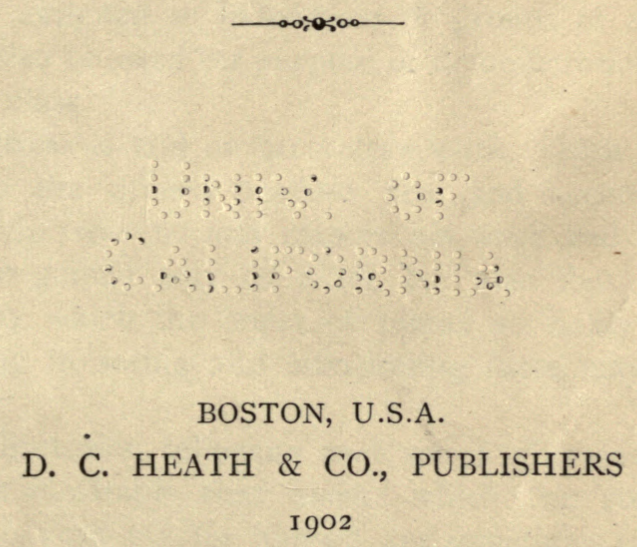




\section{BIOLOGY}

LIBRARY

G

COPYRIGHT, I902,

By D. C. Heath \& Co.

PRINTED IN

UNITED STATES

OF AMERICA

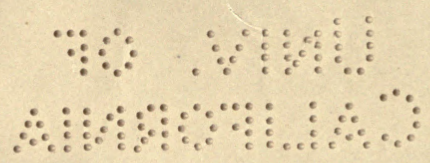




\section{PREFACE.}

ENOUGH work has been outlined in this book for a year's course in those schools that are prepared, by suitable time allotted to the subject and by laboratory equipment, to do comprehensive and thorough work. A term could be devoted to the work outlined in Chapters $\mathrm{I}$ to $\mathrm{X}$ inclusive; and where the daily laboratory periods are one hour or less, the entire school year could be profitably occupied by the course there outlined. It is, of course, presumed that the teacher will select from the book such work as the possibilities of his school warrant. It is not expected that each student will perform all of the physiological experiments herein outlined; but different experiments are to be assigned to individuals or groups of students, the results to be used for purpose of demonstration before the entire class.

Much stress is laid on laboratory work, which is to be done with the utmost possible care and accuracy, not only that the student may respect his work and that the knowledge gained may be exact, but also that the fine opportunity which the study of plants so richly affords for training in seeing and interpreting facts, may not be passed by.

In the discussion following each set of laboratory directions it is inevitable that results which the student is expected to work out for himself are sometimes told; but 
there is a large body of work for which no such aid is given, quite sufficient for training in independent and self-reliant study.

In order that the student may not be hampered with preconceptions of what he is expected to see and to represent, in choosing the illustrations care has been exercised to avoid in most instances those subjects which he is required to draw in his laboratory book. And that the main facts about the nature of plants may be kept plainly before the student, glossology has for the most part been kept from the discussions and placed in compact form in Part III.

I wish to acknowledge my special indebtedness to my colleague, Professor M. A. Barber, in conjunction with whom a large part of the course here outlined has been worked out. He has read most of the manuscript and has made many helpful suggestions.

The manuscript has been examined by Dr. C. E. McClung, Associate Professor of Zoölogy, University of Kansas; Dr. V. M. Spalding, Professor of Botany, University of Michigan; Dr. John W. Harshberger, Instructor in Botany, University of Pennsylvania; Dr. Rodney $\mathrm{H}$. True, Plant Physiologist, Department of Agriculture, Washington, D.C. ; Mr. L. Murbach, Central High School, Detroit, Michigan; Principal Maurice Ricker, High School, Burlington, Iowa.

The proof has been read by Dr. D. M. Mottier, Professor of Botany, University of Indiana; Dr. Charles $\mathrm{H}$. Clark, Phillips Academy, Exeter, New Hampshire; Dr. F. C. Newcombe, Junior Professor of Botany, University of Michigan; B. M. Stigall, Instructor in Biology, Manual Training High School, Kansas City, Missouri.

Dr. E. C. Franklin, Professor of Physical Chemistry, 
University of Kansas, has revised the paragraphs dealing with the nature of diffusion and osmosis. Dr. S. W. Williston, Professor of Paleontology, University of Chicago, has read the chapter on Plants of Past Ages; S. J. Hunter, Associate Professor of Comparative Zoölogy, University of Kansas, has read those portions of Chapter VIII dealing with the anatomy of insects; M. W. Sterling, Associate Professor of Greek, University of Kansas, has revised and contributed to the etymology of terms in the glossary and flora; Dr. W. H. Carruth, Professor of German, University of Kansas, has revised the passages translated from Sprengel.

To all of these teachers I wish to express my grateful sense of obligation for suggestions leading to the betterment of the book.

The illustrations have been made by Miss Marguerite Wise, Instructor in Botany and Zoölogy, University of Kansas, Mr. Sidney Prentice, Miss Luella Pugh, and Miss Katherine Crew. I wish to acknowledge my special indebtedness to Miss Wise, whose knowledge of the subject has greatly lightened the task of preparing the illustrations. She has made all of the wash drawings excepting Fig. 192, which is by Miss Crew. Of the original line drawings, Mr. Prentice made Figs. I0, I9, 21, 37, 45, 47, $48,93,180,198,199,201,202,203,204$. The remainder of the original illustrations and most of the copied figures were done by Miss Wise.

W. C. S.

UNIVERSITY OF KANSAS. 



\title{
CONTENTS.
}

\author{
PART I.
}

MORPHOLOGY, PHYSIOLOGY, AND ECOLOGY. CHAPTER

I. LABORATORY WORK • • • • • • • I

II. Seeds and Seedlings • • • • • 5

III. Roots • • • • • • • • • 28

IV. Buds And Stems • • • • • • • 45

V. Leaves • • • • • • • • • 75

VI. Growth And Movement . . . . . 100

VII. Modified Parts • • • • • • • I 134

VIII. Flowers • • • • • • • • • I47

IX. Dispersion of Fruits ANd Seeds • • • . 207

$\mathrm{X}$. Studies of Selected Spermatophytes • • . 218

XI. Slime Moulds, Bacteria, and Yeasts • . . 251

XII. Alge, Fungi, And Lichens • • • • 264

XIII. Mosses, Ferns, ANd Horsetalls • • • 286

XIV. Adaptation to Environment . . . . 303

XV. Plants of Different Regions • • • • 328

XVi. Plants of Past Ages . . • • • • 35 I

XVII. Classification of Plants • • • • 359 


\section{PART II.}

\section{THE HERBARIUM, LABORATORY EQUIPMENT, AND PROCESSES.}

CHAPTER

XVIII. The School Herbarium .

XIX. Laboratory Equipment • • • • • • • $37 \mathrm{I}$

XX. Reagents and Processes . • • • . . 381

PART III.

GLOSSOLOGY.

GLOSSARY • • • • • • • • • • • • 399

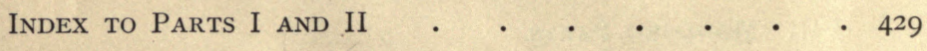




\section{INTRODUCTION TO BOTANY.}

\section{CHAPTER I.}

\section{LABORATORY WORK.}

1. Method of Study. - The study of plants, to be of much value, requires accurate observation. This is best secured by a definite and orderly record, by the student, of what he has seen. Simple drawings are usually more effective than a verbal description, and are therefore much used in the work here outlined. Students unskilled in drawing nee. not be discouraged by this requirement, for after some time of persistent and patient effort the number of those who cannot achieve passably good results is few indeed. The drawings furnish the best possible mode of expression in the study of form and structure, for they show briefly and positi ely how well the student has observed.

It is best, as a rule, not to point out the faults of the drawings to the student but to have him detect them, which he rarely fails to $b c$ able to do when asked where the faults lie. The drawin ss should be very simple, but never merely sketchy. Every line should have a meaning and should clearly indicate what it is intended to show. Only outlines are desired; shading is unnecessary, and should not be attempted except by one who thoroughly understands its application.

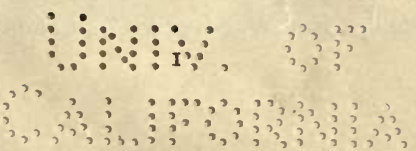


There are three simple rules which the student should keep in mind. (I) The drawings should be on a sufficiently large scale to allow the smallest details to be put in without crowding. (2) All parts must be in correct proportion with reference to one another and in right relative positions. (3) Some one dimension of the object should always be used as a measuring rod in establishing the lengths of the others. If these rules are followed, much subsequent correction will be avoided. A very serviceable notebook for the notes and drawings is afforded by the No. 2 double and reversible note covers, opening at the side, filled with a good quality of unruled linen ledger paper. ${ }^{1}$

The drawings should be symmetrically disposed over the sheet without crowding, as shown in Fig. 74. They should be made with a $6 \mathrm{H}$ drawing pencil kept quite sharp by rubbing it occasionally with a longitudinal to-andfro motion on a piece of No. o emery cloth or sandpaper. Neither a soft nor a dull pencil should ever be used. The descriptive notes, written in ink, should be on a separate sheet facing the drawings, the parts of which are to be lettered and referred to in the notes accordingly. Notes written with a pencil are liable to smirch the drawings and must not be tolerated. The student should strive for the utmost accuracy and neatness in the drawings and notes; he should have a personal pride in them, for they represent his capabilities in seeing and interpreting facts with which he has personally to deal.

2. Procedure in Drawing. - After having determined the proper scale of the drawing, that is, whether its diameters should be twice as great, three times as great, etc., as those of the object, place points to establish the limits of the 1 Made by Ghas, W. Sever \& Co., Cámbridge, Mass. 
long and short diameters, using the short diameter, for instance, as a measuring rod; and then with a very light touch of the pencil, making a barely visible line, draw the outline. If the form is not right at the first attempt, correct it before rubbing out the false line, for the latter may serve as a guide in correcting the error. When the form has been satisfactorily drawn, rub out the false lines once for all, and retrace the final outline with a firm touch so that it stands out sharply, but the pressure of the pencil must not be hard enough to dig into the paper.

Colored pencils are very helpful for giving distinct tints to the different parts of a drawing, but they should be used only where a definite purpose is to be served, as in calling attention to homologous parts in different drawings. The coloring should be done with very light cross-hatching strokes until an even light tint is produced. If satisfactory results are not obtained in this way, the color can be distributed more evenly by rubbing over the colored areas with a paper or chamois stump used by artists in crayon shading.

3. The Student at Work. - The student should think of his work as he proceeds. The drawings and notes are intended to assist him in gaining a clear conception of the problems before him, and they are good evidence of his success or failure; but he should possess his subject more completely than the drawings and notes may show. The laboratory is so much limited space in which certain conveniences for work are provided. Were it not for these conveniences, it would be better to study the subjects out of doors in their natural surroundings. The student who thinks as he works associates the subject with the natural conditions, and sees the bearing of what he is learning in the laboratory on the life of plants as they occur in nature. 
The student should never ask the teacher questions which with reasonable effort he can answer for himself; as questions arise, he should continually recur to the subject of his study as the most reliable source of information about itself.

It is a good plan, when pertinent questions are asked, to write them on the blackboard for the class to consider, and finally to use them as topics for a general discussion.

4. Field Work. - The laboratory work should, of course, be supplemented by field work. The locality where the work is to be carried on should first be visited in order to determine what the students can best learn there; then questions and directions should be written on the blackboard for the student to copy, in order that his work may have definiteness and meaning. Drawings and notes which are to count as an essential part of the course should be required in this work. In succeeding chapters, problems are given which are to be worked out in the field, but they will need to be supplemented by others particularly adapted to the specific locality. 


\title{
CHAPTER II.
}

\section{SEEDS AND SEEDLINGS.}

\author{
PROVIDING MATERIALS.
}

It is a simple matter to provide the seeds required for the work of this chapter, but there may be some difficulty in growing the seedlings on account of lack of space and equipment. If a greenhouse cannot be used, some arrangement must be made for growing the seedlings in a warm room. Boxes should be made not more than six inches deep, and white pine sawdust, or chopped sphagnum when obtainable, should be placed in these to a depth of four inches. If the classes are large and the space which can be devoted to seed boxes very limited, they may be placed one above another in tiers separated by a space of about ten inches. Moisten the sawdust throughout and then plant the seeds to a depth of about one inch in rows between two and three inches apart. After covering the seeds, press the sawdust down firmly with the palm of the hand or with a wooden block. The seeds will germinate more quickly if soaked in water over night before planting. The first sowings should be made about two weeks before the work is to begin, and then other sowings of the same kinds of seeds at intervals of a few days, in order that plenty of seedlings may be on hand in different stages of germination. The seeds need to be kept warm and well watered, but it is not necessary that they should have light until they have germinated; after that time the seedlings will grow weak and spindling if too much shaded.

\section{Lima Bean.}

OBSERVATIONS ON SEEDS AND SEEDLINGS.

I. Make drawings of the external appearance of a dry bean from the two most important points of view, showing all structural characteristics. Make drawings to the scale, 
$\times \mathrm{I} .5$; that is, the diameters of the drawings are to be $\mathrm{I} .5$ times as great as the corresponding diameters of the bean. The two most important points of view are those which best show the form of the bean, and the structures which may have some significance in the formation or germination of the seed. If beans in the pod, preserved in $2 \%$ formalin, or in $70 \%$ alcohol, are available, the data for judgment will be more complete. Compare with the dry bean one which has been soaked in water over night, and note any difference in size, form, and texture.

2. Slip the skin or seed-coats (there are usually two seed-coats, the outer, termed the testa, being thicker and harder than the inner) carefully from a soaked seed, noticing whether it is attached to the rest of the seed at any point, or simply lies in contact with it. Can any structures be seen after the seed-coats are removed which could not be seen before? If so, make drawings from the two points of view which will most clearly show them. Is there any connection between structures which are removed with the seed-coats and those lying beneath them? How do the dry and soaked seed-coats of the bean differ as to hardness and toughness? What significance do you see in the difference? In what ways is the seed protected against injury? Answer these questions in the notes, and refer to the drawings wherever they will illustrate what is said.

3. After the seed-coats have been removed, note whether the two halves of the bean, termed cotyledons, are united at any point; then carefully separate the cotyledons and place them on the table, convex side down, and in contact, at the right point, with any part from which they may have been severed. Draw as thus seen on the same scale as before.

4. Study with a lens the structures which were revealed 
by separating the cotyledons, and draw to a scale sufficiently large to bring out the details thus observed, say, $\times 4$. If any parts are folded together, make drawings to show their relationship clearly. The student should not be satisfied with his work until the drawings show the facts as clearly as he can see them by a thoughtful examination of the object.

\section{Germinating Lima Bean.}

5. Make drawings of a bean in the first stages of germination, and in various succeeding stages, identifying the parts already studied in the ungerminated seeds. Note any new structures.

6. The following terms should now be applied in the notes to the structures designated by them:-

The scar where the seed was attached to the pod is called the hilum.

The rudimentary plant formed in the seed is called the embryo.

The first leaves of the embryo are called the cotyledons.

The bud sometimes present between the cotyledons is called the plumule.

The small stem in the seed from which the cotyledons grow is called the caulicle.

The first root produced as a continuation of the caulicle is called the radicle.

All of the embryo below the insertion of the cotyledons is called the hypocotyl (including caulicle and radicle). All of the embryo above the insertion of the cotyledons is called the epicotyl.

The opening in the seed-coats near the tip of the caulicle is called the micropyle. 
7. After the drawings have been pronounced satisfactory by the instructor, impart different tints to the separate structures by means of colored pencils, using the same colors for the corresponding parts in the germinated and ungerminated seeds. Thus : color the cotyledons yellow, the plumule and whatever develops from it green, the caulicle orange, the radicle and succeeding roots red, reserve food outside of the embryo blue, and when the cotyledons contain reserve food dot their yellow color with blue.

8. In the germinating Lima bean, what structures grow most rapidly at first? Can you see any physiological reason why one structure should develop before another? How does the seedling manage to rise through the soil into the light and air?

9. Make a cross section of the main root where a whorl of rootlets arises, and show by a drawing the method of origin of the rootlets. What advantage do you see in the disposition of the rootlets in a regular order around the main root?

IO. Follow the behavior of the cotyledons, and try in this way to determine their functions. In your judgment, at what stage does the process of germination cease?

II. After two or more sets of leaves have developed, make drawings to show their position on the stem with reference to the next higher or lower set. What significance do you see in a regular arrangement of the leaves?

12. Examine the terminal bud of the seedling with a lens and determine what structures develop from it.

\section{Castor Bean.}

I3. Make drawings of the external appearance of a castor bean from the two most important points of view. Scale, $\times 2$. 
14. Carefully remove the shell or testa from a seed and note any structural details thus brought to light. Split the shelled seed longitudinally through its greater diameter, and make drawings of the internal structures. Split another shelled seed longitudinally through its lesser diameter, taking care to cut the structures already discovered exactly through the middle. Make a drawing from this point of view, sharply demarking the limits of the different structures. Make a cross section of a shelled seed a little below the middle, and draw the cut surface, bringing out clearly the outlines of the different parts. These drawings should all be made to the scale, $\times 3$.

\section{Germinating Castor Bean.}

15. Draw the castor bean in the first stages of germination, showing how the seedling protrudes through the shell. Scale, $\times 2$.

16. Remove the testa from a seed in the first stages of germination, and split it in halves longitudinally through the broad diameter. Draw from the point of view of the interior surface. Scale, $\times 3$. Split another seed in the same stage of germination longitudinally through the narrow diameter, taking care to halve all of the structures, and draw from the cut surface to the same scale.

17. Treat, in a similar manner, seedlings in later stages of germination, and call attention to any new structures which were not seen in the ungerminated seed. Follow the changes which the different parts undergo up to the stage where all reserve food materials have been used up.

18. In your notes, answer briefly the following questions: Where and how does the seedling crack the hard shell? How does the seedling get above the soil? How does the young plant get the food materials which are 
stored up for its use in germination?. How does it differ from the embryo of the Lima bean in this respect? Compare the means of protection possessed by the seed of the castor bean with that of the Lima bean.

\section{Indian Corn.}

19. Make drawings of the exterior appearance of a grain of Indian corn, from the two points of view which you consider the most important. Scale, $\times 2$.

20. Remove the skin from a soaked grain, and if any new structures are revealed, draw to the same scale. In this instance and in all grains the wall of the ovary in which the seed is formed constitutes a part of the skin.

2I. Carefully dissect out the central structures from a soaked grain and draw from the two most instructive points of view to the scale, $\times 3$.

22. With a sharp knife, make cross sections of the inner structures on either side of the center, about halfway toward each apex. Examine with a lens, and draw to a scale large enough to show all that the lens has revealed.

23. Halve a soaked grain longitudinally through the lesser diameter, making a sliding cut with a sharp knife so as to secure smooth surfaces. If the knife does not pass through the middle of the structures, carefully trim the larger piece until an exactly central section is secured. Draw the cut surface. Scale, $\times 3$. Place a drop of iodine solution on the cut surface, and after a minute draw it off with filter paper. The iodine will color the starchy parts of the seed a deep purple.

\section{Germinating Indian Corn.}

24. Make drawings of the external appearance of germinating Indian corn in successive stages of development. Scale, $\times$ I.5. 
25. Make a median longitudinal section through the lesser diameter of seedlings in various stages of germination, and draw from the point of view of the cut surfaces. The drawings should show definitely the changes which the several parts undergo as germination progresses.

26. Make cross sections of the stem of a well-advanced seedling, and drawings to show how the leaves are wrapped together, as shown by these sections.

27. What are the differences in the details of growth of Lima bean, castor bean, and Indian corn which result in the grain of corn remaining below the ground in germination, while, in the beans, the bulk of the seed is lifted above the ground? By what means does the corn seedling obtain the food which is stored in the seed?

28. After the instructor has pronounced the drawings of castor bean and Indian corn satisfactory, tint the different structures with the colored pencils, as in the case of the Lima bean, using the same color for the corresponding parts in all of the seeds and seedlings studied.

\section{General Comparisons.}

29. Make drawings on one page, for comparison, of the seeds of Lima bean, castor bean, and Indian corn, selecting the point of view which best shows the different structures, and with the colored pencils give the same colors to the corresponding parts.

30. In your notes briefly compare the corresponding parts of the seeds studied.

\section{Experiments.}

3I. With the sharp point of a penknife, scrape up a small portion from the cotyledon of a soaked Lima bean and mount in a drop of iodine solution (see page 387 ), on a glass slip, and cover with a coverglass. Examine with 
a compound microscope, using a $\frac{1}{4}$ or $\frac{1}{6}$ inch objective. Starch grains will be seen of various shades of purple, depending on the degree of action of the iodine; while other granules, whose substance, called proteid, is closely related chemically to the white of an egg, will appear from yellow to brown. These are the two chief classes of reserve food in the seed of Lima bean. The fact that starch is present in large quantities is manifest by the deep purple color which appears when a piece of cotyledon is placed in a drop of iodine. A compound microscope would therefore not be necessary to demonstrate the mere presence of starch.

32. Mount in a drop of iodine, as before, scrapings from the cut surface of soaked Indian corn, and note the presence of both starch and proteid.

33. Treat in a similar manner some scrapings from the reserve food materials of castor bean. The regular brown bodies are proteid, while the irregular yellow or brown bodies and circular masses of varying sizes are castor oil.

34. Boil in Fehling's solution (see page 385) in a test tube a dry crushed seed of Lima bean, and note whether the presence of glucose is demonstrated by the production of a red precipitate of cuprous oxide. Treat in a similar manner a seedling which is somewhat advanced in germination, and seeds and seedlings of castor bean and Indian corn. State in your notes your deductions as to the changes which the reserve materials in seeds undergo in the process of germination.

35. Place moist white pine sawdust to the depth of about an inch in four wide-mouth bottles. ${ }^{1}$

Put several grains of Indian corn that have lain in water over night into two of these. Cover the corn with about

${ }^{1}$ Such as No. $275^{\circ}$ in the catalogue of Whitall, Tatum, \& Co., Philadelphia. 
half an inch of the moist sawdust, and press down firmly. Cork the four bottles tightly (two containing the seeds in moist sawdust, and two containing sawdust only) and leave over night in a warm place. The following day fasten a piece of tallow icandle or wax taper to a wire handle; light the candle; remove the cork from one of the bottles containing sawdust only, and slowly lower the lighted candle into the bottle until it rests upon the sawdust. Leave the candle in this position for some time, and note whether there is a tendency for the flame to become extinguished. Remove the cork from one of the bottles containing the seeds and lower the lighted candle into it. If the seeds have been germinating properly, the flame will quickly expire. What do you conclude from these observations?

36. Prepare limewater as described on page 387 ; pour some of the clear liquid into a clean, wide-mouth bottle; then remove the cork from the remaining bottle of seeds, and, holding the mouth of the bottle close down over the mouth of the bottle of limewater, pour the gas from the bottle of seeds (as if pouring water) into the bottle of limewater. Cork the bottle of limewater tightly and shake vigorously. The white precipitate of calcium carbonate now appearing in the water has resulted from the reaction between the carbon dioxide gas from the bottle of seeds, and the calcium hydrate of the limewater. See whether the air in the second bottle containing sawdust only gives a like result. Shake up some limewater in a bottle containing only ordinary atmosphere, to see whether the precipitate is really produced by the gas poured from the bottle of seeds.

37. To demonstrate the identity between the gas given off by germinating seeds and a gas produced by a burning candle, or by breathing, perform the following experi- 
ments: Hold a lighted candle in the mouth of an inverted bottle until the candle is extinguished. Cork the bottle, set it right side up, and give the gas in it time to cool. Then pour limewater into the bottle and shake vigorously. The white precipitate will be produced as before. Blow the breath through a straw or glass tube into another bottle of limewater, holding the tube close to the bottom so that the breath will bubble through the limewater. After this process has continued for a short time the white precipitate of calcium carbonate will be observed.

38. It would seem from these results that a chemical process takes place in germinating seeds similar to that which occurs in our breathing, or in the burning of a candle. It will be interesting to note whether this process is associated with visible changes in the reserve food supply in the seed. Scrape a small portion from the reserve food of a soaked grain of corn, and mount under a coverglass in a drop of water; examine with a high power. Treat in a like manner some of the remnant of reserve material still remaining in the grain attached to a far-advanced seedling. The starch grains in the latter preparation show erosions like those shown in Fig. 3, page 2 I.

39. To estimate the value of the reserve materials in seeds to resumption of growth of the embryo, perform the following experiments: Plant seeds of Lima bean in sawdust, chopped sphagnum, or other suitable seed bed, and after the young plants appear, remove the cotyledons from some of them, and leave others in their normal condition. Compare the rate of growth of the two sets of seedlings. Soak grains of corn in water over night, and remove the outer food supply down to the fleshy cotyledon. Plant both depleted and normal grains in moist $\mathrm{s}$. and note their relative rates of growth. Try a similnr $\mathrm{ex}_{\mathrm{t}}$ mant 
with Lima beans, removing the cotyledons from soaked seeds, and planting the much diminished embryos, together with normal seeds, in moist sawdust. Make comparisons of the results of all of the experiments of this kind, and write out your conclusions in full. This experiment will be more certain to succeed if the sawdust has been boiled, to destroy moulds and bacteria.

40. Soak seeds of barley in water over night, and plant in moist sawdust in a wide-mouth bottle; place in the bottle a test tube containing a strong solution of pyrogallic acid and caustic potash; cork the bottle tightly by shoving the cork to a short distance below the rim of the bottle and filling in over the cork with melted sealing wax. Prepare another set of seeds in the same way, but with a potash solution only in the test tube. The pyrogallic acid in its alkaline solution absorbs the oxygen from the atmosphere in the bottle, while the caustic potash in both instances absorbs the carbon dioxide of the atmosphere, and that which is produced by the germination of the seeds; the conditions are, then, as follows: one bottle lacks oxygen and carbon dioxide, while the other lacks only the carbon dioxide. The experiment is designed to answer the question whether oxygen is necessary to germination. Let the experiment continue for several days, keeping the bottles in a dark and warm place. Record the results of your observations.

4I. Remove the glass front and the hands from a cheap alarm clock. Provide a soft pine block about an inch square, whittle one end to a taper, and drill a small hole into it, so that it will slip through the opening of the dial face and tightly over the hour-hand spindle. Fasten a Petri ${ }^{1}$ dish to 1 Vife outer face of the pine block by a melted

${ }^{1}$ See catalogues of dealers in bacteriological supplies. 
mixture of one third beeswax and two thirds rosin, taking care to center the dish with the hour-hand spindle. Pack moist pine sawdust into the dish, level with the surface, and press soaked grains of corn into the sawdust, not very tightly, broad face down, but do not cover them with the sawdust. Put on the cover of the Petri dish, and hold it in position by means of clips made of spring brass wire. (See Figs. 7 and 8.) Wind the clock and set it in its normal position; that is, with the hour-hand spindle horizontal. Prepare seeds in another dish in exactly the same manner, but fasten it so that it will stand vertically on its edge.

In the first experiment the directive effect of gravity will be neutralized by the revolution of the dish, while in the second, gravity may exercise its usual influence on the direction taken by root and shoot. Since the seeds are not covered by the sawdust, their progress in germination may be observed at any time without interrupting the experiment. The position occupied by the parts of the seedlings can easily be recorded for any period, by tracing with ink on the cover immediately over them.

\section{DISCUSSION.}

5. Nature and Purpose of Seeds. - A seed is essentially a young plant produced sexually by a flower (see page I68). The young plant has temporarily ceased to grow, and has been, or is to be, cast off from the parent plant, having first been provided with reserve food materials necessary to the resumption of growth, and with certain means of protection. The purpose of the seed is to insure the continuation, multiplication, and locomotion or distribution of the species. Many plants are too tender to survive the cold of winter, or the dry seasons of those regions where the rain does not fall for many months of 
the year. But the dry seeds of these plants can withstand great extremes of heat and cold, and do not need water to keep them alive; indeed, the ability of seeds to survive adverse seasons is due in large measure to the small amount of water which they contain. Since seeds as a rule retain their vitality for several years, in some cases, indeed, for twenty-five or even fifty years, they can, if necessary, tide a species over one or more years which are unfavorable to growth.

6. Multiplication by Seeds. - A single plant of Indian corn produces on the average about I 30 grains of corn, which, under the favorable conditions resulting from cultivation, might in the succeeding season give rise to 16,900 grains. An example of this kind will serve to demonstrate the immense capacity of multiplication by means of seeds; although under natural conditions only a small portion of the seeds produced ever result in mature plants.

7. Migration by Seeds. - Since land plants must draw their water and some other raw food materials from the soil, it is of great advantage, and even necessary, for them to be fixed in the soil by means of their roots. While the individual is thus anchored, the species is still able to move from place to place by means of the seeds. In this way, species have migrated through the long geological periods from regions which were becoming unhabitable to others which were more favorable; and by this means the borders of continents which are rising from the oceans, and newly formed volcanic or coral islands, become colonized by plants from greater or less distances. It is a matter of common observation that tracts of land which have been protected from grazing animals become inhabited in the course of a few years by plants which were never seen 
there before; and that treeless areas along the borders of streams soon become overgrown with dense groves of cottonwood and willow saplings after cattle are excluded, the seeds in some instances having come several miles from the homes of their ancestors.

8. Food and Protection. - In order fully to understand the significance of the various structures of a seed, we must keep in mind its functions of continuance, migration, and multiplication, and the conditions under which these functions must be performed. It is of great importance that the young plant in the seed be protected against unfriendly contingencies. We find that mechanical injuries are prevented either by the extreme hardness of the embryo and reserve food, as in the case of Lima bean and Indian corn; or, if the embryo and reserve food are oily and soft, by a covering of stony hardness, such as the castor bean and various nuts possess. The reserve food materials are packed tightly into the seed, and in a form which is, for the most part, insoluble in water, and on that account more certain of preservation within the seed.

In some seeds the reserve material is stored entirely within the embryo, as in Lima bean ; in others it is partly within and partly without the embryo, as in Indian corn; while in others, such as the castor bean, it lies wholly outside the embryo. But this variation in the location of the reserve food seems to have little significance so far as concerns germination, for in all cases it is finally transferred to the growing parts of the seedling; and whether this takes place before the seed is cast from the parent plant, or only during the stages of germination, is apparently indifferent to the well-being of the young plant. The facts of significance are, that the embryo plant is alive, although in a temporary state of inactivity; that it has a sufficient store of 
food materials locked in against loss; and that the plant is not inclined to spring into activity, nor are the reserve materials liable to become unlocked except in the right season and under the proper conditions for the establishment of the young plant as an independent individual,
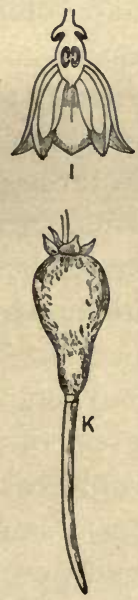

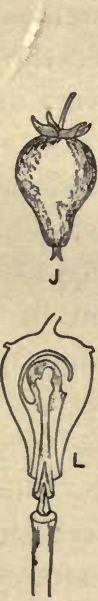

H

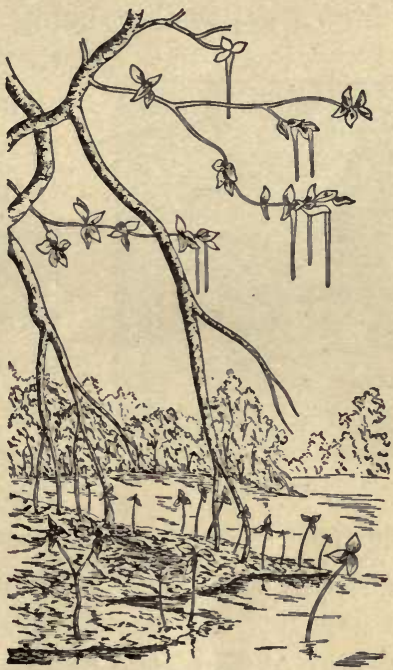

FIG. I.

Germination of the Mangrove. I, longitudinal section of the Mangrove flower; $\mathcal{F}$, the fruit; $K$, the seed germinating while yet contained in the fruit hanging to the tree (compare $H$ ) ; $L$, longitudinal section of the fruit showing the seedling separating from it; $H$, branch of the Mangrove with seedlings pendent from it and others that have fallen and taken root in the moist soil. After KERNER.

with its roots in the soil, and its leaves spread out in the sunlight and air.

9. Time of Germination. - Some seeds are capable of germination as soon as mature, and even before they are cast off from the parent plant. One may sometimes see wheat germinating in the standing ears before harvest. 
The mangrove is a notable example of a plant whose seeds habitually pass through the first stages of germination before falling from the tree (see Fig. I). In most cases, however, the seeds must go through a greater or less period of rest before they are in a condition for germination; and during this period of comparative inactivity, ferments are possibly being formed which are necessary

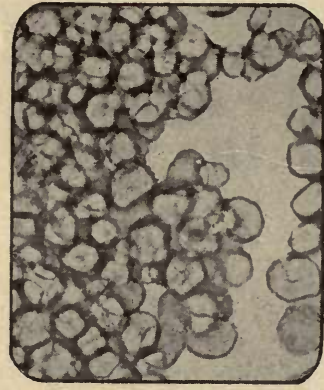

FIG. 2.

Photomicrograph of Starch Grains in a section of a grain of Indian corn. Highly magnified. to render the starches, oils, and some forms of proteid soluble in the cell sap.

10. Conditions Necessary to Germination.-Until the internal conditions are favorable through the formation of ferments, etc., the seed cannot germinate, but certain external conditions are also necessary to germination. These are the presence of oxygen, water, and a certain degree of heat. While some seeds have been known to germinate at temperatures quite close to the freezing point, most seeds germinate best between $16^{\circ}$ and $27^{\circ} \mathrm{C}$. $\left(60.8^{\circ}\right.$ and $80.6^{\circ} \mathrm{F}$.). This signifies that until a certain amount of energy in the form of heat is afforded to seeds from the outside, some of the necessary processes attending growth cannot be initiated. Neither can germination begin until the seed has absorbed sufficient water to stretch its tissues and act as the solvent for its reserve food materials. Some seeds are able to absorb water until their tissues are stretched by a force equal to about 200 pounds per square inch; and it is this stretching force which starts the increase in size of the embryo plant. If oxygen is excluded from seeds, they will 
not germinate, although all other conditions are favorable. The oxygen serves a double purpose in helping to form new soluble and diffusible compounds from the reserve materials, and in sustaining the respiration of the embryo as it becomes quickened into renewed growth. The necessity of oxygen to germination has been demonstrated by Experiment 40 , page 15 , but it is also frequently demonstrated in nature by the fact that most seeds will not germinate in a water-soaked soil ; notable exceptions are the seeds of some water plants, such as those of Nelumbo, which are able to obtain sufficient oxygen from the water.

\section{Digestive Ferments. - The} insoluble and poorly diffusible reserve materials are rendered soluble and diffusible by means of ferments present in the seeds.

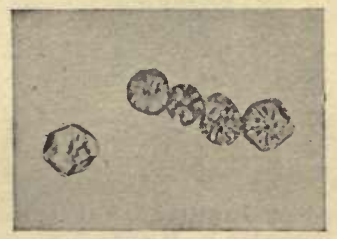

FIG. 3 .

Photomicrograph of Starch Grains from a grain of Indian corn in an advanced stage of germination.

The ferment which attacks starch is known as diastase. The result of its work can be seen by a comparison of Figs. 2 and 3 , which are photomicrographs of starch grains from ungerminated and germinating seeds of Indian corn. In Fig. 3 it is seen that the grains have been much eroded around the border and throughout their whole structure by the action of the diastase.

12. Circulation of Reserve Materials. - After the reserve materials have been rendered soluble in the cell sap, and diffusible through the cell membranes, they move from the cells in which they are stored, by the processes of diffusion (see page 36 ), to the growing regions of the embryo, there to be used in part in the building up of new tissues, and in part to be consumed by combustion or respiration. It is 
this process of respiration which consumes oxygen and gives off carbon dioxide (see Experiment 37), and in so doing

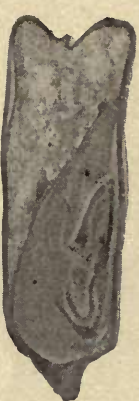

Fig. 4.

Median longitudinal section through a grain of Indian corn. The median diagonal line demarks the endosperm or reserve food of the upper half from the embryo occupying the lower half of the grain. The fleshy cotyledon constituting the larger part of the embryo surrounds the plumule and . hypocotyl above and below. Photomicrograph $\times 3$. makes active the internal energy necessary to life and growth.

In seeds having the reserve materials stored in the cotyledons, as in the Lima bean, the reserve materials need only to pass from these into the other parts of the embryo, leaving the cotyledons in a shrunken condition; but in the case of such seeds as those of corn (Fig. 4) and castor bean, where the reserve materials lie outside of the embryo for the greater part, at least, the cotyledons act as absorbing organs, and enlarge as germination proceeds so as to keep in close contact with the diminishing food supply. This is also well illustrated in the seed of the date, where the cotyledon is like that of the corn in serving chiefly as an absorbing organ.

In the seeds of the corn and date type the ferments lie partly within the cotyledon and partly within the cells which bear the food materials. As the reserve materials of the date seed, consisting chiefly of cellulose of bony hardness, become converted into sugar, they are absorbed by the cotyledon, which then enlarges and occupies the space thus vacated (see Fig. 5). In this way the cotyledon keeps in close contact with the reserve materials and transports them from the seed as fast as they are rendered soluble; 
a fact of great importance, since the action of the ferments is hindered or entirely prevented if their products are allowed to accumulate within their field of action.

A very notable example of the enlargement of the cotyledon while serving as an absorbing organ is seen in the cocoanut. Here the embryo is relatively small and lies embedded in the fleshy reserve food at the pointed end of the nut. As germination proceeds, a part of the cotyledon grows out through one of the three openings in the shell, and carries the plumule and hypocotyl out with it, while the greater part of the cotyledon remains within the seed, and as it absorbs the reserve materials it enlarges and fills the cavity (see Fig.6).

13. Direction of Growth. The root of the seedling grows downward into the soil, and the shoot (stem and leaves) upward into the sunlight and air, and it matters not in what direction the

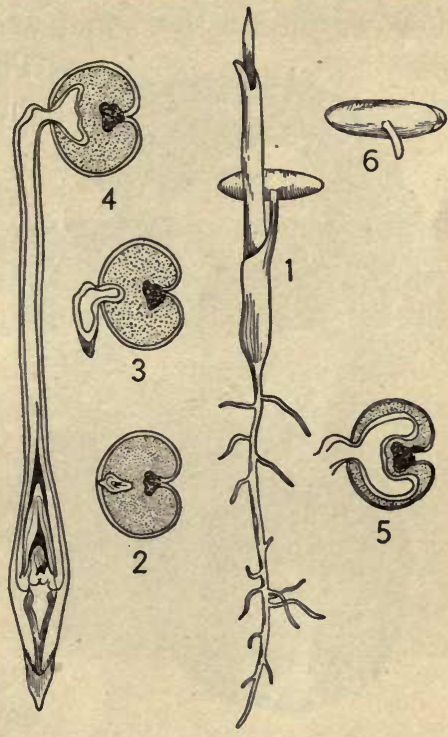

FIG. 5 .

Stages in the germination of a Date Seed: I, the young plant still attached to the seed; 2 , cross section through a seed showing the small embryo to the left embedded in the hard cellulose endosperm; 3 , cross section of a seed in an early stage of gernination; 4, section through a seedling in an advanced stage of germination. The cotyledon remains in the seed and enlarges as the endosperm is absorbed. The stem or petiole of the cotyledon depending from the seed enlarges at its base and covers the plumule. The tapering end below the plumule is the hypocotyl. 5, a later stage showing the endosperm nearly exhausted and the cotyledon filling the cavity; 6 , surface view of a seed in an early stage of germination. After SACHS. seed may be lying in the soil. The hypocotyl may be 
pointing upward, but as the root grows forth it turns sharply downward, and the shoot as it develops, sharply upward.

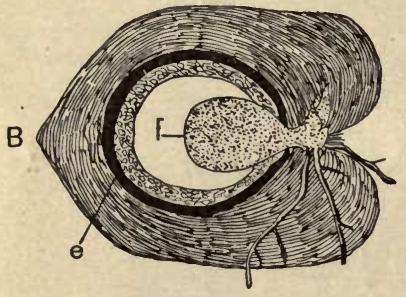

A

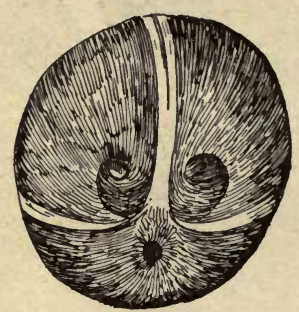

FIG. 6.

Fruit of the Cocoanut: $A$, end view of the "cocoanut" or stone of the fruit (corresponding to the stone of a peach), showing the dividing lines of the three carpels which compose the fruit. The embryo emerges through the lower opening. $B$, longitudinal section through the fruit of the cocoanut, showing the embryo in process of germination; $e$, the stone surrounding the fleshy endosperm; $f$, the enlarging cotyledon. The plumule is growing upward through the fibrous outer coat of the fruit. This coat is removed before the cocoanuts are marketed. After WARMING.
This is brought about by the influence of gravity.

In just what way gravity can exert such an influence is not known. We are accustomed to think of gravity as invariably attracting bodies toward the center of the earth, but on living and growing bodies it may exert an influence of quite another character. We might conclude, without an experiment, that gravity is the directive force; for whether seeds are germinating near the poles or at the equator, the roots always turn toward the center of the earth, and the shoots away from it. It is, however, a simple matter to eliminate the directive influence of gravity, and by observing the growth of seedlings under such circumstances to determine what effect gravity is producing under normal conditions. Figures 7 and 8 , illustrating the result of Experiment 4I, page I5, show how the influence of gravity may be demonstrated by removing it from one set of seedlings while it is still operative on another set. 
If seeds are planted in a pan of sawdust which is kept revolving rapidly in a horizontal plane, centrifugal force may be made to overcome gravity, so that the roots grow away from the axis of rotation and the shoots toward it (see Fig. 9).

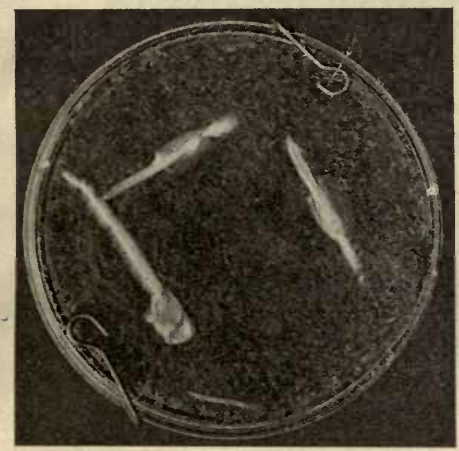

Fig. 7 .

Seedlings of Indian corn grown in sawdust in a Petri dish while revolving by clockworks one revolution per hour. The axis of revolution is horizontal, the plane of the dish vertical. Gravity as a directive agent is eliminated, and roots and shoots grow out in the direction in which they happen to be pointed.

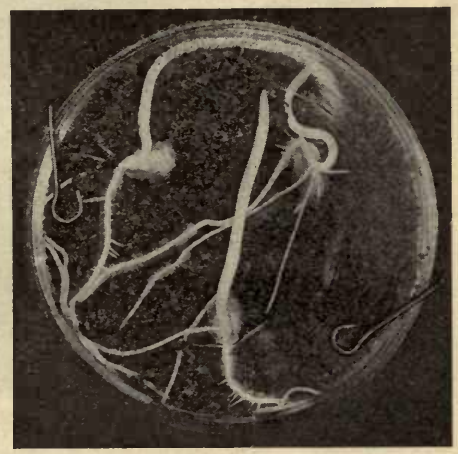

FIG. 8.

Seedlings of Indian corn grown in sawdust in a Petri dish which was kept stationary in a vertical plane in the position shown in the figure. Gravity is acting as a directive agent, and the roots find and take the downward and the shoots the upward direction, irrespective of the directions toward which they were originally pointing.

Whatever part plants, as living beings, have taken in the selection of gravity to direct their growth, a wonderful discrimination has been exercised; for, of the possibly available forces of nature, gravity is the only one which is practically constant in its strength, and in its direction of action, through all times of day and seasons, and in all positions over the earth's surface. The seedling can therefore depend with certainty on its root and shoot taking the right directions irrespective of the position in 
which the seed may be lying in the soil, or of the time or place of its germination.

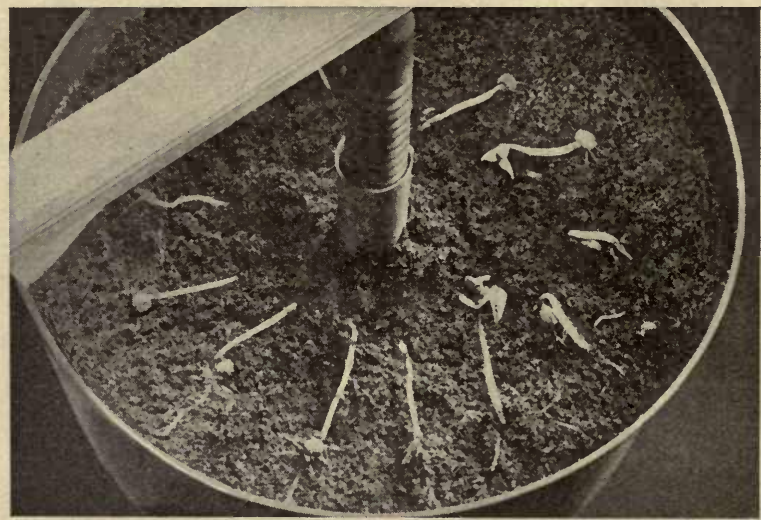

FIG. 9.

Seedlings of Indian corn, beans, and peas grown in moist sawdust in a pan I7 inches in diameter, which was kept revolving at the rate of 185 revolutions per minute. The seeds were planted beneath the surface, and the seedlings have been uncovered for the photograph.

14. Roots the First to Grow.- It has been noticed that the root first grows out and becomes established in close connection with the soil before the other parts of the embryo emerge above the surface. This insures that the seedling may not easily be dislodged from its position, and that the parts which are soon to be exposed to the drying influence of sun and winds may continually be supplied with water from the soil.

If a seedling is removed with care from a sandy loam, the soil will be found adhering to the roots in large, loose masses, and when the soil is carefully washed away in water, it will be seen to have been bound together by means of numerous fine hairs growing from the roots. It 
will be found very difficult to remove all of the particles from these hairs, so intimate is their union. The significance of this close relation will be discussed in another chapter.

15. Completion of Germination. - After the roots have become established in the soil, and the green leaves have unfolded to the sunlight, the young plant is in position to form its own food materials, and to be no longer dependent on food provided by the parent plant. The mother plant, however, often provides more than sufficient food to bring the offspring to a position of independence; for, in many instances, the reserve food is not exhausted until long after the leaves and roots are ready to take up their office of providing new food supplies. The reserve materials in the cotyledons of the oak, for example, do not become exhausted until the close of the second year's growth. The process of germination may be considered completed when the seedling is ready to provide for itself, for it would be manifestly incongruous to speak of the young oak at the beginning of the second year of its existence as still in the process of germination.

16. Size of Seeds. - The size of the seed appears to have little or no relation to the size of the parent plant. The cocoanut and cottonwood trees are both large trees when fully grown, yet the cocoanut, as we find it on our market, weighs about 750 grams (including the shell or stone, which is not a part of the seed), while a cottonwood seed as it floats from the tree weighs about $0.0015 \mathrm{gram}$. What the size and rate of growth of the plant shall be depends upon potentialities transmitted to the seed from the parent plant that are quite beyond our powers of observation. 


\section{CHAPTER III.}

\section{ROOTS.}

\section{OBSERVATIONS.}

42. Nearly fill two wide-mouth bottles with a soil composed of one third black loam, one third rotted manure, and one third sifted sand. In one bottle, plant a soaked seed of Indian corn, and in the other a soaked seed of Limia bean, and incline the bottles at an angle of $45^{\circ}$. Notice whether the roots take the same direction in both cases. If any of the roots reach the side of the bottle, note the behavior of their tips as they make their way between the soil particles. Study with a lens and note the relation of the root hairs to the soil particles. How close to the apex of the root, and how far back from the apex, do the root hairs grow?

43. Soak seeds of barley in water over night, and plant between pieces of moist carpet paper, or blotting paper, about three inches square. Prepare seeds thus for each student. Keep in a covered dish in a warm place, and do not allow the paper to become dry. After the roots have grown out for an inch or more make drawings to show the root hairs.

44. Place a piece of polished marble at the bottom of a flower pot, or, instead of the marble, a clam or oyster shell with the concave side up. Nearly fill the pot with the soil mixture above described, and plant in it a few seeds of soaked Indian corn. After a few weeks, if the corn has 
made a good growth, remove the soil from the pot and note the effect of the roots on the surface of the marble or shell. Does this teach anything as to the possible effect of the roots on the limestone constituents of the soil ?

45. To demonstrate the force with which roots absorb water from the soil, cut a groove in the form of a circle about two centimeters in diameter, by means of the edge of a three-cornered file, at the large end of a hen's egg. Carefully remove the shell within the circle, guarding against puncturing the delicate skin. File with the flat face of the file at the small end of the egg until a thin area about four millimeters in diameter has been produced, make a small hole in the shell at the thin place, and blow out the contents by means of a glass tube drawn out in a flame to a fine point. In this operation the tube must not entirely close the opening in the shell. Set the egg, large end down, in the mouth of a wide-mouth bottle which has been filled with water, and fill the egg with thin sirup colored with an aniline dye. A test tube drawn out in a flame to a fine tube is an excellent funnel for this purpose. Hold a piece of small glass tube, about one meter long, upright against the upper end of the egg and over the hole, and fasten it firmly in position, and water tight, by means of melted sealing wax. Keep the bottle filled with water, and watch the progress of the experiment. In absorbing water from the bottle the artificial cell formed by the egg and sirup acts practically in the same manner as the root hairs in absorbing water from the soil.

46. Make a cross section of a root of corn or bean and treat with phloroglucin (see page 387 ). The elements which are colored red are the water-conducting elements into which the water passes from the root hairs. They are in reality long tubes, formed by the fusion of elongated 
cells, end to end, which extend continuously through the stem into the leaves, where they branch and help to form the veins and veinlets. Make similar sections of a root, not more than three millimeters in diameter, of some woody plant, and treat with phloroglucin as before. The elements, which in this instance are colored red, consist of wood fibers in addition to the water-conducting tubes. The larger openings of the latter can easily be seen by means of a simple lens.

47. Make a cross section of a small sweet potato, which is in reality a root, and treat with the iodine solution. The section is stained purple, because it is filled with starch which supplies with food the young shoots that spring adventitiously from the root. Plant some sweet potatoes in moist sand and keep in a warm place. As the shoots develop, what change is noticed in the size of the root?

48. Make cross sections of dodder, which is parasitic on balsam, stinging nettle, or some other herbaceous plant. This material gives best results if it is taken in a young and tender condition and placed in $70 \%$ alcohol for a time, and afterwards preserved in equal parts of alcohol, glycerine, and water. Or it may be kept from the first in a $2 \%$ formalin solution. Select a section showing the penetration of the roots of the dodder into the host plant. Treat first with phloroglucin and then mount in chlor-zinc-iodide (see page $38 \mathrm{I}$ ), or mount in the latter reagent alone, and examine with the compound microscope. An examination with a simple lens even will give a fairly good idea of the intimate relation between the parasite and its host.

49. Take germinating seeds of Indian corn whose primary roots are about one centimeter long, and with waterproof India ink make marks on the roots one millimeter apart, beginning at the apices. On other roots 
make heavy, continuous lines running their full length. Place the seedlings in moist sawdust, and after a day notice whether the marks have separated at one portion more than at another, and whether the continuous mark has become broken at one portion more than at another. What do these experiments teach as to the regions of greatest elongation in roots?

50. Examine the roots of trumpet creeper, which are growing into some support. Do they appear to arise at a definite place on the stem? Do they grow directly toward the support, or do they seem to have been uncertain as to the proper direction to take? How deep do the roots penetrate into the support? If growing into a tree, do the roots seem to have penetrated to a sufficient depth to take sap from the tree? The material for this study can be secured at any time during the year. It is a good plan, however, to gather it during the growing season and keep it in jars of $2 \%$ formalin.

$5 \mathrm{I}$. Early in the spring, when the buds begin to swell, cut off a grape vine about six inches from the ground, and attach a long glass tube to the stump by means of a short piece of rubber tube. Tie the glass tube to a support so that it is held vertically. Keep note of the rapidity of the rise of sap in the tube.

52. Cut off a small branch of willow and place it in a bottle of water. Set the bottle in a warm place and keep the water replenished. Note from time to time whether roots are forming in or above the water, and if so whether they are formed in definite order.

\section{DISCUSSION.}

17. Functions of Roots. The roots. of plants have to perform the functions of fixation, mechanical support, ab- 
sorption and conduction of fluids, and storage. In following the development of the seedling, we have noticed that its first efforts are directed toward the formation of its root system. It would be hazardous for seedlings to develop parts above the ground before an anchorage has been made in the soil, for in that case the young plant could easily be torn or washed from its position by storms, and death would likely result from lack of water if the leaves

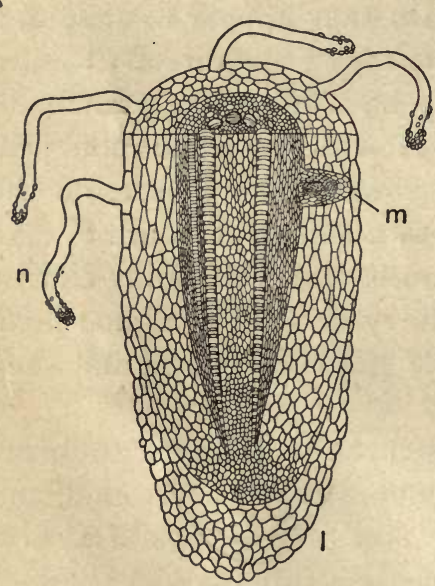

FIG. Io.

Diagrammatic representation of a cross and longitudinal section of a Root Tip. $n$, a root hair; $m$, a young lateral root; $l$, the root cap. Tracheal tubes are shown near the center in both cross and longitudinal section. Particles of soil are represented clinging to the root hairs. were spread out above the ground before connection had been established with the water of the soil.

18. Growth of the Root.As shown by Experiments 4I and 49, the root, elongating only near the apex, is directed downward by gravity. The delicate root apex, of course, meets with obstructions, but it is protected by a cushion of cells known as the root cap (see Fig. IO). Being in a state of growth, it is quickly responsive to its surroundings, and, turning aside when it meets with obstructions, it passes along the course of least resistance. While the root is thus threading its way among the soil particles, hairs are being formed on it, always a short distance back of the apex, keeping pace with it as it advances in growth and gradually dying off on the older portions. Thus the root hairs, which are the main absorb- 
ing portions of the root, are continually brought into new parts of the soil, where fresh supplies of materials, suitable for forming the food of the plant, are to be obtained.

19. Importance of Root Hairs. - The root hairs are organs of much importance, since they greatly increase the anchoring strength of the root and furnish an increased surface for the absorption of water and other substances from the soil. (See photomicrograph of root and root hairs of barley, Fig. I I.) It has been estimated that the hairs on corn roots, for instance, increase the absorbing surface about twelve times. When plants are transplanted during the growing season, after the leaves have been formed, they are quite certain to wilt, because the newer rootlets with their root hairs are broken off, even when the greatest care is exercised. The best time to transplant is, therefore, in the

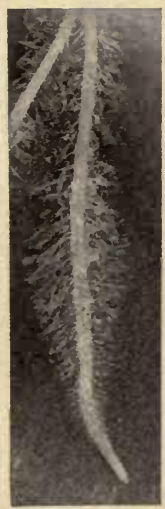

FIG. II.

Photomicrograph of a root of Barley, showing root hairs forming near the apex and dying away behind. $\times 3$. fall, after the leaves have dropped off, or in the spring, before the new growth begins.

20. The Nature of the Soil. - The value of a wide distribution of the roots in the soil lies in the fact that, aside from the benefits of anchorage, plants must take from the soil, water and certain other substances without which they could not live. The soil is therefore a subject of great interest in connection with the study of plants. There are various kinds of soils, but it may be stated in general that ordinary tillable soil consists of particles of rocks in various degrees of disintegration, intermixed with vegetable 
and animal remains which, on account of the large percentage of carbon contained in them, impart to the soil its dark color.

The process of soil formation from the disintegration of rocks can be seen to advantage in any abandoned quarry. The newly uncovered rocks are hard throughout, but those which have lain for some time exposed to the weather become so soft at the surface that they may easily be scratched, or a considerable amount of material may be scraped from them by the finger nail. After longer exposure, and particularly after the water imbibed by them has been frozen, the rocks begin to crumbleinto pieces of various degrees of fineness.

In whatever way rocks become broken down - whether by the solvent effect of water, the expansive force of freezing water, or the beating of storms; by abrasion when carried along by torrents, or when hurled to and fro by the surf, or when ground as in a mill by glaciers - the accumulated particles in time form a soil for the growth of plants. But long before the new soil is occupied by the higher plants it becomes the home of myriads of microscopic forms whose remains contribute to its richness and put it in a physical condition better adapted to the reception of the larger and more exacting plants.

21. Soil a Reservoir for Water. - The capacity of the soil to hold water is dependent on the fineness of its particles; for the finer the particles, the greater the number of small capillary spaces and the larger the surface exposed for holding water by adhesion. To take a concrete example: A cubic foot of round soil particles having a diameter of one inch would expose a total surface of 37.7 square feet, while a cubic foot of such particles one one-thousandth of an inch in diameter would present an aggregate surface 
of 37,700 square feet. The greater surface presented by the smaller particles is of further advantage in giving the water increased opportunity to dissolve out from them certain substances necessary to the food of plants.

22. Action of Root Hairs. - The root hairs place themselves in close contact with the soil particles, and conform to their irregularities of surface so completely as to embed and hold them fast. This accounts for the difficulty of washing away the soil from the roots without breaking off the hairs. The close relation of the root hairs to the soil is of great importance, for long after the capillary spaces have been emptied of their water by evaporation, the soil particles still retain a film of water about them from which the root hairs are able to draw supplies for the plant, even when the soil appears dry.

A better comprehension of the absorptive action of the root hairs will be obtained after an examination of their structure and the circumstances governing their ac-

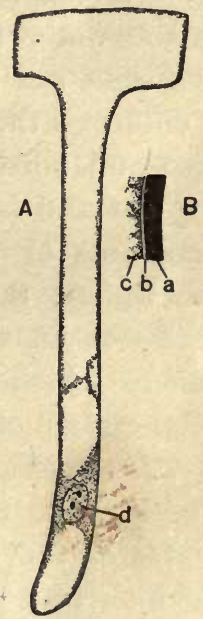

FIG. I2.

$A$, diagrammatic representation of a Root Hair; $B$, a more highly magnified detail, $a$ being the outer cellulose wall, $b$ the plasma membrane, and $c$ the cytoplasm. $d$ is the nucleus suspended in the cytoplasm. tion. They are really greatly elongated outer or epidermal cells of the roots (see Figs. I I and I2). Their outer wall, $a$ (Fig. 12, $A$ and $B$ ), is quite thin, and composed of cellulose, a substance readily permeable to water. Within the cell wall is the live part of the cell known as the protoplast (all of the granular part in $A$ ), consisting of the outer lin- 
ing membrane, $b$, called the plasma membrane, a specialized part of the cytoplasm; the cytoplasm, $c$; and the mucleus, $d$. Plasma membrane, cytoplasm, and nucleus are alive, while the cell sap, which occupies the remainder of the cell cavity (all of the clear space within $A$ ), and the cell wall are not endowed with life. The plasma membrane is readily permeable to water, but not to all substances which the water may contain in solution. Its chief service consists, not so much in keeping certain substances from entering the plant, as in prohibiting the valuable cell sap and portions of the living body of the cell from passing out and becoming lost to the plant. Thus, while vast amounts of water with substances in solution pass into the plant through the root hairs, only very small quantities of materials, useful in rendering soluble those substances which the plant needs, are permitted to pass out by the same channels.

23. The Process of Absorption. - The process of the passage into the root hairs of the substances dissolved in the water of the soil is known as diffusion. The initial force which causes this probably results from the energy of motion of the molecures and ions of the diffusing substance. Those molecules and ions which possess the greatest energy of motion, or whose size and shape best conform to the intermolecular spaces of the membrane, will traverse the membrane most rapidly. When the molecules and ions of a substance in solution are in equal concentration, that is, are in equal number per unit of volume on both sides of the membrane, and their temperature is the same, the number of them passing the membrane in both directions per unit of time will be the same. This is a state of equilibrium which can only occur between the soil and the root hairs in the case of those substances which 
are not being withdrawn from solution within the plant, or are not being used by the plant in the manufacture of new compounds. The more a substance is being employed or transformed by a plant, so that its concentration is continually diminished, the more it will enter from without; in this way the supply is adjusted to the demand.

If, on the other hand, a substance which is able to pass the plasma membrane is not being transformed by the plant, it cannot continue to enter after the concentration of its molecules and ions within the plant is equal to that in the soil water; in this way useless materials are kept from accumulating. This is true of the substances in solution, but the solvent, which in this case is water, passes most rapidly from the region of less to that of greater concentration (see Experiment 45). The passage of water through membranes from regions of lower to those of higher concentration is known as osmosis. The cell sap of the root hairs is of greater concentration than the soil water; and since this condition is maintained by evaporation from the leaves and other above-ground parts, and by the employment of some of the water in the manufacture of plant food, the water continues to enter the plant from the soil. If the water is abundant, it may enter the plant even faster than it is evaporated or used, in which case the plant cells become stretched and turgid. In this way rigidity is given to herbaceous stems and leaves. But when the water in the soil runs low, evaporation may be in excess of its movement into the plant, and wilting results.

24. Importance of Water. - Water, which is taken by land plants almost exclusively from the soil, is the solvent and vehicle of transport for all substances which enter the plant, and for those compounds as well which are manufactured within the plant; it contributes its own substance 
for the manufacture of plant food, and it further serves the plant in affording strength and rigidity to the tender herbaceous parts.

25. Elements Necessary to Plants. - There are certain chemical elements necessary to the nutrition of plants,

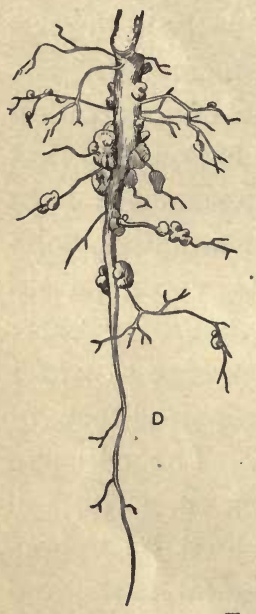

E

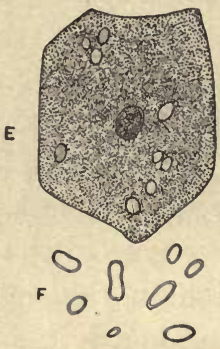

FIG. 13.

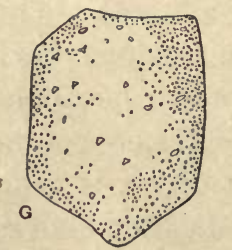

$D$, root of a leguminous plant bearing Tubercles; $E$, a cell from a tubercle containing bacteria, highly magnified; $F$, some of the bacteria more highly magnified; $G$, a cell from a tubercle after the bacteria have, in part, evidently been absorbed by the plant. After FrANK. which must be taken from the soil particles; these are calcium, magnesium, potassium, sulphur, phosphorus, and iron. Nitrogen is taken in part from the compounds of nitrogen in the.soil, and in part indirectly from the free nitrogen of the atmosphere by means of $\mathrm{mi}$ croscopic organisms which reside chiefly in the root tubercles of leguminous plants (see Fig. I3). Were it not for the fact that these elements, for the most part, are in the form of compounds insoluble in water, they would soon be washed away by the percolating water after heavy rains. They are, however, slowly rendered soluble by acids excreted by the root hairs (see Experiment $44)$, by carbon dioxide dissolved in the soil water, and by the oxygen of the soil atmosphere. It must be remembered that there are no openings in the root hairs, and only substances in solution in water can be absorbed by them. 
26. Free Nitrogen made Available. - Although about seventy-nine parts in one hundred of the atmosphere consist of free nitrogen, plants, with the exception of certain microscopic forms, are not able to use it for food until it has been combined with other elements to form some soluble compound, such as nitrates and ammonia. These are obtained largely from the decomposing remains of plants and animals. It has long been known that clover, when plowed under, leaves the soil much richer in nitrogen, and the reason for this is now well understood. There are bacteria residing in the tubercles of the clover roots (see Fig. I3), and they in some way combine the free nitrogen of the atmosphere with the other necessary food constituents provided by the clover plant, and use the substances thus formed as food. After a time the bacteria become disintegrated, and are apparently absorbed by the clover, which, in this roundabout way, obtains the nitrogen after it has been combined with other substances to form proteids. Finally, the clover decays and yields its combined nitrogen to the soil. By this remarkable coöperation of two widely different kinds of plant life the free nitrogen of the atmosphere is made available to all kinds of plants.

27. Extent of Roots. - The roots with their rootlets and root hairs form a dense plexus threading the soil in all directions. Being buried in the soil, their great extent is not easily apprehended. It has been estimated that if all the roots and rootlets of a single corn plant grown under good field conditions were placed end to end they would cover a linear mile. The roots of some plants extend to great depths, and these plants are thus able to obtain water and continue fresh and green after the surface soil has become dry and plants with shallow roots have withered away. The roots of alfalfa, for instance, sometimes penetrate the 
soil to a depth of from ten to twenty feet, this habit making it valuable for hay and pasturage in regions of scanty rainfall.

28. Path of Absorbed Substances. - The water and substances in solution pass from the root hairs toward the center of the root, where they enter tubes (see Figs. Io and 42) which conduct them through the stem into the leaves. The osmotic force in the root hairs is sufficient to lift the water in the stem to a considerable height (compare Experiment 5I), but this force is not of itself sufficient to carry the water up rapidly enough to supply the evaporation from the leaves, nor high enough to reach the tops of

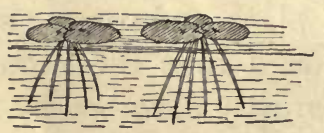

FIG. I4.

tall trees. Figure ro shows the relation between the root hairs and the water-conducting tubes (called tracheal tubes because of their resemblance in appearance to the trachea or windpipe).

29. Roots of Water Plants. The roots of water plants are much less extensive than those of land plants. In the case of such plants as Lemna and Spirodela (see Fig. 14), which float upon the water, the roots are few, short, and unbranched, and destitute of hairs. Such plants do not need an elaborate root system, since water and food substances dissolved in it are available without stint at all times.

30. Roots of Parasitic Plants. - Some plants have developed parasitic habits and attach themselves to other plants by means of their roots, having no direct connection with the soil, but depending upon their host plant for the water and other necessary food materials. If the parasite 
has no green leaves, as in the case of dodder (see Fig. I 5), it must depend upon its host for all kinds of food materials, - for the starches, sugars, oils, and proteids manufactured by its host. It is, in other words, a complete parasite. But if, as in the case of the mistletoe, it has green leaves of its own, it is entirely dependent on its host for the water and dissolved soil materials only, and is then but partly parasitic.

31. Roots of Air Plants. - The aërial roots of some tropical orchids and of other aërial plants (see Fig. I6) do not become embedded in a substratum, but grow free in the air, and they must, therefore, be able to absorb rapidly the water

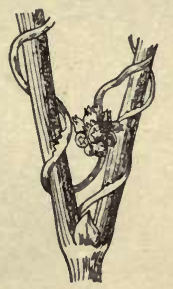

A

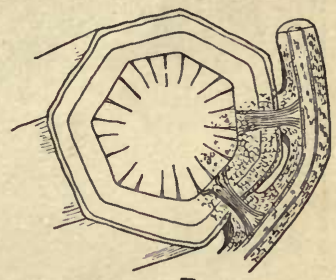

B
FIG. I5.

A, Cuscuta Europaea, or Dodder, twining about and parasitic on a hop vine and bearing a cluster of small flowers.

$B$, diagrammatic drawing of a cross section of a hop vine through the plane where the roots of the dodder enter it and penetrate to its vascular bundles. $\times$ ro. After KERNER.

which falls or gathers on them from the rain or dew. To accomplish this the outer layers of the cells of the roots are empty and their walls are perforated by minute openings through which the water can be drawn by capillarity. It may be that the water vapor of the atmosphere is condensed within these cells, but experiments on this subject have given contradictory results.

32. Prop Roots. - The prop roots growing at the basal nodes of Indian corn, and the famous prop roots of the banyan tree, grow downward to or into the soil. Supported in this way, the banyan tree is able to spread its branches over an area so large as to give shelter to an entire .village. 


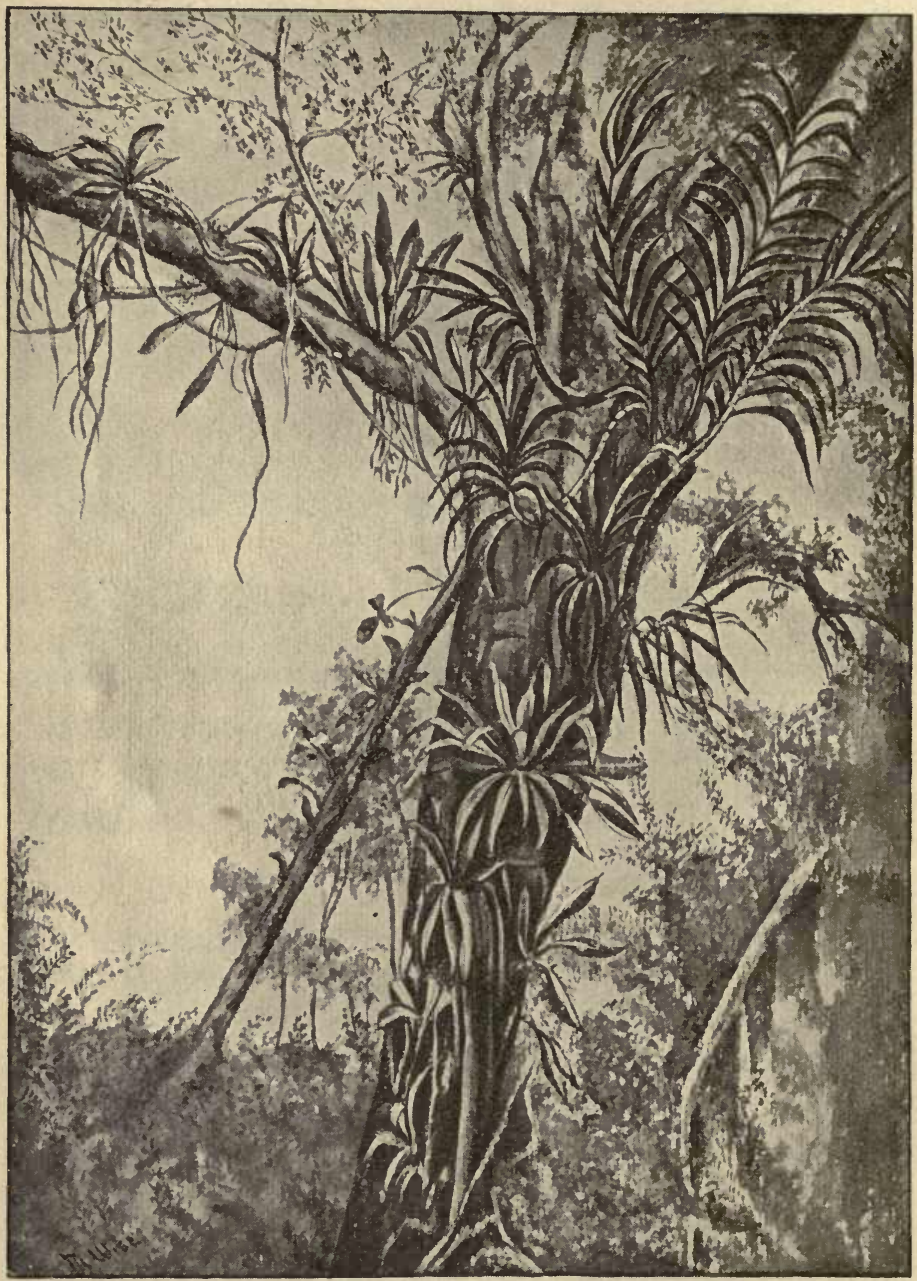

FIG. I6.

Aerophytes growing on the trunk and branches of a tree. Aërial roots seen pendent from the branch on the left. Drawn from data in Schimper's Pflanzengeographie. 
33. Clinging Roots. - The roots growing on the stems of the poison ivy and trumpet creeper (Fig. I7), for instance, do not serve an absorbing function, but are merely employed in holding the slender stems upright against a support. If these plants are growing near a tree or wall they find themselves shaded on one side, and their roots grow away from the side of greater illumination and toward the object which is shading them; in this way they are quite certain to find a suitable support.

\section{The Various Directive Forces.-}

Thus we see that roots have quite diverse functions to perform and that they show a marvelous capacity for employing various forces to direct them in their growth. If they are to grow into the soil, gravity is chosen as a guide; if into the body of some host, as in the case of dodder, the stimulus of contact is selected; if toward some object of support, either

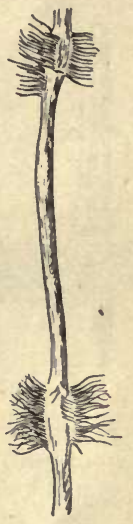

FiG. I7.

Portion of a stem of the Trumpet Creeper, showing its clinging roots. light or gravity is chosen, as is most practicable. Even to any given force the various parts of the root system may react differently; the main roots growing in the soil are directed more or less downward; the lateral roots springing from these make greater or less angles with the line of gravity; while the ultimate branches may grow in any direction, apparently without respect to gravity. In this way all parts of the soil within the range of the roots are fully occupied, which would not be the case if the entire root system were impelled by gravity in one direction. The water in the soil, also, has a directive influence on the 
growth of the roots; if the water is distributed evenly, the roots develop evenly on all sides, but if the conditions are otherwise the roots tend to follow the direction of the greater water supply.

35. Adventitious Roots. - We have noticed in the growth of seedlings that the first rootlets spring from the primary

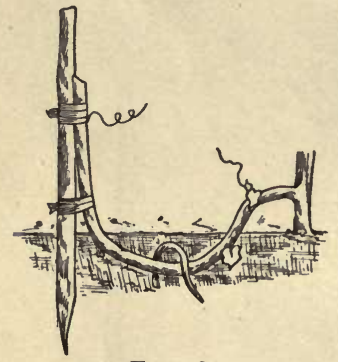

FIG. 18.

Showing the method of Layering. A branch is bent and pegged down and covered with soil. After adventitious roots have formed on the branch it is severed from its parent stock. After BARRY. root at definite angles of divergence, but that on the stems of ivy and Cuscuta their places of origin are indefinite, - in other words, these roots seem to arise adventitiously. When willow stems are cut off and placed in water, roots are formed in the same manner not far above the cut surface. The ability of plants to form adventitious roots is employed by horticulturists and florists in the propagation of many kinds of plants by the processes known as layering and cuttage. In this way may be propagated currants, gooseberries, raspberries, grapes, roses, azaleas, fuchsias, etc. (See Fig. I 8.)

36. Roots Defined. - Although roots are called upon to perform various functions, and may be modified in form accordingly, they still have certain characteristics which distinguish them from other plant members. They are members of the plant body of indefinite elongation, protected by a root-cap; and they never directly bear leaves, although capable of producing adventitious buds. 


\section{CHAPTER IV.}

\section{BUDS AND STEMS.}

\section{PROVIDING MATERIALS.}

Most of the material required in the study of buds and stems can be procured out of doors at any time before growth begins in the spring. If the study is to be taken up later in the season, twigs of horse chestnut, cottonwood, and lilac with winter buds should be secured and kept in $2 \%$ formalin until needed. In those schools where the study of botany is begun immediately after the Christmas vacation, strong shoots of the above plants should be cut off and placed in jars of water at the beginning of the term, and kept in a warm place, in order that their buds may be unfolded by the time the study of buds in their winter condition has been completed. The twigs may be crowded into the jars quite closely, and there should be several twigs of each kind for every student. The water in the jars should be frequently changed.

If Aristolochia is not at hand, it can be obtained of dealers in botanical supplies. ${ }^{1}$ Branches of Tilia (linden) can be used to good advantage for sectioning if Aristolochia cannot be obtained, and branches of elm might be used, although less advantageously. Aristolochia, however, is the best for this study. These branches and small stems of Indian corn should be taken during the growing season and placed for a week or so in $70 \%$ alcohol, and then kept in equal parts of alcohol, glycerine, and water until needed. This method of treatment makes it easier to cut good sections.

\section{OBSERVATIONS.}

53. Make drawings of a twig of horse chestnut in its winter condition, showing the relative size and position of all exterior structures. Where did last year's leaves grow on the twig?

1 Cambridge Botanical Supply Co., Cambridge, Mass. 
54. Make drawings, on a larger scale, of a terminal bud, a lateral bud, and any structures related to them. What is the relation as to position between the leaves and buds of the twig?

55. Select one of the largest buds, and pick off the bud scales carefully, so as not to injure them. Arrange them in separate groups in the order in which they were removed, each group being composed of the scales which encircle the stem once in the successive spirals, or whorls. Draw a typical scale from each of the groups. When the scales are all removed, draw the inner structures which were protected by them (scale, $\times 5$ ); first, as they stand in their natural position on the stem, and second, when removed and laid out for examination separately. If the parts are folded together make a drawing to show the manner of folding, and then spread one of them out and draw in the expanded position. Use the lens for this work, and draw in the details which can be seen with it.

56. Make cross sections of a bud, beginning near the apex and sectioning in successively lower planes until the parts protected by the scales are seen to best advantage, and then draw to the scale $\times 2.5$. Identify the different parts of the sections by the aid of the dissections already made.

57. Pay particular attention to the protection afforded the tender inner parts of the bud by means of the scales or other structures or devices. The inner parts need protection against drying, sudden freezing and thawing, attacks of parasites of various kinds, and mechanical injuries due to the beating of storms and abrasion from other causes. In what ways are these different sources of danger guarded against?

58. Make a median longitudinal section through one of 
the largest buds, and draw the cut surface. The drawing should show the outline of all the parts with perfect clearness. Do not make any lines which have no distinct significance.

59. What portion of this twig was formed last year? What portion was formed the year before? Is there any portion of the coming season's shoot present in this twig? Label the drawing of the twig according to the different years' growth, and determine by cross sections whether there is any relation between the internal structure and the age of the parts of the twig.

6o. Make drawings of buds in different stages of unfolding so that the following questions may be answered by reference to the drawings: What changes do the bud scales undergo? What finally becomes of the bud scales? Do the bud scales in any way leave their impress upon the twig? What changes do the inner parts of the bud undergo? Do the parts increase in size? in number? Do they change materially in form? Are their relative positions changed? Are any new parts produced, or does the unfolding of the bud consist simply in changes in parts already present?

6I. Turn back to twigs in winter condition and determine the location of the bud scales of last year, and of the year before that, etc., and see that your drawing takes proper account of them.

62. Count the leaf scars belonging to the different years' growth to determine whether the number of leaves produced each year is the same.

63. Determine the number of vertical rows of leaf scars, and the angular divergence of the leaves on various twigs, selecting those which have made a vigorous growth and are straight and untwisted. A good way to determine 
the number of vertical rows is to stick pins or dissecting needles into each leaf scar perpendicular to the tangent at that point, passing spirally up the stem, omitting none of the scars, until the scar is reached which stands immediately above the initial scar, that is, the one with which the start was made. When the twig is now held in front of the observer, parallel to the line of vision, the number of vertical rows of leaf scars may easily be counted and the angular divergence of the rows determined. The angular divergence of a leaf from the one next above or below it is now to be determined.

Suppose that in counting the scars it is found that in passing once around the stem the fifth scar is immediately above the initial scar; then it is plain that the angular divergence of the leaves from each other is one fifth of $360^{\circ}$, or $72^{\circ}$. But suppose the five leaves are distributed over two turns of the stem; then it is evident that they are twice as far apart as in the first case, that is, their angular divergence is now twice $72^{\circ}$, or $144^{\circ}$. If examples of this sort were multiplied, it would be seen that the angular divergence of the leaves would be that fraction of $360^{\circ}$ whose numerator is the number of times the circumference of the stem is passed over, and whose denominator is the number of intervals traversed in passing spirally from the initial scar to the one directly above it. Of what use is a definite angular divergence of the leaves?

64. Study in like manner cottonwood and lilac.

65. Make thin cross sections of a one-year-old stem of Aristolochia, and treat with phloroglucin and chlor-zinciodide, or double stain with cyanin and erythrosin (see page 384 ). Examine with a simple lens or with the low power of the compound microscope. On the outside is the epidermis, a (Fig. 19). Within this is a zone of tissues 
known as the primary cortex, extending to and including the row of cells, $b$, which is termed the starch sheath or endodermis. The groups of tissues within the endodermis make up the central cylinder or stele. The groups of tissues, $d$, which are disposed in the form of an interrupted concentric zone, are known as the vascular bundles. The tissues between the endodermis and the vascular bundles constitute the pericycle, $c$. The tissue surrounded by the vascular bundles is termed the pith,e. The tissue connecting the pith with the pericycle, and accordingly running radially between the vascular bundles, is termed the medullary rays, $f$.

In the sections of Aristolochia which are treated with phloroglucin and chlor-zinc-

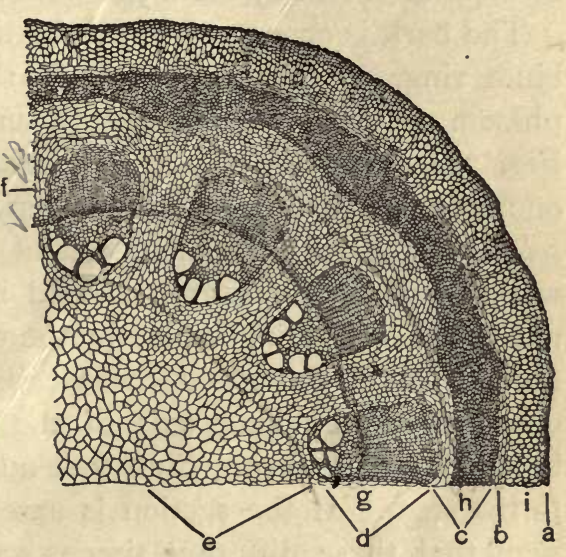

FIG. I9.

Cross section of a one-year-old stem of Aristolochia. $a$, epidermis; $b$, endodermis; $c$, pericycle; $d$, vascular bundle; $e$, pith; $g$, cambium; $h$, sclerenchyma ring of the pericycle; $i$, collenchyma; $f$, medullary ray. iodide, it is seen that the vascular bundles consist of two distinct parts - an inner part which is colored red by the phloroglucin, and possesses relatively large openings, and an outer part which is colored purple by the chlor-zinc-iodide, and whose cells are relatively small. The inner part, which is known as the xylem, is the water-conducting part of the bundle; while the outer part, termed the ploloem, is the food-conducting part. The small, brick-shaped, thin-walled cells, $g$, be- 
tween the phloëm and xylem are the cambium cells, which, during the growing season, rapidly divide and form new cells that finally become altered into the tubes, etc., of the xylem and phloëm. As the stems grow older, cambium is formed between the bundles, and thus is produced a complete ring of delicate cells which permits the bark to be separated readily from the wood.

The bark is composed of all the tissues outside the cambium ring, so that when the bark is stripped off the phloëm portion of the vascular bundles goes with it (see Fig. 21). Turning again to the section of Aristolochia, one sees that a portion of the pericycle $(h$, known as the sclerenchyma ring) is composed of cells which are thickwalled and lignified, as indicated by their being stained red by phloroglucin, or blue by the cyanin. A longitudinal section would show that these cells are elongated in the direction of the long axis of the stem and are closely bound together, being thus well adapted to give strength to the stem. If the section is carefully studied, it will be seen that these different tissues are not separated from each other by lines, such as the beginner might be tempted to use in drawing them, but that they owe their demarcation to the fact that the cells composing them differ in form and size, in the character of their contents, and in the thickness and kind of their walls.

The tissues are adapted to perform various functions by their different characters and relative positions. The epidermis has an outer wall which is infiltrated with waxy substances and is thus adapted to prevent the inner tissues from drying up; the epidermis has, therefore, a protective function. The thick-walled cells of the outer part of the primary cortex ( $i$, known as the collenchyma) are chiefly for giving strength. The inner cells of the primary cortex 
are thin-walled, and the walls are made of cellulose, a substance which is easily permeable to water and substances in solution. These cells are therefore fitted for the storage and slow conduction of materials; and since they lie near the periphery and are accessible to light, they contain the same kind of green chloroplasts which reside in the leaves, and use the energy of the light in manufacturing food materials (see page 87 ). The outer portion of the pericycle, on account of the thickness and lignification of its cell walls and of the elongation and close union of its cells, is adapted to give strength and rigidity to the stem. The inner portion of the pericycle, having thin, cellulose walls, is adapted to the storage and slow conduction of materials in solution. The phloëm portion of the vascular bundles probably carries proteid and other food materials rapidly up and down the stem as needed, being fitted for this purpose by its long, thin-walled, tubular cells, which are separated longitudinally by partitions having perforations, through which materials may readily pass. The xylem portion of the bundles carries water upward from the roots through its tracheal tubes, while the wood fibers associated with the tracheal tubes contribute to the strength and hardness of the stem. When we consider the vascular bundle as a whole we see that its conductive function is preëminent. The medullary rays carry materials radially to and from the bark and pith and to and from the vascular bundles. The pith may, in its young state, conduct materials up and down as needed, but as it gets older it dies and contains air only, or it may break down entirely.

Study also cross sections of an Aristolochia stem which is several years old and note the changes which have taken place in the different tissues since the first year.

66. Make a diagrammatic drawing of the different zones 
of tissues seen in the cross section of the stem, simply outlining them in right position and proportion, and with the colored pencils give each part having a distinct function a color of its own. For instance, color the epidermis blue, the thick-walled cells of the primary cortex red, and its thin-walled cells green, the strengthening ring of the peri-

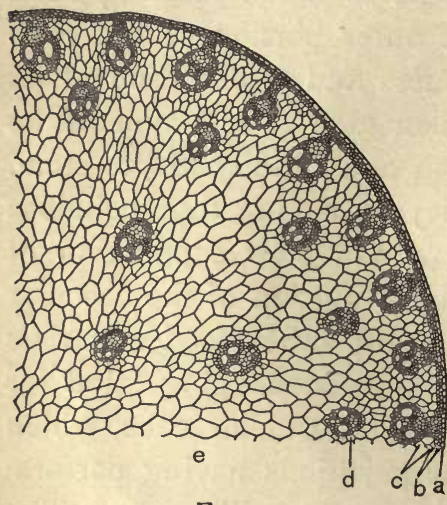

FIG. 20.

Cross section of a stem of Indian corn. $a$, epidermis; $b$, primary cortex; $c$, pericycle; $d$, vascular bundle; $e$, ground tissue.

cycle red, the inner portion of the pericycle, medullary rays, and pith yellow, the phloëm purple, the xylem orange, and leave the cambium uncolored. Run dotted lines from the different parts and at the end of each line write the name and function of the part.

67. Make sections of the stem of Indian corn where it is about one centimeter in diameter, and treat as directed for Aristolochia. Here the bundles are scattered promiscuously throughout the stem, and there is no distinction into pith and medullary rays (Fig. 20). The stem is given strength by the thick-walled cells of the primary cortex lying at the periphery, and by a sheath of somewhat similar cells around each bundle. The vascular bundles consist of the same parts as those of Aristolochia, the xylem facing the center and the phloëm the circumference. There is, however, no cambium between the two parts of the bundle, and the latter does not therefore increase indefinitely in size, but soon attains to its maximum dimensions. In corn, the 
general ground tissue in which the bundles are embedded serves the functions of the pith, medullary rays, and thinwalled portion of the pericycle and primary cortex, as stated for Aristolochia.

68. Make an outline drawing of the parts of the cross section of the stem of corn and use the colors to designate the same functions which they were made to represent for Aristolochia. Thus the epidermis would be blue as before, the thin-walled cells of the primary cortex green, while its thick-walled cells would be red; the thin-walled tissues in which the bundles are embedded yellow, the phloëm purple, the xylem orange, and the narrow zone of thick-walled tissue bordering the bundles red. The colors will assist in comparing the different parts of the stem from the standpoint of their use, blue meaning that protection is given; green, that food is manufactured, temporarily stored, and slowly conducted away as needed; red, that strength is imparted; yellow, that food is stored and slowly conducted ; purple, that food materials, and particularly proteids, are rapidly carried either up or down the stem where need is urgent; orange, that water is rapidly carried from roots to leaves; uncolored, that the cells are rapidly multiplying and increasing the diameter of the stem.

69. Plant seeds of garden balsam in moist sawdust, and keep watered with well water. As soon as the seedlings appear place one lot near a window, and another lot on a side of the room remote from the windows where the light is not intense. Note the difference in rate of growth, in the relative lengths of the internodes, and in the size of the leaves. Do the stems grow upright; if not, what force is interfering with gravity? Carefully remove some of both lots of seedlings from the sawdust and place in a 
tumbler or wide-mouth bottle containing a weak solution of fuchsin, and keep the roots covered with this solution until a red color appears in the veins of the leaves. The stain will color the tissues through which it passes and thus mark out the paths of the ascent of water. Balsam stems are sufficiently transparent to allow the tissues near the center to be seen from the outside.

70. Remove a ring of bark about an inch long from some twig or sapling which is in leaf (the willow serves excellently for this and the following experiment because its bark is strong and easily separates from the wood), and note whether the leaves wither. Watch the experiment for several days. Operate on another twig or sapling in the following manner: Make a longitudinal incision through the bark an inch or more long, depending on the diameter of the stem, and then, with a thin, smooth stick work the bark loose around the stem, inserting the stick through the longitudinal slit. Cut the wood of the stem nearly in two, and then bend the stem until it breaks, the broken ends protruding through the slit in the bark. Trim off the ends so that they will not touch each other; then hold the stem upright so that the ends are again covered by the bark, and bind splints around the outside to keep them in place. Care should be taken during this operation that the bark is not injured except by the longitudinal slit. Note the effect on the leaves in the course of a few hours. What do these experiments teach as to the region of water ascent in plants?

7I. Remove a ring of bark from a branch or sapling that can be conveniently watched, and note the result at the end of the season's growth. What do the results teach as to the region of transfer of food materials necessary to the building up of new tissues? 
72. Make thin cross sections of stems of elm, cottonwood, or other woody plants which were placed in alcohol in late summer, and treat with iodine. Can reserve food in the form of starch or proteids be made out? (The starch would be colored blue by the iodine, and the proteids from yellow to brown.) Compare with these sections others of stems of the same plants taken at the time of unfolding of the buds in the spring. What changes have taken place in the reserve materials?

\section{DISCUSSION.}

37. Upward Growth of the Shoot.-We have noticed that in the germination of seeds the shoot grows straight upward into the sunlight and air just as uniformly and persistently as the roots grow downward into the soil, and it might be inferred from this alone that the upward growth of the shoot is just as necessary to the well-being of the plant as the downward growth of the root. It has been noticed that as the shoot reaches the surface, either the cotyledons spread out in the form of thin green leaves, or the first leaves of the plumule bud quickly unfold and place their broad surfaces more or less at right angles to the light from the sky, as in the case of the Lima bean, whose cotyledons are so gorged with food materials that they are prevented from developing into useful foliage leaf forms. As the stem continues in its elongation, it puts forth new leaves until it is nearly concealed by them and is apparently subordinate to them. It may, in fact, be stated that the chief function of stems is to bear leaves, in such positions and at such distances apart as to give them free access to the sunlight and air, and to keep them in communication with the water and other raw food materials which are absorbed by the roots. 
38. Protection against Drying. - As soon as the shoot appears above the ground it is in danger of drying up, or of becoming bruised or broken. To guard against drying, the outer wall of the epidermis or exterior layer of cells becomes infiltrated with a compound of fatty and waxy substances known as cutin, and so is rendered almost impervious to water. Every one has noticed how quickly an apple shrinks and drys when its epidermis has been removed by paring, and it would be a simple matter to demonstrate that any young or succulent stem would quickly become dry if its epidermis were stripped off.

39. How Stems are Strengthened. - In order to strengthen the stem, the walls of the cells in certain regions become thickened, and sometimes woody, and the cells often become elongated and more or less interlaced, as in the case of wood and bast fibers. As evidence of the effectiveness of wood and bast in strengthening stems, let the great elasticity and strength of some of the well-known woods, such as hickory, be called to mind, and the fact that hemp rope and linen thread are made from the bast of plants.

The stress which the strengthening elements must overcome is produced usually by the wind and the weight of the crown of the plant. The force of the wind bends the stem, producing a stretching of the elements on one side and a compression on the other, while the weight of the crown produces a compressing effect simply. It is clear that if a given amount of strengthening material be distributed in the form of a hollow cylinder, all stresses of the above nature can be overcome to the best advantage. We find, accordingly, the strengthening elements of the pericycle of Aristolochia arranged in this form, and the same is true of the strengthening elements in the primary cortex of corn, and of the wood and bast fibers in the 
stems of plants in general; but in the roots, where the stress is applied more nearly as a straight pull, we find the strengthening elements in the form of a compact column at the center, a form which is best adapted to resist a longitudinal pulling stress. In the distribution of their strengthening elements, plants have been obliged to take account of many things, such as economy of materials, the free circulation of materials, and the formation of branches.

40. Conduction of Materials. - The conducting elements of the stem are quite as important as the strengthening elements. The leaves give off large amounts of water by transpiration, and must, therefore, constantly receive compensating supplies from the roots. 4 To facilitate the passage of water, long tubes are provided, extending from the roots into the leaves. These tubes, known as tracheal tubes, have relatively large openings, and their walls are thickened in various ways (see Fig. 2I) so as to strengthen them, and at the same time leave thin places for the passage of water into the tissues along their route, and to allow the passage of food materials into the tubes while they are yet in state of formation. The thin places in the tubes also serve another purpose every spring, in permitting the reserve food materials in the underground parts and lower regions of the stems to pass into the tubes and be carried rapidly upward to the unfolding buds by the ascending currents of water. The tracheal tubes have cross partition walls in them which are about eighteen inches to three feet apart; these are thin, however, and do not much retard the passage of the water.

The tracheal tubes lie in the xylem portion of the vascular bundles and are thus within the cambium ring. Accordingly when the bark is removed from a stem, the 
tracheal tubes are left intact, since the bark separates at the cambium ring (see Fig. 2I). This accounts for the fact that the leaves do not wither when the bark is removed by girdling.

While water is being supplied through the roots the most important process of the manufacture of food mate-

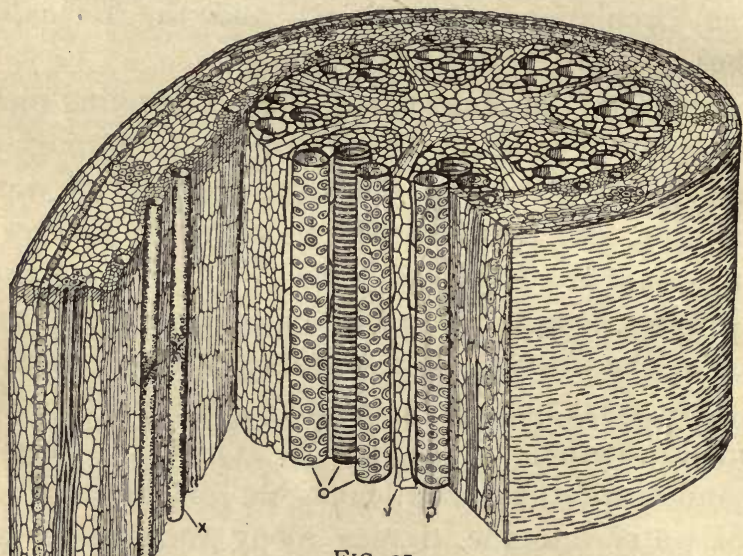

FIG. 2 I.

Diagrammatic representation of the structure of a Stem, the bark being partly stripped off at the cambium ring. 0 and $p$, tracheal tubes, which carry the water upward from the roots; thin places are seen in the walls of these tubes in the form of pits and rings. $w$ and $x$, sieve tubes which carry food down from the leaves, or up, as needed. $v$, medullary ray, which carries water and food radially to and from the bark and the wood as needed. $z$, bast fibers. Cells of the cambium ring are clearly shown to the right of the sieve tube, $x$.

rials is taking place in the leaves, and highways must be provided for the transport of these food materials downward and upward wherever growth in length or in thickness is taking place. The tracheal tubes could not answer this purpose because the upward movement of the water through them would prevent the passage of food materials 
downward. The sieve tubes in the phloëm portion of the bundle outside the cambium ring (see Fig. 2I) seem to be the highways for these food materials. The sieve tubes are thin-walled and allow the food materials to pass to and from the surrounding tissues as needed. They have cross partitions at frequent intervals to strengthen them, but the partitions are perforated so that materials may pass rapidly through them.

41. Effect of Girdling. - When a tree is girdled, the sieve tubes are removed with the bark (see Fig. 2I), and the parts of the stem and of the roots below the girdle are no longer supplied with food. While the portions of the stem above the girdle increase in thickness and length as usual, all parts of the plant below the girdle are restricted in growth because the main food supply is shut off. If the bark of a stem is removed early in the spring, before the leaves are out, the usual process of the unfolding of leaf and flower buds may still take place because the reserve materials stored in the underground parts and in the lower portion of the trunk may be carried upward by the water currents in the tracheal tubes; but the following year, if the bark has been completely removed in the girdle, and no twigs bearing leaves have been allowed to grow below the girdle, the roots will not have stored in them the food necessary to the production of new rootlets and root hairs, and thus can no longer absorb the water necessary to the resumption of growth. The advantage of girdling trees which are to be cut down lies in the fact that the stump and roots below the girdle will thereby be prevented from receiving the food which might be employed in the production of new shoots from adventitious buds on the stump or roots. In stems of the type of Indian corn and palm there can be no separation of the food-conducting and the water- 
conducting elements by the process of girdling, for the reason that the bundles are scattered promiscuously throughout the stem (Fig. 20).

42. Summary of Structure and Function.-When we review the details in the plan of construction of typical stems, we see that they are so admirably adapted to give strength

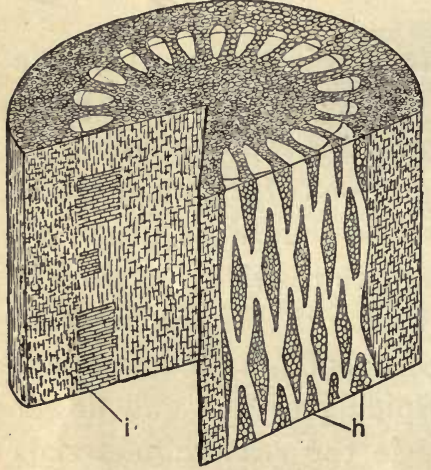

FIG. 22.

Diagram showing medullary rays from cross, tangential, and radial points of view. The rays are seen at $i$ and $h$. After HARTIG. and facilitate transport that trees can withstand the storms of centuries, and the interchange of materials between roots and leaves can continue without interruption even in trees which have reached a height of four hundred feet. We see that the strengthening elements are laid down according to approved mechanical principles, and that the conducting system is a double highway along which materials may pass in opposite directions without hindering each other in the least degree. We find that the movement of materials radially is provided for by the medullary rays, which extend individually only a short distance longitudinally, and so are prevented from transporting materials in any other than the radial direction (see Fig. 22).

43. Transporting Forces. - The forces which are concerned in carrying the water upward have not yet been demonstrated with certainty. Atmospheric pressure does not suffice to carry water to the height of tall trees; capillarity in the tracheal tubes cannot lift the water beyond the height of a middle-sized tree; and osmotic pressure in 
the roots cannot provide water fast enough to supply the loss by transpiration. Neither does the lifting power appear to be due to living cells in the roots or stem acting after the manner of a heart, or in any other way; for after these cells have been killed by poisonous solutions the water continues to rise and evaporate from the leaves. It seems probable that the leaves themselves assist in lifting the water, perhaps by osmotic action between the tracheal tubes and the parenchyma cells in the leaves; but this has not been satisfactorily demonstrated. The problem of the ascent of water, although apparently simple on the face of it, has been one of the most elusive in plant physiology. The forces concerned in the rapid movement of food materials through the sieve tubes also remain undemonstrated. Diffusion would account for slow movements, but the sieve tubes are evidently designed for conducting more rapid hydrostatic currents.

44. Direction of Growth. - We have seen that the shoot of the young seedling employs gravity to direct its course upward. After it appears above the ground, however, it does not necessarily continue in a vertical direction, but may grow more or less nearly horizontal, and may even turn downward. If the stem becomes more intensely illuminated on one side than another, it usually grows toward the region of greatest illumination, but in some climbing plants it may grow in the opposite direction and thus keep in close contact with its support.

45. Gravity as Guide. - While the plant employs gravity as a guide in bringing the shoot out of the ground, it also uses the same force in conjunction with light to guide it in placing its branches at various angles to the vertical, as its needs may require. Although it may be stated as a rule that shoots which are formed beneath the surface of 
the soil are directed upward by gravity, this is by no means always the case. Certain underground shoots of the po-

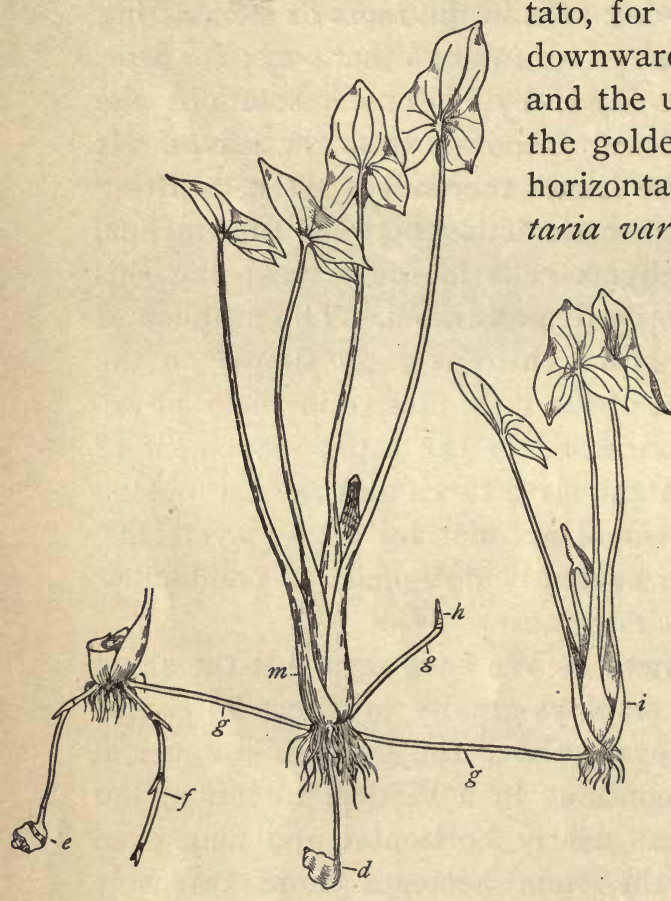

FIG. 23.

Sagittaria variabilis. $d$, last year's tuber, from which the central plant $(m)$ has sprung; $g$, offshoots from $m$; $h$, terminal bud of $g$, which has turned upward and will produce a plant like $i$; $f$, offshoot which has grown down into the mud and will produce a tuber similar to $e$, which is a tuber of the current season destined to survive the winter and then produce a new plant, as $d$ has done.

teresting example of somewhat diverse habits. Radiating in various directions from the parent plant ( $m$, Fig. 23) are horizontal underground shoots $(g)$, which finally turn upward at the apices $(h)$ and produce leaves above the surface. Roots are produced at the basal node of the upright shoots, which then become independent individuals $(i)$. Later in the season, underground shoots $(f)$ are produced which grow vertically downward for about a foot, and then turn upward at their apices for a short distance and form bulbs (e) that become filled.with 
reserve food materials, and in this position survive the winter.

In the spring the terminal shoot produced from a bulb grows vertically upward $(d)$ and sends forth leaves and flowers; while at a node a short distance below the surface of the mud, roots are formed, and also buds, from which the horizontal shoots arise, thus bringing us back to the stage at which we began the cycle. This example is an exceedingly instructive one in showing how plants may employ any force acting in a definite direction (in this case gravity), not in impelling all of its parts in one direction, but as a fixed line from which may be determined the various directions toward which its different members should grow. It is also instructive in another way: it shows that plants are not inert bodies; but are possessed of a high degree of sensibility which enables them, in a certain sense, to perceive their condition and the forces within whose range they are lying. Notice, for instance, that the horizontal shoots of Sagittaria do not grow indefinitely in the horizontal direction, but after a suitable distance from the parent plant has been traversed so that danger of crowding is avoided, they turn upward and produce leaves and roots. So, too, the downward-growing shoot, having reached a depth where the winter may be passed in safety, turns upward and produces the bulb to which is intrusted, as well as to the seeds, the life of succeeding generations.

46. Light as Guide. - Where gravity cannot be employed as a guide in achieving certain results, light may be used instead. If the stem has a climbing habit and is supported by lateral outgrowths in the form of suckers or roots which are able to obtain foothold on walls, trees, etc., the shoot employs the line of propagation of the greatest incident 


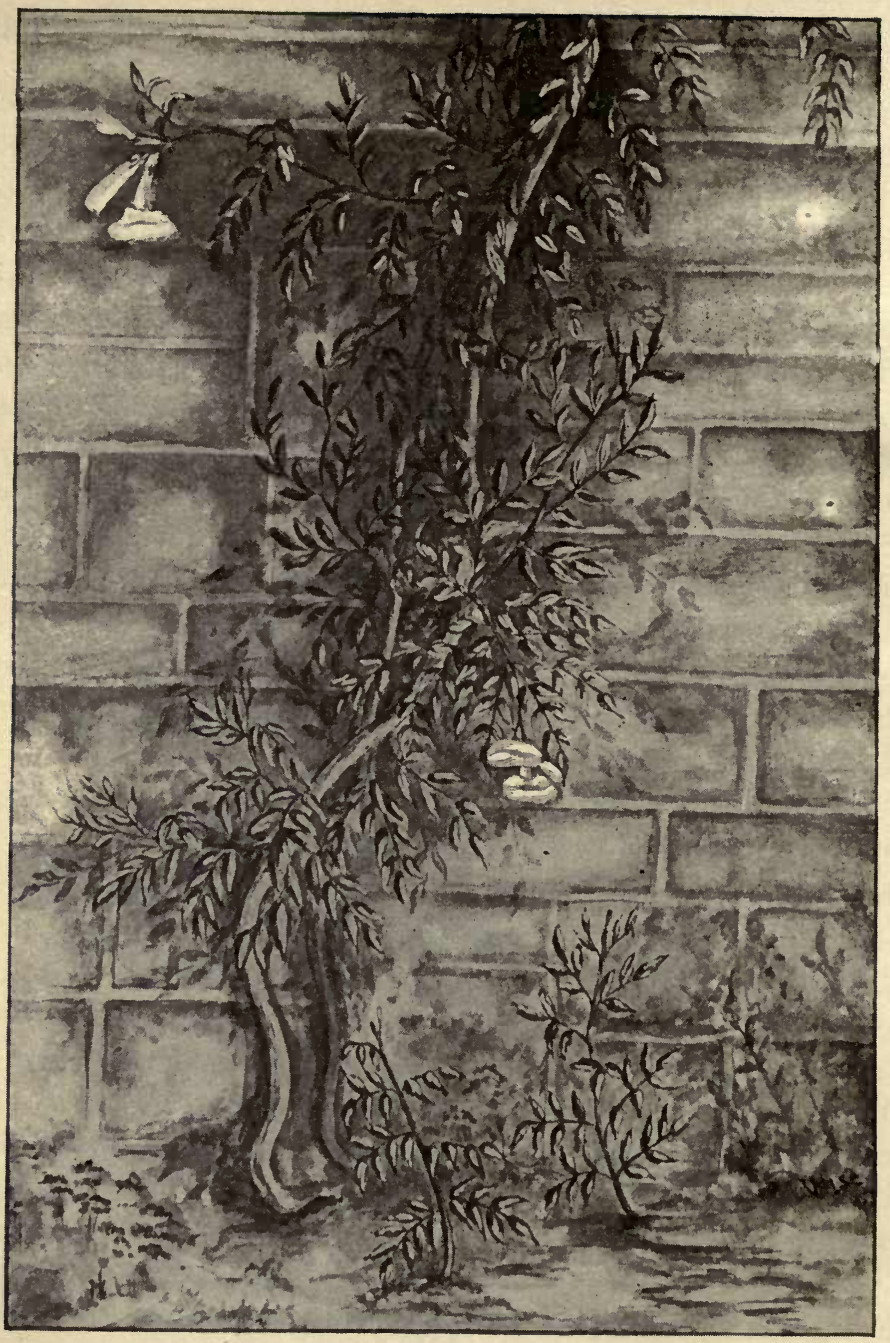

FIG. 24.

Mature plant and two seedlings of the Trumpet Creeper. The seedlings are growing toward the wall and away from the source of greatest illumination. On the mature plant the flowering branches are growing away from the wall and toward the source of greatest illumination. 
light to guide it toward its support. The common trumpet creeper, Tecoma radicans, acts in this way. Figure 24 is a drawing from nature showing seedlings which have started at some distance from the wall toward which they have sharply turned; their leaves, however, are facing the light. As this plant climbs a wall its shoots lie close against it, so that the clinging roots easily get a foothold. But the shoots that are to produce flowers as well as leaves

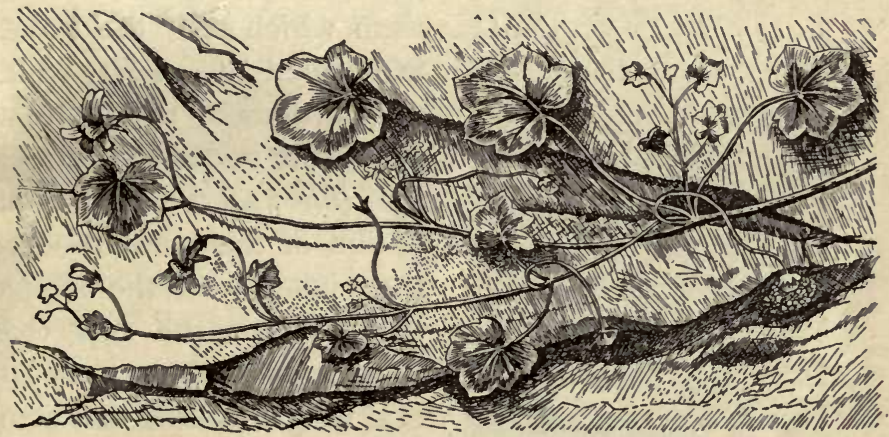

FIG. 25 .

Linaria cymballaria clambering over rocks. After KERNER.

turn toward the light instead of from it (see Fig. 24), so that the flowers may be easily detected by the insects and humming birds which assist in their cross pollination (see page I70). A no less marvelous example in which the sensibility of the plant involves a perception of its own condition (conscious recognition is of course not meant) is furnished by the behavior of the flower stems of Linaria cymballaria, a clambering plant which fastens itself to walls, etc., by means of suckers. The pedicels bearing newly opened flowers turn outward toward the source of greatest light so that the flowers are noticeable to those 
insects which are necessary to their cross pollination; but after fertilization has been achieved and the production of seeds thereby insured, the pedicels turn from the light and deposit the seed pods in the crannies of the rocks, where they may find a suitable place for their germination (Fig. 25).

47. Formation of Leaves. - We have noticed, in following the development of seedlings, that leaves are produced at definite intervals along the stem as the shoot elon-

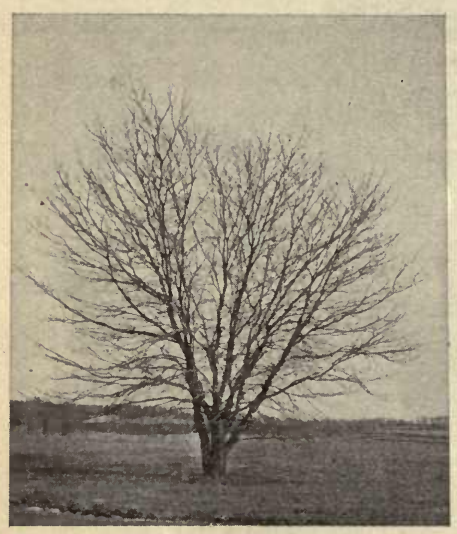

FIG. 26. gates. The segments of the stem which bear the leaves are called the nodes, while the, portions of the stem between the nodes are termed the internodes. An examination of the apical bud of the shoot, with the simple lens, reveals the fact that the leaves are begun as lateral outgrowths of the stem quite close to the growing point, the apical bud consisting, in fact, of a succession Honey locust with deliquescent trunk. of immature. nodes, internodes, and leaves.

48. Summer and Winter Buds. - In some plants, the growing point of the shoot continues to give rise to new nodes, internodes, and leaves throughout the growing season, so that when winter sets in there is a certain unripe portion of the shoot extending back from the apex which is killed by the cold, or its buds are weak, and the continuation of growth the following season devolves on buds on older portions of the stem. In such plants the crown is much branched and has no main central trunk (see Fig. 26). 
In other plants, such as the horse-chestnut, hickory, and cottonwood, the elongation of the stem ceases before the close of the growing season. In such cases a few of the last-formed internodes fail to elongate, while the leaves of some of the lower nodes subtending the short internodes grow up in the form of scales, completely enwrapping all of the parts above them, and protecting them against mechanical injury and the vicissitudes of weather. Such buds are quite certain to survive the winter, and, if stronger than the lateral buds, to continue the growth of the shoot the following spring, so that the main shoot of such plants is apt to retain its identity in the form of a central shaft extending through the crown (see Fig. 27). Buds which ripen and prepare for winter are termed winter buds; while the buds which do not ripen, and die or re-

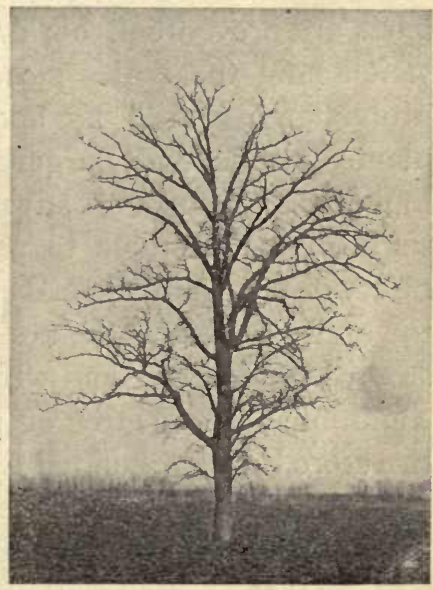

FIG. 27.

Oak with excurrent trunk. main weak in consequence, may be called summer buds.

49. Protection afforded Winter Buds. - The study of the winter buds of the horse-chestnut and cottonwood has shown that the leaves formed at the uppermost nodes are ordinary foliage leaves in an embryonic state, and consequently in need of protection. The amount and character of protection afforded to the tender parts of winter buds is quite different for different plants. As has already been observed by the student, the outer scales of the buds of 
horse-chestnut and of hickory are hard and dry and well adapted to give protection against mechanical injury, while the hairs growing over the scales tend to keep them separated slightly, and so form dead air spaces which retard the escape of water from the succulent parts of the bud, and, on account of the slight conductivity of air for heat, protect the inner parts against sudden changes in temperature. In the winter buds of the cottonwood, the tender parts are prevented from drying up by resinous substances which hold the scales closely together. The amount of protection required by buds varies greatly for different plants, and depends largely upon the ability of their protoplasts to withstand adverse conditions. The buds of some plants are able to pass through severe winters in safety without any special means of protection. Even in the arctic regions there are plants whose buds are able to survive the intense cold while in a succulent, half-formed condition. The degree of protection given to buds seems, therefore, to be regulated by the needs of the living cells.

50. Disposition of Leaves in Buds. - The manner in which the embryonic foliage leaves are packed away in the bud is not the same in all cases; but, however these details may vary, the leaves already possess in the bud the regular angular divergence on the stem which is found in mature shoots, and this facilitates their disposition in the very small compass of the bud.

51. Unfolding of Winter Buds. - The unfolding of winter buds in the spring is essentially the resumption of growth in parts already formed. The short internodes and the minute leaves quickly elongate and expand, and almost in a day the trees are again covered with foliage. Such remarkable development would be impossible if reserve food materials were not already at hand for the building 
up of new tissues. The reserve materials can be demonstrated by using tests for starch, proteids, sugar, oils, etc., chiefly in the medullary rays and wood parenchyma cells of the stems and underground parts. Before these materials are needed in the spring, those which are insoluble or poorly diffusible are changed by appropriate ferments so that they can pass by diffusion from cell to cell, or be carried more rapidly upward by the ascending currents of water. Buds which remain attached to the parent plant and are able to draw upon it for their food do not need to have the reserve materials stored within their own tissues, as in the case of seeds which are cast off from the parent and left thereafter to shift for themselves.

52. Leaf and Flower Buds. - Dissection of the terminal buds of the horse-chestnut reveals the fact that many of them contain flowers as well as leaves, while the terminal buds of the lilac may contain only flowers. In the cottonwood we find many of the lateral buds containing flowers only, while the terminal buds contain only leaves. When we inquire the reason why the growing point of certain forming buds gives rise to leaves and that of others to flowers, or to both leaves and flowers, we are unable to obtain a satisfactory answer. We know, however, that some perennial plants produce only leaf buds for a number of years, and are not able to form flowers until they have attained a certain age; we do not expect apple trees, for instance, to bear flowers until they are five years old or more. The century plant does not produce flowers until it has attained an age of twenty to thirty years. We know, too, that shoots which would otherwise terminate with winter buds containing leaves only can be made to terminate with buds containing flowers if the branch on which the shoot is borne is pruned back, or if the roots are pruned. By this 
means the fruit grower is able to increase very materially the yield of his trees. It seems from observations on different forms throughout the plant kingdom that under conditions of good nutrition, if changes occur which check mere vegetative growth, and are not inimical to the life of the plant, then the activity of the plant is apt to manifest itself in the formation of reproductive organs. The results of pruning back branches and roots illustrate this for the higher plants.

53. Position of Buds. - Buds usually occur at the apices of branches and laterally in the axils of the leaves; in the latter position they are termed axillayy, and in the former, terminal. Buds which sometimes occur beside or above the axillary buds are called accessory. Sometimes, however, buds occur without order on both stems and roots, and are then called adventitious. Both axillary and accessory buds have the same angular divergence as the leaves, and their growth under good conditions results in a symmetrically shaped plant. Always many buds are formed which never develop into shoots; or some, after lying dormant for years, may resume their growth if accidents to the plant require the production of new shoots on the old branches. Adventitious buds occur normally on the roots of certain plants, such as the white poplar, but they usually appear only as the result of injuries - as illustrated by the numerous shoots which grow forth on the stumps of felled trees.

54. The Nature of a Bud. - Whether a bud gives rise to leaves or flowers, or to both leaves and flowers, it is essentially a miniature shoot whose succession of internodes, nodes, and lateral outgrowths is but a repetition of a similar succession dating back to the germination of the seed, and constituting the whole above-ground body of the plant. 
We might expect, then, that a bud would have all of the potentialities possessed by the entire plant. How true this is, is shown by the results of the process known as budding, which is practiced by nurserymen for the propagation of various sorts of plants.

55. Propagation by Budding. - In this process, a bud, together with the bark and a very thin layer of the wood just beneath it, is removed from the plant which it is desired to propagate ( $a$, Fig. 28). A longitudinal slit about an inch long is made in the bark of the plant on which the bud is to be grown, and at the top of the longitudinal slit a cross slit is made so that the bark may be separated from thewood. The bud is then slipped
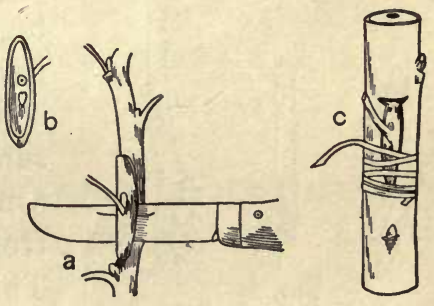

FIG. 28.

Process of Budding. $a$, cutting the bud from the parent branch; $b$, inner surface of the removed portion, showing the base of the petiole, and beneath it a bit of wood adhering to the base of the bud; $c$, the bud placed in position and partly tied in. After BARRY.

under the bark and tied into position (c, Fig. 28). After a few weeks the bud will have grown to its foster stem, and the string which binds it down is cut away.

This process is usually done in the summer or early fall, and the bud is not expected to grow until the following spring, when all the branches of the foster plant are cut away, and it alone is allowed to develop. The shoot which grows from the bud is found to possess the characters of the plant from which it was taken, and the branches which later spring from it have the same characters. In short, all of the characters of the parent plant were transplanted by means of a single bud; and so certain is this to occur 
that the process of budding is relied on for multiplying desirable varieties of many fruits and flowers, such as peaches, cherries, pears, roses, etc.

56. Propagation by Grafting. - Likewise, if a small portion of the branch bearing a bud be inserted into the stem of another closely allied plant which is rooted in the soil, after the manner represented in Fig. 29, the correspond-

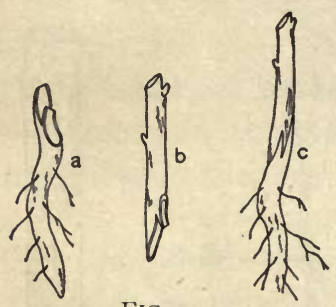

FIG. 29.

Process of Grafting. $a$, the root, and $b$, the branch, made ready for grafting; $c$, grafted root and branch. After BARRY. ing parts of the two will grow together, and the branches arising from the bud of the engrafted stem will possess essentially the same characters and bear the same kind of fruit as the plant from which the stem was taken. This processis known as grafting, and is employed in the propagation of various fruits and flowers.

We learn from facts of this kind that the powers and qualities of the plant as a whole reside in very restricted parts of it; a fact of great importance to organisms which cannot seek shelter against the vicissitudes of weather, but must stand in one place and meet the force of storms, often with the result of mutilation or even amputation of their members. It is in virtue of this fact that all of the above-ground parts of certain plants, such as Solomon's seal, goldenrod, and Dahlia, may die away and be regenerated on the return of spring from the relatively small underground portions.

57. Functions of Stems. - While a typical stem stands erect above the soil and, for its chief function, bears leaves and connects them with the roots, yet, in the economy of the plant, stems may have other forms and perform other 
services. The tuber of the Irish potato, for instance, is a portion of a stem having for its chief function the storage of materials; the stems of cacti are green, and perform the food-producing functions; the underground stems of Sagittaria and goldenrod creep along under the surface of the ground, producing new erect shoots here and there, and so serve the purpose of multiplication; the tendrils of the grape and Virginia creeper, which seem to be stems, have a supporting function. Indeed, as we shall learn in the chapter on modified parts, the members of the plant body may be put to quite as various services as the economy of the plant may require.

58. Habits of Stems. - The typical foliage stem is erect or nearly so, and stands by its own strength. Climbing plants do not possess the strength nor form of stem to enable them to acquire the erect position without the aid of some support. An examination of the structure of stems of climbing plants reveals the fact that they are relatively lacking in the strengthening elements, while the tubes for conducting water occupy a correspondingly larger space. This is what we should expect, for plants that have acquired the habit of clinging to other objects for their support have less need of strengthening elements; while their long and slender stems, which lift the foliage to a considerable height above the ground, require that a relatively large space be given over to highways for water transport. At the opposite extreme of habit stand the plants with prostrate stems, such as Euphorbia serpens. These two types of stems, the climbing and the prostrate, between which all degrees of gradation may be found, are adapted to quite different habitats, - the climbing stems to shady situations, such as dark woods, or the shady side of banks or buildings, and the prostrate stems to situations along 


\section{4 \\ Introduction to Botany.}

the borders of roads, and rocky and waste places, where they may be rooted in good soil, but are able to run out over the barren areas where their leaves will not be too much shaded by the surrounding vegetation.

59. Characterization of Stems. - When we survey their character and functions, we find that stems are members of the plant body which bear the leaves and roots, and have for their chief functions the exposure of the leaves to the light, and the transport of materials between the leaves and roots. The other functions which they perform seem to be adaptations to special conditions. 


\section{CHAPTER V.}

\section{LEAVES.}

\section{PROVIDING MATERIALS.}

Most of the observations on leaves are best made in the field or upon materials freshly brought into the laboratory. If vegetation out of doors is not sufficiently advanced to begin the study of leaves as soon as the work on buds and stems has been completed, the study of modified structures outlined in a following chapter might next be taken up, and the study of leaves postponed until the right kind of material can be obtained in abundance.

\section{OBSERVATIONS.}

In the study of leaves, make account by notes and drawings of the following points :-

73. The form of the leaf and the character of its outline.

74. The texture of the leaf and the character of the two surfaces, whether rough, smooth, hairy, etc.

75. The manner of attachment of the leaf to the stem; namely, is it broad or narrow at the base? Is the leaf. blade attached to the stem, or does a leaf stalk or petiole intervene? Are there lateral outgrowths at the base of the leaf?

76. How does the leaf vary as it grows from the bud? Do the form and size of the leaf and the character of the two surfaces vary materially?. Compare the color of leaves which are just issuing from the bud with that of mature leaves.

77. How do the leaves vary in form, size, and outline 
from the base to the summit of the stem? Draw types of the different kinds.

78. How does the angle made by the leaf with the stem vary as the leaf advances from its embryonic condition in the bud to maturity? Does the direction taken by the stem seem to affect this angle? To answer this question study the approximately horizontal and vertical branches of the same plant.

79. What is the position taken by the leaves with reference to the incident light?

Note the position of leaves at different times of the day to find out whether the movement of the area of greatest light from the east toward the west affects the positions of leaves, and if so, to what degree.

8o. Determine the angular divergence of the leaves. (See page 48.)

$8 \mathrm{I}$. Is there any relation between the breadth of the leaves and the number of vertical rows on the stem? When the vertical rows are numerous, are the leaves more narrow as a rule than when the rows are fewer?

82. Is there any definite relation between the lengths of the leaves and their vertical distances apart? It should be remembered that short leaves will permit the light to strike between them better than long ones.

83. When leaves are growing close together in the form of rosettes, how are they prevented from shading each other too much? Notice whether there is a difference between the lower and upper leaves as to their size and shape, and their angular deviation from the horizontal.

84. In trees and shrubs, do the leaves on the horizontal branches grow from buds on the lower as well as on the upper side? Do the leaves seem to assume a definite position with reference to the light from above? 
85. Is there any marked difference in position and distribution between leaves on horizontal branches and those on vertical branches?

86. Make a longitudinal section with a sharp knife through the middle of a leaf and the branch which bears it, and note its connection with the bark and wood. Remove another leaf from its stem, with a downward pull, and examine the wound with a lens to see whether the broken ends of the vascular bundles can be made out.

87. Make drawings to show the course of the veins in a grass or lily leaf, or in the leaf of any monocotyledonous plant; also drawings of veins in the leaf of a sunflower, castor bean, or other dicotyledonous plant. Bleach any thin leaf by soaking it in strong alcohol until the chlorophyll is extracted (chlorophyll is the green coloring matter in the leaf), and allowing it to lie in a saturated solution of chloral hydrate (see page $38 \mathrm{I}$ ) for several days; then mount it in a drop of dilute glycerine, and examine under the medium power of a compound microscope in order to note the ultimate branches of the veinlets.

88. Cover rapidly growing plants or branches of plants so as to keep them dark, and compare the color and size of the leaves which develop in the dark with that of leaves grown under normal conditions of illumination; compare also the lengths of the internodes formed in the two cases. How long does it take for the blanched leaves to turn green after they have been exposed to the light? Boil for a short time in water a blanched leaf which has developed in the dark and a green leaf which has grown in the light, and place in alcohol until the green leaf has become colorless; then place both leaves in a solution of iodine, and note whether one is colored more purple than the other, indicating a greater. amount of starch. Sugars, as 
well as starch, are manufactured within the leaf; but the starch is more readily demonstrable, and will therefore be considered here and in the discussion as the visible product of the leaf's work.

89. Make thin cross sections of a green leaf and mount them in a drop of water under a coverglass and study them with a high power of the microscope. Note the rounded green bodies, termed chloroplasts, lining the walls of the cells. Mount other sections under a coverglass in a drop of a saturated solution of chloral hydrate to which has been added sufficient iodine to give it a pale brown color. By this process the leaf will be bleached and rendered transparent, while the starch grains in the leaf will be stained purple. Watch the action of this reagent from the beginning, and note how the chloroplasts are gradually bleached, while the starch grains in them become more and more distinct. Treat in this way sections from a leaf taken just before sunset, and from another leaf from the same plant taken just before sunrise the next morning. Note the relative amounts of starch in the two sections. What conclusions do you reach from the results of your observations?

90. Tie a branch of a floating water plant, such as Ceratophyllum or Myriophyllum, to a glass rod or tube and set in a beaker of water, the glass rod being about an inch shorter than the depth of the water. Invert a test tube of water over the rod and plant, the cut end of the plant being uppermost and extending into the test tube. Prepare two other branches in the same manner, filling the second tube with water which has just been boiled and cooled, and the third with water which has been charged with carbon dioxide by blowing into it for some time through a glass tube; invert each tube in a beaker containing the same 
kind of water that the tube holds. Expose all three preparations to the full light of the sun. Compare the results with those obtained from a single plant placed successively under the three sets of conditions.

The first tube and beaker will contain a moderate amount of carbon dioxide, the second little or none, and the third a great deal. Watch for bubbles of gas issuing from the cut ends of the branches. Count the number rising from each in a given unit of time. The green leaves are taking carbon dioxide from the water and are dissociating the carbon and oxygen, retaining the carbon for the manufacture of starch, and liberating part of the oxygen. The frequency of bubbles in each tube is an indication of the relative amounts of starch manufactured. Set the tubes in the shade and note the effect. What do you learn from these experiments as to the conditions necessary to the manufacture of starch in the leaves?

Nearly fill a glass funnel with shoots of Ceratophyllum, Myrio-

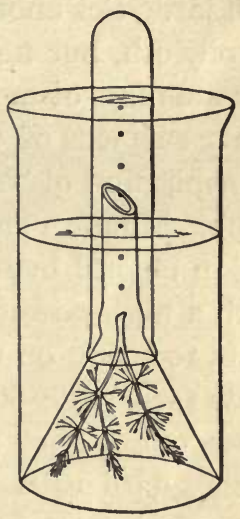

FIG. 30.

Device for collecting the gas evolved by a green plant under the influence of the sunlight. See text. phyllum, or other suitable water plant, and invert it in a tall beaker of well or spring water. The end of the stem of the funnel should be submerged for an inch or more. Fill a test tube with water and invert over the funnel (see Fig. 30). Set the preparation in the sunlight. If the tube becomes nearly half filled with gas, carefully remove the tube from the funnel, close the tube tightly with the thumb, while its mouth is kept submerged; turn its mouth upward, remove 
the thumb, and quickly thrust a glowing, but not blazing, splinter into the tube. The splinter should blaze up, indicating the presence of a large percentage of oxygen.

9I. Place a bell jar over any plant of proper size growing out of doors, having first fitted a piece of oilcloth, by cutting a slit in it, closely around the base of the plant, and having closed the slit with vaseline so that the moisture from the ground will be prevented from rising into the bell jar. Set another bell jar beside the first, on a piece of oilcloth, but not over a plant. After a time compare the amounts of moisture which have been condensed on the inner surfaces of the jars. What does this teach as to the transpiration of water from the leaves?

92. Mount a small piece of a leaf, with the under side up, in chloral hydrate-iodine (see page $38 \mathrm{I}$ ), and examine with a high power of the compound microscope. By carefully focusing on the upper surface of the mount, the stomata (see page 86 ), or openings in the epidermis, can be made out. They are made conspicuous by the starch in their guard cells being stained blue by the iodine, while the other cells of the epidermis are lacking in starch and remain unstained. The opening between the guard cells allows carbon dioxide to enter the leaf readily, and oxygen to pass out. It also permits the water to evaporate from the leaf.

93. To determine whether the stomata are necessary to the ingress of sufficient carbon dioxide for the manufacture of starch within the leaf. Select a plant, such as the lilac, whose leaves have stomata on the under side only. Keep a branch darkened for a day or two or until the leaves are found destitute of starch by the method described in Observation 89. Coat the under side of some of the leaves with a melted mixture of equal parts of cocoa 
butter and wax, and expose the branch to the sunlight until the uncoated leaves are found to contain starch. Then test sections from the coated leaves, and if these are found to be without starch, the inference is well founded that this is due to the exclusion of carbon dioxide by the closing of the stomata by the wax mixture.

94. Strip the leaves from some thrifty plant, and pick off new leaves as fast as formed. Note the result, and tell how the final effect is produced.

\section{DISCUSSION.}

60. Prominence of Leaves. - We have seen that as soon as the seedling appears above the ground its leaves unfold and turn green, and that the subsequent development of the shoot appears to consist mainly in the production of a succession of green leaves arranged in a definite order around the stem. When we look at a plant under normal conditions, in the prime of its development, it is the wealth of its foliage which impresses us most. In the whole family of grass plants, with exceptions which do not need to be considered here, the stem is very insignificant compared with the leaves. When we contemplate the billows of leaves in vast wheat and corn fields, and remember the rich harvests that are to follow, we must be impressed with the supreme value of leaves in the life of the plant and in the production of its seeds. Under normal conditions of moisture, etc., we find many plants without stems above the ground, but not without leaves. The superficial evidence is therefore plain that leaves are of paramount value in the economy of the plant. We shall see how true this is when we consider the work done by them.

61. Position of the Leaves. - We have observed that the leaves are borne in a definite order on the stem. In 
the lilac and horse-chestnut two leaves are borne at the opposite sides of each node, each successive node having its leaves at right angles to those of the node next below. In corn and other grasses the leaves are in two opposite rows, only one leaf occurring at each node. In the sunflower there are five rows of leaves, the rows being $72^{\circ}$ apart, and each leaf $142^{\circ}$ from the one next below or above it. Numberless examples could be brought forward to show that leaves are arranged in a definite order - a fact which of itself suggests that they are important members of the plant body. Not only do they have definite places of origin on the stem, but they grow outward from the stem at somewhat uniform angles with the horizontal - a fact which may suggest to the student that they sustain important relations with external forces.

62. Light Relation of Leaves. - When we stand beneath a tree we can see that the shaded portion of its crown does not bear leaves, but only the better-lighted peripheral portion. In carrying out Observation 79 we have noticed that the direction assumed by leaves appears to have some direct relation to the light; for when the plant has been illuminated more on one side than on another, the direction of those leaves which are still capable of growth becomes changed so as to expose their broad surfaces more nearly at right angles to the greatest incident light. Our experiments have taught us further that starch is formed in leaves exposed to the light, but not in those kept in the dark. Finally, when the leaves were stripped from a plant it attempted to produce others, but, being prevented in this, it died.

Such observations lead us to the conclusion that the definite arrangement of leaves on the stem and the more or less constant angle which they make with the horizontal are for the purpose of insuring that the light shall be impeded 
as little as possible in reaching them, and that in some way the light assists them in manufacturing the starch which is an important food of the plant. Having reached this conclusion, we find it all the more interesting to note how the leaves strive to intercept the light which comes their way, and how the form and size of the plant and the disposition and direction of growth of its branches are also adapted to this purpose.

The same end is attained in various ways by differ-

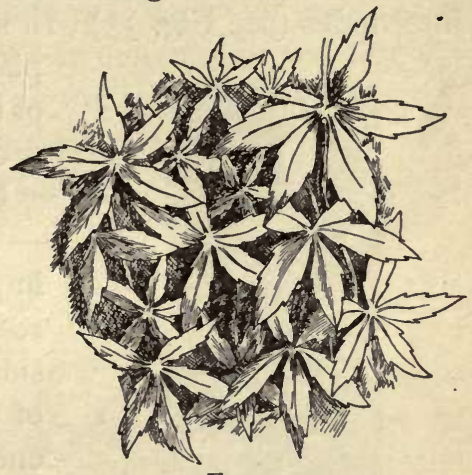

FIG. 3I.

Mosaic of Virginia Creeper leaves. The plant is climbing a wall and the blades of the leaves stand vertically.

ent plants. When plants are prostrate on the ground or have grown up over a wall, the leaves spread out more or less horizontally in the first instance, but vertically in the last, and intercept nearly all of the light which falls within the radius of the branches (see Fig. 3I). When plants are upright, the tiers of branches and leaves are so separated that the light can strike between them; when the leaves are crowded together in rosettes, the lower leaves

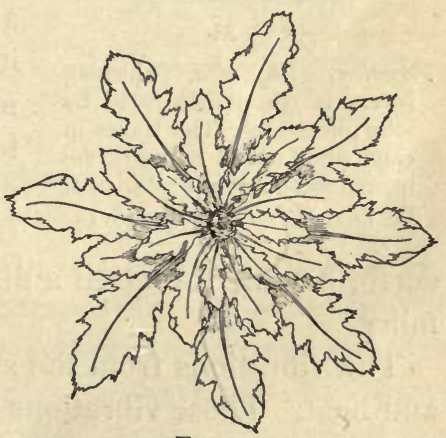

FIG. 32.

Dandelion plant viewed from above. Leaves in rosettes. grow out beyond the upper ones and produce broad surfaces only in the exposed area (see Fig. 32); or, when the 
leaves are borne in whorls at the nodes, the illumination of the lower leaves is brought about by an elongation of the internodes (see Fig. 33). In short, after whatever plan the

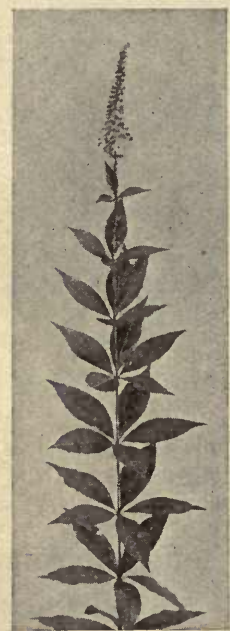

FIG. 33 .

Veronica Virginica, showing leaves in whorls separated by long internodes. The leaves in each whorl stand opposite the interspaces between the leaves of the next higher or lower whorl. plant may be built, the exposure of the leaves to the light is always provided for.

63. Sun the Source of Energy. - The sun is, of course, the one important source of light which reaches the earth from the outside universe. The heat and light of the sun are due to the intense energy of motion of its molecules; this is communicated to the ether, which fills interplanetary space, and in which all objects are embedded. (We do not know what the ether is, but there are good reasons for assuming its presence.) The ether transmits the motion communicated to it by the sun with such swiftness that in 8 minutes the disturbance is being felt on the earth, having traversed a distance of more than 93 million miles.

The vibrations from the sun are manifest to us as light and heat. Those vibrations which give us the sensation of light succeed each other with inconceivable quickness, ranging from 399 million million to 750 million million per second; those of less frequency than 399 million million, while not producing light, can be detected by their heating effect. In this way, vast amounts of energy are brought 
to the earth, it being estimated, for instance, that when the sun is in the zenith the energy communicated by it to the earth's surface amounts to one horse power for each area five feet square. It is impossible to conceive of the amount of energy coming in this way to the leaves of plants over the entire surface of the earth; but the student will find it instructive to estimate the amount for a single definite field of wheat or corn.

64. The Leaf a Manufactory. - The leaf intercepts the sun's energy and is enabled by it to manufacture the starch and other food materials necessary to the life and growth of the plant. We must therefore look upon a leaf as a manufactory which uses energy directly from the sun to do its work. To use economically the energy of the sun directly is, as yet, for

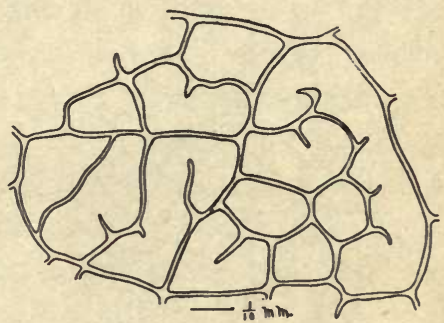

FIG. 34 .

Diagram showing the distance apart of the branches of vascular bundles in the sunflower leaf, as seen when looking through a bleached leaf with a medium power. The horizontal line shows ro $\mathrm{mm}$. magnified to the same extent as the figure. us an unsolved problem, but plants have solved it for themselves. We must examine the construction of a leaf to see how this is accomplished. We note that a typical leaf is spread out in the form of a flat lamina, which insures the interception of a maximum amount of light, and the ready ingress and egress of gases to and from all parts of the leaf. We have seen in Observation 87 that the vascular bundles ramify throughout the leaf so completely as to bring all parts of it in close communication with the water brought up from the roots, and to quickly carry out of the leaf its manufactured products (Fig. 34): And we have further seen by Observation 92 
that, while the leaf is covered with an epidermis which keeps the water from passing out of it too rapidly, there are openings in the epidermis (called stomata; singular, stoma) through which gases may pass in and out. (See Fig. 35.)

Observations 90 and 93 have taught us that carbon dioxide is necessary to the manufacture of starch by the leaf,

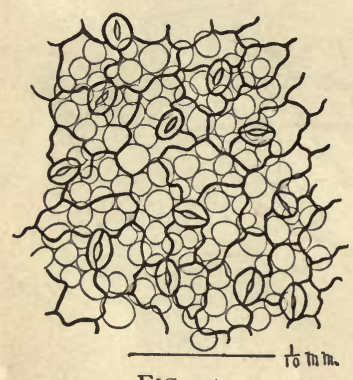

FIG. 35.

Epidermal cells with heavy outlines. The stomata are the elliptical bodies consisting of two curved guard cells with a narrow opening between. Ends of palisade cells of circular outline are seen beneath the epidermis. From leaf of Solanum rostratum seen from above. Drawn with a camera lucida. and that if the stomata are artificially closed, starch cannot be produced, although all other conditions may be favorable. From this we may conclude that the stomata are the ways through which the carbon dioxide of the atmosphere enters the leaf. Thus we see the leaf is well provided for receiving the raw materials upon which it must work. But how is the energy of the sunlight employed in transforming the raw materials into the finished food product?

If we strip off a bit of the epidermis, without bringing with it the underlying tissues, and hold it between us and the light, we see that a large percentage of the light passes through it; to such an extent, in fact, that objects may be seen through it. This fact assures us that the light may pass freely into the interior of the leaf. If now we hold an entire leaf between us and the light, we find that by far the larger part of the light has been absorbed by the leaf; in other words, most of the energy from the sun which 
reaches the leaf has not been transmitted through it, but has been arrested within it and transformed into some other form of energy. This has, in fact, taken place within the chloroplasts, which were seen in Observation 89. It is the green substance, the chlorophyll, which has enabled the chloroplasts to accomplish this. (See Fig. 36.)

65. Starch the First Visible Food Product. - We have seen by Observation 89 that starch is formed within the chloroplasts, and we must conclude that the water from the soil and the carbon dioxide of the atmosphere are brought together in them, and that the chloroplasts, employing the energy of the sunlight, transform these substances into the finished food product in the form of starch. We know that starch contains exactly the chemical elements furnished by water and carbon dioxide, and that hydrogen and oxygen exist in the same ratio in starch as in water. The chemical formula for starch is $\mathrm{C}_{6} \mathrm{H}_{10} \mathrm{O}_{5}$, while that of water is $\mathrm{H}_{2} \mathrm{O}$, and of carbon dioxide $\mathrm{CO}_{2}$. The combination of water and carbon dioxide into starch might be expressed theoretically in the following formula: $5 \mathrm{H}_{2} \mathrm{O}+6 \mathrm{CO}_{2}=\mathrm{C}_{6} \mathrm{H}_{10} \mathrm{O}_{5}+6 \mathrm{O}_{2}$; the oxygen being given off to the air again through the stomata. Doubtless the process is not as direct and simple as this (it is not definitely known what the steps in the process are), but the equation shows what excellent raw materials water and carbon dioxide are for the production of starch.

66. The Chloroplasts. - The chloroplasts (see Observation 89) are parts of the protoplast and are, of course, alive. It is their special function to arrest the energy of the sunlight, by means of the chlorophyll which they contain, and employ it in the manufacture of plant food from the raw materials, carbon dioxide and water. If Observations 89 and 92 are carefully made, it will be seen that the 
chloroplasts lie within cells and close against their walls, and that for the circulation of air there are spaces between the cells which are in communication with the stomata. A reference to Fig. 36 will show the relation of the

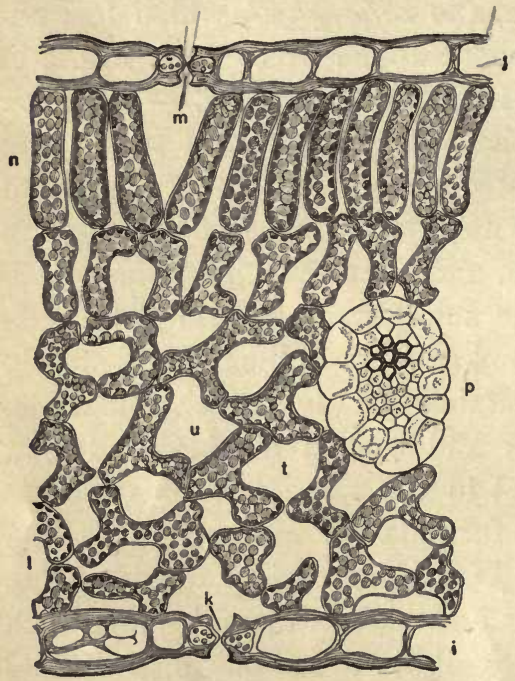

FIG. 36.

Cross section of a leaf. $j$, upper epidermis; $m$, stoma in upper epidermis; $n$, row of palisade cells containing rounded chloroplasts; $i$, lower epidermis; $k$, stoma in lower epidermis; $l$, spongy parenchyma cells containing chloroplasts; $p$, vascular bundle in cross section; $t$ and $u$, intercellular spaces. After SACHS.

chloroplasts to the rest of the leaf structure. The cells of the upper side of the leaf, termed the palisade cells, are set up in regular order with their long axes parallel to the direction of the incident light; the chloroplasts are embedded in the living substance of the protoplast which lines the cell walls, the remainder of each cell being filled with a watery cell sap.

The palisade cells are not compacted together, but have air spaces between them communicating with the stomata. The more loosely arranged parenchyma cells on the under side of the leaf, called spongy parenchyma cells, also contain chloroplasts, but they necessarily receive less light than those on the upper side and cannot manufacture so much food. The vascular bundles are seen to lie at the center of the leaf where they can readily communicate both with the palisade and spongy parenchyma 
cells, and so give over to them the water that they bring from the roots, and receive from them the products of their manufacture. Thus we see that the leaf is in reality a factory to which the raw materials are constantly being

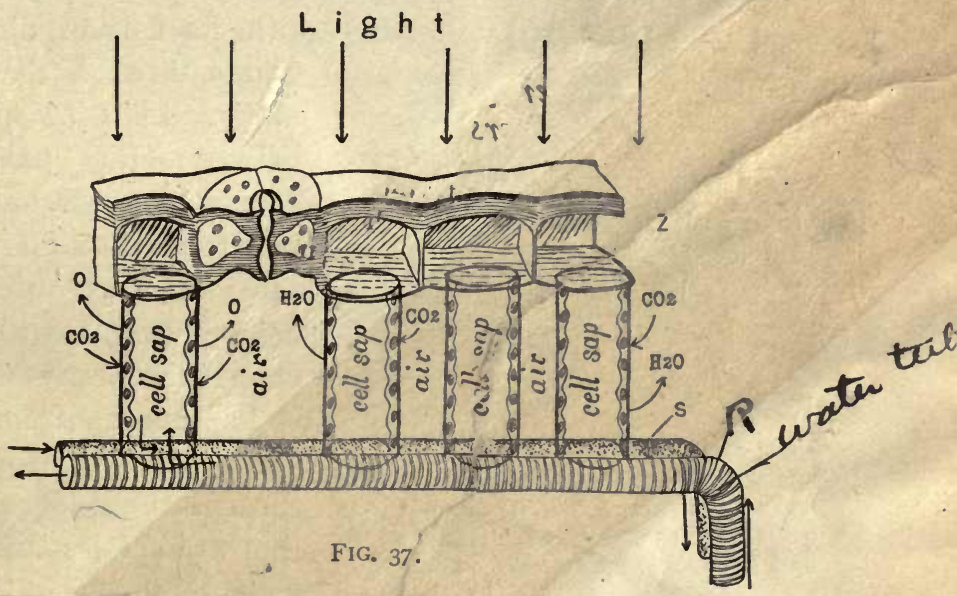

- the essential working parts of a leaf and their relation to each vi the upper epidermis containing one stoma; $S$, a sieve tube through whi the manufactured materials are removed from the leaf; $r$, a tracheal tube through which water is brought into the leaf; between these tubes and $t V_{c}$ epidermis four palisade cells are shown with chloroplasts (rounded, sho ded bodies) embedded in the cytoplasm lining their walls. The downwardbointing arrov s indicate the light energy which penetrates to and is absorbed by the chloroplasts. The gas interchange of photosynthesis and transpiration is indicated by the curved arrows.

brought, and fre' $n$ which the finished product is as constantly being tra - ported.

67. Method of the Leaf's Work. - The plan of the leaf and the method of its work will be still better understood by reference to the diagram of Fig. 37. For the sake of simplicity, only a few palisade cells covered by a small portion of the epidermis, and a sieve tube and water tube, 
are represented in the diagram. The water from the roots ascends the water tubes and passes by osmosis into the palisade cells and mingles with their sap. Since the sap bathes the cytoplasm which lines the cell wall, the water has easy access to the chloroplasts embedded in the cytoplasm (see page 36). The air with its carbon dioxide

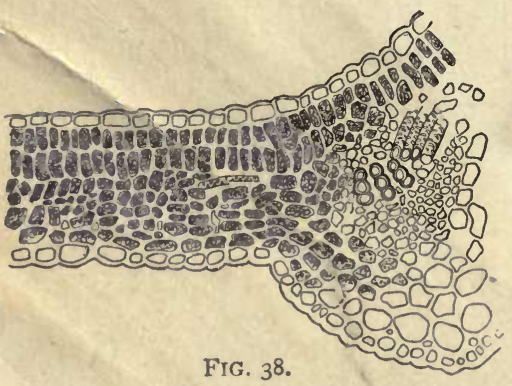

Cross section through leaf of Melilotus alba taken at sundown. The section has been treated with chloral hydrate iodine, and the dark granules in the cells are starch. enters through the stomata and fills the spaces between the palisade cells, and needs only to penetrate through their thin walls in order to come in contact with the chloroplasts.

The energy from the sun is readily transmitted through the epidermis to the chloroplasts, and the work of food making is begun. Under these conditions, the chloroplasts may become filled with starch in the course of an hour (see Fig. 38). The starch, which is the first food substance formed that can be detected by aid of the microscope, dies not long remain in the chloroplasts, but is made soluble, mainly in the form of glucose or grape sugar; and in this form, or in combination with compounds of nitrogen and sulphur to form proteids, it passes into the sieve tubes, and is carried down the stem and in part into the roots, or up the stem where buds are unfolding or flowers and fruits forming, being drawn from the sieve tubes and used for food wherever it may be needed throughout its course.

In the night, when the leaf can no longer obtain energy 
from the sun, the chloroplasts cease to manufacture starch, and, gradually becoming emptied of what they contain, by sunrise may show no further traces of it. Thus they begin each day's work unhampered by the products of their previous labors (see Fig. 39).

68. Manufacture of Proteids. - Starch and sugar represent only one class of food materials necessary to the nutrition of plants; the proteids, which contain nitrogen and sulphur and sometimes phosphorus in addition to the carbon, hydrogen, and oxygen composing starch and sugar, have yet to be accounted for. Proteids can be formed in any of the living cells, and in darkness as well as in light. They are evi-

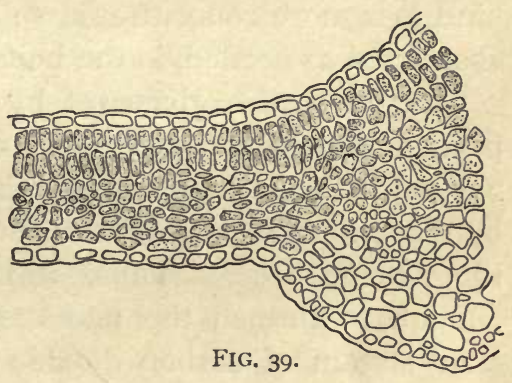

Cross section through leaf of Melilotus alba taken just before sunrise, treated with chloral hydrate iodine and showing that the starch has been removed during the night.

dently formed by the cy,toplasm of the cells from the elements of starch, combined with the compounds of nitrogen, sulphur, and often phosphorus, which have been abstracted from the soil by the roots. Since their manufacture can proceed in darkness, the energy for their production is evidently derived from the oxidation of sugar, and other soluble compounds obtained from the starch.

69. Transpiration, and Evolution of Oxygen.-Only a small per cent of the water entering the palisade cells is used in the manufacture of starch, the greater bulk of it being transpired into the intercellular spaces, and passing out of the leaf through the stomata. The openings in the 
stomata are exceedingly small, being only about $\frac{1}{178}$ millimeter in diameter (see Fig. 35), but this small size is compensated by the great number of the stomata, of which there may be, in different plants roughly estimated, from fifty to seven hundred in each square millimeter of epidermis. While the water is thus evaporated, the salts, brought into the plant in solution from the soil, are left behind in a more concentrated solution, from which they are abstracted as needed, in the building up of new compounds.

Oxygen is also given off by the leaf, and, for the most part, doubtless through the stomata; the source of this oxygen is the carbon dioxide which has been worked over by the chloroplasts, for they use only the carbon, and set the oxygen free. Thus, while the daylight is strong enough to furnish the necessary energy, there is a constant stream of carbon dioxide into, and of oxygen out of, the leaf; so that while the plant is forming its food, - and its food is likewise our food, - it is making the air purer for breathing. The process of starch formation by leaves under the influence of the sunlight is called photosynthesis - a putting together by light.

The diagram shown in Fig. 42 illustrates the mutual relations of root, stem, and leaf in the absorption, manufacture, and translocation of materials needed by plants.

70. Respiration. - After the sun goes down, photosynthesis ceases, and another process is found to be going on in the leaves; that is, the leaves are taking in oxygen and giving off carbon dioxide; this they were also doing during the daytime, but the oxygen evolved by photosynthesis predominates so much that the consumption of oxygen at that time is obscured. We see, then, that the leaves are taking in carbon dioxide and giving off oxygen during the daytime, and are taking in oxygen and giving off carbon 
dioxide all of the time, both day and night. This latter process, called breathing or respiration, is essentially the same in plants as in animals, and is as necessary to the life of the plant as to that of the animal. In the higher plants the oxygen for respiration enters chiefly through the stomata and through groups of loosely arranged cork cells, termed lenticels, which break through the epidermis in those parts where cork is being formed near the surface. The lenticels can be seen to good advantage as small rounded or elongated protuberances on many woody branches a year or more old. Since all living cells must respire, intercellular spaces are provided in which oxygen diffuses throughout the plant body. In aquatic plants these spaces are large enough to be seen with the naked eye (see the chapter on Adaptation to Environment). Although plants consume oxygen in their breathing, they give off so much more by the process of photosynthesis that the net result is a large addition of free oxygen to the atmosphere. While the process of respiration is common to both plants and animals, that of photosynthesis is peculiar to green plants.

71. Supply of Raw Materials. - Thus plants make their own food by the wonderful processes of tearing down and building up which take place in the chloroplasts. An important question in this connection is whether there is an unlimited amount of raw materials at their disposal. Water certainly seems unlimited for the larger part of the earth's surface. The necessary salts occurring in naturally rich soils are also practically inexhaustible, although they may not become soluble rapidly enough to satisfy all the demands of agriculture. Carbon dioxide occurs in the atmosphere in very small percentage, namely, between three and four parts in ten thousand of the atmosphere; 
and although it is employed by plants in making their food, it is constantly being replenished by the breathing of animals and plants, by the disintegration of plant and animal remains, by volcanic activity, and by the burning of wood and coal. Since carbon dioxide exists in such small percentage in the atmosphere, vast amounts of the latter must be sifted by the leaf before sufficient carbon can be obtained to build the body of a good-sized plant. A tree having a dry weight of, say, five thousand kilograms would contain twenty-five hundred kilograms of carbon, and to obtain this, twelve million cubic meters of air must have been deprived of carbon dioxide. The spaces between the cells of the palisade and spongy parenchyma allow a broad expanse of free cell surface for the absorption and giving off of gases, the free surfaces acting much as do the gills of a fish in absorbing the small percentage of oxygen from the water.

72. Action of the Stomata. - When there is sufficient light to enable the chloroplasts to do their work, there being at the same time plenty of water in the soil to satisfy the demands from the leaf, the stomata stand wide open (Fig. 40, $a$ ) and permit the ingress of carbon dioxide; but if the water supply is running low so that the plant is in danger of drying up, the stomata close (Fig. 40, b), even when the leaf is well illuminated. The stomata as a rule close in darkness, but rather from physical than physiological reasons. The conditions governing the action of the stomata appear to be about as follows: when the leaf is illuminated, the chloroplasts in the guard cells manufacture substances which become dissolved in the cell sap, and so alter its density and constitution. This results in an osmotic inflow from the neighboring tissues, which increases the turgidity of the guard cells, causing them to 
stretch and spread apart; but in the nighttime the chloroplasts of the guard cell no longer manufacture new material; the cells accordingly lose their turgidity and are drawn together again by the elasticity of their walls (see Fig. 40).

When the soil is dry, and the amount of water rising from the roots is much reduced, the guard cells probably lose water faster
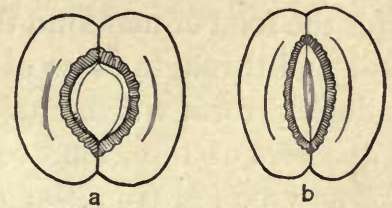
than they are able to draw it from surrounding tissues, and so are incapable of acquiring the degree of turgidity necessary to their opening. From such simple causes as these, the plant may allow the ingress of carbon dioxide when the conditions are such that it can be employed in food making, and may guard

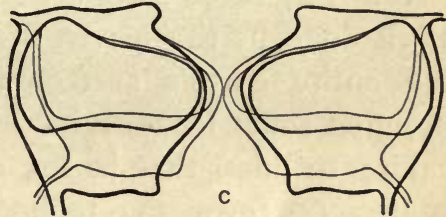

FIG. 40.

$a$, surface view of an open stoma; $b$, surface view of a closed stoma (after HANSEN) ; $c$, diagram of a transverse section of a stoma. The light lines indicate the closed position of the guard cells and the heavy line the open position (after SCHWENDENER). against too great loss of water when the supply of it is scarce.

73. The World's Food Supply. - When we consider photosynthesis from the standpoint of the world's food supply, it becomes a subject of supreme interest. The entire substance of seeds, tubers, bulbs, and roots, which directly or indirectly constitute the food of mankind, was only a few months past scattered in the form of water, soil particles, and gases of the atmosphere, no amounts of which could keep the world from starvation; and the continuance of animal life has been made possible only through the 
intervention of plants. The starch and proteids stored up in seeds, and particularly in the grains of cereals, are in quite stable condition, and are well adapted to carry plant life through months and even years of adverse conditions. Their stability and condensed condition also make them fit for the food of mankind through the months between harvests and through years of famine. It is this stable and condensed form which enables cereals to be transported to all quarters of the globe so that the people of one continent may be fed from the granaries of another.

74. Amount of Work done by Leaves. - The amount of work done by the leaves of plants in a single year over the entire earth's surface is beyond computation; but perhaps some idea of its immensity may be gained by calling to mind that in the state of Kansas alone, in the year I900, over I34 million bushels of corn and over 77 million bushels of wheat were harvested. Or, to take examples with smaller numbers, it has been estimated that one square meter of sunflower leaf may, in a single summer day, produce twenty-five grams of starch, and a single stalk of corn may in the same time send out into the ears from ten to fifteen grams of reserve food material.

75. Duration of Work of Leaves. - In some kinds of plants, such as tulips, peas, wheat, and corn, the leaves finish their work before the close of the growing season and die, having first given over to the seeds, bulbs, etc., most of their materials which could be used as reserve food. In other plants, such as our common trees and shrubs, the leaves continue their work until the close of summer. They then send over to the body of the plant, which is to survive the winter, much of the useful material contained in them; and then sever themselves from the branches by producing a layer of delicate tissue, so that 
they become torn away by their own weight, or are easily blown off by the wind. The separating layer of tissue is often of the nature of cork and then serves also to heal the wound. In evergreens, however, the leaves may remain on the branches for several years. Figure $4 \mathrm{I}$ shows a branch of pine bearing leaves some of which are three years old.

The fall of the leaf is a wise provision for the conditions of winter. When the ground is very cold or frozen, the roots are no longer able to absorb water from the soil, and if the broad transpiring surfaces of the leaves remained, the plant would

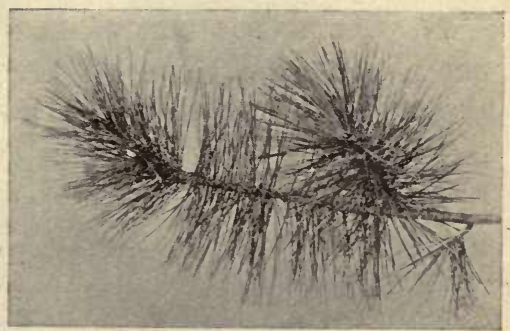

FIG. 4I.

Branch of Pine tree bearing leaves three years old. There are gaps between the leaves of each year's growth where the bud scales were.

suffer from too great loss of water; the weight of the snow also which would accumulate on the leaves would break the branches, as may sometimes be observed when early snows overtake the trees with their leaves still on.

76. Size and Form of Leaves. - Leaves show great variation in size and form. The leaves of mosses, for instance, are only a few millimeters in length and breadth, while those of the palm Raphia tadigera, growing in Brazil, have petioles from four to five meters long and leaf-blades from nineteen to twenty-two meters long and twelve meters broad. The student can at any time during the growing season find endless materials for the study of variations in leaf forms. We should not, however, look at the differences in leaf forms as expressions merely of the power to vary, for we may find that the form of the leaf is nicely 
adjusted to its size, position on the stem, and the habit and habitat of the plant. Indeed, it may be taken for granted that any member of an organism so important to the life of the individual and the continuance of the species as the leaf is would not be apt to show marked variations not in some way correlated with its functions.

77. Characterization of Leaves. - While the leaf is an outgrowth from the stem it does not grow indefinitely as stems do, but soon attains its maximum size. It seems impossible to formulate a definition that will clearly apply to all leaves; as a general characterization we might say, however, that leaves are lateral outgrowths, having buds or branches in their axils, arising in definite succession on a stem, limited in growth, and having for their chief function the manufacture of the plant's food. 


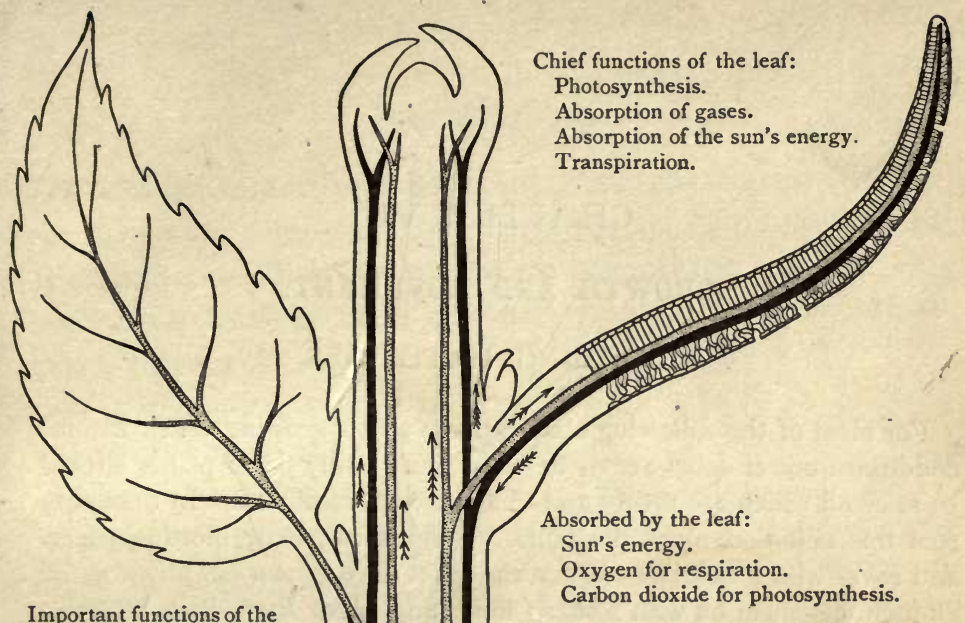
leaf in common with other plant parts:

Synthesis of proteids.

Respiration.

Digestion.

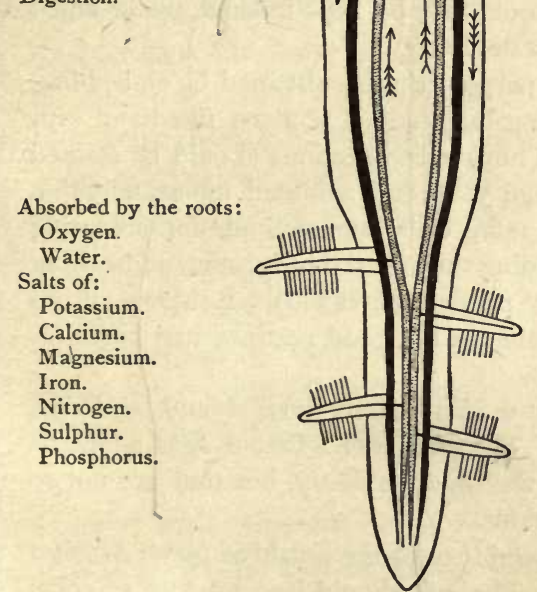

Given off from the leaf:

Oxygen from photosynthesis.

Carbon dioxide from respiration.

Water by transpiration.

FIG. 42.

Longitudinal diagram of the vegetative parts of a plant, with special reference to the absorption and translocation of materials. "In this diagram the dotted highway is the water-conducting area (xylem portion of vascular bundle), and the black highway (phloëm portion of the vascularbundle) is the area for conduction of the food made in the leaf. The arrows indicate the direction of flow in these highways. 


\section{CHAPTER VI.}

\section{GROWTH AND MOVEMENT.}

\section{PROVIDING MATERIALS.}

For most of the following observations, plants growing under natural conditions out of doors are to be employed. Only those plants should be selected which are thrifty and in a growing condition. In obtaining root tips of onion, place the bulbs on moist carpet or blotting paper, and cover with a bell jar. When the roots have grown out about $\frac{3}{16}$ of an inch, cut them off with a sharp knife and place them in a $1 \%$ solution of chromic acid in water for 48 hours; then wash in running water for half a day, and place in $20 \%$ alcohol for a few hours, and for the same length of time in $50 \%$ alcohol, and finally in $70 \%$ alcohol. After being prepared in this way the roots may be kept indefinitely in equal parts of alcohol, glycerine, and water.

Good sections for our present purpose can be obtained by embedding the roots in elder pith, and making longitudinal sections free-hand with a sharp razor (see page 377). Thin median sections should be stained in a solution of Safranin (see page 388), and mounted for examination in dilute glycerine. There are more elaborate methods for preparing uniformly thin sections by embedding the material in paraffin, and cutting the sections with a microtome (see pages $3^{8} 3$ and 386 ), but these methods are not necessary for this study, since a few good sections may serve for demonstration to the entire class.

In selecting stamen hairs of Tradescantia, only those should be chosen whose cell sap is still colorless. These hairs of different ages might be used in place of sections from the onion root tip, but they are not so satisfactory as well-prepared sections.

It is a simple matter to grow seedlings of the sensitive plant, Mimosa pudica, in pots under bell jars. The soil should be a mixture of equal parts of rich garden soil, sifted sand, and well-rotted manure. The pots should be kept in a warm place, and as soon as the seedlings appear, the bell jars should be raised at one side. The pots should be kept well watered. 


\section{OBSERVATIONS.}

95. With waterproof India ink make marks two millimeters apart on some rapidly growing stem, beginning at the apex and extending downward for several internodes. Observe from time to time whether the marks become separated farther by the elongation of the stem, and whether growth seems to be more rapid in one region than in another. Where does growth in length seem to have ceased? Compare with similar observations on the growth of roots.

96. By means of the simple apparatus illustrated in Fig. 43 observe the rate of growth of some rapidly growing stem. Compare theamount of growth in the daytime with that for the same number of hours in the nighttime.

97. By means of recorded measurements, compare the rate of growth of two plants

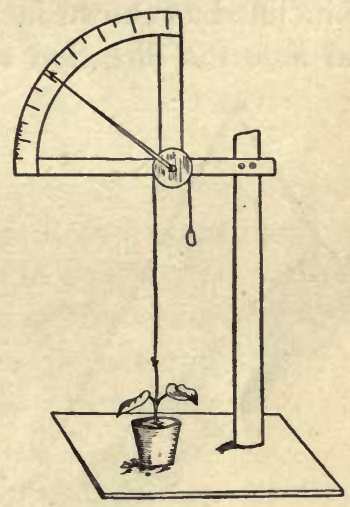

Fig. 43 .

A simple form of Auxanometer. A thread is attached to the apex of the plant and passed over the pulley and held taut by a weight which is only heavy enough to move the pointer as the plant elongates. The arc can be made of heavy manila paper and all other parts of light wood. of the same kind, one of which is in a dry soil, and the other under the same conditions, with the exception that it is kept well supplied with water. What conclusion do you reach as to the use of water in growth?

98. Cover one shoot of a plant which branches near the ground (such as the potato) with something to exclude the light, and leave the remaining shoots uncovered. After a few days compare the lengths of the newly formed inter- 
nodes of the shaded and unshaded shoots, and also the sizes of the leaves formed after the experiment was started. What is the effect of absence of light on the growth of stems and leaves?

99. Plant some seeds of Indian corn and garden bean in moist sawdust contained in a wire basket, which should be inclined as shown in Fig. 44. Keep the sawdust moist, and note the direction taken by the roots after they have

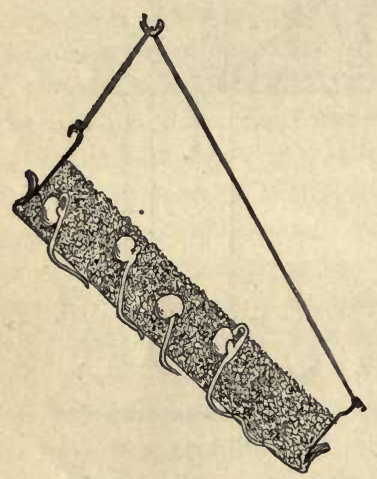

FIG. 44 . grown down through the meshes of the wire at the bottom. What does this experiment teach as to the probable behavior of roots under natural conditions?

I00. Bend over a vertical shoot of some thrifty plant, and fasten it down in such a way as to allow. freedom of action toward the apex. Observe the position finally taken by the stem and leaves. How do you account for the change of Experiment to show hydrotropism of roots. After SACHS. position observed, and what is its use?

IOI. Shade a plant so that it receives light on one side only. How do the leaves and stems behave, and to what purpose? Remove the screen after a marked change has been noticed, and note whether the different members assume their former positions.

I02. Invert a plant and notice the direction taken by its growing members. Do those parts which have ceased to grow change their positions? After a day or so set the plant upright and note whether the parts come back to their original positions.

103. Observe the behavior of the tendrils of plants 
growing under normal conditions out of doors. Try to determine what incites some tendrils to twine, and why the tendrils of Virginia creeper grow toward a support. Gently rub the tendrils of squash or wild cucumber on one side with a stick and watch for the result. Inclose some young shoots of Virginia creeper so that they are kept dark, and, after new tendrils have been formed under these conditions, note whether they grow in a different direction from that taken by tendrils which have developed under normal conditions of illumination.

I04. Note the positions of leaves of clover, Oxalis, Amorpha, etc., in the daytime and in the nighttime. Of what use are the observed changes in position?

105. Observe the direction taken by the stems of trumpet creeper. Is there any difference in behavior between those stems which bear leaves only and those which bear flowers as well as leaves?

I06. Grow in a greenhouse or under a bell jar seedlings of Mimosa pudica, and note the effect of touching the leaflets, or of shaking the entire plant. Do the leaflets change their position on being transferred from sunlight to shade, and vice versa? Do they have distinct positions for nighttime and daytime? Of what use to the plant are the actions observed?

107. Mount a young stamen hair of Tradescantia Virginica, or hairs from young portions of the stem of tomato or squash, in a drop of water on a glass slip, and examine under high power of the microscope for streaming motion of the cytoplasm. In removing hairs, some of the tissue to which they are attached should be taken with them in order to prevent their injury.

108. Cut a hole a trifle smaller than a coverglass in a piece of thick felt paper one inch square. Boil the paper 
for a short time in water, and place it at the center of a glass slip. Place at the center of a coverglass a small bit of rotting wood or bark on which is growing a slime mould plasmodium (see page 252). The piece of wood or bark should be so small that it will cling to the coverglass by its own moisture. Put the coverglass with the object downward over the hole in the felt paper, and set the preparation over a tumbler of water under a bell jar so that it will not become dry. If the experiment is successful, the plasmodium will grow out over the coverglass, and may be studied by transmitted light under high powers of the microscope. Make notes on the streaming of the protoplasm. The batterlike plasmodium can be found at almost any season of the year under the bark of moist logs or on decaying leaves which have gathered to some depth in damp woods.

\section{DISCUSSION.}

78. The Plant Cell. - When we examine very thin sections of the growing root tip of onion, for instance, under a high magnifying power, we find that they are composed of very small compartments, those near the apex being very nearly isodiametric, while those farther back are more or less elongated (Fig. 45, $A$ and $B$ ). If we were able to see through a whole root tip magnified to the same extent, we should find that each of these compartments is really a closed box to which, including its living contents, the term cell has been applied. The walls of the cells of the onion root tip are composed of cellulose, a substance well suited to form the walls of growing cells, for it can stretch and allow the cells to enlarge, and it permits liquids and gases in solution to pass through it readily. It is not alive, but has been manufactured by the live parts of the cell. The 
live part of the cell consists of the cytoplasm, a protoplasmic structure which occupies most of the cell cavity of young cells; the mucleus, which is suspended centrally in the cytoplasm in young cells, but as the cell grows older may have a lateral position (Fig.45); the leucoplasts, small dense bodies which multiply by division and have special functions recounted in the next paragraph; and the plasma membrane, a specialized part of the cytoplasm which lines the cell wall. (See Fig. I2 for details.)

\section{Functions of Cell} Organs. - The plasma membrane, cytoplasm, leucoplasts, and nucleus constitute the live part of the cell, and whatever is done by the plant as a living body is accomplished by one or more

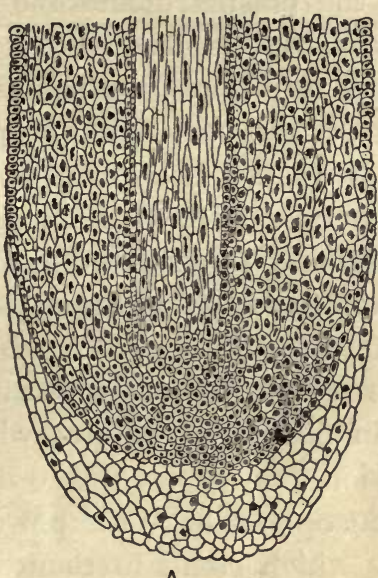

A

FrG. 45 .

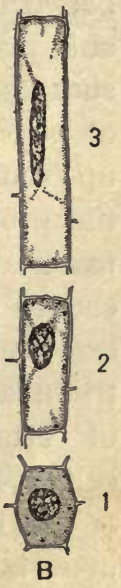

$A$, longitudinal section through the root tip of an Onion; $B$, successively older cells. $\mathbf{I}$, from immediately back of the root cap where cell division is going on; 2 and 3 , older cells, showing the modifications which I undergoes with age. In I the cytoplasm fills the cell cavity and the nucleus is relatively large. The few heavy points indicate leucoplasts. of these live parts or organs of the cell, which collectively are termed the protoplast. We have noticed in our study of roots that it is the plasma membrane which determines, to some extent, whether certain substances shall pass to or from the interior of the cell. It seems to be the guardian of the cell and the inspector of all material interchanges. It appears also to build the cell wall, and there is evidence to show that it is the receiving organ for stimuli from the 
outside world - light, heat, gravity, mechanical impact, etc., being probably first perceived and communicated by it to the other organs of the cell.

The cytoplasm is the medium of interchange of stimuli between the plasma membrane and the nucleus; it probably manufactures various nitrogenous food materials from substances furnished it by the chloroplasts, and from salts of nitrogen, sulphur, and phosphorus, which have come up from the soil. It appears to have something to do with the production of ferments which render the reserve food materials more soluble and diffusible; it is probably concerned with manifold changes of a chemical nature which are constantly taking place within the cell; and it contributes some of its own substance toward the production of certain fibrillar structures which assist in nuclear and cell division.

The leucoplasts have the power of forming starch from materials which come to them from the leaves; or when they are exposed to the light they may produce chlorophyll within themselves, and become chloroplasts; or they may produce other coloring substances than green, as seen in certain flowers and ripening fruits, and then they are termed chromoplasts. Leucoplasts, chloroplasts, and chromoplasts collectively are called plastids.

To the nucleus, in particular, is intrusted the very important function of bearing and bequeathing from generation to generation the inheritable qualities. That the embryo in an acorn shall develop into an oak instead of into another kind of plant depends in large measure on the nuclei in the cells of the embryo. The nuclei, as the bearers of the inheritable qualities, must determine how the cells shall behave under varying conditions. The nucleus has to do also with processes involving chemical changes, such as the 
formation of starch and proteids, the production of secretions, the growth of plasma membrane, and building of cell wall. While it is not absolutely known that the parts af the cell have the distinct functions here assigned to them, yet the circumstantial evidence that they do amounts almost to proof.

We find, then, that we may look upon the living cells of the plant body not only as units of structure, like the bricks of a house, but as centers of vital activity which induce and regulate whatever the plant does.

80. Advantages of Cellular Structure. - The construction of the plant body from many small cells has certain distinct advantages: it renders the body stronger, lessens liability to fatal injuries, and makes division of labor possible, so that one part of the body may protect the other parts; one part may give strength to the whole body, while other parts may be adapted for transporting or manufacturing materials, etc. Thus the business of the plant is economically and efficiently carried on.

81. Continuity of Living Substance. - While the live part of the plant body seems to be divided by the cell walls into numberless units, it is probable that these are united by minute strands of living substance, which are difficult of demonstration by means of the microscope; their existence, however, has actually been demonstrated in many cases. We are justified in the conception that the whole live body of the plant stands united by living substance, from the farthest roots to the remotest buds, although apparently severed by numberless cell walls.

82. Cell Division. - The enlargement of the plant body depends upon the multiplication and enlargement of its cells. Multiplication of the cells is brought about by their division, one cell becoming two by the formation of a new 
partition wall, and so on. In this process the parent cell must distribute to the two daughter cells resulting from its division all of the qualities and powers possessed by itself ; and to accomplish this it is important that the division of the nucleus in particular should be equal, one daughter cell receiving neither more nor less than the other. We are able to see, in following the steps in the division of a cell, that the equal partition of the nucleus is actually accomplished with astonishing care (see Fig. 46).

The nucleus becomes threadlike in structure (I); the thread is divided longitudinally through the middle, and then the double thread is broken transversely into several double rods (2); the double rods are now grouped at the equator of the cell, namely, at the central plane, where the dividing wall is to be formed ( 3 and 4 ); then the two halves of each double rod are drawn apart to opposite poles of the mother cell $(5,6$, and 7$)$, where they fuse together to form two daughter nuclei (8). Finally a wall is formed which cuts the mother cell into two daughter cells ( 8 and 9 ). This will serve as a general statement, but the details of the process are so wonderful that they have been given more fully in the description of the figure.

If we go back to the beginning in the life history of one of the higher plants, we find that it is, at first, a fertilized egg cell, of which we shall learn more in the chapter on the flower. The plant has, therefore, its beginning in a single minute cell; this divides, and its offspring in their turn divide, until the whole plant body, consisting of millions of cells, is formed by its descendants.

83. Cell Growth. - After the cells have divided, the daughter cells increase in size, and not till then does an enlargement of the plant take place. The increase in size of the cells is brought about by absorption of water until 


\section{Growth and Movement.}
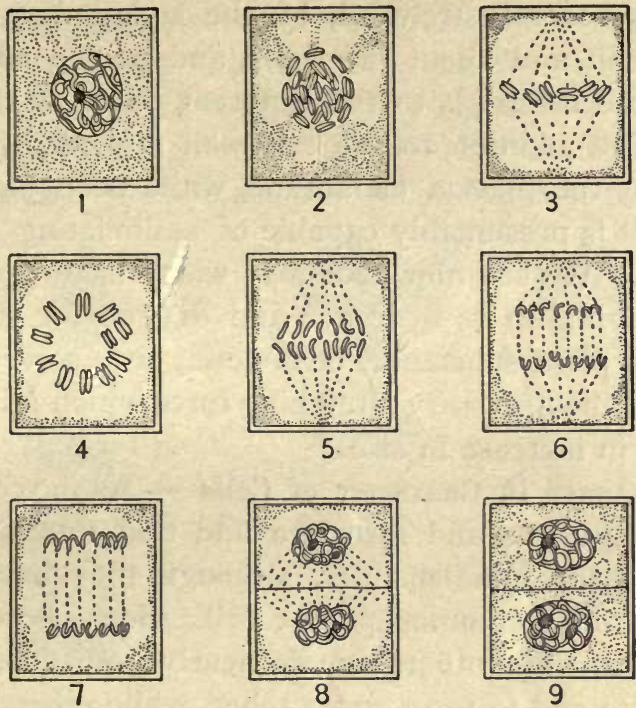

FIG. 46 .

Diagrammatic representation of Nuclear and Cell Division.

I. A cell with resting nucleus just previous to division. The cytoplasm (stippled) fills the cell cavity. The nuclear thread is shown as a tortuous band throughout the nucleus; the black body in the nucleus is the nucleolus.

2. The nuclear membrane and the nucleolus have disappeared, the nuclear thread has become divided transversely into distinct bodies termed chromosomes, and these are seen to be divided longitudinally.

3. The chromosomes have become lined up at the equator of the cell and protoplasmic threads converge from them toward opposite poles.

4. The same as 3 seen from one of the poles.

5. The longitudinal halves of the chromosomes are moving toward opposite poles.

6. A later stage, showing connecting protoplasmic threads between the receding chromosomes.

7. The chromosomes arrived at the opposite poles are fusing together end to end to form nuclear threads.

8. The nuclear threads have assumed the form of two daughter nuclei, nucleolj have appeared, the connecting threads have spread from wall to wall, and a new cell wall dividing the mother cell in halves is being formed, apparently by the connecting threads.

9. A nuclear membrane has been formed about the daughter nuclei, the connecting threads have disappeared, and nuclear and cell division is completed. 
the walls become stretched, by the laying down of new materials in and about the walls, and by the addition of appropriate materials to the different parts of the protoplast. The addition to the cell wall is evidently accomplished by the plasma membrane, while each part of the protoplast is presumably capable of assimilating materials for itself. We see how necessary water is to growth, not only in giving up its hydrogen and oxygen for the manufacture of plant substance, or in acting as a solvent, but in supplying the necessary stretching force which initiates the first step in increase in size.

84. Changes in Character of Cells. - As the cells attain their definite size and form, we find that they do not all behave in the same manner, although they have all descended from a common parent cell. Some become long and fibrous, while others remain nearly isodiametric; some fuse end to end to form water tubes, while others infiltrate their walls with waxy substances to keep water from passing through them. The cells have come to act so differently because they have been under different conditions. Those which are on the outside exposed to the air have not the same surroundings as those which lie at the interior, and the various zones of interior cells are under different conditions of exposure to air, and of tension, pressure, etc. Thus the unlike action of cells which have descended from a common ancestor may be in part acounted for.

85. Regions of Continued Growth. - In dicotyledonous plants, such as the oak, maple, elrn, etc. ( $B$, Fig. 47), the regions of continued growth lie at the apices of roots and shoots, in the cambium zone which separates the bark from the wood, and in certain zones of cells called cork cambium which give rise to the cork of the bark. In monocotyledonous plants, such as grasses $(A)$, palms $(C)$, etc., there 


\section{Growth and Movement.}

is no cambium zone similar to that in dicotyledonous plants; but in such monocotyledonous plants as the palm and smilax $(C)$ a zone of cells near the periphery of the stem remains in a dividing condition indefinitely, and thus adds to the diameter of the stem, some of the cells of the periph-
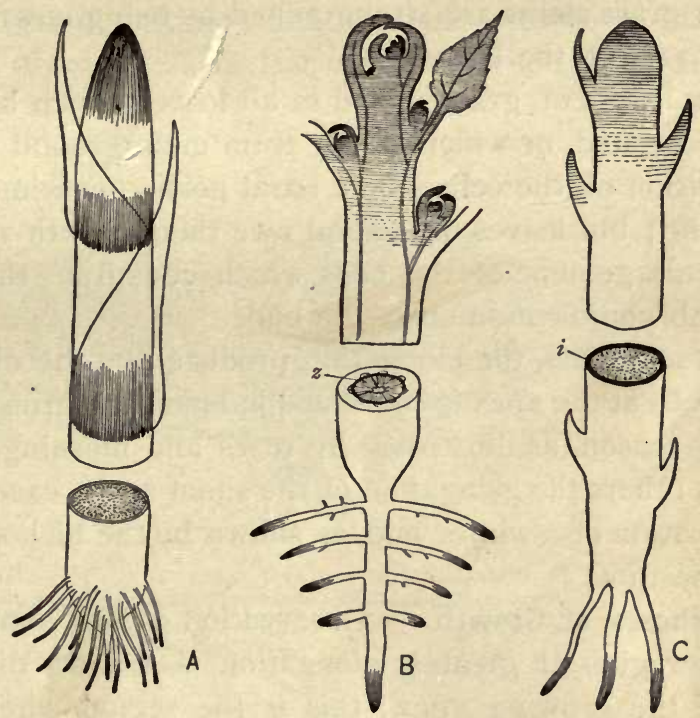

FIG. 47 .

Diagrans showing regions of continued growth in dicotyledonous and monocotyledonous plants. 'The shaded regions are capable of growth. $A$, a Monocotyledon of the grass type, growth taking place at the apices of the stem and roots and at the bases of the younger nodes; $C$, a Monocotyledon of the palm and lily type, the apices of the stem and roots and a zone $(l)$ near the periphery being in a growing state; $B$, a Dicotyledon, growth taking place at the apices of the stem and roots and at the cambium ring $(z)$.

eral zone undergoing the necessary modifications to form new vascular bundles.

In such monocotyledonous stems as those of grasses $(A)$, increase in length is also brought about by the division of the cells at the base of each node, which retain the power 
of division for a long time. It is a matter of common observation that grass stems are easily pulled apart at the nodes, and that the place of rupture is tender, succulent, and sweet; these are all characteristics of regions where cell division is rapidly going on. The tender bases of the nodes of grass stems are strengthened by being enwrapped by the bases of the leaves.

In the leaves of grasses, and in all leaves which become much elongated, or which spring from underground bulbs, etc., division of the cells of the basal portion continues for some time; but leaves in general owe their growth mainly to the enlargement of the cells which constitute them in their embryonic condition in the bud.

In some plants, the elongation produced by the division of the cells at the apex is continued indefinitely through the growing season, as illustrated by roses and morning-glory, while in others the elongation of the shoot soon ceases with the formation of a winter bud, as shown by the hickory and horse-chestnut.

86. Phases of Growth. - Observation 95 has shown us that the region of greatest elongation is a short distance back of the growing apex; this is the region where the daughter cells, produced by cell division, are increasing in size. Back of this region, elongation has ceased, and thickening of the cell walls and changes in their chemical constitution and in the condition of the protoplasts are taking place. We might, therefore, speak of three phases of growth: (I) the phase of cell division; (2) the phase of cell enlargement; (3) the phase of cell modification.

87. Conditions Necessary to Growth. - The conditions necessary to growth are essentially the same as those requisite to the germination of seeds, which is, in reality, simply a resumption of growth. Water must be at hand in suffi- 
cient quantity to render the cells turgid; food materials must be available; there must be a certain amount of external energy in the form of heat; oxygen must be present for the process of respiration, resulting in the setting free of internal energy, without which the life of the plant would become extinct; finally, there must be an inclination to cell division. This last condition is itself dependent, to

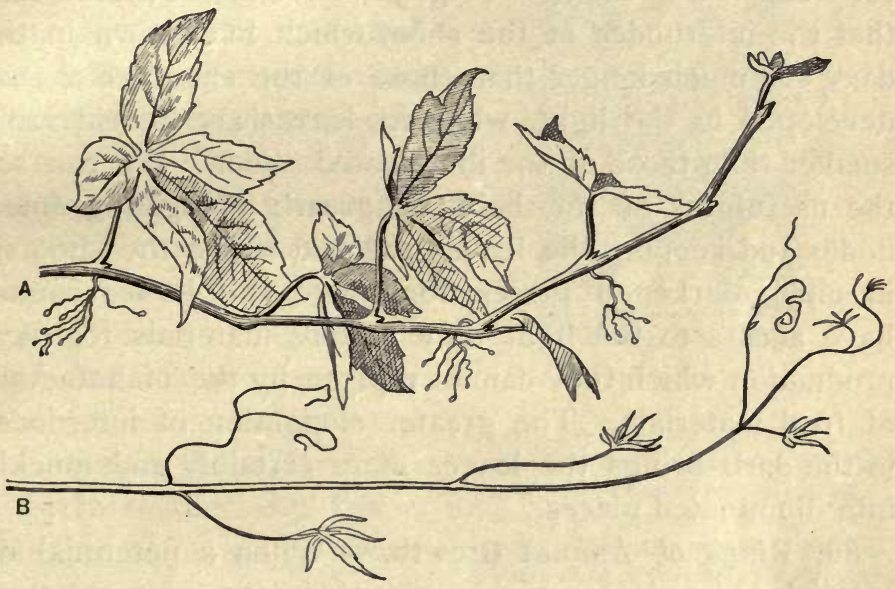

FIG. 48.

$A$, a shoot of Virginia creeper growing in the light. $B$, a shoot from the same plant growing in partial darkness.

a certain degree, on the others; but, even when these are favorable, the inclination to cell division does not always result, as we see in the cessation of growth of hickory shoots in the height of the growing season, and in the limited growth of embryos in forming seeds. The causes for this behavior are inherent in the nature of the cell, and are faithfully transmitted by cell division from generation to generation. 
88. Influence of Light and Gravity. - While light and gravity are not necessary to the immediate processes of growth, they do influence the direction and character of growth of the plant members, as we have already seen. Light has much to do with the size and form of the members. Compare, for instance, a shoot of Virginia creeper which has grown in the dark with one which has grown fully exposed to the light (Fig. 48). We see in such a case that the internodes of the shoot which has grown in the dark are much longer than those of the shoot which has developed in the light, while its leaves are considerably smaller than those of the illuminated shoot. We can see the usefulness of the habit of greatly elongating internodes and keeping the leaves reduced where the shoot is in much darkened places, for where the leaves cannot have access to the light they require materials for their production which they cannot replace by the manufacture of food materials. The greater elongation of internodes in the dark brings the leaves more certainly and quickly into illuminated places.

89. Rings of Annual Growth. - When a perennial dicotyledonous plant resumes growth in the spring, it produces more branches and leaves than it possessed the previous year. This is characteristic of branching perennial plants. In consequence of the increased transpiring surface and weight of the crown, more demands are made on the stems and roots, for they must be, stronger, and they must be able to conduct larger supplies of water to the leaves. Here is where the usefulness of the cambium ring is shown; for while the leaves and branches are being formed, the cells of the cambium ring are dividing and adding to the thickness of the stems and roots. The tissues which are first formed by the cambium are of a nature 
to meet the most pressing demand at the time; namely, for a greater water supply for the increased transpiring surface of the leaves. Accordingly we find that a great many water tubes are first formed (see Fig. 49), which communicate with the veins of the leaves and with the water tubes running out into the newly formed rootlets.

The water supply having been provided for, the cambium cells next produce wood fibers in much greater proportion. At the close of the season we find on examining the year's growth that it consists of a zone of tissues $(s)$ in which the water tubes predominate, followed by a zone $(t)$ which is more dense and firm because the water tubes in it are smaller and fewer, and the thick-walled wood fibers more in evidence. A single ring of annual growth consists then of a zone of early growth and a zone of

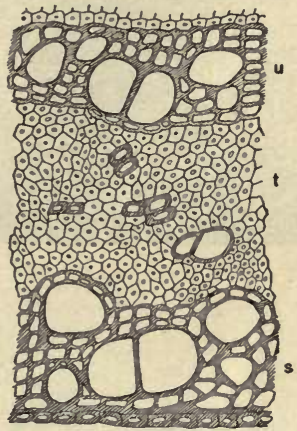

FIG. 49 .

Constitution of a ring of growth. $s$ and $u$, early growth; $t$, late growth. The lowest row of small cells in $s$ belongs to the late growth of the previous year. The early growth here consists chiefly of the tracheal tubes and wood parenchyma and the late growth chiefly of wood fibers. $s$ and $t$ constitute an annual ring. After HABERLANDT. later and denser growth, each zone having its own peculiar significance in the economy of the plant. The cambium ring makes annual additions to the bark as well as to the wood, but in much less quantity, and the additions are not demarked into rings of growth.

90. Plants without Annual Rings. - In perennial monocotyledonous stems, which branch but little or not at all, the crown of leaves does not increase materially from year 


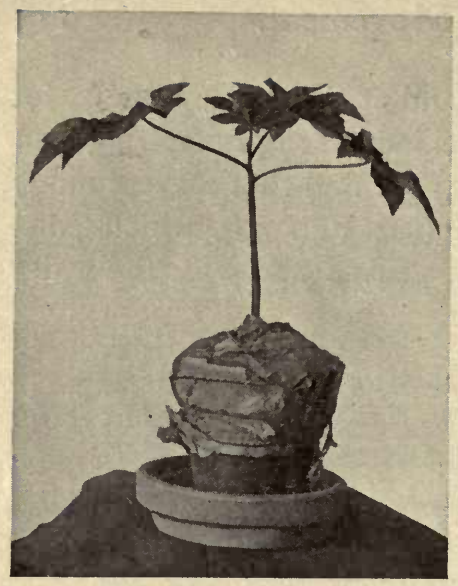

FIG. 50 .

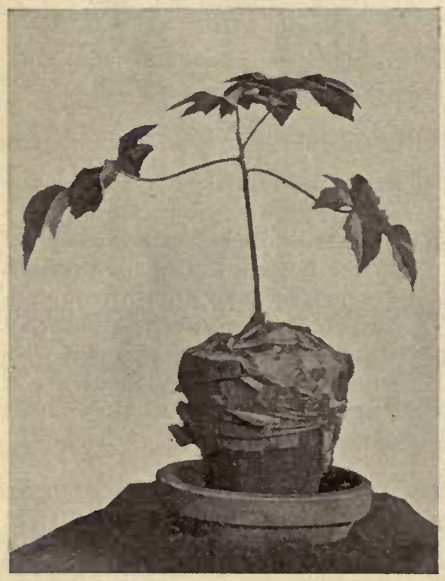

- FIG. 52.

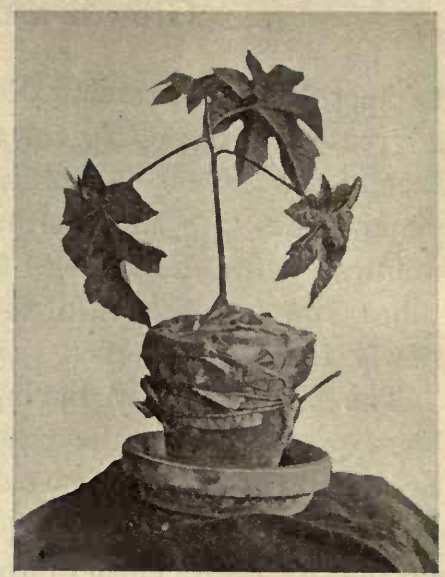

FIG. 5 I.

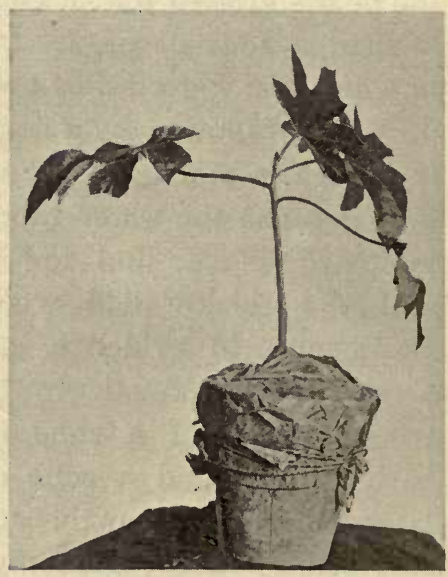

FIG. 53 .

Fig. 50. Castor bean grown under equilateral illumination.

Fig. 5I. The same plant after some hours of slow revolution on a universal axis.

Fig. 52. The same plant after a few hours of equilateral illumination, following the condition shown in Fig. 5 I.

Fig. 53. The same plant after a few hours of one-sided illumination, following the condition shown in Fig. 52. 


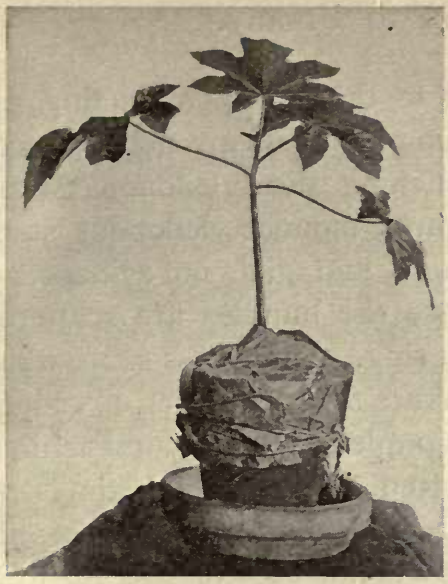

FIG. 54.

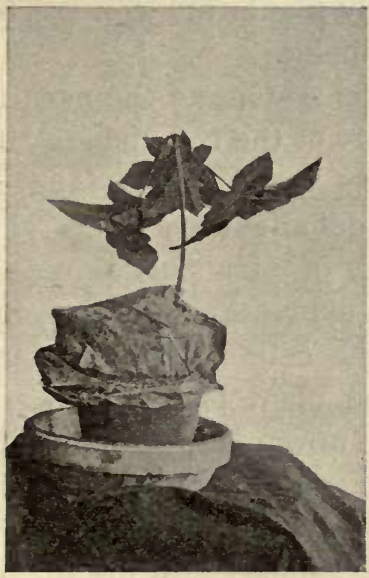

FIG. 56.

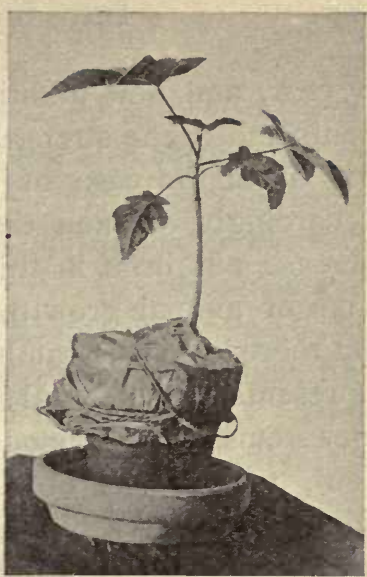

FIG. 55 .

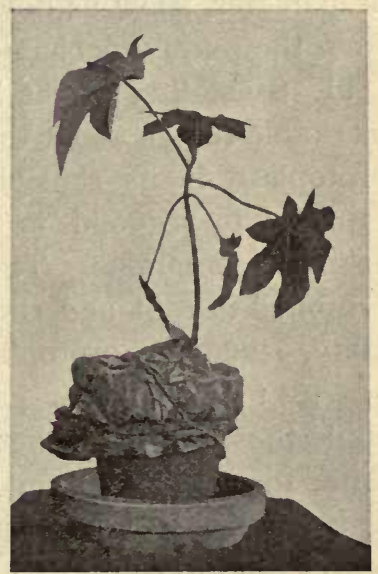

FIG. 57.

Fig. 54. The same plant after a few hours of equilateral illumination, following the condition shown in Fig. 53.

Fig. 55. Another castor bean plant grown under equilateral illumination.

Fig. 56. The same plant as shown in Fig. 55 after being inverted for a few hours under equilateral illumination.

Fig. 57. The same plant after being set erect for a few hours under equilateral illumination, following the condition shown in Fig. 56. 
to year, and new additions to the water-conducting elements and to the strengthening elements are not so necessary as in the case of branching dicotyledonous stems. Accordingly, we find that most monocotyledonous stems increase but little in thickness, and give no evidence of rings of annual growth, since the bundles are scattered, and there is no true cambium ring. The grass stem, which represents in its general structure a vast number of endogenous stems, is a marvelous example of architectural achievement, for its height is often five hundred times its diameter. If Washington Monument were built in like proportions its base would cover an area of less than one square foot. Stems of this sort, which increase but little in diameter, show us how perfectly plants are constructed from the purely mechanical standpoint.

91. Sensibility of the Protoplasts. - One of the most remarkable things about the live part of the cell, namely, the protoplast, is its sensibility to its surroundings, and its capacity to respond in certain definite ways to varying external conditions. The polarity of plants, as we see it exhibited in root and shoot, is an expression of this sensibility, since the protoplasts of the cells of the root use gravity to guide their part of the plant body downward, while those of the shoot find the upward direction by the same force. The stem of the trumpet creeper finds its way to a support through the perception of light, while the leaves are directed away from the support and toward the light by the same means.

If, by any accident, a plant becomes overturned or bent out of its normal position, the protoplasts perceive the altered relation to light and gravity, and cause the growing members to shift their positions into proper relations to these forces. Figure 50 is a photograph of a young castor 
bean plant which has been grown in a pot, and kept equally illuminated on all sides. Figure 5 I shows the same plant after revolving for eighteen hours on both horizontal and vertical axes at the same time, so that both light and gravity as a guide were useless to it. Its protoplasts appear to have perceived its abnormal condition, and have changed the position of the leaves; having no guide, however, only fruitless curvatures have resulted.

Figure 52 represents the same plant after standing eighteen hours in a normal position under equilateral illumination; the protoplasts have found their direction again, and have placed the leaves more nearly in their wonted position. Figure 53 represents this plant after being next illuminated on one side only for twenty-four hours; the protoplasts of both stem and leaves have attempted to bring the leaves into position for receiving as much as possible of the incident light. Figure 54 shows the same plant again after being equally illuminated on all sides for about twentyfour hours; since there was the same degree of light intensity on all sides, gravity has evidently been employed to bring the parts back from their one-sided position.

Figure 55 is a photograph of another plant which has grown in a pot under normal conditions. Figure 56 represents the same plant after standing inverted for about eighteen hours while kept equally illuminated on all sides by rotation on a vertical axis; a very successful attempt has been made to bring the leaves and stem back to their normal relations to light and gravity. (To see this to best advantage hold the book upside down.) Figure 57 shows the same plant again after standing erect under equilateral illumination, the stem and younger leaves having assumed approximately their original positions. 
92. Greater Sensibility of Growing Members. - In comparing these photographs, it is seen that the younger portions of the stem and the younger leaves are more responsive to the reversed position or to unilateral forces. It appears that, in the older members, the protoplasts may be less sensitive to external forces, and that it is a more difficult matter for the older and somewhat rigid parts to change their positions.

93. Cause of Movements. - The movements which we have just observed are due to a more rapid growth on one side of the leaves and stem than on the other. When the plant is inverted, the cells on the lower side of the petioles and leaf blades and on the lower side of the stems increase in size more rapidly than those of the upper side, and there is accordingly a general bending of these parts upward. When the plant is illuminated on one side more than on another, growth is most rapid on the side remote from the source of greatest illumination. When a plant which has made a one-sided growth under such circumstances is again placed so that it is equally illuminated on all sides, its parts draw back toward their normal position, provided they are not too old for growth; but in order that this may occur, growth must take place on one side more than on another, although all sides are now equally illuminated. It appears that in such cases gravity is used as a guide, and that the protoplasts persist in causing unilateral growth until the stems and leaves stand in their normal positions. The experiment illustrated in Fig. $5 \mathrm{I}$ is very instructive, for it teaches that when the directive influence of all exterior forces is removed the protoplasts are no longer able to determine a definite position for the stems and leaves.

94. Heliotropism. - As we have seen in the study of leaves, the interception of sunlight is of vital importance 
to plants, and therefore light is the most feasible guide in determining the position of leaves and leaf-bearing branches. Since, however, the source of greatest illumination hourly changes as the sun advances from east to west, it is plain that if the position of leaves is fixed once for all, the best possible light relation is not attained. The behavior of

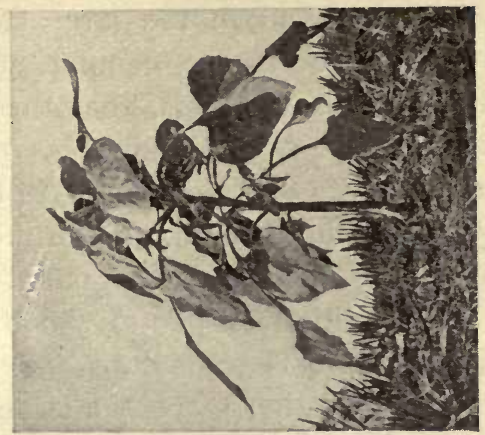



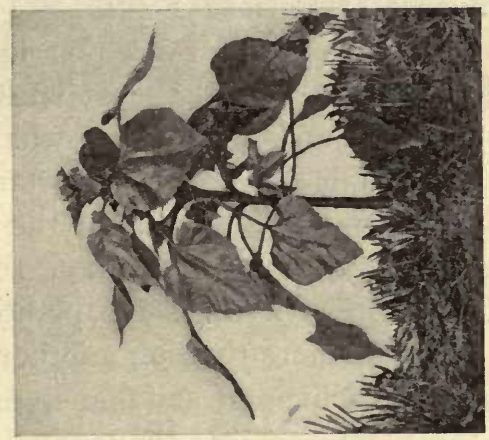

a rapidly growing sunflower shows how plants may take account of the shifting light. The three photographs of Fig. 58 show the same plant as it appeared in the

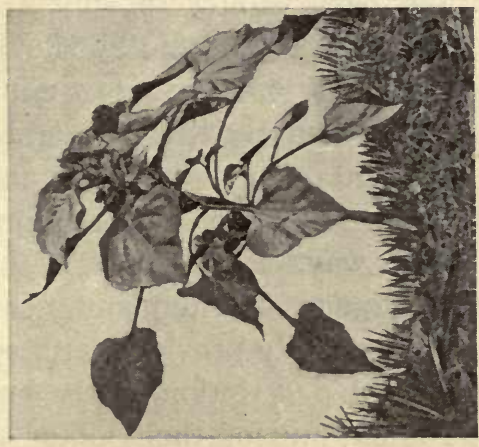

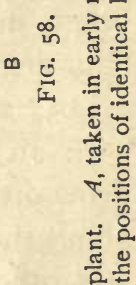

苋芯

हี ฮึ

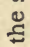

4

है 
morning, at noon, and in the late afternoon of a single day. It can be seen, by comparing the same leaves in the different photographs, that they were incessantly striving to intercept as much light as possible by keeping their broad surfaces at right angles to the changing direction of greatest illumination. Such movements, and any others caused by light, are designated as heliotropic, and the state or condition of plants which makes heliotropic movements possible is termed heliotropism. The student may observe many other plants which exhibit high degrees of motility of this kind.

95. Influence of Various Forces. - The movements exhibited by plant members, for which light and gravity are the guiding forces, are of the most vital significance to the plant; but there are movements which are directed by other means. Observation 99 has taught us that roots may disregard gravity and turn in the direction of moist areas; and we saw by Observation IO3 that by contact with an object, tendrils of some plants may be made to grow on one side more than on another so that they twine about the object if it is of suitable size and shape. There are other movements due to unequal growth, exhibited chiefly by the parts of flowers, which are induced by varying degrees of light and heat. The flowers of Tulip, Crocus, Colchicum, etc., will open in a few minutes if the temperature is raised to a marked degree, and at a constant temperature they will open in the light and close in the dark. It is a matter of common observation that the dandelion behaves in this way.

96. Motor Organs. - Old plant members which exhibit movements are usually provided with special structures for that purpose. A description of the behavior of the leaves of scarlet runner, and of their motor organs, will serve to 
illustrate this. Figure 59 represents a leaf in its night position; at $a, b$, and $c$ (Fig. 60) are the motor organs. The positions of the motor organs of the leaflets are shown for the nighttime at $B$, and for the daytime at $A$. Cross sections of a petiole and of a motor organ are shown at $C$ and $D$.

It is seen that while the petiole is made rigid by the disposition of its vascular bundles in a circle outside the center, as seen at $C$, and by the winglike outgrowths containing each a vascular bundle $g$, the vascular bundles in the motor organ are

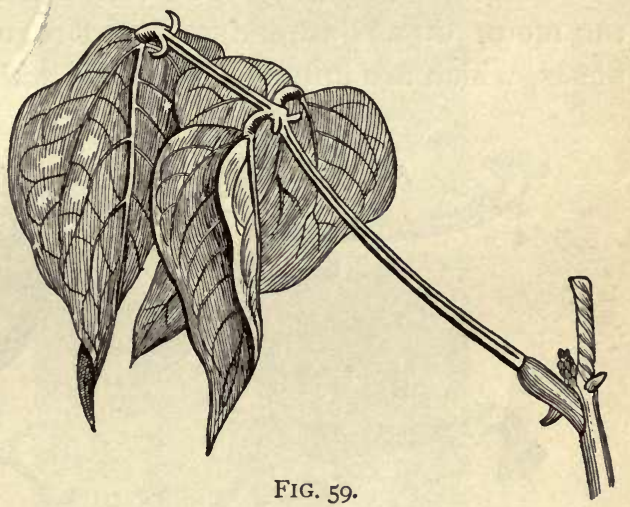

Leaf of Scarlet Runner in its night position. After SACHS.

all thrown to the center; and surrounding them is a relatively broad zone of thin-walled parenchymatous tissue which is capable of undergoing great variation in the turgidity of its cells. In the nighttime the upper portion of the parenchymatous tissue of the motor organ of the petiole becomes less turgid than the under portion, and the leaf as a whole rises in consequence; but at the same time the motor organs of the individual leaflets become less turgid on the under side, and the leaflets are made to droop. In the daytime the motor organs of the leaflets become less turgid on their upper sides, and the leaflets rise in consequence, while the motor organ of the petiole becomes less turgid on its under side and the leaf as a whole drops to a lower plane. 
Such movements are in harmony with the functions of the leaf, for the leaflets are spread out to receive the light necessary to photosynthesis in the daytime, and are folded together in the nighttime so that they are less apt to receive injury from too great radiation of heat or from the beating of storms. The variations in the turgidity of the motor organs are induced and regulated by the protoplasts, which are influenced in their action by variations in

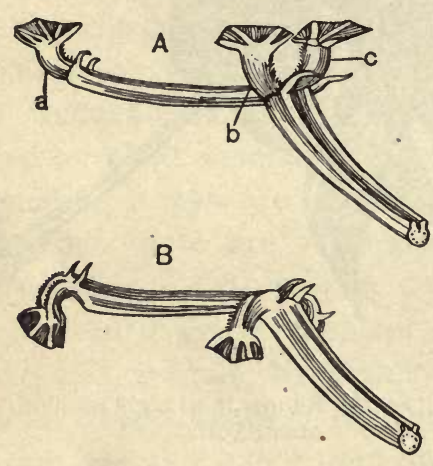

FIG. 60.
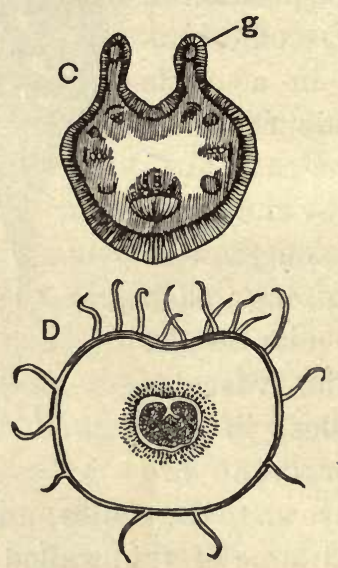

Motor organs $(a, b$, and $c)$ of Scarlet Runner in their day position at $A$, and in their night position at $B . C$, a cross section of a petiole, and $D$ of a motor organ. After SACHS.

light intensity. Periodic movements induced by alternations of day and night may be observed in the oxalises (commonly represented by the violet and yellow wood sorrel) and in many members of the pulse family.

97. The Sensitive Plant. - Movements of the leaves of some plants may be induced by contact with a solid body, by shaking the entire plant, by intense illumination, or by chemical stimulus, etc. The sensitive plant, Mimosa pridica, 
affords a most notable example. Figure 61 shows photographs of a seedling of this plant under different conditions: $A$ represents the plant in diffuse light, with its leaflets spread out to catch the light; $B$ shows the leaflets somewhat folded together as the result of a short exposure to direct sunlight; in this position the leaflets stand more nearly parallel with the incident light; $C$ exhibits the plant directly after being shaken by a strong wind.

Figure 62 represents a leaf of a mature sensitive plant in its open position at $A$, and in its closed posi-
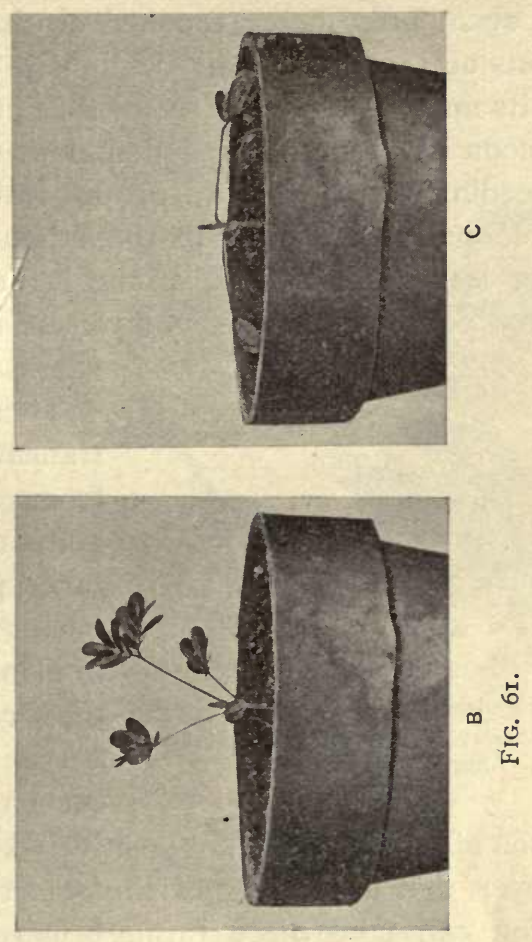

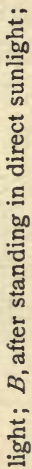

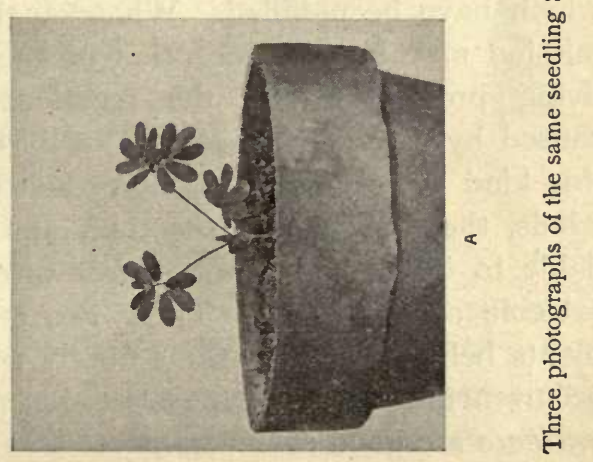


tion at $B$, after being touched or shaken. If one of the leaflets of the upper pair be lightly touched, it will rise, and its mate will quickly rise with it; the next pair of leaflets soon fold together in a similar manner, and so on with succeeding pairs, until all of the leaflets of a secondary petiole have responded to the stimulus received by the upper leaflet; indeed, all of the leaflets on all of the secondary petioles may respond to a stimulus received by a

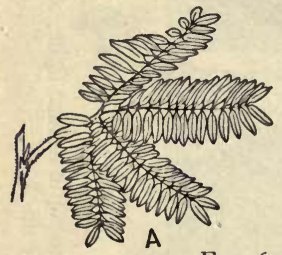

FIG. 62.

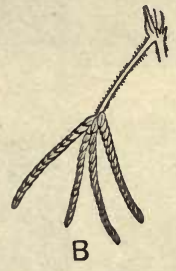

$A$, mature leaf of the Sensitive Plant fully expanded; $B$, the same after stimulation. After Duchartre. single leaflet.

There is, therefore, a transmission of a stimulus from one part of the plant body to another, such as takes place in animals by means of the nerves; but in plants there are no structures corresponding to the nerves of animals, and the exact manner of the transmission of a stimulus is not positively known. It is known, however, that in the case of the sensitive plant the transmission can take place through portions of the petiole which have been killed. Whatever the method of transmission may be, the reception of the stimulus is by the living protoplast, and the reactions which follow are caused by it. The benefits to be derived from actions of this kind are obvious : in rain or hail storms, or in strong winds, the leaflets fold together, and become much less liable to injury. Mimosa pudica affords an example of extreme sensitiveness. There are many other sensitive plants belonging to the same family which are of common occurrence; Cassia chamecrista and nictitans and Schrankia uncinata are good examples. 
98. Action of Venus's Flytrap. - In Venus's flytrap, Dionea muscipula (Fig. 63), and in sundew, Drosera rotundifolia (Fig. 65), we find still more wonderful sensibility and transmissions of stimuli. The two halves of a leaf of Venus's flytrap are capable of closing together as if they were hinged along the median line (see Fig. 64). The margin of each half is provided with from 12 to 20 teeth, and at the center of each half there are three hairs and numerous rose-

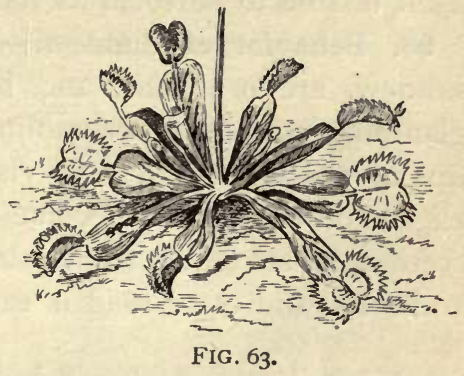

Dioncea muscipula. Some of the leaves stand open, and others are closed after stimulation by contact with insects. After KERNER. colored glands. The three central hairs are specially concerned with the reception of stimuli, for although the leaves are impassive to contact at other places, when any of the hairs have been touched, the two halves close up rapidly until the marginal teeth are interlocked. The hairs are sensitive in this way to solid bodies, but not to the wind and rain. When an insect has been caught by the closing leaves, the glands on each half pour out a digestive ferment, and the insect is held until

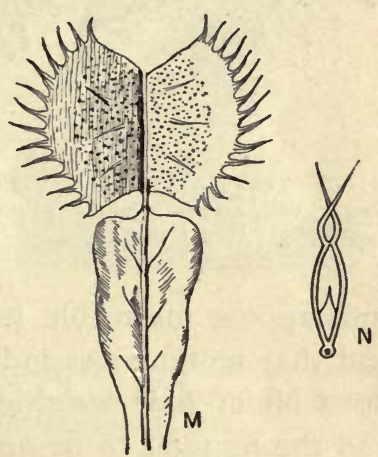

FIG. 64.

$M$, leaf of Dionca muscipula; $N$, cross section of the trap part of the leaf in its closed position. After KERNER. it is digested and absorbed, this process requiring from one to two weeks, according to the size of the insect. Then 
the leaf slowly opens and is ready for another victim. However, if the insect digested is large, the leaf may find itself unable to perform its trap function a second time.

99. Behavior of Sundew. - The leaves of Drosera, or sundew, are orbicular, and bear on their upper surface glandular structures resembling tentacles (see Fig. 65), the tips of which exude a clear viscous fluid. When an insect alights on the leaf it becomes entangled in the viscid excretion, its struggles excite the glands to greater activity, and more and more fluid is exuded; at the same time the

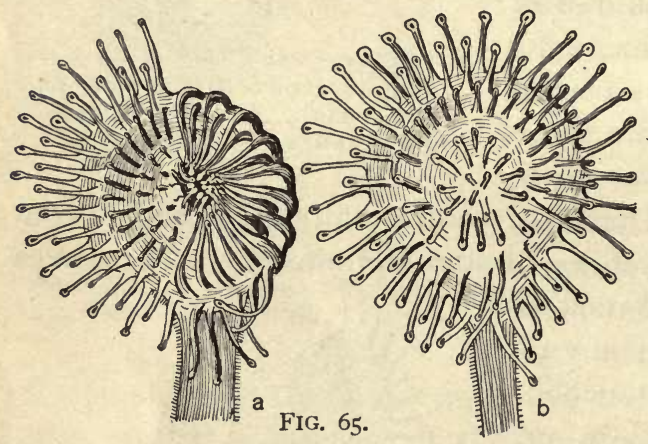

Leaves of Drosera rotundifolia. $a$, after stimulation by contact with an insect, and $b$, with all of the tentacles expanded. After KERNER. tentacles bend down over the insect, and render escape more difficult. The viscous fluid has the nature of a digestive ferment, and by it the insect is rendered soluble, and in this condiition is absorbed by the leaf. The glands appear insensible to falling raindrops, but Darwin found that motion was induced when he placed on a tentacle a bit of hair weighing only $\frac{7 \frac{1}{7} 4 \overline{0}}{0}$ of a grain. $\mathrm{He}$ found the tentacle to be quite sensitive also to very dilute solutions of nitrogenous salts; they bent downward completely when the leaves were immersed in a solution of ammonium carbonate so dilute that each gland could absorb no more than $\frac{1}{20000000}$ of a grain. This experiment serves at least to show that the sensibility of plants 
may approximate or even surpass in some respects that of animals.

100. Spontaneous Movements. - The movements of plants which have thus far been mentioned are evidently induced

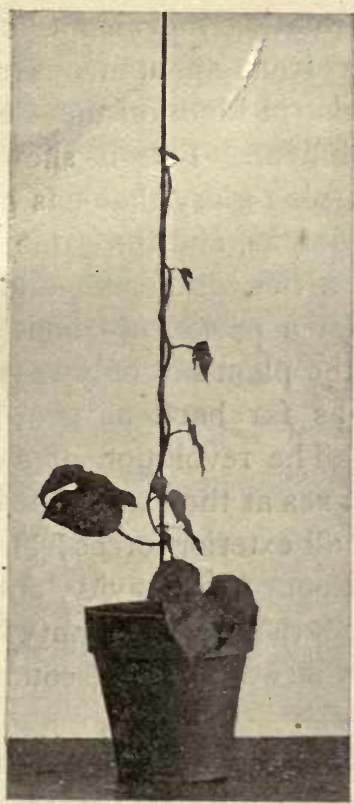

A

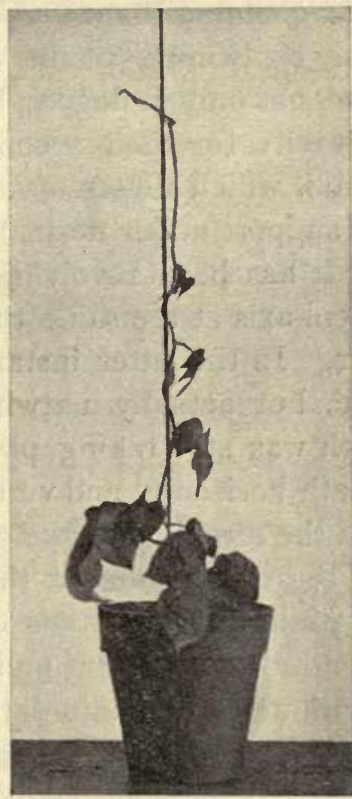

B

FIG. 66.

Two photographs of the same seedling Morning Glory. $A$, after growing under usual conditions, the light stronger from the right : $B$, after revolving on a universal axis immediately following the condition shown in $A$.

or guided by some external force, although in all cases the protoplast is the immediate cause of the movement. There is, however, another class of movements which seem to require no external stimulus other than those conditions which are necessary to the healthy existence of the plant. 
The growing apices of roots and shoots are almost constantly in motion, describing more or less irregular ellipses. Such movements are usually so slight as to be inappreciable without the use of instruments for measuring movements through minute distances.

101. Twining Plants. - The rotating movements of the apices of twining plants differ from spontaneous movements, not only in degree, but also in kind, for they depend on gravity for their accomplishment. This is shown by Fig. 66, which illustrates a morning-glory that has grown up a support under normal conditions, and the same plant after it has been revolving on a horizontal axis and on a vertical axis at the same time for a period of about eight hours. In the latter instance the plant not only ceased to twine, but actually untwined as far back as growth in length was still taking place. The revolution of a plant on both horizontal and vertical axes at the same time eliminates the directive influence of all exterior forces; but that it is the elimination of the influence of gravity, and not that of light, which has caused the plant to untwine is shown by the fact that under otherwise normal conditions it continues to twine in darkness.

102. Method of Twining. - Most twining plants twine contrary to the movement of the hands of a watch, that is, the coil facing the observer passes from the left below to the right above. Some plants, however, twine in the opposite direction, and some twine indifferently in both directions. The seedling twining plant does not show a tendency to twine for the first one or two internodes, but after that the apical portion bends over by its own weight to a position more or less horizontal, and then, if it is a left to right twiner and the apex is bent toward the north, for instance, the east side of the stem begins to grow faster 
than the others, causing the apex to bend toward the west; then the region of greatest growth is on the north side, resulting in the bending of the apex toward the south, and so on.

By movements of this sort the plant is able to feel about in space for some support, and having found it to twine about it. If growth ceased in the coils as fast as they were made, the plant would rise upward little or not at all, and the successive coils of the stem would lie near together or over each other. This, however, is prevented by the continued growth of the stem for some time after the coils have been laid down, the growth then being most rapid on the under side of the coils, causing them to rise upward and become spread apart in the form of a spiral; at the same time the stem is brought into closer contact with the support.

The benefit of the twining habit is easy to see, for those plants which possess it are able to raise their leaves to the sunlight with a very small expenditure of energy and materials for the construction of strong stems.

103. Source of Internal Energy. - How the living protoplast is able to carry out the processes of growth and movement is a mystery. We know in regard to it, however, that the protoplast ceases its activity and dies unless a certain amount of internal energy is available, resulting from the oxidation of the substance of the protoplast and of reserve materials; no amount and no form of external energy can take the place of this. The more rapidly growth and movement take place the greater the amount of materials consumed. This oxidation of the substance of the protoplast and of the reserve materials is the essential process of respiration, in plants as in animals. The active, or kinetic, energy resulting from respiration is in 
part immediately employed in the work of the protoplasts, and in part lost to the plant in the form of heat by radiation and conduction, and in the evaporation of water from the tissues. The internal energy which appears during respiration was obtained for the most part from the sun during the process of photosynthesis, and in part from the salts from the soil, and stored within the plant in the form of potential energy in starch, sugar, proteids, etc. ; and as active energy from the sun was required to form these substances, so now it is evolved when they are broken down by oxidation. If we are to understand the essential thing about photosynthesis, we must perceive it as a process of storing the sun's energy in such a form as to make it available to plants by night as well as by day, and throughout all seasons of the year.

104. Oxidation a Vital Process. - The process of respiration is not a passive oxidation, but is induced, and to a certain extent regulated, by the living protoplast. In plants, however, the regulation of oxidation is not by any means so exact as in warm-blooded animals, whose temperature is allowed to fluctuate only within very narrow limits, while the temperature of plants under normal conditions seldom differs much from that of the surrounding atmosphere.

105. Annuals, Biennials, and Perennials. - A large class of plants bears seeds the first year, and in so doing these plants send into the seed so much of their stored energy that they are unable to survive the winter, or in some cases even to continue to the end of the summer. Such plasits are known as anmuals. Many other plants store up the energy accumulated by photosynthesis in undergound parts, such as tubers, bulbs, etc., and having survived the winter produce their seeds and die at the end of the second 
season; these are known as biennials. Still other plants keep in reserve sufficient energy to maintain their individual existence year after year; these are termed perennials. The production of seeds is in the interest of the species, but not of the individual, upon which it is a severe tax, and only those plants can survive it that can retain within their own bodies a good amount of reserve energy. Some perennials further protect the individual life by alternate years of seed-bearing and sterility.

106. Length of Life. - Some perennials attain an enormous age. A Taxus tree in Kent is considered to be 3000 years old, and an Adansonia in the Cape Verde Islands and a Taxodium in Mexico appear to have lived for 6000 years. It must be remembered, however, about plants of great age, that those of their tissues which are actually alive are of comparatively recent production; the older tissues are gradually dying, while new tissues are being formed. Since perennial plants are annually rejuvenated by the formation of new tissues, it would seem that they might live indefinitely; but changes in the character of the soil, the ravages of storms and parasites, and competition with other individuals sooner or later bring their life to an end.

107. Nature of Growth. - If we were to attempt a formal definition of growth, we might say that it is any permanent change in the form and size and internal structure which is brought about by the action of the protoplasts. Growth does not always involve an increase in weight, for germi$\mathrm{n}$ ting seeds are actually decreasing in weight by the oxidatic 1 of their reserve materials; neither could temporary incr ase in size by the imbibition of water be classed as growth. 


\section{CHAPTER VII.}

\section{MODIFIED PARTS.}

\section{PROVIDING MATERIALS.}

Material for the study of the morphology of spines can be procured at any time of the year, but branches for the study of the spines of barberry should be gathered while in leaf, and either pressed or preserved in $2 \%$ formalin. The wild smilax, whose tendrils are excellent material for morphological study, should be gathered during the summer and preserved as suggested for barberry. The greenhouse smilax can, of course, be obtained at any time of the year from greenhouses; and nothing could be better than this for testing the ability of students in seeking out morphological evidence. Utricularia should be gathered from ponds and shallow lakes during the summer and preserved in $2 \%$ formalin.

\section{OBSERVATIONS.}

Most plant members are either roots, stems, or leaves, and for the sake of classification these may be taken as the morphological elements, although some structures have a different origin. To determine the origin of a modified structure, that is, whether it is a root, stem, leaf, etc., evidence along the following lines should be sought: (I) arrangement; (2) relation to other structures; (3) transitional forms; (4) construction. Thus, under $\mathrm{I}$, is there a definite arrangement, a fixed angular divergence? Under 2, does the structure have a definite and constant relation to a leaf or bud? Under 3, can forms be found which are less and less like the modified form in question, and more and more like a typical morphological element? Under 4 , 
is it composed of bark, wood, and pith, or of one or two of these? The evidence under 4 is not entirely reliable, since the modification of a structure may involve the suppression of some of its tissues. Before proceeding with the study of modified structures, write out the characteristics under I, 2, and 3, of a typical root, stem, and leaf (see Discussion I IO). It must be remembered that form and physiological function do not furnish reliable evidence as to the morphology of a modified structure; this should be tested as you proceed with the studies outlined in this chapter.

109. Make a drawing of a spine of wild plum, showing its form, size, mode of attachment to the stem, and position on the stem with reference to nodes and internodes. Show its relation to buds, branches, leaves, or leaf scars. To show the attachment to the stem, make a median longitudinal section through the spine and the branch which bears it, and draw the cut surface.

I IO. Give the morphology of the spine, that is, state what one of the morphological elements has been modified to form this structure, and give in your notes all of the evidence for your decision, as suggested under I, 2, 3, etc., of the introductory note. Refer to the details of your drawings by letters or numbers for each point of evidence. Determine the probable function of the spine, and give reasons in full for your conclusion. It is not possible to demonstrate a useful function for every structure. Some modified structures seem to have arisen either from lack of nutrition or from abundant nutrition, and in either case they may, or may not, be useful. The student should not attribute a function to a structure without good evidence.

I I I. In a similar manner, work out the morphology of the spines of the honey locust, black locust, prickly ash, and osage orange. 
I 12. Determine, as above, the morphology of the tendrils of the wild smilax and Virginia creeper, or grape.

I 13. Work out the evidence for the morphology of the spines of barberry.

I I4. Determine the morphology of all structures which are borne on the stems of Asparagus medioloides (greenhouse smilax).

I 15. What is the morphology of the prickles of rose and gooseberry?

I 16. Determine the morphology of an onion bulb.

I17. What is the morphology of the tuber of Irish potato, and of the structures borne on it?

I 18. Determine the morphology of the bladders of Utricularia or bladderwort.

\section{DISCUSSION.}

108. Diversity of Plant Forms. - The great diversity of plant forms, as we now find them, has doubtless been evolved from comparatively few and simple forms. No two plants are entirely alike, and probably no structure is exactly like any other of the same kind. Compare the leaves on a plant and note how dissimilar they are in form, size, and outline, and what endless varieties of leaves are on the different kinds of plants. Such facts as these are an expression of the extreme plasticity of the plant body, which seems to respond to external conditions and forces, and to internal tendencies, like clay to the hands of the potter; yet we must remember that the infinite variety of forms which we now see has been evolving through the untold years since life began upon the earth.

109. Morphological Elements. - After plants had advanced in their evolution from the undifferentiated body known as the thallus (see pages 257 to 285 ) to the forms 
having roots, stems, and leaves, they seem to have found in these a sufficient number and kind of members for the successful nutrition of the individual (we may consider structures of the nature of hairs and prickles as outgrowths of roots, stems, and leaves without grouping them as distinct morphological elements). We find very few plant members which may not be classified certainly as either roots, stems, or leaves, and for this reason we may speak of these members as the morphological elements, without implying that no other members may sometimes occur. Thus, when for any reason plants have need of new structures, it is their habit to modify one of these elements to meet the new demand. When Solanum jasminoides, for instance (Fig. 67), was acquiring the climbing habit it put into its petioles sensibility to contact, and power to respond in such a way as to twine about the object with which it came in contact. Or, when the turnip began to store up food for use in the succeeding year, instead of producing a new member

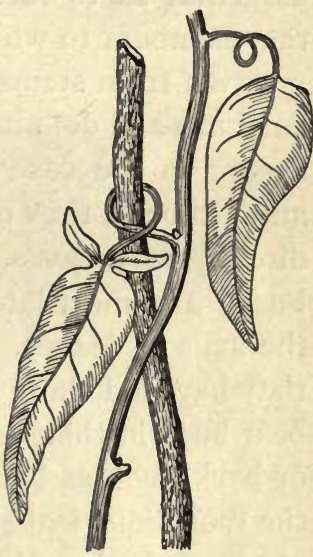

FIG. 67 .

Shoot of Solanum jasminoides, showing the leaf petioles acting as tendrils. After GRAY. as a storehouse for reserve food, its tap root was incited to increase in size sufficiently for this purpose. So, too, when buds were to be protected, internodes were kept short, and the leaves were so modified as to enwrap the tender parts in the form of tough resistant scales. Examples of this kind might be cited at great length. In acting in this way, plants have shown themselves to be wise economists, for it is certainly moving along lines of 
least resistance to modify old members rather than to produce entirely new ones.

110. Characteristics of Morphological Elements. - Since there is such diversity in form as well as in function of roots, stems, and.leaves, how are we to tell when we are dealing with these members? By a comparative study, we find that there are certain characters which appear so fundamental as to furnish reliable evidence for recognizing the members to which they belong. Thus, roots are outgrowths from stems or from other roots, and do not, as a rule, arise in definite order or definite angular divergence, except in the case of the secondary roots of seedlings; and although they may bear adventitious buds, they do not directly bear leaves. Stems directly bear roots, leaves, and buds; and most lateral stems arise either in, or just above, the axils of leaves. Leaves are borne directly on stems; they have a definite angular divergence, and they commonly bear buds in their axils. Mere outgrowths of the epidermis, or bark, such as hairs and prickles, differ structurally from the morphological elements in containing none of the parts of a vascular bundle, such as wood fibers and tracheal tubes. Whatever form, size, structure, color, or function a member may have, if it possess a set of characteristics as above stated we may classify it accordingly.

111. Modified Roots. - Roots are very commonly modified to serve as storehouses for nourishment; in such cases they consist for the most part of thin-walled tissues, to and from which the reserve materials can readily pass in solution. The dahlia and sweet potato afford familiar examples of roots of this kind. In our western plains, Ipomæa leptophylla has a storage root weighing from io to 100 pounds. The climbing roots of the trumpet creeper occur in clusters at the nodes, while those of the poison ivy occur 
in two almost continuous rows (see Fig. I7). In these cases, the real nature of the roots is not much masked. The aërial roots of orchids and the parasitic roots of Cuscuta,

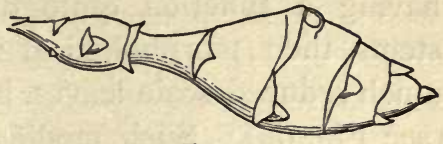

FIG. 68.

Artichoke tuber. After GRAY. although modified for absorption under special conditions, have still retained much of the appearance of typical roots. The supporting roots of the banyan, however, extending to the ground, have the general appearance of stems. but their identity is easily determined by the method and place of their origin on the branches.

112. Modified Stems. - Stems are frequently modified to grow beneath the surface, and there attain considerable size for the purpose of storage; the potato and artichoke are good examples. Although growing beneath the ground likeroots, and
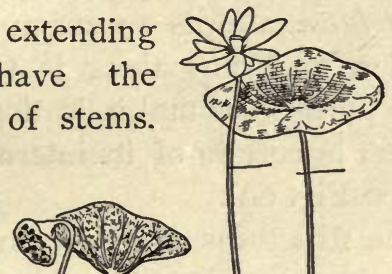
having a function quite different from that of typical stems, their morphological nature is made clear by their much reduced scale leaves, in the axils of which buds occur (see Fig. 68). Such modified stems also have a reproductive function, since, after they have survived the winter, their buds develop into new shoots. Stems which are modified for the purpose of multiplication are illustrated by the above-ground runners of the strawberry and the underground runners of the goldenrod. The yellow water lily, Nelumbo lutea, affords an example of a stem which is at first slender, and runs along in the mud beneath the water for the purpose of multiplication, and later becomes much enlarged in certain of its internodes for the purpose of storage (see Fig. 69).

The leaflike structures of Ruscus are really stems, as is shown by the fact that they do not bear buds in their axils, but are themselves borne in the axils of scales which have a regular angle of divergence on the main stem; the scales being, therefore, morphologically, leaves, and the leaflike structures, stems. But we have still further evidence, for the leaflike structures, termed cladophylls, bear flowers in the axils of scales that are evidently leaves (see Fig. 70). To sum up the evidence: The cladophyll is borne in the axil of a leaf, and itself bears a leaf and flowers. The evidence is, therefore, strong that it is a stem. The fact that it looks like a leaf and performs the photosynthetical function of a leaf should not be taken as evidence that it is not a stem, for we have already seen that the forms of plant members can vary indefinitely, and that they may be put to a variety of uses. The green, thick stems of cacti perform the double function of storage and photosynthesis, while the leaves have become reduced to spines that have entirely lost their normal function of photosynthesis. 
We can only conjecture how such modifications of form and changes of function have come about. In the case of cacti, we can see that the reduction of the leaves and the thickening of the stems have fitted them to inhabit desert regions by a reduction of the transpiring surface, and an increase of the waterstorage tissues, of which the bulk of the plant consists. In the case of Ruscus, it may be that some time back in its ancestry it became desirable to reduce transpiration on account of scarcity of water, or inability to absorb the water because of its saltiness, or because of the low temperature of the soil (see the Chapter on Ad-

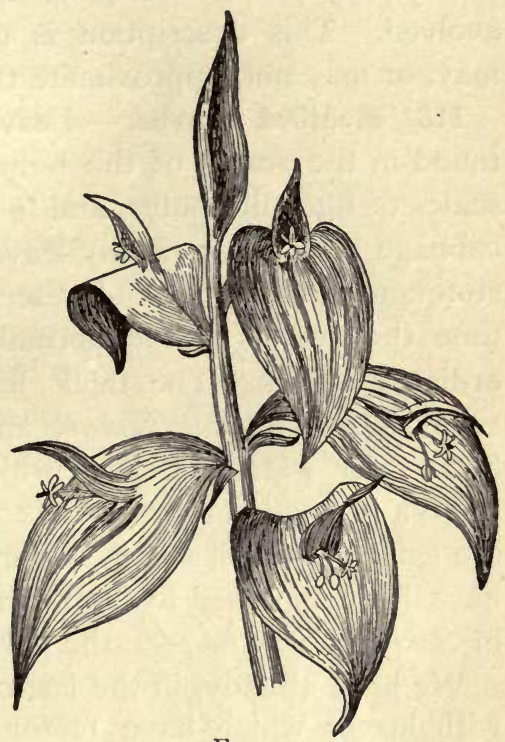

FIG. 70.

Shoot of Ruscus hypoglossum, showing leaflike stems or cladophylls. After KERNER. aptation to environment); under which conditions the plants with the smallest leaves might have fared the best and produced the greatest number of offspring. Then it might have come about in the course of time that the species was represented by individuals whose leaves were mere scales. Later, the conditions might have changed so that water could be more readily obtained; it would then have been desirable to increase surfaces for photosynthesis, but the scale leaves, being poorly nourished and reduced members, would have 
been unable to produce variations from which a selection of suitable forms could be made. The better-nourished stems, however, would have been able to do this, and finally the forms which we now see could have been evolved. This description is of course conjectural, and may, or may not, approximate the true events.

113. Modified Leaves. - Leaves modified for storage are found in the scales of the bulbs of onion, in the thicker scales of tiger lily bulbs, and in the leaves constituting the cabbage head. The fleshy leaves of Agave Americana are storehouses of water and reserve food, while at the same time they carry on the normal constructive functions of ordinary leaves. The thick leaves of succulent plants, such as Mesembryanthemum and Sedum, serve as storehouses for water. Leaves which have been modified to serve a protective function are seen in bud scales, and in the spines of cacti, and in barberry. Parts of leaves which have been modified to form tendrils for climbing, we find in Solanum jasminoides, the garden pea, etc.

We have already, in the last chapter, become acquainted with leaves which have motor organs, digestive glands, sensitive hairs, etc., for the capture of insects. There are other modified forms of leaves which entrap insects and apparently use them for food. These are the pitchers of the pitcher plants and allied forms, and the bladderlike traps of the bladderwort. In these cases, the modifications are so great and their adaptation as traps is so wonderful that they deserve a somewhat detailed description.

114. Pitcher Plants. - The pitcher plants (Nepenthes) are natives of the old world tropics. The pitcher is borne at the end of a slender prolongation of the petiole, and is probably itself a part of the petiole which has grown out in the enlarged tubular or pitcher form. At the top of the 
pitcher is a lid (see Fig. 7I), which may correspond to the blade of the leaf. The lid, in the mature pitchers, stands open, and may serve as an attraction for insects. The border of the mouth of the pitcher is rolled inward and downward, and often there are stout teeth extending downward on the inner edge of this border. Honey glands occur on the outside of the pitcher, on the inrolled border, and on the inner surface of the lid; by this means insects which alight on any portion of the pitcher are lured to the edge of the open mouth. Once over the border, the insect slips into the pitcher, which contains a fluid having digestive properties, and so the insect becomes digested and absorbed.

The pitcher plant called Darlingtonia shows a still greater degree of modification. In this plant the top of the pitcher arches over so that the mouth is directed downward (see Fig. 72 ), while extending beyond the border of the opening is a bi-

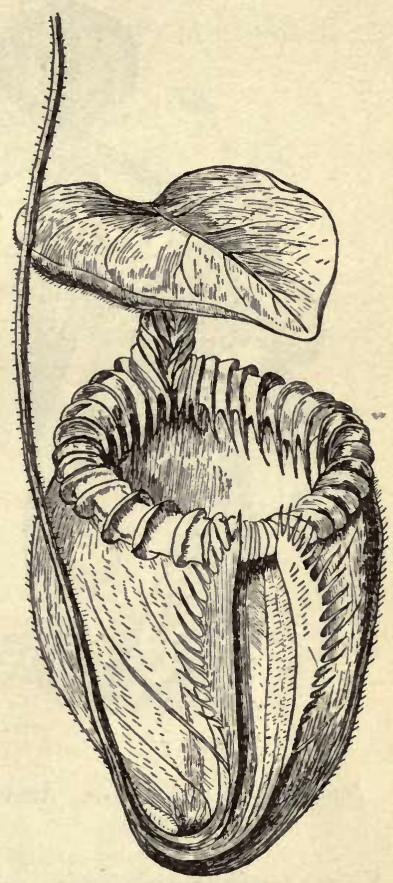

FIG. 71.

Nepenthes villosa. After KERNER. parted, brightly tinted expansion somewhat in the form of a pennant, which no doubt acts as an attraction to insects. The arched dome, or helmet, at the top of the pitcher is marked with red, and studded over with spots destitute of color, so that the light can shine through them as through a window. Nectar glands on the outer surface of 
the pitcher and under surface of the pennant lure both creeping and flying insects to the mouth of the pitcher, and after they have passed beyond the incurved border,

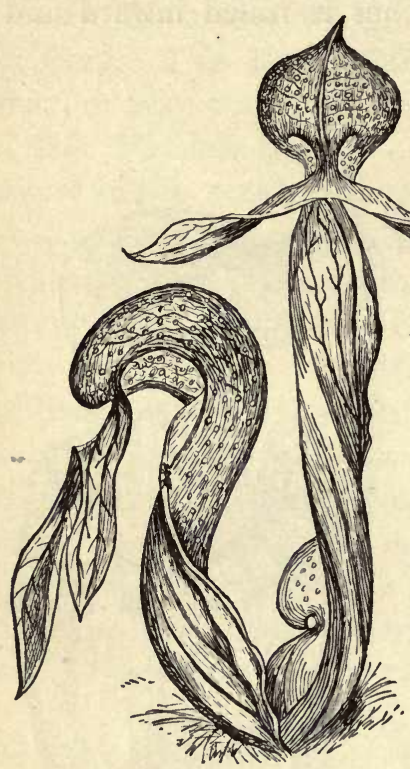

FIG. 72.

Darlingtonia Californica. After GEDDES.

which they are likely to do, they meet with a smooth surface to which it is impossible for them to cling, and are precipitated to the bottom of the pitcher, where a digestive secretion awaits them. The attempts of creeping insects to crawl out of the pitchers are frustrated by stiff, downward-pointing hairs. Winged insects can fly upward, but instead of finding the opening they are attracted by the transparent spots in the overarching helmet, against which they vainly beat until they become exhausted and fall to the bottom, where they become immersed in the digestive fluid. The pitcher plant known as Sarracenia variolaris, which is common in the southern states, although not so elaborate, has essentially the same devices as Darlingtonia, for alluring and entrapping insects.

Investigations thus far leave us in doubt whether the main function of these pitchers is the capture of insects for the food of the plant, or whether they are primarily water reservoirs to hold water that has been exuded from the plant itself, or caught from the rain in those cases 
where the pitchers stand open. Insect-catching, at any rate, appears to be one of their important functions.

It is not easy to conceive how the modifications into pitchers have come about. We know that there is in plants a capacity to vary which is apparently stimulated to activity by both internal and external causes. Useful variations would be likely to persist and become more pronounced by the production of more numerous and stronger offspring, of which those having the most useful variations would finally predominate, because better fitted to contend for soil, air, and sunlight. But how, in this case, the variation became started along the line of pitcher formation leading finally to the windowed dome, slippery surfaces, and detaining downward-pointing hairs can only be answered with conjectures.

115. Bladderworts. - Not less wonderful are the modified

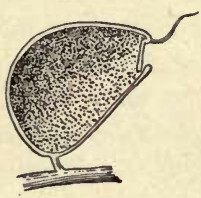

B

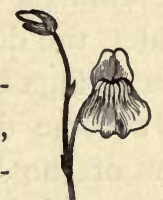

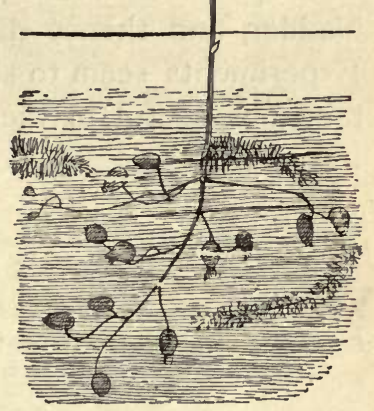

A

Utricularia grafiana. $A$, showing flower rising above the water, and below the water finely divided foliage leaves, and bladderlike leaves. After KERNER. $B$, longitudinal diagram of a bladder leaf of Utricularia vulgaris, showing trap door opening inwards.

leaves of the common Utricularia or bladderwort. This is an immersed, floating water plant (see Fig. 73, A). Some of its leaves are much divided and threadlike, and others are modified in the form of little bladderlike traps adapted to catching very minute water animals. The entrance into the cavity of the bladder is provided with a door which swings inward, but never outward, because its 
free edge swings against, and overlaps on the inside, the thickened lower border of the opening (see Fig. 73, $B$ ). Stiff hairs fringe the opening on the outside, and probably offer a place of refuge for small water crustaceans and various small larvæ when pursued by larger animals. The little animals are apt to push their way farther into the bladder, the door of which easily rises to admit them, but never swings outward to permit their escape. Finally they die, become disintegrated by bacteria inhabiting the interior of the bladders, and the soluble products of their decomposition are absorbed by the inner surface of the bladder, and thence distributed to the rest of the plant. Experiments seem to show that the animal food thus obtained is useful in the nutrition of the bladderwort. 


\section{CHAPTER VIII.}

\section{FLOWERS.}

PROVIDING MATERIALS.

If the work in botany is begun soon after the Christmas holidays, the early wild flowers will probably begin to appear by the time the work already outlined has been completed. When there is doubt of the availability of wild flowers at the required time, material placed in formalin the previous summer should be at hand; or arrangements should be made with a greenhouse for forms which can be supplied in abundance, such as sweet alyssum, Chinese primroses, Freesias, Triteleias, and single hyacinths.

It is desirable to select for preservation in formalin flowers which are somewhat stiff and leathery, such as those of the honeysuckle, trumpet creeper, Yucca, tiger lily, and some of the larger composites. The Yucca is particularly good for introducing the student to the structure of flowers, since all of its parts are large and simple in construction, and it has the further advantage of being one of the most interesting flowers in the method of its cross pollination. (See page 196.) As soon as the structure of a few typical flowers has been learned, wherever practicable, entire plants should be provided for the study of plants as a whole.

When flowers have become sufficiently abundant out of doors to admit of choice, only those forms should be selected which show well some definite facts of floral structure, adaptation to pollination, or relationships such as are exhibitcd by the different species of a genus. Nowhere in an introductory course in botany is the limited time usually available more in danger of being misapplied than in the promiscuous study of flowers without reference to some definite problem.

To illustrate: Anemone or Ranunculus might be chosen to show a simple type of flower, with parts distinct and regular; larkspur, to bring out relationship to the simple Anemone type, but with profound modi- 
fications to insure cross pollination (flowers of different ages are necessary to show all that this flower has to teach); Oxalis, to show special devices for cross pollination, each student being supplied with the two forms of Oxalis violacea, for instance, which may be found growing together in the same patch; all obtainable species of violets, to exhibit special devices for cross pollination, and considerable variations in the foliage and habits of plants having flowers of essentially identical structure. This comparative study of violets is excellent in showing how the flower, more than any other structure, gives the most reliable clews to plant relationships.

It is best to have the whole class at one time working on the same kind of flower, in order that the discussions and blackboard demonstrations, which are frequently desirable, may be founded on the experience of all of the students. The material should therefore be chosen in time to have an abundance provided for each day's work.

\section{OBSERVATIONS.}

I I9. Make a drawing of the flower, showing its position on the branch and its relation to outgrowths from the branch (a, Fig. 74).

I20. Dissect the flower into its separate parts, and make sketches showing the form of each $(d, f, g, h, i, j)$.

I21. Make a drawing of the flower on a larger scale, if necessary, showing the sets of organs in right number and proportion (b).

It is best to sketch in lightly the form of the flower before drawing its details. This can be most easily and accurately done by drawing first a circle, ellipse, square, or rectangle as a form guide, for most flowers conform in general contour more or less to one of these figures (see diagrams of Fig. 75). A flower whose general outline is circular when held before the observer with axis horizontal, as in $H$, will appear more and more narrowly elliptical as its axis is shifted toward the vertical, as in $I$ and $J$. The 

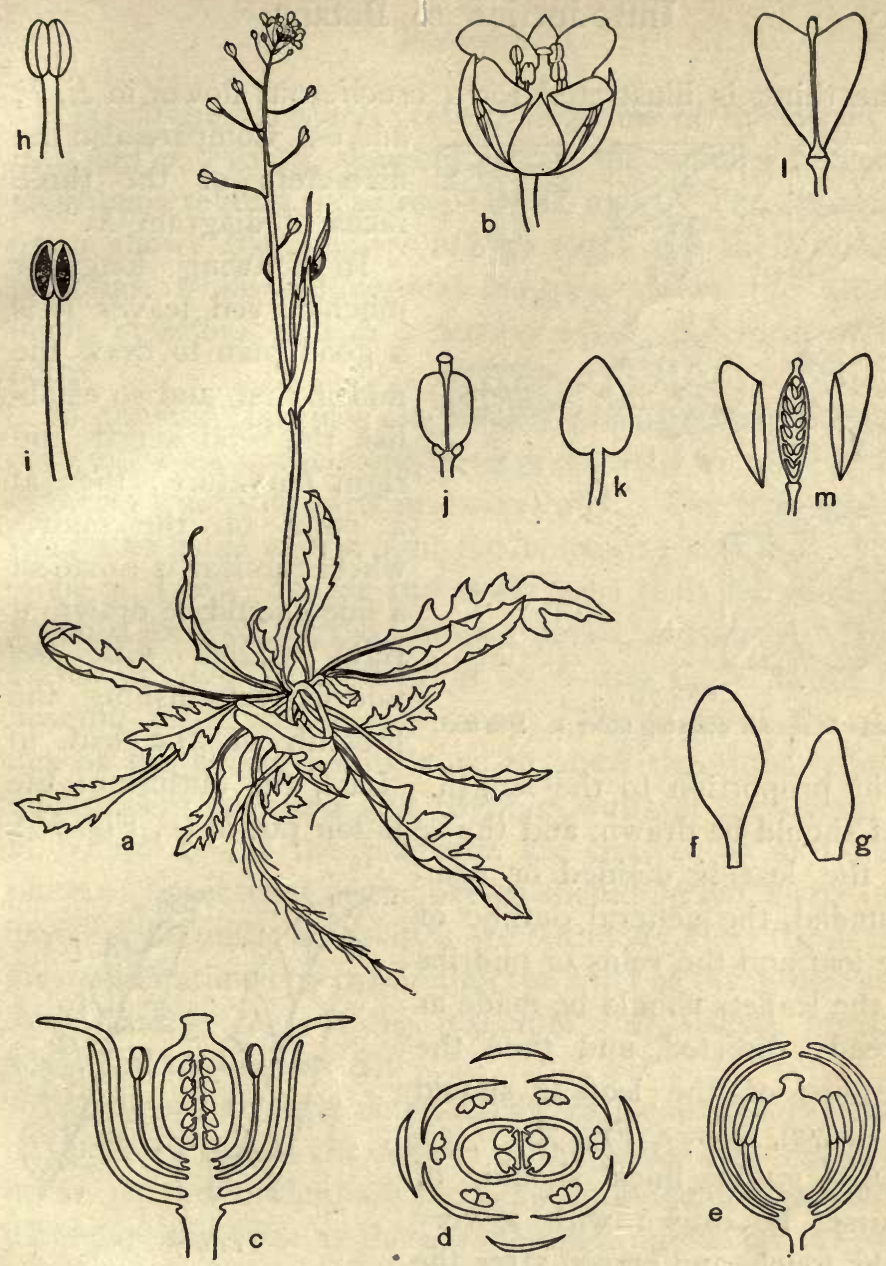

FIG. 74 .

Study of Capsella bursa-pastoris (Shepherd's purse). A type page of Student's Laboratory Book: $a$, entire plant; $b$, a flower enlarged; $c$, longitudinal diagram of an open flower; $d$, cross diagram of a flower; $e$, longitudinal diagram of a bud; $f$, a petal; $g$, a sepal; $h$, a stamen before the opening of the anther; $i$, a stamen after the anther has broken open to discharge the pollen; $j$, a pistil enlarged, with nectaries at its base; $k$, an ovule enlarged; $l$, the fruit or ripened pistil; $m$, fruit dehiscing. 
same thing is illustrated for a cruciferous flower in $E, F$,
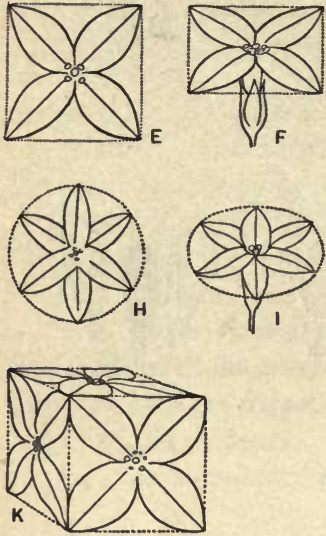

FIG. 75 .

Guide forms for drawing flowers. See text. and $G$. Compare also the diameters of the three faces of diagram $K$.

In drawing long or much-curved leaves it is a good plan to draw the midrib first, and so establish the total length and right curvature; then at a point on the midrib where the leaf is broadest a line should be drawn at right angles with the midrib, establishing the breadth of the leaf in right proportion to the length. Last, the outline of the leaf should be drawn, and the venation put in ( $d$, Fig. 76). If the leaf is divided or compounded, the general outline of the leaf and the veins or midribs of the leaflets should be made as already directed, and then the outlines of the leaflets should be drawn, as in $e$, Fig. 76.

All guide lines should, of course, be drawn with a very light touch, and erased after the flowers and leaves have been outlined.

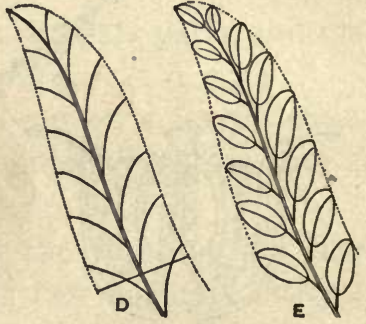

FIG. 76.

Guide forms for drawing leaves.

See text.

I22. With a sharp knife make cross and vertical sections of the ovary, and draw to a scale sufficiently large to show the manner of attachment of the ovules (in $c$ and $d$, Fig. 74). 
123. Make cross and vertical diagrams of the flower as in $c$ and $d$, Fig. 74, showing clearly the number, relationships, and relative positions of the parts. The cross diagram shows the number of the parts and their relative positions, while the vertical diagram shows the attachment, direction, and to a certain extent the form of the parts.

In drawing the cross diagrams a pair of cheap dividers carrying a lead pencil will be a great help in placing the different whorls of parts symmetrically. The guide circles should be made with a light touch, making first the central circle $a, A$, Fig. 77 , for the ovary, and then the circles $b$, $c$, and $d$ for locating the stamens, petals, and sepals. Draw dotted or very faint lines, as in $B$ and $C$, to locate the position of the walls of the ovary. Then outline the cavities of the ovary, taking care to show the right relative thickness of the outer and partition walls of the ovary, as in $D$ and $E$. If the placentæ are central, as in $D$, the place of attachment of the ovules should be left blank (see cavity $e, D$ ) until the cavities are outlined; then the ovules are to be outlined by continuing the ends of the interrupted line $(f$ and $g, D)$. If the placentæ are parietal, proceed according to diagram $E$.

Place points on the outer circles to locate the positions of the stamens and the centers of the petals and sepals, as in $A$. If the dividers are set to swing guide circles having diameters the same as those of the half circles on the protractor (Fig. 84), the guide circles may quickly be divided into the required number of equal parts. Suppose, for example, that the guide circle has the same diameter as the half circle $c$, and it is desired to divide the guide circle into five equal parts; place one point of the dividers at $c$ on the zero line and the other on the circumference $c$, 
where the $72^{\circ}$ line would intersect it. With the dividers thus set space off the guide circle into five equal parts.
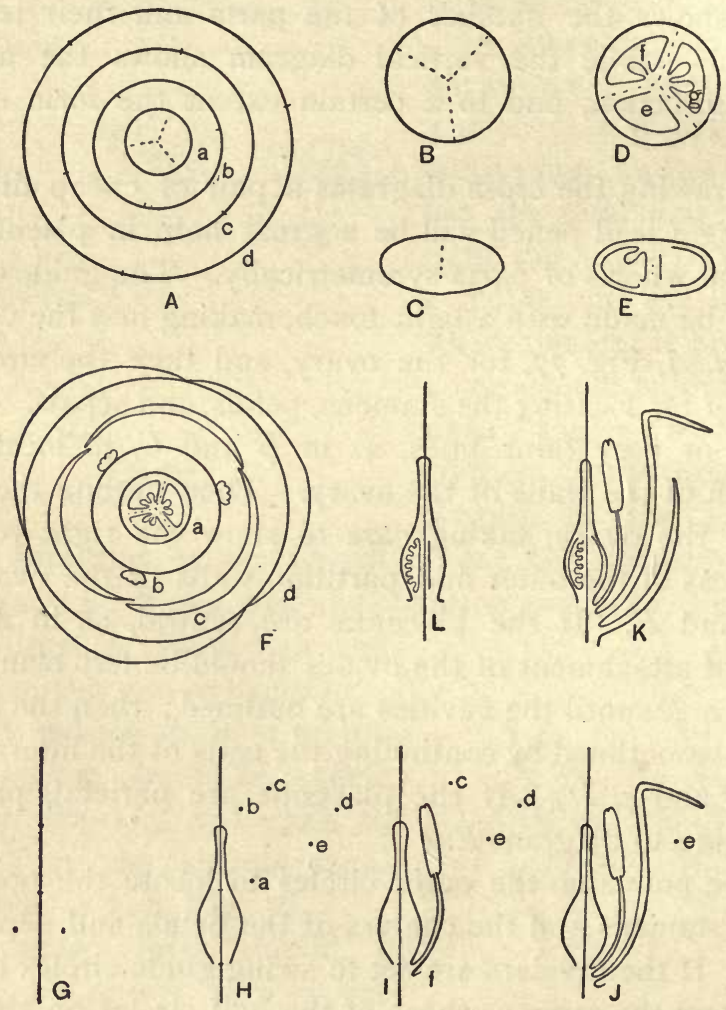

FiG. 77.

Method of making floral diagrams. See text.

In the cross diagram outline the anthers as they appear in the cross section, having the lobes face outward $(b, F)$ or inward, as the case may be in the specific flower. Represent the sepals and petals as crescentic figures ( $c$ and 
$d, F)$, having them sustain the same relation to each other as to overlapping as observed in the flower.

In drawing the longitudinal diagram make first a faint guide line, $G$, having the same relation to the vertical as the axis of the flower has in its natural position on the plant. The guide line is placed vertically in $G$, but in violet, larkspur, Baptisia, etc., it would be at some angle with the vertical. The position which the flower assumes often has an important bearing on pollination. The meaning of the irregularities of irregular flowers frequently cannot be understood until the direction taken by the axis of the flower is known. In the case of a flower having a definite upper and under side, both the longitudinal and the cross diagram should take note of this so that the structurally under side of the diagram is directed toward the bottom of the page and the upper side toward the top. Space off on this guide line the relative lengths of ovary and style, and on either side locate with points the greatest diameter of the ovary, as shown in $G$. Then outline the pistil, as in $H$, and set points $a$ and $b$ to fix the relative lengths of anther and filament, and points $c, d$, and $e$ to mark the height and greatest spread of the corolla and calyx. At the base of the ovary continue the outline upward to form the stamen, as in $I$, and continue the outer line of the filament upward at the base to form the petal, as in $I$ and $J$, and continue in like manner to form the sepal as in $K$. Construct the other half of the diagram in the same manner.

In outlining the cavities of the ovary, if the placentæ are central, draw first the inner border of the outer wall, as in the right half of the pistil of diagram $K$, and then continue this line to form the ovules, as in the left half. If the placentæ are parietal and there is more than one 
cavity in the ovary, draw the line near the center, fixing the thickness of the central partition wall, as in the right half of diagram $L$, and then continue this line to form the ovules, as in the left half. Erase guide lines when the diagrams are completed.

By constructing the diagrams as here directed the parts of the flower will be represented as continuous tissues, as they should be. There should not, for instance, be a line separating the ovules from the placentæ, or the pistil, stamens, petals, and sepals from the receptacle. Unless one has good judgment of distance and proportion the guide lines and points here suggested will be found necessary for the required degree of accuracy.

The chief features of irregularities in irregular flowers should be indicated in the cross and longitudinal diagrams.
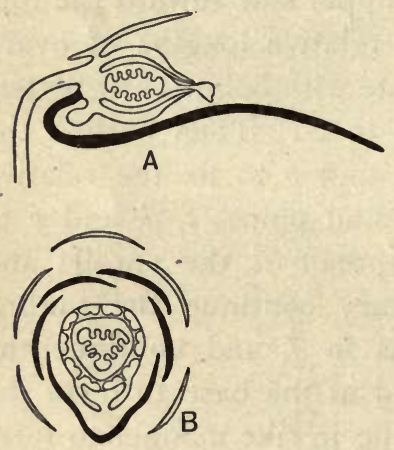

FIG. 78.

$A$, longitudinal, and $B$, cross diagram of the flower of the violet.

Taking the violet for an example: in the longitudinal diagram the spur of the lower petal, one of the nectaries projecting into the spur, the onesided stigma, and the anthers with sterile tips conniving around the style should be shown in right proportion and position. In the cross diagram the spur of the lower petal and the nectaries projecting into it should be shown, and the fact that the stamens are syngenesious should be shown by the close proximity of the anthers and the continuous line from the back of one anther to that of another (see Fig. 78).

In the larkspur, to take another example of an irregular 
flower, the upper sepal with its long spur and one of the upper petals with its spur projecting into that of the upper sepal may be shown in the longitudinal diagram, as in $A$, Fig. 79. In the cross diagram the fact that the upper sepal and the two upper petals each form a tube below. may be indicated by dotted lines, as in B, Fig. 79 .
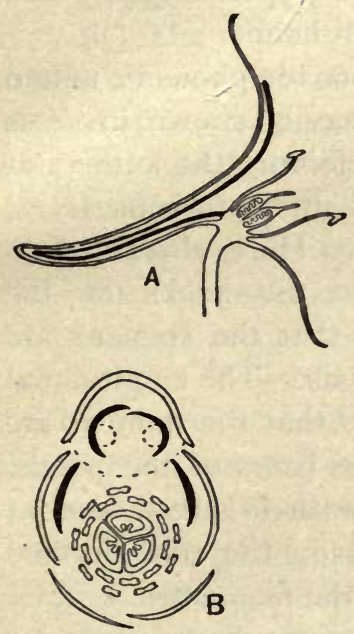

FIG. 79 .

$A$, longitudinal, and $B$, cross diagram of the flower of the larkspur.
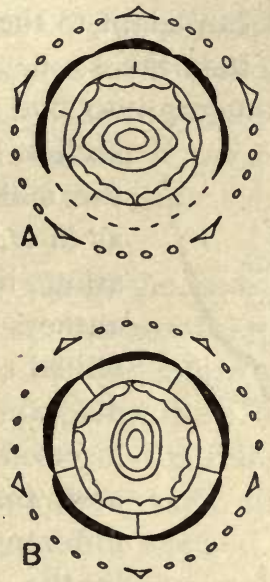

FIG. 80.

Diagrams of composite flowers. $A$, cross diagram of a flower with ligulate carolla; $B$, cross diagram of a flower with tubular corolla; $C$, longitudinal diagram of a flower.

The method of diagraming composite flowers is shown in Fig. 8o. $A$ is a cross diagram of a flower with ligulate corolla. The stamens are shown to be syngenesious by lines uniting the backs of the anthers, and the radiating lines from the backs of the anthers signify that the stamens are inserted on the corolla. The dotted line completing the petaline whorl indicates that the corolla is tubular 
below. In the sepaline whorl the five triangular figures are meant to indicate that in all probability five sepals are represented in the circle of hairs, scales, awns, etc., collectively termed pappus in these flowers.

$B$ is a cross diagram of a flower with tubular corolla. Diagram $C$ is a type of either ligulate or tubular flower. These diagrams are to be considered as types simply, which the student can adapt to the flower in hand.

The fact that the stamens are monadelphous or united by their filaments into one group may be shown in cross section by lines joining the anthers at

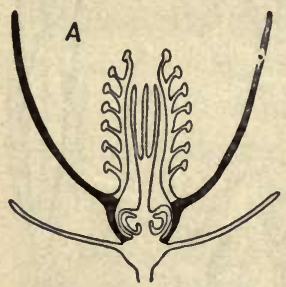
the middle of their contiguous faces, as in $B$, Fig. 8I. Here also the radiating lines from the backs of the anthers indicate that the stamens are united to the petals. The longitudinal diagram $A$ shows that the stamens are inserted near the bases of the petals,

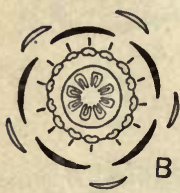

FIG. 8I. and that the anthers are borne at different heights on the tube formed by the union of the filaments.

The type diagrams here given, together with those of Fig. I23, will serve

$A$, longitudinal, and $B$, cross diagram of a mallow flower.

to show the student a concise method of representing the various irregularities and unions of parts.

I24. With colored pencils tint the calyx in the diagrams green, corolla yellow, receptacle orange, ovary and style blue, stigma purple, anther red, and ovules red. The anthers and ovules are widely enough separated not to be confused by having the same color. By giving them the same color it is meant to signify that they both contain essential organs for reproduction, namely, the anthers con- 
Flowers.

taining the pollen which supplies the sperm, and the ovules containing the eggs.

125. Study the appearance of the sets of parts in bud and in blossom, and record with drawings and notes the changes which take place from bud to fruit (see drawings in Fig. 74). Work out these points according to the following outline:-

$A$. (I) The manner in which the calyx affords protection to the other parts of the bud, by its character and its method of enwrapping them. (2) The changes in the character and functions of the calyx. Does it persist through the continuance of the rest of the flower, or even through the formation of the fruit?

$B$. (1) The position of the corolla in the bud. (2) How the petals are related to each other, and how they enfold the parts within. Does the corolla in the bud afford any protection to the stamens and pistils? (3) How the petals change in size, form, texture, and color as the bud unfolds; at what stage the petals seem to have completed their mission.

C. (I) The positions of the stamens in the bud. (2) The changes in the position and size of the stamens as the bud unfolds. (3) At what stage of the flower the anthers open and discharge their pollen. Do the stamens change their position with reference to the stigma as the bud unfolds? Do these positions seem to prevent or insure self fertilization?

$D$. (I) The changes in the size and position of the style. (2) The stage at which the stigma has sufficiently developed and has assumed the right position to receive the pollen. Could self-pollination take place in the bud? Are the changes undergone by the style and stigma in the interest of cross or self-fertilization?

$E$. Whether the position taken by the open flower has 
any direct bearing on pollination, and whether in the open flower the relation of calyx or corolla to the stamens and

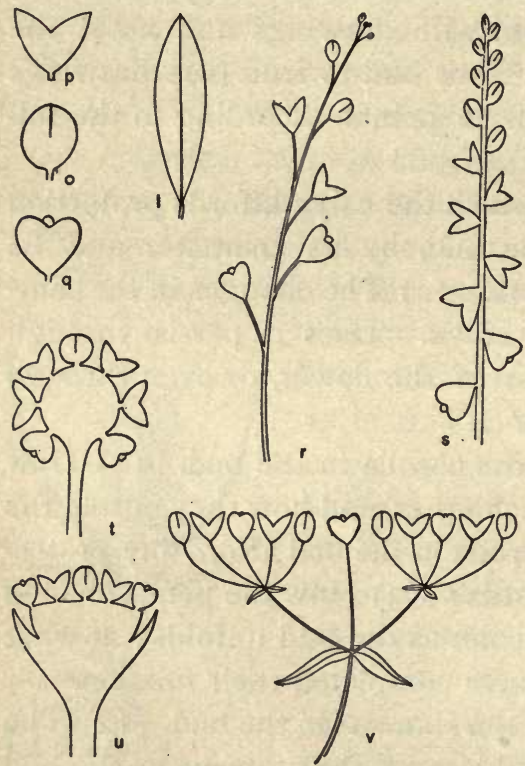

FIG. 82.

Conventional diagrams to represent a leaf $l$, flower bud $o$, open flower $p$, fruit $q$, raceme $r$, spike $s$, head of the clover type $t$, head of the composite type $u$, a compound cyme, $v$; either cluster to the right and left of the central flower would represent a simple cyme.

pistil has anything to do with pollination.

$F$. If there are irregularities of structure try to find their significance.

I26. Having become familiar with the method of working with flowers, study the plant as a whole, and make diagrams to show the habit of plants, and the manner in which the flowers are borne.

In showing the habit of the plant and the character of the inflorescence, conventional forms may be used. Thus $l$ of Fig. 82 may stand for a leaf, $o$ for a flower bud, $p$ for an open flower, and $q$ for a fruit. Using these symbols, the chief types of inflorescences may be represented as in diagrams $r$ to $y$ in Figs. 82 and 83. $r$ represents a raceme, in which the axis of the inflorescence elongates with age, giving rise to new flowers as growth in length proceeds, each flower having its own stalk or pedicel. $s$ represents a spike or ament, which differs from a raceme in having the flowers sessile upon the common axis. $x$ is a diagram 
of a corymb, whose older flowers differ from those of a raceme in having their pedicels elongated so that a somewhat flat-topped flower cluster is produced. $y$ stands for an umbel, having the pedicels of nearly equal length, and inserted at about the same height on the common axis. $u$ represents a head of the composite type, and $t$ a head of the clover type with the receptacle much exaggerated.

The raceme, spike, corymb, umbel, and head are called indeterminate;

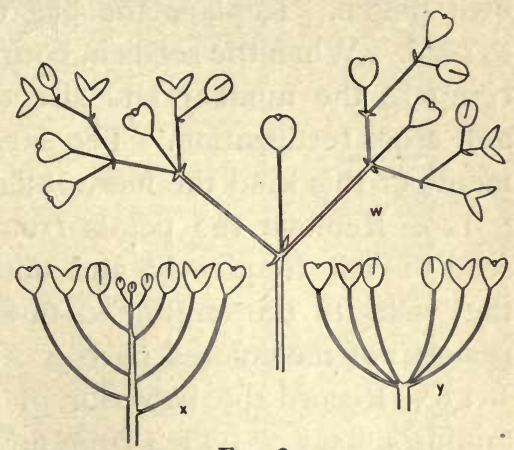

FIG. 83.

$w$, a crowded cyme or fascicle; $x$, a corymb; $y$, an umbel. they have this feature in common, that the older flowers are at the base or periphery, and the younger flowers are at the apex or center of the inflorescence.

In another type of inflorescence known as the cyme ( $v$ and $w)$ the terminal or central flower is the oldest. This sort of inflorescence is termed determinate, since the common axis is not indefinite in elongation as in the raceme and spike. A compact cyme like that of the Sweet William is called a fascicle, and one that is still more compact so as to simulate a head is termed a glomerule.

127. Tie paper bags over flowers, so that neither wind nor insects can disturb them, and note whether they are able to achieve self-fertilization.

128. Before the stamens have discharged their pollen, remove them from a flower, and then by means of a camel's hair brush transfer to the stigma pollen from a flower of another plant of the same kind. Tie a bag over the flower 
and allow it to go to seed in this way. Tie a bag over another flower, while it is yet in bud, to keep foreign pollen away, and when the flower has opened pollinate it with its own pollen. Replace the bag and allow the flower to go to seed. When the seeds in both experiments have formed, compare the number and size of seeds produced by self and cross fertilization. The larger the number of experiments of this kind the more reliable are the results.

129. Remove the petals from flowers which bees are seen to visit frequently, and note whether the number of the visits to the mutilated flowers is influenced by the removal of the colored parts.

I30. Record the behavior of bees and butterflies in obtaining nectar or pollen from flowers, noting whether these insects would be apt to be the cause of cross pollination.

I3 I. Make a record of some flowers which open only in the nighttime. Do they become more fragrant at that time? Are they of light or dark hue? What particular sorts of insects visit them?

132. Make a record of some flowers which are open only in the daytime. After closing at night do they open again?

133. Watch the behavior of the stigmas of . catalpa flowers. At what stage in the development of the flowers do the stigmas spread apart? Do you find both open and closed stigmas in the older flowers? Touch the open stigmas with a brush or stick, and note their behavior. Dust pollen on other open stigmas and note the result. Do the stigmas behave alike in both cases? The experiment should be kept under observation for some time before the final record is made.

I34. Touch the bases of the filaments of barberry flowers, and note the behavior of the stamens. Would pollination be likely to occur without the aid of insects? 


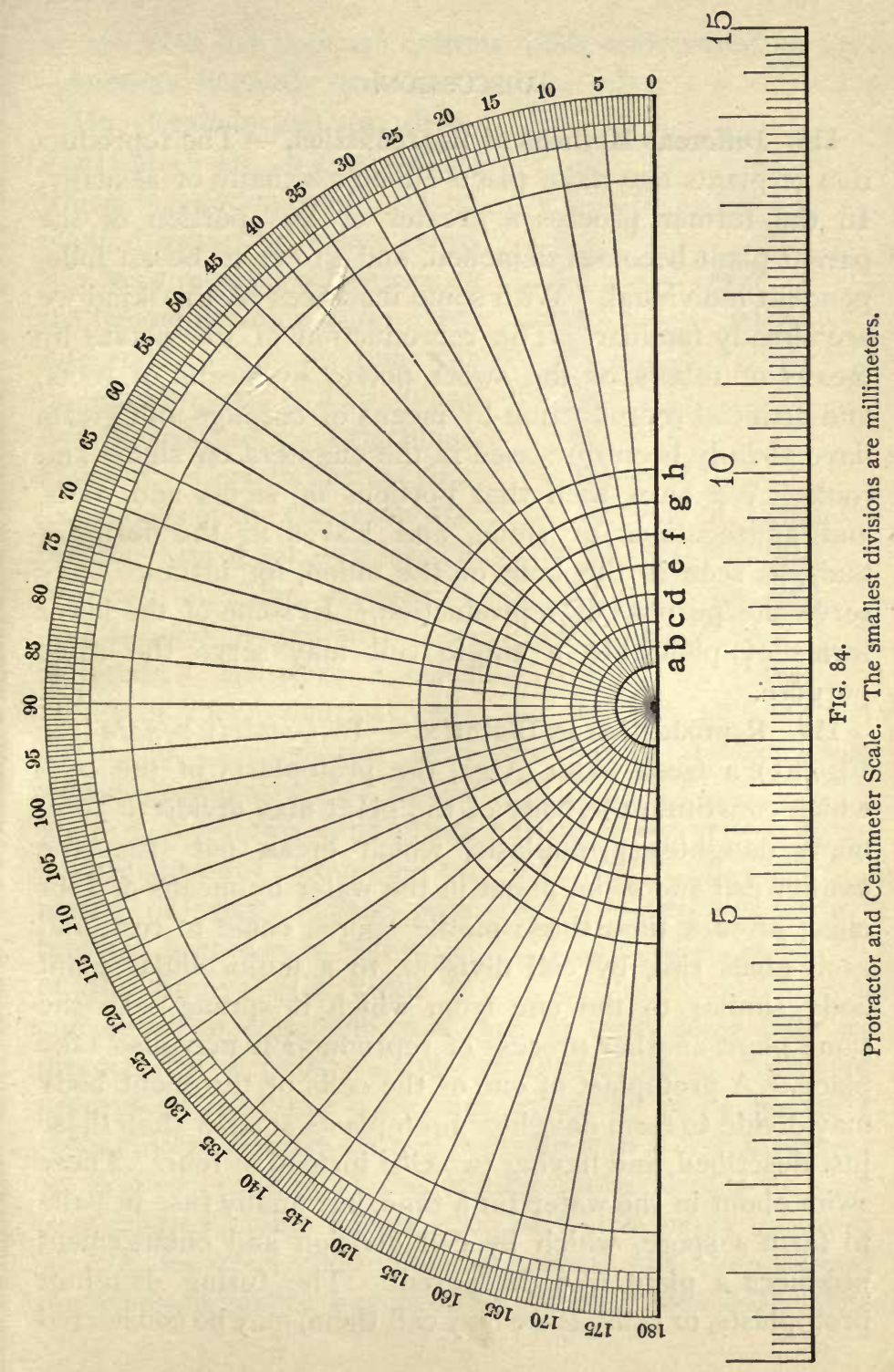




\section{DISCUSSION.}

116. Different Methods of Reproduction. - The reproduction of plants may take place either asexually or sexually. In the former process a greater or less portion of the parent plant becomes detached, and grows to be an independent individual. With some instances of this kind we are already familiar. The reproduction of the potato by means of tubers, of the sweet potato by means of roots, and artificial reproduction by means of cuttings and grafts have already been discussed in the chapters on stems and roots. We have seen that portions of stems and roots, and aggregations of stems and leaves in the form of buds, as seen in the bulb of the onion, for instance, may serve the purpose of reproduction. In some of the lower forms of plant life a single cell may serve the same purpose:

117. Reproduction in Ulothrix. - In Ulothrix zonata (see Fig. 85), a fresh-water Alga, the protoplasts of the cells which constitute the body of the plant may divide to form many daughter protoplasts, which break out from the mother cell and swim about in the water by means of four cilia; after a time these motile spores come to rest, and each gives rise, by cell division, to a multicellular plant body similar to the one from which it sprang. In the same plant another process of reproduction may also take place. A protoplast of one of the cells of the plant body may divide to form daughter protoplasts smaller than those just described, and having two cilia instead of four. These swim about in the water for a time, but finally fuse in pairs to form a spore, which by cell division and enlargement produces a plant like the parent. The fusing daughter protoplasts, or cells, as we may call them, may be considered 
Flowers.

as sex cells, but they are entirely alike, and cannot be considered as distinctly male and female.

\section{Reproduction in Oedo-} gonium. - In Oedogonium, which is another low form of plant growing in water, there is a very marked difference in the two cells which unite. By reference to Fig. 86 this will be made clear. The plant body consists of a multicellular filament. Certain of the cylindrical cells of the filament grow into the form of spheres(termed oogonia; singular, oogonium), and become gorged with food materials. In some species others of the cylindrical cells

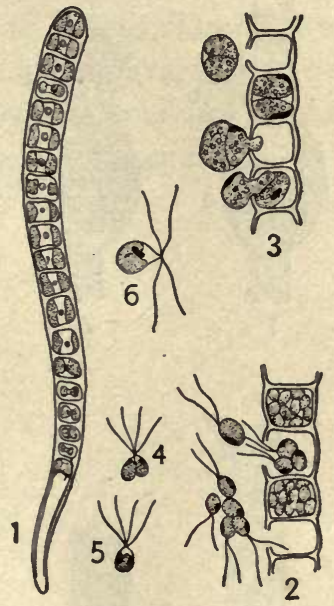

FIG. 85 . divide to form shorter cells Reproduction in Ulothrix zonata. whose protoplasts also divide each to form two protoplasts having power of locomotion by means of cilia. These pass through openings in the walls $\mathbf{I}$, a young filament; 2 , four cells of a filament whose protoplasts have divided to form small zoospores, seen escaping from two of the cells; 3 , protoplasts escaping to form large zoospores; 4 and 5 , the fusion of two small zoospores; 6, a large zoospore. After DODEL-PORT. of the parent cell, swim to and enter an oogonium, and one of them fuses with its protoplast. A thick wall is now formed about the fused protoplasts, and the body thus protected is known as a resting spore. After a time, depending to a certain extent on external conditions, the resting spore divides to form four ciliated motile bodies, each of which finally grows to be a filamentous plant like the parent. In Oedogonium, therefore, sexuality has become clearly developed. One 
reproductive cell is much larger than the other, is stored with food materials, and is stationary, while the smaller re-
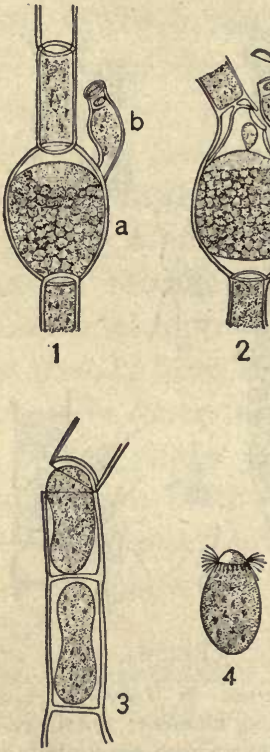

FIG. 86.

Reproduction in Oedogonium ciliatum. I, $a$, an oogonium in which is borne the large egg cell; $b$, an antheridium in which the sperm cell occurs; 2 , showing the sperm cell after it has entered the oogonium and is fusing with the egg; 3 , showing the protoplasts in two cells of an Oedogonium filament ready to emerge and become asexual swarm spores; 4, an asexual swarm spore. After PRINGSHEIM. productive cell is motile, seeks the larger cell, and fuses with it. The larger cell may therefore be designated the female reproductive cell or $\mathrm{egg}$, and the smaller cell the male reproductive cell or sperm.

119. Functions and Relationships of Floral Structures. The essential parts of a flower consist of the sperms and the eggs, and the organs which bear and protect them. Figure 87 is a diagrammatic representation of a typical flower. At $b$ is a sepal, one of the external members of the flower, which taken collectively constitute the calyx. The calyx enwraps the other parts of the flower in the bud and gives them some degree of protection, and sometimes it is brightly colored and helps to make the flower conspicuous. It is not necessary to the production of seeds, and is sometimes wanting.

At $c$ is a petal. The petals constitute the corolla; they are usually white or brightly colored; in the bud they enwrap the inner members and thus help to protect them, and in the open flower they are advertisements to insects, 
humming-birds, etc., which, as we shall see, may be of service in the production of seeds. The petals are not absolutely necessary to reproduction, and are often wanting.

At $d, e$ is a stamen, made up of $d$, the filament, and $e$, the anther. The anthers contain small grains, known as pollen grains, which produce the sperm, and are therefore absolutely necessary to the production of seeds.

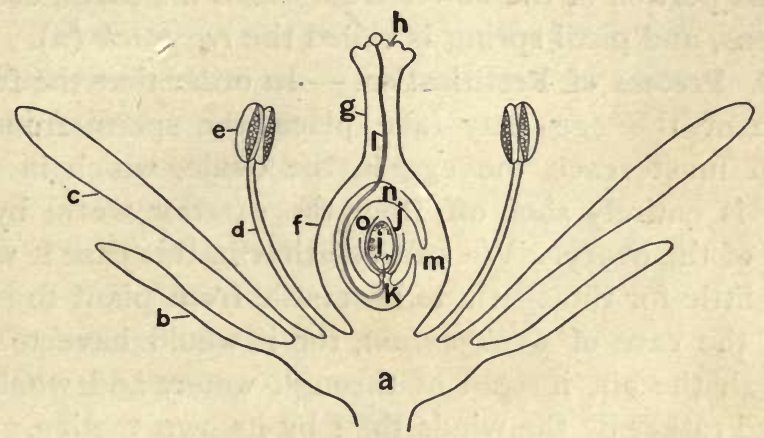

FIG. 87.

Longitudinal diagram of a Flower. $a$, the receptacle; $b$, sepal; $c$, petal; $d$, filament; $e$, anther (filament and anther constitute the stamen) ; $f$, ovary; $g$, style; $h$, stigma (ovary, style, and stigma constitute the pistil) ; $l$, pollen tube descending through the style to the ovule; $n$, cavity of the ovary; $j$, the ovule consisting of the stem or funiculus, $m$; two coats with an opening, $k$, through them (micropyle) at the lower end; the nucellus, $o$, and the embryo sac, $i$.

At $f, g, h$ is the pistil, which is composed of the enlarged basal portion $(f)$, known as the ovary, the more or less slender prolongation of this $(g)$, called the style, and a portion of the style $(h)$ near the apex, termed the stigma, which is usually somewhat rough and sticky so as to catch and hold the pollen. Sometimes the style is very short, and the stigma appears to rest on the top of the ovary. - Within the ovary and growing to it are the ovules (one shown at $j$ ), which are destined to become the seed. The 
ovule contains a large cell, or spore (i), which produces the egg, and is therefore necessary to the production of seed.

The flower may be lacking in both calyx and corolla, but if it have stamens and pistil, or only pistil, provided pollen is brought to it from some other flower of the same kind of plant, it may still bear seed. For this reason the stamens and pistil are called the essential parts of the flower.

That portion of the flower from which the calyx, corolla, stamens, and pistil spring is called the receptacle $(a)$.

120. Process of Fertilization. - In order that the fertilization of the egg may take place, the sperm from the pollen must reach the egg in the ovule, which in most cases is entirely shut off from the exterior world by the walls of the ovary. We can see that in this case it would avail little for the sperm to be motile from plant to plant, as in the case of Oedogonium, for it would have to pass through the air, instead of through water, and would be carried rather by the winds than by its own motion. The process of the transference of the sperm has therefore been modified to meet the aërial environment. The anthers burst open, and the liberated pollen is then blown about by the wind, or is carried from flower to flower by insects which are seeking nectar or pollen for food for themselves or their young; or in some cases the anthers may touch the stigmas and transfer to them their pollen, or the pollen may drop from the anthers upon the stigmas; while in water plants it may be carried to its destination by means of movements of the water.

After the pollen has been transferred to the stigma (as at $m$, Fig. 88) it imbibes moisture from the moist and sugary stigmatic surface, and increases in size so that finally the inner membrane (represented by the innermost line of the pollen case) pushes through the outer coat (thick, and 
Flowers.

shaded with diagonal lines) either through thin places designed for the purpose, or through crevices caused by swelling of the inner parts, and grows forth in the form of a slender tube $(t)$. This penetrates between the cells of the tissues of the style, from which it derives nourishment, until it reaches the cavity (u) of the ovary, in which the ovule $(j)$ is contained, and then it seeks the opening (micropyle) through the coats of the ovule, and, passing through this, reaches the spore $(s)$ called the embryo sac, in which the egg $(k)$ is borne. Then the pollen tube breaks open, and the sperm $(n)$, which has been passing down within the tube as it elongated, fuses with the egg (see Fig. 89, where $h$ is the egg cell, $t$, pollen tube, and $u$ the uniting sperm and egg nuclei). This fusion of the two sexual elements is called the fertilization of the egg.

The egg and the sperm are both protoplasts, - that is, they both consist of plasma membrane, cytoplasm, nucleus, and

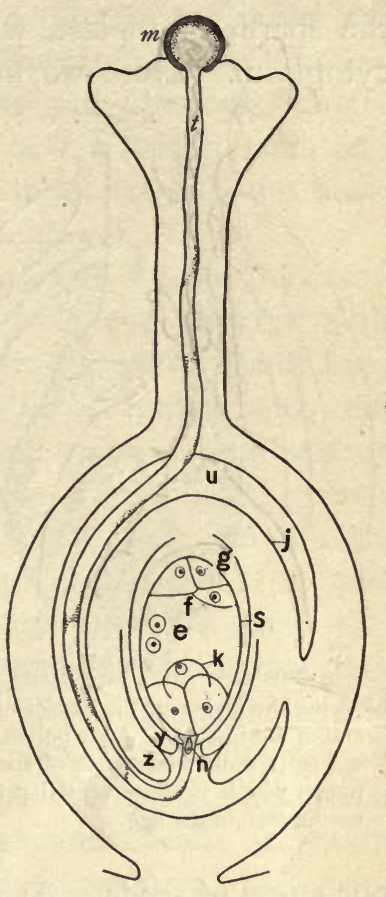

FIG. 88.

Showing the descent of the Pollen tube and its entrance into the Ovule. $m$, pollen grain on the stigma; $t$, the pollen tube; $n$, the sperm cell which has descended the tube and is ready to penetrate the embryo sac, $s$, and fuse with the egg cell, $k$. Below $k$ are two cells termed the synergids; $e$, two nuclei which fuse and form the secondary nucleus of the embryo sac, from the division of which the tissue of the endosperm arises; $g$, the nucellus; $f$, antipodal cells; $z$, outer, and $y$, inner coat of the ovule; $u$, cavity of the ovary. 
plastids, the nucleus making up by far the greater part of the sperm protoplast, while the egg is relatively rich in cytoplasm. Here we have evidence that the nucleus in

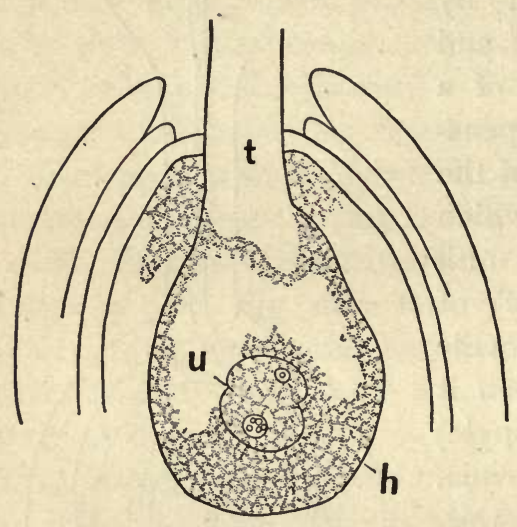

FIG. 89.

Showing the fusion of the nuclei of the Sperm and of the Egg cell. $t$, the pollen tube; $h$, the egg cell; $u$, the coalescing of sperm and egg nuclei which is the essential process of the fertilization of the egg.

particular is the bearer of the inheritable qualities, since the offspring may inherit more of the peculiarities of the plant furnishing the sperm than of the plant bearing the egg.

121. Result of Fertilization. - After the egg has been fertilized it forms a wall about itself, and begins a series of cell divisions which result in the formation of the embryo, with which we have already become acquainted in our study of seeds. At the same time, within the embryo sac other cells are being produced which become gorged with food materials. This food-bearing tissue is called the endosperm. The reserve food remains either entirely outside of the embryo, as in the case of the castor bean, or it may become absorbed by the cotyledons about as fast as it is produced, as illustrated by the Lima bean; or it may be only partly absorbed by the embryo, as in Indian corn.

The fertilization of the egg stimulates not only the growth of the embryo and the accumulation of reserve food, but it also incites the growth of the ovule as a whole 
and the further development of the ovary, and, in some cases, of the receptacle and calyx. If the egg is not fertilized, the whole flower soon drops off; but once fertilization is achieved, the ovary persists and develops into the mature fruit. Evidently there is here a transmission of a stimulus from the fertilized egg to all parts of the ovary and even to other structures of the flower.

122. Time required for Fertilization. - The distance traversed by the pollen tube varies from one to a few millimeters in the case of the smaller flowers to about forty centimeters through the long styles of Indian corn, commonly known as the silk. The time which elapses between the deposition of the pollen on the stigma and the fusion of the sperm with the egg may therefore vary from a few hours to several days. In the case of the pine, for other reasons, the interval extends through an entire year.

123. Relative Value of Asexual and Sexual Reproduction. - If we consider the relative cost to the plant of the asexual and the sexual methods of reproduction, we can see that the sexual method is much the more expensive in materials and energy. The calyx and corolla are costly contrivances, and the production of nectar and aromatic substances for the allurement of insects, and of styles and elaborate stigmatic surfaces, has been at the cost of much valuable material; but, added to this, most of the pollen does not reach the stigma and never takes part in fertilization, and is therefore an entire loss.

The struggle for existence among plants is too great to permit any extravagance that does not bring with it some amply compensating benefits; and we might therefore conclude that the expensive and elaborate contrivances which we find attending the sexual method result in some great advantage. It will perhaps add to the clearness of the 
discussion to state at once that the devices of flowers are chiefly for the purpose of securing the coöperation of two individuals in the production of offspring, and that the offspring thus produced are stronger and better equipped for the struggle for existence than those which arise by the division of a single individual.

This has been clearly shown by experiments by Darwin, which were carried out through a period of many years, and in which a large number of species was employed. These experiments show that if the egg is continually fertilized by the sperm from the same flower (self fertilization), or from another flower of the same plant (cross fertilization in a narrow sense), through a number of generations, the resulting individuals are smaller and weaker, and produce smaller and a less number of seeds than those individuals which have for an equal number of generations resulted from the fertilization of eggs by sperms which have been brought from the flowers of different plants (cross fertilization in a more exact sense), provided the plants taking part in the cross fertilization were not descended from the same immediate ancestors, and were therefore not closely related.

Darwin concluded from his experiments "that the mere act of crossing by itself does no good. The good depends on the individuals which are crossed differing slightly in constitution, owing to their progenitors' having been subjected during several generations to slightly different conditions, or to what we call in our ignorance spontaneous variation."

It having been ascertained that cross fertilization as compared with self fertilization is of immense advantage to the species, the manifold devices which have been evolved to prevent self fertilization and insure cross fertilization are subjects of great interest and meaning, and the 
greater value of the sexual as compared with the asexual method of reproduction becomes apparent.

124. Historical Summary. - It appears from the writings of Aristotle and Theophrastus (about 350 B.c.) that only vague notions were entertained at that time about the essential nature of flowers. It was a common practice of the palm culturists of that time to shake the branches of the staminate trees over the flowers of the pistillate trees (see page 175 for definition of staminate and pistillate), in order to-increase the production of fruit, and Theophrastus remarks that there was obviously a great difference in the flowers; but he seems not to have attempted to find out wherein the difference consisted. As late as A.D. 60 Pliny writes, in speaking of the date palm, that the pollen dust is the material of fertilization, and that naturalists say all trees and even herbs have the two sexes, but knowledge of the subject was at that time indefinite and conjectural. It was Camerarius ( $1665-172 \mathrm{I}$ ) who was the first to show by definite experiment that the coöperation of the pollen is necessary to the formation of an embryo within the ovule.

Kœlreuter (I733-I806) observed the nectar and noted the aid of insects in pollination; and he produced hybrids by the transference of the pollen of one species to the stigmas of the flowers of another species. Conrad Sprengel ( $1750-1816)$ noted that cross fertilization between different flowers and between flowers of different individuals was of common occurrence in nature, and he said that, judging from the construction of flowers; nature seemed to have intended that no flowers should be fertilized by their own pollen; and he concluded further that the various peculiarities of structure in flowers have a definite relation to insects in effecting pollination. While Sprengel discovered the common occurrence of cross fertilization by 
means of wind, insects, etc., the beneficial effects of this process, so definitely determined by the experiments of Darwin, were evidently unsuspected by him.

125. Sprengel's Discoveries. - Sprengel's book, entitled "The Discovered Secret of Nature in the Structure and Fertilization of Flowers" (Berlin, I793), is of great interest to the student of botany, not only because it contains an exposition of many important discoveries, but also because it reveals an inquiring and unprejudiced mind, capable of placing facts in their true relationships, and of understanding their meaning. A few paragraphs from Sprengel's book are here given as an illustration of his method of reasoning.

"While in the summer of 1787 I was attentively examining the flower of Geranium sylvaticum, I found that the lowest part of its petals was provided on the inside and on the two borders with fine and delicate hairs. Persuaded that the wise Author of nature has not created even a single hair without a definite object, I considered what purpose these hairs might possibly serve. And in this it soon occurred to me that if one assume that the five drops of nectar, which are secreted by the same number of glands, are set aside for the food of certain insects, one would then not find it improbable that provision should be made to preserve this nectar from injury by rains, and that these hairs had been placed there for this purpose. . . .

"Each drop of nectar sits upon its gland immediately under the hairs which occur on the borders of the two adjacent petals. Since the flower stands upright and is rather large, raindrops necessarily fall into it when it rains. But none of the falling raindrops can reach a droplet of nectar and mix with it, since it is held back by the hairs which occur over the droplet of nectar, just as a drop of 
sweat which has run down a man's forehead is caught by the eyebrow and eyelashes, and kept from entering the eye. An insect, on the contrary, is not in the least hindered by these hairs from getting at the droplet of nectar.

"I then investigated other flowers, and found that various sorts had something in their structure which seemed to serve this same purpose. The longer I pursued this investigation, the more I perceived that those flowers which contain nectar are so constructed that insects indeed can get at it very easily, but that the rain cannot spoil it. I therefore concluded that the nectar of these flowers is secreted, at least principally, for the sake of insects, and in order that they may enjoy it pure and unspoiled, it is protected against the rain.

"In the following summer I investigated the forget-menot (Myosotis palustris). I found that this flower not only has nectar, but also that the nectar is fully protected against the rain. But at the same time I was struck by the yellow ring which surrounds the mouth of the corolla tube, and contrasts so beautifully with the sky-blue limb of the corolla. Might possibly, thought I, this circumstance also relate to insects? Might nature perhaps have colored this ring for the special purpose of showing insects the way to the nectar receptacle? With this hypothesis in mind I examined other flowers and found that most of them corroborated it. For I saw that those flowers whose corolla is colored in any way differently from the general coloring always have these spots, figures, lines, or splashes of distinct color where the entrance to the nectar receptacle occurs. From the part I conceived the whole. If, thought $\mathrm{I}$, the corolla is colored differently in any place especially for the sake of insects, its whole coloring must also be for their sake; and if any distinct color of a part of a corolla 
is for the purpose that an insect which has alighted on the flower may easily find the right way to the nectar, so the color of the corolla as a whole is for the purpose that the flowers possessed of such a corolla may be recognized

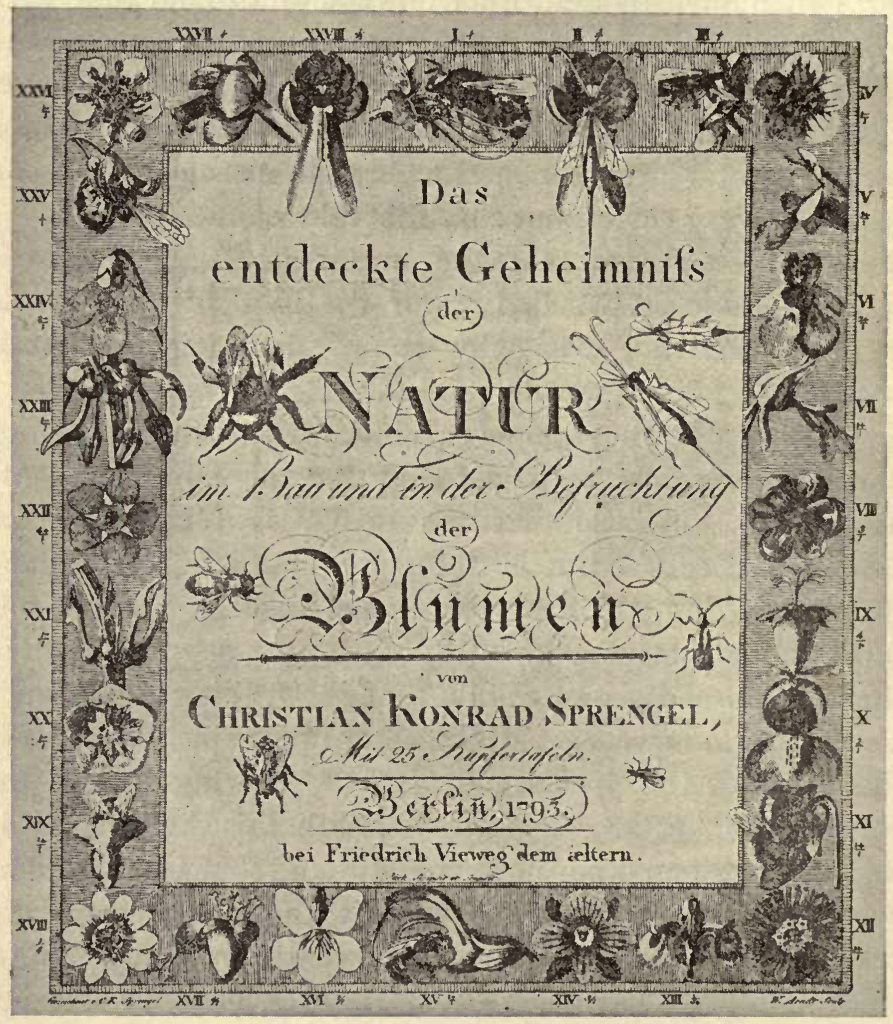

FIG. 90.

Reduced facsimile of the title-page of Sprengel's book, "The Discovered Secret of Nature in the Structure and Fertilization of Flowers."

from afar as receptacles of nectar by insects which are flying about in quest of food. 
"While in the summer of 1787 I was studying some species of Iris I soon found . . . that the nectar is fully protected from the rain, and that there are specially colored places which lead the insects to the nectar. But I found still more, namely, that the flowers cannot possibly be fertilized in any other way than by means of insects, and in fact by insects of considerable size. ... My investigations ever more and more convinced me that many, indeed perhaps all, flowers which have nectar are fertilized by the insects which obtain food from this nectar."

Figure 90 is a reduced facsimile of the title-page of Sprengel's book; the various flowers of the border give some idea of the variety of forms worked out by him.

126. Devices for Cross Fertilization. - The ability of plants to adapt their members, by modifications of form and structure, to various conditions and ends, is perhaps best shown in the construction and behavior of their flowers. The end which most flowers seek to attain, as long since pointed out by Sprengel, is cross fertilization, and the agents to which they have to adapt themselves are wind, insects and other animals, and water. In order that cross fertilization may be achieved it is of paramount importance that self fertilization should be prevented, and we accord ingly find special devices having this end in view.

The chief of these devices are as follows: (I) Only one sex is represented in each flower, but both sexes occur on the same plant. The flowers which contains the stamens are called staminate, while those which contain the pistils are called pistillate; the flowers in this case are called monocious (see Glossary for derivation of terms). (2) The pistillate flowers only are borne on one plant, while the staminate flowers are borne on another plant; such flowers are called dicecious. (3) The flowers contain both sexes, 
but (a) the pistils and anthers are not mature at the same time. Thus the stigmas may be ready to receive the pollen, but the anthers are not ready to break open and discharge
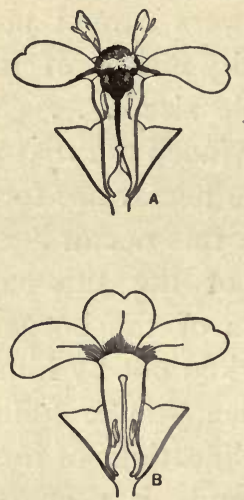

FIG. 9r.

Cross pollination of Primula, a dimorphic flower. In $A$ a bee is gathering nectar at the base of the pistil while its head is in contact with the anthers inserted in the throat of the corolla. In gathering nectar from the long-styled flower, $B$, the pollen on its head will be deposited on the stigma, and at the same time pollen from the low stamens will be brushed off on its proboscis at the right height to be transferred to the stigma of a shortstyled flower as in $A$. - WISE. the pollen, or vice versa; such flowers are called dichogamous. If the stamens mature first, the flowers are proterandrous; if the pistils mature first, they are proterogynous. (b) The stamens and pistils may differ greatly in length, so that the pollen would not be apt to attain to the stigmas of the same flower. Flowers of this kind are called dimorphic if the stamens and pistils are each of two different lengths, or trimorphic if of three different lengths. (c) The relative position of stamens and pistils may in other ways be such as to keep the pollen from the stigma, or other parts of the flower may intervene between them.

The devices to prevent self fertilization are also in the interest of cross fertilization. If the flowers are diœcious, insects in going from one plant to another are quite certain to carry pollen from the staminate flowers to the stigmas of the pistillate, or the wind may accomplish the same thing. An insect in visiting dichogamous flowers would, in the case of proterandry, carry pollen from the stamens of 
the younger flowers to the stigmas of the older, or vice versa in case of proterogyny. In dimorphic flowers the pollen from short anthers would quite certainly be deposited by insects on the stigmas of the short pistils, as in going from $B$ to $A$, Fig. 9I.

Adaptations for cross fertilization are perhaps best seen in the various adjustments of flowers to the agents which are to transport the pollen and deposit it on the stigma. These agents, as has been stated, are wind, insects and other animals, and water.

127. Adaptation to Wind. - Those flowers which depend upon the wind for transporting the pollen are characterized

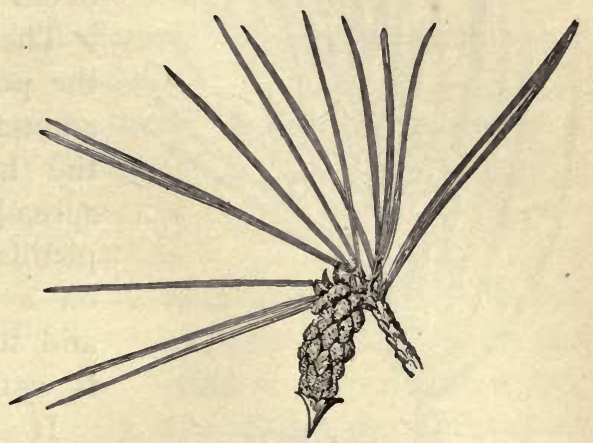

FIG. 92.

Staminate catkin of the Pine.

by protruding stamens and a relatively large expanse of stigmatic surface; this is well shown by Indian corn, whose staminate flowers surmount the plant and send forth numerous pendent stamens which offer their pollen to the wind. The long silken styles which protrude beyond the husks of the pistillate inflorescence, or ear, present a large surface for arresting the pollen as it is being carried about by winds.

The pines, poplars, and willows illustrate the same thing in somewhat different ways. The pollen of the pine is borne in great abundance in the staminate catkins (see Fig. 92), and each grain is provided with two balloon-like expansions containing air, which contribute to its lightness 
and sailing qualities. The pistillate inflorescence is in the form of a cone (Fig. 93, a) whose scales are broadly expanded and collect the pollen as it settles down upon

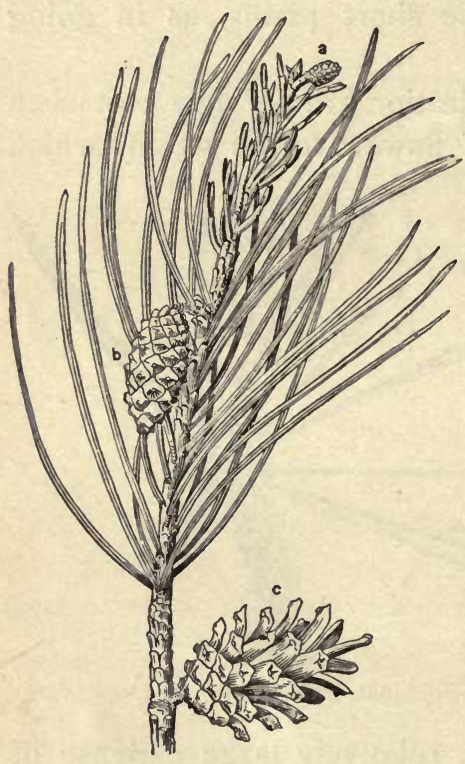

Fig. 93.

Pistillate inflorescence of the pine. At $a$, young cone of the current spring ready to receive pollen; $b$, cone which the previous spring was like $a ; c$, cone one year older than $b$; here the scales have spread apart and the seeds have dropped out.

·

fact that allurements for insects in the form of brightly colored corollas, nectar, and fragrant odors are not necessary, and are accordingly not produced.

128. Cross Pollination by Water. - In the case of flowering plants which are entirely submerged in water, the pollen frequently has the same specific gravity as the water, and 
is readily dispersed from flower to flower at any depth. In the case of Vallisneria spiralis (see Figs. 95-96) the pistillate flowers rise to the surface on slender stems. The staminate flowers, which are formed under the water, break loose from their stems and float on the surface, the

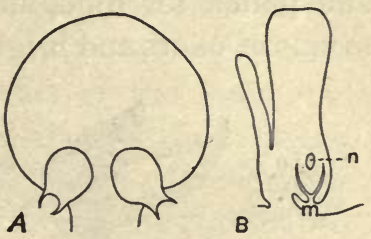
calyx consisting of boat-shaped sepals which buoy the stamens above the surface, and allow them to float high and dry. The stamens bend outward beyond the rim of the calyx, and when the stami-

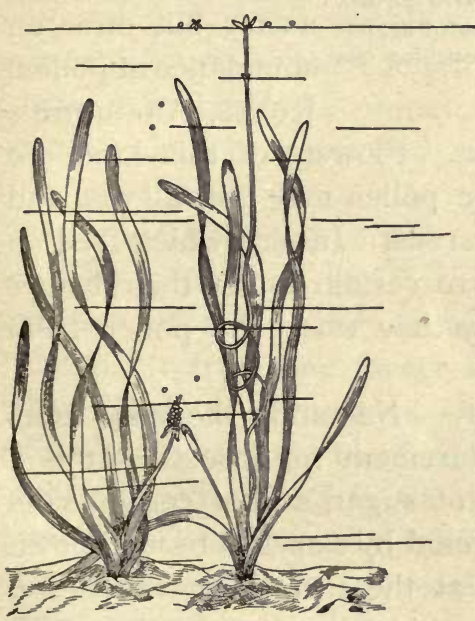

FiG. 95 .

Vallisneria spiralis. The plant on the left bears staminate flowers which are breaking away and rising to the surface. The plant on the right bears a pistillate flower rising on a long stem to the surface.After KERNER. nate and pistillate flowers are brought together by the wind or currents of water, the anthers come in contact with the stigmas and effect their pollination. The stem which bears the pistillate flowers then coils itself spirally and draws the flower to the bottom of the water, where the seeds may mature undisturbed.

129. Adaptations to Insects. - The most wonderful modifications of flowers are found in those forms which are adapted to cross pollination by means of insects. These have had to 
provide for the allurement of insects by offering them nectar and pollen for food, and for attracting their attention by means of odors and bright colors; and at the same time they

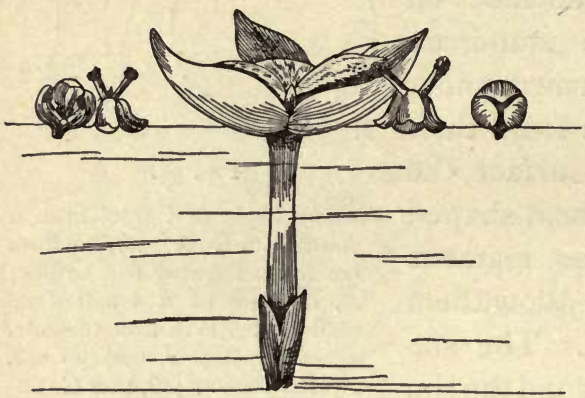

FIG. 96.

Pistillate and staminate flowers of Vallisneria spiralis. On the right a staminate flower has floated against the pistillate flower and an anther is touching one of the stigmas. After KERNER.

have had so to construct and arrange their parts that insects in securing food would necessarily carry the pollen from one flower to the stigma of another.

130. Allurement by Pollen. - Some flowers secrete no nectar but offer an abundance of pollen. Roses, Anemones, and poppies are of this sort. Flowers of this kind are more or less erect so that the pollen may not fall out, and the stamens are usually numerous. Insects which feed on the pollen of such flowers are certain to get their bodies dusted over, and in this way they carry the pollen from flower to flower.

131. Allurement by Nectar. - Nectar is the most common and most important allurement for insects. It is a more or less watery solution of sugar, and of certain salts and aromatic substances, secreted by a special tissue known as the nectary, and expelled at the surface by transfusion through the epidermis, by breaking down of the tissues, or through a special opening of the nature of a stoma. The nectar either remains clinging to the surface of the nectary or it gathers in large drops and falls into a nectar receptacle provided for it, as in the case of violets, where horn- 
like outgrowths from the two lower stamens secrete the nectar and pour it into a cup formed by the base of the lower petal.

The nectaries may occur on any part of the flower, but they are most frequently found at the bases of the stamens, petals, and ovaries, and rarely on the calyx. In the plum and peach they form a thick inner lining of the cup-shaped receptacle. In Nasturtiums the nectar is secreted in a long spur from the calyx.

Some flowers of simple construction expose their nectar freely to all sorts of insects, but others conceal it in various ways so that it is accessible only to insects of certain kinds. A frequent device is to have some parts of the corolla close over the way to the nectar so that small insects which would not assist in cross pollination are excluded, and only those which are strong enough to push aside the barrier or have proboscides of proper construction to thrust past it can obtain the nectar and accomplish the transference of the pollen.

The student is quite certain to find that irregularities and complexities of floral structure are in the interest of the protection of the nectar or pollen and the transference of the latter from one flower to another.

132. Color and Fragrance. - As Sprengel pointed out, flowers not only provide food for insects, but they also furnish advertisements in the way of brilliant colors and agreeable odors to notify insects from afar where food awaits them, and lines, spots, etc., of special color to show insects coming to the flowers the direct way to the nectar. It appears from various experiments that the odor is most effective in attracting insects from a considerable distance, and that on the nearer approach of the insect the color becomes an important guide. There seems to be no doubt 
that insects have a keener sense of smell than our own, and are attracted by the odors of flowers which we cannot detect; but their vision, except at short distances, is not sharp. They, however, appear to appreciate differences in color at a distance where the forms of objects are still indistinct to them.

133. Sense of Smell in Insects. - The nerves of insects that are sensitive to odors ramify and come to the surface

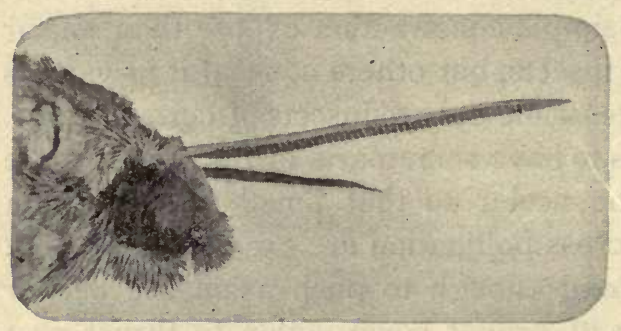

FIG. 97 .

Photomicrograph of the head of a Sphinx Moth, $\times 3$, showing the antennæ in which are located the nerves of smell, and the large compound eye. The long proboscis is coiled like a watch spring out of sight beneath the eye. in the antennæ, which are understood to be their organs of smell (Fig.97). That the sense of smell may be very keen in insects is shown by the fact that they can go unerringly to inconspicuous and concealed flowers which to us may be scentless.

134. Sense of Sight in Insects. - The eyes of insects are compound, - that is, they are composed of numerous smaller eyes all grown together, and in communication with the same optic nerve, and therefore really constituting one organ The number of single eyes or facets may amount to twenty-five thousand. The end of each facet has the appearance of a convex hexagonal disk. Figure 98 represents a longitudinal section of the eye of a cockroach which may be taken as a type of the compound eye. At $f$ is a lens-like body, clear as glass, whose outer face is at the surface of the eye. The light passing through this 
next traverses the crystal cone $k$, which is also a transparent lens. The nerve tissue $l, m$, and $n$, is in immediate communication with these lenses, and transmits the light stimuli to the main optic nerve, and thence to the nerve ganglia which represent the brain.

The compound eye of an insect does not form an image in the same manner as does the human eye, in which divergent rays of light from any point are brought to a focus on the retina, and a relatively bright inverted image is formed. Insects can, therefore, not see as well as we can. This appears to be borne out by the action of insects, which do not seem to appreciate the forms of objects well until they are quite near to them.

Since the main object of insects' Diagram of the compound Eye visiting flowers is that of obtaining food for themselves or their young, their relations to flowers will be better appreciated when the construction of their food-gathering apparatus is understood; and for this purpose the bees and butterflies will

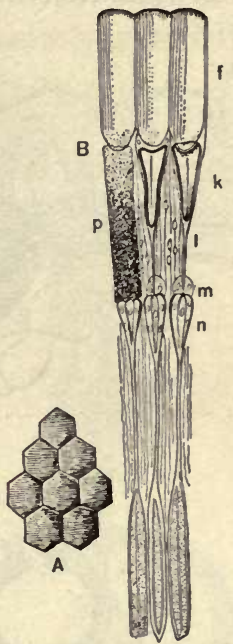

FIG. 98.

of an Insect. $A$, a surface view of some of the simple eyes united to form a compound eye. $B$, a longitudinal diagram of three simple eyes. See text. The image formed is said to be a mosaic, each simple eye contributing a distinct portion. After LUBBOCK. be chosen for examples, because they represent two kinds of insects which are most important to flowers.

135. Relation of Butterflies to Flowers. - Butterflies sip the nectar only, and do not make use of the pollen; although in getting the nectar they incidentally transfer pollen from flower to flower. They do not carry away 
and store nectar for themselves or their young, but take only what they immediately desire for food. They do not, on the whole, seem to require much food in the imago or butterfly state (the student will remember that in the first

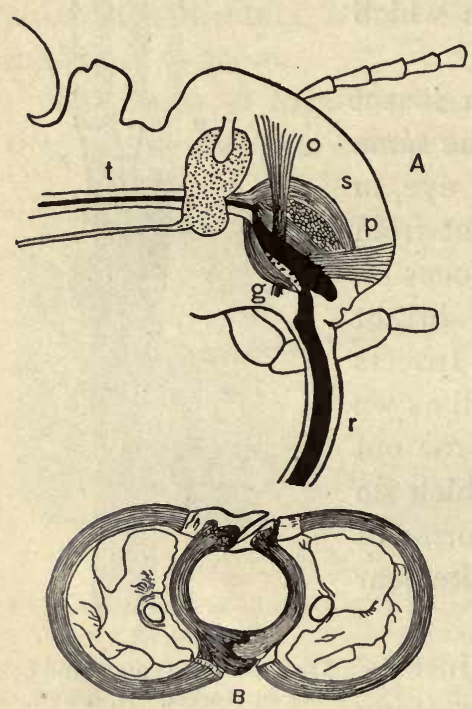

FIG. 99.

$A$, longitudinal diagram of the head of a Butterfly. $0, s, p, g$, muscles operating the suction apparatus; $r$, the proboscis, the dark band indicating the suction cavity; $t$, œsophagus; $B$, cross diagram of butterfly's proboscis. The central cavity is for suction and the lateral cavities carry muscles, nerves, and air tubes. After BURGESS. stage of their existence after hatching from the egg they are caterpillars), although they are sometimes so eager for the nectar that they may be caught in the hand while obtaining it.

The organ by means of which the nectar is secured is known as the proboscis ; it is long and slender, and contains muscles, nerves, and air tubes, and a cavity for sucking up liquids (Fig. 99, $A$ and $B$ ). When the proboscis is not in use, it is tightly coiled like a watch spring, and tucked away in small space beneath the head (compare Figs. 97 and 104). The proboscis is very flexible and is admirably constructed for probing for concealed nectar or for reaching to the bottom of flowers with long tubes, such as those of the morning glory, petunia, etc. Flowers which are especially adapted to butterflies and moths. (the difference between butterflies and moths being inconsequential) are so constructed that the proboscis comes in contact 
with both anthers and stigmas, so that the pollen is quite certain to be transferred from one flower to the stigmas of another.

136. Relation of Bees to Flowers. - Bees gather and store up both nectar and pollen for themselves and their young, and are more indefatigable than all other insects in their visits to flowers. Their mouth parts are so constructed that they can transfer pellets of pollen, by means of their mandibles, directly to the so-called mouth or opening into the pharynx, whence it passes through the œsophagus into the stomach; or they can adjust an elongated suction apparatus to the mouth opening, and by means of it suck up nectar from more or less concealed nectaries.

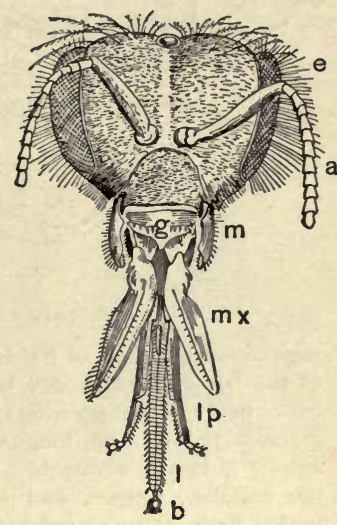

FIG. 100 .

Since bees depend almost entirely upon flowers for their food, and are at the same time of inestimable value to plants in accomplishing cross pollination, it may be taken for granted that the structures of

The head and mouth parts of a honey bee. $e$, compound eye; $a$, antenna; $m$, mandible; $g$, flap over the mouth; $m x$, maxilla; $l p$, labial palpus; $l$, tongue or ligula with button-like extremity $b$. After CHESHIRE. bees, and of flowers visited by them, have been evolved side by side, and have influenced each other by their interdependence. The mouth parts of the bee (Fig. 100) are used, not only for collecting food, but also in the construction of the comb, and in other manifold duties pertaining to the care of the young.

The bee flies from flower to flower until the honey stomach (see Fig. IOI) is extended to about $\frac{3}{18}$ of an inch in length and $\frac{2}{18}$ of an inch in breadth; it then flies to the hive, 
and by a contraction of the honey stomach empties its load of honey into the cells of the comb; then, urged by an

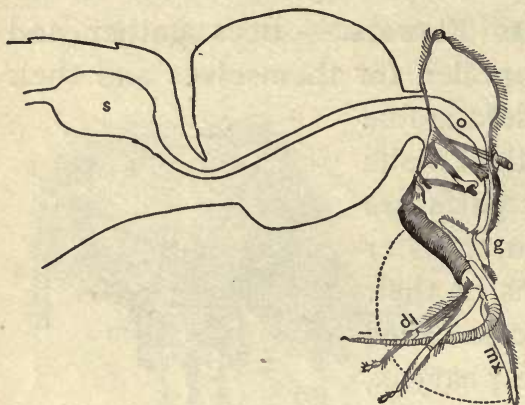

FIG. IoI.

Longitudinal diagram of the head and a part of the body of the honey bee. $g$, the flap over the mouth opening; $m x$, maxilla; $\not p$, labial palpi; $l$, the tongue; $o$, the œesophagus; $s$, honey stomach. When at rest the maxillæ, tongue, and labial palpi are folded back as indicated by the dotted line. After CHeshire. irresistible instinct, it flies forth to repeat the process again and again until darkness sets in. It can be seen at once that the industry of the bee places it in the forefront of insects useful in the cross pollination of flowers.

As has been said, bees also collect pollen as an important food for themselves and their young. Some pollen may be incidentally swallowed with the nectar, but most of it is deftly transferred to the hind legs, where it is sometimes heaped up in large masses, having been rendered adhesive, if necessary, by being mixed with nectar. Figure 102 is a photomicrograph of two legs of a honey bee, one loaded with pollen and the other empty. Some other bees, notably the green bees which frequent the yellow pond lilies at early morning,

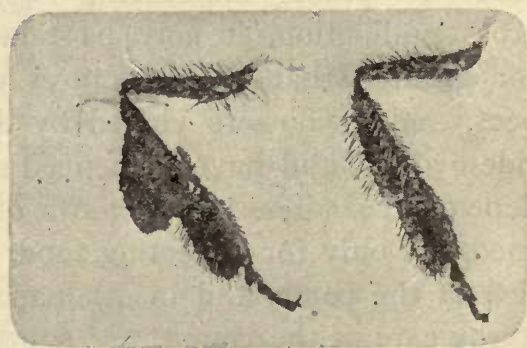

FIG. I02.

Photomicrograph of two posterior legs of Honey Bees, without pollen on the right, and with a load of pollen on the left. $\times 3$. 
have long curved hairs on the hind legs which serve as a sort of basket for the pollen, as shown in Fig. 103, a and $b$.

Having now become somewhat acquainted with the equipment of butterflies and bees for dealing with flowers, it will be well to consider a few special cases showing how the need of the insect for food and of the flower for cross pollination are mutually satisfied.

\section{Cross Pollina-} tion of Datura. - Many flowers possess long tubes at the bottom of which the nectar is stored; such flowers usually have the way to the nectar obstructed by a constriction of the tube, or by outgrowths in the form of hairs, etc., so that only
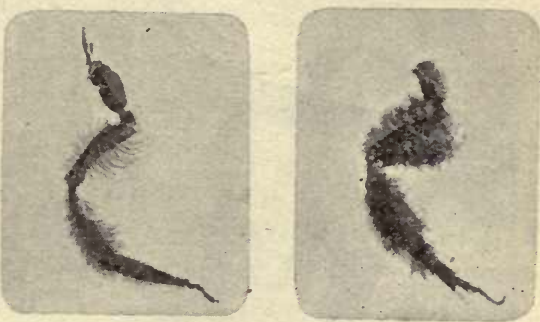

FIG. 103.

$A$, leg of a wild bee with hairs serving as baskets for the collection of pollen; $B$, the same laden with pollen. Photomicrograph $\times 4$. those insects having long proboscides can reach it. The common jimson-weed (Datura stramonium) is an excellent illustration of this. The corolla is about five centimeters long, and the cavity of the tube is nearly closed at about the middle of its length by the insertion of the filaments there. When the flower opens in the evening, it emits a strong musky odor, and a large drop of nectar is already present in the bottom of the tube; so that large sphinx moths, leaving the places of seclusion occupied by them during the day, are attracted by the strong odor and white color of the flowers.

Flying swiftly from flower to flower, the moth thrusts 
its long proboscis to the bottom of the tube and secures the nectar; and while it is tarrying briefly at each flower, keeping itself poised by the swift vibration of its wings, it is pretty certain to touch with its proboscis both anthers and stigmas, which stand close together at about the same height near the mouth of the corolla. Both cross and self

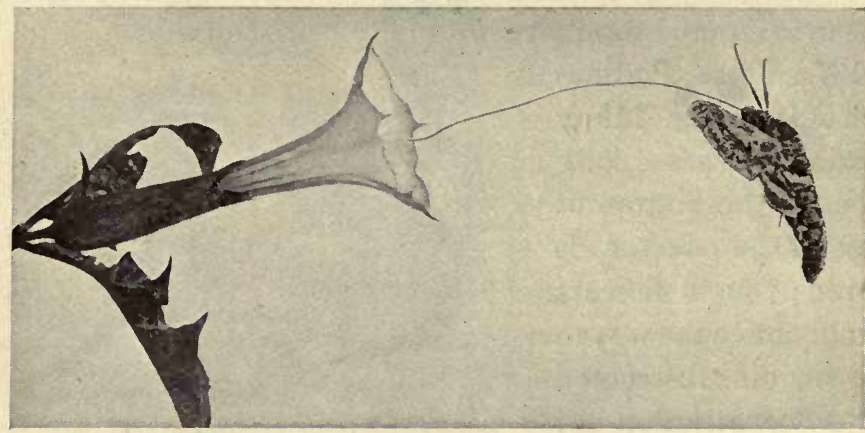

FiG. I04.

Sphinx moth and flower of Datura stramonium, posed to show the relative lengths of the flower and of the moth's proboscis. Reduced rather more than one-half.

pollination might be brought about in this way, but, as Darwin has shown, the foreign pollen would probably possess the greater potency, and cross fertilization would be apt to result. Figure 104 is a photograph of a sphinx moth and Datura flower, posed to show the relative lengths of the moth's proboscis and the corolla tube.

138. Cross Pollination of Salvia. - In the Salvias, or sages, we find several contrivances working together for a common end. The corolla is tubular below and twolipped above, the lower lip serving as an alighting place for bees, and the upper forming a protective covering for the stamens and style. There are two stamens of peculiar construction set one on either side of the mouth of the 
corolla (see Fig. 105). The filament $(f)$ is very short and bears a two-armed connective $(c c)$, the upper arm being long and thread-like and supporting a pollen-bearing anther, while the lower arm is short, spatulate, and sterile. By pressure on the lower arm the upper arm may be rotated

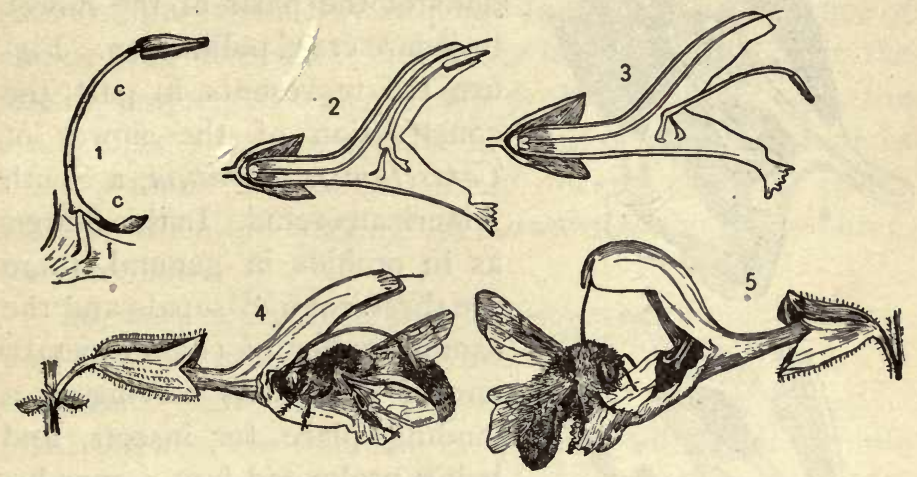

FIG. 105.

Pollination of Salvia glutinosa. r, a stamen. The upright column to the left of $f$ is the filament. $c, c$, the connective, anther bearing above and sterile below. 2 and 3 , longitudinal diagrams of a young flower, showing the anther in its natural position in 2 , and pushed down by a bee by pressing on the lower part of the connective, in 3. 4 , a bee visiting a younger flower; the anthers pushed down upon its back. 5 , a bee visiting an older flower; the style having become elongated and pendent touches the bee's back. After KERNER.

downward, the short filament acting as a fulcrum. When a bee which has alighted on the lower lip attempts to thrust its suction apparatus into the tube of the corolla, its head presses against the lower arm, and the two anthers are rocked forward until they press against the bee's body and discharge pollen upon it (3 and 4). In the younger flowers the styles remain close under the upper lip (diagrams 2 and 3 ), but as the flowers get older the styles bend down so that visiting bees would necessarily rub their backs against the stigmas (5). In this way the 
bees transfer pollen from the younger flowers to the stigmas of the older (4 and 5).

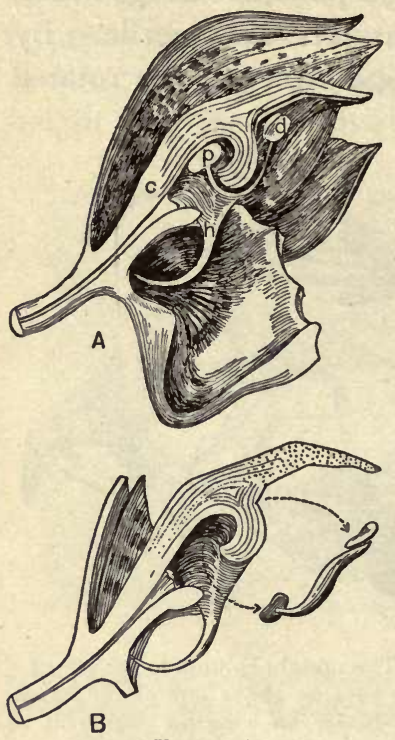

Fig. I06.

$A$, longitudinal diagrams of the flower of Catasetum tridentatum. $c$, the column; $h$, the sensitive spur; $p$, pollinium; $d$, viscid disk. $B$, projection of the pollinium and viscid disk after the stimulation of the spur by the touch of an insect. After KERNER.

139. Cross Pollination of Orchids. - In orchids we find some of the most wonderful modifications of the parts of the flower to secure cross pollination. Figure 106 represents, in part, the construction of the flower of Catasetum tridentatum, a South American orchid. In this flower, as in orchids in general, there are three colored sepals and the same number of colored petals, one of the petals serving as a landing place for insects, and being prolonged into a spur below for the conservation of the nectar. The irregular central body $c$, known as the column, is composed of the pistil confluent with the single stamen. The upper part of the column bears two pollen sacs, each of which contains a mass of pollen, $p$. The pollen masses are connected by means of an elastic band with a body termed the viscid disk $d$, which is really a modified portion of the stigmatic part of the column. Running down from the central portion of the column are two slender horns, $h$, standing in the way of insects which would gather the nectar or eat the fleshy parts of the flower.

As soon as an insect's head touches one of these horns, 
a stimulus is transmitted to the column resulting in the sudden rupture of the tissues connecting the viscid disk with the rest of the column. The viscid disk is then jerked forward by means of the elastic band with sufficient force to pull the pollen masses, termed pollinia, from their pollen sacs and hurl them forward, disk foremost, to a distance of one or even three feet; but if the insect is standing in the line of projection, which would probably be the case, the viscid disk is thrown against its head or thorax and sticks there. Thus the insect is made to carry away the pollen masses, which are sticking out forward as the insect enters the next flower.

The flower just described is known as the male flower, that is the pistil is abortive and does not bear seed. If the insect next visits a female flower, having a perfect pistil but abortive stamens, the pollinia will be thrust into a concave structure known as the stigmatic chamber, which has an adhesive surface capable of holding the pollinia with sufficient tenacity to wrest them from the insect. Then the pollen tubes grow into the ovary and cross fertilization of the eggs is accomplished.

In this flower we see a marvelous correlation of modified parts to attain a definite purpose ; but most wonderful of all is the sudden transmission of a stimulus due to the touch of the insect at one definite part of the flower, and a correspondingly sudden dissolution of. the tissues which hold the viscid disk in place.

140. Cross Pollination of Asclepias. - In the genus Asclepias, we find an adaptation to cross pollination by insects scarcely less wonderful than that of the orchids. Asclepias cornuti (Fig. I07), common everywhere in this country, is perhaps the best species for demonstrating this. As shown in Fig. 108 the sepals $(s)$ and petals $(p)$ are reflexed; the 
stamen's $(r)$ are joined throughout their length, and are united to a thick and flat structure $(t)$ at their apices, known as the stigmatic disk, which is also united with

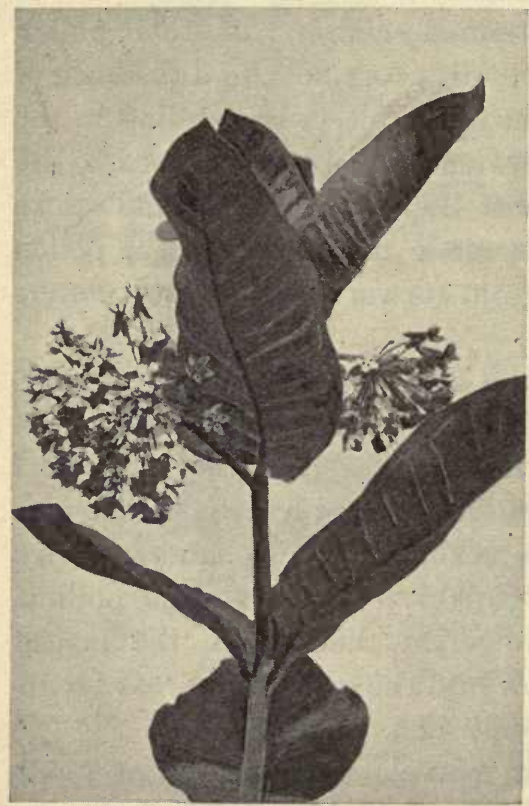

FIG. 107.

Asclepias cornuti. the top of the two pistils $(n)$. The pistils are entirely inclosed by the stamens and the stigmatic disk. Five spreading, hollow receptacles $(v)$ for the nectar grow out and upward from the bases of the stamens.

Each pollen sac contains a compact mass of pollen grains $(w)$ which never become separated from one another, and so constitute what is termed a pollinium. The two contiguous pollinia of adjacent anthers are united by horny rods $(x)$ which converge upward and join with a horny dark body $(y)$ known as the corpusculum, which is hollow and has a slit along its outer face. This slit is relatively broad at the bottom, and tapers toward the top, thus forming a clip in which the feet of the insects get caught. Between each pair of anthers there is a deep recess closed by two vertical lips which stand wider open at the bottom than at the top, and the recess also narrows at the top. The opening between the lips at 
the top stands exactly beneath the slit in the corpusculum.

The surface of the flower is slippery, so that when a bee, for instance, visits it, a good foothold is not obtained until the bee slips its foot into the recess between the anthers, termed
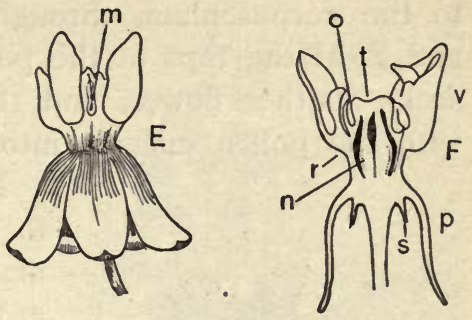
the stigmatic chamber $(m)$. Having obtained a foothold, the bee thrusts its sucking apparatus into the hollow nectar receptacle and obtains the nectar which has invited it to the
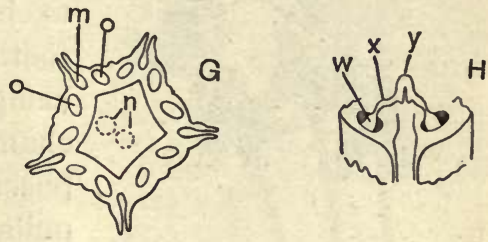
flower. When the bee, however, seeks to go to another flower, its foot slips upward and becomes caught in the slit in the corpusculum. A struggle now ensues which usually
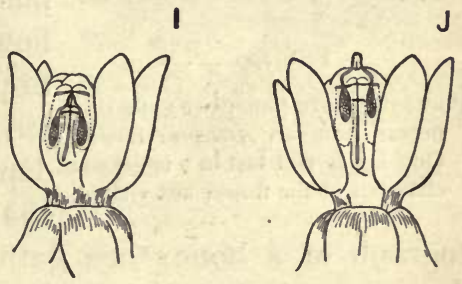

FIG. 108.

Diagram of a flower of Asclepias cornuti. $E$, surface view of a flower, showing the opening into the stigmatic chamber at $m$, the upward-pointing nectar receptacles, and the reflexed petals. $F$, longitudinal diagram of a flower; $s$, sepal; $p$, petal; $n$, one of the pistils; $r$, stamen and nectar receptacle growing from it ; $o$, pollen sac; $v$, nectar receptacle, with stigmatic chamber between it and the cavity containing the pistils. $G$, cross diagram of a part of a flower; $m$, stigmatic chamber; 0 , pollen sac; $n$, apices of the pistils. $H$, diagram showing the relation of the pollinia to the stigmatic chamber; $w$, pollinium ; $x$, connecting rod or retinaculum. $y$, corpusculum or clip. The pollinia are in the pollen sacs and the clip stands over the stigmatic chamber. $I$, semi-diagrammatic view of a flower, showing the pollinia in position (as if the pollen sacs were transparent); outline of pollen sacs shown with dotted lines; at the top of the pollen sacs are slits through which the pollinia are to be pulled out. $\mathcal{F}$, showing the pollinia partly removed from the pollen sacs. - WISE. 
results in the bee pulling the two pollen masses, united to the corpusculum, through the narrow slits (as in $I$ and $J$ ) at the tops of the pollen sacs; and thus laden, it seeks another flower, and there slips its foot, together with the pollen masses, into the stigmatic chamber.

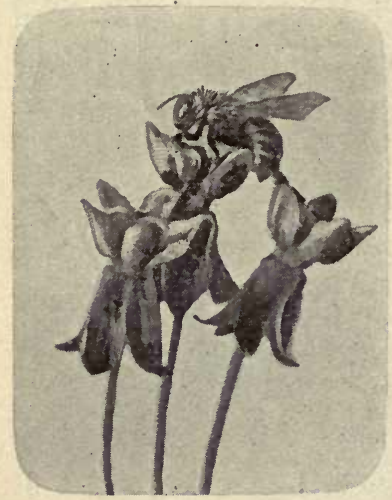

FIG. IO9.

Now when the bee attempts to leave the flower, the pollen masses become tightly wedged at the narrow apex of the chamber, and a hard pull is required to break them loose from the foot. Finally, as the foot is being drawn from the stigmatic chamber it catches into the corpusculum directly above and pulls out a second pair of pollen masses. Thus the bee goes from flower to flower and from plant to plant, repeatedly pulling pollen masses from their sacs and depositing them in the stigmatic chambers. Figure rog is a pho-

Photograph of a honey bee gathering nectar from an Asclepias flower. One leg is still fast in a stigmatic chamber of the flower last visited.

tograph of a honey bee gathering nectar from Asclepias flowers. One of the hind legs is still held in the stigmatic chamber of the flower which the bee has just deserted. Referring to Diagram $G$, note that the pollinia are removed by the bee from the pollen sacs 0,0 , and deposited in another flower in the stigmatic chamber $m$. The bee always inserts its foot in $m$ both in removing the pollinia and in depositing them.

While the honey bee is the most important cross pollinator of this plant, butterflies and wasps are also of service. After the pollinia have been deposited in the stigmatic 
Flowers.

chamber, they put forth pollen tubes which penetrate to the tips of the styles, and then turn downward and find their way to the ovules.

A useful insect is rarely held in captivity by the flower, while weak insects, or their legs which have been pulled off in their struggle to free themselves, are often found hanging to corpuscula that have not been removed from their original places.

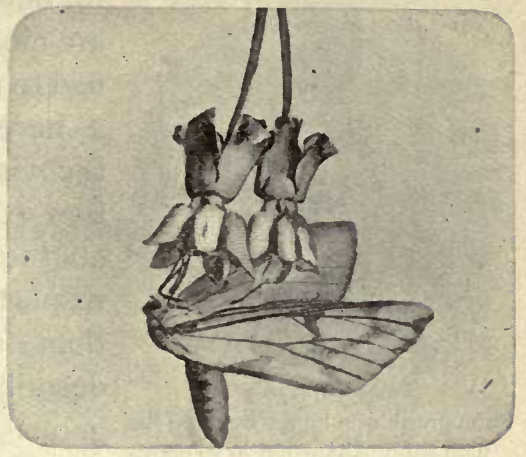

FIG. IIo.

Photograph of a Cabbage Butterfly caught by its legs in the corpuscula of two Asclepias flowers and unable to escape.

Figures I IO and II I illustrate instances of this kind.

Rarely, indeed, a honey bee, too eager in its search

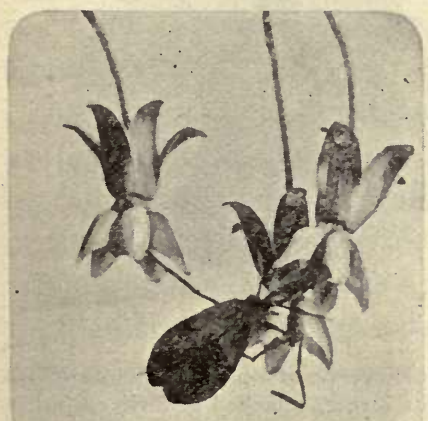

FIG. III.

Photograph of a Moth with its legs caught in the corpuscula of three Asclepias flowers, one leg having been pulled off in its vain efforts to extract the pollinia and escape. for nectar, becomes caught in many flowers at once, and is unable to extricate itself (Fig. I I2).

Figure II 3 is a photomicrograph of a pair of pollinia attached to their common corpusculum, and Figure II 4 is a photograph of a bee's leg with four corpuscula and two pollinia attached. There the second corpusculum has caught on one arm from the first, and so on. 
141. Cross Pollination of Yucca. - Thus far we have taken

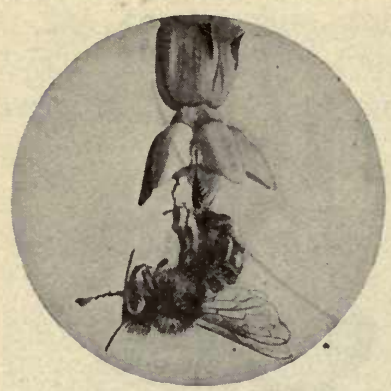

FIG. II2.

Photograph of a honey bee that has died from exhaustion in its efforts to free itself after its legs had been caught in several stigmatic chambers at once. for our illustrations flowers which are more or less profoundly modified to secure cross pollination as a necessary incident attending the visits of insects. We shall now examine an instance of quite another character, and in some respects even more wonderful than those which have been described. The flowers of the genus Yucca, representatives of which are commonly found in gardens, depend almost entirely upon the Pronuba moth for their pollination. The structure of the flower is very simple and readily understood. The perianth is of the liliaceous type, there being three sepals and three petals, all of a creamy white color. In some of the Yuccas these droop forward and form bell-shaped flowers, while in others they are more widely spreading. The six stamens consist of fleshy, outward-curving filaments surmounted by small anthers. The pistil extends beyond the stamens, and the three carpels are im-

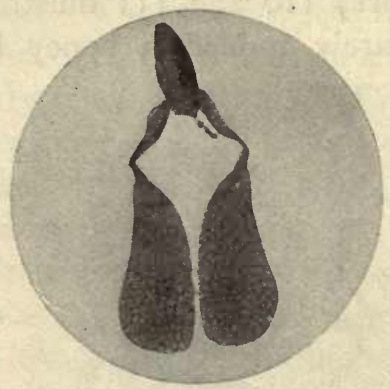

FIG. II3.

Photomicrograph of a pair of pollinia of Asclepias cornuti attached to their corpusculum, as they appear when withdrawn from their pollen sacs. Photographed by transmitted light, and on account of the opacity of the corpusculum the slit in it is not shown, but a portion of a leg of a small insect is pendent from the slit. The pollen grains of which the pollinia are composed can be made out. $\times 15$. 
perfectly united at the top, leaving a tube entirely open at the apex. The inner surface of this tube is stigmatic. The stigmatic tube does not open directly into the cavities of the ovary, but sends off three very narrow branches, each of which communicates with the cavity of a carpel. Accordingly, when pollen is once deposited on the inner surface of the main stigmatic tube, the pollen tubes find easy access to the ovules in each of the three carpels. The pollen is sticky and hangs together in masses, so that it is not adapted to being carried by the wind, and it is apparently impossible

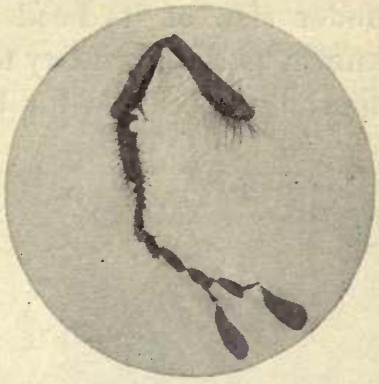

FIG. II4.

Photomicrograph of the leg of a honey bee with a chain of four corpuscula clinging to it. The last two corpuscula are still bearing one pollinium each; the remaining six pollinia have doubtless been deposited by the bee in stigmatic chambers. $\times 5$. for it to get to the stigmatic tube without some outside agent.

A small amount of nectar is secreted, but it is excreted at the very base of the pistil, so that insects seeking it would be far removed from the stigmas. Indeed, the low position of the nectar would seem rather to lead insects away from the stigmas. The flowers are borne in compound racemes high aloft on a strong woody shaft, and, because of their rather strong odor when new buds are opening in the evening and their white color, they are quite certain to make their presence known to insects flying in the twilight (see Fig. I I 5).

If we take these facts as our clew and attentively watch these flowers about eight o'clock in the evening, the method of cross pollination will be made clear. A white moth, 


\section{I98 Introduction to Botany.}

known as the Pronuba moth, is seen to mount a stamen, scrape together the sticky pollen, and pack it against the under side of its head by means of a spinous structure known as the maxillary tentacle, which seems to have been specially developed for this purpose, for in other moths it

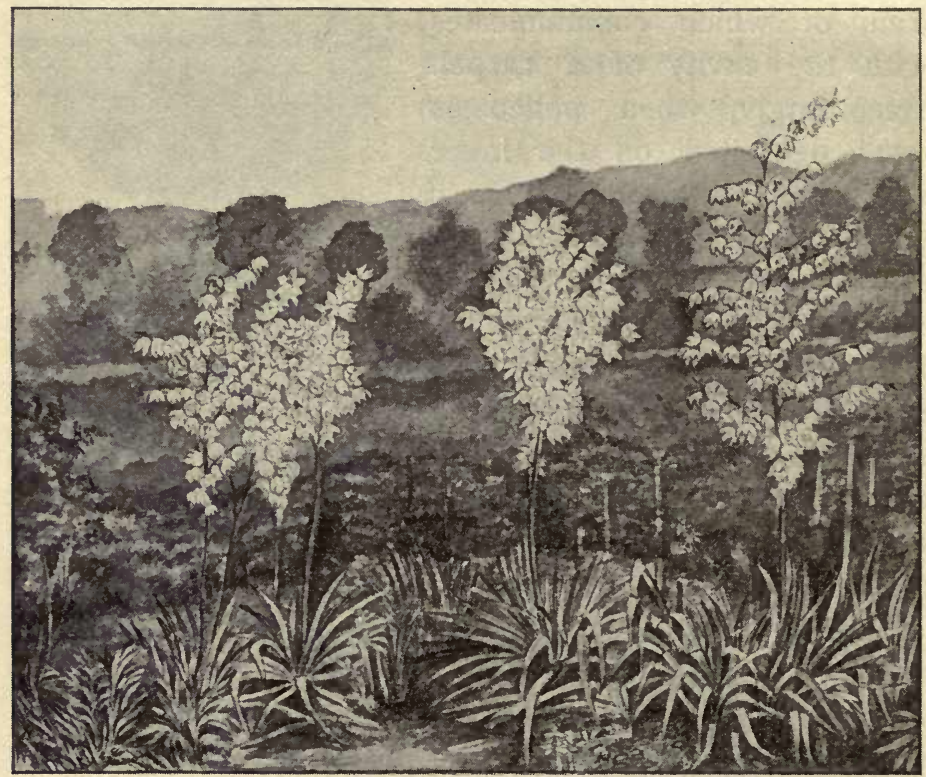

FIG. II5.

Yuccas in the twilight. Drawn from a photograph.

is a mere vestige. In gathering the pollen it hooks its tongue over the end of the stamen, evidently to secure a better hold (see Fig. I 16). Having become well loaded with pollen, as shown in the photomicrograph of the moth's head (Fig. I I 7), it descends the stamen and flies to another flower. There it places itself on the pistil between two of 
the stamens (see Fig. I I 8) and thrusts a slender ovipositor through the wall of the ovary and into the cavity occupied by the ovules.

Having deposited an egg, it ascends the pistil, and by means of the maxillary tentacles and tongue, which at other times are coiled around the load of pollen, as seen in Fig. II 7 , it rubs pollen down the inner surface of the stigmatic tube. Figure II9 is a flashlight photograph of a moth

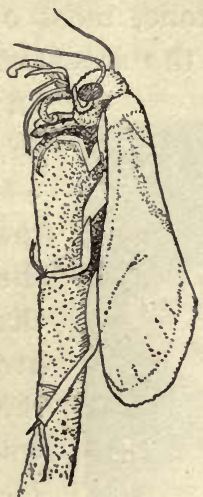

FIG. II6.

Pronuba moth gathering pollen. After RILEY.

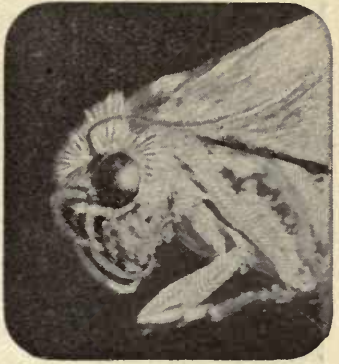

FIG. II7.

Photomicrograph of the head and fore part of the body of a Pronuba moth, showing the tongue and maxillary tentacles coiled under a mass of pollen held beneath the head. $\times 6$. performing this act. The moth then descends the pistil, and standing between another

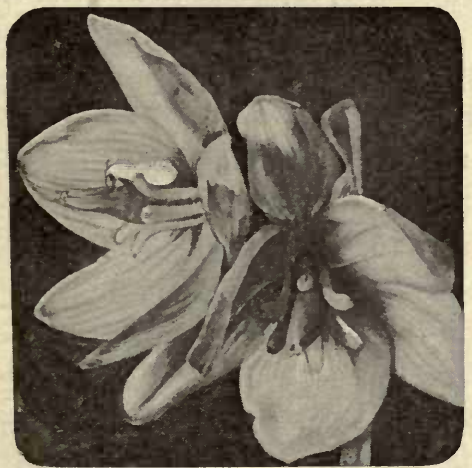

FIG. II8.

Pronuba moth depositing its eggs in the ovary of a yucca flower. Flashlight photograph taken about 8.30 P.M. pair of stamens it deposits another egg within the ovary; then it ascends the pistil and rubs pollen on the stigmatic surface as before. This process is repeated until it may be that each of the six lines of ovules is provided with an egg, and the process of pollination has been as many times accomplished.

The full meaning of this wonderful series of opera- 
tions will not be understood until subsequent developments have been followed. Since the process of pollination has been so thoroughly done, most of the numerous ovules become fertilized and the seeds begin their development.

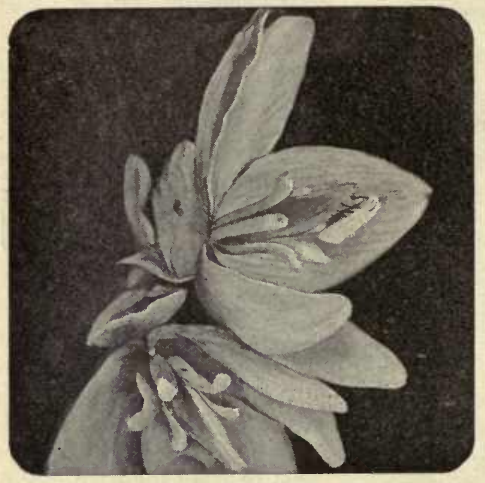

FIG. II9.

Pronuba moth rubbing pollen down the stigmatic tube of a yucca flower. Flashlight photograph taken about 8.30 P.M.

In the meantime the moth eggs hatch into larvae, which find their food in the developing seeds. But the seeds are so numerous that the larvae reach their growth, gnaw a hole in the seed-pod and escape, while many uninjured seeds still remain in the pod. The larva spins a thread by which it descends to the ground, and, burrowing beneath the surface, it passes the winter in its pupal state, emerging as a fully developed moth at the time of the flowering of the Yucca the following summer.

It appears that the mature moth takes no food, unless it secures some of the nectar of the Yucca blossoms in which it is wont to pass the day, with its head close to the bottom of the flower where the nectar is excreted. It does not eat the pollen which it gathers, and it seems certain that it is prompted to place the pollen in the stigmatic tube after each act of oviposition solely by the instinct to provide for its young; for it is readily understood that if the ovules are not fertilized the seeds would not develop and the larvæ would be without food.

The Yucca flower, instead of having elaborate devices 
to secure cross pollination, simply prohibits self pollination by its tubular stigmas and its relatively short and reflexed stamens; and then, the sticky pollen and an abundance of ovules being provided, the performance of pollination is intrusted to the wise instinct of the Pronuba moth; and not pollination simply, but cross pollination, for it has been noticed that it is the habit of the moth after securing the pollen to fly to another flower before it begins to lay its eggs. We wonder how such an instinct could have been evolved, and how the moth and the plant came to be so intimately associated and so absolutely necessary to each other's existence. It seems certain that they have come through the long years of their race history together, and that each has been affected by the modifications of the other.

Sufficient illustrations have now been given to show the student that there is a wide and attractive field for study in the structure and behavior of flowers; for they afford us not only the best evidence of the relationships of plants (see Chapter XVII), but they also reveal to us a mutually beneficent association of plants and animals, and the marvelous plasticity of plants in molding the forms of their parts and responding to external forces and internal conditions in such a way as to meet any required end.

142. The Morphology of a Flower. - In doing the work in the chapter on Modified Parts, the student has become familiar with the methods of seeking out morphological evidence, and he should now test, with all the evidence obtainable by him, the following statement of the morphology of a flower: A flower is a branch with much shortened internodes (termed the receptacle) whose growth in length is terminated by the production of spore-bearing leaves (stamens and carpels); the most complete flowers also having modified leaves in the form of sepals and petals. 
In order to understand the morphology of the parts of a flower we must refer back to simpler and older types of vegetation. The Lycopodiums or club mosses are representatives of an ancient group of plants which reached its

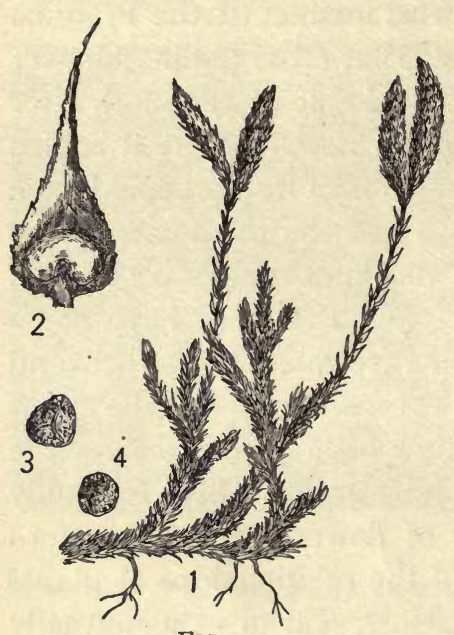

FIG. I20.

I, Lycopodium clavatum, the leaves aggregated into cones at the apices of the branches; 2 , a leaf from the cone, with a sporangium in its axil; 3 and 4, spores from the sporangium. After WOSSIDLO. maximum development in the Carboniferous period. There are good reasons for the belief that the Lycopodiums and the flowering plants are offshoots from a common ancestral stock, the Lycopodiums being, of the two, much less modified and more primitive in character. We find in them that the stem is thickly beset with small, awl-shaped leaves (Fig. I20). Near the apex of the stem some of the leaves bear spore cases or sporangia at their bases. In certain species the spore-bearing leaves or sporophylls are broader and longer-pointed than the foliage leaves, but there may be all degrees of gradation between the two forms. In other species there may be no difference in appearance between the foliage leaves and sporophylls. In some species the sporophylls are aggregated into a cone, in others not.

In the Lycopodiums the sporangia and spores are of one kind only; but in the somewhat nearly related genus Selaginella, there are two sorts of sporangia, borne each on the stem in the axil of the sporophyll, which in general 
does not differ from a foliage leaf in appearance. One kind of sporangium contains relatively small spores termed microspores (Fig. I2I) which on germination give rise to a rudimentary plant body called prothallium, bearing sperm cells; and the other sort of sporangium contains relatively large spores, the macrospores (Fig. I2 I), which on germination produce a prothallium bearing egg cells. The sporangium containing macrospores is termed macrosporangium, and the sporophyll subtending it macrosporophyll, while the corresponding parts relating to the microspores are called microsporangium and microsporophyll.

Now, in a flower, since the pollen grain produces

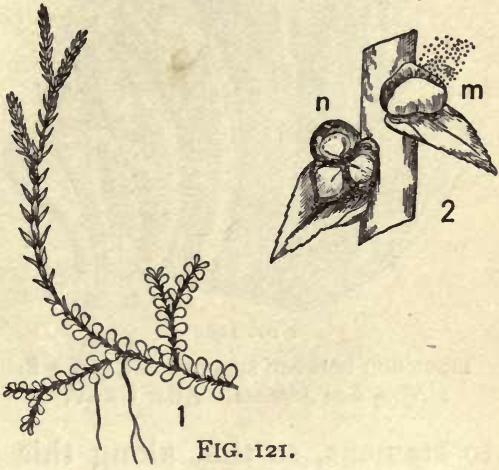

I, Selaginella. Toward the summit of the plant the leaves are more pointed, and they become aggregated into a cone-like group at the apex. 2, Microsporangium, $m$, discharging microspores; $n$, macrosporangium containing macrospores. After STRASBURGER. the sperm, it must be a microspore, the anther a microsporangium, and the entire stamen a modified microsporophyll. The large cell in the ovule (termed embryo sac) which produces the egg must be the macrospore, and the ovule the macrosporangium, while the pistil evidently corresponds to a macrosporophyll with the edges infolded and grown together forming an inclosed chamber. Each sporophyll taking part in the formation of a pistil is called a carpel. Or the pistil may be composed of more than one sporophyll united, as indicated by the number of styles, stigmas, or groups or rows of ovules. When composed of a single sporophyll or carpel the pistil is said to be simple, when of 
more than one, compound. The sepals and petals may be either modified foliage leaves or barren sporophylls. Evi-

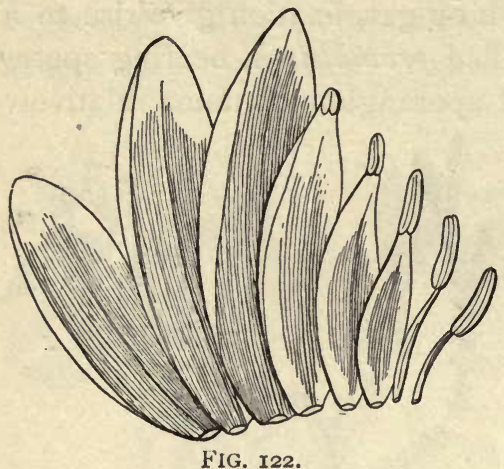

Transition between stamens and petals in Nymphcea odorata. After GRAY. dence that they may be the latter is found in the fact that all gradations of transition between stamens and petals occur in the sweet-scented pond lily, Nymphaa odorata (Fig. I22), and in various double flowers. But on the other hand all degrees of gradation may be found from foliage leaves, through sepals and petals to stamens, so that along this line of evidence we cannot come to a positive conclusion. Since in the relatively ancient-Lycopodiums and Selaginellas there may be no difference in appearance between the foliage leaves and sporophylls, and in others there may be all degrees of gradation between them, it would seem that foliage leaves and sporophylls have had a common origin.

The relative positions of the different parts of flowers may show considerable variation, as seen in diagrams of row $d$, $A, B, C, D$, of Fig. I23. When the parts arise near together on the apex of the receptacle $(d, A)$, the pistil is said to be superior and the flower hypogynous. When the outer part of the receptacle is prolonged into a tube or cup carrying the sepals, petals, and stamens above the insertion of the pistil $(d, B)$, the pistil is called half inferior and the flower perigynous. When the zone of the receptacle bearing sepals, petals, stamens, and carpels is prolonged in the form of a hollow tube or cup which forms 
the outer wall of the ovary $(d, C)$, the pistil is called inferior and the flower epigynous. In determining the
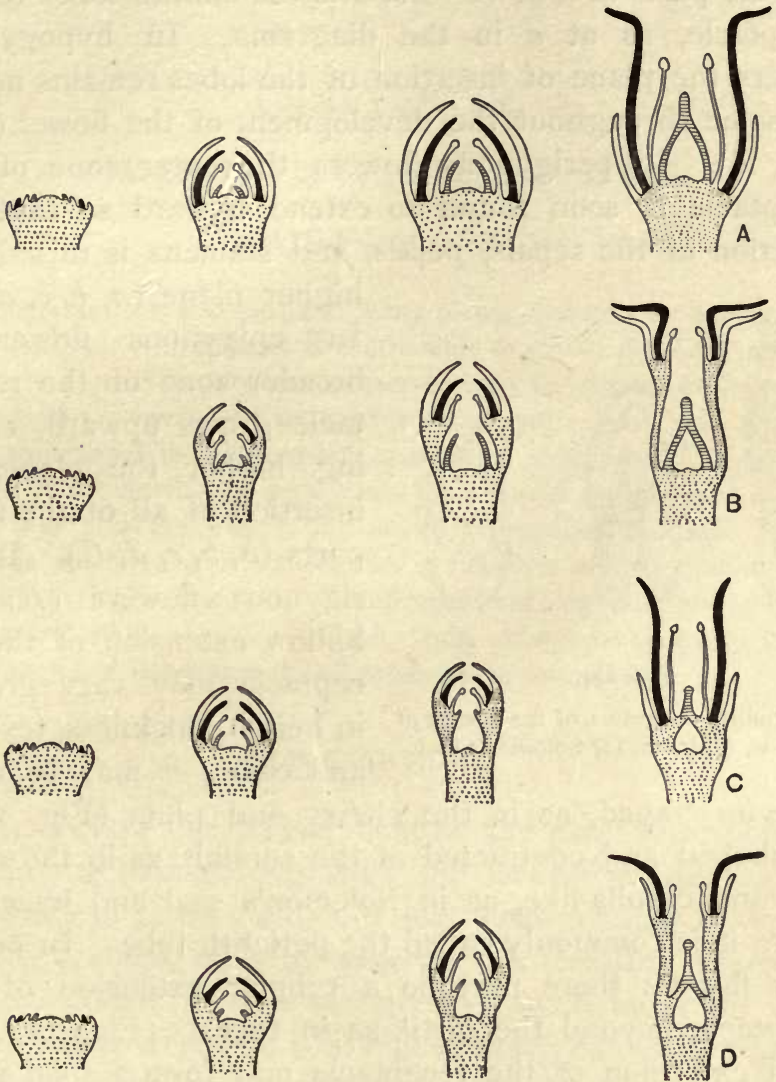

$a$

b

FIf. I23.

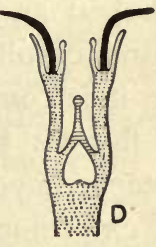

$d$

Diagrammatic representation of the embryology of $(A)$ hypogynous, $(B)$ perigynous, and ( $C$ and $D$ ) epigynous flowers. The receptacle is dotted, the sepals and stamens are unshaded, the petals are black, and the pistil ruled horizontally. In such flowers as $B$ and $D$ the tubular extension of the receptacle, commonly called the tube of the corolla, may be considered a distinct member of the flower, termed the ring leaf. (See Ganong, "The Teaching Botanist.") After GANONG. 
identity of the parts of perigynous and epigynous flowers their embryology must be called in evidence. It is seen that the parts of a flower first arise as minute lobes of the receptacle, as at $a$ in the diagrams. In hypogynous flowers the plane of insertion of the lobes remains nearly the same throughout the development of the flower $(a, b$, $c, d, A)$. In perigynous flowers the outer zone of the receptacle is soon found to extend upward so that the insertion of the sepals, petals, and stamens is raised to a

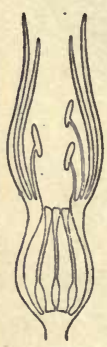

a

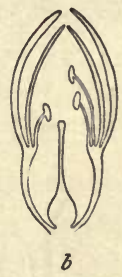

FIG. 124.

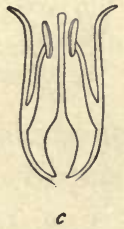
higher plane $(a, b, c, d, B)$. In epigynous flowers a broader zone of the receptacle grows upward, carrying higher the planes of insertion of all of the floral parts $(a, b, c, d, C)$. In perigynous flowers $(B)$ the hollow extension of the receptacle may vary greatly in height, thickness, texture, Longitudinal diagrams of the flowers of (a) rose, (b) plum, (c) Solomon's seal. and color; it may be thick and cup-shaped, as in the cherry and plum (Fig. 124); urn-shaped and contracted at the summit, as in the rose; thin and corolla-like, as in Solomon's seal and hyacinth, where it is commonly called the perianth tube. In epigynous flowers there may be a tubular extension of the receptacle beyond the pistil, as in fuchsia (Fig. 123, D); or an extension of the receptacle may form a solid shaft surmounting the ovary, bearing sepals, petals, stamens, and styles at its summit, as in Iris. But in this instance, and in some others where the pistil is inferior, it may be that the inner portion of the outer wall of the ovary is carpellary tissue adherent to the hollow receptacle. 


\section{CHAPTER IX. \\ DISPERSION OF FRUITS AND SEEDS.}

\section{PROVIDING MATERIAL.}

Since the fruits and seeds of many plants mature after the work of the school year is completed, it is advisable to gather the most interesting forms as they mature, and preserve them for the work of the succeeding year. Seeds and fruits with wings, barbs, hooks, etc., may be dried and stored in boxes or any convenient receptacles. Seeds with delicate hairs serving as parachutes, etc., should be gathered shortly before their pods break open, and stored in pasteboard boxes. When the pods are large, it is a good plan to tie them so they cannot burst open on drying. The pods of milkweed and dogbane, for instance, may be treated in this way. Fleshy fruits should be gathered in different stages of development and preserved in formalin.

\section{OBSERVATIONS.}

I35. Follow the development of the pistil after pollination, in the cases of some of the flowers studied in the laboratory or in the field, and note what changes it, or any parts connected with it, undergo. Interesting examples may be found in the strawberry, anemone, rose, crab apple, gooseberry, plum, larkspur, violet, oxalis, geranium, milkweed, spurge, cottonwood, hornbeam, hop hornbeam, oak, walnut, hazelnut, maple, sumac, catalpa, climbing bittersweet.

136. Make drawings of fruits having hooks, anchors, etc., by means of which they cling to animals and become scattered by them. Typical forms of this sort, of common 
occurrence, are the cocklebur, burdock, black snakeroot, common beggar ticks, etc.

I37. Make drawings of seeds or fruits which are adapted to being carried about by the wind. Good examples are dandelion, maple, elm, milkweed, dogbane, pine, goldenrod, and feathergrass.

I38. Make drawings and notes showing the method of dispersion of the seeds of some water plants, such as those of pond lilies.

I39. In your notes discuss briefly the following questions: Of what advantage to the species is it for plants to produce edible fruits? Would the minute ripened pistils of the strawberry, or the small seeds of the fig, grape, gooseberry, etc., be apt to be broken when the seeds are eaten? Of what advantage is the bitter taste of the seeds of the orange? What common plants probably have their seeds scattered by birds?

I40. Determine the morphology of the various devices for dissemination in the fruits and seeds studied, and record observations and conclusions in your notes.

I4I. Count the number of seedlings which spring up in one square foot of ground, and as they grow older note how many of them succumb in the competition for food and light.

\section{DISCUSSION.}

143. Importance of Seed Dispersion. - A moment's reflection will convince the student that the dispersion of the seed to some distance from the parent plant is of vital importance to the continuance and well-being of the species. If the seeds were to fall immediately beneath the plant, a crowd of offspring would result, growing so close together as to deprive each other of a sufficient amount of sunlight 
and raw materials from the soil. Or, if the parent were a perennial, it would overshadow and starve the seedlings. Even with some means of seed dispersion, there is apt to be greater competition between individuals of the same species than between those of different species, because plants of the same species require the same proportions of the different soil constituents, and, having the same habit of growth, crowd each other more than the same number of individuals of different species distributed over the same area would be apt to do. One need only compare the number of seedlings of a particular species appearing above the soil in a given area with the number of those which actually reach maturity in the same area to be convinced of the struggle which is taking place among them, with fatal results except to the relatively few individuals which are stronger and more rapid growers than their fellows. Any devices which aid in dispersing the seed help to ameliorate these conditions.

144. Migration by Seeds. - The dispersion of seeds and other reproductive bodies has been of vital importance to plants in another way: The climate of the earth has been undergoing profound changes through the long geological periods. In some places a temperate, or even a warm, climate has given way to a frigid one; and then after a long time the temperate climate has returned. By means of fruits and seeds capable of dispersion, plants have been able to recede before the advancing cold, when without this power they would have become extinct. And finally when the warmer climate returned, they have been able to follow the retreating ice back toward the arctic circle.

In the same way they have taken possession of land which has risen on the borders of continents, or far out in the oceans in the form of volcanic or coral islands, and of 
the rich deposits which have accumulated at the mouths of rivers. Much of the variation resulting in the great multiplicity of form and structure which we now see has originated in the different environments found by plants by means of their migrant seeds.

We see, then, that from many points of view it is important to plants to produce reproductive bodies capable

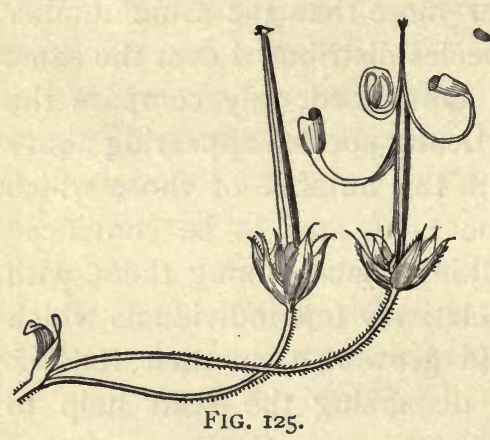

Elastic carpels of Cranesbill; seed being thrown on the right. After KERNER. of dispersion by their own movements or by natural agents outside of themselves, such as the wind, running water, birds, insects, etc.; and we may expect to find a great variety of devices intended to facilitate the scattering of fruits and seeds.

145. Dispersion by Elastic Tissues. - The seeds of the wild cranesbill are scattered by a sudden springing outward of the valves of the carpels. This action is caused by the outer layers of cells being more succulent and shrinking more on drying than the inner layers. The ripened seeds lie loosely in the inflated lower portion of the carpels, and are consequently thrown out when the carpels spring upward (see Fig. 125).

When the fruits of the common Euphorbias are picked and placed on a table in a dry room, the carpels suddenly spring apart as they become dry, and hurl the seeds with considerable force to various parts of the room. Of course they act in the same way under natural conditions out of doors. 
The behavior of the seed pods of the wild and cultivated touch-me-nots is familiar to every one. Here the valves of the pods suddenly coil elastically when touched, and scatter the seeds.

In many plants the carpels break apart in such a way as to allow the seeds to lie loosely in them, so that when the plant is violently shaken by the wind, or when it suddenly rebounds after being bent down by passing animals, the seeds are thrown from their position. In many composite plants the dry fruits which lie loosely on the receptacle are scattered in this way, the stems having become dry and elastic by the time the fruits have ripened.

A very curious case where the elasticity of succulent - tissues is employed is found

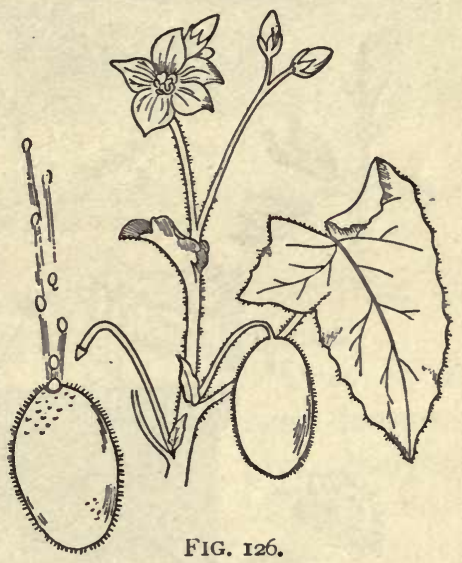

A branch of squirting Cucumber, showing a fruit falling off and ejecting the seeds. After KERNER. in the squirting cucumber (Fig. 126). When the fruit ripens, a portion of it in the form of a plug continues with the stalk, becomes separated from the surrounding tissues while still remaining attached to the stalk, and is drawn out like a stopper when the fruit drops off. Then the swollen mucilaginous contents which had kept the walls of the fruit in a state of tension are forcibly ejected, together with the seeds, by the sudden contraction of the walls.

146. Dispersion by Winds. - A common device designed to employ the wind as an agent of dispersion is found in 


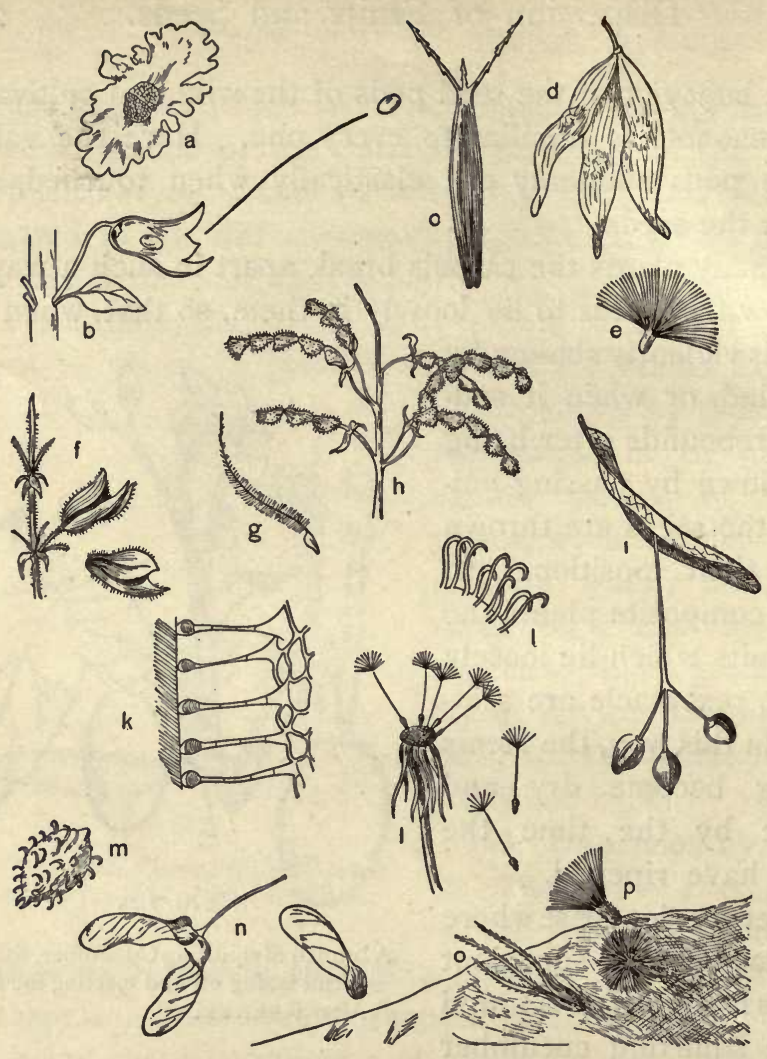

FIG. I27.

Various devices for Seed Dispersal. Arrangements for dispersal by means of the wind are shown at $a$, seed of a Bignonia; $d$, fruits of Ailanthus; $e$, seed of Sulix myrsinitis; $g$, fruit of Geum montanum; $i$, fruits of Tilia, where a bract serving as a wing adheres to the fruiting peduncle; $l$, fruits of Taraxacum, where the modified calyx or pappus acts as a parachute; $n$, fruits of Acer. Devices for catching hold of passing animals are shown at $c$, fruit of Bidens bipinnata; $h$, Hedysarum Canadense, the jointed fruits of which are beset with hooks, as shown at $j ; m$, fruit of cocklebur. Sticky glandular hairs covering the calyx containing ripened fruits occur in $f$, Salvia glutinosa; the magnified hairs are shown adhering to an object at $k$. Hurling the fruits from a catapult formed by the persistent calyx and elastic pedicel occurs in Teucrium Euganaum at $b$; here projection occurs when the plant is shaken, etc. Creeping of fruits by the hygroscopic movement of arms and hairs: 0 , Egilops ovata; $p$, Crupina vulgaris. After KERNER. 
those seeds and fruits with outgrowths in the form of sails or wings. In the case of elms, maples, Ailanthus, etc. $(a, d$, n, Fig. 127), the border of the ovary grows out in the form of a membrane; and

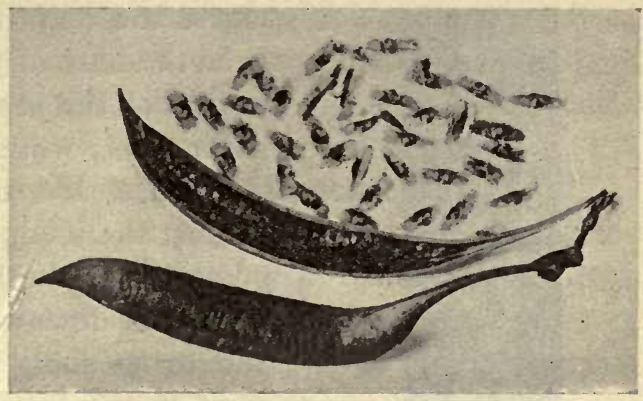

FIG. I28.

Seed pod of Trumpet Creeper broken open and winged seeds fallen out. Reduced. in the seeds of the Catalpa and trumpet creeper (Fig. 128) the walls of the seeds behave in a similar manner.

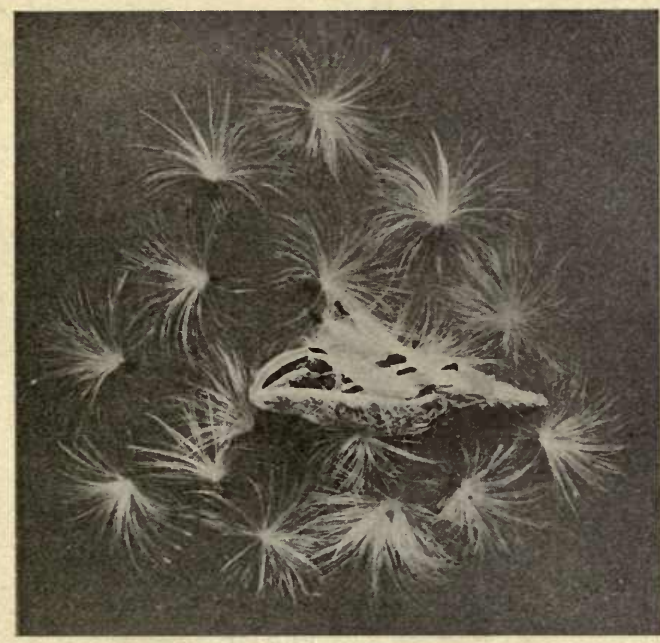

FIG. I29.

Seed pod of Asclepias cornuti and escaping seeds. The tuft of hairs on each seed acts as a parachute. Reduced.
One of the best devices to give buoyancy to light seeds or fruits is an outgrowth of hairs, such as is found on the fruits of dandelion or Anemone, or on the seeds of the poplars, milkweeds (Fig.129), cotton of commerce, etc.

In the cottonwood (Populus monilifera), 


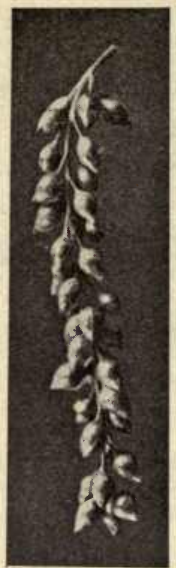

F1G. I30.

Seed pods of the Cottonwood just before breaking open. Reduced.

where the hairs are an outgrowth from the base of the seed, the total weight of the seed and hairs is $\mathbf{I . 5}$ milligrams; while in the seed pod the hairs are closely appressed against the seed, but after the pod breaks open the hairs begin to dry and bend downward and outward, and in so doing they assume the form of a parachute and spread the seeds apart at the same time, so that the slightest puff of wind carries them away. On a single tree the pods may be breaking open in succession and offering their seeds to the wind for a space of two months, so that the seeds are quite

certain to be borne away by winds from all directions. In strong winds, such seeds must be carried to very great distances (Fig. I3I).

In the tumbleweeds another method has been devised for employing the wind in scattering the seeds. These plants have a rounded general contour; when the seeds have ripened, the whole plant dies and breaks off close to the ground, and is easily rolled by the

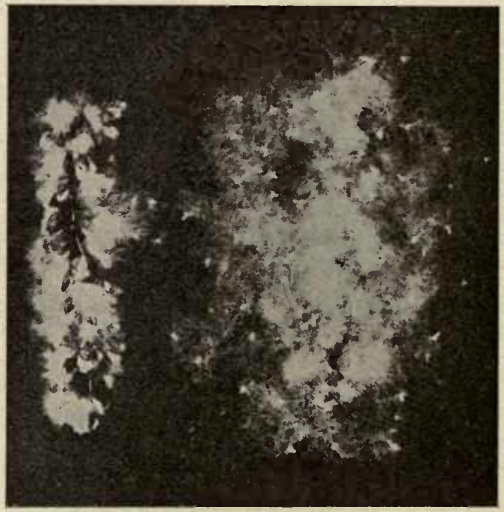

FIG. 13I.

On the left seed pods of Cottonwood breaking open and the hairs on the seeds beginning to spread apart and push the seeds out of the pods; on the right, a later stage, the seeds ready to be wafted away by the wind. Reduced. 
wind across open spaces, the seeds being scattered from their pods during the journey. It is this tumbling habit which helps to make the Russian thistle such a troublesome pest.

147. Dispersion by Birds. - The birds are the most effective agents among animals for distributing seeds. They often swallow berries and other pulpy fruits whose seeds are too small or hard to be broken up in the gizzard, and are finally ejected undigested and capable of germination. Such berries are usually inconspicuous and unpalatable until the seeds are ripe, when they take on bright colors, are often fragrant, and their pulp becomes agreeable to the taste, with the evident design of attracting those animals which may aid in scattering the seeds.

Small seeds are often carried from place to place while embedded in the mud clinging to the feet of birds and other animals; thus Darwin found that eighty-two seeds germinated from the mud taken from the feet of a single partridge. The small floating seeds of water plants often cling to water fowl as they rise for flight, and are carried to other waters. Those birds which are able to sustain swift and prolonged flight must be particularly efficient in scattering seeds over broad areas. Pigeons and cranes, for instance, can fly about forty miles per hour, and swallows and peregrine falcons can cover a hundred miles in the same length of time.

148. Dispersion by 0ther Animals. - Seeds of various plants are carried away and stored by squirrels and other animals. Most of these seeds are doubtless eaten, but some of them are dropped in transit. In such cases the service of carriage seems to be dearly paid, but we may be sure the expense is not unwarranted, since nut-bearing trees and shrubs have been able to maintain themselves 
successfully in competition with other plants. The acorns, which, in spite of their astringent taste, are palatable to many nut-loving animals, are often carried away by them; and they are also stored by woodpeckers for the sake of the insect larvæ which frequently occur in them. On account of their bulk and weight, the acorns are doubtless occasionally dropped to the ground, and eventually succeed in becoming oak trees.

Hooks, barbs, and sticky glandular hairs for clinging to passing animals are frequently found on the fruits of many families of plants (see $h, j, k, m$, and $c$, Fig. 127). Every one is familiar with the fruits of the cocklebur and burdock, which cling in great numbers to the tails and legs of cattle and horses; and the clinging qualities of the fruits of black snakeroot, beggar-ticks, and bur-grass are too familiar to need more than passing mention. In Mentzelia, the leaves, stems, and fruits, are covered with anchor-like hairs which become fastened to garments or to animals so tenaciously that large portions of the plant may be broken off and carried away at once.

149. Dispersion by Water. - The nut-like fruits of the yellow water lily, Nelumbo lutea, are borne in top-shaped receptacles which are at first upright, but, as the fruits ripen, bend downward so that the nuts drop out or are shaken out by the wind, and sink at once to the bottom of the water (Fig. 69). Later in the season the receptacles break away from their dry stalks and float about on the water, carrying with them the few nuts which are still held captive. In this way a considerable distance from the parent plant may have been traversed by the time the receptacles have become sufficiently softened to set the remaining nuts free.

The fruits and seeds of many genera of water and 
marsh plants are able to keep afloat for a long time and become scattered about by the wind, finally sinking and taking root in the mud. The fruits of the cocoanut and other palms may float for a long time in the sea water, and are still able to germinate after having been cast up on far-distant coasts.

These few examples will serve to introduce the student to the almost endless variety of devices for dispersing seeds and fruits, examples of which he can readily find at the proper season. 


\section{CHAPTER $\times$.}

\section{STUDIES OF SELECTED SPERMATOPHYTES.}

In Chapter VIII general directions are given for the study of flowers. The studies which follow are intended to show how in a course of limited time certain plants may be selected to bring out facts of particular interest regarding the structure, behavior, and relationship of plants. In this work Kerner and Oliver's "Natural History of Plants" and Müller's "Fertilization of Flowers" are most useful books of reference.

\section{Naias flexilis.}

This plant flowers in summer, and material should be gathered then and kept in formalin for class use.

The flowers are of special interest on account of their great simplicity. There are no devices to secure pollination by means of insects, for the plant is entirely submerged, and rooted, and pollen is carried to the stigmas by water currents. In this genus the flowers are monœecious or diøcious. The staminate flower consists of a single stamen terminating the floral axis, surrounded by a hyaline membrane, and this in turn by a tubular leaf, both of which constitute a double perianth. The pistillate flower consists of a single ovule having the two ovular coats ordinarily found, and an enveloping leaf which may be considered as a carpel.

Draw staminate and pistillate flowers as seen under a hand lens. 
The form of the plant is interesting, since it is plainly adapted to its submerged habitat (see page 323). Take fresh plants from water and note how quickly they dry up. The lack of a waterproof epidermis probably enables this plant to absorb gases in solution throughout its surface.

Do the seeds float when ripe, or sink at once to the bottom?

This is apparently one of the lowest of the Monocotyledons. Its simple staminate and pistillate flowers remind one of the sporangia of Pteridophytes, and it seems to be on the direct line of ascent from Pteridophytes to Spermatophytes. The evidence, however, is not sufficient for a definite conclusion.

\section{Arisæma triphyllum.}

Make a drawing to show the habit of the entire plant. What terms would you apply to the leaves as to their habit and form? Are they simple or compound, radicle or cauline? (See Glossary.). The enlarged underground part is a corm stored with reserve food.

The inflorescence is unique. The slender, naked peduncle is called a scape. The leaf-like envelope of the inflorescence, funnel-shaped below, overarching above, is termed a spathe. The fleshy floral axis inclosed in the spathe is called a spadix.

The flowers are very simple and destitute of a perianth. A single staminate flower consists of four stamens, and a pistillate flower of a single pistil. Note whether they are monœcious or diœcious, proterandrous or proterogynous (see Glossary).

Make a drawing of an inflorescence with the spathe cut away on one side to reveal the interior. Halve a pistil longitudinally and draw the ovules in position. Draw a 
stamen on a large scale, showing the manner of the dehiscence of the anthers. Draw a stigma on a large scale, showing the character of its surface.

Of what use is the spathe? Note the behavior of insects while visiting the flowers. Can you see any use for the sterile upper part of the spadix? Can it, among other uses, serve the attractive function of a corolla?

The student will find it interesting to observe the behavior of this plant throughout the season. Examine plants in their natural habitat, and find answers to the following questions: What becomes of the spathe and sterile portion of the spadix? What kind of fruit does the ripened pistil become? When and in what way do the seeds become planted in nature? At what time of the year do the leaves wither away? After the above-ground parts have withered and the corm has entered into its winter rest, can the inflorescence and leaves of the following season already be found in an embryonic condition in the apical bud of the corm?

Cut the corm in two and put a drop of iodine on the surface. Does a purple color ensue, indicating starch as reserve food? The corm contains a very acrid substance. In tasting it only a very small piece should be placed for a moment on the tongue, for if much is put into the mouth and chewed the result is exceedingly painful. This acrid substance is doubtless a protection against the depredation of animals, for when it, being volatile, has been driven out by boiling or drying, the corms are found to be edible. Exceedingly minute and sharp crystals of calcium oxalate occur abundantly in the corms, but they do not cause the pain when the fresh corms are eaten, as some have thought.

If obtainable, study Arisama dracontium and note the 
points of dissimilarity which cause these two Arisamas to be classed as different species.

Erythronium albidum (or other species).

Make a diagram of the flower, following the general directions on pages I48-151. Make a drawing to show the habit of the entire plant, including the bulb and roots. How deep does the bulb lie in the ground? How close to the ground do the leaves originate? What is the light relation of the leaves? Do both sides of the leaf receive the light energy about equally? How early in the spring do the leaves appear? What advantages do you see in the very early development of this plant? In its chosen habitat is it at a disadvantage later in the season? Is it apt to be much shaded by other plants? Make observations in the field to determine how leaves and flowers come through the ground. Is the flower upright or in a nodding position when it first appears? Does the flower open as soon as it appears? Of what use is the nodding position of the flower? Do insects visit the flowers, and if so see whether they appear to be in quest of pollen or nectar. Can you find nectar and nectaries? In what ways are nectar and pollen protected from the rain? At what time in the development of the flower do the anthers dehisce? Are the flowers at all proterogynous or proterandrous? Do the anthers touch the stigmas, or could self pollen fall upon them?

Note the further course of the plant through the season. Do many seeds mature in the capsule? How are the seeds scattered? When do the leaves ripen and wither away?

The buds of Erythronium Americanum and Erythronium albidum are each successive year formed deeper in the ground by means of an offshoot which bears a new bulb at 
its end. Erythronium mesochorium does not produce offshoots, but each year a new bulb is formed which pushes down a little below the parent bulb within which it remains inclosed, so that the scars of last year's roots may be found a little higher than the new roots of the current season:

After the plant has reached the age of flowering the rudiments of next year's leaves and flowers are already formed in the bulb before the leaves of the current season ripen and wither away. With plenty of food stored about them in the thick coats of the bulb the young leaves and flowers are able to unfold quickly in the first days of spring. The advantage of an early start in the spring is evident for a low plant whose habitat is grassland, where it is in danger of being browsed after the grasses appear, or woodland, where the foliage of trees shuts out the light.

The way in which the two leaves enfold and protect the flower bud, while the hard tip of the outer leaf breaks a way through the ground, is admirable.

Sprengel says of the European species, Erythronium dens-canis, that the thin basal part of the ovary secretes the nectar, the inner perianth segments having two auricles at the base of each, between which and the nectary the nectar is conserved. Loew and Briquet conclude that the nectar is secreted by the lower part of the perianth segments below the auricles. See how the species in hand agrees with these statements.

The hanging position of the flower seems to unfit it for self pollination or cross pollination by wind. The coloration of the perianth and the secretion of nectar point to insects as agents for cross pollination. Bees are known to be frequent visitors.

The Erythroniums are excellent for showing the main characteristics of the lily family. Other genera should be 
studied to show the range of variations within the family, and on what grounds the members of a family are grouped into genera. Among cultivated plants, hyacinth, tulip, Yucca, and lily, and of wild flowers some species of Allium, Nothoscordum, Polygonatum, and Camassia might be studied.

Most of the flowers of the lily family allure insects by means of pollen or nectar, or both, and by their fragrance or bright colors. Where the flowers are small, they are often massed in a more or less compact inflorescence for a greater color effect, as in the case of hyacinth and Camassia. Frequently cross pollination is assisted by proterandry or proterogyny. The Yucca is one of the most interesting members of the lily family in the method of its cross pollination (see page 196).

\section{Hypoxis erecta.}

Make diagrams of the flower. In what essential respects does this flower differ from that of Erythronium? What evidence of relationship do you find? In what way does the flower bid for insect visits? Is there any device to prevent self pollination? Do the anthers stand at the right height to accomplish self pollination? Do the anthers and stigmas of one flower mature at the same time? Could self pollination take place in a bud or in a withered flower?

\section{Sisyrinchium angustifolium.}

Make conventional diagrams of the flower. Examine both buds and flowers to see whether proterandry or proterogyny exists. At what age of the flower do the anthers dehisce? Can self pollen reach the stigmas at any time while the flower persists? Note how the styles and stigmas change as the flower advances from bud to maturity.

Make observations in the field to note how long a flower 
remains open and what sorts of insects visit it. What part of this plant do you find to be perennial? Note the origin and arrangement of the leaves.

Compare this plant with Erythronium, noting in what respects the flowers differ so that they are not classed in the same family, and in what respects they seem to be related. Which flower would you say is probably the more primitive? In what ways is this flower farther removed than Hypoxis from the lilies? Make on one page longitudinal diagrams of the flowers of Erythronium, Hypoxis, and Sisyrinchium, and refer to them in a brief discussion of the essential characteristics of the families to which they belong.

\section{Iris Germanica (or other species).}

Show with a simple outline drawing the habit of the whole plant. What evidence do you find to indicate that the thick underground part (rhizome) is a stem? Does this part increase in size from year to year? What method of growth has the above-ground leaf-bearing stem? (See diagrams on page III.) The leaves are called equitant (Latin equitans, riding), because the outer are astride the inner. Can you see any advantage in this habit in respect to protection or strength, or in any other way?

The flower is adapted in a unique way to secure cross pollination by means of insects, particularly bees. The use of the specific positions and forms of the parts will become more apparent after the student has seen bees gathering the nectar. By observation get information about the following questions: Where does the bee alight on the flower? Where is the nectary? What does the bee do to get the nectar? How long must be the tongue of the bee to empty the nectary? (See description of the mouth parts 
of the bee on page I85.) Can other insects than bees gather the nectar? Would introrse anthers serve as well as extrorse anthers in this flower? How are pollen and nectar protected from the rain? Are there specially colored parts which might serve to guide insects to the nectar? Show in a longitudinal diagram how a bee would necessarily become dusted over with pollen, how the adjacent stigma is prevented from receiving it, and how on entering another flower the stigma is quite certain to become pollinated.

Let the student see what he can add to or correct in the following quotation from Sprengel's book (see the account of his book on page I72): "Suppose now that a bumblebee becomes aware of a distant Iris xiphium which it has not yet seen; it flies to the flower, attracted by its exquisite qualities. When near to the flower the bee sees that it is indeed mostly violet, but that those three parts which most project are blue and have a beautiful yellow spot in the middle. So these three parts attract the special attention of the bee, not only on account of the afore-mentioned facts, but also because it finds they are exactly the places upon which it can alight; therefore it comes down upon one of these parts nearest to it. Now since the arched sepal lies close against the style these appear to be a single piece; but since the bee knows the significance of the yellow spot,- namely, that it indicates the place where it must enter the flower, - it pays no heed to that appearance but works itself in between the style and sepal.

"Now nature, who found it necessary to close the flower so that no raindrop could run into the nectary, has in this case so arranged it that the trouble to the bee is somewhat lessened; namely, the style is stiff and immovable while the sepal may be pressed downward easily, springing elasti- 
cally back again, however, as soon as it is set free. Consequently the weight of the bee helps it in creeping in, since it assists in pressing down the sepal. If one consider the case reversed, - namely, that the sepal could not be pressed down while the style could be bent upward, - then the bee would have more trouble, for its weight would be of no help and it would need its whole strength to press back the style. Now when the bee has crept through the entrance, the sepal again springs upward and the space between it and the style becomes less, as it was when the bee crept in. Then, when directed by the inner part of the nectar guide, it creeps down to the nectary, the sepal presses the bee against the style, consequently against the anther lying close to this, and so the bee rubs the pollen clean off with its hairy back. After it has removed with outstretched tongue the nectar obtainable here, the bee backs upward and out of the flower. In doing this it is pressed by the sepal against the stigma lobe (which occurs on the style where the sepal is close to it), however not against its upper but its under side, and therefore without consequence to pollination. After the bee has crept from this third part of the flower it flies to one of the remaining two. In creeping in it is pressed by the sepal against the style so that it rubs hard against the stigma lobe with its back, and thus it transfers the pollen from its back to the upper surface of the stigma lobe, which is the truly stigmatic part, and fertilizes the second third of the flower, or that cell of the ovary which corresponds to this third. In this manner the bee flies from one third of the flower to another, and from one flower to another, and fertilizes them with the pollen which it has taken from the third or from the flower last visited."

Since Sprengel's observations were made it has been 
found that-other wild bees of smaller size are on the whole more efficient than the bumble-bee in pollinating the Iris. Butterflies sometimes visit these flowers ; but since they sip the nectar without bodily entering the flower, they perform no service. Certain beetles have been found to pierce the nectary from the outside so that the nectar flows out and is wasted. Such insects may be looked upon as robbers. Many other flowers besides the Iris suffer depredations of this kind.

Compare the flower of the Iris with that of Sisyrinchium or Nemastylis, and write an account of the modifications from these simpler types which the Iris has undergone, specially adapting it to cross pollination by bees. The account should be illustrated by simple diagrams clearly showing the main facts.

\section{Cypripedium pubescens (or other species).}

Make drawings of the flower and dissected parts from the best points of view to clear up the following points: The number and form of the sepals and petals; the form of the stamens and pistil and their position relative to the other parts; where insects (small bees in particular) enter the flower and where they leave it. How, on leaving the flower, bees collect the sticky pollen on the upper part of their bodies; and how after this, on leaving another flower, they leave this pollen on its stigmas and then gather another load of pollen.

Why do not insects leave the flower by the same opening through which they enter it? Find the nectar-secreting hairs at the base of the large hollow petal termed the lip. Would the other two petals and the sepals probably serve in attracting insects? Is the nectar protected from the rain? By what devices is the pollen protected against 
waste? Has the flower an odor that we can perceive? (See page 182 about the sense of smell in insects.) Describe the color plan in this flower.

Study Cypripedium in its native habitat. Is it common or of infrequent occurrence? Can you see reasons for what you find in this regard? Tie a string about the base of a newly opened flower to mark it, and note how many days the flower lasts. Note what insects visit the flower and whether their visits are frequent, and watch them enter and leave the flower. Note what per cent of the flowers become fertilized and produce seeds.

The Orchidaceæ, to which family Cypripedium belongs, show marked affinities to the Liliaceæ, Amaryllidaceæ, and Iridaceæ (see studies of Erythronium, Hypoxis, Sisyrinchium, and Iris). Comparing the flower of Cypripedium with that of a lily, we find that they are similar in having three outer and three inner perianth parts, and three carpels composing the pistil; but we find that the ovary of the Orchidacex is inferior, the upper half of the flower is unlike the lower half, the sepals and petals are irregular, and the stamens are reduced in number and apparently joined to the style. The different genera of the family show marked specialization. of the flower to secure cross pollination by insects (see-description of Catasetum on page 190). In Cypripedium the lower petal or lip (morphologically the upper petal but turned downward by a twist in the ovary) forms an overarched cavity into which the insect must go to secure the nectar. While the insect very naturally enters the cavity through the large opening on top, it finds itself prevented from going out that way by the dome-like form of the upper part. Two relatively small openings on either side of the united style and stamens do not present this difficulty, and the anthers 
and stigmas are so placed that, as the insect goes out through one of these, pollen is rubbed off on the upper part of its body, and afterward, on leaving another flower, this pollen is left on the stigmas and a new supply is removed. The device to secure cross pollination here worked out is admirable for its simplicity and efficiency. We wonder why this plant, and orchids in general, do not occur more commonly, since cross pollination has been so well provided for by them, and a vigorous brood is in consequence to be expected. It will be worth while in following up this question to observe whether the necessary insect visits actually take place. The flowers of Cypripedium insigne are said to last forty days and those of Cypripedium villosum seventy days. Does this long life of the flower seem to indicate uncertainty on the part of insect visits? After fertilization has been accomplished we find that the seeds resulting are numerous and very minute. The large number of course increases the chance of offspring, but the diminutive size of the seeds nust result in great loss before and during germination. The student can think out for himself in what ways loss would be likely to occur.

\section{Salix. (Obtainable species.)}

What sort of habitat do the willows prefer? Note the form of the willow, its habit of branching, and the character of its branches. Cut off a small willow branch and place it in a jar of water in a warm room, and note how adventitious roots are formed after a time. The branches of some willows are quite brittle, and falling off frequently become rooted in the mud, and in this way multiplication may take place. How early in the spring do the leaves and flowers appear? Which appear first? The flowers 
are diœcious. Do both kinds appear at the same time? Can the pollen be blown about readily by the wind, or is it sticky, so as to require the aid of insects in pollination?

What is the nature of the stigma? Do both staminate and pistillate flowers secrete nectar? Prove the following statement from Sprengel: "Whoever examines the pistillate flowers of willows will find a drop of nectar on their nectaries." Draw a staminate and a pistillate inflorescence showing the form of the bracts subtending the flowers. Draw a staminate and a pistillate flower from the best point of view to show all of the parts. Make a drawing of a mature fruit, showing how the capsule breaks open and how the seeds are disseminated.

Populus monilifera (or other species).

Draw a branch showing the difference between flower buds and leaf buds as to position, form, and size. Note how the buds are protected. Draw a leaf in position on the branch, showing its light relation, - that is, its position relative to the strongest incident light. Where do these trees most abound? Draw staminate and pistillate inflorescences, showing their position with reference to the vertical. Show the forms of the scales subtending the flowers. Draw staminate and pistillate flowers from the best point of view to show the number, form, and position of the parts. Is the pollen sticky, or is it dry and easily carried about by the wind? Draw the stigmas on a large scale, and decide whether they are well adapted to catch and hold pollen wafted to them by the wind. Note whether insects visit both pistillate and staminate flowers. When the capsules are ripe, notice how they break open, how the hairs on the seeds spread apart, and how the seeds are scattered. 
Studies of Selected Spermatophytes.

Now compare the poplars and willows and tabulate their similarities and dissimilarities so as to bring out the grounds for classifying them in the same family but in different genera.

\section{Ranunculus abortivus.}

State the character of the habitat in which this plant grows. Draw its different kinds of leaves from base to apex, including the leaves of the involucre subtending the flowers. What advantage is given to the lower leaves by their long petioles? Draw a flower so as to show clearly sepals, petals, stamens, and pistils. Study a longitudinal section through the center of a flower, and make a longitudinal diagram according to the directions on page 153 . Do the anthers all dehisce at the same time? Do stigmas and anthers mature at the same time? Can nectar be found? Is the pollen of a nature to be carried by the wind? Would self pollination probably take place? Study a species of Myosurus in the same manner. How does it differ from, and what has it in common with Ranunculus? What advantages do you see in the elongation of that part of the receptacle which bears the pistils? Would this habit insure the self pollination of all the pistils?

\section{Delphinium. (Obtainable species.)}

Note the habitat, habit, and - size of the plant. Draw a typical leaf. Draw an inflorescence showing correctly the position of the flowers and the direction of the spur. Dissect a flower and identify the different parts. Discover what purposes the irregularities in the parts serve. (For suggested diagram of the flower, see Fig. 79.) Get a good understanding of the positions of the different parts in flowers of various ages from bud to withering flower. 
Answer from observation the following questions: How is the pollen protected? Where is the nectar secreted and stored up? How is the nectar protected from the rain? To what sorts of insects is the nectar accessible? Do the anthers all discharge their pollen at the same time? What positions do the anthers assume before and after discharging their pollen? Do the anthers and stigmas of a flower mature at the same time, or does the flower afford an example of proterandry or proterogyny? Do the styles and stigmas assume different positions at different ages of the flower? Now make three longitudinal diagrams, one of a newly opened flower, one of a middle-aged flower, and one of a fading flower, showing the different positions of the stamens and styles. Observe the positions of insects in visiting these flowers. Do they proceed from young to older flowers of the inflorescence or vice versa? What order of procedure would be best to insure cross pollination between flowers of different plants? How long must the insect's proboscis be to reach the bottom of the nectary? In what ways are these flowers adapted to attract insects?

Compare Delphinium with Ranunculus. What similarities have they which warrant their classification in the same family? What dissimilarities compel a classification in different genera?

The flower of the larkspur is of peculiar interest on account of the specialization of its parts for definite purposes. The changes in the positions of stamens and styles at different periods are wonderful exhibitions of the power of movement of plant parts to accomplish specific results.

\section{Dicentra cucullaria.}

What is the habitat of this plant? What is the character of its underground parts? Why are the leaves called 
ternately compound? Describe the leaflets. What term would you apply to the inflorescence? (See page 158.) Examine a bud. What sort of a calyx has it? Note what becomes of the calyx about the time when the flower opens. Examine the interior of the spurs of the subcordate outer petals. Do they contain nectar? Is the nectar apparently secreted by the spur itself, or are there outgrowths from the stamens (as in the violet), which serve as nectaries? Are the flowers fragrant? Notice how the two inner petals, united at their apices, protect the anthers and stigmas. Would these petals be pushed aside by bees in quest of nectar? Do they spring back after being pushed aside? Note the actions of bees in visiting these flowers. Do the anthers dehisce while the flowers are still in bud? As soon as the pollen is discharged, are the stigmas ready to receive it? Could self pollination possibly take place? Would it make any difference in the problem of pollination whether insects work from the bottom of the inflorescence upward or vice versa? Dissect a flower and draw the different parts. Make longitudinal and cross diagrams of a flower.

Corydalis. (Obtainable species.)

Study according to the outline for Dicentra. Compare Dicentra and Corydalis. Why should they not be classified under the same genus?

Capsella bursa-pastoris.

Draw an entire plant, using the symbols for leaves, flowers, and fruits suggested on page I 58. Draw a typical basal leaf and stem leaf. Draw a single flower showing a combined side and top view. Dissect a flower and draw one of each set of parts. Find the term tetradynamous in the glossary. Can it be applied to this flower? Draw a 
cross section of the ovary, showing the attachment of the ovules. (It is a good plan to select a somewhat mature fruit for this purpose.) What are the relative positions of anthers and stigmas? Are stigmas and anthers mature at the same time? Is self pollination liable to take place? Find the nectaries. Is the flower fragrant? When insects visit these flowers, would they be likely to cause self or cross pollination? Make a drawing showing how the capsules break open, and how the seeds become scattered.

This plant has become naturalized from Europe. It occurs in nearly all parts of the world and shows a great aptitude in taking possession of waste places. It has a great advantage over most other herbaceous plants in being able to withstand low temperatures and to send forth its leaves and flowers in the first warm days of late winter or early spring. (See on page $3 \mathrm{II}$ an account of Cochlearia belonging to the same family.) Watch for it at such times and note how much it is in advance of other plants. Now note down all of the points in its structure and habits which help to make it a successful competitor with most other plants in all parts of the world. (Read the chapter on Plants of Different Regions.) Compare other genera in this family with Capsella.

\section{Prunus chicasa (or other species).}

Study the trees in their native habitat at the time of blooming. What is the character of the inflorescence? At what stage of development are the leaves when the tree is in blossom? Tell the different means by which the flowers are made conspicuous. Draw a flower from the point of view which best shows the form and relative positions of all the parts. What is the position of the anthers with reference to the stigmas? Do the anthers 
all mature at about the same time? Can self pollination take place spontaneously, or is the aid of insects necessary? Where is the nectary? What kinds of insect visitors do you find? Examine flowers of different ages for exudation of nectar. Make a cross section of the ovary. How many cells and how many ovules do you find? Make a longitudinal diagram of a flower. Estimate approximately the percentage of flowers that fall off without forming fruit. How do you account for the facts?

Compare a peach flower with that of the plum. In both cases what part of the ovary forms the pulp of the fruit, and what part forms the stone? What reasons do you see for classifying the plum and peach in the same genus? Compare the flower and fruit of the apple with that of the plum and peach. What are the reasons for classifying the apple in the same family with the plum and peach, but not in the same genus? In what details would the plum flower need to be modified to become like the apple flower? How does the following statement agree with what you are able to observe: The fruit of the apple is composed of the ovary (the core) immersed in and adhering to the fleshy receptacle. For comparison make on one page longitudinal diagrams of the flowers and fruits of the peach or plum and apple and point out the corresponding parts in flowers and fruits.

\section{Fragaria Virginiana.}

Draw the entire plant. What term would you apply to the underground stem? What term to the leaves? Describe the leaflets as to form, margin, texture, etc. Show by drawings how multiplication other than by seeds takes place. Examine a patch of wild strawberries in 
bloom. Are the flowers all alike? Are the pistils simple or compound? What term would you apply to the ripened pistil? What is the morphology of the fleshy part of the fruit? What modifications would the ripened pistil of the strawberry have to undergo to be like that of the plum? Compare the flowers of strawberry and plum. Why are these plants classified in the same family? Why not in the same genus? What changes would the flowers of the plum need to undergo to become like those of the strawberry? How much of the fruit of the blackberry is formed by the ripened pistils?

\section{Rosa Arkansana (or other species).}

Describe the habit of the plant. Draw an entire leaf. What descriptive terms would you apply to it? Are the flower buds formed on branches of the current year or on older branches? At what time of day do the flowers open? Do insects visit these flowers? Do you find them gathering nectar or pollen? Notice the relative positions of anthers and stigmas. Would self pollination probably take place? Make a longitudinal diagram of a bud showing how the floral envelopes protect the inner parts and how the latter are packed in small space. Make longitudinal and cross diagrams of an open flower. Follow the subsequent development of the fruit. What changes would be necessary to make the flower of the strawberry like that of the rose? What would be required to make the flower and fruit of the rose like that of the apple?

The fruits of the rose are eaten by birds and the seeds scattered uninjured. The bright color of the ripened receptacle or "hip" makes it conspicuous in fall and winter. Mice are fond of the rose fruit, but since they gnaw into and destroy the seeds they are not useful in 
seed dispersal as birds are. It seems, however, that mice are often deterred from climbing the bushes by the downward-pointing prickles, and then eat only those fruits which are accidentally broken off.

The rose family is one of the most important, since it supplies our best fruits and some of our most beautiful flowers. Write a review of the plants of this family which you have studied, embracing the following points: The difference in size and habit shown by the various genera. The different kinds of leaves. The divers forms and character of the receptacles. The essential similarity of the flowers of the family.

Astragalus caryocarpus (or other species, and species of other genera in the same family).

Draw a leaf. Is it simple, or palmately or pinnately compound? Draw an inflorescence showing the position in which the flowers stand with reference to the vertical. Draw the different kinds of petals. The upper petal is called the standard or vexillum; the two lateral petals are termed the wings or ale; the two united lower petals constitute the keel or carina. When a bee visits one of these flowers, it alights on the wings and keel. Watch a flower at such a time to see what transpires. What occurs after the bee leaves the flower? Press down upon the wings and keel with the finger tip or with a pencil and note how stigmas and anthers are exposed. Notice what happens after the pressure is removed. Study the mechanism by which the spring-like action of the wings and keel is brought about. Now write down the different purposes fulfilled by the standard, wings, and keel. Note the positions of anthers and stigmas in bud and in blossoms of different ages. Where is the nectar secreted? What 
path must the insect's proboscis follow in reaching it? Note all devices concerned with the protection of the nectar and pollen. Make a cross diagram of the flower, and in it show accurately how the wings and keel are locked together. Does the form of the calyx seem to have any definite relation to the peculiarities of structure of the other parts? Compare the flowers of the different genera of the family studied. Compare the different members of this family which you have studied, as to size and habit, forms of leaves, details of floral structure, and character of fruit. What features are common to the family, and in what features do you find the greatest variations?

\section{0 xalis violacea.}

Draw an entire plant, including the bulb and roots. (Some of the leaves may be omitted.) What term would you apply to the leaves, bulb, and inflorescence? Note how the leaflets are folded as they emerge from the bud, and notice the day and night positions of the mature leaflets. Look for the two forms of flowers. (Read about dimorphic flowers on page I76.) Make longitudinal diagrams showing exactly the positions of stamens and styles in the two forms. Note how the petals are wrapped in the bud. At what time of day do the flowers open? After once opening, do they close and open again? Note the positions of flowers and leaves in cloudy and stormy weather. What meaning do you attach to their behavior? Note the way in which the seeds are ejected from the capsules. Compare Oxalis violacea with Oxalis stricta, or other obtainable species.

\section{Acer. (Obtainable species.)}

Dissect an inflorescence and find how much of it constitutes a single flower. Examine inflorescences from dif- 
ferent trees and find whether staminate, pistillate, and perfect flowers occur. Make longitudinal diagrams of the different kinds of flowers. Draw a stamen and a pistil on a large scale as seen under a lens. Study with a lens and show by a drawing the position and character of the stigmatic surface. Do insects visit these flowers? Are the maple flowers adapted to cross pollination? What is the advantage in this case in having the flowers appear before the leaves? Follow the development of the pistil to the mature fruit and state what changes the pistil undergoes in ripening. What part of the pistil forms the wings? Do all of the ovules become seeds as a rule?

\section{Viola. (Obtainable species.)}

Study all obtainable species of the violet. State the character of the habitat of the different species studied. Draw a typical leaf. How are the leaves rolled up in the bud? Draw a rootstock. What evidence do you find that it is morphologically a stem? What is the nature of the roots? Draw a flower in right position with reference to the vertical. Study the face of a flower. Is there an opening through which an insect can enter or thrust its proboscis? Is pollen visible? Dissect a flower. What part constitutes the spur? The horn-like projections from the two lower stamens secrete the nectar and pour it into the spur. Do you find nectar there? Do the anthers dehisce toward the outside or inside? What becomes of the pollen after the anthers break open? What is the use of the scale-like tips of the stamens? Is the stigma so shaped and situated as to receive self pollen? Sum up those facts about the flower which relate to its pollination. How are the pollen and nectar protected against waste? As the flower is constructed, would it be just as well for it 
to point directly upward or downward? (We want to find out by this question whether the pose of the flower is correlated with its form and construction.) Draw the different parts of a flower. Make cross and longitudinal diagrams of a flower. (A suggested method of treatment in diagraming a violet is given on page 154 , but the student should make each diagram accurately show the characteristics of the species in hand.) Note that the pistil of the violet is one-celled but compound, as shown by the three placentæ. Study the inconspicuous cleistogamous flowers that appear later on short peduncles. Show how self pollination is accomplished in these flowers. Do the early and conspicuous or later cleistogamous flowers appear to be the more fruitful? It seems from a study of the violet that the cleistogamous flowers are remarkably successful in accomplishing self fertilization; the evil results which might be expected to follow are apparently kept from accumulating in the species by the occurrence of cross fertilization in the early, conspicuous flowers.

Write a short essay on the genus Viola, covering habitat, time of blooming, form of plant, forms of leaves, coloration of flowers, variations in details of floral structure in the different species, relative fertility of conspicuous and cleistogamous flowers, æsthetic considerations.

Let the student test his thoroughness and accuracy by comparing the results of his work with the following interesting account by Sprengel of his own discoveries relating to the violet:

"The nectar receptacle is the end of the spur of the corolla. At first I could not understand why in Viola canina I should find nectar there but not on the nectary; but finally I perceived that this occurs naturally, for the end of the spur has the form of an arch which curves 
about the drops of nectar excreted by the nectary. Thus it attracts the drops from many sides and consequently more powerfully than the nectary does, and the drops of nectar must follow the stronger force and flow from the nectary into the end of the spur. And here from the same cause the nectar must remain and not flow down and out of the corolla as it tends to do on account of its weight.

That the nectar is fully protected from all injury by the rain is apparent. Even if a drop of rain should get near to the opening of the spur, it could not enter; but in order that it may not even approach the opening the two middle petals have hairs just where they can be most effective. Thus when raindrops have fallen upon the upper petal and, running down it, have united into a single drop, the latter is arrested as soon as it has reached these hairs. It is therefore utterly impossible for a raindrop ever to reach the nectar.... In Fig. 13 [in Sprengel's book] one sees the greater part of the nectar guides on the lowest petal. This figure and Fig. 8 show how the veins run a bit into the opening of the spur. Therefore a bee were as stupid as a fly if it did not know how to find the nectar as soon as it has alighted on the flower. Now how is this violet pollinated? In order properly to answer this question, which for several years was an apparently insolvable riddle for me, I must make the reader somewhat more closely acquainted with this flower. The five anthers surround the pistil and conceal it so that one sees no more than the reflexed end of the style. They are not grown together but touch one another and appear to be a single body. The filaments are somewhat fleshy; the two lowest have each a projection just as fleshy which extends into the spur; the ends of the projections, as said, secrete the nectar. Each filament has an 
anther consisting of two pollen sacs on its inner side facing the pistil. Each stamen has at its apex an appendage which consists of a thin, dry, yellow membrane having a slight amount of elasticity. However, these appendages do not merely lie side by side around the style as the filaments do, but in part over one another, so that they appear even more like a single body than the filaments do. One sees that the appendages of the two lateral anthers are covered by those of the upper and two lowest, and that the appendage of one of the two lowest lies in part upon that of the other. Thus the stamens, together with the appendages, have the form of the upper conical part of a funnel, from the lower opening of which the style sticks out and at the same time completely fills and closes this opening. The part of this funnel which is formed by the filaments I propose to call the upper, and that formed by the appendages the lower part.

"The pollen revealed by the anthers after they have opened is of a very peculiar kind, for while the pollen of other nectar-bearing flowers clings somewhat firmly and is of such a nature as to be comparable to rather moist flour, that it may not be blown away by the wind or scattered when the wind shakes the flowers, the pollen of the violet, on the contrary, is completely dry and does not cling at all to the pollen sacs after they have opened. It is thus like the pollen of those flowers that are pollinated by the wind, although this agent is not here employed. Still it is not as fine as the latter and is more like flour than like actual pollen. The two pollen sacs of each filament have a prominent border at the upper end and on the sides, but not at all below where the appendage begins. Thus the dry pollen is hindered by nothing from falling from the upper into the lower part of the funnel. That just this 
must happen can be seen when the quality of the pollen is considered, together with the fact that the flower is at the arched end of a long stem and must in consequence often be shaken by the wind. Since the opening at the lower end of the funnel is closed by the style, the pollen that has fallen into this part cannot escape through the opening. One may imitate the wind as best he can; one may blow into the flower or shake it ever so hard, provided the funnel is not injured or pressed upon in so doing, and still not a grain of the pollen is brought to light. Since, now, the projecting bent end of the style is the stigma, the arrangement of this flower must seem perfectly absurd to one who knows about the pollination of flowers by mechanical means only; for all other parts of the pistil become pollinated, which does not have the slightest influence upon fertilization, and precisely the stigma alone, which must necessarily be pollinated if fertilization is to follow, is excluded from pollination. If, then, we were to conceive of pollination as taking place solely by mechanical means, we should have to believe either that fertilization never takes place in this flower, which, however, is against the facts, or that the flower, notwithstanding its possession of all the parts required in natural fertilization, is fertilized by God in an unnatural way and by a miracle, just because its parts are so strangely and impractically constructed and arranged. And that is the same as saying, as we must think, that on account of the mistake which He has made in the construction of this flower God punishes Himself by the necessity of performing a miracle on each successive individual. Now if we are neither to deny the existence of anything that is, nor affirm the existence of the impossible, nothing remains for us but to resort to insects. And since the bees, which, as we have heard, visit this flower, have already so 
often done us good service, it is to be hoped that they will not desert us in this extremity also. The end of the style is bent in such a way that it makes a somewhat sharp angle with the style, but its base is somewhat crooked and much more slender than the rest. On this account it is very easily lifted up; but as soon as released it falls back into its accustomed position. Now when a bee crawls upon the upper petal and sticks its head into the rather large space between the stigma and lowest petal, in order to thrust its proboscis into the nectar receptacle, it lifts with its head the style, and with it the appendage of the upper anther. In this way an opening is made in the funnel, through which the pollen falls out. Thus the bee becomes dusted with the pollen and necessarily transfers a part of it to the stigma, and in this way the pistil becomes fertilized. After it has consumed the nectar the bee crawls back again, and then the style falls back into its accustomed place, and likewise gradually the appendage of the upper filament. Thus the opening of the funnel gradually closes, although not so tight and snug as before the visit, probably because fertilization necessarily follows from the first visit.

"This method of pollination of this flower discovered and described by me enables the reader to answer various questions relating to the structure of the flower which he would otherwise have to leave unanswered. The easier questions that come up in regard to the structure of other nectariferous flowers I will not touch upon, for example: Why the flower secretes nectar, why it has a colored corolla, why it has dark lines upon a light background, why it is possessed of an agreeable odor, why the nectar is so well protected against the rain. But I will bring forward the following: Why does the flower grow 
upon a long upright stem which, however, bends over and downward at its upper end? Answer: First, in order that no raindrop can get at the nectar; for if the stem were quite straight and the flower stood upright in consequence, the end of the spur where the nectar occurs would be the lowest part of the flower, and raindrops which fall into the flower would flow down into the spur and mix with the nectar and spoil it. But since the upper part of the stem bends down, the flower hangs down; and the end of the spur is the highest part of it, into which no raindrop can flow. Second, in order that when the flower is shaken by the wind, which must often occur on account of the length of the stem, the pollen may fall into the lowest part of the funnel. If the stem were straight and the flower had an upright position, the pollen would fall into the part of the funnel which would then be the lowest, that is, the upper part formed by the filaments ; so it would lie there when a bee visits the flower, and never be brought upon the stigma.

"Why does the pollen have the peculiar quality described, and why is it so different from the pollen of other nectarbearing flowers? Answer: In other nectariferous flowers the pollen is to be rubbed off by insects, and so it clings somewhat firmly in order that the wind may not blow it away. In this flower, however, it is to collect in the lower part of the funnel in order to be able to fall out when a bee makes an opening. So if it remained in the anthers, the flower would never be fertilized. Why is the base of the style so slender? Answer: In order that the bee may lift up the style the more easily. But why is this base a little crooked? and why does the retroflexed end of the style make a somewhat acute instead of a right angle with the style? Answer: Both serve the same purpose as the last- 
mentioned condition. The direction of the thrust which the bee makes against the retroflexed end of the style is about parallel with its longer straight part. This thrust is intended to lift up the style in a direction about at a right angle with its long axis. Now one who has a conception of mechanics will see that this would not occur so easily if the slender base of the style were straight and its retroflexed end made a right angle with it. The retroflexed end of the style makes an oblique angle with its long axis, and consequently with the direction of the thrust, for the very same reason that the surface of the wings of a windmill makes an oblique angle with the direction of the wind. And to take a still more pertinent example, which also relates to the crooked base of the style, let one imagine, since the style has a similarity to a crutch, that one were to have a crutch.made exactly after the pattern of it. At the very first trial of it he would rue his idea, for should he put his weight upon it the crutch would slip out and he would fall. Finally, why does the membranous appendage of the upper filament lie in part upon the appendages of the two middle ones, and why not the latter or one of them upon the former? Answer: In order that it may the more easily be pushed up by the bee through the agency of the style.

"Now I will relate how I discovered the method of pollination of this flower. An observation and an experiment helped me to it in the spring of last year. I saw that the flowers were visited by bees, and I wanted to imitate the effect which they make upon the style; for I had for a long time conceived that the whole secret must lie behind the form of the style in virtue of which it can so easily be bent upward and afterward falls back again. After many fruitless efforts it finally happily occurred to me to give the plucked flower in this experiment precisely the same 
position which nature has given it. That was beginning at the right end of the question, for as soon as I lifted the flower so high that it stood above the level of my eyes, in order to be able to look into it from below, the pollen fell in large amount out of the funnel, like writing sand from the sand-box, as soon as I lifted the style with a slender stick. This phenomenon, which actually startled me, because I had not expected it, was to my understanding what a flash of lightning on a dark night is to the eyes, it revealed on a sudden the whole secret to me."

\section{Oenothera speciosa (or other species).}

What is the habitat of this plant?

Describe the leaves. What sort of an inflorescence has it?

Observe the arrangement of the floral parts in the bud. At what time of day do the flowers open? How long does a flower last? Describe the calyx of an open flower. Do the anthers dehisce before or after the flower opens? Are the stigmas ready to receive the pollen as soon as the flower opens? Draw the upper part of the style before and after the branches spread apart. Note the position of the style branches when the flower first opens. What are the relative positions of anthers and stigmas? Could self pollination take place if cross pollination does not? What significance do you attach to the cobwebby pollen? Do the flowers become more fragrant in the evening? Where is the nectar secreted? How long must an insect's proboscis be to get it? At what time of day do insects visit these flowers? With their present habits, would it be as well for these flowers to be any other color than white? Make a longitudinal diagram of a flower and a cross diagram of an ovary. Compare with the types of flowers of Fig. I23. To what type does this flower belong? What 
significance do you see in the different positions of the flower in bud, after opening, and after pollination? Sum up those facts which relate to pollination.

Asclepias cornuti (or other species).

Read the account of Asclepias cormuti on page I9I. What is the nature of the underground parts? Describe the leaves and inflorescence. Break off a leaf or stem and note the exudation of latex. Notice later how the dried latex protects the wound. Examine the exterior of a flower. Can the pistils be seen? Find the entrance to the stigmatic chamber (see Fig. 108). Slip the point of a needle into a stigmatic chamber and pass it upward and out at the top, so as to remove the corpusculum and attached pollinia (see Fig. II3). Notice the slit-like opening into the anther cavities through which the pollinia are extracted. Draw the pollinia as seen under a lens. Study cross sections of a flower at different heights, and construct a diagram showing the main facts learned. In cutting the cross sections pollinia will frequently be seen lying in the stigmatic chambers where they have been deposited by insects, and in the older flowers pollen tubes may be seen penetrating the apex of a style. Study the construction of the spur-like nectar receptacles growing from the stamens. Observe the behavior of insects at work on the flowers. Catch a bee with pollinia adhering to it and study with a lens the way in which the pollinia are made fast to the insect. Point out the deviations from the simpler types which the parts of this flower have undergone, and show the purposes which these modifications serve.

\section{Taraxacum officinale.}

Is this plant annual, biennial, or perennial? What is the nature of its underground parts? What descriptive terms 
would you apply to its leaves? Make a longitudinal section through the center of an inflorescence which has been only a short time in bloom, and notice the unopened buds at the center and the fully expanded flowers at the margin. The disk from which the flowers grow is the receptacle of the inflorescence (not a flower receptacle). Examine under a lens the apex of an unopened flower and note the number of notches in the corolla indicating the number of petals. What changes does the corolla undergo as it expands into the open flower? Remove an entire unopened flower from the receptacle of the inflorescence, taking care not to break off the ovary, lay it on the stage of the microscope, and carefully dissect it with needles while looking at it through the lens. How many stamens do you find? Where are the filaments attached? How many style branches are there? What relation do they bear to the anthers? Do the anthers dehisce toward the inside or outside? Notice the relative heights of stamens and styles in successively older flowers. Note the behavior of the style branches up to the time of the withering of the flower. How is the pollen pushed out from the tube formed by the united anthers? The stigmatic surfaces being along the inner or opposing faces of the style branches, is it possible for self pollination to take place at any time? The nectary occurs near the base of the style, and the nectar is expelled into the tubular part of the corolla. See whether the corolla tube ever becomes filled with the nectar. Can insects probe between the filaments to reach the nectar? Observe the behavior of bees on these flowers. To what parts of their bodies does the pollen adhere? Now state in precise terms all of those facts which have a bearing on the cross or self pollination of this plant. When an inflorescence has once expanded, 
does it ever close again? Tie a string loosely around a peduncle to mark it, and observe the behavior of the inflorescence on cloudy and bright days, and at different times of the day, beginning at early morning. Try to conceive the significance of what you find.

Follow the changes occurring in the parts of the flower up to the time of the scattering of the fruit. Make drawings to show the main facts, and in connection with them tell what these facts signify.

Erigeron. (Any obtainable species.)

Study the inflorescence and flowers as directed under Taraxacum, and compare the two genera by means of drawings and notes. 


\section{CHAPTER XI.}

\section{SLIME MOULDS, BACTERIA, AND YEASTS.}

\section{PROVIDING MATERIALS.}

The materials needed for the work of this chapter can be obtained at any time of the year, as directed under the observations. It is a good plan, however, to gather the sporangia of slime moulds whenever they are found in their prime, and preserve them as directed on page 370.

\section{Slime Moulds.}

\section{OBSERVATIONS.}

142. Hunt beneath the bark of rotting logs or amongst the moist leaf mould of woods for yellowish or gray slimy growths; the plasmodia of slime moulds. Cut off a portion of the log bearing the plasmodium, expose to the light and make note of the result. Allow another portion of the log bearing the plasmodium to dry in the dark, and note the result.

143. Place some of the plasmodium under a bell jar between pieces of moist felt paper (carpet paper will answer) and keep the preparation in the dark, and moist but not wet. The plasmodium will probably grow out over the paper, and its translocation should be observed from time to time. Make moist chamber preparations of the plasmodium as directed under Observation I08, page 103. Watch the circulation of the protoplasm with a medium power of the compound microscope, and make drawings in which the direction of movement is indicated by arrows. 
144. The preparation under the bell jar will finally enter into a resting condition, and sporangia containing spores may be produced. Examine the sporangia in a drop of water with a high power of the microscope, and draw the spores. Draw the sporangia as seen under low power.

\section{DISCUSSION.}

150. The Nature of Slime Moulds. - The slime moulds, or Myxomycetes, are very low organisms which stand at the
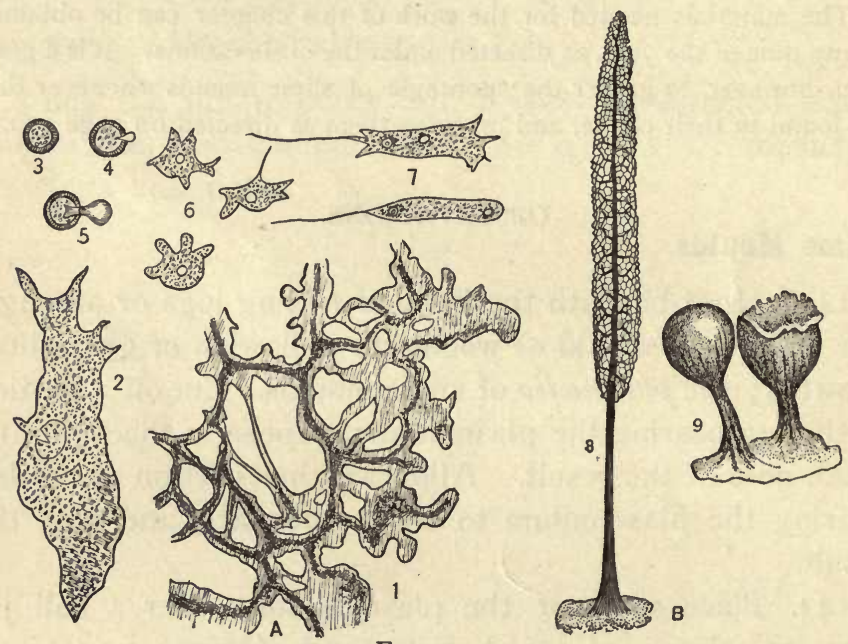

FIG. I32. $^{2}$

Myxomycete or Slime Mould. I, a bit of plasmodium. 2, a small plasmodium. 3 , a spore. 4 and 5 , protoplast escaping from the spore. 6 and 7 , stages succeeding 5, motile protoplasts which finally fuse to form 2 and $\mathbf{~}$. 8 and 9, different forms of sporangia bearing spores such as 3 . After PRANTL and MASSEY.

boundary line between the lowest plants and animals, but the character and nature of formation of their spores seem to warrant their classification among plants. In its vegetative or plasmodium state (see Fig. I 32) a slime mould creeps 
about in moist and dark places, avoiding the light and being attracted by moisture; but when it is ready to form its spores, it creeps away from its former habitat and seeks the light at the surface of the log or of whatever substratum it is inhabiting. While in the plasmodium stage, it sends out arms here and there that may surround and engulf bits of organic matter, which it digests and employs as food. It does not possess chlorophyll, and is not, like green plants, able to manufacture its own food; for this reason it is restricted in its habitat to the remains of higher plants which yield the organized materials necessary to it.

151. Formation of Spores. - When ready to produce spores, the plasmodium puts forth outgrowths which become sporangia, the forms of which vary with the species (see Fig. I32, 8 and 9). At the surface of the sporangia a crust, and sometimes a variously reticulated framework, is formed, while the interior protoplasm breaks up into numerous spores. Sometimes, among the spores, threads are formed which twist and untwist with the changing hurnidities of the atmosphere, and thus aid in loosening the spores preparatory to their dissemination by the wind.

When a spore falls into a wet place, its protoplast, which is the essential part of it, creeps out from its wall, and (Fig. 132, 7) being provided with a cilium, swims about in the water. After a time the cilium becomes absorbed, and the protoplast creeps about and obtains particles of food by surrounding them bodily (6). While in this condition it may multiply by repeated division. Finally, many protoplasts may fuse together and form a larger multinucleated body similar to the slimy plasmodium with which this description began (see Fig. I32).

It often happens that when the substratum is drying up the plasmodium changes into dry masses of various shapes, 
which again form plasmodia under proper conditions of humidity, etc. Such dry masses may be incited to resume their active creeping stage by being kept between moist felt paper under a bell jar.

\section{OBSERVATIONS.}

\section{Bacteria and Yeasts.}

145. Put hay, cabbage leaves, or beans to soak in a beaker of water, and leave the infusion standing in a warm place until the liquid becomes cloudy. Then examine a drop under a high power; minute bodies of various forms will be seen, some of them in rapid motion. The smaller rounded, rod-shaped, or corkscrew-shaped bodies are bacteria, the smallest known plants.

146. Boil a potato and cut it in two with a knife which has been sterilized by baking in an oven. Dip into the above infusion the point of a needle which has been sterilized in the flame of an alcohol lamp or Bunsen burner, and then lightly make a few scratches with the point of the needle across the full length of the cut surface of the potato. Put the inoculated potato under a bell jar or other dish, together with some moistened filter paper to keep the potato from becoming dry. Examine the preparation from day to day. Mount under a coverglass some of the growths which appear, and examine with a high power. Doubtless growths will appear on the potato outside the scratches, because not enough care has been taken to exclude the possibility of accidental inoculation; but the growths along the scratches will be sufficiently definite for our present purpose.

The experiment might be varied by touching one half of the potato against some dusty place in the room, and 
placing the other half beside it under the bell jar. Record your observations in your notes. Thread-like growths may appear among the spots of bacterial colonies; these are Fungi, which are higher in the scale of life than the bacteria. They should be left to form their spores undisturbed for use in a subsequent study.

147. Put milk into a clean flask, plug tightly with a wad of cotton, and steam in a steamer for half an hour on each of three successive days. The milk should be found to continue sweet for an indefinite period.

148. Remove on the point of a needle a very small portion of bacterial material from Observation 146 (or take a small drop of the culture of Observation 145) and place it in a drop of water at the center of a glass slip, and stir the drop with a needle to thoroughly distribute the bacteria. Let the water evaporate, and then pass the slip, preparation side up, three times through the flame of an alcohol lamp, each passage through the flame occupying about one second. This fixes the bacteria to the slip. Place a drop of a $\mathrm{r} \%$ aqueous solution of fuchsin on the preparation, and wash it off after a minute in running water, or by moving it about in a dish of water. Then pass the preparation two or three times quickly through $60 \%$ alcohol to decolorize the background. Let the preparation dry and mount in Canada balsam (see page 390). Draw the different forms of bacteria clearly brought out by the stain.

I49. Soak a piece of yeast cake over night in a saucer of water, and then mount a very small portion of it in a drop of water under a coverglass. Spread the yeast out in a thin layer by giving the coverglass a circular motion by means of the point of the finger, covered with a clean cloth to avoid smirching the coverglass. Examine with a 
high pơwèr. À various assemblage of small objects will be seen. Run a drop of iodine under the coverglass; the objects colored blue or almost black by this reagent are starch grains, which make up the bulk of a cake of yeast, while the round or oval yellowish bodies are yeast plants. The still smaller rods and rounded bodies are bacteria. Some of the yeast plants will be found in the process of reproduction by budding (Fig. 133,a). Yeast plants are efficient in making bread light, as will be shown in the discussion.

150. Mix about two teaspoonfuls of molasses with a pint of water and add a small piece of yeast cake. Fill a wide-mouth bottle with the mixture and invert it in a dish of the same. To keep the liquid from running out of the bottle while inverting it, fill the bottle quite full and place a piece of stiff paper over the mouth and hold the paper in position with the tips of the fingers while inverting the bottle and placing it in the dish; then remove the paper. Keep the preparation in a warm place. After a time bubbles of gas will arise and displace the liquid. When a sufficient quantity of gas has been secured, it can be demonstrated to be carbon dioxide by the methods detailed on page 13. The solution will now be found to have an alcoholic taste, and, if it is properly distilled, alcohol can be obtained strong enough to burn.

\section{DISCUSSION.}

152. Nature of Bacteria. - The bacteria are the smallest of known plants, the rounded forms being about .ooor $\mathrm{mm}$. in diameter; the oblong and rod-shaped forms are no greater than this in their short diameter, as a rule, and their long diameter is from $\mathrm{I} \frac{1}{2}$ to 5 times as great. They are therefore still very small in appearance, even when magnified with the highest powers of the microscope, and it is difficult 
to determine the details of their construction. They are enveloped by an extremely thin translucent membrane. Whether they have nuclei is a question which is still in

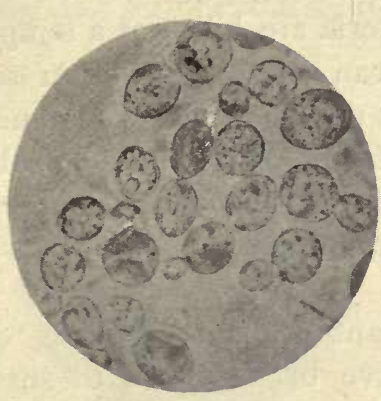

a

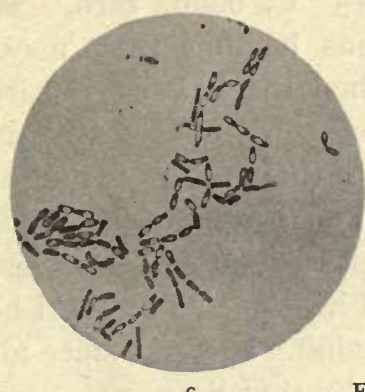

FIG. I33.
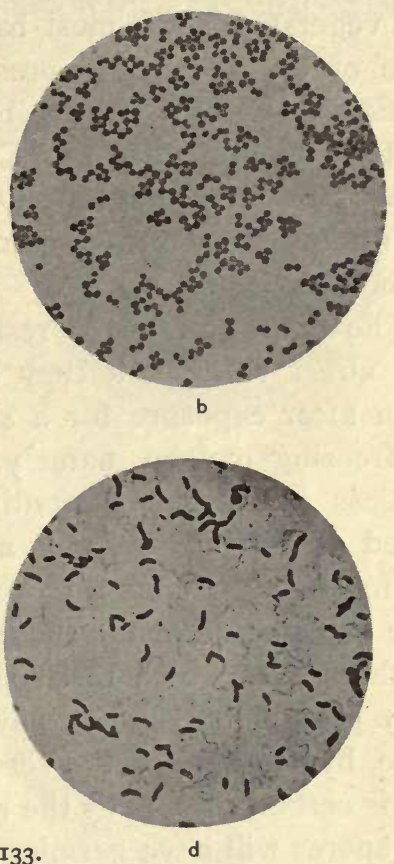

$a$, yeast plants, some of them budding; $b$, micrococci (singular, micrococcus) from the air ; c, Bacillus subtilis, showing internal spore-formation; $d$, bacilli (singular, bacillus) of Asiatic cholera, with motile flagella. Photomicrographs $\times 740$. After GÜNTHER.

doubt. It is thought by some that the bulk of their bodies is made up of nuclear material.

153. Methods of Reproduction. - Bacteria reproduce by division, one becoming two, two four, and so on. Asexual spores are also formed, either by the ordinary cells pro- 
duced by division assuming a spore character, or by the cell contents rounding off, expelling water, and entering into a condition in which great extremes of heat, cold, and desiccation can be withstood (see Fig. 133,c). While in the vegetative state, most bacteria are killed at a temperature of $65^{\circ} \mathrm{C}$., their spores can stand $100^{\circ}$, or in some cases $130^{\circ} \mathrm{C}$., of dry heat; but no spores can withstand an air-free steam heat of $120^{\circ} \mathrm{C}$. for half an hour. This high temperature is achieved by generating the steam under $\mathrm{I} \frac{1}{2}$ atmospheres' pressure in an apparatus known as an autoclave.

The spores are very resistant to cold; freezing does not affect them, and they have been known to survive even after exposure for a short time to the temperature of freezing oxygen, namely, $213^{\circ} \mathrm{C}$. below zero. By a process known as discontinuous heating they may be killed at $100^{\circ} \mathrm{C}$. By this method the heating is carried on for half an hour on each of three consecutive days. The explanation of the success of this process is that most of the spores which resist the first heating will probably have germinated, and so have passed into a less resistant state, by the time of the second heating. The third heating is certain to destroy the remaining bacteria, since all of the spores will have germinated by that time.

154. Forms of Bacteria. - In form, bacteria are round, oblong, rod-shaped, or spiral (Figs. 133-134). But while exceedingly simple in the construction and contour of their bodies, the results of their activities are quite diverse, and of vast importance to other plants and animals.

155. Nutrition of Bacteria. - Although bacteria may produce pigments of various colors, they do not form chlorophyll and cannot obtain their living by employing the energy of the sunlight to build their food; they must, 
therefore, live upon materials already organized, or they must utilize some source of energy other than the sunlight for the construction of their food. Most bacteria have adopted the former course, and obtain their sustenance from
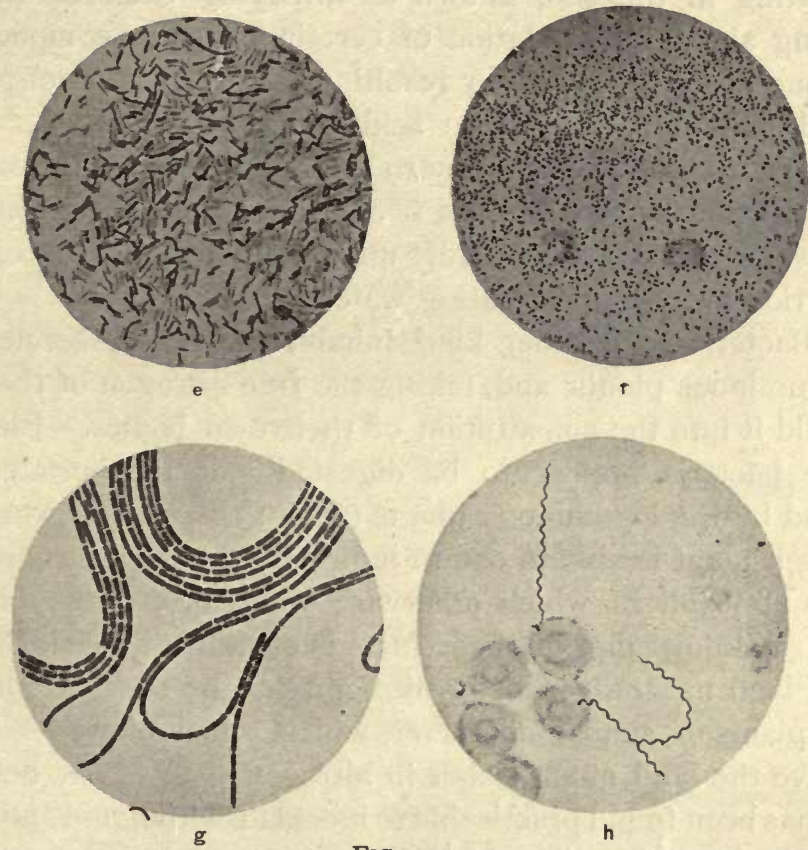

FIG. 134.

$e$, bacilli of typhoid fever; $f$, bacilli of chicken cholera; $g$, bacilli of splenic fever; $h$, spirillum of recurrent fever, and red blood corpuscles. Photomicrographs $\times$ 740. After GÜNTHER.

the bodies or products of plants and animals. Others, however, are able to induce the oxidation of compounds of nitrogen, sulphur, iron, etc., and appropriate the heat thus produced for the fixation of carbon from carbon dioxide, much as green plants do by means of the sunlight. To 
show the importance of bacteria in the economy of nature, a brief statement will be made of some of the things which are known to be accomplished by them.

156. Bacteria of Economic Importance. - There are forms existing in the soil, known as nitrifying bacteria, which bring about the oxidation of certain nitrogen compounds brought down by rains or resulting from the decay of plant and animal bodies, nitric acid being produced, $-\mathrm{a}$ substance absolutely necessary to the nutrition of plants. Its production by the bacteria is of great importance, since it exists in the soil in relatively minute quantities and is easily carried away by percolating water.

Bacteria of another kind inhabit the root tubercles of leguminous plants, and, taking the free nitrogen of the air, build it into the constitution of their own bodies. Finally the bacteria appear to be digested and appropriated as food by the leguminous plants (Fig. I3). In this way the green plant is able to obtain indirectly the free nitrogen of the atmosphere, which otherwise would be inaccessible to it. A knowledge of these facts is of utility to agriculture, for land naturally poor in nitrogen can be sown to alfalfa or plants of similar character, which, when plowed under, leave the land much richer in nitrogen than it was before. It has been found practicable to inoculate with liquid cultures of the bacteria soils which do not naturally contain them. The importance of these bacteria to leguminous plants growing in soils poor in compounds of nitrogen is shown by the experiment illustrated in Fig. 135 .

Other forms are instrumental in the production of vinegar from alcohol, in the proper ripening of cheese, and others add to the agreeable flavor of butter, as has been efficiently demonstrated in the dairies of Finland and Denmark.

157. Disease-producing Bacteria. - There are other forms 
which are injurious instead of beneficial, such as those producing consumption, diphtheria, typhoid fever, lockjaw, blood poisoning, bubonic plague, etc. (Figs. I33-I34). These diseases are brought about by poisons produced by the bacteria within the body. Great advances in surgery and in the treatment and prevention of diseases have been made by an understanding of the life history and habits of these microscopic forms of life. It is now known, for instance, that gangrene and blood poisoning, which formerly often followed in the wake of surgical operations, were brought about by bacteria clinging to the surgeon's knife, or which were in the water, bandages, etc., used in dressing the wound. Now every instrument or object employed in such operations is thoroughly sterilized, and the healing of the wound goes forward without complications. So, too, it is

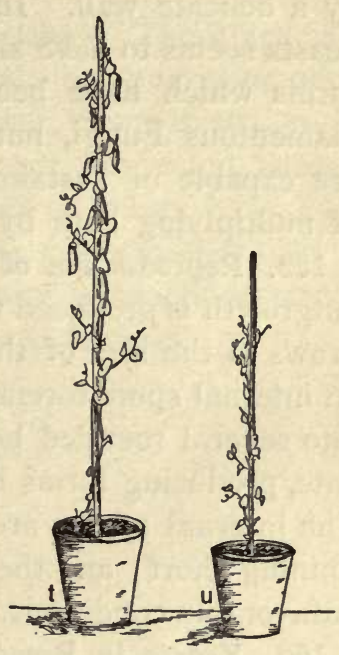

FIG. I35.

Experiment showing the importance of nitrogen-fixing bacteria to leguminous plants. $t$, peas grown in a nitrogen-free soil with bacteria; $u$, the same in all respects, but without bacteria. After FRANK. now known that the expectorations of consumptives and the dejecta of typhoid patients are teeming with the bacteria causing these diseases, and that unless the bacteria are destroyed by suitable poisons or heat they may spread disease.

158. The Nature of Yeasts. - Yeasts are low forms of plants which, like bacteria, are destitute of chlorophyll, and are dependent for their food upon materials built up 
by green plants. They are rounded or ellipsoidal (Fig. $133, a$ ), and are about .0OI 5 to .01 5 millimeter in diameter. Each individual consists of a single protoplast, surrounded by a delicate wall. Investigation into the life history of yeasts seems to have shown that they are really spore-like forms which have been produced by certain species of filamentous Fungi, but however this may be, the yeasts are capable of sustaining an independent existence, and of multiplying both by budding and by internal spores.

159. Reproduction of Yeasts. - In budding, a knob-like outgrowth is produced which finally becomes separated and grows to the size of the individual from which it sprang. In internal spore-formation, the cell protoplasm breaks up into several rounded bodies that escape and finally germinate, producing forms like the one from which they sprang. The internal spores are apt to be formed when the food is running short; and they are evidently useful in tiding over unfavorable conditions.

160. Yeasts in Bread-making. - Yeasts obtain part of their food from weak solutions of sugar, and in so doing convert the sugar into alcohol and carbonic acid; their usefulness in the raising of bread is due to this action. When the sponge is made, the ferment known as diastase, which was produced in the grain of wheat for the purpose of digesting the starch when the seed germinates, carries on the process of starch digestion in the sponge, changing a part of the starch into grape sugar. Then the yeast plants begin their action on the sugar, and the carbon dioxide produced along with the alcohol becomes entangled in the sticky mass and causes it to puff up. If, now, while the gas is still forming. the flour is worked in with the sponge, the gas is produced at all points throughout the mass of dough and raises it. The alcohol which is produced 
at the same time is driven off in the process of baking. If the bread is allowed to stand too long before baking, the alcohol is attacked by bacteria which change it into acids, and the dough is soured.

161. Yeasts in Alcoholic Fermentation. - There are various sorts of yeasts which produce alcohol, and some other little-known substances that give flavor, bouquet, etc., in the manufacture of beer, wine, and other fermented liquors. In recent years these various kinds of yeasts have been grown separately, and are used in the production of distinct kinds of beer and wine. Some yeasts seem also to be the cause of disease in man and the lower animals. 


\title{
CHAPTER XII.
}

\section{ALGE, FUNGI, AND LICHENS.}

\author{
PROVIDING MATERIALS.
}

Algæ and Fungi should be gathered in both vegetative and fruiting conditions and preserved in formalin. Many of the fresh-water Algæ do well in glass jars of water kept in a well-lighted place in the laboratory. Spirogyra and Oedogonium are very amenable to this sort of treatment. Reproductive stages will be found in abundance in early spring and summer. Vaucheria can usually be obtained in greenhouses, growing on flower pots, etc., at any season of the year. Out of doors it should be sought on moist and shady banks near the water's edge; and when found in good condition to show the method of sexual reproduction it should be preserved in formalin. One may secure Pleurococcus at any time of the year on the shady side of trees, etc., bread mould and Fungi. of a similar character as directed under Observation 160, and rusts, smuts, and mildews in great abundance at almost any time during the growing season. The last may be preserved either dry, in formalin, or in $70 \%$ alcohol. Toadstools and their kind can be collected in pastures and open woods at any time during the growing season when the weather is not too dry. Lichens are to be found on trees, old fences, and on rocks at any time.

\section{OBSERVATIONS.}

\section{ALGÆ.}

I5I. Cut, from the north side of a tree, bark which is covered with a green mealy growth; moisten with water, and place on wet filter paper under a bell jar for several hours. Observe the character of the growth, first with the naked eye and then with a simple lens. Scrape up 
some of it with the point of a knife, and notice whether it readily breaks up into small particles. Observe the tree on which it was growing. Is the growth always on the north side? How far up the trunk does it extend?

152. Scrape up a small portion of the growth with the point of a knife, taking care not to remove the bark, and mount under a coverglass in a drop of water. Place a clean cloth over the forefinger and move the coverglass about gently, in order to spread out the preparation in a thin film. If the pressure of the finger is too great, the little plants will be broken. Examine with a high power, and pick out what appears to be a single individual. How do you account for the clusters of individuals which are found? Does the green color occur in definite chloroplasts? Treat the preparation with chloral hydrate-iodine. Does starch appear? Is there any difference in this respect between material which has been kept in the dark and that which has been exposed to the bright light of the northern sky, but not to the direct rays of the sun? Can you tell why some trees seem to possess this growth more than others?

I53. Examine some of the green filamentous plants which occur in ponds, lakes, or small running streams. Do they float freely in the water or are they fastened down in any way? How do you account for the bubbles of gas which collect about these plants in the sunlight? Is there any difference in this respect between plants which are freely exposed to the sun and those which are deeply shaded? If so, what is the significance of the difference?

I54. Pick up some of the material and spread it out in the hand. Can you distinguish the individuals of which it is composed? Can you distinguish the parts of which the 
individuals are composed? Leave some of the plants out of the water for a time, and note what occurs to them.

155. Spread out a few of the individuals in a drop of water under a coverglass and examine with a medium power of the microscope. Do the parts of which the individuals are composed differ from each other in any essential way? Note the character of the walls and of the contents. Is a nucleus to be seen? What is the form of the chloroplasts?. Treat a preparation with chloral hydrate-iodine, and note whether starch is demonstrated. Does the nucleus become more prominent for a time while the protoplasm is dissolving?

I56. Examine some of the green, filamentous, felty growth which abounds on moist shady banks. Is it ever found in sunny situations? Examine some of the filaments, and try to determine how they are held together to form the felty mass. Are they anchored to the earth, or do they simply grow over its surface?

I57. Mount some of the filaments in a drop of water under a coverglass. Are they composed of cells, or does each filament appear to be one large cell? Locate the chloroplasts. Can more than one nucleus be found?

I58. If living near the seacoast, observe the bladderwrack. How is it fastened to its substratum? Is there any special device to keep the free parts buoyed up in the water? Examine the enlarged ends and press them between thumb and finger while observing them with a lens. Make thin sections through the swollen pitted ends, mount in a drop of water under a coverglass, and examine with a medium power. Small cavities, or conceptacles, should be seen opening exteriorly. In our common bladder-wrack, Fucus vesiculosus, these cavities will be found to contain either the eggs or the sperms, this species being diœcious. 
I59. Make sections through the enlarged bladder-like portions of the plant. Do they contain air or water? What is their evident purpose? Make sections through the other portions of the plant, and examine under the microscope. Although the plant has a brown color, it really contains chlorophyll, whose character is masked by the brown coloring matter associated with it in the chloroplasts. By what means is this plant held in proper condition to catch the sunlight?

\section{FUNGI.}

I60. Moisten stale bread in water and place it under a bell jar in a warm place. To be more certain of obtaining what is wanted, place under the bell jar some lemon pulp and rind from which the juice has been squeezed, or pieces of partly decayed sweet potato or banana. Various sorts of growths will appear, but the filamentous growths which in a few days bear minute spore cases on thread-like stems, are the forms desired for this study.

16I. Determine whether the filaments making up the body of the growth penetrate the substratum or simply lie on its surface. Examine with a lens the spore cases, or sporangia, of different ages. With a pair of forceps, carefully pull off sporangia of different stages of development, and mount under a coverglass in a drop of $70 \%$ alcohol. (Alcohol is used instead of water because air bubbles adhere to the material when water is used, but after mounting in alcohol a drop of water may be placed on the slide in contact with the coverglass, and as the alcohol evaporates the water will take its place.) Note the appearance of the spores in the sporangia of different ages. Why do the old sporangia appear black?

162. Soak a cubical block of stale bread in water, sow 
spores from the old sporangia over all sides of it, and keep it in a moist atmosphere under a bell jar. When the sporangia appear, note whether they all stand upright, or whether the direction taken by them bears a definite relation to the surface of the bread. Expose them to the light so that they will be illuminated on one side more than on another, and note whether the direction taken by the stems which bear the sporangia is affected in any way.

163. Examine the surface of a rusted leaf of wheat, oats, or any of the wild grasses. Does the rust appear on the veins or between them? Examine with a lens and note whether the epidermis of the leaf has been broken by the rust. Can any growths not belonging to the leaf be seen?

164. Make thin sections of the leaf across the rusted spots, and mount under a coverglass in a drop of water. If the leaf is dry, soak it for a few hours in water before sectioning. The section of the leaf should be so thin that the spores of the rust and the cells of the leaf immediately beneath may readily be seen. The parts may be rendered more transparent by mounting the section in a saturated solution of chloral hydrate. Notice the course of the thread-like part of the rust which penetrates the interior of the leaf.

165. Does the rust appear to produce any serious mechanical injury to the tissues of the leaf? In what ways is the injury to the plant probably produced? Does the rust appear to have any means of manufacturing its own food independently of the plant on which it is growing? What have you observed in regard to the ravages of rust in wheat fields, etc.?

166. Examine a toadstool growing in the woods or pasture. Dig away the soil carefully at its base to see 
what sort of connection it has with the substratum. Can smaller toadstools be found just above or beneath the soil ?

167. Break off the cap of the toadstool and lay it on a piece of blue paper where it will not be disturbed, and after a day or so remove the cap and note what has happened.

168. Make thin sections across the gills, and mount in a drop of $70 \%$ alcohol, and with a high power look for the spores growing from the gills. Examine with the same power some of the deposit found on the paper of Observation 167 , and compare with the spores found growing to the gills.

169. Examine the bark of old trees in a wood for the discolored spots and scale-like growths known as Lichens. Is the body of the Lichen all alike, or does some part appear to be spore-bearing? Cut off the Lichen from the bark and soak for a day in water, then make thin sections through the spore-bearing part and mount in a drop of water. Of what is the body of the Lichen found to consist? Are there any parts containing chlorophyll? Observe the slender sacs in which the spores are borne. How do Lichens probably obtain the water necessary to their growth? Do they make a slow or a rapid growth ?

\section{DISCUSSION. - ALGÆ:}

162. Nature of Algæ. - The Algæ, of which we have seen examples in the green growth on trees, in the filamentous green growths in water and on shady banks, and in the bladder-wrack of the ocean, are low forms of plants of very simple construction, except in the case of the larger seaweeds. They consist for the most part of single cells, or of rows of similar cells joined end to end to form filaments. They contain chlorophyll, and so are able to make 
use of the carbon dioxide of the air, or of the water in which they reside, in the same manner as do the more complex green plants. Having need of the sunlight, they usually float near the surface if their habitat is water; but if they live on the land, they are adapted only to moist and shady situations. They have no waterproof protective covering such as is furnished
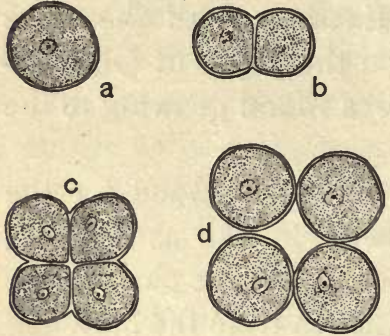

FIG. I36.

Pleurococcus viridis. $a$, a single individual $; b, c$, and $d$, various stages in cell division. The chloroplasts are so crowded that no attempt has been made to distinguish them in the figure. by the cuticle of the higher land plants, and accordingly quickly dry up if exposed to a dry atmosphere.

163. Pleurococcus Viridis. Pleurococcus viridis, which we found as a mealy green growth on the north side of trees, is one of the simplest of the Algæ. As has been seen, an individual consists of a single globular cell, having an outer transparent wall of cellulose, a nucleus which is difficult to see, and a few, relatively large chloroplasts occupying the bulk of the cell (Fig. 136). This single, minute individual is equipped to sustain an independent existence, being able to perform within its small compass the necessary nutritive and reproductive functions. But since it consists of a single cell, it is unable to construct special systems for the separate functions; and it is therefore restricted to habitats which by their nature protect it against too great transpiration, the beating of storms, and other sources of mechanical injuries. The thin and delicate wall necessary to allow the ingress of its raw food materials will not permit it to exist in places which are dry or fully exposed to the sun. We may 


\section{Algæ, Fungi, and Lichens.}

look upon it as one of the simplest forms which have essayed to appropriate the energy of the sunlight for the manufacture of their food; and it, or some form not distantly related to it, probably represents the very primitive organism from which the higher plants have been evolved.

Pleurococcus multiplies by cell division, one becoming two, two four, etc., as in the case of the bacteria. When examined under the microscope, the individuals are usually found adhering in groups in a manner which suggests that they are on the border land of unicellular and multicellular forms.

164. Spirogyra. - Among the forms of filamentous Algæ taken from fresh water, Spirogyra and Oedogonium are quite likely to occur. The method of reproduction of Oedogonium has already been described on page 163 , and Spirogyra (Fig. 137) will now be used as a type of filamentous Algæ. Each individual consists of similar cells joined end to end. The cytoplasm lines the cell wall, leaving a large vacuole filled with cell sap. Embedded in the cytoplasm are one or more elongated and spirally coiled chloroplasts. A rather large nucleus is suspended at the center of the cell by arms of cytoplasm which extend to certain bodies, known as pyrenoids, embedded in the chloroplasts. When a filament is mounted in a drop of chloral hydrate-iodine, starch is found to be clustered about the pyrenoids, and at no other places in the chloroplasts. We may conclude from this that while the chloroplast is undoubtedly manufacturing food throughout its whole body, the pyrenoids are the centers of accumulation of reserve materials in the form of starch (Fig. 137). When actively growing filaments are kept in the dark for a few days, the starch disappears. The chloroplasts utilize the sunlight in the same manner as do the chloroplasts in the 
leaves of higher plants. The student may be able to perceive some advantage in the spiral course of the elongated chloroplasts with reference to the absorption of light.

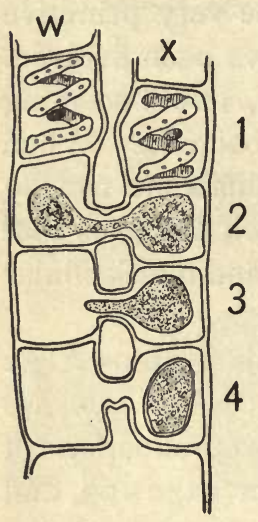

All cells of the filamentous individual perform the same nutritive functions; any one of them may take part in the formation of spores; and all bear practically the same relation to the outer world. The exterior wall of the filament, and the partition walls between the cells, are of cellulose, and permit liquids and gases to pass readily through them. Since they possess no waterproof covering, the filaments quickly dry up when taken out of the water. Being buoyed up by the water and freely floating in it, very little FIG. 137.

$v$, a single cell of Spirogyra, showing the spiral chloroplasts containing numerous rounded pyrenoids. The nucleus is suspended at the center of the cell. $w$ and $x$, two conjugating filaments; at $\mathrm{I}$, an early stage in the formation of a connecting tube between the two cells; at 2 , the tube has formed and the protoplast from a cell of filament $w$ is passing into the corresponding cell of filament $x$ and is fusing with its protoplast; at 3, a later stage; at 4, a spore has formed from the fused protoplasts. After SACHS.

stress is ever exerted on the filaments, and special strengthening devices are unnecessary.

165. Reproduction of Spirogyra. - Spirogyra shows a degree of sexuality in the method of its reproduction. Two filaments which happen to be lying in close proximity put forth outgrowths from one or more of their cells which finally meet, and the walls separating them become absorbed. The entire protoplast 


\section{Algæ, Fungi, and Lichens.}

in the conjugating cell of one filament passes through the tubular connection and fuses with the protoplast in the conjugating cell of the other filament, all of the conjugating cells of one filament being receptive, and of the other contributive. The two fused protoplasts organize a wall about themselves, and become a resting spore which is able to endure desiccation and other adverse conditions. After a period of rest the spore germinates and produces a filamentous individual similar to those from which it sprang (Fig. 137). The filament which bears the spores may be considered the female and the other the male, but there is no structural differentiation into egg and sperm.

166. Vaucheria. - Vaucheria is a filamentous Alga of another character. It grows either in water or on moist and shady banks. Each individual is a tubular branched filament which in its vegetative state is not divided by cell walls. One end of the filament is modified in such a way as to serve as an anchor to the substratum (Fig. 138). The protoplast which lines the tubular filament contains many nuclei and probably consists of many fused protoplasts. Small rounded chloroplasts are found in great numbers in the filaments. The wall is of cellulose without much waterproofing, and the plant cannot flourish away from water or moist places. The branched filaments have the habit of interweaving, and so furnish each other mutual support.

167. Reproduction of Vaucheria. - Vaucheria reproduces by both the sexual and asexual methods. In the latter process the protoplasm accumulates in a swollen end of the filament, which becomes demarked from the rest of the filament by means of a transverse wall. The swollen end breaks open and the mass of protoplasm passes out and swims about for a time by means of numerous cilia that 
grow out over its surface. Soon it comes to rest and organizes a wall over its surface, and after a brief period it germinates, producing a new branched filament, with an expansion at one end for anchorage to the substratum.

In the sexual method of reproduction, two forms of outgrowths are produced on the same filament; a rounded

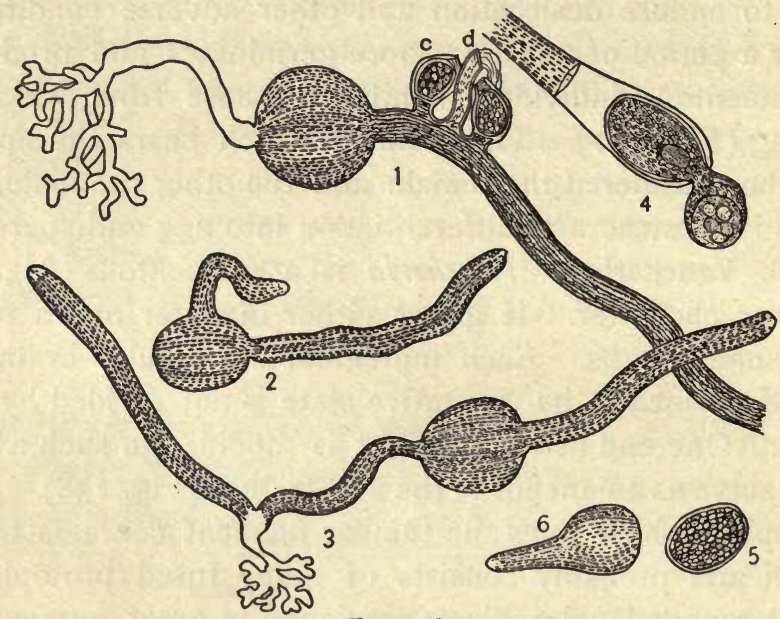

FIG. 138.

Vaucheria sessilis. I, a filament-bearing oogonia (c) and an Antheridium (d). The rounded portion to the left represents the spore from which the filament has sprung. The clear branched portion is a clinging organ by which anchorage is made with the substratum. 2, a germinating spore. 3, a later stage. 4 , an asexual spore escaping from its parent cell. 5, the same after it has come to rest and surrounded itself with a cell wall. 6 , the spore, (5) in the first stage of germination; 2 and 3 are later stages. After SACHS.

one (the oogonium), which is separated from the parent filament by a wall, and contains the egg, and a slender outgrowth (the antheridium), containing the sperms at its apical portion (see Fig. 138). The cells containing the egg and those containing the sperms break open at their apices, and the sperms swim out by means of two cilia 
with which each is provided, and, seeking the egg, one of them fuses with it and accomplishes its fertilization. However, proterandry may occur, so that the egg is often not fertilized by sperms from the neighboring antheridium. The egg while still contained within its cell then produces a thick wall about itself, and passes through a resting period before germinating. Vaucheria is a step in advance of Spirogyra in the evolution of sexuality, for its reproductive elements are quite sharply differentiated into egg and sperm.

168. Fucus. - The large brown marine Alga known as wrack-weed or bladder-wrack (Fucus vesiculosus) grows anchored to the rocks between high and low tide levels. Its branches are somewhat flattened, and possess air bladders which buoy it up in the water, and thus enable it to expose a broad surface to the light. It is multicellular, and its tissues are somewhat differentiated, the central part consisting of slender cells with large spaces between them containing mucilage, and the outer tissues being made up of smaller rounded cells, the peripheral layer of which has the nature of an epidermis. Chloroplasts reside in the outer tissues, but their green color is modified by a brown coloring matter associated with the chlorophyll. Notwithstanding this, the chloroplasts of Fucus are active in photosynthesis and sustain the same relation to the sunlight in the manufacture of food materials as do the chloroplasts of green plants.

169. Reproduction of Fucus. - Reproduction is effected sexually by the production of eggs and sperms, borne in minute cavities of the plant body, known as conceptacles (Fig. 139). In the species vesiculosus the sperms and eggs are borne in different plants, but in other species they both occur in the same conceptacle. The eggs, 
eight in number, are borne in small sacs growing out from the walls of the conceptacle (see Fig. I39); and the sperms are formed in elongated cells produced on hair-like outgrowths. Both eggs and sperms become discharged
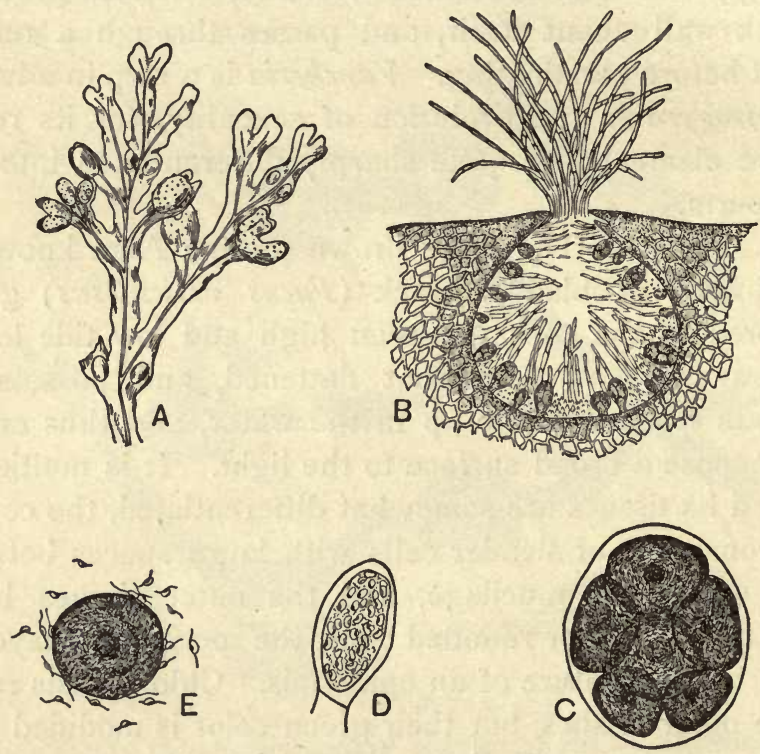

FIG. I39.

Fucus. $A$, portion of a frond; $B$, section through a conceptacle; $C$, oogonium with eggs; $D$, antheridium with sperm cells; $E$, an egg with sperm cells swimming about it. After THURET.

from their conceptacles into the surrounding water, and the sperms seek the eggs, being able to swim about by means of two cilia. One of the sperms penetrates the egg and effects its fertilization. The egg then forms a wall about itself and is capable of immediate germination.

Some of the marine Algæe closely related to Fucus attain as much as a hundred feet in length, and produce lateral 
Algæ, Fungi, and Lichens.

outgrowths resembling leaves and performing the functions of leaves; so that both in the construction of the vegetative parts and in the method of reproduction we find in them close affinities to the higher plants.

\section{FUNGI.}

170. Character of Fungi. - Turning now to the fungi, we find plants which are characterized by their lack of chlorophyll, and a consequent inability to utilize the energy of the sunlight in the manufacture of their food. We find, therefore, that they are either parasitic or saprophytic, that is, they obtain their food from living or dead plants or animals. They are closely allied to the Algæ in their structure and modes of reproduction, and may be their degenerate descendants.

171. Mucor, or Bread Mould. - The common black bread moulds, Mucor mucedo and Mucor stolonifer, are good representatives of the saprophytic kinds of Fungi. The firstnamed Mucor is composed throughout its whole vegetative body of a much-branched unicellular filament which ramifies partly through the substratum and partly over its surface, forming a somewhat felty coating. The wall of the filament is thin and permits the transfusion of liquids. The portion of the Fungus which is embedded in the substratum excretes a ferment that renders organic substances soluble and adapted to absorption and assimilation. In this way the Fungus is able to devour the starchy and proteid substances of bread, vegetables, fruits, etc., and even the cellulose composing cell walls. Such ferments are extracted in large quantities from Fungi and sold on the market in the form of digestive extracts or tablets.

172. Reproduction of Mucor. - Having accumulated a sufficient amount of food materials, it proceeds to form its 
reproductive bodies. Portions of the branched filament become cut off by cell walls, and these demarked portions
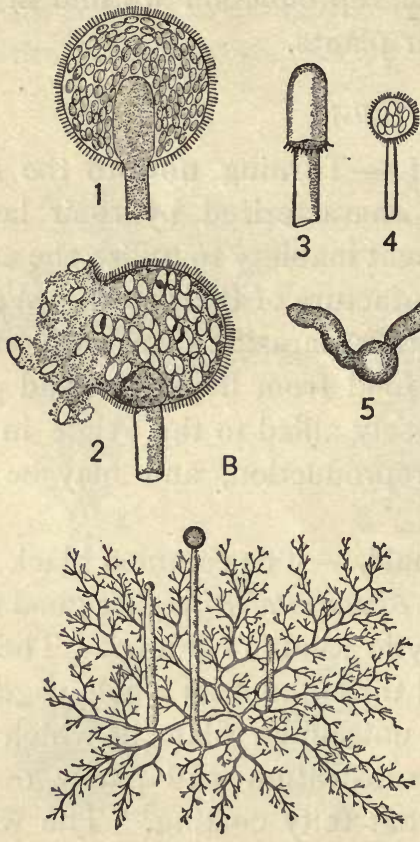

A

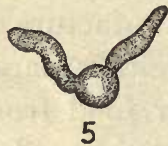

FIG. I40.

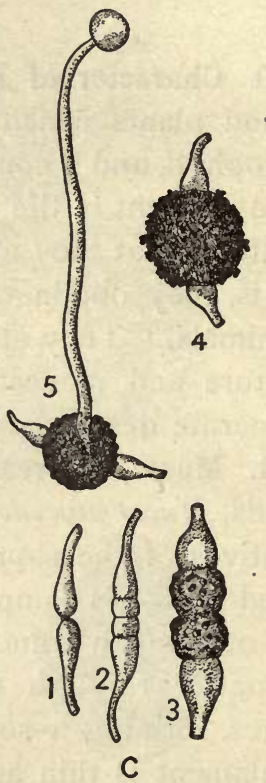

Stages in the life history of Mucor mucedo. $A$, an entire plant bearing sporangia on upright stalks. $B, \mathrm{I}$ and 2 , a sporangium before and after breaking open to discharge the ellipsoidal spores; 3 , the upper part of the stalk after the sporangium has broken away; 5 , a germinating spore which is to produce a plant like $A$. $C$, r, two conjugating branches of the thread-like mycelium; 2, a later stage, showing two end cells demarked by cell walls; 3 , a later stage where the wall separating the two end cells from each other has become dissolved, so that the contents of the two cells have fused to form a resting spore; 4 , the mature resting spore; 5 , the germinating resting spore giving rise to a single sporangium bearing relatively few spores, as shown in $B, 4$. After BREFELD.

send forth branches which grow outward from the substratum, sometimes to a distance of several centimeters, 


\section{Algæ, Fungi, and Lichens.}

and finally produce enlargements at their ends which become separated from the rest of the branch by a cell wall (Fig. 140). The protoplasm in these enlarged ends divides into many spores, which finally break out from the surrounding wall and become scattered, giving rise on their germination to new filamentous individuals.

When the substratum supplies an abundance of food materials, resting spores may be produced sexually. In this process the ends of two branches meet, and a partition wall is formed in each, a short distance back from the ends. The end walls then become dissolved, and the contents of the short terminal cells fuse together, thus constituting a single cell, which then enlarges considerably, produces a thick outer wall, and enters into a period of rest. When this sexually produced spore germinates, the thick outer wall becomes broken, and the inner wall and contents grow forth and produce a branched filament which sooner or later gives rise to asexual spores as above described (see Fig. 140). By the asexual spores, which are produced in almost countless numbers, the fungus becomes broadly scattered, while the less number of sexual spores serves to carry the species through adverse conditions.

173. Rusts. - The rusts on wheat and other grasses are interesting representatives of a large class of Fungi which obtain their living at the expense of other live plants. Observations 163 and 164 have shown us that the rusty streaks appearing on the leaves of wheat, for instance, are really masses of the oval spores of the fungus (Fig. I4I, $A$ ) which break through the epidermis and become blown about by the wind. The spores are borne on the filamentous vegetative part of the Fungus (Fig: I4I, $B$ ), which ramifies amongst the parenchyma cells of the leaf 
and appropriates the materials manufactured there. These spores, called summer spores, or uredospores, when brought

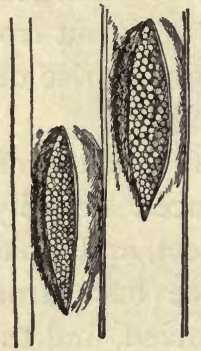

A

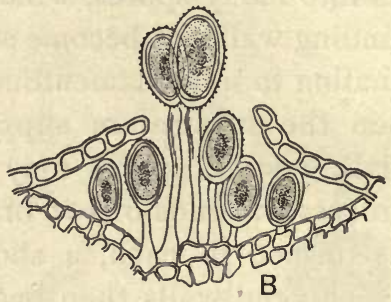

FIG. I4I.

$A$, clusters of uredospores of wheat rust breaking through the epidermis between the parallel veins of a leaf of wheat. $B$, a cross section through one of the spore clusters of $A$, showing the uredospores highly magnified.

to other leaves by the wind, put forth slender sprouts which enter the leaf through the stomata, and thus within the

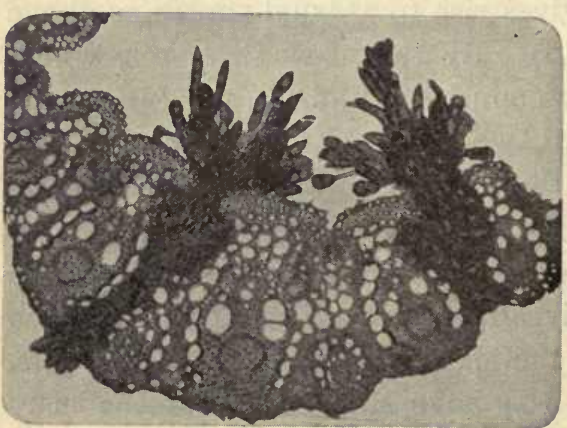

FIG. I42.

Photomicrograph of a cross section of a grass leaf parasitized by Puccinia. The mycelium of the fungus extends through the leaf and bears clusters of teleutospores at the upper surface. leaf new rust plants are started, which in turn produce summer spores.

Later in the season spores are formed which are two-celled, pear-shaped, and darker in color (Fig. I42); these, known as the winterspores, or teleutospores, survive the winter, and the following spring germinate and produce filaments, each bearing several small spores (Fig. I43) which may grow into the leaves of barberry 


\section{Algæ, Fungi, and Lichens.}

bushes where these abound, producing chains of spores, termed aecidiospores, in cup-shaped cases (Fig. 144, B). These spores become discharged, and when blown to the surface of a leaf of wheat they grow into it, and finally summer spores appear at the surface of the leaf, as already described.

It appears, then, that the rust of wheat produces three kinds of spores and requires two kinds of plants to run the full course of its existence. The winter spores, however, can establish themselves in the leaves of wheat without the intervention of the barberry, and the summer spores can also survive the winter and reproduce the fungus on wheat the following spring.

Other parasitic Fungi of common occurrence which often do great damage to the plants of the field and garden are the srnuts and mildews.
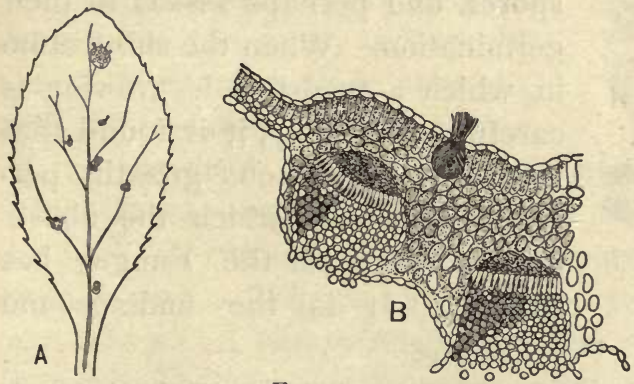

FIG. 144.

$A$, leaf of barberry bearing aecidiospores of wheat rust. $B$, cross section through the barberry leaf, showing groups of spores immersed in the tissue of the leaf. ( $B$ after DE BARY.)

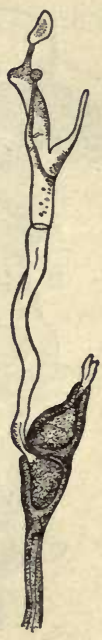

FIG. I43.

Germinating teleutospore of wheat rust. After Tulasne.

174. Toadstools. - The toadstools are too commonly observed to require description here, and only a brief account of their structure and ways of life will be necessary. The stem and 

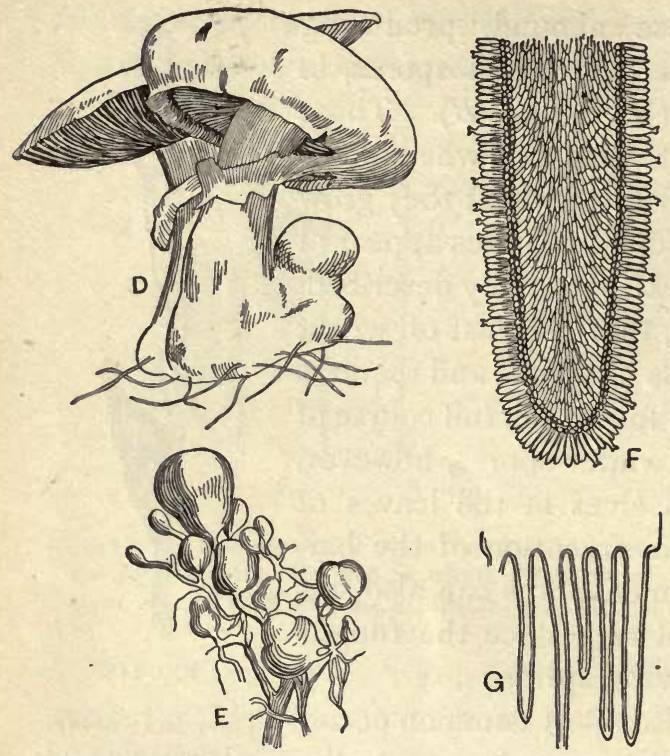

cap are composed of multicellular filaments so closely woven and grown together as to form a false tissue (Fig. 145). On both surfaces of the gills minute spores are borne which become projected to the g r o u nd, a s shown by Observation i67. Or in some cases

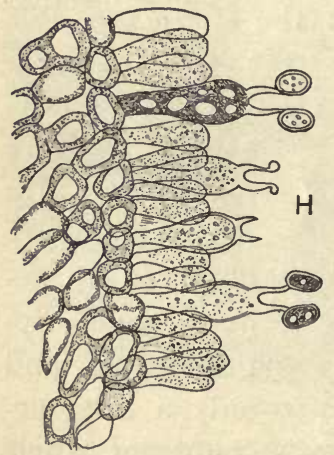

both cap and stem deliquesce, forming a fluid mass which engulfs the spores, and perhaps assists in their germination. When the substratum in which a toadstool is growing is carefully dug away, it is found that there are filamentous growths permeating it from which the aboveground part of the Fungus has sprung. It is the underground

FIG. I45.

Agaricus campestris or mushroom. $D$, mature plant with stalk bearing an expanded cap, from which gills are pendent. $G$, a cross section of some of the gills, slightly magnified, and $F$, one of the gills in cross section more highly magnified, showing the gill to be fringed on both surfaces with stalks bearing spores. $H$, a more highly magnified detail of a portion of $F$, showing the rounded spores. $E$, young mushrooms, to become like $D$. After SACHS. 
part that has accumulated the food necessary to maintain the very rapid growth which toadstools are so well known to make. The student will notice by a careful examination of the substratum that it is very rich in vegetable remains in the form of mouldering leaves, stems, roots, etc., whose substance the toadstool is able to digest by means of ferments excreted from its underground filaments.

But the toadstools and their allies do not always restrict themselves to this innocent mode of life, for it may happen that underground filaments, ramifying through the rich mould of a forest in quest of food, penetrate the roots of a tree, and entering the woody tissues, digest and appropriate them for food (Fig. I46). So the Fungus advances deeper into the roots, and up into the stem, sapping their strength, until the tree may easily be broken

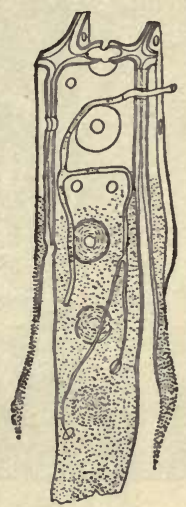

FIG. 146.

Destruction by Fungi of a cell of pine wood. The branched filament is a part of the mycelium of the Fungus. The upper part of the wood cell has not yet been disintegrated by the Fungus, as has the lower dotted part. After R. HARTIG. off or upturned by the wind. These Fungi also gain access to the interior of the trees through wounds in the bark, or through the broken branches of the aboveground parts. (Figure 147 shows a portion of a trunk of a tree which has suffered from the ravages of Fungi.) In some localities forest trees suffer considerable damage from foes of this kind; and not only trees, but timbers also which have already been put to purposes of construction. 


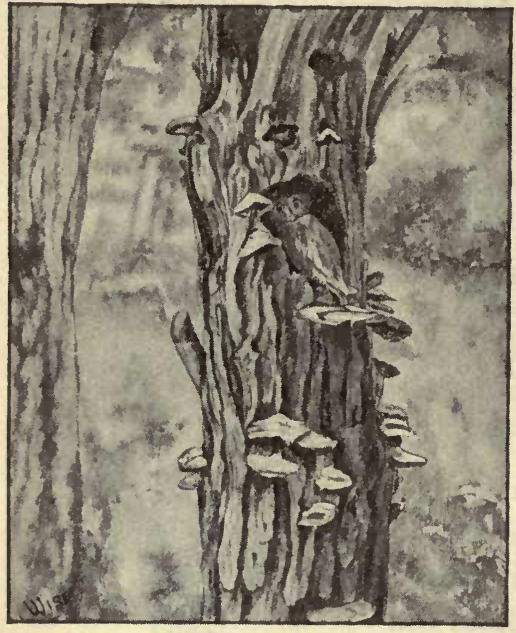

FIG. I47.

Trunk of a tree in process of destruction by Fungi. Fungi belonging to the genus Polyporus growing out from the trunk. Interior of the tree rotten and hollow and used as a nest by owls.

Besides multiplying by spores, the toadstools may be disseminated by the separation of bits of the masses of filaments which occupy the substratum; in this way mushrooms are artificially propagated.

\section{LICHENS.}

175. Nature of Lichens. - The Lichens afford a unique example of parasitism by Fungi ; for a Lichen is not a distinct plant, but rather a community of Fungus and Algæ. The little Pleurococcus with which we are already acquainted is often associated with a Fungus in this way. The Fungus undoubtedly derives its food from materials manufactured by the Algæ, but at the same time it

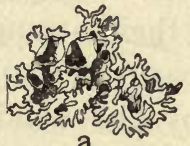

a

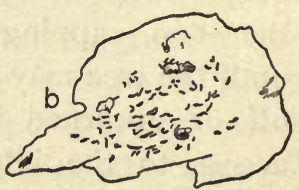
does the Algæ no bodily harm, except that which might result from the tax on their industry. It is thought by some that there is a fair exchange of benefits between the

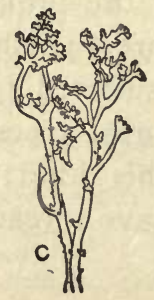

FIG. 148.

Different forms of Lichens. $a$, Parmelia colpodes; $b$, Graphis scripta on bark of tree; the elongated black spots are the Lichens; $c$, Cladonia furcata. 


\section{Algæ, Fungi, and Lichens.}

Fungus and the Algæ, the Fungus extracting water and salts from the substratum and sharing it with the Algæ; but it must be remembered that the Algæ are able to flourish, perfectly well without the intervention of the Fungi, while those Fungi which form Lichens are, with few exceptions,

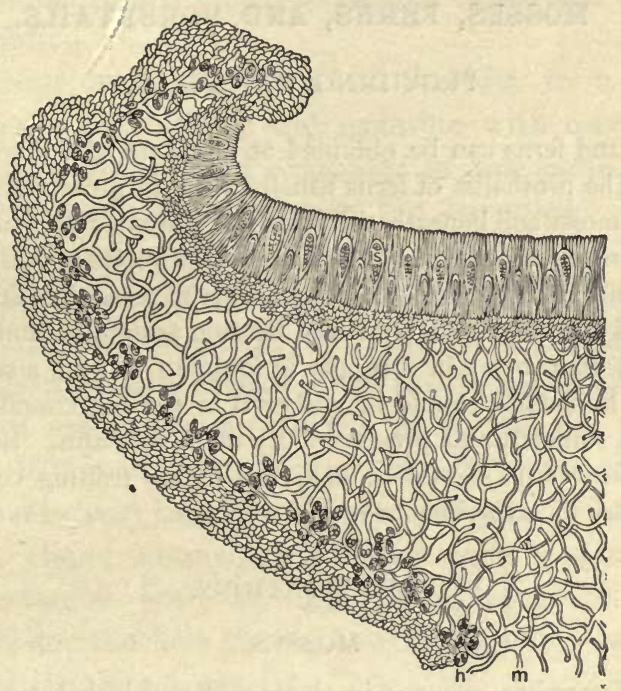

FIG. I49.

Section through the fruiting part of a Lichen. The stratum of dark rounded bodies (h) represents the algal part of the Lichen; $m$, the thread-like hyphæ of the Fungus constituting the bulk of the body of the Lichen; $s$ (at the top in one of the sacs), sacs or asci in which the spores of the Fungus are borne. After SACHS.

not known to exist without the assistance of the Algæ, with which they associate themselves. Figure 148 shows different forms of Lichens, and Figure 149 represents a cross section through the body of a Lichen, revealing the tissue formed by the filaments of the Fungus and the embedded Algæ. 


\section{CHAPTER XIII. \\ MOSSES, FERNS, AND HORSETAILS.}

PROVIDING MATERIALS.

Mosses and ferns can be obtained at any time of the year in greenhouses. The prothallia of ferns usually grow in great quantities in the shady and moist soil beneath the benches. Mosses may also be found in good condition in the shade and shelter of woods in early spring and summer, and even in the winter season, in some localities. When mosses and fern prothallia are found bearing antheridia and archegonia, it is a good plan to lay in a supply in formalin or $70 \%$ alcohol. Equisetums, or horsetails, should be gathered in fruiting condition in early spring and summer, and preserved dry or in formalin. Some mosses, ferns, and horsetails should be collected in the fruiting condition and preserved dry for experiments with the spores.

\section{OBSERVATIONS.}

MOSSES.

170. Examine mosses in their natural habitat. Separate a single plant carefully from its associates and from the substratum. What is the character of the members which connect it to the substratum? What is the nature of the substratum; is it moist or dry?

I7r. Examine mosses which are in fruit. The fruiting stage will be recognized as the slender stem growing from the apex of the leafy stem and terminating with a capsule which bears the spores. Examine the spore capsules of different ages. Notice how they normally break open. Examine the opening with a lens, and shake out some of the spores and examine them with a lens. 
172. Examine with a lens the apices of some of the leafy stems which are not bearing capsules. A diligent search is likely to reveal two kinds of structures, - very small flask-shaped bodies, termed archegonia, which bear the eggs, and club-shaped bodies, termed antheridia, which contain the sperms. Make drawings of all the members thus far studied.

I73. Mount archegonia and antheridia in a drop of water under a coverglass, and examine with medium and high powers. Mount and examine, in the same manner, some of the spores contained in the capsules. Draw as seen with both low and high powers.

174. Boil pieces of soft brick, and, after cooling, place them in a dish of water so that they are but little submerged. Scatter the spores over the brick, cover with a bell jar, and set in strong diffuse light, but not in direct sunlight. When delicate green filaments (protonemata, singular protonema) begin to appear over the brick, scrape off some of them, mount in a drop of water under a coverglass, and examine with medium and high powers. Leave the brick under the bell jar, replenish the water as needed, and observe developments.

175. Remove a leaf from a moss, mount in a drop of water under a coverglass, and examine with a high power. How many cells thick is the leaf? What is the form of the chloroplasts? Are stomata present? Place a drop of chloral hydrate-iodine on the slip in contact with the coverglass, and draw out the water with a piece of filter paper placed against the opposite edge of the coverglass. As the chloral hydrate-iodine replaces the water, watch the effect upon the chloroplasts. Does starch appear in them? Do plants which have been kept in the dark give a different result? 
176. Dig up a clump of mosses in fruit, and place it in a shallow dish containing sufficient water to keep the bottom of the clump moist. Cover it with a bell jar, and after a few hours remove the bell jar and observe the behavior of the capsules as the atmosphere about them becomes less humid.

FERNS.

177. Examine ferns in their natural habitat. Note exposure to the sun, and the nature of the soil,- whether it is moist or dry. Dig up a plant and observe the character of the underground parts.

178. Examine the back of the leaves for the rounded or linear clusters (sori, singular sorus) of sporangia. Select a portion of a leaf which shows by the brown color of the sporangia that the spores are ripe, and place it under a bell jar on a piece of moist white filter paper. After a few hours remove the bell jar and examine the paper with a lens to see whether the spores have become scattered. Even old herbarium specimens might answer the purpose.

I 79. Scatter spores over pieces of brick and keep moist under a bell jar, as directed for the spores of mosses. After a few weeks the spores should have germinated, and the green bodies resulting, known as the prothallia, should be examined under a microscope. How are they anchored to the brick? After a time archegonia and antheridia should be observable on the under sides of the prothallia. Take care of the experiment until young fern plants begin to grow erect from the prothallia.

\section{HORSETAILS.}

I80. Note the character of the habitat in which horsetails flourish. Dig up some of the plants and observe the nature of the roots and the relation of the plants to each 
other by means of the underground parts. Examine the cylindrical stems. Are there any structures growing from the nodes which may be morphologically leaves? Halve a stem longitudinally from top to bottom and note its construction.

181. Examine the sporangia which are borne in cone-like clusters at the tops of the stems. Scatter some of the dry spores on a glass slip and examine with a medium power. Gently breathe on the slip and note the result. Mount some of the spores in a drop of water under a coverglass and examine with a high power.

\section{DISCUSSION.}

MOSSES.

176. Character of Mosses. - The shoot of the moss

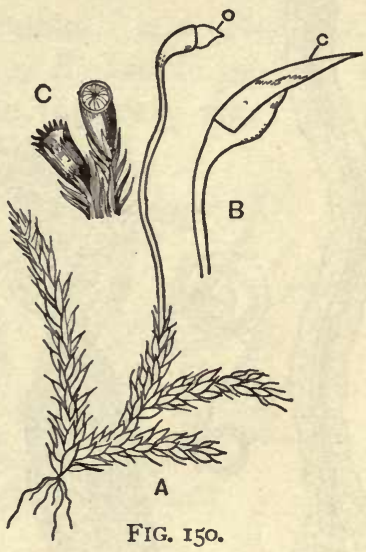

$A$, entire moss plant, the leaf-bearing gametophyte with rhizoids, bearing the sporophyte, i.e. the capsule and its stem ; 0 , the operculum, which falls off and allows the spores to escape; $B$, the capsule surmounted by the calyptra $(c)$; $C$, capsules with the fringe of teeth open and closed. In part after KERNER.

plant is differentiated into

stem and leaves of very simple construction. Filamentous outgrowths, called rhizoids, anchor the plant to the substratum, and have the absorptive function of the roots of the higher plants (Fig. I 50). Because of their simple construction and lack of an effective protection against too great transpiration, the mosses are mostly restricted to moist and shady situations, although some species occur in exposed localities, having acquired the power of reviving after long periods of desiccation. The leaves of 
mosses perform the same functions as do those of higher plants.

177. Reproduction of Mosses. - An examination of the apices of moss shoots reveals the fact that archegonia and

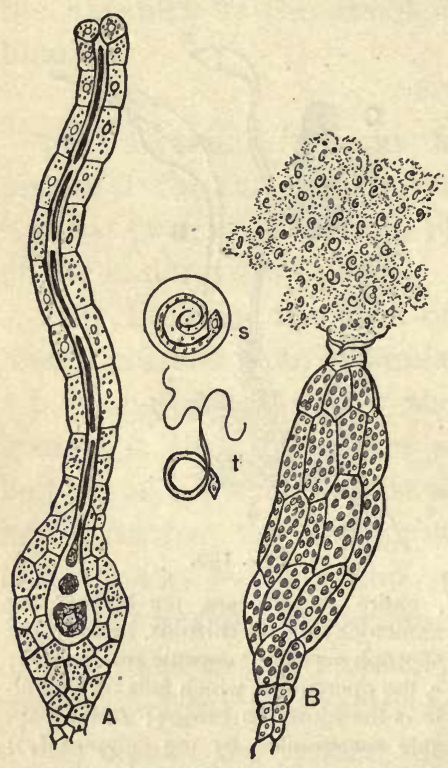

FIG. I5I.

$A$, archegonium with egg cell at the base of the cavity, and $B$, antheridium of a moss. At $s$ and $t$ are sperms; these are seen escaping from the apex of $B$. All highly magnified. After SACHS. antheridia (Fig. I5I) are produced there, in some species both on the same plant, and in others on different plants. The archegonia and antheridia are small and obscure, and would be overlooked by the casual observer. The wall of the antheridium breaks open at its apex (Fig. I 5 I, $B$ ), and the sperms ( $s$ and $t$ ) being freed, swim about in the dew or rain which has collected over the plants; and being attracted by some chemical substance secreted within the archegonia (Fig. I $5 \mathrm{I}, A$ ) they enter these through their hollow apical elongations, and one sperm in each case fuses with the egg.

The fertilized egg immediately germinates without leaving its position in the archegonium, and the rod-shaped embryo, as it elongates, breaks off the narrow upper part of the archegonium and carries it as a sort of cap called the calyptra (Fig. I $50 B, c$ ). At the same time the lower part of the embryo pushes its way down into the tissues of the parent plant, from which it continues to draw food until 
the time of its maturity; that is, until the capsule containing the ripened asexual spores has been formed at its apex. The asexual spores are capable of germination as soon as formed, but, instead of producing a leafy moss plant immediately, they first form filamentous outgrowths called protonemata, resembling the filamentous Algæ (Fig. I 52, $A$ and $B$ ). Finally buds (Fig. $152, B$ ) are formed on the protonemata, which develop into the leafy moss plant.
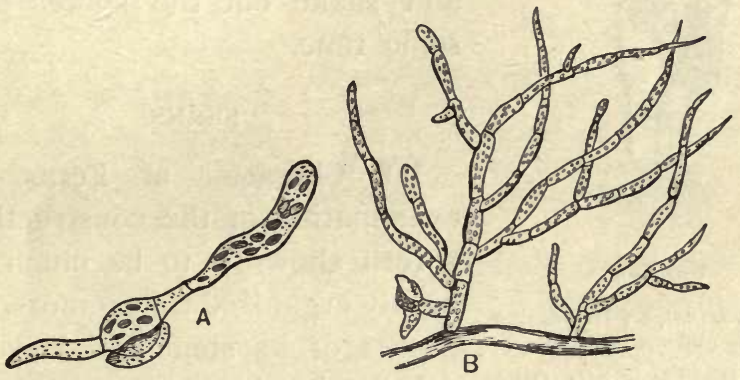

FIG. 152.

$A$, germinating moss spore; $B$, protonema produced by further development of $A$. To the left, near the base, is a bud which is to become a moss plant. After SACHS.

The life history of a moss exhibits an alternation of two sorts of generations; the leaf-bearing generation, with its archegonia and antheridia, known as the sexual generation, or gametophyte, and the leafless asexual generation, or sporophyte, namely, the capsule and its stalk, which grows from the fertilized egg in the archegonium and produces spores asexually. It should be observed that the asexual generation in its young state bears chlorophyll, and must therefore be able to manufacture a portion of its food, at least for a time.

178. Dissemination of Spores. - It will be seen that after the top, or operculum (Fig. I50, O), of the sporangium has 
broken away the spores are still kept from falling out by means of a fringe of teeth (Fig. I 50, C); these are more or

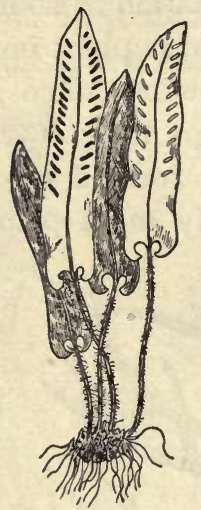

FIG. 153 .

Scolopendrium, a fern bearing oblong clusters of sporangia (sori, singular sorus). After STRASBURGER. less hygroscopic in different species. They close the opening of the capsule in a humid atmosphere and open outward to allow the spores to be shaken out in a dry atmosphere. The winds probably dry the teeth and shake out the spores at the same time.

\section{FERNS.}

\section{Character of Ferns. - An} examination of the construction of a fern shows it to be much more highly organized than a moss. The tissues of its stem and leaves are differentiated to perform separate functions, as we have seen to be the case in the higher plants, and it has true roots. The leaves not only perform the usual photosynthetic function of green leaves, but they may also bear spores asexually on their under surface (Fig. I 53); and since no part of the fern plant bears sperms or eggs, we must look upon it as an asexual generation, or sporophyte, which in the moss we saw to be leafless (namely, the capsule

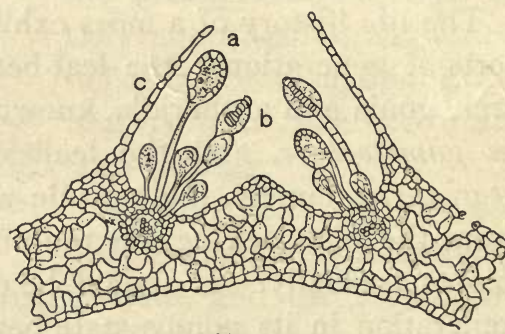

FIG. I54.

Cross section through a sorus of Scolopendrium. $a$ and $b$, sporangia containing spores (the spore is the unicellular stage of the gametophyte) produced asexually; $c$, the protective covering or indusium. After STRASBURGER. 
and stalk bearing it) and more or less parasitic on the sexual generation.

180. Reproduction of Ferns. - The sporangia (Fig. I54) and the spores contained in them are, in some of the ferns, direct descendants of a single epidermal cell. In other ferns they originate in several cells of the epidermis and deeper-lying tissues. When the spores
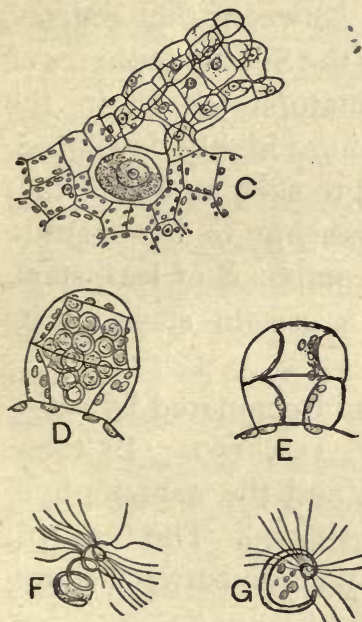

FIG. I56.

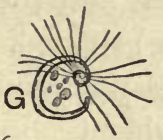

$C$, archegonium of a fern with its egg cell ; $D$, an antheridium with sperm cells; $E$, an empty antheridium; $F$ and $G$, motile sperms. After STRASBURGER.
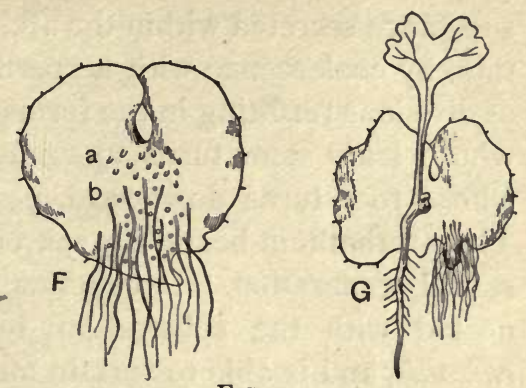

FIG. I55.

$F$, prothallium or gametophyte of a fern seen on its under surface. The hairs are the rhizoids. At $a$ are archegonia, at $b$ antheridia. $G$, a prothallium bearing a young fern plant or sporophyte. After STRASBURGER.

are ripe, the sporangia break open elastically on imbibing moisture and throw the spores to some distance. When the spores germinate, they do not immediately produce the fern plant, but instead, a small thin body known as the prothallium (Fig. I 55), which lies flat on the substratum. The prothallium, which may be a centimeter or less in diameter, bears on its under surface eggs in archegonia, and sperms in antheridia (see Fig. 1 56).

The sperms are minute ciliated . bodies. They become discharged from the antheridia and swim in the rain water or dew in quest of the egg, being attracted by a 
substance secreted within the archegonium. After fertilization, by coalescence with a sperm, the egg begins a series of divisions resưting in the formation of a young fern plant, whose leafy stem turns upward and seeks the light, and whose root turns downward, penetrating the substratum. The prothallium bears the sex organs and constitutes the sexual generation. It contains chlorophyll, and is connected with the substratum by absorbing hairs called rhizoids, and is able to sustain an independent existence, in some instances for several years.

181. Comparison of Asexual and Sexual Generations. We note that while the sexual generation is the more conspicuous in the mosses, it becomes subordinate in size and differentiation in the ferns. In this respect the ferns may be looked upon as intermediate between the mosses, together with other lower plants, and the Spermatophytes or seed-bearing plants. In the Spermatophytes, as in the ferns, the asexual generation is the more highly developed, the sexual generation being reduced to such an extent that it requires good powers of the microscope to demonstrate it; while the asexual generation is composed of leaf, stem, root, and all of the flower up to a certain stage of its development.

These facts can be summarized and compared by reference to the diagrams of Figs. 157, 158, I 59. In these figures the sporophyte is unshaded and the gametophyte (after the germination of the spore) shaded. The fern will be considered first, since in it the distinction between sporophyte and gametophyte is most evident.

When the fern spore (Fig. I 57, $d$ ) germinates, a multicellular body $(A)$ is produced (prothallium) bearing sex organs, namely, archegonia $(a)$, containing each an egg cell, and antheridia $(b)$, bearing sperms. Since the pro- 
thallium bears the sex organs, it is rightly called the sexual generation, or gametophyte. The egg, having been fertilized by fusion with a sperm (see paragraph 180), undergoes segmentation; and the cellular division thus begun continues indefinitely, resulting in a conspicuous fern plant (Fig. I 57 $B, e$ ) which bears spores asexually. As has been said, the fern plant has no sex organs, and is therefore called the asexual generation, or sporophyte.

During the cell divisions which are immediately concerned in the formation of the spore, the number of the chromosomes entering into the constitution of the nucleus is reduced by one-half (see page I09 for a description of cell and nuclear division), so that the spore contains only one-half as many chromosomes as do the cells which make up the body of the fern plant, but precisely the same number as the cells of the prothallium or gametophyte. Therefore we look upon the spore as the one-celled stage of the gametophyte. When the nucleus of the sperm cell fuses with that of the egg cell, the number of chromosomes now entering into the

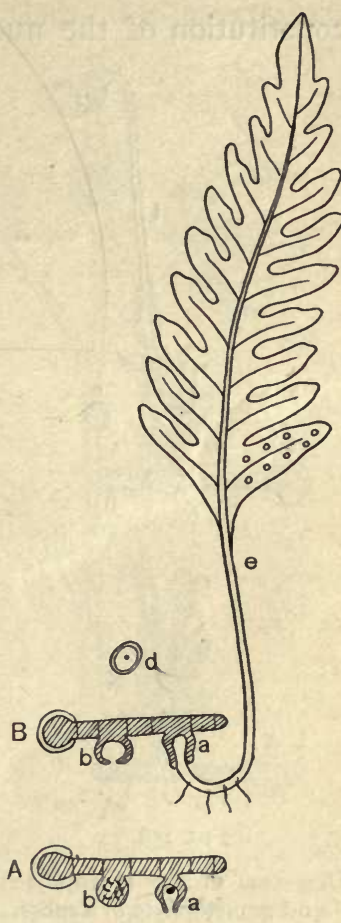

FIG. 157 .

Diagrams of the sporophyte and gametophyte of a fern. The spore $d$ produced asexually on the fern plant $e$ (sporophyte) is the beginning of the gametophyte. All of the gametophyte produced by the germination of the spore is shaded; all of thesporophyte is left unshaded. $A$, the prothallium (gametophyte) bearing an archegonium (a) with its egg, and an antheridium $(b)$ with its sperms. The fern plant $e$ grows from the fertilized egg. $B$, a later stage than $A$. 
constitution of the nucleus of the fertilized egg is doubled

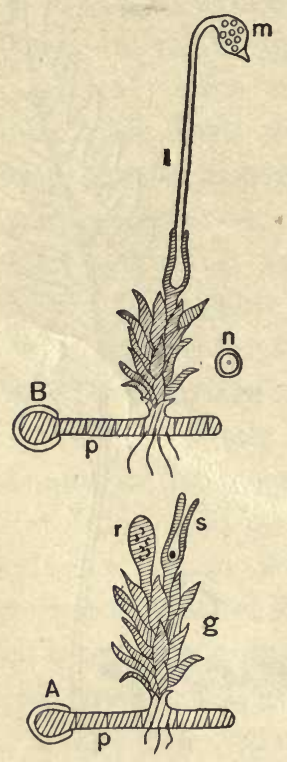

FIG. 158 .

Diagrams of the sporophyte and gametophyte of a moss. The spore $n$ produced asexually in the capsule $m$ (which with its stalk $l$ is the sporophyte) is the beginning of the gametophyte. All of the gametophyte produced by the germination of the spore is shaded; all of the sporophyte is left unshaded. $p$ in $A$, the protonema from which springs the moss plant $g$ (gametophyte together with $p$ ), bearing an archegonium $(s)$ with its egg, and an antheridium $(r)$ with its sperms. $B$, a later stage, the capsule or sporophyte having sprung from the fertilized egg. by this union, so that the fertilized egg contains the same number of - chromosomes as do the nuclei of the cells which make up the body of the fern plant or sporophyte springing from the fertilized egg. Therefore we look upon the fertilized egg as the one-celled stage of the sporophyte.

When the moss spore (Fig. I 58 , $n$ ) germinates, a multicellular body is produced (protonema, $p$ ) from which springs the moss plant $(g)$ bearing antheridia $(r)$ and archegonia $(s)$ at its summit. Evidently, therefore, the entire body resulting from the germination of the spore is the gametophyte. The egg after fertilization produces the slender stalk $(l)$ and capsule $(m)$, namely, all of the unshaded part of Fig. I $58, B$, which must be the sporophyte, since it is produced in the same manner (namely, from the fertilized egg within the archegonium) and has the same position in the life cycle as has the fern plant in the life cycle of the fern. It is true that the stalk and capsule appear to be an organic part of the moss plant, but from the evidence before us we must conclude that 


\section{Mosses, Ferns, and Horsetails.}

they are neither more nor less than the sporophyte parasitic upon the gametophyte.

We see that in proceeding from the moss to the fern, or from a lower to a higher type of vegetation, the gametophyte becomes much reduced in size and complexity of structure, while the sporophyte relatively advances in these respects (compare shaded and unshaded portions of Figs. I 57-1 58). Turning now to the Spermatophytes or flowering plants, the most complex and specialized of plants, and the latest product of plant evolution, we find two kinds of spores (read paragraph 142, page 20I), the pollen spores and the embryo sac spore within the ovule. The fact that the embryo sac is a spore is

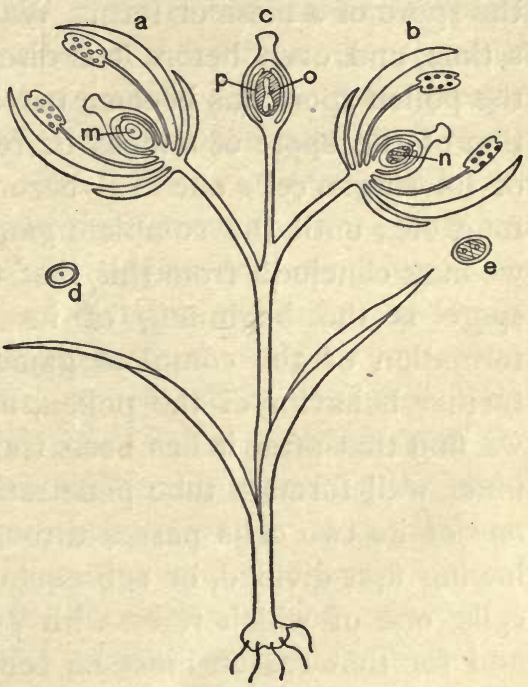

FIG. 159.

Diagrams of the sporophyte and gametophytes of a flowering plant. The pollen spore $(d)$ produced asexually on the plant (sporophyte) is the beginning of the male gametophyte, and the embryo sac spore $m$, also produced asexually on the sporophyte, is the beginning of the female gametophyte. All of both gametophytes ( $e$ and $n$ ) produced by the germination of both kinds of spores is shaded; all of the sporophyte is left unshaded. There is no differentiated archegonium and antheridium on the gametophytes. $b$ and $c$, later stages than $a$. The young sporophyte or embryo (o) has sprung from the fertilized egg. At $p$ is the endosperm.

not so evident from its appearance as from its behavior.

In tracing the life cycle of a fern and a moss we began with the germination of the spores, and we shall begin at 
the same place in the life cycle of a flowering plant. The pollen spore consists of a single cell (Fig. 159, $d$ ), as does the spore of a moss or fern. We find, however, that after a time, and even before it is discharged from the anther, the pollen spore has become two-celled $(e)$. The germination of the spore of a moss or fern began by the division of its single cell, one cell becoming two, two becoming four, etc., until the complete gametophyte is formed; and we may conclude from this that the division of the pollen spore is the beginning of its germination and of the formation of the complete gametophyte. Following the further behavior of the pollen, as described on page 166 , we find that after it has been transferred to the stigma its inner wall forms a tube penetrating to the ovule, and that one of its two cells passes through the tube to the ovule, having first divided, or subsequently dividing, to form two cells, one of which fuses with and fertilizes the egg cell, and for that reason must be considered a sperm. From this we are led to the conclusion that the three cells resulting from the germination of the pollen spore constitute a gametophyte. The great elongation of the inner wall of the pollen spore producing the pollen tube does not have its counterpart in the germinating spores of ferns and mosses. The pollen tube functions in part as an antheridium, although being apparently not its homologue.

The behavior of the embryo sac spore will now be traced. It is a single large cell $(m)$ which never becomes discharged from the place of its formation within the ovule. Usually before the descent of the pollen tube its nucleus divides and the daughter nuclei continue the process of division until eight nuclei have been formed, four of them taking position at the micropylar and four at the opposite end of the embryo sac $(n)$. One from each 
of these groups then moves toward the center of the embryo sac (see Fig. 88), and the two fuse, forming one, which by repeated division gives rise to the endosperm tissue of the seed. A plasma membrane is organized about each of the remaining nuclei of the two groups, including with each some of the cytoplasm of the embryo sac, thus forming definite cells. When a sperm cell leaves the pollen tube, it is found to penetrate and fuse with one of the three cells at the micropylar end, resulting in its fertilization and subsequent division and ultimate formation of the embryo $(o)$ within the seed. We may conclude from this that the cell with which the sperm fused is the egg, and that it, and the other cells resulting from the germination of the embryo sac spore, including the endo$\operatorname{sperm}(p)$, constitute a gametophyte, which has no differentiated archegonium. As has been said, the division of the fertilized egg results in the formation of the embryo $(o)$. This is an early stage of a new sporophyte, and we may say that, just as in the case of the ferns and mosses, the fertilized egg marks the close of the gametophyte part and the beginning of the sporophyte part of the life cycle of Spermatophytes. The sporophyte part of the life cycle continues through the germination of the seed and on through the growth of the plant up to the formation of pollen spore and embryo sac spore, which marks the beginning of the gametophyte part of the life cycle.

We see that the flowering plants have two sorts of gametophytes, - male, produced by the germination of the pollen spore, and female, formed by the germination of the embryo sac spore. The same thing is true of the Selaginellas (standing in the scale of evolution between the ferns and flowering plants), in which the microspores produce male, and the macrospores female, gametophytes (see page 202). 
The fact that an alternation of sporophyte and gametophyte occurs in the life cycle of plants from mosses to flowering plants, that homologous memibers can be traced, and that there is a definite law of progression, - namely, an increase in the sporophyte and a decrease in the gametophyte (compare shaded and unshaded portions of Figs. I 57, I 58, I 59), - affords important evidence of a common origin of these different classes of plants, fitting in with geological evidence pointing to the same conclusion.

\section{HORSETAILS.}

182. Character of Horsetails. - Horsetails, or Equisetums, are interesting not only because of their peculiar form, but because they are the sole representatives of a large and ancient group whose fossils are much in evidence in the coal measures. Like the ferns, they attain in the tropics a much greater size than we are accustomed to see in the temperate zones. The cylindrical, hollow, green stem bears at each node a whorl of leaves, which are united into a tube at their bases. The leaves are much reduced in size and have lost the normal function of leaves, the photosynthetic function being performed by the stems, whose exterior cells are well supplied with chloroplasts, and whose epidermis is perforated with numerous stomata. The surface of the stem is encrusted with silica, which gives protection against mechanical injuries. The plants occur in clumps, or large masses, on banks and in low places near streams or pools, their gregarious habit being largely due to running underground stems.

183. Reproduction of Horsetails. - Asexual spores are produced at the apices of the stems in sporangia which are borne on the under side of umbrella-like leaves, termed sporophylls, aggregated in the form of a cone (Fig. I60). 
The sporangia split open after the manner of anthers and the spores are readily shaken out by the wind. The spore is peculiar in having its outer coat split into four ribbon-like bands which coil and uncoil with the varying humidity of the atmosphere. On drying, the bands uncoil, and on imbibing moisture they coil up again. Perhaps the coiled bands, when they come in contact with a suitable object, may serve to anchor the spores in a moist place where the conditions for germination are good; or in dry situations, where

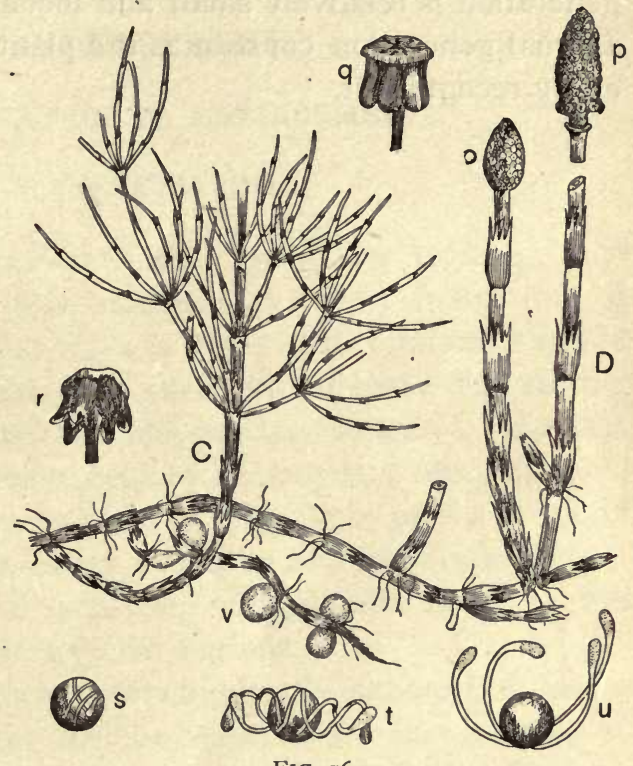

FIG. I6o.

Equisetum arvense. $C$, a sterile shoot; $D$, fertile shoots; $o$ and $p$, clusters of sporangia; $q$ and $r$, sporophylls bearing sporangia; $s, t$, and $u$, spores, with spiral bands uncoiled at $t$ and $u ; v$, tubers on the underground stem. After Wossidlo.

the bands are spread out, they may serve as sails to catch the wind so that the spores may be carried to more favorable situations. The bands may also prove useful in binding several spores together; for since the spores on germinating give rise to prothallia bearing only eggs or sperms, the prothallia being diœcious, it would be obviously in the interest of the fertilization 
of the eggs for several spores to germinate in close proximity.

As in the case of the ferns, the prothallium or sexual generation is relatively small and inconspicuous, while the asexual generation constitutes the plant proper as we generally recognize it. 


\section{CHAPTER XIV.}

\section{ADAPTATION TO ENVIRONMENT.}

\section{OBSERVATIONS.}

182. Examine plants which grow in dry regions; note whether much or little branched, the form and texture of the leaves. In what ways is rapid transpiration guarded against? Are there any parts which serve for storing water? Where are the chloroplasts located? If these plants were removed to regions of abundant rainfall, would they have any advantage over the plants of that region? Can you see wherein they would be at a disadvantage?

183. Compare, if possible, with plants native to salt marshes or to alpine or polar regions.

I84. Examine plants growing partly submerged in the water, such as water lilies or Sagittarias. In what way do you find them better adapted to living in water than are plants which grow on the land? Would they be at any disadvantage on the land? If so, in what ways?

I85. Make cross sections of the stems and underground parts of water lilies and Sagittarias, and note how the air is given access to the submerged parts.

186. Examine plants such as Lichens, Pleurococcus, etc., growing on the bark of trees. Do they at times become quite dry? Have they special devices to keep them from drying up? Pour water over some of these plants which have become dry. Do they quickly absorb it? How are they adapted for the rapid absorption of water? 
187. Weigh a piece of dry Spanish moss. Dip it into water for a moment and then shake and filter away the water which clings to its surface, and weigh again. Does it absorb water quickly enough to profit much by the rains which fall upon it?

\section{DISCUSSION.}

184. Advantages of Complex Structure. - Under normal conditions of soil and climate, the complex body which we find in the higher plants is better adapted to utilize materials and forces than the plants of simpler construction, such as mosses and Algæ. This is due to the fact that the differentiation of the plant body into distinct kinds of tissues has permitted division of labor, so that one part of the plant may rise into the free atmosphere and light, and obtain to best advantage what they have to offer, while another part may burrow into the soil, and draw from its reservoirs of water and mineral substances. The aboveground parts of such plants are protected against too great transpiration by waterproof outer layers of cells in the form of epidermal or cork tissues. They are equipped to withstand the stress of storms by strong wood and bast tissues; and the relatively long distances between the aërial and subterranean supplies of food are connected by suitable systems for transportation (see chapters on Roots, Stems, and Leaves).

185. Result of Unfit Environment. - We see at onae how well adapted these plants are to the position in which we find them; but if they were submerged in water they would die. The waterproof tissues on their exterior which are so beneficial to them in their natural habitat would prevent the relatively small amount of oxygen dissolved in the water from being taken up in sufficient quantities to 
support the respiration necessary to the life of the protoplasts, and the plants would drown.

But beside these dying plants others, native to the water, would be thriving, well able to obtain their necessary oxygen from the relatively small amount in the water, because their bodies (in the case of Ceratophyllum and Myriophyllum, for instance) are divided into slender segments, which, like the gills of a fish, expose a large surface for absorption; and because the very slight amount or total absence of waterproofing on their surfaces permits a ready interchange of gases.

If, however, we remove the water plant and place it on the land, it also soon dies; but now not for lack of oxygen, for it can obtain more of this than before, but for lack of sufficient water, since the plan of its construction which adapted it to the water now allows it quickly to dry up. It is clear from these illustrations that if a plant is to succeed in life, or if it is to live at all, it must be adapted by its form and construction to the habitat in which it finds itself.

186. Kinds of Habitats. - We find some plants living in the water, others on the land, that is, with roots in the soil and shoots in the air; and still others entirely in the air without connection with the soil, such as some tropical orchids. If we examine any one of these classes of habitats, we find that it offers wide ranges of variation. The water may be deep or shallow, still or running, hot, as in hot springs, or cold, fresh or salt, turbid or clear. The soil may vary greatly as to its chemical composition or physical condition; it may be clayey, loamy, sandy, gravelly, rocky, etc. ; it may contain little or much of common salt; it may be warm or cold, depending in part on the foregoing conditions. The air may be relatively quiet 
or boisterous, warm or cold, humid or dry, foggy or clear, and rare or dense, depending on elevation.

Also in considering the environmental conditions to which plants must become adapted we must take account of the maximum, minimum, and average temperatures for the year; maximum, minimum, and average rainfall; time of greatest rainfall, whether during the growing season or during the period of vegetative rest; the direction of the prevailing winds and their maximum and average forces; and of the intensity and quality of the light as it varies from low to high elevations, and from the equator to the poles. Indeed, the conditions appear to be too numerous and varied for us to assign to each its proper value; but plants feel the influence of each factor and accommodate themselves to it. We shall here discuss simply the main factors with which plants have to deal, - namely, water supply, light, temperature, atmosphere, soil, and relation to animals and to other plants.

187. Water Supply. - Variations in the water supply are more effective in requiring adaptive changes in the forms and construction of plants than variations in any other factors (see pages 37 and I Io, for a discussion of the value of water to plants). When there is plenty of water available in the soil, but not to the extent of saturation, we find plants much branched above and below ground, with a large expanse of leaf surface; the stomata occur frequently on both sides of the leaf, but the greater number usually occur on the under side. The great majority of plants in regions having a temperate climate and abundant rainfall are of this character.

188. Effect of Scarcity of Water. - A scarcity of water necessitates certain profound changes from this type which are designed to reduce transpiration; for if plants are un- 
able to absorb much water, it would be fatal to them to permit rapid transpiration. Accordingly we find under these circumstances a diminution in the transpiring surface by a reduction in the number and extent of the branches and leaves, as in the case of cacti, for example. There is also usually an increase in the thickness and waterproofing qualities of the outer wall of the epidermis, a restriction of the stomata to the under sides of the leaves, a depression of the stomata below the surface of the leaf, as in the leaves of the India rubber tree, a reduction in the size of the intercellular spaces, and frequently the aboveground parts are covered with hairs, scales, etc., which retard transpiration and reduce the intensity of illumination. There is also frequently an occurrence of specialized cells and tissues for the storage of water, and of cells containing mucilage, which assists in retaining water within the plant.

189. Conditions affecting Absorption. - There are certain conditions under which plants absorb water with difficulty even when it is present in abundance. Thus, if there is a large percentage of salts dissolved in the water, or a large amount of humic acid produced by the disintegration of organic remains, as in peat bogs; or if the soil and soil water are cold, plants absorb the water with great difficulty. Under such circumstances they must be modified to reduce transpiration just as if little water were present.

190. Effect of Submergence in Water. - We find quite another class of modifications in those plants which are rooted in earth saturated with water, such as the mud at the bottom of ponds, etc. In these plants there is not so much need of retarding transpiration, but access of air to the submerged parts must be provided for. Accordingly we find the stomata nearly all transferred to the upper side 
of floating leaves, such as those of the water lily, and a large increase in the extent of the intercellular spaces for the conduction of air from the leaves through the stems into the underground parts.

In plants entirely submerged in the water we usually find the stems and leaves very much branched for the purpose of exposing a large surface for the absorption of oxygen and carbon dioxide from the water, and at the same time there is a reduction in or an entire obliteration of the roots, since such plants throughout their whole surface can absorb whatever the water affords them. There is also a reduction in the amount of waterproofing, a diminution or disappearance of the stomata, and a decrease in the waterconducting elements.

191. Light. - Although light is quite as important as water for the life of the higher independent plants, the variations in its quality and intensity over the earth are not so great as to cause or to require the profound modifications which we find produced by variations in the water supply. It is a commonly observed fact that plants which sprout in cellars have long internodes and reduced leaves. Experiments have shown that if the intensity of the light be increased up to a certain point, the size of the leaves produced increases; but that passing above a certain degree of intensity of illumination the leaves attain a less and less size.

192. Effect of Dim Light. - Experiments with Scolopendrium officinarum showed that in the dark the leaves reached a length of 76 millimeters and a breadth of II millimeters (Fig. 16r, r), while in a light about one-eighth as intense as direct sunlight the leaves grew to be 228 millimeters long and 25 millimeters broad (2); and in a still stronger light having about one-third the intensity of full 
sunlight the leaves attained a size of 152 millimeters in length and 20 millimeters' breadth (3).

Sempervivum tectorum, which grows naturally in sunny situations, and bears fleshy leaves in rosettes near the ground, is considerably modified when grown in a light about one-sixth as strong as direct sunlight. Under this condition the internodes elongate so that the rosette grouping of the leaves is lost, the leaves formed are smaller, and the amount of chlorophyil is less.

We can see that some of the changes in habit here recorded are directly adapted to the variations in the intensity of illumination. In a very dim light it is advantageous to the plant to lengthen the internodes at the expense of all other parts, so that the leaves may more surely and quickly be brought into places of greater illumination. Another
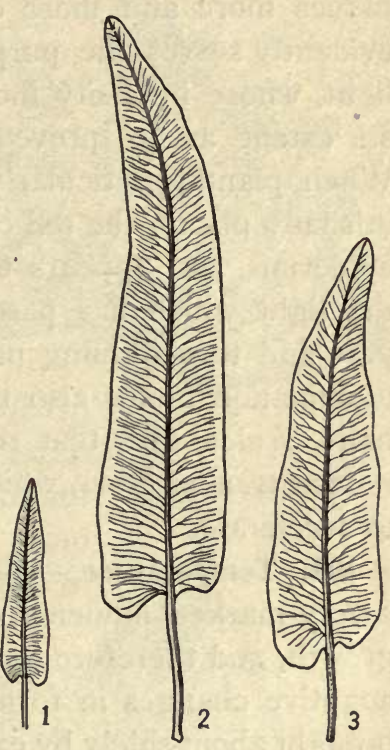

FiG. I6r.

Leaves of Scolopendrium officinarum. 1 , grown in the dark; 2 , grown in $1 / 8$ full sunlight; 3 , grown in $1 / 3$ full sunlight. directly adaptive effect due to variations in light intensity is the production of chloroplasts nearer the surface in dimly lighted situations. This is well illustrated by our wild Smilaxes, which grow in the shade of woods, and have chloroplasts in the cells of the epidermis where they can absorb the dim light to best advantage.

193. Effect of Intense Illumination. - Plants may adapt themselves to varying degrees of illumination in still 
another way. Plants of the same species growing at progressively higher elevations on the mountains may produce in the cells of the epidermis and deeper-lying tissues more and more of a red coloring matter, which evidently serves the purpose of cutting off a part of the light, whose intensity increases with the altitude to such an extent as to prove destructive to the chlorophyll. When plants, particularly those of prostrate habit, grow in shady places, the red color is apt to occur in the lower epidermis, and appears to serve the purpose of arresting the light which has passed the upper tissues, reflecting part and transforming part into energy of heat. Intense illumination seems also to give rise to the production of hairs, scales, etc., that reflect part of the light, and also reduce transpiration, which the intense illumination tends to accelerate.

194. Temperature. - Variations in temperature may exert a very marked influence on the processes of nutrition and growth, and therefore on the size of plants; but no visible adaptive changes in form and structure are known to be brought about solely by exposure to different temperatures. On the contrary it frequently happens that the plants of the polar regions or cold mountain peaks have the same general appearance and construction as those of hot deserts. But although different degrees of heat may not give rise to adaptive changes in form, they do produce very radical adaptive alterations in the qualities of the protoplasts, for it is well known that plants of tropical and temperate regions would quickly succumb to the low temperatures which plants of polar regions are known to endure.

195. Resistance to Cold. - The plants of the Siberian forests, for example, withstand temperatures as low as $60^{\circ} \mathrm{C}$. $\left(76^{\circ} \mathrm{F}\right.$.) below zero. Kjellman, the botanist of the Vega 
Expedition, relates the following remarkable instance of resistance to cold by Cochlearia fenestrata, a member of the mustard family, shown in its natural size by Fig. I62.

The cold was very persistent, and often fell lower than $46^{\circ}$ below zero, Centigrade. The plant in question grew upon the summit of a rather high sand hill near Pitlekai,

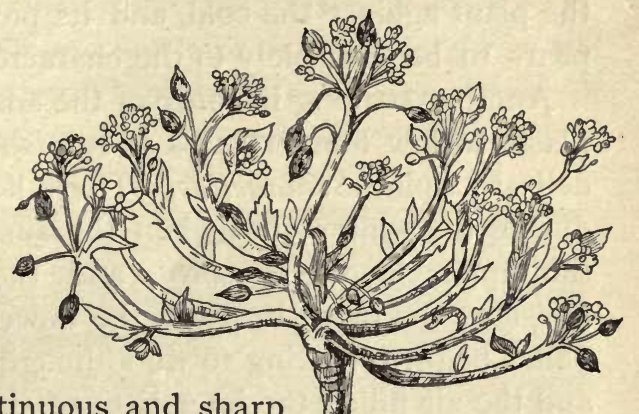
exposed to the continuous and sharp north and northeast winds. It began to bloom in the summer of 1878 , but its full quota of flowers was far from complete when winter arrived and stopped its further development, the inflorescence then consisting of flower buds in different stages of development, newly opened flowers, faded flowers, and more or less ripened fruit. There were only a few shriveled remains of the basal rosette of leaves, but the upper leaves were alive and fresh. In this

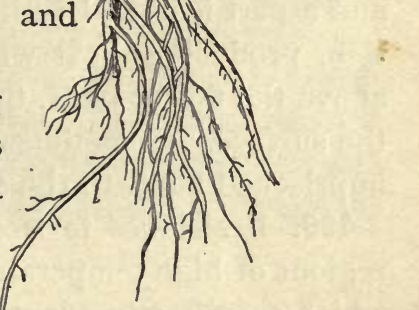

FIG. I62.

Cochlearia fenestrata, natural size. After KJELlmaN. condition the plant was overtaken by winter and exposed to its full severity. One might expect it to be destroyed under such circumstances, but this was not the case; for when the summer of 1879 began, the plant resumed its development where it left off at the beginning of winter: the flower buds unfolded and 
new inflorescences arose in the axils of the fresh upper leaves.

In this instance, there is no special device to protect the plant against the cold, and its power of resistance appears to be due solely to the character of the protoplasts.

Another striking instance of the adaptation of the protoplasts to low temperatures is furnished by the small and delicate alpine Soldanellas. These lie buried in the snow during the winter; but when the summer sun first melts the upper layers of snow, and the percolating water moistens the earth beneath, the flower buds of the Soldanellas begin to spring forth, although the earth and water and the air filling the interstices of the snow must be near the freezing point. As growth continues, a part of the reserve materials stored in the prostrate leaves and subterranean rootstock is used in the formation of new tissues, and a part is united with oxygen in the process of respiration, producing heat which melts the snow immediately above the flower buds, thus enabling them to unfold above the surface. Sometimes the buds blossom out while still imprisoned beneath the snow (Fig. 163).

196. Resistance to Heat. - The plants of deserts and regions of high temperatures show no modifications of form which are directly adaptive to heat alone, but they are able to withstand very high temperatures because of the nature of their protoplasts. At Lahore and Multan, India, the maximum temperatures are respectively $50.9^{\circ}$ and $52.8^{\circ} \mathrm{C}$. $\left(123.6^{\circ}\right.$ and $127^{\circ} \mathrm{F}$.), and in the sun probably from $63^{\circ}$ to $70^{\circ} \mathrm{C}$. $\left(145.4^{\circ}\right.$ to $158^{\circ} \mathrm{F}$.) ; and the plants of those regions have of course become adapted to these extremes. At some places near the equator the soil temperature is known to have risen to $80^{\circ} \mathrm{C}$. $\left(176^{\circ} \mathrm{F}\right.$.), and in one instance even to $84^{\circ} \mathrm{C}$. $\left(183.2^{\circ} \mathrm{F}\right.$.). A species of Ipomoea has been 
found in bloom where the surrounding soil had a temperature of $69^{\circ} \mathrm{C}$. $\left(156.2^{\circ} \mathrm{F}\right.$.).

Regarding the adaptation of plants to various degrees of heat, the general statement may be made that nowhere on the earth is it too cold and nowhere too hot (except at the mouths of volcanoes, etc.) for plants to live and grow, in virtue of the adaptive capacity of their protoplasts.

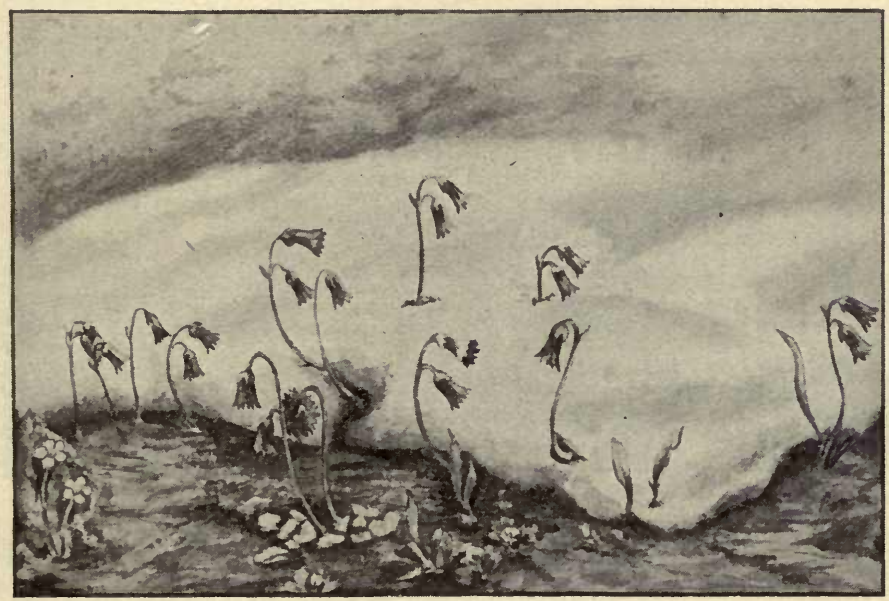

FIG. I63.

Alpine Soldanellas growing through the snow. After Kerner.

197. The Atmosphere. - Variations in the pressure of the atmosphere, so far as artificial experiments teach us, bring about marked adaptive modifications. As the pressure is diminished, the rate of growth increases, more numerous branches are produced, and the leaves are larger. These changes apparently bear a direct relation to the diminished amount of oxygen; for just as animals must breathe faster at high elevations where the atmosphere is 
rare, so plants may be expected to have need under like circumstances of increasing their surfaces for the more rapid absorption of oxygen. But in nature, at high elevations, there are many circumstances which must be taken into account by plants. The rarefaction of the atmosphere increases illumination, radiation, and transpiration, and these circumstances tend to modify the influence of decreased pressure.

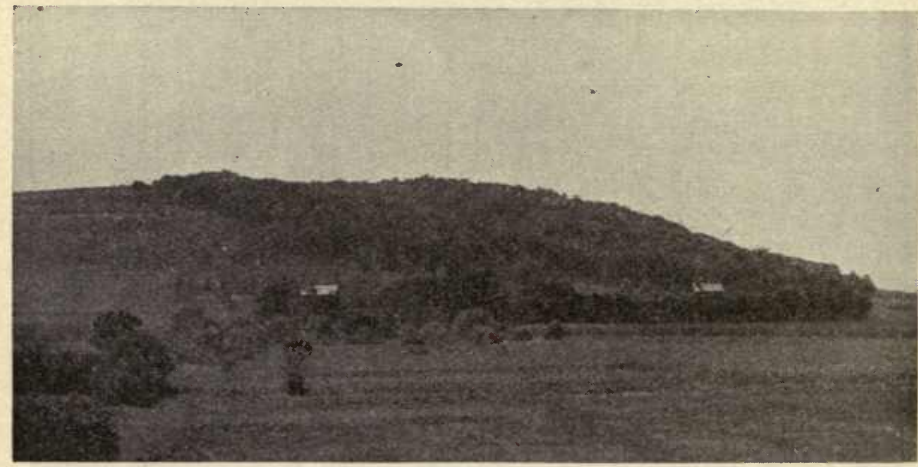

FIG. 164 .

Showing the north slope (on the right) of a hill densely wooded, while the south slope (on the left), which is exposed to the drying summer winds, is destitute of trees.

198. Effect of Winds. - The wind has much to do in determining the kinds of plants which shall grow in a windswept locality, and the wind also brings about adaptive changes by causing a diminished growth of the plant as a whole, and notably of the leaves; but these modifications are probably brought about indirectly through the increase in transpiration caused by winds. Instances are common where the windward side of a hill is nearly or quite destitute of trees, being clothed merely by low-growing herbs, grasses, and shrubs (Fig. 164). 
We have already seen in the chapters on Flowers and Dispersion of Seeds how adaptive modifications have enabled plants to employ the wind in the scattering of pollen and seeds. Plants with these modifications are more apt to abound in windy situations. .

199. The Soil. - The different kinds of soils affect the form and structure of plants chiefly in the capacity of soils as water reservoirs. Coarse-grained soils with large interstices allow the water to percolate readily through them, and retain but little for the use of plants. Accordingly, plants which inhabit such soils must be modified so as to reduce transpiration and to store water. On the other hand, soils which are fine-grained, and particularly such as are rich in clay or humus, hold large percentages of water, and the plants growing in them are not so apt to possess special devices to restrict transpiration.

200. Effect of Excess of Salts. - If large percentages of soluble salts occur in the soil, they influence the osmotic conditions and render the absorption of water by the roots more difficult; and it may happen in such cases that even with plenty of water at hand plants may be in danger of drying up. The same sorts of adaptive modifications, designed to retard transpiration, as we find occurring when the soil is dry, are accordingly brought about.

201. Other Plants and Animals. - In the preceding chapters, numerous illustrations of modifications which are adaptive to animals and to other plants have already been discussed. The student may refer to the modifications of leaves to serve as traps, of stems, roots, and leaves for purposes of climbing, of flowers, fruits, and seeds, for the dispersion of pollen and seeds by means of insects and other animals. The discussion of parasitic roots in Chapter III, and of Fungi and Lichens in Chapter XII, has called 


\section{Introduction to Botany.}

attention to parasitic habits of life; and now an instance of another character will be given to illustrate the sharp competition which is so common among plants.

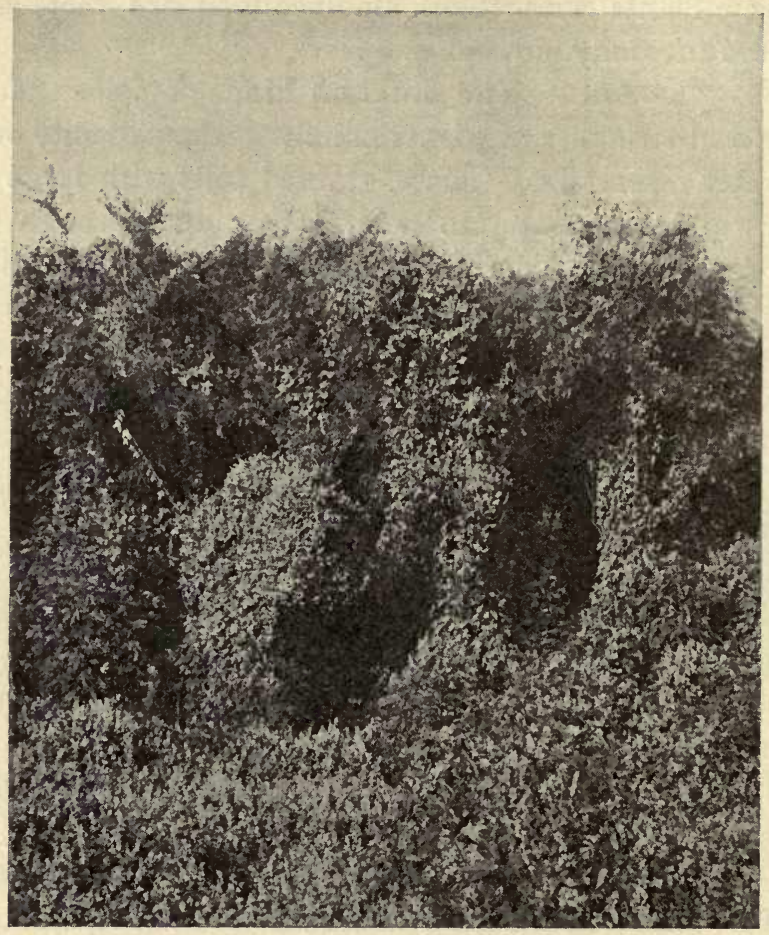

FIG. 165 .

Photograph of Sicyos angulatus clambering over undershrubs in the foreground and over willow trees in the background. The plant is bedecked with its clusters of white flowers.

The one-seeded bur cucumber, Sicyos angulatus, has had certain of its branches modified in the form of tendrils which are so sensitive to contact that when they touch the branches of other plants they twine about them, drawing 
the coils tight to obtain a firm hold. In this way this plant is enabled to reach the tops of trees and spread itself out over their crowns; or it may cover the tops of thickets of undershrubs, greatly to its own advantage in obtaining light. The tendrils are so quick to take advantage of any suitable object of support that within a few minutes after they have perceived its presence by contact with it they may have made at least one turn about it. Figure 165 shows undershrubs in the foreground, and willows in the background, almost completely hidden by the foliage and white flowers of this plant, demonstrating how efficiently it has employed its method of using other plants as supports.

202. Protective Adaptations. - Factors in the environment which are a source of danger to plants have resulted in adaptive modifications of various sorts. Not infrequently branches and leaves become modified to form spines which may serve as a means of protection against marauding animals. In desert plants, such as the cacti, whose succulent tissues are greedily sought by animals, these modes of protection are of vital importance. Still other plants find protection in bitter and poisonous secretions.

One of the most wonderful devices to secure protection against the destruction of leaves by leaf-cutting ants is found in the Brazilian tree, Cecropia adenopus. When one shakes the tree, an army of ants pours forth from small openings in the branches and makes a vicious attack on the intruder. These are the most warlike of known ants, and their bite is very painful. Their chief service to the tree is the prevention of the depredations of leaf-cutting ants, which abound in these regions and are wont to strip the leaves of unprotected trees clean to the midrib, carry- 
ing the pieces to their nests for the purpose of working them into a pulp which they plant with a certain kind of fungus, constituting their chief or sole food. Figure 166

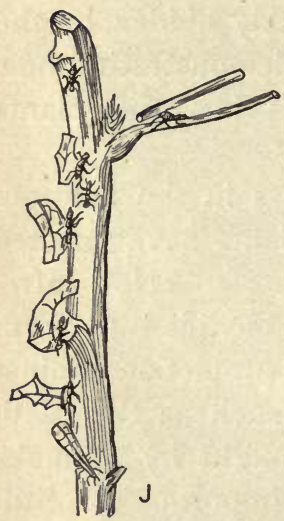

FIG. I66.

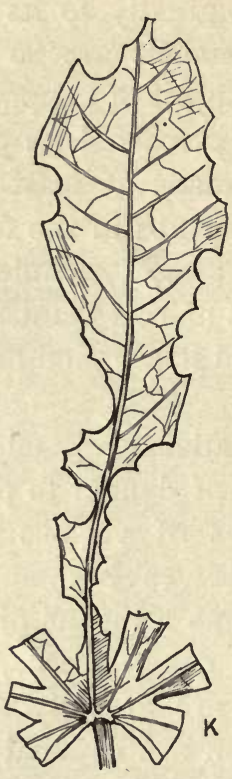

shows the way in which these ants destroy the leaves.

The soldier ants that protect the Cecropias against the leaf-cutting species do not, however, serve without pay, for these trees provide them with both food and shelter. The $C_{e}$ cropia branches are hollow at the center, the cavity being divided by numerous cross partitions (Fig 167, $A$ ). Just above the axils of the leaves are thin places (Fig. $167 B, a$ ) in the stem where the tissues are lacking in woody

elements and are easily

F, leaf-cutting ants walking off with their plunder; $K$, a leaf showing damage from leaf-cutting ants. After A. MÖLLER. cut through by the ants. The ants also find no difficulty in perforating the cross partitions so that they can pass from chamber to chamber. The food provided for the ants is produced at the bases and under sides of the petioles in the form of minute egg-shaped bodies, rich in proteids and fats. These bodies are renewed as often as the ants remove and carry them to their nests (see Fig. 168). 
The adaptive modifications of the Cecropia having a bearing solely on the problem of protection, are the thin places through which the ants burrow an entrance, and the never failing supply of food at the bases of the petioles. The hollow stem is found in many plants, and is the outcome of economy of materials in stem construction.

203. Most Potent EnvironmentalFactors. - Instances showing adaptations to special conditions might be cited at great length, but the few cases already given suffice to show the power of plants to meet such conditions. It has already been suggested that of all the factors to which plants must accommodate themselves, the water supply is the most potent in molding their form and visible constitution. Indeed, so great has this influence been that

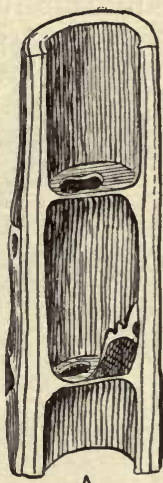

A

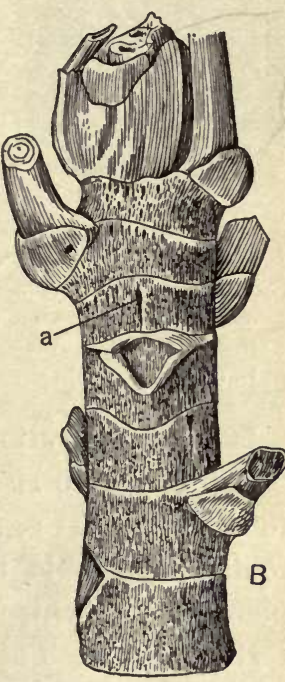

FIG. I67.

$A$, longitudinal section through a portion of a young stem of Cecropia adenopus, showing partitions cut through by ants; $B$, apex of a young stem of Cecropia, showing at $a$ a thin place through which the ants cut a way to the hollow interior. About one-half natural size. After SCHIMPER.

those plants which have to guard against loss of water by transpiration are very different in appearance from those which find plenty of water at their disposal and may transpire it unstintingly without danger to themselves. The former class of plants are termed xerophytes, or if growing in a salty substratum they are termed halophytes (see paragraph 208), while plants of the latter class are called hydrophytes. 


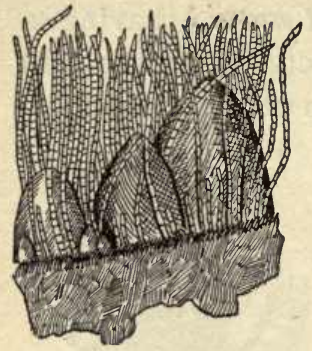

FIG. I68.

Food bodies of Cecropia adenopus, somewhat magnified. After SCHIMPER.

Plants which grow under ordinary conditions of moderately moist soil and average humidity of atmosphere, and are not subjected to prolonged drought during the growing season, are termed mesophytes. These have less pronounced characteristics of the hydrophytes. Most of the mesophytes cast off their leaves at the close of the growing season, and many of them die back to the ground and survive as bulbs, tubers, etc. Such mesophytes are also termed tropophytes.

204. Xerophytic Conditions. - The following physical conditions give rise to xerophytes: A dry soil, such as is found in desert regions; a low temperature of the soil, the roots being able to abstract but little water, even when plenty is present, from soils whose temperature approaches zero; soils, such as those of peat bogs, abounding in humic acids, these acids in some way diminishing the absorptive power of roots.

205. Halophytic Conditions. - The halophytes have been evolved in soils whose water contains more than $0.5 \%$ of salts in solution, as is the case along seacoasts, in salt marshes, and in the dry beds of ancient salt waters. A low relative humidity, a high temperature, and a low pressure of the atmosphere all hasten transpiration, and assist in the evolution of xerophytic and halophytic characters.

206. Hydrophytic Conditions. - The hydrophytes have been produced under conditions opposite to those above enumerated; namely, in soils abundantly provided with water even to saturation and submergence, of sufficiently high temperature to enable the roots to absorb the water 


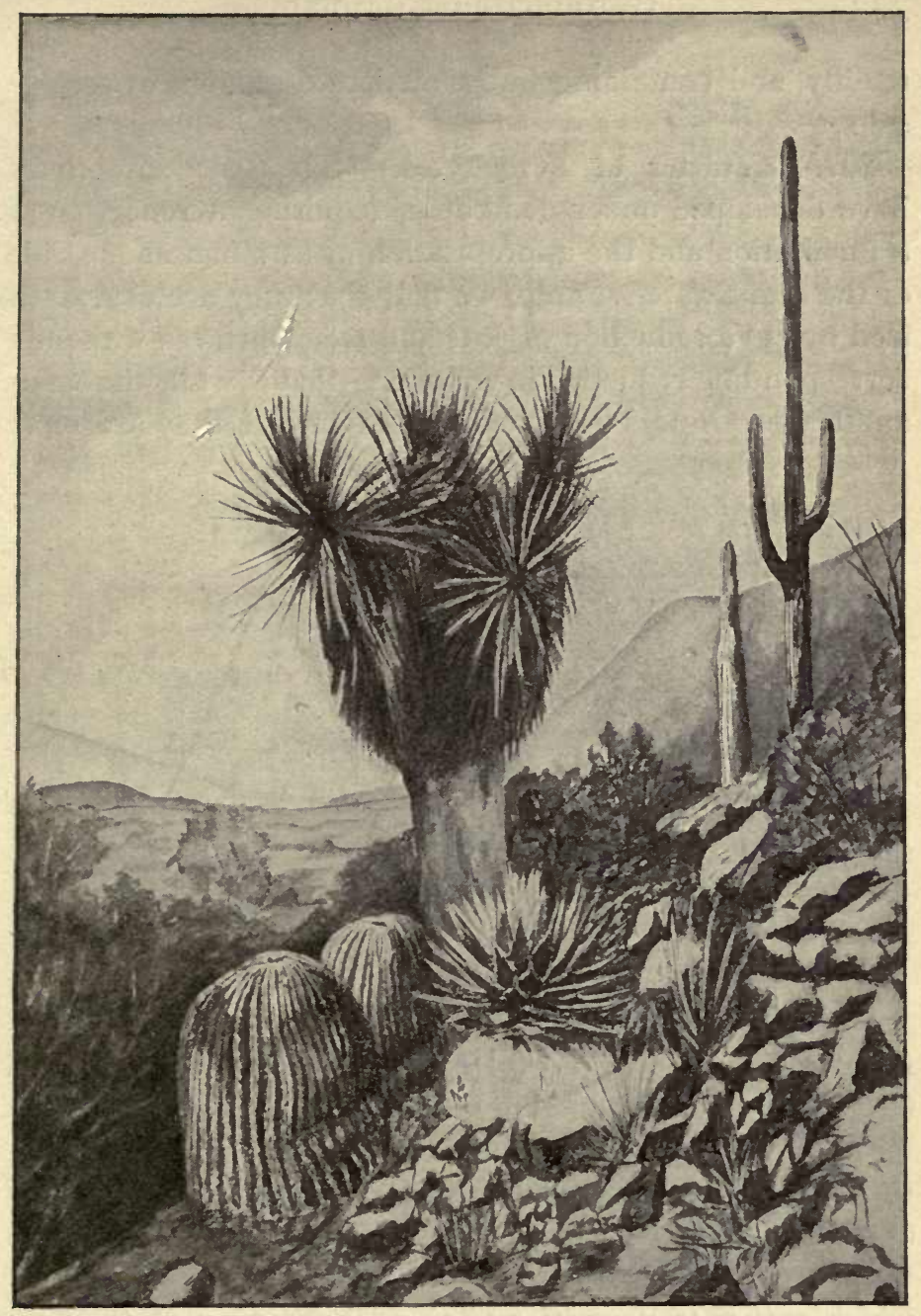

FIG. I69.

North American Xerophytes. In the foreground Cereus ingens (dome-shaped); to the right of this Agave; back of these an arboreus Yucca; in the background to the right, Cereus giganteus. 
readily, and containing but a moderate amount of soluble salts.

207. Character of Xerophytes. - Since the xerophytes have developed under conditions requiring a reduction of transpiration and the appropriation of as much as possible of the scant water supply, we find that they are characterized by (I) a reduction of leaf and stem surfaces without a corresponding reduction in volume, - that is, while the stems and leaves are less branched and expanded, they are more

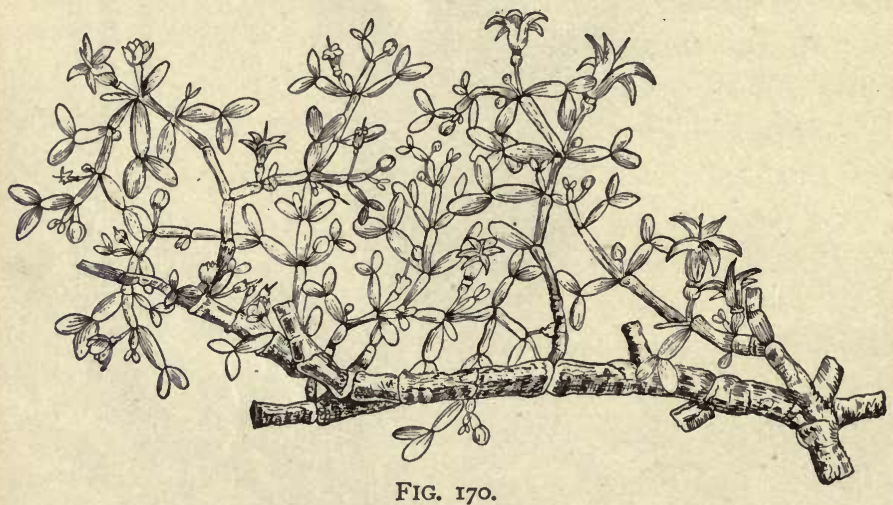

Zygophyllum cornutum from the Algerian desert. After ENGLER.

fleshy, as in Cactus, Portulacca, and Mesembryanthemum (Fig. 169); (2) a reduction of intercellular spaces so that more room may be obtained for the storage of water, the transpiring surfaces being reduced at the same time; (3) increase in tissues which absorb, conduct, and store water; (4) frequently a covering of hairs, scales, etc., which reduce transpiration; (5) a lengthening of the palisade cells; (6) depression of the stomata beneath the surface, and (7) the production of mucilage which assists in retaining the water within the plant. 
Adaptation to Environment.

208. Character of Halophytes. - The halophytes have essentially the same character as the xerophytes; for although they grow in soils abundantly supplied with water, their. roots absorb it with great difficulty.

Whatever be the cause of the difficulty to plants in obtaining water, the methods of adaptation to the unfavorable conditions for absorbing water are essentially the same, as will be seen by comparing Zygophyllum cormutum (Fig. I70), a plant from the Algerian desert, Batis maritima (Fig. I7I), growing on wet, salt, tropical beaches, and Cassiope te-

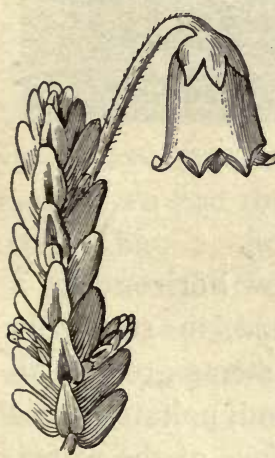

FIG. I72.

Cassiope tetragona, bearing small, leathery, in-rolled leaves; from Greenland. After WARMING.

tragona (Fig. I 72), growing in the cold soil of Greenland. In each of these plants

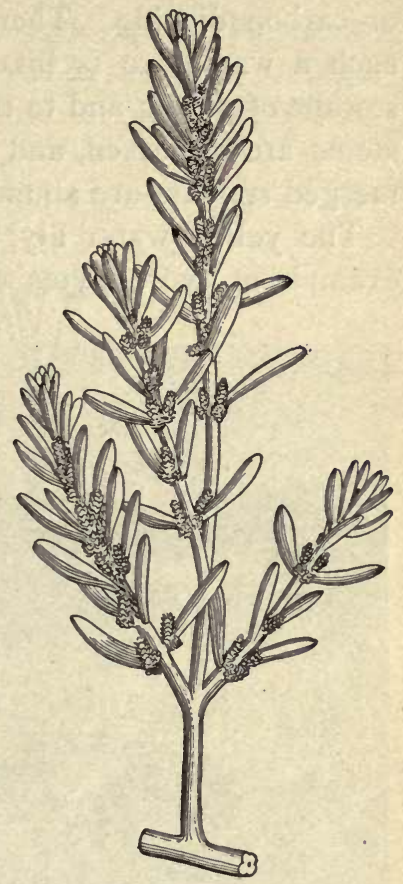

FIG. I7I.

Batis maritima. Halophyte from a tropical sea beach. After DAMMAR. the reduction of the transpiring surface is very marked.

209. Character of Hydrophytes. - The hydrophytes are abundantly supplied with water and do not need to provide special devices to guard against its loss. But because they are often in part, or wholly, submerged in water they are in danger of suffering from lack of sufficient oxygen 
or carbon dioxide. Therefore we find them modified in such a way as to facilitate the entrance, circulation, and storage of gases; and to this end the surfaces of leaves and stems are increased, and in the parts which are not submerged stomata are abundantly provided.

The yellow water lily, Nelumbo lutea, serves as a good example of hydrophytes which are partly submerged (Figs.

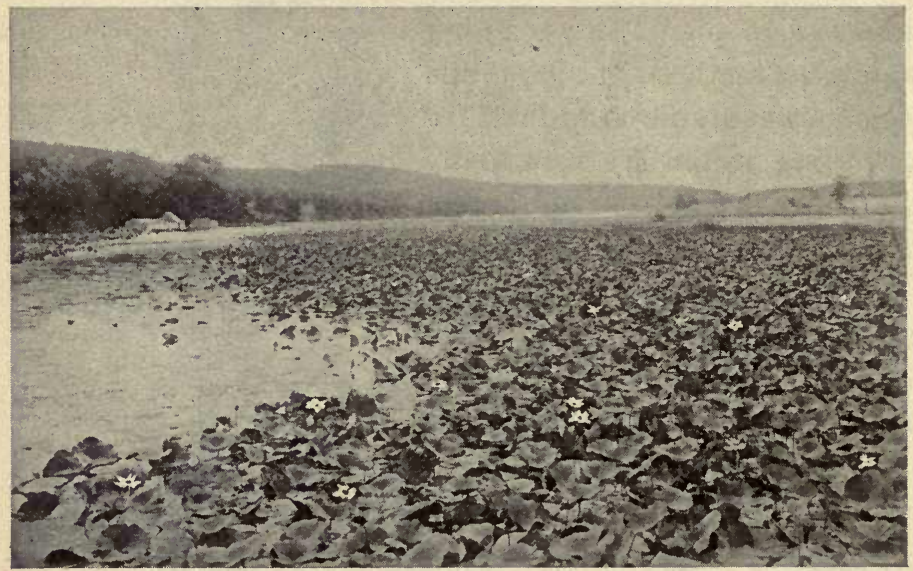

FIG. I73.

Nelumbo lutea growing in a lake.

69 and 173). Its underground stems grow horizontally a few inches below the surface of the mud, in relatively shallow and still water. Leaf and flower stems grow vertically from the horizontal stems. The round, peltate leaves which are first produced float on the surface of the water, while most of the later leaves rise for some distance above it, so that the light can pass under and strike those which are floating. The stomata are very numerous on the upper side of the leaf, but are found very rarely on the under 
side where they would become stopped up by the water, even in the case of the taller leaves in the time of high water. The upper surface of the leaf is coated with wax, so that the water which falls upon it rolls off without wetting it and stopping up the ways through the stomata. The intercellular spaces in the leaf are large and numerous and communicate with tubular spaces in the ribs of the leaf which are continuous with still larger. spaces in the petioles, horizontal stems, roots, and tubers. The submerged parts are thus in free communication with the atmosphere.

If we examine roots which have been carefully dug up, we find that they extend but a short distance beneath the mud; indeed, the water-conduct-

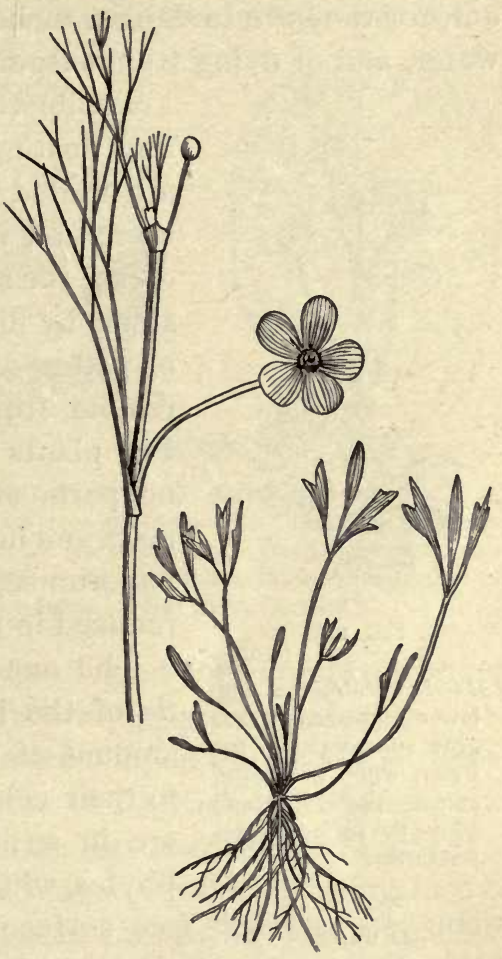

FIG. 174. ing and absorbing ele- Ranunculus fuitans. The lower figure shows ments throughout the the land form and the upper figure the water whole plant are very form, $2 / 3$ natural size. After SCHIMPER.

small compared with those found in xerophytes or even in mesophytes.

Thus we see that where water can be had without stint, no special provision is made for its absorption, conduction, 
or conservation. Plants in such situations can afford to be indifferent to water in their form and construction, but their attitude toward the atmosphere must be quite different, for they are in danger of being cut off from it by the water, and of dying from suspended respiration.

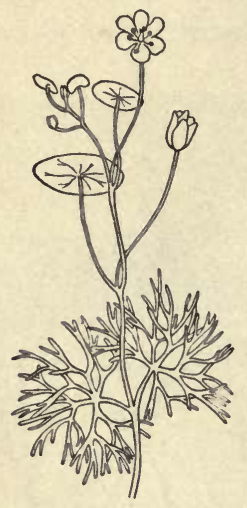

FIG. I75.

Cabomba Caroliniana. The lower dissected leaves grow submerged in the water, while the upper entire leaves extend above the surface. After BELZUNG.

Plants which are entirely submerged and floating in the water must absorb all needed substances from it. It is, therefore, necessary for them to increase the surfaces of their leaves and stems by dividing them into thin rods or plates, and to have no tissues very remote from the absorbing surfaces. For plants built after this plan, with no parts extending into the air, stomata and intercellular spaces lose their importance, and are lacking or much reduced in number and extent.

The one most general characteristic of the hydrophytes is their large amount of free surface in proportion to their volume. In this respect they are in striking contrast to the xerophytes, which frequently have no more than $\frac{1}{30} \overline{0}$ as much free surface for a given volume as the hydrophytes.

210. Origin of Xerophytes, Halophytes, and Hydrophytes. - The xerophytes, halophytes, and those hydrophytes which belong to the Phanerogams, ferns, and mosses, have probably descended from mesophytes which have been able to vary sufficiently to adapt themselves to permanent extremes of moisture and dryness; and so, migrating into habitats of one extreme or the other, they 
have been able to thrive away from the sharp competition which prevails among plants in more favorable situations.

It is not surprising to find that plants which have shown such great adaptability to environment as have the xerophytes and the hydrophytes, are able to undergo radical modifications in form and structure even in the lifetime of a single individual, if the conditions under which they
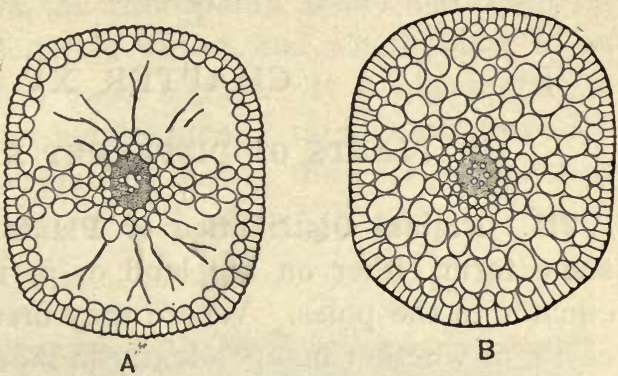

FIG. I76. are growing become much altered. Ranunculus fluitans, for instance, which has its leaves divided into fine filamentous segments when growing in the water, produces leaves with much broader divisions when growing on the land (Fig. 174). So, too, the submerged leaves of Cabomba Caroliniana are finely divided, while the floating leaves are entire (Fig. 175).

The stems of Callitriche stagnalis when growing in the water have intercellular spaces occupying about half of their volume, while the intercellular spaces of stems of the same species found growing on the land are relatively quite small (Fig. I76).

Having become aware of the ability of plants to undergo adaptive changes, we are prepared to understand in our study of the plants of different regions, and of past ages, how plants have been able to migrate from one latitude to another without suffering extinction. 


\section{CHAPTER XV.}

\section{PLANTS OF DIFFERENT REGIONS.}

211. Original Distribution of Plants. - Plants occur in some form, either on the land or in the water, from the equator to the poles. Where they first originated on the earth, or whether independently in more than one locality, is not known. It is known, however, that when they had advanced sufficiently in the course of their evolution to form tissues which could be preserved as fossils, they were fairly uniformly distributed throughout all latitudes, the earth down to Middle Tertiary time having a temperate climate, even within the arctic circle. After that time, snow and ice accumulated in and beyond the arctic circle, and extended far southward in the form of moving glaciers. Land plants had then to move southward by means of their seeds and spores, or become exterminated. When the ice again retreated toward the poles, only those exiled plants which were able to adapt themselves to extremes of temperatures moved northward. We accordingly find that the floras of the tropical, temperate, and frigid zones are dominated by different kinds of plants.

212. Factors governing Floras. - Within each zone there are factors, other than temperature; which sort the vegetation into groups of various characters. These factors are water supply, relative humidity of the atmosphere, winds, and nature of the soil. 
Whether the vegetation of a region shall consist largely of trees and shrubs, or of grasses and low herbs, depends for the most part upon the depth and amount of the soil water. Trees will predominate in regions where the vegetative period is warm, the atmosphere humid and comparatively quiet, particularly in winter, and where there is an unfailing subterranean water supply. It is unessential to trees when, and with what frequency, rains occur, provided they are of sufficient amount during the year to replenish the soil reservoirs.

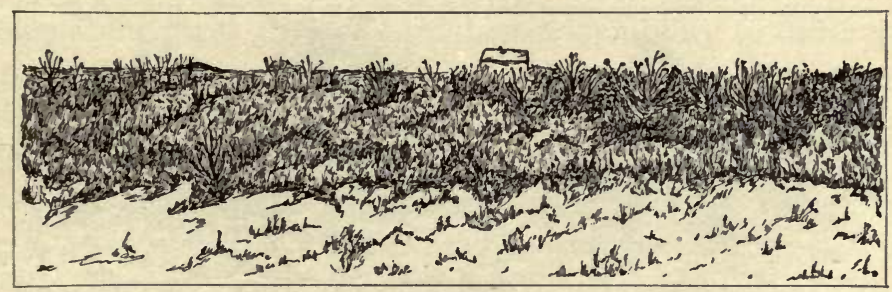

FIG. I77.

Drawing from a photograph of a neglected tree claim in western Kansas.

Since the roots of trees go deep, they can avail themselves of water supplies which are beyond the reach of grasses and other plants with shallow roots. The fact that the roots of trees extend to considerable depths enables them to become established along the banks of streams in regions where the annual rainfall is too scant to penetrate beyond the superficial layers of the soil. The water from streams percolates some distance beyond their beds, so that fringes of timber may follow water courses for hundreds of miles through prairie or even desert regions,

Trees may be made to grow where they would not naturally, by keeping the surface soil loose and mellow with the plow, so that rains may percolate to some depth 
and that the water thus caught may be retained by the mulch of loose soil at the surface. The success of tree claims in the semi-arid regions of the United States depended largely upon the degree of persistence in this mode of treatment. Figure $\mathrm{I} 77$ is a drawing from a photograph of a claim which was planted with trees and neglected, while Figure 178 shows the result of cultivation in the neighborhood of the neglected claim.

In regions where the rains occur frequently during the growing season, though not in sufficient amount to produce

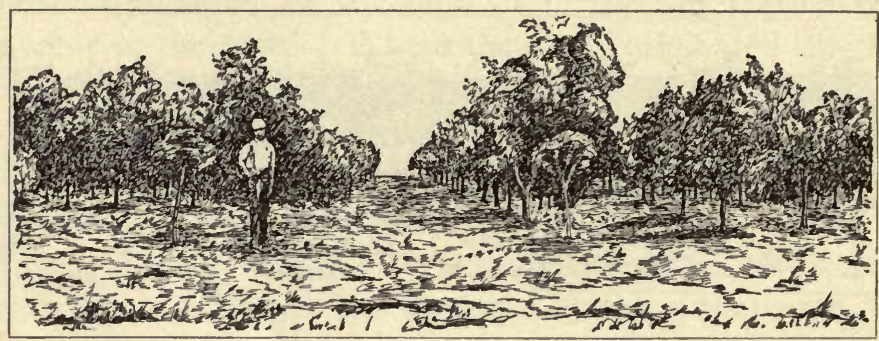

FIG. 178.

Drawing from a photograph of a tree claim in western Kansas that has been properly cultivated.

a perennial water supply in the deeper strata of the soil, grasses predominate, while trees, with the exception of those of xerophilous character, such as occur in savannas, are absent. The dry atmosphere and strong winds which are apt to occur in treeless regions are much less inimical to grasses than to trees, because the former keep close to the ground, where the adverse conditions are less pronounced.

The character of the soil may also impress its stamp upon the flora of a region. Thus, to take examples from a single genus, Enothera laciniata occurs in dry sandy soils, Enothera rhombipetala is a prairie plant, and Enothera Mis- 
souriensis abounds along the crests of limestone hills. Grasses, and other plants with creeping underground stems, are well adapted to take possession of the shifting soil of sand dunes where competition with other plants less suited to such situations is not sharp, and in this indirect way the unstable sandy soil gives a distinct character to its vegetation.

If all plants were able to accommodate themselves equally well to different kinds of climate and soils, we might expect to find them uniformly distributed, excepting where broad waters intercept their migration; but they show great diversity in their adaptability to different external factors, most of them being unable to traverse deserts or lofty mountains. Some are unable to maintain themselves where the annual precipitation falls below a certain amount, where the ranges of temperature pass certain maximum and minimum limits, or where the prevailing winds exceed a certain velocity. It is because of its bearing on this diverse capacity that the subject of plant distribution is one of high importance in the study of plant life.

213. Vegetation in the Tropics. - In the tropics the temperature is uniformly high, excepting in the mountains, averaging from $20^{\circ}$ to $28^{\circ} \mathrm{C}$. $\left(68^{\circ}\right.$ to $82.4^{\circ} \mathrm{F}$.) throughout the year. In general the tropics are regions of high annual rainfall (see rainfall map, Fig. I79), the precipitation along the coast and in the mountains amounting in some localities to 4 or 5 meters. The greater part of Central and South America has an annual rainfall of I 30 to 200 centimeters, and for the most part of the East Indian Archipelago, and of the east coast of tropical Asia, the annual precipitation exceeeds 200 centimeters. In tropical Africa there is a zone $10^{\circ}$ broad where the rainfall amounts to 130 to 200 centimeters. 
$33^{2}$ Introduction to Botany.

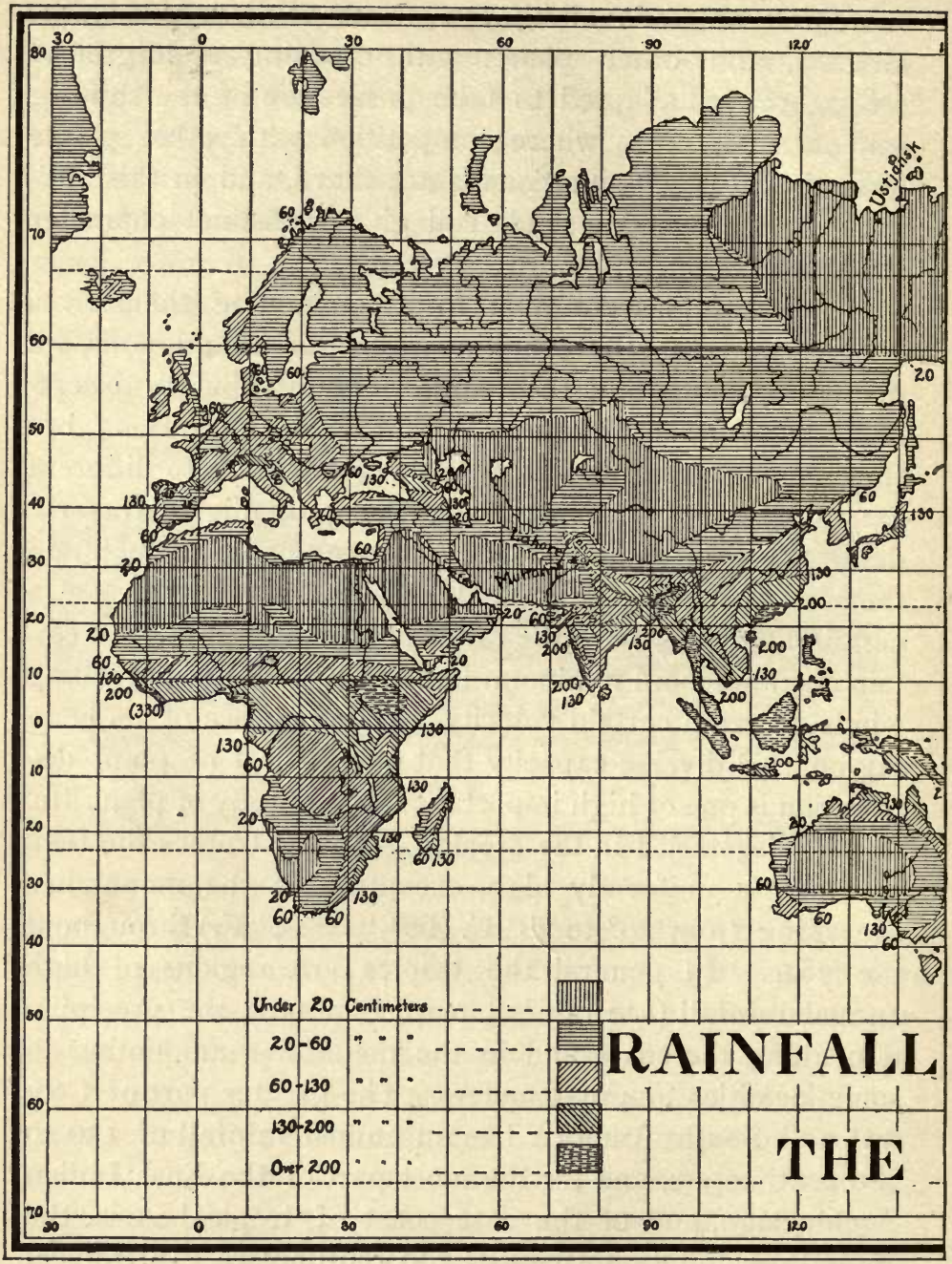

FIG.

Rainfall Map of the World. After data 


\section{Plants of Different Regions.}

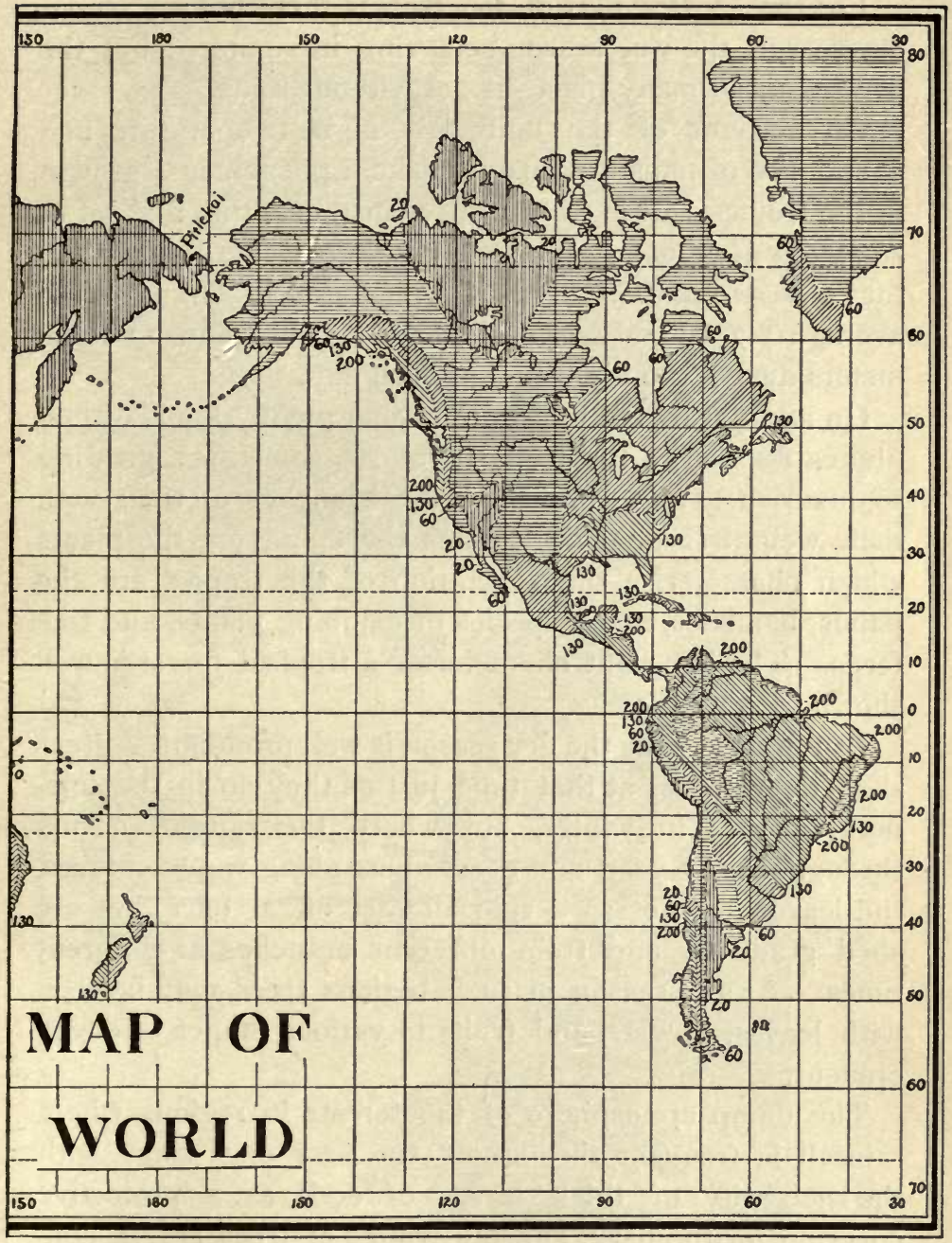

I79.

in SCHIMPER's Pfianzengeographie. 
For the greater part of the tropics there is a wet and a dry season, the wet season occurring in summer; but the dry season in many places is not without rains.

On account of the uniformly high temperature and abundance of moisture, plants make a phenomenal growth in the tropics. It is recorded, for instance, that a shoot of Bambusa grew nearly 8 meters within a single month, and that a Dendrocalamus (Fig. I 80) increased in length on the average 7.7 millimeters during the daytime and I 3 millimeters during the nighttime of each day.

On account of the moist atmosphere many kinds of epiphytes flourish in the tropical forests, sometimes growing so luxuriantly as to break off the branches of trees with their weight (Fig. I6). Besides the epiphytes, the plants which characterize the vegetation of the tropics are the palms, bamboos, many species of climbing plants, and tree ferns. The luxuriant character of a tropical forest is well shown by Fig. I8I.

In regions where the dry season is well pronounced, trees shed their leaves at that time just as they do in the temperate zones in winter, but where the rainfall occurs throughout the year, and everywhere along water courses, the leaves are not as a rule all cast off at once, but are shed gradually and from different branches at different times. At any season in such regions trees may be seen with leaves, flowers, and fruits in various stages of development.

The damp atmosphere of the forests in regions where rainfall is frequent throughout the year does away with the necessity of thick layers of cork as a protective covering for the bark, and the trunks of trees are, in consequence, relatively smooth and frequently green in color. To the thinness of the bark is probably due the peculiar 


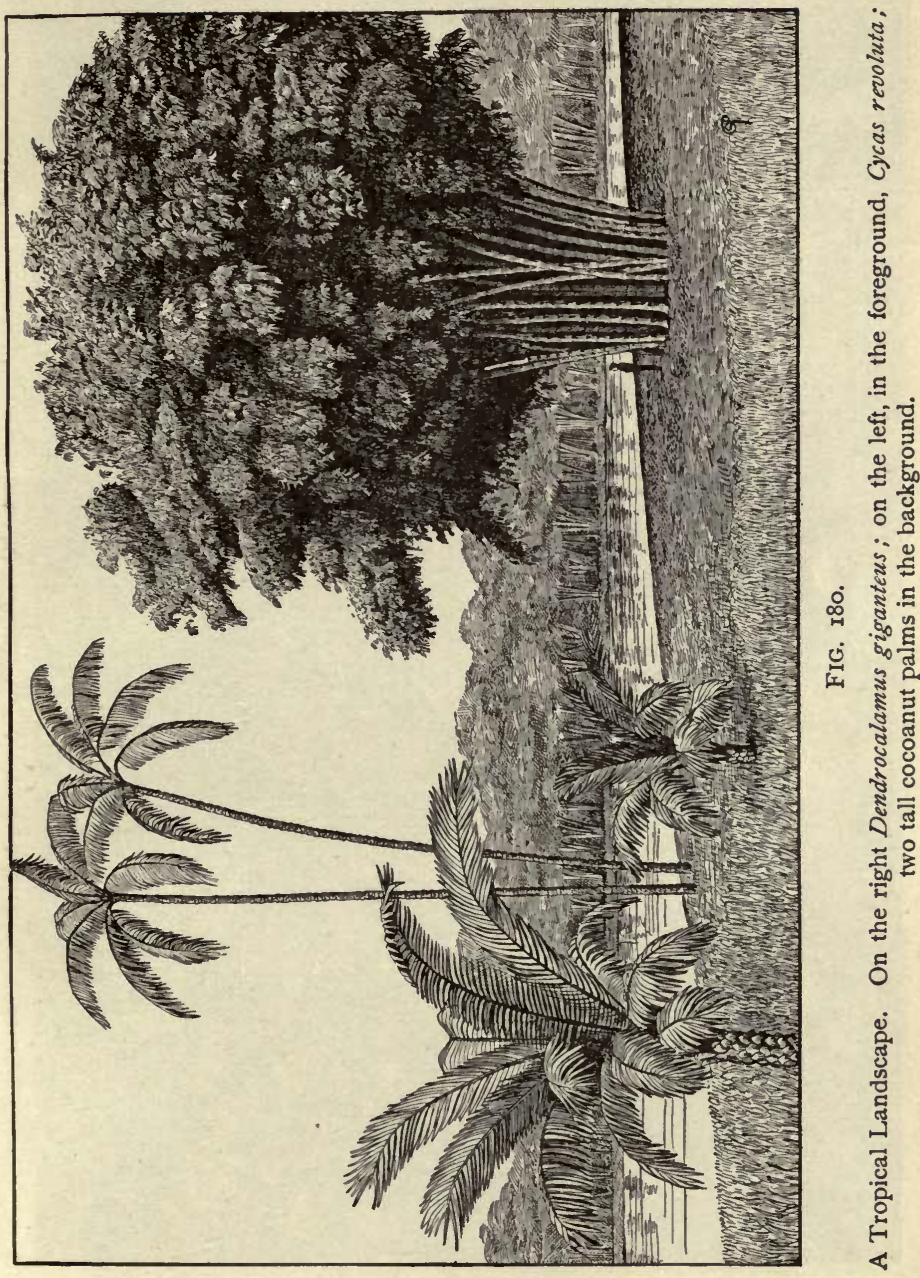




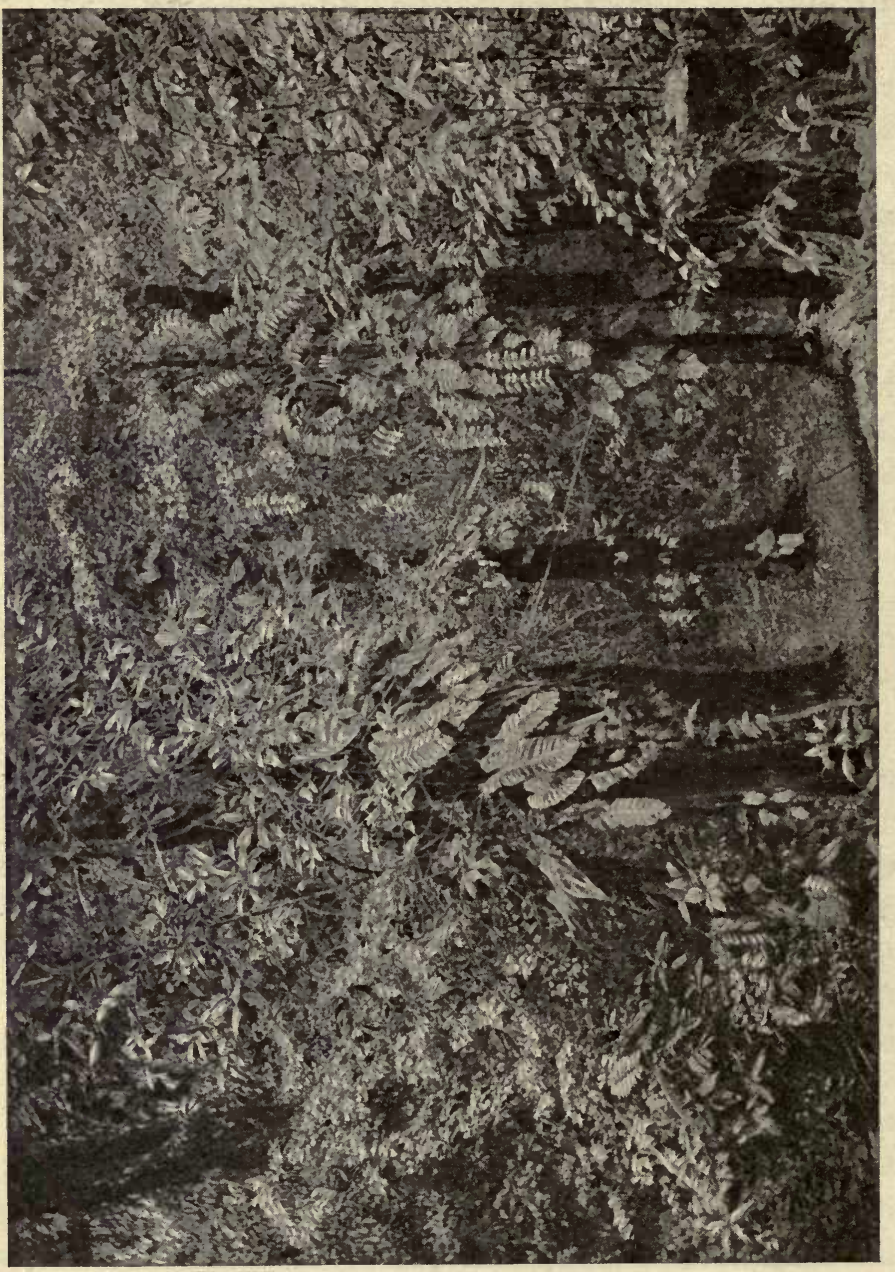




\section{Plants of Different Regions.}

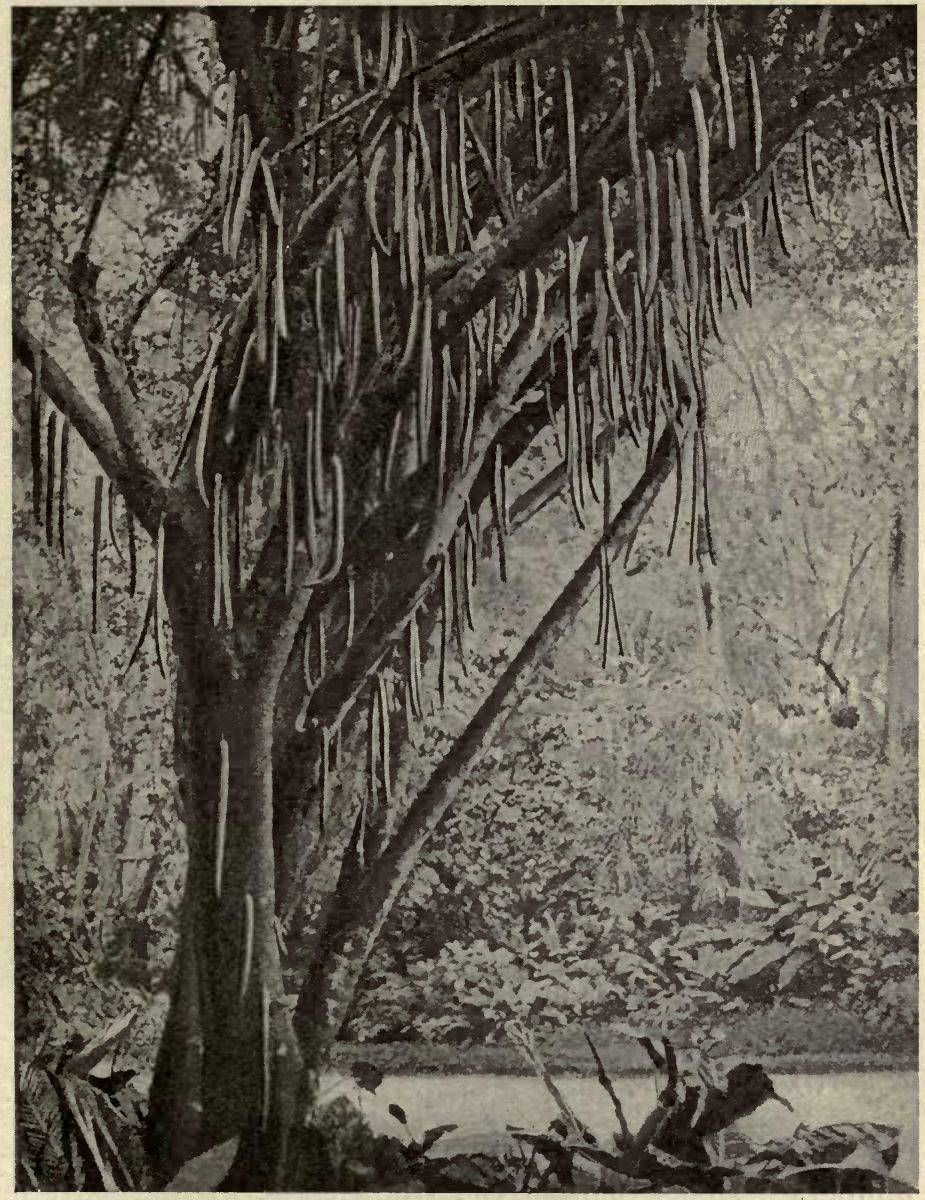

FIG. 182.

Parmentiera cereifera, bearing fruit on the trunk and old branches: Ceylon. After SCHIMPER's Pfanzengeographie. 
phenomenon of flowers and fruits borne on the old branches and even on the trunks of trees, as shown in Fig. 182.

In the moist forests the buds of trees do not prepare for their period of rest by the production of elaborate scales,

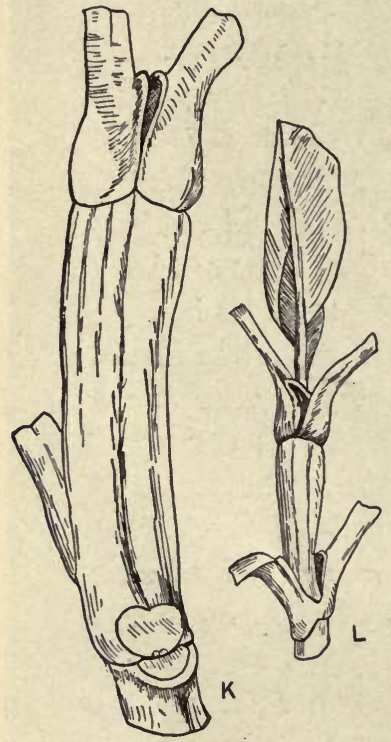

FIG. 183 .

$K$, young shoot, with apical bud, of Tabernaemontana dichotama; $L$, young shoot of Clusia grandiflora (?). After P. GROOM. such as are found in the winter buds of trees of temperate regions, and the resumption of growth of resting buds does not differ essentially from the uninterrupted apical elongation of shoots at the height of the growing season (Fig. 183).

214. Vegetation in Temperate Regions. - The temperate regions are characterized by much greater extremes of temperature than occur in the tropics, the minimum for the winter months running in some places far below the freezing point, and the maximum in summer rising as high as $50^{\circ} \mathrm{C}$. ( $122^{\circ} \mathrm{Fahr}$.). Those portions of the temperate zones which lie next the tropics gradually blend with the latter in their physical characteristics and vegetation. The amount of precipitation is much less in the temperate zones than in the tropics, the greater part of Central Asia and large parts of Europe and western North America having an annual rainfall of 20 to 60 centimeters, while the deserts of North America and the greater part of the Sahara and of the great deserts of Asia, where the 


\section{Plants of Different Regions.}

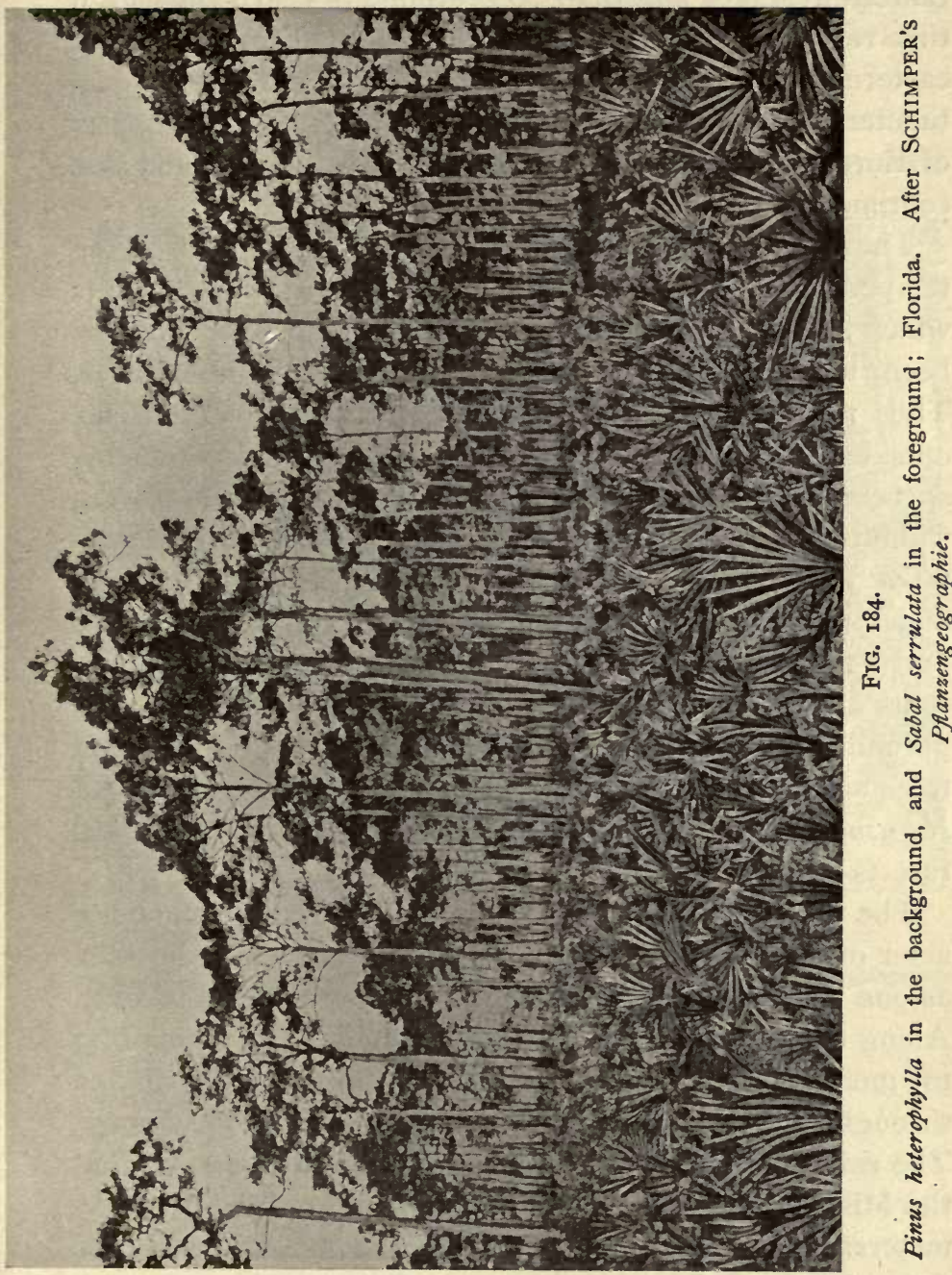


rainfall averages less than 20 centimeters yearly, lie within this region. A large part of western Europe and of eastern North America has a rainfall of 60 to I 30 centimeters, while in some of our Southern states, and in parts of Europe, the precipitation ranges between 130 and 200 centimeters annually.

The low winter temperature of the greater part of the temperate zones precludes the presence of many plants which are at home in the tropics, but which have thus far been unable to adapt themselves to low temperatures. Thus palms, bamboos, bananas, etc., do not venture far outside of the tropics, but in their stead we find pines, firs, spruces, and other conifers, and forests of oaks, hickories, walnuts, and beeches, which in the tropics have representatives, as a rule, only in the mountains. How the tropical and temperate forms mingle in the southern part of the north temperate zone is shown by Fig. 184 , which represents dwarf sabal palms and pine trees occupying the same ground. In Florida and Louisiana the boughs of forest trees are often bedecked with luxuriant growths of Tillandsia usneoides, an epiphyte which is also native to tropical forests (Fig. I85).

The effect of the amount of annual rainfall on the character of the vegetation in the temperate zone may be seen as one passes across the United States from east to west. Along the Atlantic and Gulf coasts the rainfall, amounting to more than Ioo centimeters annually, is distributed throughout the year, with the maximum amount in summer. The rainfall gradually diminishes toward the west, until at the Mississippi River the annual amount is about 60 centimeters. Along the coasts, and-in the mountains of the Atlantic states, the rainfall being sufficient to keep the soil moist at considerable depths, forest trees abound, so that 
the early settlers were obliged to make clearings with ax and grub hook in order to obtain land suitable for agriculture.

But as the rainfall diminishes toward the west and north, we find the forests giving way to prairies, the grasses

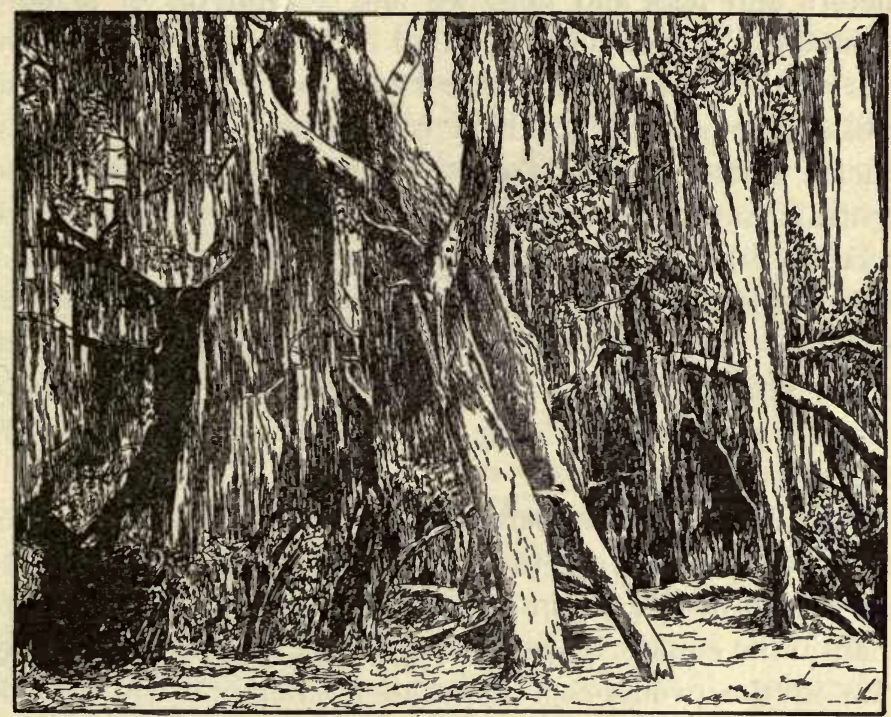

FIG. 185 .

Tillandsia usneoides covering and pendent from Quercus virens. After photograph by WEBBER.

and herbaceous plants which characterize them being able to obtain sufficient moisture from the summer rains to flourish where trees, with their larger demands on moisture, are unable to subsist, excepting along water courses. Westward from the Mississippi to the Rocky Mountains the rainfall, which occurs for the most part in the spring, early summer, and fall, steadily diminishes, and the grasses and 
herbs, and the trees following the streams, are represented for the most part by forms specially adapted to withstand dry weather.

Beyond the Rocky Mountains, to the Sierra Nevadas, there is a high plateau where the rainfall is about 30 centimeters and less, and here we find xerophytes of a more pronounced type, such as cacti, Agaves, and sage brush. Across the mountains, on the Pacific slope, the rainfall increases, amounting to about 93 centimeters in the north Pacific states, and to 45 centimeters in the northern and central parts of California, where, however, the rainfall occurs in winter and so occasions a peculiar form of vegetation especially adapted to these conditions. The shrubs and trees have leaves which are commonly leathery and often entire, and so adapted to reduce transpiration and perform their food-building function through the dry summer season.

215. Light in Temperate Regions. - The intensity of light diminishes from the equator to the poles; but since the length of the day in summer is greater in the temperate than in the equatorial regions, the total amount of illumination in the twenty-four hours of a summer's day may be greater in the temperate regions. In latitude $30^{\circ}$ on the longest summer day the sun is above the horizon 13 hours and 56 minutes; in latitude $50^{\circ}, 16$ hours and 9 minutes; in latitude $60^{\circ}, 18$ hours and 30 minutes; and in latitude $66 \frac{1}{2}^{\circ}, 24$ hours. Accordingly, in passing from the equator to the poles, the diminishing energy of the sun which comes to plants in a given period is, in part, compensated by the increasing length of time in which the energy is available each day.

216. Vegetation in Arctic Regions. - The arctic region is characterized by its low average temperature, its long 
winter night and long summer day, and its cold, dry winds. During the winter night, the land is shrouded in ice and snow which is frequently not sufficiently melted for vegetation to appear before about the first of July. July, which has an average temperature varying in different localities from $3.8^{\circ} \mathrm{C}$. to $8.8^{\circ} \mathrm{C}$. $\left(38.8^{\circ}\right.$ to $47.8^{\circ} \mathrm{F}$.), and even rising as high as $13.4^{\circ} \mathrm{C}$. $\left(56.1^{\circ} \mathrm{F}\right.$.) at Ustjansk, is the warmest month. The sun is entirely above the horizon for 65 days in latitude $70^{\circ}$, and for 134 days in latitude $80^{\circ}$, and, during part of these periods, the sum of the sun's energy available to plants for each twenty-four hours may be even greater than the available amount at the equator at the same time. Because of the cold, the shortness of the vegetative period, and the dry winters, trees are able to make only a feeble growth, and they do not exist far beyond the arctic circle.

About the first of July, when the snow and ice have melted in places, and sufficient heat has accumulated in the soil, vegetation in general suddenly starts into new growth. Kjellman, the botanist of the Vega Expedition, says of the beginning of the vegetative period within the arctic circle: "It is here not the same as in the more southern latitudes, where one species after another gradually comes to maturity. In the high north there are no spring, summer, and autumn floras composed of various plants blooming at different times, as farther to the south; in the polar regions everything, or nearly everything, springs into life at once; development begins everywhere at the same stage and proceeds with equal rapidity, so that all flowering plants are suddenly and at the very beginning of the vegetative period decked in their summer attire."

The vegetative period lasts only till the end of August, there being but two months of each year during which 
plants can accumulate food and produce seeds. Kjellman says of the close of the growing season: "An arctic landscape at the advent of winter is most nearly like a southern region which has been desolated by a heavy frost before its season. Many plants are interrupted at the height of

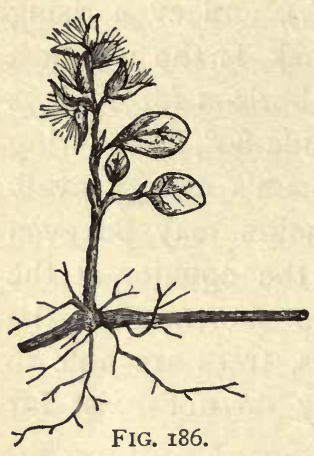

Salix polaris. Natural size. After SCHIMPER. their growth and stand with frozen lifeless leaves, with swelling flower buds, with half-open or entirely open flowers, and with half or entirely ripened fruit. No preparation has been made for the winter rest; while plants are in full activity, they are paralyzed by the stiffening cold." But how well the plants of this region are able to withstand a catastrophe that would prove fatal to those of lower latitudes has already been recounted on page $3 \mathrm{I} I$.

It might be expected that the plants of arctic regions would prepare for winter by ripening the new branches and producing protective scales for the young buds; but yet it seems clear that making the protoplasts, in and of themselves, resistant to the severe cold is the better plan, for the short vegetative period necessitates the rushing forward of the processes of vegetation and reproduction, and affords no time for special defensive preparations.

As would be expected, growth is very slow in the polar regions; measurements made on the willow, Salix polaris (Fig. I86), showed that in most instances, by the end of the growing season, shoots had increased from I to 5 millimeters in length, and at most from 5 to I I millimeters. A forest tree which was 83 millimeters thick at the base was found to have 544 rings of annual 
growth, the rings having an average breadth of 0.15 millimeter.

The polar regions possess no families of plants peculiar to themselves, the plants which appear there being dwarfed and xerophytic representatives of families which dominate the north temperate zone.

217. Vegetation of Desert Regions. - The deserts occupy a considerable area of the earth. In north Africa the Sahara desert alone nearly

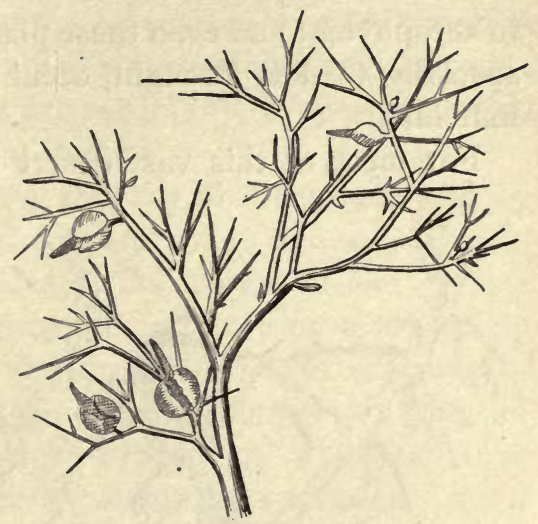

FIG. 187 .

Zilla spinosa, slightly reduced. From the Sahara desert. After PrantL. equals the United States in size, and its area is more than

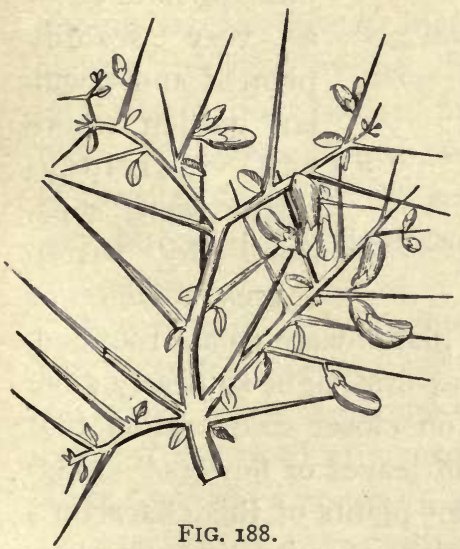

Alhagi maurorum. From the Sahara. After TAUBERT. doubled by the deserts of Arabia and of south and central Asia. To this must be added a large tract between the Sierra Nevada and Rocky Mountains, a narrow strip east of the Rocky Mountains, a large portion of central and southwestern Australia, and a narrow strip along the western border and in the south central part of South America. The total area of these desert regions equals approximately the area of the North Ameri- 
can continent. They have a rainfall of less than 30 centimeters annually, and, for the most part, are habitable only to xerophytes; and even these plants, which are constructed specially to defy drought, occur as more or less isolated individuals.

For most of this vast desert region, the sole cause of

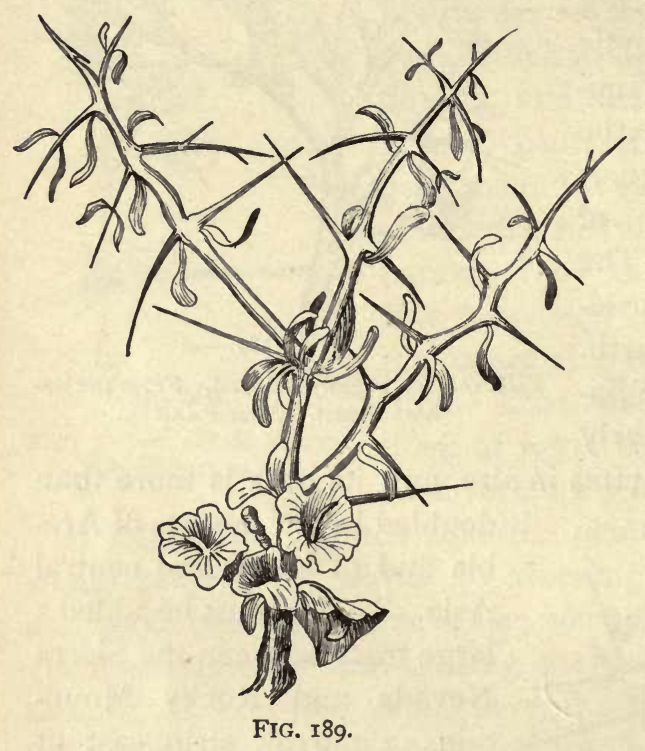

Sarcobatus Baileyi. From western North America; a desert plant. After Coville. the scant vegetation is lack of water, the soil being rich enough to support a luxuriant plant population. Schimper says of the Sahara desert near Biskra: "The firm and mostly clayey soil between the oases presented the appearance of a very scantily planted and peculiar garden where the individual plants were separated by barren spaces a meter or more in breadth. Most of the plants were small, rounded, dense shrubs which appeared at first sight so nearly alike that it was a surprise to find on closer examination that they possessed different kinds of leaves or flowers." (Figures 187,188 , and 189 represent plants of this character.) In the same region occurred a plant of quite different character belonging to the gourd family, whose thick succulent 
stems bore large leaves which remained green throughout the summer. It seemed to possess no special provision to prevent transpiration, for the leaves on severed branches soon withered. It was able to survive and remain green and turgid because of its extraordinarily long roots, which absorbed moisture at great depths.

The deserts of our own country are characterized by Yuccas, thick-leaved Agaves, and cacti, whose succulent green stems assume various forms from oval and globose to columnar, the latter forms sometimes rising to the height of trees (Fig. 169). In the cacti, the leaves are reduced to protective spines, and the chloroplasts are borne in the superficial cells of the cortex of the stems, while the major part of the tissues of the stem serves as a storehouse for water.

218. Vegetation of Mountain Heights. - The physical conditions vary materially from the base to the summit of high mountains. As a rule, the amount of precipitation increases up to a certain height, the atmospheric pressure diminishes as altitude increases, and the amount of heat energy absorbed by the atmosphere also diminishes; the intensity of illumination, however, increases with the altitude. It follows from these conditions that objects exposed to the direct rays of the sun warm up quickly, but on the withdrawal of sunlight they also quickly radiate their heat into space.

The amount of transpiration from plants increases with the elevation because of the increased illumination and decreased atmospheric pressure, together with the increased force of the wind at successively greater heights. At the same time the amount of absorption by the roots is apt to be lessened by the relatively low average temperature of the soil. 
348 Introduction to Botany.

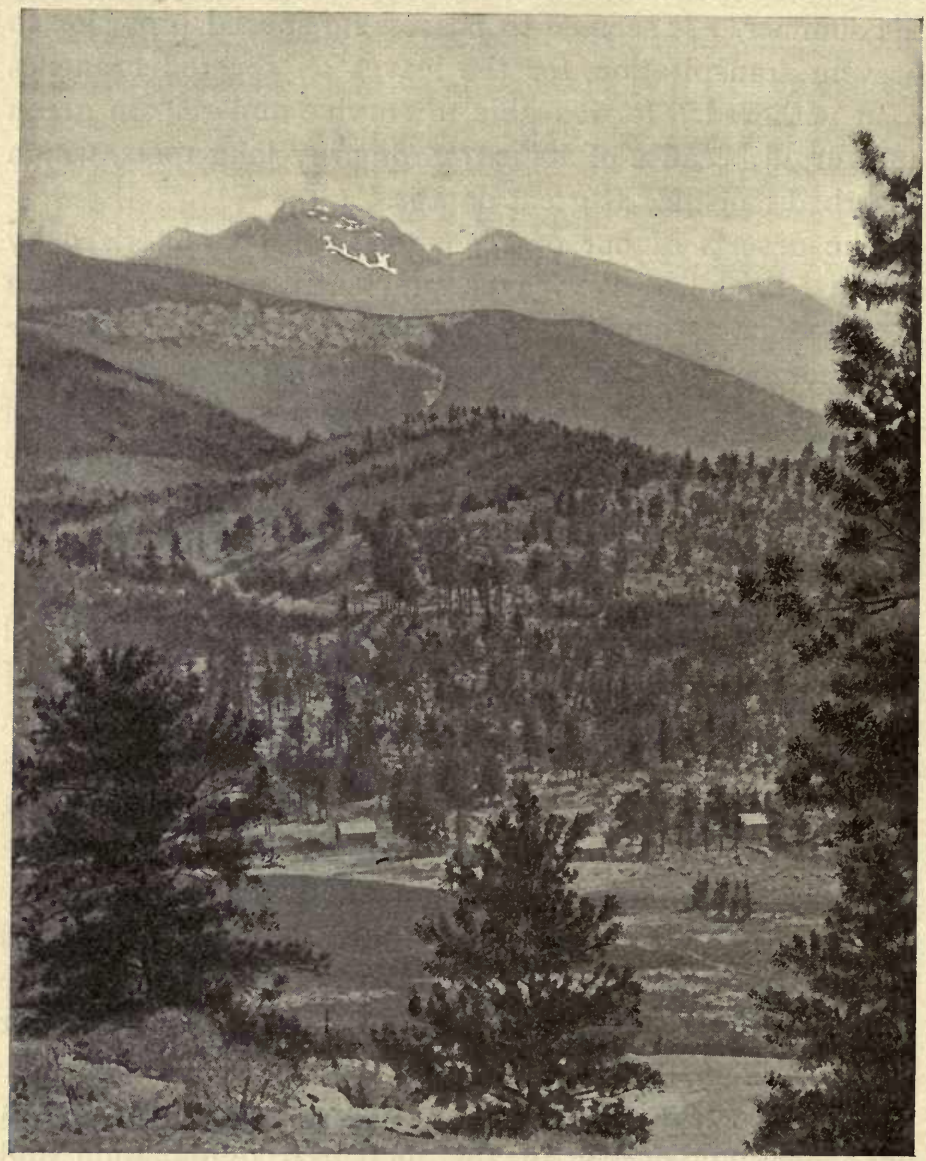

FIG. Igo.

Trees at different elevations on a mountain side. The largest trees occur near the base of the mountain. The dark zone near the summit of the middle range is a belt of trees, the upper border of which is the timber line, no trees growing beyond this. Above the timber belt is Long's Peak with its perpetual snow. 
Up to a certain height the vegetation may be more hygrophilous than in the lowlands, on account of increased rainfall; but higher up the factors which accelerate transpiration and lessen the rate of absorption necessitate special provision for the reduction of transpiration, and xerophytes begin to predominate similar to those which characterize desert and polar regions. Trees are stunted in their growth beyond a certain height, and finally an elevation is reached beyond which they are unable to exist. The line where the growth of trees ceases is known as the timber line (see Fig. 190).

One finds in ascending mountains in the tropics, that the vegetation gradually changes in its aspect, the plants at the base of the mountains being tropical, those farther up are warm temperate and temperate forms, until, at the height of perpetual snows, polar plants appear. Indeed, some of the same species of plants that occur in the polar regions may be found near the summit of tropical mountains.

Where representatives of the same species occur at different elevations, it is found that at successively greater heights the internodes become more and more shortened, the leaves smaller, thicker, and tending to cluster in rosettes, and the roots longer, so that the identity of the same species at the two extremes would not be recognized but for the intervening forms (see Fig. I9I).

When we compare the plants of tropical, temperate, and frigid zones, and of desert and mountain regions, we find that their chief modifications of form and structure have special reference to the available water, and that the same general modifications are found to serve wherever reduction of transpiration becomes necessary. The modification of the protoplasts of arctic plants, so that they are able to 
withstand very low temperatures, even at the height of their vegetative condition, is of particular interest, for it

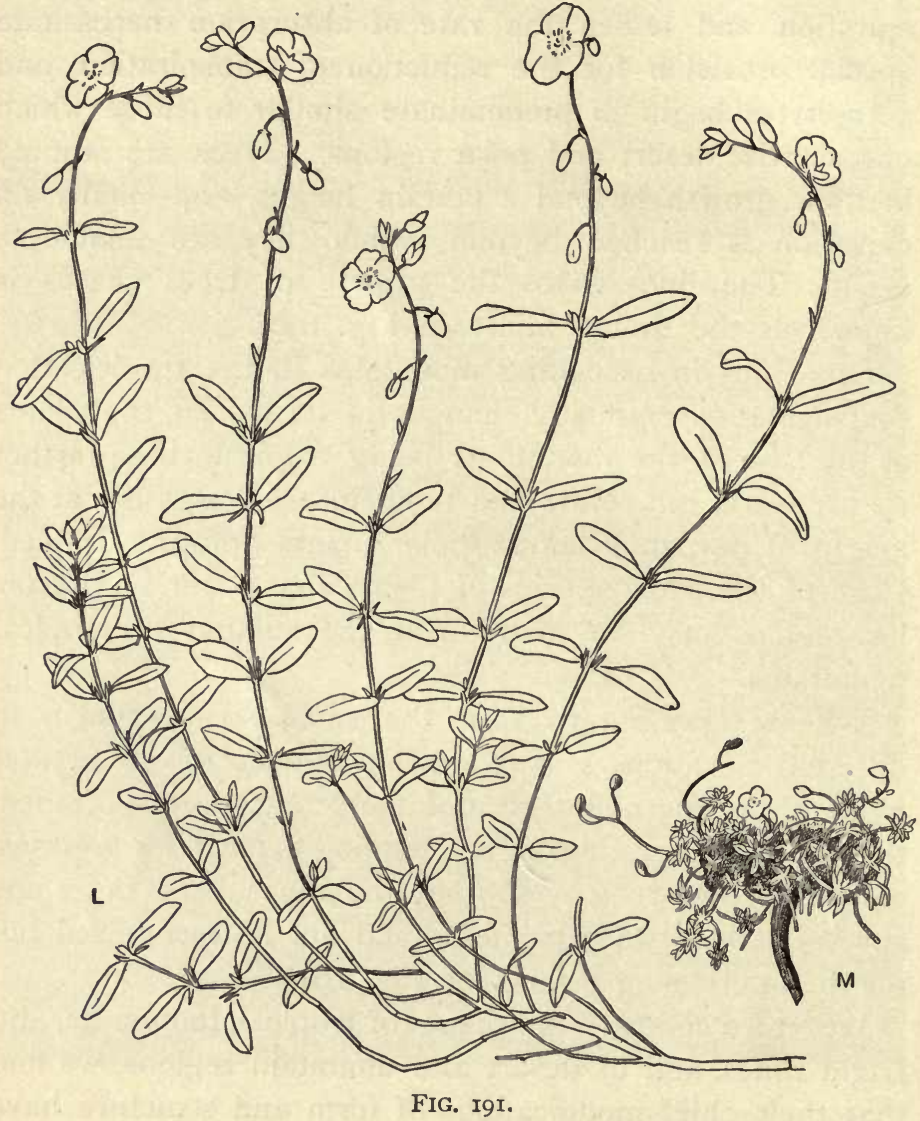

Helianthemum vulgare. $L$, the lowland form; $M$, the mountain form; both rather less than one-half natural size. After BonNIER.

suggests the almost limitless possibilities of modification wherever necessity urges with penalty of extinction. 


\section{CHAPTER XVI.}

\section{PLANTS OF PAST AGES.}

219. Antiquity of Plants. - Only approximate estimates can be made of the length of time which has elapsed since the waters began to lay down the stratified rocks of the Cambrian period, whose fossils contain the earliest evidence of the life of the earth. An average of the estimates of those best qualified to judge is between twenty-five million and seventy-five million years. In all probability life existed untold ages before this time in very simple forms which were not of a character to leave their impress in the rocks. If the great quantities of graphite existing in the rocks of the Algonquin period are of plant origin, as is probable, plant life must at that early time have already attained an enormous quantitative development before much complexity of structure had been evolved.

220. Primitive Physical Conditions. - The physical conditions were probably such as to incite and sustain rapid growth and multiplication. The atmosphere was charged with aqueous vapor and carbon dioxide, a part of which has since been locked up in limestone, coal, etc., and the interior heat of the earth may have contributed to maintain a uniformly high temperature. The oceans during the Cambrian, Ordovician, and Silurian periods undoubtedly abounded in Algæ, but because of the uncertainty in the preservation of their tissues as fossils, or of their leaving impressions in the rocks, positive identification of Algæ is 
seldom possible. In the Silurian rocks, however, have been found the silicified remains of a large plant which appears to be closely related to the modern Laminarias; Fucus also, described on page 275 , being a near relative. Dawson named it Nematophyton, and wrote of it: "When we consider that Nematophyton was a large tree, sometimes attaining a diameter of two feet and a stature of at least twenty before branching, that it had great roots and gave off large branches, and that it was an aërial plant, probably flourishing in swampy flats, that its seeds are so large and complex as hardly to be regarded as mere spores, we have evidence that there were in this early Paleozoic period plants scarcely dreamt of by modern botany."

221. Devonian Plants. - In the rocks of the Devonian period we find remains of representatives of nearly all forms of the higher Cryptogams, the tree ferns (Fig. I92) being of most frequent occurrence. In the forests of the Devonian occurred near relatives of the modern Lycopods or ground pines, and a genus of Gymnosperms, known as Cordaites, having leaves about 50 centimeters long (Fig. 192). All conditions were favorable for an abundant growth of plant life, and the waters must have teemed with forms which have left no impression in the rocks. The greatest petroleum-bearing strata in the world belong to the Silurian and Devonian periods, and since the source of petroleum, natural gas, etc., is evidently to be attributed to plant as well as to animal remains, the amount of organic materials built up by the plants of those periods is incalculable. (It will be remembered that animals obtain their food already organized from plants.)

222. Carboniferous Plants. - The Carboniferous period is of particular interest because the remains of its vegetation constitute the chief part of the coal supply. The 


\section{Plants of Past Ages.}

softer coals still give clear evidence of their origin, for they appear to be chiefly the carbonized masses of the stems and leaves of ferns, Lycopods, and Calamites (see Fig. 192). Even those coals which have been so much altered from their original condition that the naked eye is unable to detect their vegetable origin are found by means of the

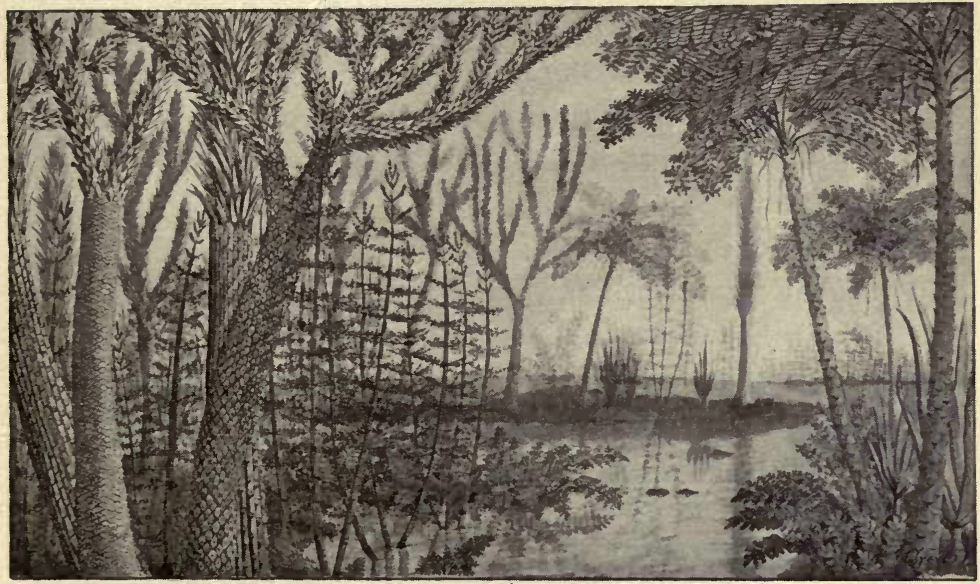

FIG: I92.

Carboniferous Plants. In the foreground on the left Lepidodendrons and Sigillarias (relatives of the modern Lycopods) ; just back of these Calamites, with branches in whorls (relatives of the modern horsetails). On the right tree ferns; in the - background, in addition to the foregoing, Cordaites (low with upright leaves).

microscope to contain the cells and tissues of plants. Many of the ferns of this period attained the size of trees, and the Lepidodendrons reached a height of 50 to 75 feet. The Lycopods, as many as eighty species of which have been found, also grew to great size. The Calamites, which are near relatives of the modern horsetails, also attained great proportions and apparently occupied the inundated 
flats along the borders of the forests of ferns, Sigillarias, etc. (see Fig. 192).

The coal-bearing areas of the United States are about 300,000 square miles in extent, and about 50,000 square miles of this are workable. The coal seams in the workable fields average from 4 to ro feet in thickness, and in some localities they reach a maximum thickness of 60 to Ioo feet. Some idea of the enormous amount of vegetation embodied in these deposits may be obtained when it is remembered that their thickness is only, roughly speaking, about $7 \%$ of the original depth of the vegetation which formed them. During this period there seems to have been a fairly uniform and temperate climate throughout North America, Europe, and Asia, even as far north as Greenland, Scandinavia, and Nova Zembla.

223. Permian Plants. - As time advanced into the Permian period, the giant Lepidodendrons and Sigillarias became rare; the Calamites were still abundant, and the tree ferns even more so than in the Carboniferous period. The Gymnosperms were represented by the Cordaites, Gingko, and conifers related to the modern yews and spruces. The ferns dominated the landscape, while the Cycads and conifers were not abundant and no Angiosperms had yet appeared.

As the Paleozoic era drew to its close, the Lepidodendrons, Sigillarias, and Calamites, which had been the dominant land vegetation, became almost extinct, the tree ferns became less abundant, and the Cycads and conifers gained ascendency.

224. Triassic Plants. - In the Triassic period the conifers were forming dense forests and the Cycads (Fig. I 80) were common; the tree ferns were becoming more rare, and only a few straggling Sigillarias still persisted. The 
smaller forms of ferns occurred in great abundance, and the genus Equisetum appeared with representatives whose stems grew to be four inches in diameter.

225. Jurassic Plants. - In the Jurassic the Cycads appear to have reached their highest development, occurring as far north as Greenland and Spitzbergen, whereas at the present time they are restricted to the warm regions of Mexico, the West Indies, Florida, Africa, and India. The conifers of this period were more like those of the present time. There is some evidence that Monocotyledons had already been evolved before the close of this period. The fact that ants, bees, wasps, flies, and possibly butterflies appeared at this time affords collateral evidence that the flowering plants were already at hand to supply food in the form of pollen and nectar.

226. Cretaceous Plants. - The beginning of the Cretaceous period is noted for the apparently sudden appearance of the noble Sequoias of twenty-six species; and of Dicotyledons, represented by the Sassafras, poplars, and Rhododendrons. By the middle of the Cretaceous period a characteristically modern flora had been evolved, represented, in addition to the trees named above, by the willows, oaks, maples, elms, beeches, chestnuts, palms, and numerous other forms. It is a fact of great interest that the plants of this period occur as fossils in northern latitudes all around the world; and we may be quite certain that there was a continuity of land masses in the vicinity of the arctic circle at that time. The Sequoias, which are now restricted to California and Oregon, occur as fossils in the Cretaceous strata of Greenland, England, France, Germany, Italy, Siberia, Alaska, and Kansas. The northern hemisphere at that time was like a vast botanic garden in which were growing side by side plants which are now dis- 
tributed in various climes. Cycads, palms, and tree ferns were near neighbors to oaks, beeches, and chestnuts, and with them were found the, at present, cosmopolitan Compositæ and Leguminosæ.

227. Tertiary Plants. - The Cenozoic era began with the same characteristics which distinguished the Cretaceous period. Early in the Tertiary period grasses first appeared, and with them land animals made a notable advance, the ancestors of our domestic animals then distinctly appearing. By the middle of the Tertiary, grasses were carpeting the earth as we now find them. The climate was evidently becoming cooler, for palms and other tropical forms were growing less abundant. Toward the close of the Tertiary, the tropical forms had become appreciably less numerous in the north; and we may be certain that the lowering of the temperature, which in the Quaternary culminated in the glacial period, was now decidedly manifest.

228. Exodus Southward in the Quaternary. - In the Quaternary period occurred the notable exodus of plants from the land of their origin in the north southward across the continents in front of the advancing accumulation of ice and snow. The cause of the changed conditions which resulted in the movement of glaciers as far south as southern Illinois and northern Kansas, is not fully determined; but there is no doubt that such glaciation took place and produced a profound effect on plant distribution.

In North America there were no barriers to prevent the migration of plants southward, but in Europe and Asia the east and west trend of the mountain ranges caused the extinction of many species which were unable to maintain themselves in the cold mountain altitudes long enough to start their progeny on the southern slopes. Thus the gigantic Sequoias (Fig. 193) were exterminated in Europe 


\section{Plants of Past Ages.}

and Asia, but were able to find a congenial home in Oregon and California.

In all probability the northern flora in its southern migration attempted to disseminate itself throughout the breadth of the continent, but some forms found themselves more readily adaptable to the Atlantic slopes, some to the Mississippi valley, and others to the Rocky Mountains and the regions west, so that now we find.more or less distinct

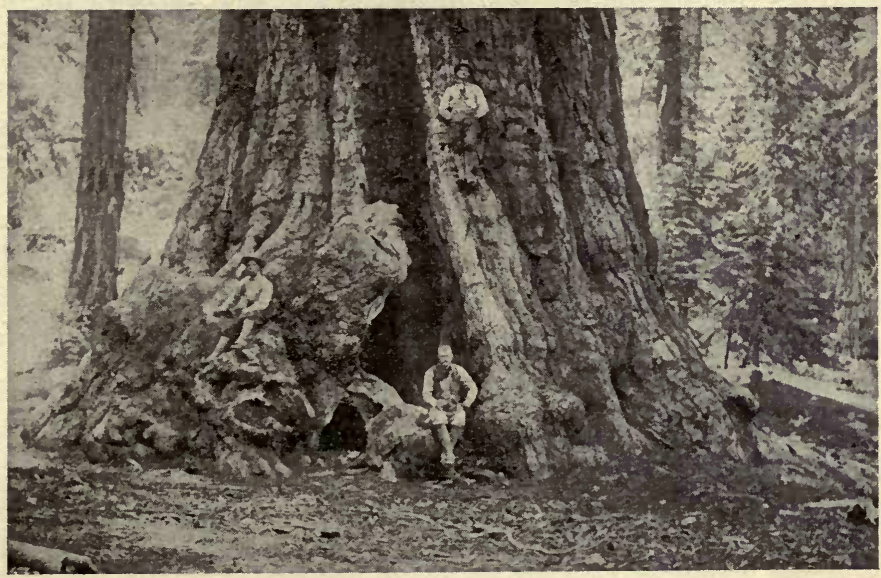

FIG. I93.

Base of the giant Sequoia "General Grant." Photograph by SQUIRES.

floras in these different regions. Along the eastern coast of Asia, including the Japan islands, the physical conditions approximated those of our Atlantic slopes, and we find that in these regions, remote from each other, very similar floras have been evolved from the common stock which came to them during the migration in the glacial period.

229. Migration Northward. - When, for some reason, 
the conditions changed so that the sun's heat was able, finally, to melt the ice back to the arctic regions, and the plant pioneers sought to occupy again the land from which their ancestors had been driven, the mountain barriers in Europe and Asia interposed difficulties to which the American species were not subjected. The greater hardships in both Europe and Asia, first in the southern and then in the northern migration, have resulted at the present time in a richer flora in the New World than in the Old. There is evidence that man himself was present during at least a part of the Quaternary period, and was a witness of these migrations, which, however, doubtless took place very slowly, and were no more recognizable than the trend of plant history is at the present time.

If we put the geological record in evidence along with the structural affinities of plants, we find we have reasonable ground to conclude that plants have evolved from the low and simple types, at first probably of microscopic size, to the complex forms which by a division of labor among their tissues are able to cover the surface of the land, and, rising and branching in the atmosphere, to appropriate increased amounts of the sun's energy; and thus while fulfilling their own destiny they have prepared the earth for the habitation of man. 


\section{CHAPTER XVII.}

\section{CLASSIFICATION OF PLANTS.}

230. Basis of Classification. - The number of diverse plants is so enormous that a systematic classification is a necessity for their identification and for a concise indication of their relationships. It is plain that since plants are descended from generation to generation it is best, if possible, to classify them according to their lineal descent; those plants which give evidence of a recent common ancestor being placed together in a group, and assemblages of this sort which appear still further back to have descended from a common form, being classed under a still more comprehensive family, and so on in widening tribes of remoter relationships until the ultimate groups are finally embraced under one all-comprehensive designation, the Vegetable Kingdom. Properly to determine the degrees of relationship among plants is, in many cases, impossible, and our classification on grounds of consanguinity can be only approximately right.

As we have already learned, plants have been subjected to great vicissitudes in their long residence on the earth; many forms have become extinct, while many others have wandered far from their ancestral homes, and have become profoundly modified under-changed environments; and other modifications have come about by intercrossing. The chain of evidence may, therefore, be incomplete or obscure. Where, however, forms are related by lineal descent, there 
are certain to be some characteristics common to all which afford satisfactory evidence of the relationship. It is clear to every one that the different kinds of roses are nearly related; and when one compares the flowers and fruits of cherries and plums, the evidences of consanguinity are very striking, but the points of similarity between roses and cherries, for example, are fewer, and for their detection require one to be somewhat familiar with the proper lines of evidence.

231. Cause of Variation. - If it is true that forms of plants, as we now find them, have descended from fewer and simpler primitive types, as appears to be the case from structural evidence and geological record, then an inherent capacity to vary under changed environment, or under other conditions which we do not understand, must be one of the fundamental causes of the evolution of diverse forms from one or few generalized types; and an intercrossing between forms which have persisted in definite lines of variation must have contributed to the same result. Gaps occur in the lineage because those forms whose variations are best adapted to the environment have, in the competition for room and food, crowded out other forms less favorably adapted. It is these gaps which enable us to classify plants into more or less circumscribed groups. We cannot, of course, follow plants in a state of nature from generation to generation, and in this way make positive record of their parentage and relationships. We have to take them as we find them, and classify them according to the circumstantial evidence of their similarities and dissimilarities.

232. Grouping into Orders, Genera, etc. - In classifying plants, we say that those plants belong to the same species which are so nearly alike as evidently to have sprung from 
seeds or asexual propagative bodies, such as tubers, rhizomes, etc., of the same parent plant, or from different plants which in their turn sprang from a common parent, and so on back for an indefinite number of generations. Deviations from the type of the species which originate when seeds from the same pod, for instance, or buds from a common stock, are germinated and grown under different conditions of soil or climate, are called varieties. Our different kinds of cultivated apples and potatoes are good examples. Varieties which tend to come true from seeds are called races. Then those groups of species which are sufficiently alike to indicate a common ancestry at some time in their history are classed under one genus (plural, genera), and the groups of genera of evident rela-

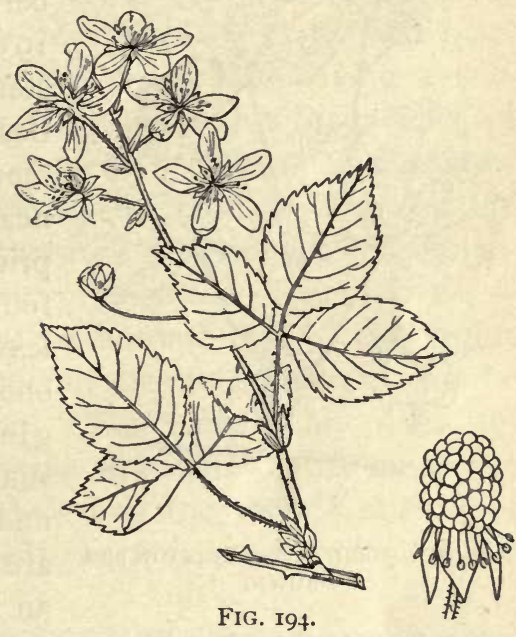

Rubus villosus. After BRITTON and BROWN. tionship are classed under a common order, or family, and so on.

We may find good illustrations of what has just been said in the different kinds of wild raspberries and blackberries. The high bush blackberry, Rubus villosus (Fig. 194), is a shrub with erect or recurved stems from three to four feet long, having stout recurved prickles; the leaflets of the three to five foliate leaves are ovate oblong, with margins closely serrate and with sharp points, and they 
are pubescent on the under side. The flowers are white, from three-fourths to one inch broad, and the black pulpy fruit is from one-half to one inch long, and has a pleasant flavor. The individual plants are essentially alike, their common ancestry is very evident, and we class them ac-

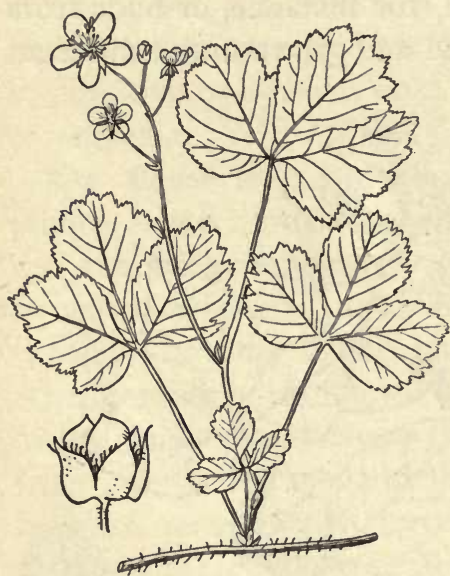

FIG. I95.

Rubus hispidus. After BRITTON and BROWN. cordingly, under one species.

The running swamp blackberry, Rubus hispidus (Fig. 195), has slender creeping stems, beset with weak bristles. From the creeping stems more or less erect branches, nearly or quite destitute of prickles, arise to a height of four to twelve inches, and bear leaves of three, rarely of five, obovate leaflets whose margins are irregularly and sharply serrate above the middle. The white flowers are one-half to two-thirds of an inch across, and the black, sour fruit is less than one-half inch long.

No one would consider that these two blackberries had arisen by suckers or seeds from the same stock, and they are accordingly classified under different species; but still they have so many structural characteristics in common that they apparently sprang from a common ancestor somewhere back in their lineage. We note, for instance, that each has flowers consisting of a deeply five-parted calyx with a shallow, broad tube; five petals, numerous distinct stamens inserted on the calyx, several two-ovuled carpels inserted on a convex receptacle, which in ripening become 
little one-seeded stone fruits or druplets that adhere and form an aggregate fruit. Because of these similarities we classify them under the same genus, Rubus. Rubus villosus and Rubus hispidus have many other relatives among - the blackberries, raspberries, and dewberries, with distinctive characters, but all sufficiently alike to indicate a close relationship.

If we turn to the strawberries we find evidences of relationship between them and the blackberries and raspberries, the flowers of the strawberry also having a fiveparted, broad and shallow calyx, but with a bract between each division; numerous stamens with slender filaments, numerous carpels on a convex receptacle. Thus far the resemblances are striking and afford evidence of relationship; but we also find some pronounced dissimilarities, the carpels in ripening do not become fleshy stone fruits, but dry, one-seeded nutlets, and the receptacle grows to be large, fleshy, and fragrant. The relationship does not seem close enough to class the strawberry under the genus Rubus, and we accordingly group the various species of it under the genus termed Fragaria, on account of the fragrant receptacle.

The similarities which we find between the raspberries, blackberries, and strawberries indicate a remote common ancestry, and we therefore place the genera Rubus and Fragaria under the same family, Rosacea, rose family. While it can be seen that a classification on such a basis is natural in contradistinction to artificial, and does, to a large extent, indicate true degrees of relationship, yet it must be a matter of judgment as to where the lines shall be drawn between species, genera, etc., which must be more or less arbitrarily exercised.

There are still more comprehensive grades of classifica- 
tion than the orders or families, these being grouped into sub-classes, these into classes, and the classes into subkingdoms, which finally are comprised under the vegetable kingdom.

233. Best Evidence of Relationship. - It is evident that those structures which are apt to vary considerably under changed environment, in the lifetime of a single individual, do not furnish good evidence as to relationships. Thus, individuals of Helianthemum vulgare, which are known to be from the same stock and consequently of the same species, exhibit the different forms shown in Fig. I9I when grown at different altitudes. It is seen that the size and habit of the plant are greatly altered, while the character of the flowers is quite the same. Many instances could be brought forward to show that the vegetative parts, - that is, those parts which are actively concerned in the life of the individual, - are much more unstable under changed environment than the reproductive parts which do not contribute toward the maintenance of the individual, but are only concerned with the continuance of the species. It is because of the greater fixity of character of the flowers, seeds, and fruits, that chief reliance is to be placed on them in determining relationships. 
PART II.

THE HERBARIUM, LABORATORY EQUIPMENT, AND PROCESSES. 



\section{CHAPTER XVIII.}

\section{THE SCHOOL HERBARIUM.}

234. Character of the Herbarium. - An herbarium containing representatives of the most interesting plants in the region of the school will be found useful in many ways. It would be well to attempt to obtain the complete flora of the region, but first of all those plants and parts of plants should be collected which possess a special interest. To take a single illustration, the different species of violets growing in the region should be represented as being among the most cherished of our spring flowering plants, and as affording, in their differences and similarities, a good idea of what is meant by species. Those plants and plant parts should be represented in the herbarium which best show the different methods of securing cross fertilization, of scattering seeds and fruits; different habits of climbing, various modes of protection against too rapid transpiration, or against mechanical injuries.

Plants of widely different habitats should be represented, such as those growing in dry places, in moist soil, in wet places, and in water. It is better to have each kind of plant as completely represented as possible, the plant in flower being accompanied with fruits, seeds, and seedlings. It is readily perceived that an herbarium which is thus replete with information about the life and ways of plants has a high educational value. Now that amateur photography has become so general it would probably not be difficult to have the herbarium accompanied with photographs of plants in their natural habitat. A map of the region might also be prepared with the habitat of the different plants indicated thereon.

Those students who are interested in making private collections should be encouraged to do it, but it is perhaps best not to require individual collections of students as an organic part of the course; partly for the reason that a large part of such work is merely mechanical and therefore requires time that could be more profitably spent in studying plants as living beings. The few students who would cherish such collections and profit by them would willingly undertake their preparation as extra work. 
235. Collecting and preparing Materials. - In collecting materials for the herbarium, a tin collecting box is very useful, since in it specimens may be kept in a fresh condition for two days or longer. The collecting boxes can be obtained from the dealers in botanical supplies, or they may be made of the following dimensions at the tinner's : Length 17 inches; diameters of ends, which are oval, 4 by 6 inches; on one of the broad sides there should be a hinged lid, $4 \frac{1}{2}$ by i 5 inches, fastening with a spring catch; rings should be soldered on near the ends, to which a strap could be fastened for suspending the case over the shoulders. It is well to coat the case, inside and out, with paint or Japan varnish. For digging up plants, a strong garden trowel or weed digger is very serviceable.

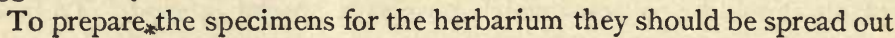
between two sheets of thin porous paper I $1 \frac{1}{2}$ inches wide by $16 \frac{1}{4}$ inches long, called specimen sheets, taking care that the parts overlap each other as little as possible, and that the leaves are not crumpled, and then the specimen sheets should be placed between two or more sheets of thick carpet or blotting paper, termed driers. Specimens thus prepared may be stacked one above another to the depth of a foot or so, a flat board being placed on top of the pile, and on the board rocks or other weights amounting to 40 or 50 pounds. If the objects are too long for the specimen sheets, they may be bent once or even twice like the letter N. Thick stems, tubers, etc., should be pared down to requisite thinness, but so as to preserve their form.

The damp driers should be replaced by dry ones every day for about a week, or until the specimens appear quite dry. The damp driers should be spread out in the sun or hung about a stove, and it were better in changing the driers to put on the fresh ones while they are still warm from this treatment. Some plants with mucilaginous juices, such as the spiderworts, dry very slowly and are apt to discolor badly. This can be obviated to a certain extent by ironing them with hot irons while lying between the specimen sheets. Very fleshy plants such as the cacti should be slit open longitudinally and the pulp scraped out before placing them in the press. The heads of composite flowers whose ray florets would not otherwise come under pressure in the press should have rings of cotton placed around them to obviate this difficulty. If parts of plants must necessarily overlap in the press, pieces of porous paper should be placed between them.

When dry the specimens are to be glued, with a good quality of pre- 
pared fish glue, to heavy linen ledger paper $11 \frac{1}{2}$ by $16 \frac{1}{4}$ inches in size. The regulation quality and size of this paper is kept in stock by the dealers in botanical supplies. The glue should be thinly spread over the back of the specimen with a soft, flat brush, and after the glued specimen has been laid in position on the herbarium paper a sheet of thin paper, and then a flat board surmounted by a weight, should be placed over it. Many sheets with specimens attached may be stacked in this way while the glue is drying.

The name of the plant (genus and species written in one line), should be written in the lower right-hand corner of the herbarium sheet, together with the locality where found and the date of collection; the character of the habitat should also be stated, as, for instance, whether the soil was clayey or loamy, wet or dry; whether the situation was shady or fully exposed to the sun, and so on. The name of the collector should also be given. It is a common practice to have labels about $1 \frac{1}{2}$ by $3 \frac{3}{8}$ inches, with the name of the collector or of the school printed in plain letters across the top, and on these to write the name of the plant and other data. The labels should be made of strong, but thin, white paper, and should be fastened at the lower right-hand corner of the herbarium paper by coating them thinly with glue along the borders only.

One species only should be mounted on a single sheet of herbarium paper; but several specimens of the same species, illustrating different stages of growth or variations in different habitats, might well be mounted together, each specimen being accompanied by its own record. The specimens thus prepared are then to be inclosed in a folded genus cover of heavy manila paper, which should be about $\frac{1}{4}$ inch broader and longer than the herbarium paper. All the species of a genus should be included under one cover unless too numerous.

Ferns, horsetails, club-mosses, and other Cryptogams of large size should be pasted to the herbarium paper in the same manner as described above for Phanerogams. The horsetails and club-mosses are apt to adhere with difficulty, but may be fastened on more securely with narrow strips of gummed paper. Mosses, liverworts, Lichens, and Fungi in general should be inclosed loosely in envelopes, which are to be glued at their centers only to the herbarium paper. The envelopes can be made in any desired size by doubling strong and thin white paper, folding back the loose top edges, and folding the ends under for about an inch of borders.

Delicate Algæ demand a special method of treatment. Herbarium 
paper is cuit into pieces $4 \frac{1}{16}$ by $5^{\frac{3}{4}}$ inches in size. The Alga to be mounted is placed in a pan of water, and the piece of herbarium paper, while resting at its center on the tips of the fingers and thumb, is submerged beneath the Alga and carefully brought up against it, care being taken as the paper is lifted from the water that the Alga spreads out equally on all sides. If success is not attained at first the process should be repeated until the branches of the Alga are symmetrically distributed. The paper is then laid on a drier, and a piece of old muslin over the Alga, and then over all a drier, board, and weight. The driers are to be changed as usual, but the cloth is not to be removed until the specimen is dry. When dry, the Alga sticks to the herbarium paper without further assistance, but not to the cloth, which has been employed for that reason.

Very delicate specimens, such as the sporangia of some Myxomycetes, need to be protected against crushing. A piece of the substratum to which they are growing, such as a dried leaf, twig, or rotting $\log$, should be glued to a piece of cardboard, and then strips of cork or strawboard should be fastened to the card, on two sides of the specimen, to support a cardboard cover. Thus protected, the specimen is to be inclosed in a folded envelope fastened to the herbarium paper as already described.

It is the practice of some botanists to fasten the envelopes containing mosses, etc., and the small sheets of paper on which the Algæ are mounted, to herbarium paper I I $\frac{1}{2}$ by $8 \frac{1}{8}$ inches in size, which is just one-half the standard size. The specimens should be kept in dustfree wooden cases or tin boxes. A wooden case, with tightly fitting door, and provided with thin shelves about three inches apart, is very convenient. 


\section{CHAPTER XIX.}

\section{LABORATORY EQUIPMENT.}

236. Tables. - In a school where the study of plants is to be seriously pursued there should be a working room equipped with tables of such size, and so arranged, as to give the students plenty of room and light for their work. The ordinary school desks are not of suitable construction and arrangement for laboratory work, and where a special laboratory cannot be provided, it would be a good plan to fasten boards, about eighteen inches wide, to the wall beneath the windows, by means of strong brackets, to serve as working tables. Even where there is a special workroom, such tables will be found very serviceable. The main thing in any case is to have a flat working table, well lighted by diffuse light, which will afford plenty of elbow room for each student.

237. Microscopes. - Much of the observation in an introductory course in botany can be done with the naked eye, but for part of the work a good simple lens is a necessity. ${ }^{1}$ It is economical to have cheap pine blocks made as stands for the lenses, after the manner of Fig. 196, rather than to purchase the stands of the dealers. Ordinary 2 by 4 inch pine scantling is dressed smooth, and cut into two lengths of 8 and 3 inches respectively. It is better to bevel the ends of the short pieces. The short piece $(b)$ is to be fastened to the long piece $(a)$, as shown in Fig. I, by means of glue and a screw driven through from the lower face

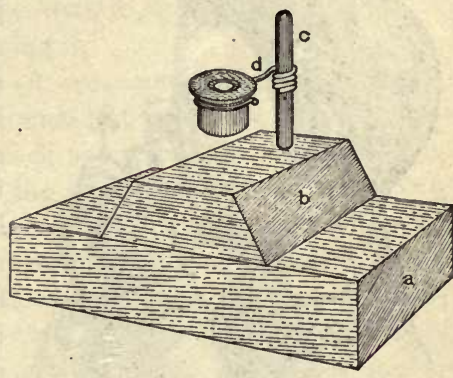

Fig. $x 96$.

A simple dissecting microscope.

See text. of the long piece. A brass rod $(c)$ about $\frac{3}{16}$ inch in diameter and $3 \frac{1}{4}$ inches long is driven into the short block, as seen in the figure, to a

1 The doublet lenses manufactured by Bausch \& Lomb, Rochester, N.Y., are satisfactory for this purpose, those of $\frac{3}{4}$-inch focal length being the best for general work. 
depth of $\mathrm{r}$ inch - a hole of slightly smaller diameter having first been bored to receive it. Spring brass wire of $\frac{3}{32}$-inch diameter is then cut into lengths of 17 inches, and each piece shaped to serve as two lens holders according to the following directions :-

Clamp one end of a wire nail of about $\frac{11}{6} \frac{1}{4}$-inch diameter firmly in an iron vise. Place the brass wire with its middle against the wire nail, and wrapping from both ends, make a coil of ten turns about the nail, and cut the wire in two at the middle of the coil ; then at $I \frac{1}{4}$ inches from the center of the coil of each half bend the wire into a loop to receive and firmly hold the doublet lens. When completed $(d)$, the spiral coil should grip the rod in the block firmly enough to hold the lens at any height, but without binding too tightly to prevent an easy vertical adjustment. The block should be given one or two coats of shellac into which enough lampblack has been stirred to make a black varnish. No glass stage or mirror is necessary, for the shellac affords a black background against which thin sections, etc., are strongly contrasted.

238. Dissecting Needles. - Dissecting needles can be made as follows: Grasp a strong needle in a pair of pliers and thrust it, eye

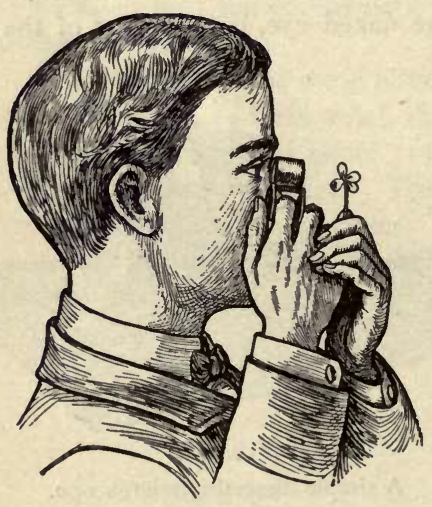

FIG. 197.

Showing the manner of holding lens and object. foremost, into a handle of soft wood or into a smooth twig with very small pith. Hard wood handles, such as can readily be prepared from butchers' skewers, should have holes drilled into them to receive the needles. A good drill for this purpose is made by breaking the eye from a needle a size larger than those which are to be inserted, and rubbing the broken end into the form of a drill point on an oil stone. The needle should be pushed into the handle a slight distance beyond the bottom of the hole made with this drill.

239. Manner of Using Lenses. For much of the work requiring the use of a lens the dissecting block will not be needed, the lens simply being held between the thumb and forefinger of one hand while the object is held in the other hand in the same manner. To prevent vibration, 


\section{Laboratory Equipment.}

the middle, ring, and little fingers of the hand holding the lens should rest, in a closed position, in the palm of the hand holding the object, as shown in Fig. 197. It is to be remembered that the object, and not the eye or the lens, should receive the best illumination; for the object becomes visible only by the light which is reflected from its surface through the lens and into the eye. The best light is that which comes over the shoulder or from one side, and the student, if facing a window, should turn to one side when making observations. For some work the dissecting stands are a great convenience, particularly when minute structures require separation with the needles. In such a case the object should lie upon the dissecting stage, and the lens should be placed in the wire holder and adjusted to a proper height to give a sharp image of the object; then both hands will be free to manipulate the specimen with the needles while it is viewed through the lens. In using the needles, the hands should rest upon the shelves made for that purpose at the ends of the block.

240. Care of the Lens. - The lens must be kept clean and bright. It will be noticed that if the fingers come in contact with its faces a filmy spot is left which excludes much of the light when the lens is again used. To remove such spots, breathe upon the glass and quickly polish it with a clean soft cloth. The student should always see that the lens is in good condition before using it.

241. The Compound Microscope. - A compound microscope is not an absolute necessity in a beginning course in botany, but it is very useful in gaining a clear comprehension of some primary facts of plant structure and physiology with which the student should early become acquainted. A good compound microscope with a very satisfactory outfit can now be purchased by schools for $\$ 25$ or less, and there is no reason why every school in which botany is taught should not be supplied with at least one. The optical parts of a compound microscope, which are most useful for a general study of tissues and cell contents, are a r-inch eyepiece and a $\frac{2}{3}$-inch and a $\frac{1}{6}$-inch objective. The objectives should be fastened to a double nosepiece in order that they may quickly be shifted.

242. Use of Compound Microscope. - When thin sections or very minute objects, such as starch grains or cell contents in general, are being studied, the object should be seen by transmitted light, - that is, by light which is reflected from the mirror ( $o$, Fig. 198), below the stage $(n)$, through the object $(l)$, and into the objective $(k)$. The surfaces 
of opaque objects are to be seen by light which is reflected directly from their surfaces into the objective. The compound microscope dif-

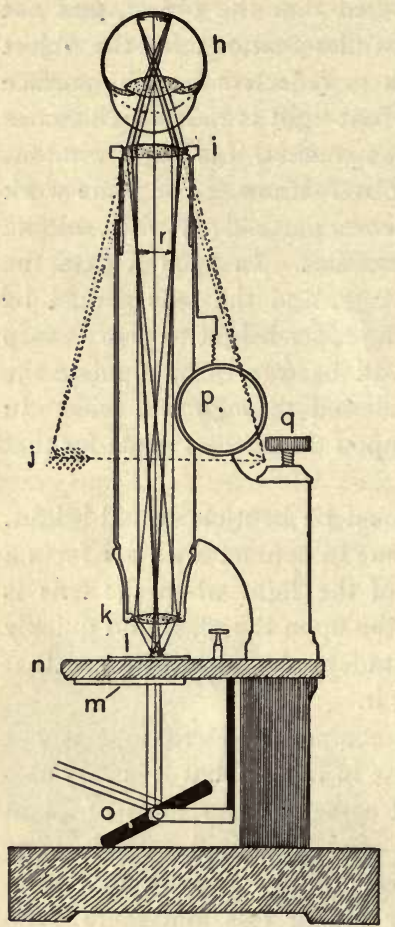

FIG. 198.

Diagram of a Compound Microscope, showing the manner of the formation of the image. See description in the text. fers from the simple lens essentially in that the light, which passes from the object into the objective, is brought to a focus in the tube of the microscope, at the level of the eyepiece diaphragm, forming an enlarged real image $(r)$, which in turn is magnified by the eyepiece (i). Thus, the eyepiece serves as a simple lens and magnifies as at $(j)$ an enlarged image of the object. It will be seen that it is of utmost importance that the lenses of eyepiece and objectives should be kept perfectly clean, otherwise the light will be too much absorbed to produce a clear image. In examining cell contents or thin sections of plant tissues with the compound microscope, the objects should be mounted in a drop of water or other reagent, as the case may require, on a clean glass slip, and covered with a perfectly clean coverglass.

243. Preparing Material for Observation. - In becoming acquainted with the use of a compound microscope, starch from a potato will do for examination. Having washed a slip and coverglass and polished them with a clean cloth, set the coverglass on edge until wanted, and lay the slip flat upon the table; then cut a potato in two and scrape off a very small portion of the pulp with the point of a penknife. Transfer the material on the knife to a small drop of water placed at the center of the glass slip, and cover with the coverglass. In doing this, grasp the coverglass by its opposing edges between the thumb and forefinger, and place its lower edge against the slip a little to the left (if the coverglass is held in the left hand) of the object; then place the middle finger 
of the hand holding the coverglass against the lower edge of the latter, to keep it from sliding to the left ; gradually lower the coverglass, giving it additional support by means of a dissecting needle held against its lower surface. As it comes in contact with the fluid, see that no air bubbles become entangled; if there seems to be danger of this, briskly move the coverglass up and down, by means of the dissecting needle, until the bubbles become broken. The reason for lowering the coverglass gradually instead of simply dropping it over the object is to prevent the formation of bubbles, which would make the preparation more difficult to study.

244. Manipulating the Microscope. - Having properly mounted the object, place the slide on the stage (Fig. 198, $n$ ) of the microscope so that the object stands over the center of the opening in the stage. Set the microscope in a convenient position (an upright one, when the object is mounted in a thin fluid), and, by means of the concave surface of the mirror, reflect diffuse light from a white cloud or other bright portion of the sky (never use direct sunlight), so that the object is illuminated from below. This is done while looking directly at the object outside the microscope. When the object is seen to be illuminated, swing the $\frac{2}{3}$-inch objective into position, and rack the body of the microscope down until the front face of the objective is within about $\frac{1}{4}$ inch of the object; then look into the microscope and slowly rack the body up until the object comes into focus. Finally, make the image sharp by means of the micrometer-screw fine adjustment $(q)$. If the field does not appear bright on first looking through the microscope, only a slight adjustment of the mirror is likely to be necessary. If the image does not appear as the body of the microscope is drawn upward, it can at least be told when the surface of the coverglass is in focus, by means of flecks of dust which are quite certain to be present, and the slide can then be moved slowly until the object is brought into position.

The potato pulp will appear as a more or less indistinct mass, but free starch grains will be found suspended in the water. Adjust the slide so that a group of the free grains is exactly in the center of the field, rack the body of the microscope upward and swing the 1 -inch objective into position, then rack downward until the objective nearly touches the coverglass. In racking downward always observe the objective to see that it is not forced against the coverglass. Now look through the microscope and raise the objective by means of the 
micrometer screw. The image should soon appear, for the $\frac{1}{6}$-inch objective is in focus when its front lens is very close to the coverglass.

It will be noticed that there is a diaphragm $(m)$ beneath the stage of the microscope, with openings of different sizes, or, in case of an iris diaphragm, with a single opening which can be enlarged or contracted. The larger openings give greater illumination, and the smaller, sharper definition. It is a good rule to employ the smallest opening which permits sufficient illumination with a given object and source of light. If the diaphragm is of the revolving kind, care must be taken that the opening employed is centrally placed, as indicated by the stop which clicks into position. When scraped or powdered material has been mounted in a drop of fluid under a coverglass, it may be spread out in a thin even layer by rubbing the coverglass around with gentle pressure, by means of the finger covered with a clean cloth.
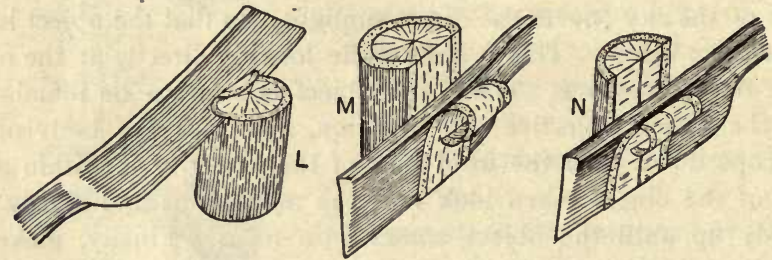

FIG. 199.

Positions of the razor with reference to the stem in cutting: $L$, a cross section; $M$, a longitudinal tangential section; $N$, a longitudinal radial section.

245. Cutting Thin Sections. - Thin sections need to be cut from roots, stems, leaves, etc., in order to study their cellular structure. In the case of stems and roots, cross sections, and longitudinal sections through the center, called longitudinal radial sections, are the most instructive, but it is often desirable to prepare longitudinal sections outside the center, termed tangential sections (Fig. 199). In the case of leaves, cross sections of the blade, at any desired place, answer the usual purposes.

A good, half hollow-ground razor - that is, one with a thick blade ground slightly concave on both sides - is more effectual in section-cutting than the plano-concave razors sold by the dealers in microscope supplies. Material which is fresh may be sectioned at once, but dry 


\section{Laboratory Equipment.}

material should be well soaked in warm water before using. It is still better, after soaking dry roots, stems, or pieces of dry wood, to place them in equal parts of alcohol, glycerine, and water, for a week or so before sectioning.

To cut a cross section of a stem or root, trim one end squarely across with a sliding stroke of a sharp knive; then hold the object between the thumb and forefinger, flood the upper face of the razor with water or $50 \%$ alcohol, and rest it on the forefinger with the point against the object; regulate the thickness of the section to be cut by raising or lowering the forefinger, and cut the section with a long forward stroke (see Fig. 200). The sections usually need to be made as thin as they can possibly be cut. The upper face of the razor is kept wet in order

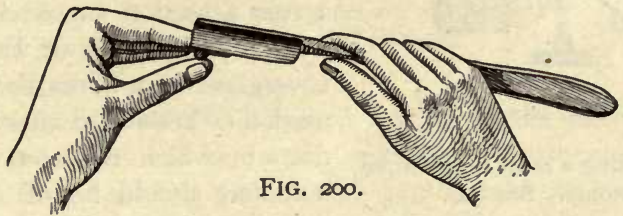

Showing the method of holding the object and razor in cutting sections free-hand.

that the sections may easily slide over the blade without crumpling or rolling up. As the sections are cut, they should be transferred to a dish of water, and should not at any time be allowed to dry.

Leaves and some succulent stems and roots need to be embedded in elder pith before sectioning, and for such purpose a good supply of dry pith should be kept on hand. A piece of the pith not more than an inch in length is halved longitudinally with a sliding stroke of a knife, while held on a table firmly between the thumb and fingers to keep it from breaking. If a leaf is to be sectioned, a strip of it is placed between the pieces of pith, and then leaf and pith are sectioned together, the razor being kept wet as before, preferably with $50 \%$ alcohol. If succulent stems and roots are to be sectioned, longitudinal $\bigvee$-shaped grooves are cut

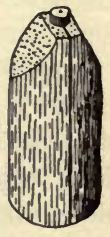

FIG. 20r.

Method of inclosing an object in elder pith. in both pieces of pith, of proper size to clamp the material firmly (Fig. 201). 
A simple microtome, which can be clamped to the laboratory table, will be found of great service. ${ }^{1}$

In cutting sections with such an instrument, the razor is to be held

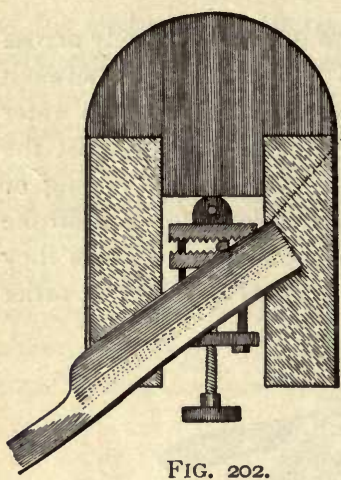

FIG. 202. firmly, edge and back, against the glass top, and given a sliding forward motion, as indicated by the arrow in Fig. 202. The object, embedded in elder pith, if necessary, is clamped in the object holder, which, as the sections are cut, is raised by means of a micrometer screw to the desired thickness of the sections.

246. Mounting the Sections. - As a rule a section should be mounted for study in a drop of water under a coverglass, and, if reagents are to be used, they are/added afterward. The

Method of using a razor on a simple microtome. See text. drop in which the section is to be mounted should be of such a size that it will fill the space under the coverglass and no more; if too much has been added, draw away the surplus with filter paper; if not enough, place a small drop on the slip close to the coverglass on the side where the water comes to the edge, and then draw the drop into contact with the coverglass by means of a dissecting needle or small stick. If the drop is placed at first in contact with the coverglass it may run over its upper surface; if it is placed on the side where the water does not fill out to the edge, it is likely to entangle air bubbles when it runs under. If no reagents are to be used, the preparation may be kept from drying by applying a drop of $50 \%$ glycerine to one edge of the coverglass, so that it may take the place of the evaporating water.

247. Applying Reagents. - Any reagent miscible with water, such as a solution of iodine, may be made quickly to replace the water in which the section is mounted, by placing a drop of the reagent on the slip in contact with the edge of the coverglass, and then placing a strip of filter paper in contact with the opposite edge; in this way the water is drawn out and the reagent flows in and occupies its place.

248. Sharpening Knives and Razors. - No satisfactory results can

${ }^{1}$ See catalogue of Bausch \& Lomb, Rochester, N.Y. 
be obtained in section cutting without sharp knives and razors. An oilstone should therefore be provided, and a fine hone and strop for razors. ${ }^{1}$ To sharpen an ordinary pocket knife, hold the under face of the blade at an angle of about ten degrees with the stone, and impart a sliding back and forth motion, honing the two sides alternately until a keen edge is produced, keeping the stone well oiled with mineral oil.

The razor should be kept in such condition that at any place on its edge it will readily cut in two a hair held between the thumband forefinger. If it will

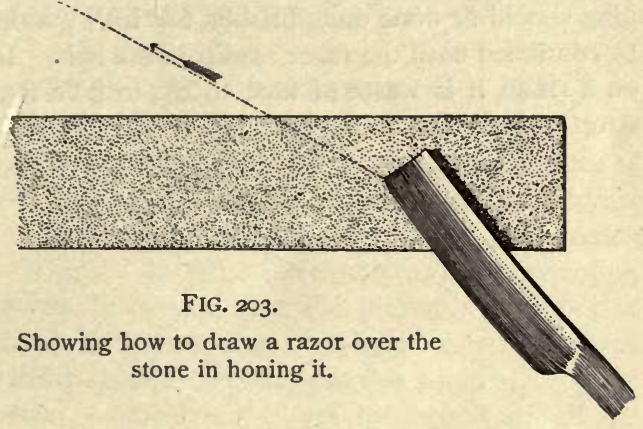
not do this, it may only need stropping on the prepared leather of the strop. To tell whether it should be honed on the stone before stropping, moisten the ball of the thumb and pass it with gentle pressure longitudinally along the edge of the razor; if the edge, throughout its length, produces

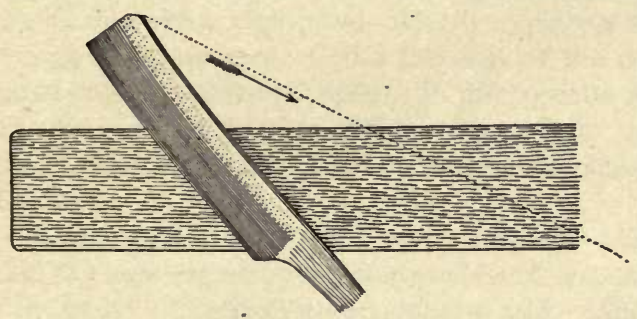

FiG. 204 . the sensation of taking hold of the skin, the use of the stone will not be necessary, but otherwise the razor should be honed on the oilstone until it responds properly to the test. In honing, Showing how to draw a razor over the strop.

oil the stone with mineral oil; hold the blade of the razor flat on the stone and slide it edge foremost along the full length of the stone, imparting a longitudinal as well as a forward motion to the razor, so that it glides for its full length from point to heel over the stone, as shown in Fig. 203. Then turn the razor on its back, so that the other side lies on the stone, and slide the razor forward and longitudinally, edge foremost, as before.

1 One of the best for this purpose is the Torrey combination strop and hone. 
When the honing is completed, wipe the oil from the blade and strop the razor on the prepared leather strop, holding it flat upon the strop, and pushing it, back foremost, along the strop, at the same time drawing it from heel to point for its full length, as shown in Fig. 204. Then turn it on its back and repeat the process for the other side. The stropping should be done quite briskly, but with gentle pressure, and should be continued until the razor readily cuts a hair. If a razor needs honing on a stone, it is waste of time to try to give it a proper edge on the strop without first honing it. 


\section{CHAPTER XX.}

\section{REAGENTS AND PROCESSES.}

Chloral Hydrate. - Dissolve 8 parts of chloral hydrate in 5 parts of distilled water, - namely, in the proportion of 8 grams of chloral hydrate to 5 grams or 5 cubic centimeters of distilled water (since I cubic centimeter of distilled water weighs I gram). Used to clear plant tissues, or, in conjunction with iodine, to demonstrate the presence of starch in leaves, etc. (See Chloral Hydrate-Iodine.) Whole leaves and thick sections can be very quickly cleared by boiling in this solution.

Chloral Hydrate-Iodine. - Dissolve 5 parts by weight of chloral hydrate in 2 parts by weight of a weak solution of iodine. (See Iodine Solution.)

In using this reagent as a test for starch in thin sections of leaves, etc., mount the section in a drop of water under a coverglass; focus the section with a high-power objective; place a drop of the solution on the glass slip in contact with the edge of the coverglass, and place a piece of filter paper in contact with the opposite side of the coverglass. In this way the solution will be drawn under the coverglass, and its progressive action in clearing the section and staining the starch can be followed.

Chloroiodide of Zinc. (Chlor-zinc iodide.) - Dissolve zinc to saturation in concentrated hydrochloric acid and evaporate the solution to the consistency of concentrated sulphuric acid. Add potassium iodide to saturation, and then as much iodine as can be taken up. The solution should have a decidedly reddish brown color.

Since only a small quantity of this reagent is likely to be needed, it is perhaps best to buy it ready prepared, from dealers in microscope supplies. It should ba kept in the dark, well stoppered. By this reagent cellulose membranes are stained violet to purple. Starch is stained the same color and swollen. Lignified, corky, and cutinized membranes are stained a golden yellow. Proteid cell contents and protoplasts are stained yellow to brown. It can be used in connection with phloroglucin, as described under that reagent. 
Chrom-Acetic Fixative. - Dissolve I gram of chromic acid in 100 cubic centimeters of distilled water, and add 0.5 gram of glacial acetic acid.

When it is desired to study the construction of the protoplasts in embryonic tissues, as in root and shoot tips, or in Algæ, Fungi, etc., the fresh material should be submerged in the above killing and fixing solution from $I$ to 2 days. By this treatment the protoplasts will be made to keep the form which they had while living. The fixative should penetrate the material quickly, so that root and shoot tips should be hardly more than 3 millimeters or $\frac{1}{8}$ inch long. Anthers and ovaries should be cut open at both ends. Ovules should be removed from the ovary with only a narrow strip of tissue adhering to them. Leaves should be cut into narrow strips, not more than 3 millimeters broad, etc.

A relatively large amount of the fixative should be used; for example, to a depth of 4 centimeters in a test tube 2 centimeters in diameter, for fixing about ro root tips.

After fixing the material tie it up in thin muslin and wash for 6 hours, or over night, in running water, or in water which is frequently changed. Then gradually dehydrate and harden the material by keeping it for 2 hours in each of the following grades of alcohol : $20 \%, 30 \%, 40 \%, 50 \%$, $60 \%, 70 \%$. It may then stay in $70 \%$ alcohol until wanted. If it is to be kept a long while before using, it would be better to take it from the $70 \%$ alcohol and preserve it in equal parts of $95 \%$ alcohol, glycerine, and water. If the material is to be stained with safranin (which see), or first sectioned and then stained, it may go directly into the stain from $50 \%$ or $70 \%$ alcohol, or from the mixture of alcohol, glycerine, and water ; and then may be mounted for immediate study in equal parts of glycerine and water, or it may be mounted permanently in glycerine jelly (which see).

It is frequently very desirable to embed root and shoot tips, anthers, ovaries, ovules, etc., in paraffin preparatory to cutting very thin and serial sections of them. To learn how to do this read under Imbedding in Paraffin, and Cutting Paraffin Sections.

Cutting Sections. - Directions for cutting sections free-hand will be found on page 376 .

Sections from hard tissues, such as the shells of nuts, may be cut as thin as possible by means of a hack saw, and then brought to the necessary thinness between two oil stones, using water, instead of oil, as a lubricant. 
Cutting and Mounting Paraffin Sections.-For cutting paraffin sections some form of microtome must be used.1 The smaller student forms may be made to perforın very efficient service in paraffin sectioning.

After the material has been imbedded, as described under Imbedding in Paraffin, page 386 , cut out a small block of paraffin containing the object to be sectioned; melt a small piece of paraffin on the end of a pine block I centimeter square in cross section and about 2 centimeters long, and while the paraffin on the end of this stick is still melted, press into it and firmly against the stick the paraffin block containing the object. The paraffin block should be so placed on the stick that when the latter is fastened upright in the object carrier of the microtome, the microtome knife will cut the sections in the desired direction through the object. Heat a wire nail or dissecting needle, and melt the base of the paraffin block superficially, to seal it more firmly to the stick. Submerge the stick in cold water to harden the paraffin, and fasten it in the object carrier of the microtome, having first set the carrier near its lowest position. Adjust the stick so that the top of the paraffin block just touches the under surface of the knife. Set the knife at right angles to the bed of the microtome. Trim the face of the paraffin block which faces the knife parallel with the edge of the knife, and to about I millimeter from the object, and trim the opposite edge of the block in the same manner, so that the two edges are parallel, and parallel to the knife edge. Then trim the remaining two sides of the block rather close to the object. The sections should now adhere in a ribbon as they are cut; Io micromillimeters is a good average thickness for the sections ( 1 micromillimeter $=\frac{1}{1000}$ millimeter). In wielding the knife see that the knife carrier runs easily in its bed (using oil if necessary), rest the elbow upon the table, and make short strokes with a wrist motion simply. Transfer the ribbons of sections to a clean tray, keeping the side down which was down in cutting, and finally mount the sections on the glass slip in the same position.

To mount the sections on a glass slip, spread a few drops of albumen water over that portion of the slip which is to be occupied by the sections, the slip having first been washed with soap and water, rinsed, and polished by rubbing it vigorously with a clean cloth. If the albumen water has a tendency to creep away from the place where it has been

${ }^{1}$ For description of microtomes see catalogue of Bausch \& Lomb, Rochester, N.Y. 
spread out, the slip has not been thoroughly cleaned. It is a good plan to keep the slips in concentrated sulphuric acid saturated with bichromate of potash contained in a pint Mason jar, and then to rinse and polish them as needed. The albumen water is made as needed from a stock solution. To make the stock solution, shake together equal parts of the white of one egg and glycerine, and add to this mixture a small amount of salicylate of soda to keep it from spoiling. Allow the mixture to stand over night in a tall cylinder, and skim off the impurities which rise to the top. To make the albumen water, add I drop of the stock solution to about ro cubic centimeters of distilled water. Make the albumen water afresh every few days, as the old solution becomes turbid or is found to contain a precipitate.

Having spread the albumen water on the glass slip, cut a piece of the desired length from the ribbon of sections and lay it upon the albumen water, and so on until nearly the entire breadth of the slip is occupied, in case rectangular coverglasses are used and it is desired to study the sections in series in the order in which they were cut. With a piece of filter paper filter away most of the albumen water, and while doing it arrange the sections at the center of the slip. Then place the slip to dry on the copper plate of the paraffin imbedding apparatus described under Imbedding in Paraffin. Place asbestos paper or felt paper between the slip and the copper plate until the slip is heated to a little below the melting point of the paraffin. It is best to let the slips remain thus for an hour or more.

Before staining the sections stand the slide on end in a tumbler of xylene to dissolve the paraffin. (After the sections have been mounted on the slip it is the custom to call the entire preparation a slide.) The sections should adhere to the slip during this and all subsequent manipulations. Rinse off the xylene in a tumbler of $95 \%$ alcohol and stain the sections as directed under Staining and Sealing in Balsam.

Cyanin and Erythrosin for Double Staining. - Sections of plant tissues having both cellulose and lignified walls may be double stained in the following manner: Place the sections for a few hours or over night in a saturated solution of cyanin in $95 \%$ alcohol; then rinse the sections in a dish of $95 \%$ alcohol until the cyanin ceases being washed out in clouds. (This should be within a few moments.) Then transfer the sections to a saturated solution of erythrosin in clove oil. Leave them there only a moment and then place them in a dish of xylene and mount almost immediately in Canada balsam as described under Stain- 


\section{Reagents and Processes.}

ing and Sealing in Balsam. By this process of staining the lignified walls should be blue and the cellulose walls red. If a proper differentiation is not obtained by the time ratios here given, a little experimentation will show what the time ratios should be for the specific material.

Fehling's Solution for Demonstrating Grape Sugar (Glucose).Make three stock solutions, which are to be preserved in separate bottles.

I. I7.5 grams of copper sulphate dissolved in 500 cubic centimeters of distilled water.

2. 86.5 grams of sodium-potassium-tartrate (Rochelle salts) in 500 cubic centimeters of distilled water.

3. 60 grams of sodium hydrate in 500 cubic centimeters of distilled water.

To prepare for use, mix I volume of each of the three stock solutions with 2 volumes of distilled water; for example, mix ro cubic centimeters of each of the solutions with 20 cubic centimeters of water (50 cubic centimeters in all). The mixture should have a clear blue color.

Place the material to be tested in the mixture in a test tube and boil for a few moments. If glucose is present, a red or orange precipitate of cuprous oxide will be formed.

Glycerine Jelly. - Soak for 2 hours 6 parts by weight of best gelatine in 2 parts by weight of distilled water, and add 7 parts by weight of pure glycerine. Add to each 100 grams of this I gram of crystallized or concentrated carbolic acid. Warm the mixture over a water bath and stir it until it is clear. Then strain it through filter paper placed in a funnel in a steamer or incubator to keep the mixture fluid enough for filtering. Wet the filter paper with distilled water before pouring in the mixture. Keep the jelly well stoppered and free from dust.

This is an excellent mounting medium for microscope objects. Use it as follows: Put a small piece of the jelly on a glass slip and slowly warm the slip over the flame of an alcohol lamp, or other suitable flame, until the jelly melts. Then place in the melted jelly the material to be mounted, which has first been brought into concentrated glycerine as directed under Safranin. Clean and warm a coverglass and carefully place it over the preparation by standing it on one edge and gradually lowering it so as not to entangle air bubbles. (See directions for doing 
this on page 374.) The jelly hardens on cooling and holds the coverglass firmly. After a few months the edge of the coverglass should be cemented to the slip by a thick solution of shellac in $95 \%$ alcohol, adding to each ro cubic centimeters of the solution 7 drops of castor oil.

Imbedding in Paraffin. - If material is to be imbedded in paraffin, it must be passed from the $70 \%$ alcohol, or the mixture of alcohol, glycerine, and water (see under Chrom-Acetic Fixative), through gradual degrees of concentration into a solvent of paraffin, such as chloroform, and finally into pure paraffin having a melting point close to $52^{\circ}$ C. Proceed as follows: From the $70 \%$ alcohol, or from the alcohol, glycerine, and water mixture, transfer the material successively to $80 \%$ alcohol, $95 \%$ alcohol, absolute alcohol, equal parts of absolute alcohol and chloroform, pure chloroform, a second pure chloroform, leaving it in each grade about 2 hours. Then transfer the material to a vial, and pour over it enough chloroform to cover it, but no more, and add a shaving of paraffin. Continue to add paraffin, a little at a time, until no more can be dissolved at the room temperature. Now place the vial on the copper plate of the paraffin bath described below, with enough asbestos paper or felt paper between it and the plate to keep it just below the melting point of pure paraffin, and add a few more bits of paraffin to the vial. Leave the vial standing thus until the chloroform is entirely evaporated, which will be when the paraffin no longer has a sweetish taste. If the paraffin should tend to solidify while driving off the chloroform, remove some of the paper separating the vial from the copper plate, for the paraffin should be kept in a fluid state. Pour the material, paraffin and all, into the dish of paraffin of the paraffin bath, and leave it there from 24 to 48 hours in order thoroughly to infiltrate the tissues with the paraffin. Then pour the material and melted paraffin into a small paper tray, made by turning up the edges of stiff writing paper for about half an inch. Heat dissecting needles in a blue flame, and arrange the material in orderly rows, leaving enough space between the pieces so that each piece can be cut from the block with a good border of paraffin about it. Now float the tray in a dish of cold water; blow upon the surface of the paraffin to harden it; and submerge the tray as soon as the surface film of paraffin can bear the weight of the water. In cutting out a piece of paraffin with an object imbedded in it, preparatory to mounting it on a microtome (see under Cutting and Mounting Paraffin Sections), score the paraffin block deeply on both sides with a knife 


\section{Reagents and Processes.}

in order to keep it from cracking across the other specimens in the block.

The paraffin bath can be simply made and operated as follows: Nearly fill a tumbler or other relatively tall dish with melted paraffin, and after the paraffin has hardened, set a copper plate over the tumbler, and leave one corner of the plate projecting far enough to permit a Bunsen burner or kerosene lamp to be placed under it. The flame should be kept at a height to melt the paraffin to a depth of about half an inch. When the material which has been gradually brought into concentrated paraffin as above directed is poured into the melted paraffin of the bath, it will sink to the bottom of the melted portion, where its infiltration will be completed just at the temperature of the melting paraffin; which is as it should be.

The object of gradually bringing the material into a solvent of paraffin and of infiltrating with paraffin slowly is to keep the protoplasts from shrinking together and to make the infiltration more complete.

Iodine Solution. - Dissolve 0.5 gram of potassium iodide in a few cubic centimeters of water, and add iodine until no more can be taken up. As a reagent for starch, dilute to a light brown color. For demonstrating proteids and protoplasts, dilute much less than for starch, - namely, to a red-brown color. By this reagent, starch is stained violet to dark blue, and proteids and protoplasts yellow to brown. Lignified, cutinized, and corky membranes are stained yellow.

Lime Water. - Pound up unslaked lime; place this in a bottle to one-third its capacity, fill the bottle with water, cork it and shake thoroughly. Keep the bottle stoppered, and after the lime has settled decant or filter off the clear liquid. Keep in a tightly stoppered bottle. Used to demonstrate $\mathrm{CO}_{2}$, which combines with it to form a white precipitate of calcium carbonate. .

Phloroglucin. - Dissolve in 15 cubic centimeters of $95 \%$ alcohol as much phloroglucin as is held on the point of a penknife. Use as a test for lignified membranes in the following manner: Place the material to be studied in the solution for a few moments; then transfer to a drop of water on a glass slip, and put on a coverglass. Place a drop of concentrated hydrochloric acid on the slip in contact with the coverglass. As the acid diffuses through the water, the lignified membranes will be stained pink.

The cellulose tissues of the section may now be stained purple by replacing the reagent under the coverglass with chloroiodide of zinc. 
To do this, place a drop of chloroiodide of zinc on the slip in contact with the coverglass, and place a piece of filter paper in contact with the opposite side of the coverglass. By this means the acid will be drawn from under the coverglass and the chloroiodide of zinc will occupy its place.

Safranin. - Dissolve safranin to saturation in $95 \%$ alcohol and dilute with an equal bulk of distilled water. This is very useful in staining sections of plant tissues, or for staining unicellular or filamentous Algæ or Fungi which have been fixed in chrom-acetic fixative (which see). The material should lie in the stain over night, or even for 24 hours. It should then be transferred to a small amount of $50 \%$ alcohol, and strong alcohol added to this drop by drop until the color in the material has a transparent quality, but is still quite evident. The material may now be mounted in a drop of dilute glycerine under a coverglass for immediate study with a microscope, or permanent mounts may be made in glycerine jelly as follows: Transfer the material from the alcohol rinsing bath to equal parts of glycerine and water, and leave it in a place free from dust until the glycerine has become concentrated by the evaporation of the water. Then mount it in glycerine jelly, as described under that head.

Staining Paraffin Sections, and Sealing in Balsam.- Having mounted paraffin sections as directed under Cutting and Mounting Paraffin Sections, and having dissolved away the paraffin in xylene and rinsed off the xylene with $95 \%$ alcohol, as there directed, the slides may be set in a tumbler or Stender dish containing safranin, for about 6 hours, or over night, then quickly rinsed in $95 \%$ alcohol, then placed in a tumbler of xylene, thence mounted in balsam as directed below. Or the sections may be double stained with cyanin and erythrosin, practically as directed under that head; leaving the slides over night in a tumbler of cyanin solution, then rinsing in a tumbler of $95 \%$ alcohol; by means of a drop tube covering the sections with clove oil saturated with erythrosin, while holding the slide horizontal ; after a moment draining off the clove oil and rinsing the slide in xylene, and then sealing the preparation in balsam.

But where the embryonic tissues of root and shoot tips, and developing pollen grains, ovules, etc., are to be studied with special reference to the construction and behavior of the protoplasts, the most beautiful and serviceable results in staining are achieved by the three-color method, employing safranin, gentian violet, and orange $\mathrm{G}$, made by Gribler and 
obtainable in this country from dealers in microscope supplies. The stains are made as follows :-

1. A saturated solution of safranin in $95 \%$ alcohol, diluted with an equal bulk of distilled water.

2. A saturated solution of gentian violet in distilled water.

3. A saturated solution of orange $G$ in distilled water, diluted with 5 times its bulk of distilled water.

The safranin and gentian violet should be kept ready for use in covered tumblers or Stender dishes, and the orange in a drop bottle.

In addition to the stains there should be conveniently at hand -

4. A drop bottle containing absolute alcohol.

5. A drop bottle containing clove oil.

6. A tumbler of xylene.

7. A tumbler of $95 \%$ alcohol acidulated with I drop of concentrated hydrochloric acid.

Proceed with the staining as follows:-

1. Set the slide upright in the dish of safranin for a few hours or over night. (The paraffin having been dissolved away in xylene, and the latter having been rinsed off in a dish of $95 \%$ alcohol.)

2. Rinse the slide quickly in water and place it in the dish of acidulated alcohol (No. 7 above) until the safranin ceases to come away in clouds. The sections should appear almost decolorized.

3. Place the slide in the dish of gentian violet for 10 minutes.

4. Rinse off the gentian violet quickly with water and flood the sections with orange $\mathrm{G}$ from the drop bottle for 4 seconds.

5. Rinse off the orange with water, and thoroughly dehydrate by holding the slide slanting, sections upward, and dropping absolute alcohol over the sections from the drop bottle.

6. Set the slide in a horizontal position and drop clove oil from the drop bottle over the sections. The preparation should now be watched under low power of the microscope, and when the gentian violet has lost its too great intensity and has a transparent quality, drain off the clove oil and set the slide in the dish of xylene and let it remain there until ready to seal the mount in balsam. If the sections have been successfully stained, the cytoplasm will be stained from gray to orange, the resting nucleus violet, the nucleolus red. In the dividing nucleus the chromosomes will be red and the spindle fibers violet. Cutinized membranes will be red, lignified membranes blue, and cellulose membranes will be almost colorless. 
To seal the preparation in Canada balsam, remove the slide from the dish of xylene and place it in a horizontal position. Place a small drop of balsam to the left end of the group of sections. Grasp a thoroughly clean and dry coverglass between the thumb and forefinger of the left hand. Rest the lower edge of the coverglass on the slide close to the drop of balsam, and lower it to the right over the sections. Support the upper end of the coverglass by means of a dissecting needle held in the right hand, and let the coverglass down slowly, so as to drive all air bubbles forward and out from under it. During this process keep the coverglass from sliding toward the left by means of a needle. If the coverglass is of the oblong or square form it is often needful, when it has nearly reached the horizontal position, to grasp its edges by means of the thumb and middle finger of the left hand and press it down against the slide with the tip of the forefinger, with a sliding movement from the left toward the right end of the coverglass. In this way the balsam, if scantily put on, is forced to the extreme edge of the coverglass.

The slide should now be kept at about $52^{\circ} \mathrm{C}$. for several days to harden the balsam. 
83.

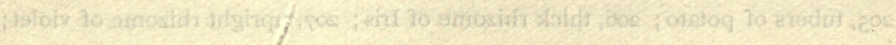
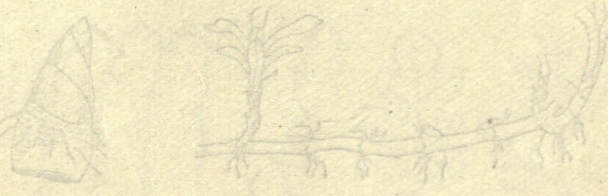

sos

PART III.

GLOSSARY.
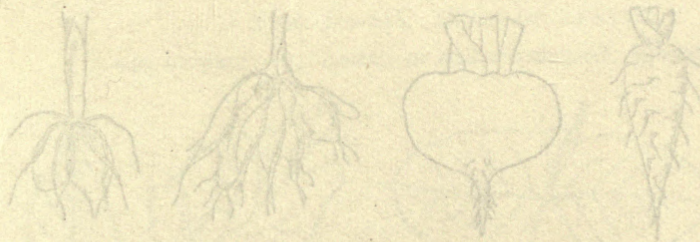


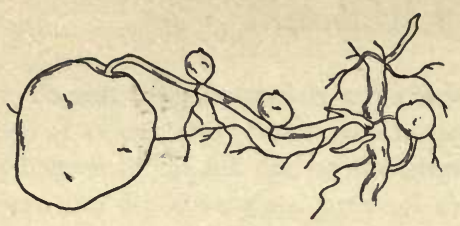

205

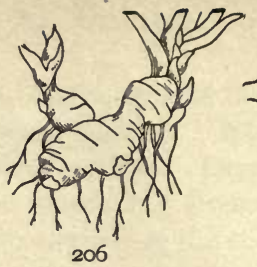

206

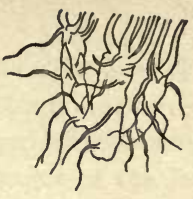

207

Figs. 205-2I3. FORMS OF UNDERGROUND STEMS.

205 , tubers of potato; 206 , thick rhizome of Iris ; 207 , upright rhizome of violet;

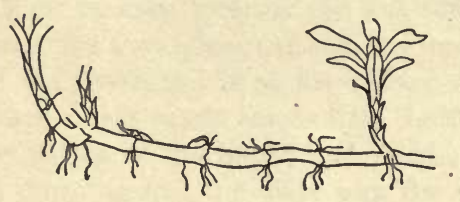

208

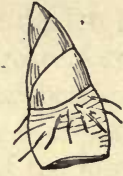

209

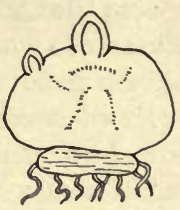

2IO

208, running rhizome of goldenrod; 209, corm of Trillium; 210, longitudinal section of a corm or solid bulb;

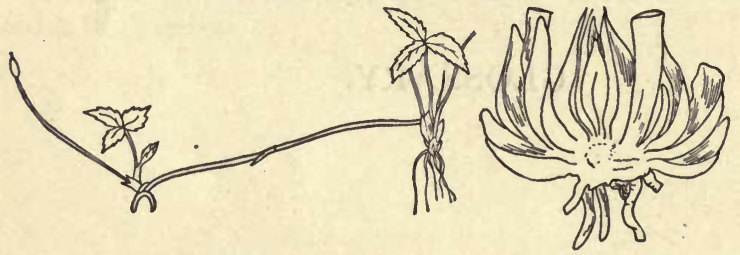

$2 I I$

212

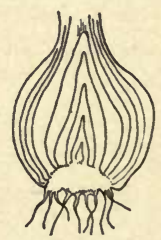

$2 I_{3}$

2II, runner or stolon of strawberry; 212 , scaly bulb of tiger lily; 213 , tunicated bulb of onion.

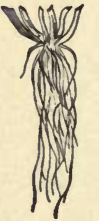

2I4

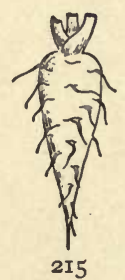

215

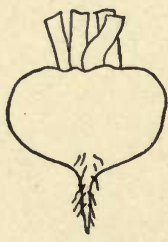

216

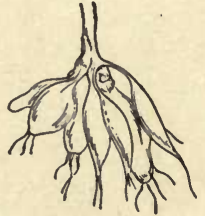

217

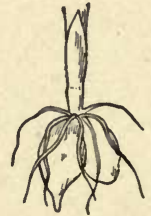

218

FIGS. 214-2I8. FORMS OF ROOTS.

214 , fibrous roots; 215 , conical root; 216 , napiform root; 217 , tuberous roots, the individual roots fusiform; 218 , fibro-tuberous roots. 


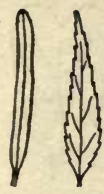

219220

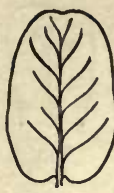

$22 I$

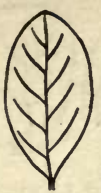

222

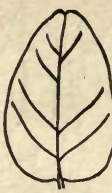

223.

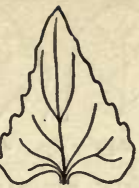

224

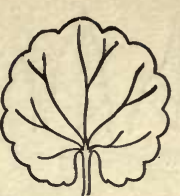

225

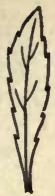

226

FIGS. 219-228. FORMS OF LEAVES.

219, linear; 220, lanceolate; 22I, oblong; 222, elliptical ; 223, ovate; 224 , cuneate; 225, orbicular; 226, oblanceolate; 227, reniform or kidney-shaped; 228, spatulate.

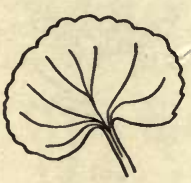

227

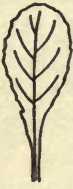

228

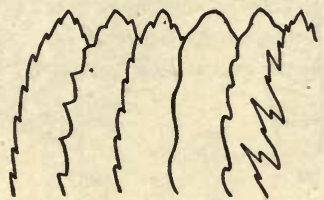

$229 \quad 230 \quad 231 \quad 232 \quad 233234$

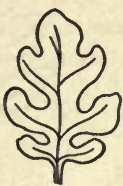

235

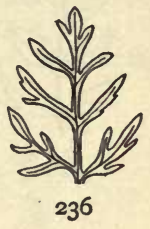

236

FIGS. 229-239. LEAF MARGINS.

229, serrate; 230, dentate; 23I, crenate-serrate. The divisions would be called crenate when rounded, with apices pointing outward instead of upward. 232, undulate; 233 , sinuate; 234 , pinnately lobed or incised; 235 , more deeply pinnately lobed, or parted; 236 , pinnately divided; 237 , palmately lobed; 238 , palmately parted; 239, palmately divided.

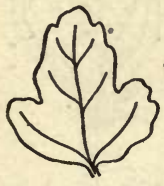

237

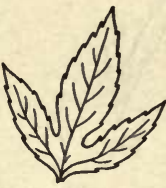

$23^{8}$

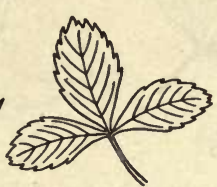

239

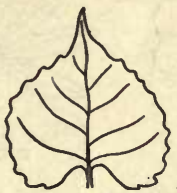

240

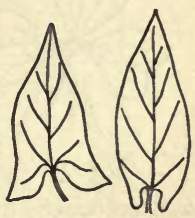

$24 I \quad 242$

Figs. 240-246. BASES OF LEAVES. 240 , cordate; $24 \mathrm{I}$, hastate or halberd-shaped; 242 , auriculate ;

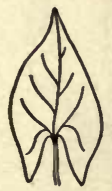

243

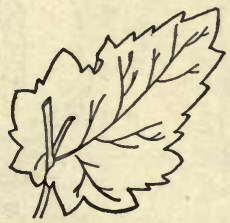

244

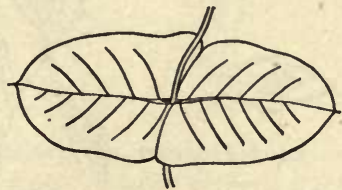

245

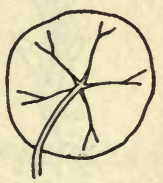

246

243 , sagittate ; 244 , perfoliate ; 245 , connate-perfoliate ; 246 , peltate. 


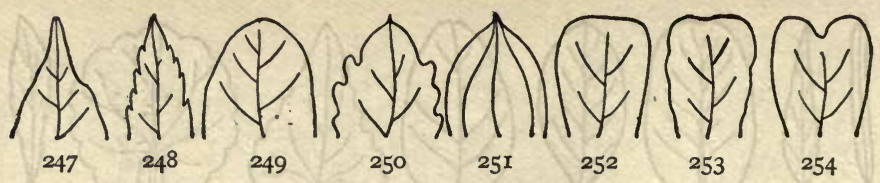

FIGS. 247-254. APICES OF LEAVES.

247, acuminate; 248, acute; 249, obtuse; 250, mucronate; 25I, cuspidate; 252 , truncate; 253 , retuse; 254 , emarginate.

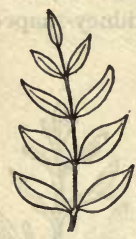

255

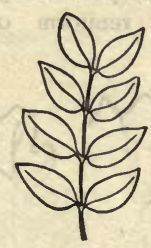

256

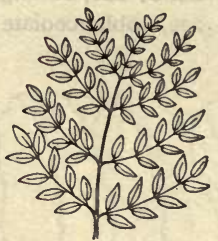

257

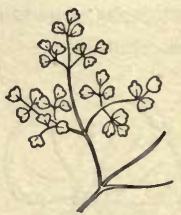

$25^{8}$

Figs. 255-260. CoMpound Leaves.

255 , oddly pinnately compound; 256, abruptly pinnately compound; 257 , bipinnately compound; $25^{8}$, ternately decompound (the two primary divisions on the right are similar to the completed one on the left);

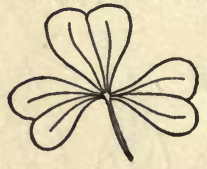

259

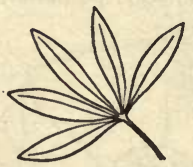

260

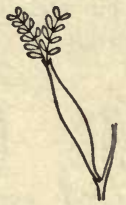

$26 r$

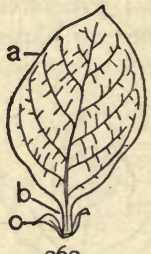

262

259, palmately trifoliate; 260 , palmately five foliate; 261 , a phyllodium, the petiole expanded in the form of a leaf; 262 , the parts of a leaf: $a$, the blade; $b$, the petiole; $c$, the stipules;

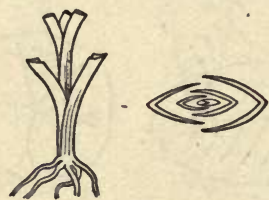

263

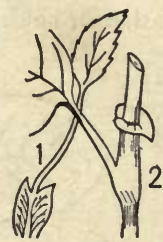

264

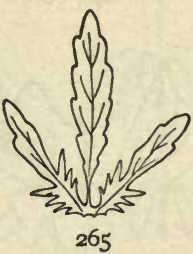

265

263, equitant leaves of Iris; 264 , I, stipules adherent to the base of the petiole; 264,2 , stipules clasping the stem and forming an ocrea; 265 , leaf-like stipules. 


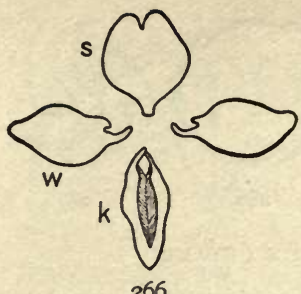

266

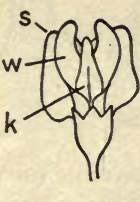

267

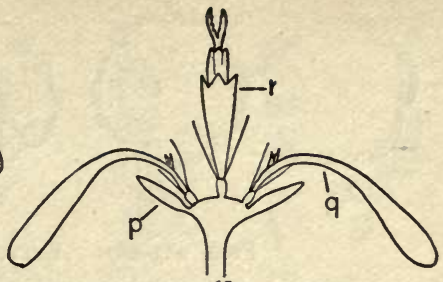

268

FIGS, 266-282. FORMS OF COROLLAS.

266, papilionaceous: $s$, the standard (in the sense of flag or pennant); $w$, the wings; $k$, the carina or keel; 267 , a papilionaceous flower seen from the under side, lettering as in $266 ; 268$, longitudinal diagram of the inflorescence of one of the Compositæ: $p$, bract of involucre; $q$, ligulate ray flower; $r$, tubular disk flower;

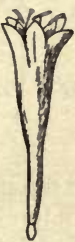

269

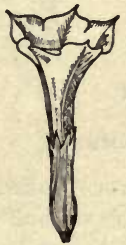

270

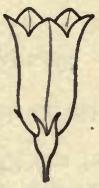

27 I

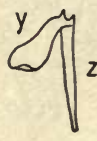

272

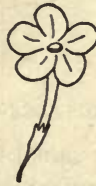

273

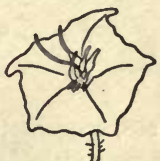

274

269, trumpet-shaped; 270, funnel-form; 271, campanulate; 272 , parts of a petal: $y$, the limb; $z$, the claw; 273 , salver-shaped; 274 , rotate;

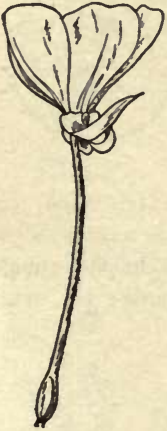

$27^{8}$

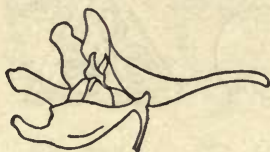

275

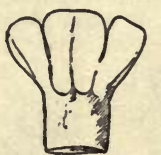

279

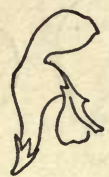

276

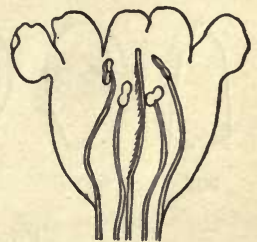

277

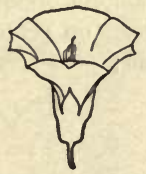

280

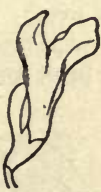

$28 I$

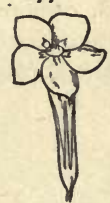

282

275, bilabiate-personate; 276, bilabiate; 277, inflated-tubular corolla of Pentstemon split open and showing the sterile filament between the four fertile ones; 278 , flower of evening primrose with inferior ovary and so-called calyx tube prolonged above it; 279, tubular-rotate; 280 , rotate-funnel-form; $28 \mathrm{I}$, bilabiate, with helmeted calyx; 282, trumpet-salver-form. 


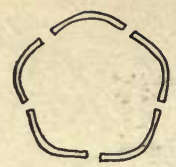

283

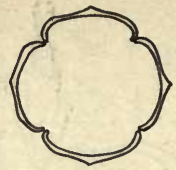

284

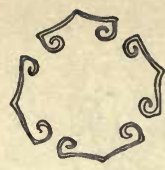

285

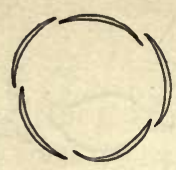

286

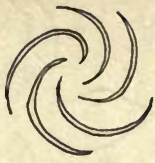

287

Figs. 283-287. Astivation OF the Petals.

283 , valvate; 284 , induplicate ; 285 , involute; 286 , imbricate ; 287 , convolute.

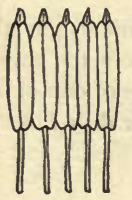

288

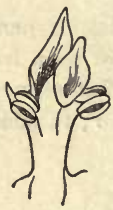

289

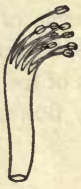

290

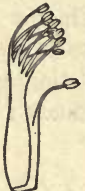

$29 \mathrm{I}$

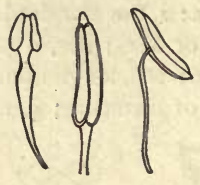

292293

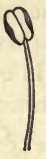

295

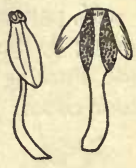

$296 \quad 297$

Figs. 288-297. Pertaining to Stamens.

288, syngenesious, united by the anthers; 289 , gynandrous, united to the pistil ; 290, monadelphous, united by the filaments ; 29r, diadelphous, united in two sets ; 292, anther innate; 293, anther adnate; 294, anther versatile; 295, anther dehiscing by longitudinal slits; 296 , anther dehiscing by terminal chinks or pores; 297 , anther dehiscing by uplifted valves.

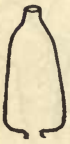

298

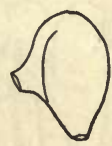

299

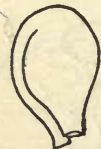

300

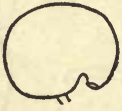

3 이

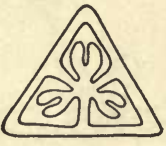

302

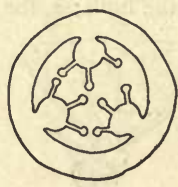

303

Figs. 298-308. Pertaining to OvUles.

298 , orthotropous ovule; 299 , amphitropous ovule; 300 , anatropous ovule; 301 , campylotropous ovule; 302 , ovules on central or axial placentæ; 303 , ovules on parietal placentæ, pistil compound;

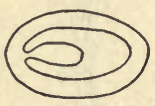

304

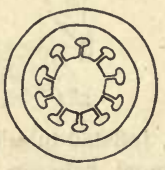

305

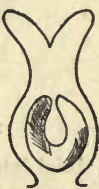

306

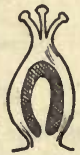

307

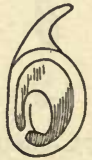

308

304 , ovules on parietal placenta, pistil simple; 305 , ovules on a free central or axial placenta; 306 , ovule suspended ; 307 , ovule erect; 308 , ovule ascending. 


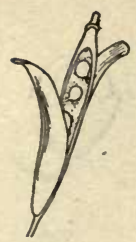

309

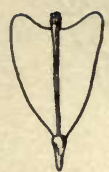

310

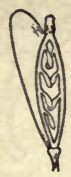

3II

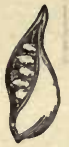

$3^{12}$

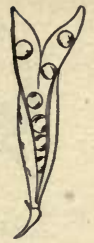

313
3I4

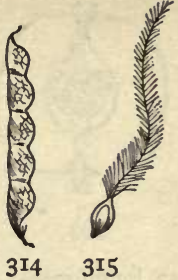

Figs. 309-340. Pertaining to Fruits.

309, silique; 310, sillicle; 3 II, one carpel of 3 Io removed, showing ovules; $3^{12}$, follicle; $3^{13}$, legume; $3{ }^{14}$, loment $; 3^{15}$, achene, with persistent plumose style;

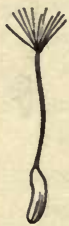

3 I6

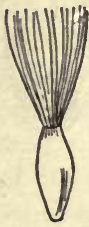

$3^{17}$

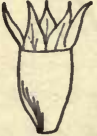

318

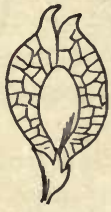

319

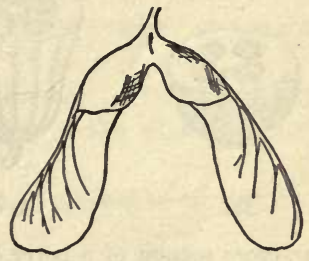

320

3I6, achene with hairy pappus (calyx) borne on a long beak; $3 I 7$, achene with capillary pappus; $3^{18}$, achene with pappus in the form of scales; $3^{19}$, samara, winged all around; 320 , double samara of maple;

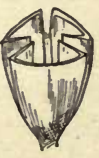

321

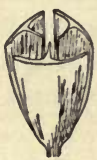

322

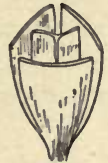

323

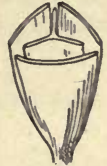

324

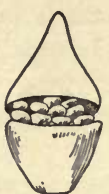

325

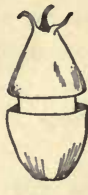

326

321 , septicidal dehiscence; 322 , loculicidal dehiscence; 323 , septifragal dehiscence; 324 , another mode of septifragal dehiscence; 325 , circumscissile dehiscence ; 326 , utricle ;

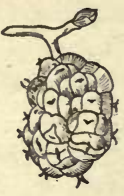

327

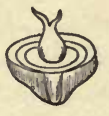

328

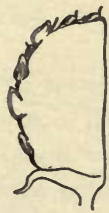

329

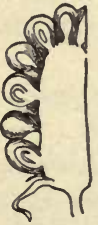

330

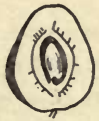

$33^{r}$

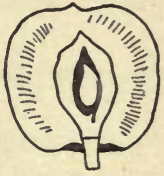

$33^{2}$

327 , multiple fruit; 328 , section of a single fruit from $327 ; 329$, longitudinal section of an accessory fruit (strawberry); 330 , longitudinal section of an aggregate fruit (blackberry); $33 \mathrm{I}$, a single stone fruit from $330 ; 332$, longitudinal section of a drupe (peach) ; 


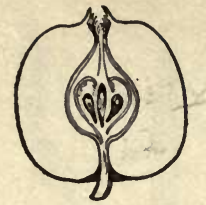

333

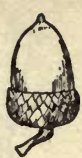

334

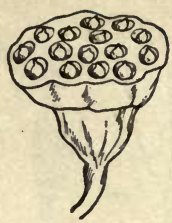

335

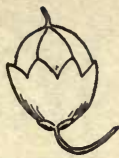

336

333 , longitudinal section of a pome (apple) ; 334 , nut of the acorn type; 335 , receptacle and nuts of the water lily; 336 , a berry;

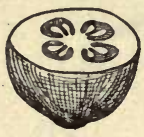

337

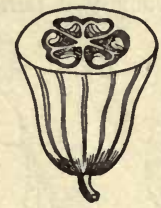

$33^{8}$

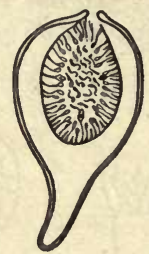

339
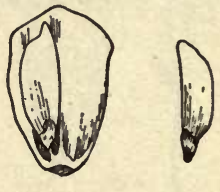

340

337 , cross section of a berry (cranberry) ; $33^{8}$, cross section of a pepo or gourdfruit; 339 , longitudinal section of a syconium or fig-fruit; 340 , an ovule-bearing scale of the pine with one ripened ovule or seed in position, the other seed removed and shown by itself on the right. 


\section{GLOSSARY.}

In giving the derivation of words in the Glossary, the abbreviation $\mathrm{L}$. signifies that the root word is Latin, and Gr. that it is Greek; pr.=pronounced; pl.=plural.

Acaulesc'ent (Gr. a, without; L. caulis, a stem): with little or no apparent stem above ground.

Acces'sory fruit (L. accessus, an accession or increase): a fruit having the receptacle or other parts as an important part of the whole, as in the strawberry. Fig. 329.

Accumb'ent (L. accumbere, to lie down or recline) : applied to cotyledons in the seed when lying against the hypocotyl.

Achene', pr. a-kēn' (Gr. a, without or not; chainein, to gape open) : a small, dry and hard, indehiscent, one-seeded fruit consisting of a single carpel. Figs. 316-318.

Achlamyd'eous (Gr. a, without; chlamys, a cloak) : without calyx and corolla. Acic'ular (L. acicula, diminutive of acus, needle) : slender or needle-shaped. Acu'leate (L. aculeus, a sting or prickle) : prickly, as the stem of the rose. Acu'leolate: somewhat prickly.

Acu'minate (L. acuminare, to sharpen): gradually tapering to a point. Fig. 247.

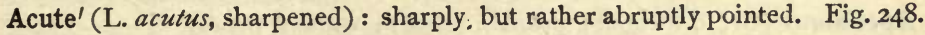
Ad'nate (L. adnascor, to grow to): united or growing together - applied only to the union of unlike parts, as of stamens to petals. When the term is applied to anthers, it signifies that they are attached for their whole length to the inner or outer face of the filaments. Fig. 293.

Adventi'tious (L. adventicius, unusual) : out of the usual place - applied to buds and roots.

Estiva'tion (L. astivare, to spend or pass the summer): the arrangement of the parts of the flower in the bud. Figs. $283-287$.

Ag'gregate fruit (L. ad + gregario, to collect into a flock or herd, from grex,

a flock or herd): a fruit having the carpels assembled over a common receptacle, as in the blackberry. Fig. 330 .

A'late, pr. $\bar{a}^{\prime}$ late (L. ala, a wing): winged, or having a thin expansion like the fruit of an elm. Fig. 319.

Albu'men (L. albus, white) : reserve food materials stored in a seed.

Albu'minous : having albumen. 
Allia'ceous (L. allium, garlic): smelling like garlic or onions.

$\mathrm{Al}^{\prime}$ veolate (L. alveolus, a hollow or cavity) : pitted so as to appear somewhat

like a honeycomb.

Am'ent (L. amentum, thong or strap) : a bracted unisexual spike.

Amphit'ropous (Gr. amphi, around; trope, a turn): applied to ovules or seeds which are half inverted, with the point of attachment near the middle of one side. Fig. 299.

Amplex'icaul (L. amplecti, to encircle ; caulis, a stem): applied to petioles or stipules encircling the stem. Fig. 264, 2.

Anat'ropous (Gr. ana, up; trope, a turn): having the ovule inverted as shown in Fig. 300.

Androg'ynous (Gr. aner, andros, man; gyne, woman): having staminate and pistillate flowers on the same inflorescence.

An'giosperm (Gr. aggeion, vessel; sperma, seed): plant having seeds inclosed in an ovary.

An'nual : enduring for a year. Annual ring: that portion of the wood of stem or root produced by one year's growth. Fig. 49 .

An'nular (L. annulus, a ring) : disposed in a circle.

An'ther (Gr. antheros, flowery): that portion of the stamen containing the pollen. Fig. 87 .

Antherid'ium (the Greek diminutive ending idion + anther): the organ of cryptogams which produces the sperms. Figs. $\mathbf{I}_{5} \mathbf{I}$ and $\mathbf{1}_{5} 6$.

Antherozo'id (Gr. zoon, animal; eidos, resemblance + anther): motile sperms produced in some cryptogams and in Cycads. Fig. 156. Same as Sperm. Anthe'sis (Gr. anthesis, flowering): the time or condition of expansion of the flower.

Apet'alous (Gr. a, without ; petalon, a leaf) : without petals.

Apic'ulate (L. apiculum, a little point) : ending in a short, sharp, but not hard point.

Appressed' ${ }^{\prime}$ : close and flat against.

Arach'noid (Gr. arachne, spider, or spider's web ; eidos, resemblance) : cobwebby.

Archego'nium (Gr. arche, beginning; gone, race): the organ of cryptogams and gymnosperms which produces the eggs. Figs. ${ }_{5} \mathbf{I}$ and 156.

Are'olate (L. areola, diminutive of area): divided into small spaces.

$\mathrm{Ar}^{\prime}$ il : an expansion arising from the base of a seed and enveloping it, as in the seed of the white water lily. Ar'illate : having an aril.

Ascend'ing : rising obliquely. Ascending ovule : ascending from just above the base of the ovary. Fig. 308.

Asexual spore: a spore produced by the division of a mother cell without previous cell union. 


\section{Glossary.}

Aur'icle (L. auricule, diminutive of auris, ear): an ear-shaped appendage. Fig. 242.

Auric'ulate : provided with auricles.

Awn: a bristle-like appendage.

$A x^{\prime}$ il (L. axilla, armpit): the angle formed between the axis of a stem and any part arising from it. Ax'illary: situated in an axil.

$A x^{\prime}$ is (L. axis, axle): the central line of an organ.

Barbed (L. $b a r b a$, beard): provided with rigid points or bristles, usually reflexed. Bar'bellate: finely barbed.

Bar'bulate : finely bearded.

Bast : the fibrous part of the bark.

Bearded : awned, as spikes of wheat; or having tufts of hairs.

Berry : a fleshy or pulpy fruit, such as a gooseberry, grape, tomato, cranberry, etc. Fig. 337.

Bi-, bis- : Latin, in compound words signifying twice.

Bident'ate (L. dens, a tooth): having two teeth.

Bien'nial (bi- + L. annus, a year) : a plant which requires two years to complete its life cycle; usually flowering and fruiting the second year only, and then dying.

Bi'fid (L. bi- + findere, to cut): two-cleft.

Bila'biate (L. bi- + labium, a lip) : divided into two lips. Figs. 275 and 276. Biloc'ular (L. bi- + loculus, a compartment): two-celled.

Bipin'nate (L. bi- + pinnatus, feathered; pinna, a feather): having both primary and secondary divisions of a compound leaf pinnate. See Pinnate. Figs. 255-257.

Bladdery: thin and inflated.

Blade : the expanded portion of a leaf. Fig. 262, $a$.

Bract (L. bractea, a thin plate): a modified or reduced leaf subtending a flower, or belonging to an inflorescence. Fig. 268, $p$.

Bract'eate : provided with bracts.

Bract'eose : having numerous or conspicuous bracts.

Bract'let: a secondary bract, occurring on the pedicel of a flower instead of subtending it. Bract'eolate: having bractlets.

Bulb (L. bulbus, a bulb): a subterranean bud with fleshy scales or coats. Figs. 212 and 213.

Bulbif'erous (bulb + L. fero, I bear) : bearing bulbs.

Bulb'let: a small bulb, usually one borne above ground on the stem.

Bulbous: having the character of a bulb.

Cadu'cous (L.caducus, falling) : falling off very early; applied to petals which fall as the flower opens. 
Cal'lus (L. callosus, hard) : a hard protuberance; or the hardened coating which forms over the end of a cutting which has been inserted in the soil. Calyp'tra (Gr. calyptra, a veil): the membranous covering over the capsule of a moss, formed from the apex of the archegonium. Fig. x 50, c.

Ca'lyx (Gr. calyx, a cup): the outer whorl of floral envelopes. Fig. 87 . Campan'ulate (L. campana, a bell): bell-shaped. Fig. 271.

Campylot'ropous (Gr. campylos, curved; trope, a turn): applied to an ovule which has curved so that the micropyle is about on a plane with the hilum. Fig. 3or.

Canalic'ulate (L. canalis, a canal or channel) : longitudinally grooved.

Canes'cent (L. canescens, growing gray): hoary from a gray pubescence.

Cap'itate (L. caput, the head): head-shaped, or collected into a head. Fig. $82, t$ and $u$.

Cap'sule (L. capsula, a small box): the spore-case of mosses; a dry fruit of more than one carpel, which opens to discharge its seeds. Figs. 321-324.

Car'inate (L. carina, a keel) : having a median longitudinal projection on the lower surface.

Car'pel (Gr. carpos, fruit) : a simple pistil, or a single element of a compound pistil; a single female sporophyll.

Car'uncle (L. caruncula, diminutive of caro, flesh): a wart or protuberance near the hilum of a seed, as in seed of castor bean.

Caryop'sis (Gr. karyon, nut; opsis, resemblance): a seed-like fruit with the capsule closely adherent to the seed, as in corn and other grasses.

Cat'kin: same as Ament.

Caud'ate (L. cauda, a tail) : having a tail-like appendage.

Cau'dex (L. caudex, stem or trunk): the main axis of a plant; also the perennial rootstock of an otherwise annual plant. Figs. 206 and 207.

Caules'cent (L. caulis, stalk or stem): having an apparent stem above ground. Cau'line, pr. kaw'lin or kaw'line: belonging to the stem.

Ces'pitose (L. cespes, turf): matted, or growing in tufts.

Chaff : a dry or membranous scale or bract.

Charta'ceous (L. charta, paper): having a papery texture.

Chlo'rophyll (Gr. chloros, grass-green; phyllon, a leaf): the green coloring matter of plants.

Chlo'roplast (Gr. chloros + plastos, molded): the plastid or grain of protoplasm which contains the chlorophyll. Fig. 36 .

Chro'moplast (Gr. chroma, color + plastos): plastid containing other coloring matter than chlorophyll.

Chro'mosome (Gr. chroma + soma, a body): one of the strongly staining bodies of definite number into which the body of the nucleus divides in nuclear division. Fig. 46 . 


\section{Glossary.}

Cil'iate (L. cilium, eyelid): fringed with hairs.

Cil'iolate: fringed with minute hairs.

Cine'reous (L. cinis, cineris, ashes): ashen or grayish.

Cir'cinnate (L. circinare, to make round): coiled downward from the tip. Sometimes spelled with one $n$.

Circumscis'sile (L. circum, around; scindo, scissus, to split): dehiscing by transverse circular cleavage. Fig. 325 .

Cla'vate (L. clava, a club) : club-shaped; thickening toward the apex.

Claw : the narrowed base of certain petals. Fig. 272, $z$.

Cleft: split nearly to the middle. Fig. 235.

Cleistog'amous (Gr. kleistos, shut or closed; gamos, marriage): applied to flowers which do not open, but become self-fertilized in the bud.

Coales'cent (L. coalesco, to grow together): having organs of the same kind grown together.

Coated : applied to bulbs, such as the onion, having inwrapping scales.

Coch'leate (L. cochlea, snail or snail shell): spirally coiled.

Cohe'rent (L. cohaereo, to cling to) : applied to organs of the same kind which are united, as in the union of the petals to form a gamopetalous corolla.

Com'missure (L. commissura, a joining together): the face along which one carpel joins another, as in the Umbelliferæ.

Co'mose (L. comosus, hairy): having a tuft of hair.

Com'plicate: folded upon itself.

Com'pound: consisting of two or more united similar parts. Compound ovary: consisting of two or more united carpels.

Compressed' : flattened laterally.

Condu'plicate : folded together lengthwise.

Cone: fruit of the pine, etc., with ovule-bearing scales. Figs. 93 and 94.

Con'fluent: blended into one.

Con'ical (Gr. konos, a cone) : round and tapering to a point. Fig. 215.

Conif'erous (Gr.konos, a cone; L. ferre, to bear): cone-bearing.

Con'nate (L. con-, with, together; natus, born): united, particularly at the base. Connate-perfoliate: applied to leaves which are united at the base around the stem. Fig. 245 .

Connect'ive: portion of the filament which connects the two lobes of an anther. Fig. 292.

Conni'vent: converging or coming into contact.

Con'volute : rolled up longitudinally.

Cor'date (L. cor, heart) : heart-shaped, with the point remote from the place of attachment. Fig. 240.

Corm (Gr. kormos, a trimmed tree trunk) : a solid bulb-like expansion at the base of some stems below ground. Figs. 209 and 210. 
Corol'1a (L. corolla, diminutive of corona, a crown or garland): the inner whorl of the floral envelope, that is, the petals taken collectively, whether distinct or united. Fig. 87 .

Coro'niform (L. corona, a crown; forma, form) : shaped like a crown.

Cor'ymb (L. corymbus, a cluster of flowers or fruit): a flat-topped or merely convex cluster of flowers whose pedicels arise at different heights on the main axis, the older flowers being outermost. Fig. $83, x$.

Cor'ymbose : in corymbs, or corymb-like.

Cos'tate (L. costa, a rib): having one or more longitudinal primary ribs.

Cotyle'dons (Gr. kotyledon, any cup-shaped hollow or cavity) : the first leaves of an embryo.

Creeping : running along or over the ground and rooting. Fig. 211.

Cre'nate (L. crena, a notch) : having margins with rounded teeth. Fig. 23I.

Cren'ulate: finely crenate.

Crusta'ceous (L. crusta, a crust): hard and brittle.

Cryp'togam (Gr. kryptos, hidden; gamos, marriage): plant without flowers in the usual sense, and without true seeds, such as the ferns, mosses, and lower plants.

$\mathrm{Cu}^{\prime}$ cullate, pr. kü'kull-ate (L. cucullus, cap or hood): hooded or hoodshaped.

Culm (L. culmus, stalk or stem, especially of cereals): the hollow stem of grasses.

$\mathrm{Cu}^{\prime}$ neate (L. cuneus, a wedge) : wedge-shaped. Fig. 224 .

Cus'pidate (L. cuspis, a point): terminating in a hard and sharp point. Fig. $25 \mathrm{I}$.

Cylindra'ceous (Gr. kylindros, a roller) : somewhat cylindrical.

Cyme (Gr. kyma, anything swollen, a wave or billow): a flat or convex flower cluster similar to a corymb, but having the innermost flowers oldest. Fig. 82, v. Cy'mose : cyme-like, or bearing cymes.

Cy'toplasm (Gr. kytos, a hollow vessel; plasma, anything formed or molded): the general protoplasm of the cell exclusive of nucleus and plastids.

Decid'uous (L. decidere, to fall down): falling off; not evergreen.

Decom'pound : more than once compound or divided. Figs. 257 and $25^{8}$.

Decum'bent (L. decumbens, reclining) : reclining as if too weak to stand, and tending to rise at the apex.

Decur'rent (L. decurrens, running down): applied to a leaf whose base extends downward below the insertion and forms a sort of wing along the stem.

Decurved': curved downward.

Deflexed' : curved or bent abruptly downward. 


\section{Glossary.}

Dehis'cence : definite mode of opening of capsules and anthers. Figs. 295297 and $321-326$.

Dehis'cent (L. dehiscere, to yawn) : splitting open in a definite way.

Del'toid : shaped like the Greek letter $\Delta$ (delta).

Den'tate (L. dens, a tooth): toothed, usually with the teeth directed outward. Fig. 230. Dentic'ulate : minutely dentate.

Depau'perate (L. depauperatus, impoverished) : much reduced in size.

Depressed $d^{\prime}$ : somewhat flattened from above.

Deter'minate : applied to the growth of stems where increase in length is terminated by a winter bud; applied to inflorescences where flowering begins with a terminal or inner bud, as in cymes. Fig. 82, v.

Di-, dis-: Greek prefix signifying two or twice.

Diadel'phous (Gr. di- + adelphos, brother): said of stamens when combined in two sets. Fig. 29I.

Dian'drous (Gr. di- + aner, andros, man): having two stamens.

Dicarp'ellary (Gr. di- + karpos, fruit) : composed of two carpels.

Dichog'amy (Gr. dicha, asunder; gamos, marriage): the condition of flowers

whose stamens and pistil do not mature simultaneously, preventing selffertilization.

Dichot'omous (Gr. dicha + temnein, to cut) : forking regularly in pairs.

Dicotyle'don : a plant having two cotyledons.

Did'ymous (Gr. didymos, twin): occurring in pairs.

Didyn'amous (Gr. di- + dynamis, power): applied to stamens when in two pairs of unequal length.

Diffuse' : widely or loosely spreading.

Dig'itate (L. digitus, a finger) : applied to a compound leaf whose leaflets are all borne at the apex of the petiole. Figs. 259 and 260.

Digitately: applied to leaves which are lobed, cleft, parted, or divided in digitate manner, as in Figs. 237-239.

Dim'erous (Gr. di- + meros, a part) : applied to a flower having all of its parts in twos.

Dimor'phous (Gr. di- + morphe, form) : occurring in two forms. Fig. $9 \mathbf{I}$. Diœ'cious, pr. di-ê'shus (Gr. di- + oikos, a house) : having only unisexual flowers, the staminate and pistillate flowers being borne on different individuals.

Dis'coid (Gr. diskos, a round plate; eidos, form) : resembling a disk; applied to the heads of Compositæ which are composed of disk flowers only.

Disk : the central part of the head of Compositæ as opposed to the margin. Dissect'ed : deeply cut into many divisions.

Dissep'iment (dis- + L. salpire, to inclose): the separating membrane or wall in a compound ovary or fruit. 
Dis'tichous (di- + Gr. stichos, a row or verse): in two vertical rows.

Distinct' : separate, not united.

Divar'icate (di- + L. varicare, to straddle): widely diverging.

Diver'gent: inclined away from each other.

Divi'ded : lobed or segmented to the base or midrib. Figs. 236 and 239.

Dor'sal (L. dorsum, the back): the surface of a member turned away from

the main axis; thus, the dorsal surface of a leaf is its under surface.

Drupa'ceous : producing or having the form of a drupe.

Drupe (L. drupa, an overripe olive): a fleshy or pulpy fruit with the inner portion hard or stony. Figs. 331 and 332.

E- or ex-: in compound words meaning out from, without, or destitute of.

Ebe'neous : black as ebony.

Ebrac'teate : without bracts.

Ech'inate, pr. ek'inate (L. echinus, a hedgehog): beset with bristles; like a hedgehog.

Effuse' $^{\prime}$ : very loosely spreading.

Egg: the female cell which after fusion with the sperm develops into an embryo or new plant.

Ela'ter (Gr. elater, a driver): a spirally marked thread borne among the spores of some liverworts and slime moulds.

Ellipsoi'dal (Gr. elleipsis, ellipse; eidos, form): shaped like an ellipse.

Emar'ginate: having a decided terminal notch. Fig. 254.

Em'bryo (Gr. embryon, an embryo): the rudimentary plant within a seed.

En'docarp (Gr. endon, within; karpos, fruit): the inner layer of a matured ovary.

En'dogen (Gr. endon + genos, descent or birth): a plant among the Spermatophytes without a true cambium ring, as in grasses and monocotyledonous plants in general.

Endog'enous : pertaining to an endogen; arising from deep-seated tissues. Entire' : with undivided margin.

Ephem'eral (Gr. ephemeros, daily; from epi, over, and hemera, day) : lasting only one day.

Ep'icarp (Gr. epi, upon or over; carpos, fruit) : outer layer of a mature ovary. Epicot'yl (Gr. epi + cotyle, hollow vessel): the young shoot above the cotyledons.

Epider'mis (Gr. epi + derma, skin): the outer protective layer of cells of leaves, young stems and roots, and fruits. In old stems and roots the epidermis becomes permanently replaced by cork.

Epig'ynous (Gr. epi + gyne, woman): growing from the summit of the ovary, or apparently so. Fig. 123, $C$. 


\section{Glossary.}

Ep'iphyte (Gr. epi + phyton, plant): a plant which grows upon other plants, but not parasitically; an air plant. Fig. I6.

Eq'uitant (L. equitare, to ride): said of leaves which are folded longitudinally so as to bestride those next above and within, as in Iris. Fig. 263 .

Erose' : (L. e, out; rodere, to gnaw): irregularly jagged as if gnawed.

Essential organs : stamens and pistils.

Evanes'cent (L. evanescere, to vanish): of short duration.

$\mathrm{Ev}^{\prime}$ ergreen: bearing green foliage throughout the year.

Exalbu'minous (L. ex, without + albumen, which see): said of seeds having the reserve materials stored entirely within the embryo, as in Lima bean.

Excur'rent (L. ex, out; currens, running): applied to the midrib or veins of a leaf when they project beyond the margin; also to a stem or trunk when it persists as the main axis to the top. Fig. 27.

Exfo'liate (L. ex, from; folium, leaf) : to peel off in scales or thin layers.

Ex'ogen (Gr. ex, out; genos, descent or birth): a plant with a true cambium ring which adds new wood to the outside of that already formed.

Exog'enous : belonging to the exogens; having the structure of an exogen.

Exsert'ed (L. exserere, to stretch out or forth): applied to stamens which protrude beyond the tube of the corolla.

Exstip'ulate (L. ex, in the privative sense of without; and stipulate): without stipules.

Ex'tine, pr. ex'tin or ex'teen (L. exter, on the outside): the outer membrane of a pollen grain.

Extrorse' (L. extra, on the outside; versus, toward): facing outward; applied to anthers which occupy the outside of a filament.

Fal'cate (L. falx, sickle or scythe): sickle- or scythe-shaped.

Farina'ceous (L. farina, meal) : containing starch; starchy.

Far'inose : covered with a mealy powder.

Fas'cicle (L. fasciculus, diminutive of fascis, a bundle) : a close bundle or cluster of flowers, leaves, stems, or roots.

Fastig/iate (L. fastigium, gable end or top): upright and parallel in clusters.

Fave'olate (L. favus, honeycomb): honeycombed.

Ferru'ginous (L. ferrugo, iron rust): rust color.

Fer'tile (L. fertilis, capable of producing fruit) : applied to flowers with pistils, or to anthers with pollen.

Fi'brillose (L. fibra, a small fiber or filament): covered with hair-like appendages.

Fi'brous : containing or consisting of fibers. 
Fil'ament (L. filum, thread): the usually slender stalk which supports the anther; any thread-like object or appendage.

Filament'ous : composed of filaments or threads.

Fil'iform: slender and thread-like.

Fim'briate (L. fimbria, fringe): having the margin beset with slender processes. Fimbril'late : minutely fringed.

Fis'tular (L. fistula, a pipe or reed): hollow and cylindrical.

Flac'cid, pr. flak'sid (L. flaccus, flabby) : weak and without rigidity.

Flex'uous (L. flexus, bent) : zigzag; bent altęrnately from side to side.

Floccose' (L. floccus, a flock of wool): having tufts of soft hair.

Floral-en'velopes: calyx and corolla.

Folia'ceous (L. folium, leaf) : having the shape or texture of a leaf.

Fo'liar : leaf-like; pertaining to a leaf.

Fo'liate: provided with leaves; bi-foliate, two-leaved; tri-foliate, three-leaved.

Fo'liolate: provided with leaflets.

Fol'licle (L. folliculum, a small bag): a fruit of one carpel dehiscing along the ventral suture, to which the seeds are attached. Fig. 312.

Follic'ular: like a follicle.

For'nicate (L. fornix, a vault) : having scale-like appendages which converge and close the tube of the corolla.

Free : not adnate to other organs.

Frond (L. frons, a leaf) : the leaf of ferns and other cryptogams, or the shoot of Lemanceæ and other Spermatophytes which is not differentiated into stem and leaf.

Fruit : the ripened ovary and its contents; or, in a broader sense, the ripened ovary and contents, together with any structures which by adhesion are an integral part of it.

Fuga'ceous (L. fugax, fleeing): soon fading or falling off.

Ful'vous (L. fulvus): dull yellow or tawny.

Fu'nicle (L. funiculus, diminutive of funis, a cord): the little stalk which connects the ovule or seed with the placenta.

Fun'nelform: applied to corollas with a tube gradually enlarging from the base. Fig. 270.

Fu'siform (L. fusus, a spindle; forma, shape): tapering toward each end, spindle-shaped. Fig. 217 .

Ga'lea (L. galea, a helmet): the helmet-shaped upper lip of labiate flowers. Fig. 276. Ga'leate: helmet-shaped; having a galea.

Gam'etophyte (Gr. gametes, a spouse; phyton, plant): the generation which bears the eggs and sperms, and gives 1 ise to the sporophyte. Figs. 157, 158 , and 159 . 


\section{Glossary.}

Gamopet'alæ (Gr. gamos, marriage or union; petalon, a flower leaf): plants having the petals united.

Gamopet'alous: having the petals more or less united.

Gamoph'yllous (Gr. gamos + phyllon, a leaf): having leaves united by their edges.

Gem'ma (L. gemma, a bud): a young bud; an asexual bud-like body of Hepaticæ.

Gemmip'arous (L. gemma + parere, to produce): bearing gemmæ.

Gen'era : plural of genus.

Genic'ulate (L. geniculum, little knee, or joint, diminutive of genu, knee): bent abruptly at an angle.

Ge'nus (L. genus, a race): the smallest natural group containing distinct species.

Geotrop'ic (Gr. ge, the earth; trope, a turning) : relating to or evincing geotropism.

Geot'ropism: the property or state of a growing member or organ which enables it to respond to gravity as a guide to the direction which it shall take.

Gib'bous (L. gibbus, humped): swollen or humped on one side.

Gla'brate (L. glaber, smooth): nearly smooth or becoming smooth with age. Gla'brous: smooth; without hairs.

Gland (L. glans, an acorn) : an organ of secretion, or a small prominence apparently having a secreting function.

Glan'dular: bearing glands, or of the nature of a gland.

Glau'cous, pr. glaw'kus (Gr. glaukos, bluish gray): covered with a bloom or powder, as a plum or cabbage leaf.

Glo'bose, glob'ular (L. globus, a sphere) : spherical or nearly so.

Glochid'iate (Gr. glochis, a point of an arrow): having barbs.

Glom'erate (L. glomus, a ball) : collected into a head, or compactly clustered. Gluma'ceous: resembling or provided with glumes.

Glume (L. gluma, hull or husk): an outer husk or bract on the spikelet of grasses.

Gran'ular (L. granum, grain): consisting of or resembling small grains. Grega'rious (L. grex, gregis, a herd): growing in clusters.

Gymnosper'mæ (Gr. gymnos, naked; sperma, seed): plants with naked ovules, as the conifers. Fig. 94 .

Gymnosper'mous : bearing naked ovules and seeds.

'Gynæ'cium (Gr. gynaikeion, woman's apartment, from gyne, woman, and oikion, house): the pistil or pistils of a flower taken collectively.

Gynan'drous (Gr. gyne, woman; aner, andros, man): having the stamens borne upon the pistil. Fig. 289 . 
Gy'nobase (Gr. gyne + basis, a pedestal) : an enlargement or prolongation of the receptacle bearing the gynæcium.

Gy'nophore (Gr. gyne + phoreo, I carry): a more or less elongated pedicel carrying the pistil.

Hab'it (L. habitus, appearance) : the general appearance of a plant.

Hab'itat (L. habitatio, dwelling): the kind of locality in which a plant grows. Hal'berd-shaped: same as hastate.

Haloph'ilous (Gr. hals, halos, salt, the sea; philein, to love): adapted to a salty substratum.

Hal'ophyte (Gr. halos + phyton, plant) : a plant abounding in salty soils.

Has'tate (L. hasta, a spear): arrow-shaped, with the basal lobes pointing outward instead of downward. Fig. 24I.

Hausto'rium (L. haurire, haustum, to drink): the sucker-like rootlet of parasitic plants, as of the dodder.

Head : a dense cluster of sessile or nearly sessile flowers on a short axis.

Fig. $82, t$ and $u$.

Herb (L. herba, herbage) : a plant without a persistent woody stem above ground. Herba'ceous: having the characters of an herb.

Hermaph'rodite (from Hermaphroditus, who, in mythical story, was joined in one body with Salmacis): having stamens and pistils in the same flower.

Hesperid'ium (Gr. Hesperides, names of mythological characters said to own a garden of golden apples) : a berry with a thick but not hard rind, such as the orange and lemon.

$\mathrm{Hi}^{\prime}$ lum (L. hilum, a trifle or little thing) : the scar or place of attachment of the seed.

Hir'sute (L. hirsutus, hairy): beset with rather stiff hairs.

His'pid (L. hispidus, bristly): beset with stiff hairs or bristles.

Hoar'y : grayish from a fine pubescence.

Homog'amy (Gr. homos, one and the same; gamos, marriage): simultaneous maturity of pollen and stigmas in a perfect flower.

Homog'amous : bearing but one kind of flowers.

Hood'ed: shaped like a hood.

Host: a plant which nourishes a parasite.

Hy'aline (Gr. hyalinos, of glass): colorless, translucent.

Hy'brid (L. hybrida, a mongrel): a plant produced by the crossing of two species.

Hy'drophyte (Gr. hydor, water; phyton, a plant): a plant adapted to an aquatic or moist habitat.

Hydrophyt'ic: relating to hydrophytes. 
Hy'pha (Gr. hyphe, a web): one of the thread-like elements of the body of a fungus.

Hypocot'yl (Gr. hypo, under; cotyle, a hollow): the axis of an embryo below the cotyledons.

Hypocotyle'donary: below the cotyledons and above the root.

Hypog'ynous (Gr. hypo, under; gyne, woman): inserted beneath the pistil or gynæcium and free from it. Fig. 123, $A$.

Im'bricate (L. imbricatus, covered with gutter tiles): overlapping like the shingles on a roof. Fig. 286.

Immersed': grawing below the surface of water; embedded.

Imper'fect : applied to a unisexual flower.

Incised': sharply and irregularly cut into. Fig. 234.

Inclu'ded : not extending beyond the surrounding envelopes.

Incomplete' : wanting in one of the floral envelopes.

Incum'bent (L. incumbens, leaning on): said of cotyledons when reflexed so that the back of one lies against the hypocotyl.

Indef'inite: applied to stamens when inconstant in number, or too numerous for easy counting. Said of an inflorescence whose main axis continues to elongate as the buds unfold, as in a raceme; applied to the growth of branches which continue to elongate throughout the growing season.

Indehis'cent (L. in, not ; dehiscens, gaping) : remaining closed, or not opening by valves or along regular lines.

Indeterm'inate : not absolutely terminated, as when no flower ends the axis of an inflorescence.

Indig'enous (L. indiges, native) : native to a country; not introduced.

Indu'plicate (L. in, toward; duplicare, to double): arranged around an axis without overlapping, with the edges turned inward. Fig. 284.

In'durated (L. induratus, hardened): hardened.

Indu'sium (L. indusium, an under garment): the covering over the groups of sporangia or sori of ferns. Fig. 154 .

Inequilat'eral : having right and left sides of different extent.

Infe'rior: lower or anterior. Inferior ovary: having the other floral organs apparently growing from it, at or near its summit. Fig. 123, $C$ and $D$. Inferior calyx : growing below and free from the ovary. Fig. 123, $A$.

Infla'ted : bladdery.

Inflores'cence (L. inflorescere, to begin to blossom): the general arrangement of the flowers on the flowering axis.

In'nate (L. innatus, inborn): said of an anther when joined by its base to the apex of a filament. Fig. 292.

Insert'ed : growing out of or attached to. 
In'ternode (L. inter, between; nodus, a knot): the portion of the stem between the nodes or places where the leaves arise.

Intramar'ginal: within and near the margin.

Introrse' (L. intra, on the inside; versus, toward) : facing inward or toward the axis.

Invol'ucel (L. diminutive of involucrum, a wrapper): a secondary involucre. Involu'cral : pertaining to an involucre.

Involu'cre (L. involucrum, a wrapper): an assemblage of bracts subtending a flower or flower cluster. Invol'ucrate: having an involucre.

In'volute (L. involvere, involutum, to wrap up) : rolled inward. Fig. 285. Irreg'ular: applied to flowers whose individual members of a set of organs are unlike in form.

Junc'oid (L. juncus, a rush ; Gr. eidos, resemblance): rush-like.

Keel : the two anterior or lower petals of a papilionaceous corolla which are united in the form of a keel of a boat. Figs. 266 and 267, $k$.

Key or key'-fruit: a winged fruit, such as of the maple or ash; a samara. Fig. 320.

Kid'ney-shaped : crescent-shaped, with rounded ends; reniform. Fig. 227.

La'biate (L. labium, lip) : having the limb of calyx or corolla divided as in Fig. 276. Belonging to the Labiatæ.

Lac'erate : irregularly cleft or jagged, as if torn.

Lacin'iate (L. lacinia, the flap of a garment) : provided with a jagged, irregular fringe.

Lamel'la (L. lamella) : a thin plate or scale.

Lan'ceolate: lance-shaped; narrow and tapering toward each end, as in Fig. 220.

La'tent (L. latens, hidden) : applied to buds which lie dormant for a number of years without losing their power of developing when conditions are favorable.

Lat'eral: borne on the side of an organ.

La'tex (L. latex, juice): the milky secretion of such plants as Euphorbia. Laticif'erous (L. latex + ferre, to bear): latex-bearing.

Lax: loose and slender.

Leaf'let: one of the separate divisions of a compound leaf.

Leaf-stalk: the stem or petiole on which the leaf is borne. Fig. 262, b.

Leg'ume (L. legumen, pulse or any leguminous plant) : a pod dehiscing into two valves and having the seeds all attached on one side. Fig. 313.

Legu'minous : pertaining to a legume or to the order called Leguminosæ. 


\section{Glossary.}

Lentic'ular (L. lens, lentis, a lentil or its seed which has a double convex outline): shaped like a double convex lens.

Lig'ulate: provided with a ligule.

Lig'ule (L. ligula, a little tongue) : a strap-shaped body such as the limb of the ligulate corolla of the Compositæ. Fig. 268, $q$.

Lilia'ceous : lily-like; pertaining to the Liliaceæ.

Limb: the border or expanded part of a gamopetalous corolla; the blade of a leaf or petal. Fig. 272, $y$.

Lin'ear: long and narrow with parallel margins. Fig. 219.

Lip : one of the divisions of a bilabiate corolla or calyx. Fig. 276.

Lobe: any segment, but particularly a rounded segment of an organ.

Lobed or $10^{\prime}$ bate : divided into lobes.

Loc'ular (L. loculus, a little compartment) : having cells.

Loc'ulicidal (L. loculus, a cell or compartment; cadere, to cut): dehiscent or breaking open through the dorsal suture. Fig. 322 .

Lo'ment: a legume which is contracted between the seeds. Fig. $3 \mathbf{I} 4$.

Lu'men (L. lumen, a light, an opening): the cavity of a cell.

Lu'nate (L. luna, the moon): shaped like a half or crescent moon.

Lu'nulate: diminutive of lunate.

Ly'rate (Gr. lyra, a lute or lyre): lyre-shaped; spatulate and lobed, with the smaller lobes toward the base.

Mac'rospore (Gr. makros, long or large; spora, seed): the larger spore of some Pteridophytes, which gives rise to the female gametophyte; the embryo sac of Spermatophytes.

Macrospor'ophyll (Gr. makros + spora + phyllon, a leaf) : carpel, carpellary leaf.

Mar'ginal: along or near the edge.

Mar'ginate: provided with a border or margin of distinct character.

Membrana'ceous, or mem'branous : thin and somewhat transparent.

Menis'coid (Gr. meniskos, a crescent; eidos, resemblance) : concavo-convex, like a watch crystal.

Mer'icarp (Gr. meros, a part; karpos, fruit): one carpel of an umbelliferous fruit.

-merous : in composition relating to a certain number of parts, as 2-merous, having two parts.

Mes'ophyll (Gr. mesos, in the middle; phyllon, a leaf) : the thin-walled parenchyma cells which make up the greater part of the interior of a leaf.

Mes'ophyte (Gr. mesos + phyton, a plant) : a plant adapted to a habitat having a moderate amount of humidity in atmosphere and soil.

Mesophyt'ic:-relating to mesophytes. 
Mi'cropyle (Gr. mikros, small; pyle, a gate): the passage through the coats of an ovule through which the pollen tube enters; the spot on or opening through the coats of a seed corresponding to or identical with the opening of the ovule. Fig. 88.

$\mathbf{M i}^{\prime}$ crospore (Gr. mikros, small; spora, seed): the smaller spore of certain Pteridophytes, which gives rise to the male gametophyte; the pollen grain of Spermatophytes.

Microspor'ophyll (Gr. mikros + spora + phyllon, a leaf): a leaf bearing microspores; a stamen.

Mid'rib : the central or main rib of a leaf.

Monadel'phous : (Gr. monos, one; adelphos, brother): applied to stamens when united by their filaments into a tube or column. Fig. 290.

Monil'iform (L. monile, necklace; forma, shape): like a string of beads.

Monocotyle'don (Gr. monos, one; kotyledon, a hollow): a plant having but one cotyledon.

Monocotyle'donous : having but a single cotyledon.

Monœ'cious, pr. mo-ne'shus (Gr. monos, one; oikos, household): having stamens and pistils in separate flowers on the same plant.

Mucilag'inous : slimy; composed of mucilage.

Mu'cro (L. mucro, sword): a sharp and abrupt tip.

$\mathbf{M u} \mathbf{u}^{\prime}$ cronate : ending abruptly with a sharp point. Fig. 250.

Mul'tifid (L. multus, many; findere, to split): sleft into many segments.

Mul'tiple fruit : a fruit consisting of many ripened pistils consolidated into a mass, as in the mulberry. Fig. 327.

Mu'ricate (L. murex, a pointed stone) : rough with short hard points. Muric'ulate : finely muricate.

$\mathrm{Na}$ 'ked : destitute of covering, as buds without scales.

$\mathrm{Na}$ 'piform (L. napus, turnip; forma, shape): turnip-shaped. Fig. 216.

$\mathrm{Na}$ 'tant (L. natans, swimming): floating submerged in water.

Nec'tar (Gr. nektar, the drink of the gods): a sweetish fluid excreted from some parts of a flower for the attraction of insects.

Nectarif'erous (nectar + L. ferre, to bear or produce) : nectar-bearing.

Nec'tary: any part of a flower which secretes and excretes nectar.

Nec'tar recep'tacle : a part of a flower into which the nectar is excreted and conserved, as the spur of violets and larkspurs.

Nerva'tion: the manner in which the nerves are arranged in a leaf.

Nerve : a slender unbranched vein or rib.

Neu'ter (L. neuter, neither the one nor the other): sexless, as a flower having neither stamens nor pistil.

Neu'tral : applied to flowers having neither stamens nor pistil. 


\section{Glossary.}

Nod'ding : hanging downward.

Node(L. nodus, a knot): that part of a stem which normally bears a leaf or leaves. Nodose': having many or conspicuous nodes or knotty swellings.

$\mathrm{Nu}$ 'cleus (L. nucleus, kernel or inner part): a well-defined, and in young cells, central body of a cell, which is thought to be the bearer of the inheritable qualities. Figs. 12, 45, and 46.

Nut : a hard, indehiscent, usually $\mathrm{I}$-seeded fruit, as the walnut and acorn. Fig. 334. Nut'let: a small nut.

Ob-: a Latin prefix indicating inversion.

Obcla'vate (L. ob-, inverse; clavatus, club-shaped): club-shaped, and attached at the thicker end.

Obcompressed ${ }^{\prime}$ : compressed dorsiventrally instead of laterally.

Obcon'ical (L.ob- + conus, a cone) : conical and attached at the narrower end.

Obcor'date (L. ob- + cor, cordis, the heart): heart-shaped and attached at the narrower end.

Oblan'ceolate (L. ob- + lanceola, a little lance) : lanceolate with the broadest part toward the apex. Fig. 226.

Oblique', pr. obleek' or oblike' (L. obliquus, slanting): slanting or unequal sided.

$0 \mathrm{~b}$ 'long: much longer than broad and with parallel sides. Fig. 221.

Obo'vate (L. ob- + ovatus, egg-shaped): egg-shaped and attached at the smaller end. Obo'roid : an obovate solid.

Ob'solete (L. obsoletus, worn out): much reduced or rudimentary.

Obtuse': blunt or rounded at the end. Fig. 249.

Ochroleu'cous, pr. ok-ro-lü'kus (Gr. ochra, yellow earth; leukos, white): yellowish white.

$0^{\prime}$ crea (L. ocrea, a legging) : a tubular stipule, or a pair of stipules united in the form of a tube. Fig. 264, 2.

0 'create: provided with an ocrea.

Odd'ly pin'nate : pinnately compound and having a terminal leaflet. Fig. 255.

Offic'inal (L. officina, a workshop): used in medicine or the arts.

Off'set or off'shoot: a lateral shoot which takes root and starts a new individual. Fig. $2 x x$.

0'ospore (Gr. oon, an egg; spora, seed): the fertilized egg cell.

Opaque': dull; not transparent.

Oper'culum (L. operculum, cover, lid): the upper half of a capsule which dehisces transversely. Figs. 150 and 325 .

Op'posite : said of leaves when two stand on opposite sides at a node.

Orbic'ular (L. orbicularis, circular) : flat with circular contour. Figs. 225 and 246. 
Orthot'ropous (Gr. orthos, upright; trope, a turning): applied to an erect ovule or seed having the micropyle at the apex. Fig. 298.

O'val (L. ovum, an egg): broadly elliptic.

0'vary: that part of the pistil which contains the ovules. Fig. 87 .

$0^{\prime}$ vate: having the outline of a hen's egg, with broader end down. Fig. 223. O'void: a solid with an oval outline.

$0^{\prime}$ vule: the body which, after the fertilization of the egg, becomes the seed. Fig. 87. Ovulif'erous (ovule + L. ferre, to bear): bearing ovules.

Pal'ate (L. palatum, the palate): in a bilabiate corolla a projection from the lower lip closing the throat. Fig. 275 .

Pale (L. palea, chaff) : chaffy scales on the receptacle of some Compositæ. The inner bract of a spikelet in the inflorescence of grasses (in this sense most commonly called palet).

Palea'ceous : chaffy.

Pal'et: the inner bract in the spikelet of grasses.

Palisade' cells (L. palus, a stake) : in leaves or stems elongated chlorophyllbearing cells with long axis perpendicular to the surface. Fig. 36 .

Pal'mate (L. palma, the hand): lobed or divided, with the sinuses directed toward the petiole. Figs. 237-239.

- Pal'mately: in a palmate manner.

Pan'icle (L. panicula, a tuft): a compound raceme or corymb.

Pan'icled or panic'ulate: borne in a panicle.

Papiliona'ceous (L. papilio, butterfly): having a winged corolla somewhat resembling a butterfly, as found in the pea and locust. Figs. 266 and 267. Papil'la, pl. papillæ (L. papilla, a nipple): a minute rounded projection.

Pap'illose : covered with or resembling papillæ.

Pap'pus (L. pappus, an old man; or a woolly, hairy seed of certain plants): thistledown; the hairy calyx-limb of certain Compositæ. Figs. 316-318.

Par'asite (Gr. para, beside; sitein, to feed): an organism subsisting on another called the host.

Parasit'ic: living as a parasite on another organism.

Paren'chyma (Gr. para, beside; en, in; chein, to pour): applied to cells which are more or less isodiametric.

Pari'etal (L. paries, a wall or partition wall) : applied to ovules attached to the wall of the ovary instead of to the axis. Figs. 303 and 304 .

Part'ed : cleft nearly to the base or midrib. Fig. 235 .

Par'tial: sometimes used in the sense of secondary.

Pathogen'ic (Gr. pathos, disease; genos, descent): producing disease.

Pec'tinate (L. pecten, a comb): having narrow close divisions like the teeth of a comb. 


\section{Glossary.}

Ped'ate (L. pes, pedis, a foot): palmately parted or divided, with the lateral segments again cleft.

Ped'icel (L. pediculus, a little foot): the stalk of a single flower of a cluster. Ped'icellate: having a pedicel.

Pedun'cle : the stalk of a solitary flower, or the primary flower stalk of a cluster. Pedun'culate: borne on a peduncle.

Pellu'cid (L. per, through; lucidus, clear, luminous) : transparent or nearly so.

Pel'tate (L. pelta, a shield) : shield-shaped, and attached at its lower surface instead of by its margin. Fig. 246.

Pen'dulous : applied to an ovule which hangs downward from the side of the ovary.

$\mathrm{Pe}^{\prime}$ po (Gr. pepon, a kind of gourd or melon) : a kind of berry with a firm, hard rind, such as the pumpkin or gourd. Fig. 338.

Peren'nial (L. perennis, that lasts the whole year through; from per, through; annus, the year): lasting from year to year.

Per'fect: applied to flowers having both pistil and stamens.

Perfo'liate (L. per, through; folium, a leaf): said of a leaf having the stem apparently passing through it. Fig. 244 .

Per'ianth (Gr. peri, about; anthos, a flower): calyx or corolla, or both collectively when present.

Per'icarp (Gr. peri, about; karpos, fruit) : the matured ovary or its wall.

Perigyn'ium (Gr. peri + gyne, woman): the inflated sac inclosing the ovary in Carex.

Perig'ynous : borne above the base of the ovary but not at its summit. Fig. $123, B$.

Persis'tent: continuing long, as sepals on the maturing fruit or leaves remaining on their branches through the winter.

Per'sonate (L. personatus, masked): applied to a bilabiate corolla having the throat closed by a prominent projection or palate. Fig. 275 .

Pet'al (Gr. petalon, a leaf): one of the parts of the corolla. Fig. 87.

Pet'aline, pr. pet'al-in : pertaining to or resembling a petal.

Pet'aloid: colored and resembling a petal.

Pet'iolate (L. petiolus, a little foot or leg): having a petiole.

Pet'iole : the footstalk of a leaf. Fig. 262, $b$.

Phænog'amous (Gr. phainein, to show; gamos, marriage): having flowers in the ordinary sense of the word, and producing seeds.

Phan'erogam or phaneroga'mia (Gr. phaneros, evident; gamos marriage): a plant or class of plants having flowers in the ordinary sense, and producing seeds.

Photosyn'thesis (Gr. phos, photos, light; synthesis, a putting together): the formation of starch and sugars from carbon dioxide and water by means 
of the chloroplasts, which derive their energy for the work from the light absorbed by the chlorophyll.

Phyllo'dium (Gr. phyllon, leaf; eidos, form): a petiole expanded into the form of a leaf blade. Fig. 261 .

Phyl'lotaxy (Gr. phyllon, leaf; taxis, arrangement): the angular distribution of leaves on the stem.

Phy'tomer (Gr. phyton, plant; meros, part): a node with its leaf or leaves, and the internode subtending it.

Pi'lose (L. pilus, a hair): beset with soft hairs.

Pin'na (L. pinna, a feather): one of the primary divisions of a pinnate, or pinnately compound, leaf.

Pin'nate: compound, with the leaflets arranged on each side of a common petiole. Figs. 255 and 256 . Oddly pinnate : with a single terminal leaflet. Fig. 255. Abruptly pinnate : all of the leaflets in pairs, and no terminal leaflet present. Fig. 256.

Pinnat'ifid (L. pinna + findere, fidi, to cut): divided in a pinnate manner, but not as far as the midrib. Fig. 235 .

Pin'nule: a secondary pinna.

Pis'til (L. pistillum, a pestle): the female spore-bearing leaf, or leaves united, of Spermatophytes, consisting of ovary, stigma, and commonly style. Fig. 87.

Pis'tillate : having pistils, and, as the term is commonly employed, destitute of stamens.

Pitted: having small depressions over the surface.

Placen'ta (L. placenta, a flat cake): that part of the interior of the ovary which bears the ovules. Figs. 302-305.

Plasm or plasma (Gr. plasma, anything formed or molded) : protoplasm; the living part of the plant or animal body.

Plasma membrane: the limiting protoplasmic membrane at the exterior of plant cells which are destitute of cell walls, or the membrane which lies in immediate contact with the wall when the latter is present. Fig. I 2.

Plasmo'dium : a multinucleated mass of protoplasm destitute of cell walls, and capable of amoboid movement. Fig. 132.

Plas'tid (Gr. plastis, plastidos, a creator) : a differentiated portion of the cell protoplasm having definite work to do, such as the manufacture of starch, chlorophyll, and other pigments.

Pli'cate (L. plicare, to fold or plait): folded like a fan.

Plu'mose (L. pluma, a feather): having hairs on each side like the plume of a feather.

Plu'mule (L. plumula, a little feather): the first bud of the embryo.

Pod : a dry and dehiscent fruit, such as a legume or silique. Figs. 309 and 313. 


\section{Glossary.}

Pol'len (L. pollen, fine flower): the microspores of Spermatophytes which are borne within the anthers, and give rise to the sperms or fertilizing cells.

Pol'len cells or sacs : the cavities of the anther which contain the pollen.

Pol'len grain : a single microspore of Spermatophytes.

Pol'len tube: the tubular outgrowth from the pollen grain, which penetrates the pistil and conducts the sperm into the ovule. Fig. 88.

Pol'linate : to apply pollen to the surface of the stigma.

Pollinif'erous (L. pollen + ferre, to bear): pollen-bearing.

Pollin'ium: a coherent mass of pollen, as in the milkweed and orchids. Fig. II 3 .

Polyadel'phic (Gr. polys, many; adelphos, a brother): having the stamens united into several bundles or brotherhoods.

Polypet'alous (Gr.polys, many; petalon, a leaf) : having several separate petals.

Poma'ceous (L. pomum, a fruit of any kind): like an apple or pear; producing pomes.

Pome : a fruit of the apple, pear, and quince type. Fig. 333 .

Pore : any small aperture, as in some anthers for the discharge of pollen.

Por'ous or porose' : pierced with small openings or pores.

Poste'rior : next to or toward the main axis; superior.

Pos'ticous (L. posticus, posterior): situated on the outer side of a filament, as an extrorse anther.

Præmorse' (L. pre, before; mordere, to bite): appearing as if bitten off.

Prick'le : outgrowths of the epidermis or of the bark, with sharp point.

Prismat'ic (L. prisma, a prism): having the form of a prism; angular with flat sides.

Procum'bent (L. pro, forward; cumbens, lying down): lying on the ground. Prolif'erous (L. proles, offspring; ferre, to bear): producing offshoots.

Pros'trate: lying flat upon the ground.

Proteran'drous or protan'drous (Gr. proteros, first; aner, andros, a man): having the anthers mature before the stigmas of the same flower are ready to receive the pollen.

Proteran'dry or protan'dry: the condition of having the anthers mature before the stigmas.

Proterog'ynous or protog'ynous (Gr. proteros, first; gyne, a woman): having the stigmas ready to receive the pollen before the anthers of the same flower are mature.

Proterog'yny or protog'yny: the condition of having the stigmas mature before the anthers.

Prothal'lus or Prothallium (Gr. pro, before; thallos, a young shoot or sprout): the gametophyte of ferns and other Pteridophytes, resulting from the germination of an asexual spore. Fig. 155 . 
Prox'imal (L. proximus, nearest, next) : the part nearest the axis.

Pter'idophyte (Gr. pteris, pteridos, a kind of fern, from pteron, a feather; phyton, a plant): ferns and their allies.

Puber'ulent (L. puber, of ripe age, pubescent): minutely hairy.

Pubes'cence : a covering of short soft hairs.

Pubes'cent (L. pubescens, reaching maturity, growing hairy): covered with soft or downy hairs.

Pul'vinate (L. pulvinus, a cushion): having the form of a cushion or pad.

$\mathrm{Pul}^{\prime}$ vinus : the motor organ at the base of some leaves and leaflets, as in scarlet runner. Figs. 59 and 60.

Punc'tate (L. punctum, a point): marked with depressions, translucent glands, or colored spots.

Punctic'ulate : minutely punctate.

Pun'gent (L. pungens, piercing) : ending in a sharp and rigid point.

Pus'tular (L. pustula, a pimple): having blister-like elevations.

Pus'tulate: as though blistered.

Puta'men, pr. pew-tā'men (L. putamen, shells or peels): the shell of a nut; the stone of a stone fruit.

Pyram'idal: shaped like a pyramid.

Race : a variety of such fixity as to be reproduced by seeds.

Raceme' (L. racemus, a bunch of grapes) : a simple inflorescence, elongating at the apex, with pedicels of the individual flowers of about equal length. Fig. 82, $r$. Rac'emose : borne in racemes; raceme-like.

$\mathrm{Ra}^{\prime}$ chis or rhachis (Gr. rhachis, a backbone): the axis of an inflorescence, compound leaf, or frond.

$\mathbf{R a}^{\prime}$ diate (L. radius, spoke of a wheel) : divergent from a common center; bearing ray flowers.

Rad'ical (L. radix, a root): belonging to or proceeding from the root, or from the base of the stem close to the ground.

Rad'icle: the name sometimes given to the whole of the hypocotyl, but more properly restricted to the embryo root at the tip of the hypocotyl.

Radic'ulose: bearing rootlets.

$\mathrm{Ra}$ 'dix : the root developed from the radicle.

$\mathrm{Ra}^{\prime}$ phe or rha'phe, pr. rā'phy (Gr. rhaphe, a suture or seam): the continuation of the seed stalk along the side of an anatropous ovule. Fig. 300 .

Ray : the marginal portion of a composite flower; a branch of an umbel.

Ray flower or flor'et: an outer flower of the head of the Compositæ, whether tubular or ligulate. Fig. 268. 
Recep'tacle (L. receptaculum, a reservoir): the axis of a flower, from which spring sepals, petals, stamens, and pistils; or the axis which bears the separate flowers of a head. Fig. 87, $a$, and Fig. 82, $t$ and $u$.

Recurved' : curved backward or downward.

Redu'plicate (L. reduplicatus, doubled): doubled back; applied to leaves in the bud with edges valvate and turned outward.

Reflexed': abruptly bent downward.

Reg'ular (L. regularis, according to rule): applied to flowers having all the parts of a whorl uniform in shape, as when the petals are all alike, etc.

Ren'iform (L. renes, the kidneys; forma, shape): kidney-shaped. Fig. 227.

Repand' (L. repandus, bent backward or turned up); applied to leaves having slightly undulating margins. Fig. 232.

$\mathrm{Re}^{\prime}$ pent (L. repens, creeping) : prostrate and rooting. Fig. $2 \mathrm{II}$.

Resinif'erous (L. resina, rosin; ferre, to bear) : resin-bearing.

Retrorse' (L. retro, back; versus, toward) : bent downward or backward.

Retuse' (L. retusus, blunted) : having the apex rounded and slightly indented.

Figs. 221 and 253.

Rev'olute (L. revolvere, revolutum, to roll back or unwind) : rolled backward or downward.

Rha'chis : see Rachis.

Rha'phe : see Raphe.

Rhi'zoid (Gr. rhiza, a root ; cidos, form) : a hair serving as a root in mosses and Hepaticæ.

Rhizome' (Gr. rhizoma, a mass of roots, a stem or race): a prostrate unders ground stem, rooting at the nodes, and erect and leaf-or stem-bearing at the apex; or short and variously thickened, quite variable in appearance. Figs. 205-210.

Rhom'bic or rhomboid'al (Gr. rhombos, a spinning top) : shaped like a rhomb, - namely, a quadrilateral figure with parallel and equal sides which may be either oblique or perpendicular to each other.

Rib : a prominent vein. Ribbed : having prominent parallel veins.

Rin'gent (L. ringens, gaping): wide open, as the mouth of a bilabiate corolla.

Root'stock : see Rhizome.

Ros'trate (L. rostrum, a beak) : provided with a point or beak.

Ro'tate (L. rota, a wheel) : wheel-shaped; flat and circular. Fig. 274.

Rotund': rounded in outline.

Ru'fous (L. rufus, red or reddish): reddish, yellowish red, or tawny.

Rugose' (L. ruga, a wrinkle): wrinkled; having the veinlets sunken and the spaces between elevated, as in the leaves of sage. 
Run'cinate (L. runcina, a large saw): deeply incised, with the lobes pointing toward the base of the petiole, as in the dandelion leaf. Fig. 32.

Run'ner: a prostrate lateral offshoot rooting at the nodes. Fig. 21 .

Sac'cate (L. saccus, a bag) : bag-shaped.

Sag'ittate (L. sagitta, an arrow) : arrow-shaped; having two acute lobes at the base like the barbs of an arrow. Fig. 243 .

$\mathrm{Sal}^{\prime}$ ver-form or sal'ver-shaped : tubular, with a spreading border. Fig. 273. Sama'ra (L. samara or samera, the fruit of the elm) : an indehiscent winged - fruit like that of the maple and elm. Figs. 319 and 320.

Sapona'ceous (L. sapo, soap): soapy or slippery to the touch.

Sap-wood: the new wood of exogenous woody plants, of lighter color and of less specific gravity than the older heart-wood.

Sca'brous (L. scaber, rough): rough to the touch.

Scan'dent (L. scandens, climbing) : climbing in any manner.

Scape (L. scapus, a shaft or stem): a leafless floral axis or peduncle rising from the ground.

Sca'pose : bearing or resembling a scape.

Sca'riose or sca'rious (L. scariosus): dry and membranous.

Scor'pioid (Gr. skorpios, a scorpion; eidos, resemblance): having the axis of the inflorescence coiled to one side.

Scor'pioid-cyme: a cyme whose lateral branches occur alternately on opposite sides.

Scurf: bran-like scales over the epidermis.

Seed: the ripened ovule and its integuments.

Seg'ment : one of the parts of a cleft or divided member; one of the similar parts of a series.

Sep'al or se'pal (L. separ, separate): one of the divisions of the calyx.

Sep'ticidal (L. septum + cadere, to cut): a method of dehiscence in which the pod is divided through the middle of the partitions. Fig. 321 .

Septif'ragal (L. septum; frangere, fractum, to break): a mode of dehiscence in which the valves break away from the partition walls. Figs. 323 and 324.

Sep'tum, pl. septa (L. septum, a wall or fence) : the division wall of an ovary. Ser'rate (L. serra, a saw) : having forward-pointing marginal teeth. Fig. 229.

Ser'rulate: finely serrate.

Ses'sile (L. sessilis, sitting) : not provided with a stalk.

Seta'ceous (L. seta, a bristle): bristle-like.

Se'tiform: having the form of a bristle.

Setose' : beset with bristles or bristly hairs.

Se'tulose: resembling or beset with fine bristles. 


\section{Glossary.}

Sex : pertaining to a differentiation into male and female.

Sex'ual-genera'tion: the stage or generation in the life cycle which bears the eggs and sperms, as the prothallus of ferns. Figs. 157, 158, and 159. Sheath : a tubular covering, such as the lower part of the leaves of grasses. Sheathing: inclosing after the manner of a sheath.

Shoot: the ascending axis or branch therefrom with its leaves.

Shrub : a woody plant smaller than a tree. Shrubby : resembling a shrub. Sil'icle (L. silicula, a little husk or pod): a short pod scarcely longer than broad. Fig. 310.

Silique', pr. si-leek' (L. siliqua, a husk or pod) : an elongated pod divided by a partition and opening by two sutures, as in the Cruciferæ. Fig. 309. Silky : beset with a soft and straight pubescence.

Simple: undivided; applied to pistils and fruits consisting of a single carpel.

Sin'uate or sin'uous (L. sinuosus, full of windings or curves): having a strongly wavy margin. Fig. 232.

Si'nus (L. sinus, a curve, hollow, or fold): the recess between two lobes.

So'rus, pl. sori (Gr. soros, a heap) : a fruit dot, or cluster of sporangia in ferns.

Spadi'ceous (Gr. spadix, a palm branch): resembling or bearing a spadix.

Spa'dix: a spike with a fleshy axis, as in calla lily and Jack-in-the-pulpit. Fig. 346.

Spathe, pr. spāth (Gr. spathe, a spatula) : a large bract or pair of bracts inclosing a flower or inflorescence. Fig. 346.

Spat'.ulate (L. spatula, a broad piece, or a palm branch) : narrowing toward the base from a rounded summit. Fig. 228.

Sperm (Gr. sperma, a seed): the male reproductive cell.

Sperm'atophyte (Gr. sperma, spermatos, a seed; phyton, a plant): a plant bearing true seeds with differentiated embryos.

Spi'cate (L. spica, an ear of grain, as of corn or wheat) : borne in or resembling a spike.

Spike: an inflorescence having the flowers nearly or quite sessile upon an elongated axis. Fig. 82, s. Spike'let: a small or secondary spike.

Spin'dle-shaped : see Fusiform.

Spine (L. spina, a thorn): a sharp woody outgrowth from a stem.

Spi'nose or spi'nous: beset with spines.

Spi'nulose : beset with small spines.

Sporan'gium (Gr. spora, a seed; aggeion, a vessel) : a sac containing spores.

Fig. 154 .

Spore : a reproductive cell of Cryptogams capable of growing into a mature plant. 
Spur: a hollow extension of some part of a flower, usually for the purpose of conserving the nectar.

Squa'ma (L. squama, a scale): a scale of any kind.

Squa'mula : a reduced scale.

Squar'rose, pr. skwŏr'rōs or skwŏr-rōs' (L. squarrosus, rough, scurfy): having spreading and projecting processes.

Squar'rulose: diminutively squarrose.

Sta'men (Gr. stemon, a thread): the male spore-bearing leaf of Spermatophytes, consisting of anther and filament. Figs. 87 and 288-297.

Stam'inal: relating to or consisting of stamens.

Stam'inate: usually applied to flowers having stamens but no pistils.

Stamino'dium (stamen + Gr. eidos, resemblance) : a sterile or abortive stamen or its homologue, in which the anther is wanting or undeveloped. Fig. 277.

Stand'ard: the upper dilated petal of a papilionaceous flower. Fig. 266, s. Stel'late (L. stella, a star): star-shaped.

Ster'ile (L. sterilis, barren, unfruitful): applied to a flower without a pistil, or to a stamen wanting an anther.

Stig'ma (Gr. stigma, a point): that part of the pistil which is adapted to receive and hold the pollen and permit the penetration of the pollen tube. Fig. $87, h$. Stigmat'ic: pertaining to a stigma.

Stipe (L. stipes, stalk or trunk) : the leafstalk of a fern; the stalk at the base of some pistils, lifting them above the insertion of the other floral organs; the stem which bears the cap or pileus of toadstools and their kind.

Stip'itate: having a stipe.

Stip'ular (L. stipula, stem or stubble): pertaining to a stipule.

Stip'ulate: having stipules.

Stip'ule (L. stipula, stem or stubble): one of two similar appendages frequently occurring on either side of the insertion of a leaf on the stem. Fig. 262, c.

Sto'lon (L. stolo, a sucker or shoot springing from the base of a plant): any basal shoot which is disposed to lie prostrate or bend downward and strike root. Fig. $2 \mathrm{II}$.

Stolonif'erous (L. stolo + ferre, to bear): producing stolons.

Stoma, pl. sto'mata (Gr. stoma, a mouth): an orifice in the epidermis of leaves and some other plant members, bounded by two guard cells, which usually have the power to draw apart or close together as the interchange of gases or the reduction of transpiration through the orifice may be demanded. Figs. 35 and 40.

Stone-fruit : a fruit with a central stone, as in the peach and plum. Fig. 332. Striate (L. stria, a furrow): marked with fine longitudinal grooves and ridges. 
Strict (L. strictus, close, straight): straight and upright.

Stri'gose (L. strigosus, thin and meager): beset with appressed, sharp, straight, and stiff hairs or bristles.

Strob'ile, pr. strob'il or stro'bile (Gr. strobilos, a cone): an inflorescence with imbricated scales, as in the hop and the pine cone. Fig. 93.

Style (Gr. stylos, a pillar) : the elongation from the top of the ovary, usually present, which bears the stigmas. Fig. 87 .

Stylopo'dium (Gr. stylos, a pillar; podion, diminutive of pous, podos, foot):

a disk-like expansion at the base of a style, as in the Umbelliferæ.

Sub-, a Latin prefix in compound botanical terms, usually signifying somerwhat or slightly.

Su'bulate (L. subula, an awl) : awl-shaped.

Suc'culent (L. suculentus, full of juice or sap) : juicy or sappy.

Suffrutes'cent (L. sub, somewhat; frutex, a shrub): slightly woody at the base.

Suffru'ticose (L. sub, somewhat or under; fruticosus, shrubby): woody near the base, and producing annual herbaceous shoots.

Sul'cate (L. sulcatus, furrowed): grooved or furrowed.

Sulfu'reous or sulphu'reous: pale yellow.

Supe'rior: applied to the ovary when the other floral organs are borne on the receptacle beneath it; applied to the other floral organs when they are borne on an elongation of the receptacle or so-called calyx tube, above the ovary, or are apparently adherent to the ovary. Fig. 123.

Suspend'ed : applied to an ovule which hangs from the top of the cell of the ovary. Fig. 306.

Su'ture (L. sutura, a seam) : an apparent juncture or seam of union; a line of dehiscence.

Syco'nium (Gr. sykon, a fig) : a fruit consisting of a fleshy hollow receptacle lined with numerous flowers, as in the fig. Fig. 339.

Symmet'rical: having the same number of parts in the successive circles of thoral organs.

Syngene'sious (Gr. syn; with, together; genesis, generation or birth): said of stamens which are united by their anthers. Fig. 288.

Tailed : having a slender terminal prolongation.

Tap-root: a primary descending root forming a direct continuation of the radicle. Fig. 215.

Terete' (L. teres, rounded) : having a circular transverse section; cylindrical. Ter'minal : pertaining to the apex, as a bud at the end of a shoot.

Ter'nary (L. ternarius, consisting of threes): occurring in threes.

Ter'nate (L. terni, by threes): in threes. 
Ter'nate-pin'nate : applied to compound leaves when the secondary petioles occur in threes at the summit of the primary petiole.

Tes'ta (L. testa, a brick or tile): the usually hard and brittle outer coat of a seed.

Testa'ceous : brick-red.

Tetradyn'amous (Gr. tetra, four; dynamis, power): having four long and two shorter stamens, as in the Cruciferæ.

Tetrag'onal (Gr. tetra + gonia, an angle): four-angled.

Thal'loid : resembling or consisting of a thallus.

Thal'lophyte (Gr. thallos, a sprout; phyton, a plant): a plant having no clear differentiation into stem and leaf.

Thal'lus, pl. thalli : a vegetative body having no distinction into stem and leaf. Throat: the opening into the tube of a gamopetalous corolla.

Thyrse, pr. thers (Gr. thyrsos, the staff of Bacchus twined round with ivy and vine shoots): a dense panicle like that of the lilac or grape.

Thyr'soid (thyrse + Gr. eidos, resemblance) : resembling a thyrse.

Thyr'sus: same as thyrse.

To'mentose (L. tomentum, a stuffing for cushions of wool, hair, or feathers): covered with dense and matted woolly hairs.

Torose' (L. torosus, fleshy): cylindrical with alternate swellings and contractions. Tor'ulose : diminutive of torose.

Tor'us (L. torus, a bed): the receptacle of a flower. Fig. 87, $a$.

Tri (Gr. or L. prefix tri, three) : in compound words, three or thrice.

Trian'drous (tri + Gr. aner, "andros, man): having three stamens.

Trich'ome (Gr. trichoma, a growth of hair): any hair-, scale-, or bristle-like outgrowth from the epidermis.

Tri'fid (L. tri, three; findere, fidi, to split): three cleft.

Trifol'iate (L. tri, three; folium, a leaf): said of compound leaves of three leaflets, as in clover and oxalis. Fig. 259.

Trimor'phic or trimor'phous (Gr. tri, three; morphe, form): occurring under three forms, as when the stamens and styles of different flowers are long, short, and intermediate.

Triter'nate (L. tri + terni, by threes): occurring in groups of threes three times.

Tropoph'ilous (Gr. trope, a turning; philein, to love): adapted to change of condition, such as deciduous trees, or plants whose above-ground parts die away on the approach of winter or dry season.

Trop'ophyte (Gr. trope + phyton, plant): plant which sheds its leaves or whose above-ground parts die away on approach of winter or dry season.

Trun'cate (L. truncatus, shortened): ending abruptly. Fig. 252. 
Tu'ber (L. tuber, a protuberance or tumor): a short and thick underground stem beset with numerous "eyes," like the potato. Fig. 205.

$T \mathbf{u}^{\prime}$ bercle (L. tuberculum, diminutive of tuber): a small, knob-like outgrowth such as occurs on the roots of Leguminosæ for the habitation of nitrifying bacteria. Fig. 13. Tuber'culate : beset with tubercles.

Tuberif'erous (L. tuber + ferre, to bear): tuber-bearing.

Tu'berous : producing or resembling tubers.

Tu'berous root: a root thickened and tuber-like. Figs. 217 and 218.

Tuft'ed : growing in clusters or clumps.

Tumes'cent : (L. tumescens, swelling up) : somewhat tumid.

Tu'mid (L. tumidus, swollen): swollen.

Tu'nicate (L. tunica, an under garment): having concentric coats like the onion.

Tur'binate (L. turbinatus, cone-shaped): shaped like a top or inverted cone.

Tur'gid (L. turgidus, swollen): swollen.

$\mathrm{Um}^{\prime}$ bel (L. umbella, a sunshade): a kind of indeterminate inflorescence (older flowers toward the circumference) having the pedicels of the individual flowers radiating from about the same plane on the common peduncle. Fig. $83, y$.

$\mathrm{Um}^{\prime}$ 'bellate : occurring in umbels; pertaining to an umbel.

$\mathrm{Um}$ 'bellet: a secondary umbel.

Umbellif'erous (L. umbel + ferre, to bear): producing or bearing umbels. Umbel'liform (L. umbel + forma, shape): having the form of an umbel.

Unarmed': destitute of prickles and the like.

Un'dulate (L. undulatus, wavy): with a wavy surface or margin; repand. Fig. 232.

Une'qual : applied to stamens of diverse lengths.

Unguic'ulate (L. unguis, a nail or claw): having a claw or a narrow, stalklike base.

Uni- (L. unus, one): in composition signifying one or single.

$\mathrm{Ur}^{\prime}$ ceolate (L. urceolus, diminutive of urceus, a pitcher): hollow and cylindrical or ovoid, and more or less contracted below the mouth.

$\mathbf{U}$ 'tricle (L. utriculus, diminutive of uter, utris, a bag or bottle of animal skin): a small, bladder-like body; a small, thin-walled, one-seeded fruit. Fig. 326.

Valv'ate (L. valva, a wing or fold of a folding door): opening by valves; joining at the edges without overlapping. Fig. 283.

Valve : one of the parts into which a dehiscent capsule splits. 
Vas'cular ( $\mathrm{L}$. vasculum, a small vessel) : having vessels or ducts for the conduction of water and elaborated sap.

Vein : one of the branching strands of vascular tissue in a leaf or other organ. Ven'tral (L. venter, the belly) : pertaining to the side of a member which faces the axis from which it springs; in floral organs the side facing the center of the flower; the upper surface of leaves.

Ven'tricose : inflated or swollen on one side more than on the other.

Ver'nal (L. vernalis, of or belonging to spring) : occurring in spring.

Vernation: the arrangement of leaves in the bud.

Verrucose' (L. verruca, a wart): covered with wart-like elevations.

Ver'satile (L. versare, to turn round): applied to an anther which is attached to the filament at its center only and may easily swing about, as in the tiger lily. Fig. 294 .

Ver'ticil (L. verticillus, diminutive of vertex, a whirl, eddy, or whirlpool):

a circle of leaves or flowers around a stem at the same node. Fig. 33 .

Vertic'illate : arranged in a verticil or whorl.

Ves'icle (L. vesicula, diminutive of vesica, a bladder or blister): a small, bladder-like body or cavity.

Vesic'ular : composed of or covered with vesicles.

Vexil'lum (L. vexillum, a banner or flag): the upper petal of a papilionaceous flower; the standard. Fig. 266, $s$.

Vil'lous (L. villus, a shaggy hair): bearing long and soft hairs.

Vir'gate (L. virgatus, made of twigs): wand-shaped, straight and slender.

Vis'cid (L. viscidus, clammy): sticky or clammy.

Vit'ta, pl. vittæ (L. vitta, a band or chaplet worn round the head): an oil

tube found in the carpels of the Umbelliferæ.

Volute' (L. voluta, a spiral scroll): rolled up in any way.

Whorl, pr. hwurl : an arrangement of leaves or floral organs, etc., around the stem at one plane. Fig. 33 .

Wild : growing spontaneously without cultivation.

Wing: a membranous expansion of an organ or member, an ala. The lateral petal of a papilionaceous corolla. Fig. 266, w.

Woolly : clothed with long, coiled, or matted hairs.

Xeroph'ilous (Gr. xeros + philein, to love): adapted to a dry climate or habitat.

$\mathrm{Xe}$ 'rophyte, pr. zē'ro-fi te (Gr. xeros, dry; phyton, a plant) : a plant adapted by its structure to a dry habitat. 


\section{INDEX.}

Absorbed substances, path of, 40.

Absorption, conditions affecting, 307 ; process of, 36.

Accessory buds, 70.

Acer, 238.

Adventitious buds, 70 .

Adventitious roots, 44.

Ecidiospores, 281.

Aërial plants, roots of, 41.

Agaricus, 282.

Agave, 142, 821.

Ailanthus, 213.

Air plants, roots of, 41.

Alæ, 237.

Albumen water, 384 .

Alfalfa, roots of, 39 .

Algæ, 264; nature of, 269.

Alhagi maurorum, 345.

Amaryllidaceæ, 228.

Ament, 158.

Anemone, 147, 180, 207 ; seeds of, 213.

Angular divergence, determination of, 47 .

Annuals, 132.

Anther, 165.

Antheridium, 274, 287, 290.

Ants, leaf-cutting, 317, 318.

Archegonia, 287, 288, 290.

Arctic regions, vegetation in, 342 .

Arisæma triphyllum, 219.

Aristolochia, study of, 48 ; cross section of stem of, 49.

Aristotle, 171.

Artichoke, 139.

Asci, 285.

Asclepias cornuti, 192, 248; bee gathering nectar from, 194; cross pollination of, 191; diagram of flowers of, 193; seeds of, $195,213$.

Asexual and sexual generations, comparison of, 294.

Asparagus mediololdes, 136.

Astragalus caryocarpus, 237.

Atmosphere, 313.

Axillary buds, 70 .

Bacteria, cultures of, 254 ; disease-producing, 260 ; economic importance, 260 ; forms of, $257,258,259$; nature of, 256 ; nitrifying, 260 ; nitrogen-fixing, 261 ; reproduction of, 257 ; staining, 255 .

Balsam, 53.

Bambusa, 834.

Banyan, 139; prop roots of, 41.

Barberry, 136, 160.

Bark, diagram of, 58 ; effect of removal of, 59.

Bast, diagram of, 58 ; use of, 56 .

Batis maritima, 323.

Bean, castor, 8; Lima, 5.

Bee, diagram of head of, 186; head of, 185 ; pollen baskets of, 186,187 ; relation of, to flowers, 185.

Beech, Cretaceous, 355.

Beggar-ticks, 208, 216.

Biennials, 132.

Bittersweet, 207.

Black locust, 135.

Bladderwort, 142, 145, 146.

Bladder-wrack, 266.

Bud, nature of, 70 .

Budding, 71.

Buds, disposition of leaves in, 68 ; leaf and flower, 69 ; position of, 70 ; summer, 66 ; unfolding of leaves of, 68 ; winter, 66 .

Burdock, 208, 216.

Burr-grass, 216.

Butterflies, relation of, to flowers, 183.

Butterfly, diagram of head of, 184; proboscls of, 184.

Cabomba Caroliniana, 326, 327.

Cacti, 73 ; stems of, 140.

Cactus, 322.

Calamites, $353,354$.

Calclum oxalate, occurrence of, in A risæma, 220.

Callitriche stagnalis, 327.

Calyptra, 289.

Calyx, 164.

Cambium, 50 ; diagram of, 58 ; nsefulness of, 114.

Camerarius, 171.

Capsella bursa-pastoris, 149, 233.

Capsules, of moss, 286. 


\section{Introduction to Botany.}

Carbon dloxide, percentage of, in atmosphere, 93 ; test for, 13.

Carboniferous plants, 352, 353.

Carina, 237.

Carpels, 201.

Cassia chamæcrista, 126.

Casslope tetragona, 323.

Castor bean, 168; experiments with, 116, 117, 118.

Catalpa, 207, 213.

Catasetum tridentatum, 190.

Caulicle, 7.

Cecropia adenopus, $317,318,319$; food bodies of, 820 .

Cell, definition of, 104; division, 107 ; diagram illustrating division, 109 ; growth of, 108 ; organs of, 105.

Cells, changes in character of, 110 .

Cellular structure, advantages of, 107 .

Cellulose, reserve, 22.

Central cylinder, 49 .

Centrifugal force, influence of, 25.

Ceratophyllum, 79, 305.

Cereus ingens, 321 ; giganteus, 321.

Chestnut, Cretaceous, 355.

Chinese primrose, 147.

Chloral hydrate, 381 .

Chlorolodide of zinc, 381 .

Chlorophyll, 87.

Chloroplasts, 87, 88, 106.

Chrom-acetic fixative, 382 .

Chromoplasts, 106.

Chromosomes, 109

Cladonia furcata, 284.

Cladophyll, 140.

Classification of plants, 359.

Clinostat, how to make, 15 .

Clover, root tubercles of, 39 .

Cochlearia fenestrata, 811 .

Cocklebur, 208, 216.

Cockroach, 182 ; diagram of eye of, 183.

Cocoanut, seed and seedling of, 23.

Cocoanut palm, 335 .

Colchicum, 122.

Cold, reslstance to, 310 .

Collecting, 368.

Collecting case, how to make, 368 .

Collenchyma, 50.

Color, 181.

Composite flowers, method of diagraming, 155.

Compound eye, 182.

Conceptacle, 266, 275.

Conduction, 57.

Continuity of living substance, 107.

Cordiates, 352, 353, 354 .

Corn, Indian, 10; prop roots of, 41.
Corolla, 164; ligulate, 155 ; tubular, 155.

Corpusculum, 193, 195 .

Corydalis, 233.

Corymb, 159.

Cottonwood, $67,68,207$; buds of, 69 ; dispersal of seeds of, 218,214 .

Cotyledons, 6,7 .

Crab apple, 207.

Cranesbili, elastic carpels of, 210.

Cretaceous period, equable climate in, 355 .

Crocus, 122.

Cross fertilization, 170; devices for, 175 .

Cuscuta, 139 ; roots of, 44 .

Cutin, 56.

Cuttage, 44.

Cutting sections, 376, 377, 382, 383.

Cyanin and erythrosin, 384 .

Cycads, 354, 355.

Cypripedium pubescens, 227 ; insigne, 229.

Cytoplasm, 105; functions of, 106.

Dahlia, 72.

Dandelion, 208.

Darlingtonia, 143, 144.

Darwin, 170.

Date, seed and seedling of, 23.

Datura, cross pollination of, 187.

Deliquescent trunk, 66.

Delphinlum, 231.

Dendrocalamus, 334, 335.

Desert regions, cause of, 346 ; ralnfall of, 346 ; vegetation of, 345 .

Determinate inflorescence, 159.

Devonian plants, 352 .

Diagrams, floral, 152.

Diagrams of leaves, flowers, inflorescences, $158,159$.

Dicentra cucullaria, 232.

Dichogamous, 176.

Dicotyledons, flrst appearance of, 355 ; regions of growth in, 110.

Diffusion, nature of, 36 .

Digestion, 21.

Dimorphic, 176.

Diœclous, 175.

Dlonæa muscipula, 127.

Dispersion by anlmals, 215; birds, 215 ; elastic tlssues, 210 ; water, 216 ; winds, 211.

Dispersion of fruits and seeds, 207; importance of, 208.

Dissecting needles, how to make, 372 .

Dissecting stands, how to make, 371 .

Distribution, original, of plants, 328 .

Division of cells, 107.

Dodder, 41 ; roots of, 30.

Dogbane, 208. 
Double staining, 384 .

Drawing, 1; procedure in, 2 ; rules for, 2.

Drosera rotundifolia, 127, 128.

Egg, 164, 167 ; fertilization of, 167, 168 .

Elder pith, inclosing objects in, 377.

Elements necessary to plants, 38 .

Elm, 208; Cretaceous, 355; dispersal of seeds of, 213.

Embryo, 7; condition of, in seed, 18; formation of, 299.

Embryology of flower, 205.

Embryo sac, 165, 167, 203.

Embryo sac spore, behavior of, 298.

Endodermis, 49.

Endosperm, 168.

Energy, source of, 84 ; source of internal, 131.

Environment, adaptation to, 303; result of unfit, 304.

Environmental factors, most potent, 319 .

Epicotyl, 7.

Epidermis, 48 ; function of, 50,56 .

Epigynous, 205, 206.

Equisetum, 355; arvense, 301.

Erigeron, 250.

Erythronium, albidum, 221; Americanum, 221 ; dens-canis, 222 ; mesachorium, 222.

Euphorbia, 210 ; serpens, 73.

Evergreens, leaves of, 97.

Excurrent trunk, 67.

Family, 361.

Fascicle, 159.

Feathergrass, 208.

Fehling's solution, 385 .

Ferments, 21; position of, 22 ; removal of products of, 23.

Fern, diagram of sphorophyte and gametophyte of, 295.

Ferns, 286; antheridium and archegonium of, 293; character of, 292 ; gametophyte of, 293 ; prothallium of, 293 ; rhizolds of, 294 ; sperms of, 293.

Fertilization, process of, 166 ; result of, 168 ; time required for, 169 .

Field work, 4.

Filament, 165.

Fixative, chrom-acetic, 382.

Floral diagrams, 152, 154, 155, 156.

Floras, factors governing, 328 .

Flower buds, 69 .

Flower, embryology of, 205; longitudinal diagram of, 165 ; morphology of, 201.

Flowers, gulde for drawing, 150.

Food, character and location of, in seeds, 18; demonstration of value of, 14 ; reserve, 12 .
Forces, influence of varlous, 122.

Fragaria, 363 ; virginiana, 235.

Fragrance, 181.

Freesia, 147.

Fucus, vesiculosus, 266, 275; reproduction of, 275 .

Fungi, 264; character of, 277; ravages of, 283 ; saprophytic, 277.

Funiculus, 165.

Gametophyte, 294-300 ; of mosses, 291.

Genera, 360 .

Genus, 361.

Geranium, 207; sylvaticum, 172.

Germination, completion of, 27 ; necessary conditions for, 20 ; time of, 19.

Gingko, 354 .

Girdling, advantage of, 59 ; effect of, 59 ; process of, 54 .

Glomerule, 159.

Glucose, test for, 12.

Glycerine jelly, 385 .

Goldenrod, 72, 73, 140, 208.

Gooseberry, 207.

Grafting, 72.

Grape sugar, test for, 12.

Grape vine, rise of sap in, 31.

Graphis scripta, 284.

Grasses, 111; tertiary, 356.

Gravity, as directive force, 43 ; as guide to growth, 61 ; influence of, 24,114 ; to eliminate effect of, 16 ; nentralizing influence of, 25.

Ground tissue, 53.

Growth, apparatus for measuring, 101 ; conditions necessary to, 112 ; diagram illustrating, 111 ; direction of, 23,61 ; nature of, 133 ; phases of, 112 ; rings of annual, 114 ; regions of continued, 110.

Growth of cells, 108.

Gymnosperms, early representatives of, 354 .

Habitats, kinds of, 305 .

Habits of stems, 73.

Halophytes, 319 ; character of, 323 ; origin of, 326.

Halophytic conditions, 320 .

Hazelnut, 207.

Head, 159.

Heat, resistance to, 812 .

Helianthemnm vulgare, $350,364$.

Heliotropism, 120; definition of, 122.

Herbarium, character of, 367 ; preparing specimens for, 368 .

Hickory, 67, 68.

Hilum, 7.

Honey glands, 143. 
Honey locust, 135.

Honeysuckle, 147.

Hop hornbeam, 207.

Hornbeam, 207.

Horse-chestnut, $67,68,82$; study of, 45 .

Horsetails, 286, 300, 353 ; prothallia of, 301 ; reproduction of, 300 .

Hyacinth, 147, 206, 223.

Hydrophytes, 319 ; character of, 823 ; origin of, 326 ; most general characteristics of, 326.

Hydrophytic conditions, 320 .

Hydrotropism, diagram illustrating, 102.

Hypocotyl, 7.

Hypogynous, 204, 205.

Hypoxis erecta, 223.

Imbedding in paraffin, 386.

Indeterminate inflorescence, 159.

Indian corn, 168; constitution of stem of, 52 ; cross section of stem of, 52 ; inflorescence of, 177 ; stem of, 59.

Insects, adaptations to, 179 ; sense of sight of, 182 ; sense of smell of, 182 .

Internal energy, source of, 131.

Internodes, 66.

Iodine solution, 387.

Ipomca, 312; leptophylla, 138.

Iridaceæ, 228.

Iris, 175 ; germanica, 224 ; xiphium, 225.

Jimson-weed, cross pollination of, 187.

- Keel, 237.

Kœlreuter, 171.

Lahore, maximum temperature at, 812 .

Larkspur, 147, 154, 207.

Layering, method of, 44.

Leaf bud, 69.

Leaf, cross section of, 88 ; diagram of essential parts of, 89 ; summary of functions of, 99 ; work done by, 85 , and pages following.

Leaves, amount of work done by, 96 ; characterization of, 98 ; continued growth of, 112 ; disposition of, in buds, 68 ; duration of, 96 ; formation of, 66 ; guides for drawing, 150 ; lifting power in, 61 ; light relation of, 82 ; modified, 142 ; position of, 81 ; prominence of, 81 ; relation of, to roots, 99 ; significance of deflnite arrangement of, 82 ; size and form of, 97 .

Lemna, 40.

Lenses, how to use, 372 ; care of, 373.

Lenticels, 93.

Lepidodendrons, $353,354$.

Leucoplasts, 105, 106.

Lichens, 264 ; nature of, 284.
Life, length of, 133.

Light, 308; as guide to growth, 63 ; effect of dim, 308; effect of intense, 309 ; influence of, 114 ; in temperate regions, 342 ; nature of, 84.

Ligulate corolla, 155.

Lilac, 82.

Liliaceæ, 228.

Lima bean, 168 ; study of, 5 .

Lime water, 387 ; use of, 18.

Linaria cymballaria, 65.

Living substance, continuity of, 107.

Lycopodium, 202, 204.

Lycopods, 353.

Macrosporangium, 203.

Macrospore, 203.

Macrosporophyll, 203.

Maple, 207, 208; Cretaceous, 355.

Materials, conduction of, 57 .

Medullary rays, 49 ; diagram of, 58,60 ; function of, 51 ; movement in, 60 .

Melilotus alba, cross sections of leaf of, 90, 91 .

Mentzelia, 216.

Mesembryanthemum, 142, 322 .

Micropyle, 7, 165, 167.

Microscope, compound, 373,374 ; diagram of compound, 374 ; how to use, $372,373,374$, 375 ; simple, 371.

Microsporangium, 203.

Microspore, 203.

Microsporophyll, 203.

Microtome, method of using simple, 378.

Migration, 17; by seeds, 209.

Mildews, 264.

Milkweed, 207, 208.

Mimosa pudica, 124, 126 ; preparing seedlings of, 100.

Mistletoe, 41.

Modified leaves, 142.

Modified roots, 138.

Modified stems, 139.

Monocotyledons, regions of continued growth in, 110 ; time of appearance of, 355 .

Monœcious, 175.

Morning glory, 184.

Morphological elements, 134, 136 ; characteristics of, 138.

Moss, diagram of sporophyte and gametophyte of, 296.

Mosses, dissemination of spores of, 291 ; gametophyte of, 296 ; illustration of, 289 ; reproduction of, 290 ; sexual and asexual generations of, 291 ; sporophyte of, 291.

Motor organs, 122.

Mountain heights, physical conditions of, 347 ; vegetation of, 347 . 
Mounting sections, 383.

Movements, causes of, 120 ; spontaneous, 129.

Mucor mucedo, 277, 278; reproduction of, 277.

Mucor stolonifer, 277.

Multan, maximum temperature at, 312.

Multiplication, 17.

Myosotis palustris, 173.

Myosurus, 231.

Myriophyllum, 79, 305.

Myxomycete, 252; formation of spores of, 253.

Naias flexilis, 218.

Nasturtium, 181.

Nectar, 180, 181 ; allurement by, 180 ; glands, 143 ; receptacle, 180.

Nelumbo, 140.

Nelumbo lutea, 324 ; dispersion of seeds of, 216.

Nemastylis, 227.

Nematophyton, 352.

Nepenthes, 142, 143.

Nitrogen, how obtained by plants, $38,39,260$.

Nodes, 66.

Nucellus, 165.

Nucleus, 105 ; functions of, 106 ; as bearer of inheritabie qualities, 168.

Nymphæa odorata, 204.

Oak, Cretaceous, 355 ; reserve materials in cotyledons of, 27.

CEdogonium, 163, 164, 166, 264.

CEnothera Missouriensis, 330 ; laciniata, 330 ; rhombipetala, 830 ; speciosa, 247.

Onion, preparing root tips of, 100.

Oogonium, 163, 274.

Operculum, 291.

Orange, 208.

Orchidaceæ, 228.

Orchids, cross pollination of, 190 ; roots of, 41 .

Order, 361.

Organs of the cell, 105.

Osage orange, 135.

Osmosis, 37.

Osmotic pressure, 60, 61 .

Ovary, 165.

Orule, 165.

Oxailis, 148, 207 ; stricta, 238; violacea, 238.

Oxidation a vital process, 132.

Oxygen, evolution of, 91 ; necessity of, in germination of seeds, $15,21$.

Palisade cells, 88.

Palm, 111; Cretaceous, 355; stem of, 59; Sabal, 339 ; trees, 335.
Pappus, 156.

Paraftin, imbedding in, 386; oven, how to make simple, 386 ; sections, cutting and mounting, 383.

Parasitic plants, roots of, 40.

Parmelia colpodes, 284.

Parmentiera cereifera, 337.

Pencils, colored, use of, 3 ; how to sharpen, 2.

Perennials, 182.

Pericycle, 49 ; function of, 51 .

Perigynous, 204, 205, 206.

Permian plants, 354.

Petal, 164.

Petunia, 184.

Phloëm, diagram to show, 58, 91 ; function of, 51.

Phloroglucin, 387.

Photosynthesis, 92 ; essential nature of, 132.

Pine, 177, 208; pistillate inflorescence of, 178 ; staminate catkin of, 177.

Pines, 339.

Pistil, 165, 203 ; compound, 204 ; half inferior, 204 ; inferior, 205 ; simple, 203 ; superior, 204.

Pistillate, 175.

Pitcher plants, 142.

Pith, 49; elder, inclosing objects in, 377; function of, 51 .

Plant forms, diversity of, 136.

Plants, antiquity of, 351 ; classification of, 359 ; Carboniferous, 352 ; Cretaceous, 355 ; Devonian, 352 ; Jurassic, 355 ; of past ages, 351 ; Permian, 354; Tertiary, 356.

Plasma membrane, 105; function of, 105.

Plasmodium, streaming of protoplasm in, 104.

Plastids, 106.

Pleurococcus, 264, 284; viridis, 270.

Pliny, 171.

Plum, 135, 207; diagram of flower of, 206.

Plumule, 7.

Poison ivy, 138.

Pollen, allurement by, 180 ; of pine, 177.

Pollen grain, 165 .

Pollen tube, $165,167,169$.

Pollinium, 190, 192, 197.

Poplar, 177.

Poplars, Cretaceous, 355.

Poppy, 180.

Populus montlifera, 213, 230.

Portulacca, 322.

Potato, .73, 136, 139.

Preparing materials for herbarium, 368.

Prickiy ash, 135.

Primary cortex, 49 ; function of, 50 .

Primitive physical conditions, 351.

Primula, 176. 
Pronuba moth, 196-201.

Protective adaptations, 317.

Proteid, test for, 12.

Proteids, 96; manufacture of, 91.

Proterandrous, 176.

Proterogynous, 176.

Prothallia, 288.

Prothallium, 203.

Protonema, 287.

Protoplasm, streaming of, 103.

Protoplast, 105; sensibility of, 118.

Prunus chicasa, 234.

Puccinia, 280.

Quaternary, exodus southward in, 356 ; northward migration in, 358.

Quercus virens, 341.

\section{Raceme, 158.}

Races, 361.

Radicle, 7.

Rainfall, effect of annual, 340 .

Rainfall map, 332, 333.

Ranunculus, 147; abortivus, 231 ; fluitans, $325,327$.

Raphia tædigera, 97.

Raw materials, supply of, 93 .

Reagents, 381 ; applying, 378.

Receptacle, 165, 166.

Relationship, best evidence of, 364 .

Reproduction, different methods of, 162 ; relative values of asexual and sexual, 169.

Reserve materials, circulation of, 21 ; location of, in stems, etc., 69.

Respiration, 92 ; essential process of, 131.

Resting spore, 163.

Rhizoid, 289.

Rhododendrons, Cretaceous, 355.

Ring leaf, 205.

Rings of annual growth, 114; constitution of, 115.

Root hairs, 26 ; action of, 35 ; importance of, 33 ; relation of, to water-conducting tubes, 40 ; structure of, 35.

Roots, adventitious, 44 ; aërial, 41 ; clinging, 43; definition of, 44; demonstration of erosive action of, 28 ; demonstration of region of growth of, 30 ; directive forces affecting, 43 ; early formation of, 26 ; extent of, 39 ; force of absorption by, 29 ; functions of, 81 ; modified, 138 ; necessary substances absorbed by, 99 ; parasitic, 40 ; prop, 41 ; region of growth of, 30 ; relation of, to leaves, 99 ; of water plants, 40.

Rosa Arkansana, 236.

Rose, 180, 207; diagram of tlower of, 206.
Rubus hispidus, $362,363$.

Rubus villosus, $361,363$.

Ruscus, 140, 141.

Rusts, 264, 279.

Safranin, 388.

Sagittaria, 62, 78 .

Sahara, vegetation of, 346 .

Salix, 229 ; polaris, 344.

Saits, effect of excess of, 315 .

Salvia, cross pollination of, 188 .

Saprophytic fungi, 277.

Sarcobatus baileyi, 346 .

Sarraceniatvariolaris, 144.

Sassafias, Cretaceous, 355 .

Scape, 219.

Scarlet runner, 128.

Sclerenchyma ring, 50.

Scolopendrium, 292 ; officinarum, 309.

Sealing in balsam, 388 .

Section cutting, $376,382,383$.

Sedum, 142.

Seed-coats, 6 .

Seed dispersion, Importance of, 208.

Seedlings, providing for students' use, 5 .

Seed, protection of, 18.

Seeds, dispersion of, by birds and other antmals, 215 ; by elastic tissues, 210 ; by winds, 211; various devices for dispersion of, 212 ; importance of dispersion of, 208 ; method of planting, 5 ; migration by, 209 ; multiplication and migration by, 17; nature and purposes of, 16 ; size of, 27 .

Selaginella, 202, 203, 204.

Self fertilization, 170 .

Sempervivum tectorum, 309.

Sensitive plant, 124, 125, 126.

Sepal, 164.

Sequoia, 355; distribution of, in Cretaceous period, 355 ; extermination of, in Europe and Asia, 856 ; photograph of, 357.

Sexual and asexual generations, comparison of, 294.

Sharpening knives, etc., $378,879$.

Shoot, upward growth of, 55.

Shrankia uncinata, 126.

Siberian forests, 810 .

Sicyos angulatus, 316.

Sieve tubes, 59 ; movement in, 61 .

Sigillarias, 353,354 .

Sisyrinchium, 224, 227.

Slime moulds, 251 ; formation of spores of, 253 ; nature of, 252.

Smilax, 111, 134; chloroplasts in, 309 .

Smuts, 264.

Snakeroot, 208, 216.

Soil, 315 ; effect of character of, 330 ; forma- 
tion of, 34 ; nature of, 33 ; as reservoir for water, 34.

Solanum jasminoides, 187, 142.

Soldanellas, 312, 813.

Solomon's seal, 72; diagram of flower of, 206.

Sorus, 288.

Spadix, 219.

Spathe, 219.

Species, 360.

Sperm, 164, 167.

Spermatophytes, asexual and sexual generations of, 294, 297, 299; studies of selected, 218.

Sphinx moth, 182 ; and Datura flower, 188.

Spike, 158.

Spirodela, 40.

Spirogyra, 264; reproduction of, 272.

Spongy parenchyma, 88.

Spontaneous movements, 129.

Sporangia, 202.

Sporophyll, 202, 203, 300.

Sporophyte, 294-300 ; of moss, 289, 291.

Sprengel, 171.

Spurge, 207.

Squash, tendrils of, 103.

Squirting cucumber, 211.

Staining paraffin sections, 888.

Stamen, 165.

Stamens, 201.

Staminate, 175.

Standard, 287.

Starch, 96 ; formation of, 87 ; sheath, 49 ; test for, 12.

Stele, 49.

Stem, chief function of, 55 ; diagram of, 58 ; summary of functions of, 60 .

Stems, characterization of, 74 ; functions of, 72 ; habits of, 73 ; modiffed, 139.

Stigma, 165.

Stigmatic chamber, 193.

Stigmatic disk, 192.

Stimulus, transmission of, 126.

Stomata, 86 ; action of, 94 ; determination of necessity of, 80 .

Strawberry, 207.

Streaming of protoplasm, 103.

Strengthening elements, 56 .

Study, method of, 1.

Style, 165.

Sugar, grape, test for, 12.

Sumac, 207.

Sundew, 128.

Sunflower, hellotropism of, 121.

Superior pistil, 204, 205.

Sweet alyssum, 147.

Syngenesious stamens, 155.
Tabernæmontana dichotama, 338.

Tables for laboratory, 371.

Taraxacum officinale, 248.

Taxodium, 133.

Taxus, 133.

Tecoma radicans, 65 .

Teleutospores, 280.

Temperate regions, light in, 342 ; rainfall of, 338,340 ; temperature of, 338,340 ; vegetation in, 338 .

Temperature, 310.

Terminal buds, 70 .

Testa, 6.

Theophrastus, 171.

Tillandsia usneoides, 840,341 .

Timber line, 348, 349 .

Toad stools, 281.

Touch-me-not, 211.

Tracheal tubes, 57; diagram of, 58.

Tradescantia, study of hairs of, 100, 103.

Translocation, diagram to show, 99.

Transpiration, 91.

Transplanting, time for, 33.

Transporting forces, 60 .

Tree claims, $329,330$.

Triassic plants, 354 .

Trimorphic, 176.

Triteleia, 147.

Tropical forest, 336 .

Tropics, mean temperature of, 331 ; rainfall of, 331 ; vegetation in, 331,334 .

Trumpet creeper, 65, 108, 138, 218 ; clinging roots of, 43.

Tubercles on roots, 38 .

Tubular corolla, 155.

Tulip, 122, 223.

Twining, method of, 130 ; benefit of, 181 .

Twining plants, 130.

Ulothrix zonata, 162.

Umbel, 159.

Uredospores, 280.

Utricularia, 134, 136, 145:

Vallisneria, 179, 180.

Variation, cause of, 360 .

Varieties, 361.

Vascular bundle, 49 ; diagram of, 49 ; funce tion of, 51 ; position of, in leaves, 88 .

Vaucheria, 264, 278.

Vega expedition, 810

Venus's flytrap, 127.

Vexillum, 237.

Viola, 239-247.

Violet, 154, 180, 207. 


\section{6}

\section{Introduction to Botany.}

Virginia creeper, 78, 136; effect of darkness on, 113 ; experiment with tendrils of, 103. Viscid disk, 190.

\section{Walnut, 207.}

Water, cross pollination by, 178 ; demonstration of region of rise of, 29,53 ; current, diagram to show, 99 ; effect of submergence in, 307; importance of, 37 ; effect of scarcity of, 306 .

Water lily, 140.

Water plants, roots of, 40.

Water supply, 306.

Wild cucumber, tendrils of, 103.

Willow, 177 ; adventitious roots of, 44 ; Cretaceous, 355.

Wind, adaptation to, 177.

Winds, effect of, 314 .
Winter buds, protection afforded, 67.

Wood, use of, 56.

Xerophytes, 319 ; character of, 322 ; North American, 321 ; origin of, 326.

Xerophytic conditions, 320.

Xylem, diagram to show, 58, 91; function of, 51.

Yeast, culture of, 256; in aicoholic fermentation, 263; in bread-making, 262 ; nature of, 261 ; reproduction of, 262.

Yucca, 147; arboreus, 321 ; cross pollination of, 196-201; in twilight, 198.

Zilla spinosa, 345 .

Zygophyllum cornutum, 322, 323. 


\section{INTRODUCTION TO BOTANY}

\section{KEY AND FLORA}

BY

\section{WILLIAM CHASE STEVENS}

PROFESSOR OF BOTANY IN THE UNIVERSITY OF KANSAS

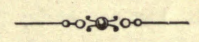

BOSTON, U.S.A.

D. C. HEATH \& CO., PUBLISHERS 
Copyright, I902,

By D. C. Heath \& Co.

PRINTED IN

UNITED STATES

OF AMERICA 


\section{MANNER OF USING THE KEY AND FLORA.}

THE manner of using the Key and Flora in finding the names of plants will best be learned by following a few examples. Since the Dogtooth Violet is one of the first to bloom in the spring, we will begin with it.

Turn to the Key on page 7 , and begin with Class I. Since our plant has its ovules inclosed in an ovary, it can not be found under this class, in which the plants (such as firs, pines, spruces, etc.) have naked ovules. (See Botany, page 177, par. 127, and Figs. 93 and 94.)

But under Class II we find the ovules inclosed in an ovary, and we see at a glance that our plant belongs here under Subclass I, since the parts of the flower are in whorls of three, and the leaves are parallel veined. (Compare with Subclass II at the bottom of the page.) Now read after A, and if the terms are not understood, look them up in the Glossary (Botany, pages 399428). Clearly our plant has a corolla, and can not come under A. Reading next after B, we note that the flowers of our plant are not on a spadix and are provided with two whorls of floral envelopes which may be considered as calyx and corolla, and we accordingly look for it under B. (Read after C, to be sure that it could not belong there.)

Under B are three lines beginning with "Perianth," and we see that our plant belongs under the second of these, which reads, "Perianth, wholly free from the ovary," etc.

Thus we are brought to the Liliaceæ, page 19, and we turn directly to that page and read the description of the family, and finding that our plant conforms to it, we follow the key to the family to find the genus to which our plant belongs. Evidently we must hunt at once under the line "Not woody climbers." The flowers of our plant are not umbellate, nor borne in racemes or spikes, and so we pass at once to the third line, beginning "Flowers." Reading under this line we find, "Stems leafy only at the base; flowers single and erect." But our flower is not erect, and so we pass to the next line, where the flower is said to be nodding. This is true of our flower, and it is evidently to be looked for under Genus VII, Erythronium.

We read the description of this genus on page 22, and find that our plant belongs there. We next read the description of the two species there given to determine to which of these our Dogtooth Violet may belong.

Let us take for our next example the Maple, which also blooms early in the spring. Turn again to the Key on page 7. Clearly the maple belongs in Class II; and under this we find that we can pass directly to Subclass II, since the stem of the maple has distinct zones of bark, wood, and pith, and the calyx (the corolla is absent in the maple) is 4-5 lobed or parted.

Under Subclass II, on pages 8, 9, and IO, we find three main divisions, headed with A, B, and C. After A we read, "Corolla, and sometimes calyx, wanting," while in the other divisions both calyx and corolla are present. Since the corolla is lacking in the maple, we must look for it under A. Here 


\section{Using the Key and Flora.}

we find two large groups, one having "flowers monœcious or diœcious, staminate and sometimes pistillate, flowers in catkins," and the other having "flowers not in catkins." The maple, of course, belongs under the latter. Here we find two main groups, headed, "Pistil more than I," etc., and "Pistil r," etc. Our plant belongs under the latter, where the plants are grouped as "herbs," or "shrubs and trees." The maple being a tree, we proceed under the latter heading.

The maple has a pair of ovules in each cell of the ovary, and the fruit is a double samara, and we accordingly find that we must look for it in the genus Acer, in the family Sapindaceæ, on page 74. Having traced a plant to its family (in this case the Key directed us at once to the genus), it is a good plan to read the description of the family, to make certain that the plant has been rightly traced. Now read the description of the genus, Acer, and of the species under it, and determine to which one of these our maple belongs.

As a final example we will trace the single-flowered Sweet Alyssum of the greenhouses. Beginning with the Key on page 7 , as pefore, we find our plant goes to Subclass II, and under this to division B on page 8. Under B we find three main groups, numbered (I), (2), and (3), depending on the number of stamens. Our plant must be under (3), "Stamens not more than twice as many as the petals," etc. Under this division we find two chief groups, designated by $(a)$, "Ovary superior," etc., and (b), "Ovary inferior," etc. (on page ro). Clearly our plant comes under $(a)$. Here we find four groups, depending on the number and character of the pistils. Our plant goes to the last of these, "Pistil I, compound, as shown by the number of cells, placentæ, styles, or stigmas." (Read about compound pistils on pages 203 and 204 of the Botany.)

In alyssum we conclude that two carpels compose the pistil, because there are two cells, each having a row of ovules; thus indicating two placentæ and two carpels. A pistil may be one-celled and still be compound as indicated by the placentæ or rows of ovules. The violets afford a good example of this.

Under "Pistil I, compound," etc., there are two groups, one having the " ovary I-celled," and the other with "ovaries 2 or more celled." Our plant comes under the latter, and under "Flowers nearly or quite regular"; and since the stamens of alyssum are tetradynamous, we find that we must look for it under the family Cruciferæ, page 47 .

Following the Key to the family, we find we are led to Division II, under "Flowers, white"; and since the pods of our plant become orbicular, we are taken to Genus XIV, Alyssum, We now turn the pages of the family until we find this genus (on page 53).

When only a few genera have been given under a family, a key to the family is not given, and the proper genus is to be determined by reading the description of each under that family.

To understand the significance of the accent marks used with the names of the families, genera, and species, read the footnote on page $\mathbf{I} 2$.

This Spring Flora does not pretend to be complete for any region. Its purpose is to afford the student sufficient drill during the spring term to make him thereafter independent in the use of the larger Floras. The habit of looking up the names of plants leads incidentally to a knowledge of many interesting facts about plant structures and relationships. 


\section{SYNOPSIS OF THE MAIN GROUPS OF THE VEGETABLE KINGDOM.}

\section{Subkingdom I. Thallophyta. Thallophytes.}

Body of the plant not differentiated into root, stem, and leaf, as the terms are used in connection with the higher plants. No vascular bundles present. Under the Thallophytes are classified the following main groups : -

A. Myxomycetes, or slime-moulds, which, in their vegetative state, are masses of protoplasm without cell wall, called plasmodia. Multiplication by means of asexual spores. Destitute of chlorophyll, and dependent upon organic materials produced by other plants. See Botany, page 252.

B. Alga. Unicellular or multicellular, with true cell wall. Cells either all alike, or some modified to perform special functions. In most instances possessed of chlorophyll, and providing their own food. Reproduction by division, or by asexual or sexual spores. See Botany, page 269.

C. Fungi. Unicellular or multicellular, with true cell wall. Cells either all alike, or some modified slightly for special functions. Destitute of chlorophyll, and living as parasites or saprophytes. - The cells are usually threadlike, and are termed hypha. The masses of threads forming the vegetative body of the fungus are termed collectively the mycelium. The hyphæ often fuse where they come in contact and form a tissue, such as is found in the toadstools. Reproduction by division, or by asexual or sexual spores. See Botany, page 277 .

\section{Subkingdom II. Bryophyta. Mosses. Liverworts.}

Plant body multicellular, and in the higher forms differentiated into stems and leaves; but there are no true roots, absorption from the soil being carried on by hairs, known as rhizoids. Chlorophyll present. Vascular bundles imperfect or wanting. In the life history of an individual both sexual and asexual generations occur, the former being the more conspicuous, and bearing the sexual organs, antheridia and archegonia. Reproduction by buds, asexual spores, and fertilized egg cells. See Botany, page 289 . 
Subkingdom III. Pteridophyta. Vascular Cryptogams. Ferns, Horsetails, Club-mosses.

Plant body differentiated into true roots and leaf-bearing shoots. True vascular bundles are present. Reproduction as in the Bryophytes. The germination of asexual spores results in small prothallia, which bear sexual reproductive organs. The asexual generation or sporophyte is the more conspicuous. See Botany, page 292.

Subkingdom IV. Spermatophyta. Seed-bearing plants. Flowering plants.

Plant body differentiated into true roots and leaf-bearing shoots. Special shoots, with shortened internodes and modified leaves, form flowers for the purpose of reproduction. After the fertilization of the egg the embryonic leafbearing plant (the embryo in the seed) is formed, but soon temporarily ceases to grow, and remains in an inactive condition until the germination of the seed. Sexual generation, or gametophyte, reduced to one or a few cells within the pollen grain and ovule. See Botany, page $\mathbf{1} 66$. 


\title{
KEY TO SOME FAMILIES OF SPERMATO- PHYTES.
}

\author{
CLASS I.
}

GYMNOSPERMAE. Ovules not inclosed in an ovary.

Fruit usually a cone, or berrylike by coherence of scales.

CONIFERE, page I2.

\section{CLASS II.}

ANGIOSPERME. Ovules inclosed in an ovary.

SubClass I. Monocotyledons. Embryo with I cotyledon. Stem having no distinct zones of bark, wood, and pith. Parts of the flower usually in whorls of 3. Leaves mostly parallel-veined.

A. Flowers on a spadix or fleshy axis, without calyx or corolla, and without chaffy scales or glumes.

Marsh herbs with long linear or swordlike leaves, and flowers in cylindrical terminal spikes.

TYPHACEE, page 15

Terrestrial plants; flowers on a spadix surrounded by a spathe. ARACEe, page r8.

B. Flowers not on a spadix; provided with calyx, or both calyx and corolla.

Perianth adherent to ovary throughout its length, or apparently so, i.e. ovary inferior. Stamens I or 2, gynandrous, flowers very irregular. ORCHIDACE 1 , page 26. Stamens 3 ; anthers extrorse, opening lengthwise. IRIDACEA, page 25.

Stamens 6. Flowers borne on a scape from a bulb. Amaryllidace.te, page 24 Perianth wholly free from the ovary.

Segments of the perianth all nearly alike in form and color, but in the genus Trillium having 3 green sepals and 3 withering persistent petals.

LiLIACEN, page Ig.

Perianth of 3 green sepals and 3 deliquescent petals. Сомmelinace

C. Flowers with sepals and petals reduced to mere scales and bristles, and inclosed in scalelike bracts or glumes.

Glume, a single scalelike bract; stems solid.

Cyperaces, page 18.

Glumes of 2 sorts in pairs; stems usually hollow.

GRAMINE/E, page 15 .

Subclass II. Dicotyledons. Embryo with a pair of opposite cotyledons. Stems having distinct zones of bark, wood, and pith. (In herbaceous plants the wood zone is often not hard nor well pronounced.) Parts of the flower mostly in whorls of 4 or 5 . 
A. Corolla and sometimes calyx wanting.

- Flowers monœcious or diøcious; staminate, and sometimes pistillate flowers in catkins.

Leaves oddly-pinnate. Fruit, a nut inclosed in a husk. JUGLANDACE 2 , page 27.

Leaves simple. Fruit, a I-celled, many-seeded pod. Seeds downy-tufted at one end.

Salicace E, page 29 .

Leaves simple. The 2-7-celled ovary becoming a I-celled, I-seeded nut, often associated with an involucre, as in the oak and hazel nut.

CUPUlifere, page 30 .

Flowers not in catkins.

Pistil more than I, ovary, or its cells, containing I or only a few ovules.

Pistils few to many, and distinct or nearly so, calyx petallike. Flowers not in panicles.

RANUNCULACE

Pistils 3 to 6, calyx petallike. Flowers in drooping panicles.

Menispermacee, page 44 .

Pistil r, simple or compound.

Herbs (sometimes shrubs or trees).

Ovary I-celled, inferior; ovules 2-4.

Santalace $A$, page 34 .

Ovary I-celled, invested by the tube of the corollalike calyx.

Nyctaginace e, page 36 .

Ovary I-celled, free from the commonly petallike calyx; stipules sheathing the stem at the nodes.

Polygonacee, page 35 .

Ovary mostly 3 -celled. Plants usually exuding a milky secretion when wounded.

EUPHORBIACEE, page 72 .

Shrubs or trees (sometimes herbs).

Ovules, a pair in each cell of the ovary.

Fruit a double samara.

Fruit a r-celled, I-seeded samara.

Acer in Sapindacese, page 74.

Oleace $x$, page 90 .

Ovules single in each cell of the 1-2-celled ovary.

Style single; anthers generally opening by uplifted valves.

Style 2-cleft.

LAURACE $E$, page 44.

URTICACEE, page 33 .

Ovules single in each cell of the 2-5-celled ovary. RHAmnaceE, page 75 .

B. Corolla and calyx present, the former of separate petals, i.e. polypetalous.

(I) Stamens more than ro, and more than twice the sepals or lobes of the calyx.

Pistils numerous, separate, and concealed in a hollow receptacle.

Rosa in Rosacese, page 62.

Pistils more than $\mathrm{I}$, separate, surmounting a more or less convex receptacle.

Stamens apparently inserted on the calyx, distinct. ROSACE $E$, page 56 .

Stamens inserted on the receptacle.

Filaments shorter than the anthers, stamens numerous, sepals 3 , petals 6. Trees.

ANONACEE, page 38.

Filaments longer than the anthers.

Flowers diocious; woody climbers with small flowers.

MENISPERMACE

Flowers perfect, petals deciduous. RanUnCULACE.F, page 38.

Pistils several, cohering in a ring; stamens numerous and monadelphous.

MalvaceE, page 78. 


\section{Key.}

Pistil I as to the ovary, styles and stigmas sometimes several.

Ovary simple, $\mathrm{r}$-celled, 2-ovuled.

ROSACE $E$, page 56 .

Ovary simple; numerous ovules on I parietal placenta; leaves lobed and peltate.

Podophyllum in Berberidacee, page 43.

Ovary compound, I-celled.

Placenta central.

Placentæ parietal, 2 or more.

Portulacaces, page 36 .

Ovary 2-several-celled, free from the calyx.

Stamens 5-10 adelphous.

Stamens monadelphous.

Papaverace e, page 45 .

Tiliace e, page 78 .

Malvace

Ovary 2-5-celled; calyx borne at the summit, trees or shrubs, with stipulate leaves.

Plants without stipulate leaves.

(2) Stamens of the same number as the petals and opposite them.

ROSACEE, page 56 .

SAXIFRAGACE E, page 54 .

Ovary I-celled; style 3-cleft; sepals 2.

Portulacacee, page 36 .

Ovary I-celled; style simple.

Ovary 2-6-celled.

BERBERIDACE $E$, page 42.

Calyx lobes mostly obsolete; plants climbing by coiled tendrils, or suckerlike disks.

VITACE 5 , page 76 .

Calyx 4-5-cleft; small shrubs or trees.

RHAMNACEE, page 75 .

(3) Stamens not more than twice as many as the petals; alternate with them when of the same number.

(a) Ovary superior; not adherent to the calyx.

Pistils 2 or more, and separate.

Stamens coherent by their anthers, and united to a fleshy disk surmounting the two pistils.

ASCLEPIADACE 5 , page $9 \mathrm{I}$.

Stamens distinct on the receptacle. Leaves punctate.

Stamens separate on the receptacle. Leaves not punctate.

RUTACEE, page $7 \mathrm{x}$.

RANUNCULACE $Æ$, page 38 .

Pistils 2-5, somewhat united at the bases of the ovaries; trees or shrubs.

SAPINDACE/E, page 74 .

Pistil I and simple, with I parietal placenta, or sometimes 2-celled, with

a row of ovules in each cell.

LEGUMINOSE, page 65.

Pistil I, compound, as shown by the number of cells, placentæ, styles, or stigmas.

Ovary I-celled.

Corolla irregular; petals 4 , somewhat united; stamens 6 , and diadelphous.

Fumariaces, page 46.

Corolla irregular; petals and stamens 5 ; placentæ 3 , parietal.

Corolla nearly or quite regular.

VIOLACEe, page 79.

Ovule I; styles or stigmas I-3. Shrubs or trees.

ANACARDIACE. 5 , page 73 .

Ovules more than $I$ on a central or basal placenta; herbs with tumid nodes.

Caryophyllaces, page 37 .

Ovary 2 or more celled.

Flowers irregular; ovary 3-celled.

SAPINDACE $E$, page 74 .

Flowers nearly or quite regular.

Stamens tetradynamous (sometimes fewer than 6); petals 4 .

CRUCIFERE, page 47. 
Stamens to (rarely only 5 ), somewhat monadelphous at base, leaves 3 -foliate, or more or less lobed and divided.

Geraniacese, page 70 .

Stamens 5, carpels 3, pod inflated. SAPINDACE

(b) Ovary inferior, being more or less adherent to the calyx.

Ovary $\mathrm{x}$-celled, many-seeded; 2 parietal placentæ.

SaXifragace e, page 54 .

Ovary 4-celled (sometimes 2-celled); pollen cobwebby.

ONAGRACE $\approx$, page $8 \mathbf{r}$.

Ovary 2-5-celled; petals 5; fruit a 2-several-celled pome; shrubs or trees.

ROSACE $A$, page 56 .

Ovary 2-celled; style $I$; ovule $x$ in each cell; petals 4 ; fruit a 2 -seeded drupe.

CORNACE , page 86 .

Ovary 2-celled; styles 2; $x$ ovule in each cell; petals 5 .

UMBELLIFERE, page 82.

C. Corolla and calyx present, the petals more or less united (gamopetalous).

(I) Stamens more numerous than the corolla lobes.

Pistil 1.

Ovary I-celled, with I parietal placenta.

Ovary 3-12-celled.

LEGUMINOSE, page 65.

Stamens free from the corolla, or nearly so.

Stamens borne on the base of the corolla.

Ovary 5-celled; stamens monadelphous at base.

ERICACEE, page 86.

EBenacee, page 90.

Pistils several, their ovaries united a ring

(2) Stamens as many as the lobes of the corolla, and opposite them.

Ovary I-celled, several-seeded; style r.

Primulacee, page 88.

(3) Stamens of the same number as the corolla lobes (or fewer), and alternate with them. (a) Ovary inferior, being surmounted by the other parts.

Stamens united by their anthers into a ring; flowers in an involucrate head.

Stamens separate, and free from the corolla, or nearly so.

Сом POSITE, page ir6.

Campanulacese, page 115 .

Stamens inserted separately on the corolla.

Ovary 3-celled. Stamens $1-4$.

Ovary 2-5-celled.

VALERIANACEe, page II4.

Leaves opposite, with stipules, or in whorls.

Leaves opposite, without true stipules.

Rubiacefe, page iro.

CAPRIFOLIACEE, page II2.

(b) Ovary superior, or free from the other parts.

Corolla irregular.

Ovary 4-lobed, with I central style; corolla labiate.

LABIATE, page 99.

Ovary 4-celled, but not lobed, with terminal style; corolla somewhat 2-lipped.

VERBENACE E, page 98.

Ovary I-celled, with free central placenta. Calyx and corolla 2-lipped. Aquatic herbs. LentiBulariacee, page ro5.

Ovary I-celled, with 2 or 4 parietal placentæ; corolla more or less 2-lipped.

OROBANCHACEA, page 106. 


\section{Key.}

Ovary and pod 2-celled by the meeting of 2 parietal placentæ. Calyx and corolla more or less 2-lipped. Trees or woody climbers.

BignONIACEA, page IO7.

Ovary and pod 2-celled; placentæ axillary; seeds mostly few on hooked projections.

ACANTHaCee, page 108.

Ovary 2-celled; placentæ axillary, ovules mostly numerous, not on Corolla regular. hooked projections. SCROPHULARIACEe, page ro3.

Ovaries 2, united only at the top to a fleshy disk; stamens monadelphois; pollen in horny masses. AsClepiadacese, page $9 x$.

Ovary I.

Deeply 4-lobed; chiefly rough, hairy herbs.

BORAGINACEA, page 96.

Entire, I-celled, with 2 parietal placentæ (sometimes appearing 2-celled by union of placentæ); usually hairy herbs with toothed, lobed, or divided leaves.

HYDROPHYLLACE. 2-celled; stamens 2-4, ovules borne on hooked projections.

ACanthaceer, page 108. 2-celled; stamens 4; flowers in spikes; chiefly stemless herbs.

Plantaginacee, page iog.

2-celled; stamens 2; trees or shrubs.

Oleacese, page go. 3-celled; style 3-lobed; corolla lobes 5 ; stamens 5 .

Polemoniacea, page 94.

2-4-celled; $1-2$ ovules in each cell; twining or slender trailing herbs; leaves alternate. Convolvulaces, page 92. 2-4-celled, with $x$ erect ovule in each cell; style slender, stigma mostly 2 -lobed, stamens 4 .

VERBENACEF, page 98. 2-celled (rarely 3-5-celled); many ovules on axillary placenta.

SOLANaCeE, page ror. 


\section{MANUAL OF SOME SPRING FLOWERING PLANTS OF THE CENTRAL AND NORTH- ERN STATES.}

\section{SUb-KINGDOM SPERMATOPHYTA. . Seed-bearing Plants.}

Plant body differentiated into true roots and leaf-bearing shoots. Special shoots with shortened internodes and modified leaves form flowers for the purpose of reproduction. Pollen grains (microspores) containing the male gametophyte are borne in the anther sacs (microsporangia) on modified leaves, known as the filament, and the embryosac (macrospore) containing the female gametophyte is borne in the ovule (macrosporangium), which grows from a much modified leaf, termed the carpel (see Botany, page 203). After the fertilization of the egg in the embryo-sac by the sperm from the pollen grain (see Botany, page 166), an embryo plant, with minute stem, leaf or leaves, and root fundament, is formed, which soon temporarily ceases to grow, and remains in an inactive condition until the germination of the seed.

\section{Class I. - GYMNOSPERMÆ.}

Ovules borne upon an open scale or disk, and not in a closed ovary; fruit usually a cone, or berrylike, by coherence of scales. Trees or shrubs, usually with needle-shaped or scalelike evergreen leaves.

\section{CONÍFER 瓜. PINE FAMILY.}

Resinous trees or shrubs, usually with narrow, scalelike or needleshaped evergreen leaves. Wood without tracheal tubes (water tubes) after the first year's growth, the ring of growth consisting of comparatively large cavitied tracheids (wood fibers with numerous circular thin

NoTE. - The vowel is long in the accented syllable when the accent mark is inclined with its apex to the left, and short when the accent is turned to the right. 
places or pits), produced in the spring, and smaller cavitied tracheids of the later growth. Flowers monœcious, rarely diœcious, borne in catkins, or solitary, destitute of calyx or corolla.

Leaves needle-shaped, in clusters of $2-5$.

Pinus I.

Leaves deciduous, soft and needle-shaped, many in a fascicle.

Leaves small, closely appressed; branchlets 2-edged and flat.

LARIX II.

Leaves awl-shaped, not closely appressed.

THuYa III.

JUNIPERUS IV.

\section{PINUUS. Pine.}

(The classical Latin name.)

Leaves of two kinds, the first or primary leaves being in the form of bud scales, from the axils of which the secondary, evergreen, needleshaped leaves arise, on very short stems, in clusters of 2 to 5 . Staminate catkins borne at the base of the shoot of the current season ; each stamen borne in the axil of a minute scale; anthers 2-celled, dehiscing longitudinally. Pistillate catkins, solitary or in clusters, borne just beneath the terminal bud, or laterally on the young shoot; carpellary scales in the axils of a persistent bract, and bearing a pair of inverted ovules at its base. Fruit, a cone maturing the second autumn.

I. Pinus Stróbus, L. (Latin name of a tree bearing an odoriferous gum.) White PINe. Leaves in clusters of fives. Cone scales but little thickened at the end and not sharp pointed. Cones trom 4 to 6 inches long, about $I$ inch thick before the scales spread, often slightly curved. A large forest tree, becoming sometimes I75 feet tall.

2. Pinus echinàta, Mill. (L., echinatus, prickly ; from echinus, a hedgehog.) Yellow Pine, SpRUCe PINE. Leaves in clusters of two and three. Cone scales thickened at the apex, with prominent transverse ridge, and slender, straight, deciduous prickle; cones about 2 inches long and less than I inch thick before the scales spread.

3. Pinus rígida, Mill. (L., rigidus, rigid, stiff.) PITch PINE. Leaves in clusters of threes, rarely of fours, dark green and rigid. Cone scales thickened at the apex, with transverse ridge and recurved prickle. Cones from $I \frac{1}{2}$ to 3 inches long, becoming nearly globular when the scales spread; often borne in clusters.

4. Pinus sylvéstris. (L., sylvestris, belonging to the woods; from sylva, a wood.) Leaves in clusters of twos; cone scales thickened the apex; without sharp points; cones tapering. Introduced from Europe, and much planted as an ornamental tree.

\section{LÀRIX. Larch.}

(The ancient name, probably Celtic.)

Slender trees with soft, needle-shaped, deciduous leaves, borne many in a fascicle. Catkins in early spring, terminating short spurs on 
branches of the previous year. Pistillate catkins crimson or red in flower. Cones maturing the first year; their scales thin, without prickly tips, and persistent.

I. Larix Americàna, Michx. TAMARACK, HACKMATACK, AMERICAN BlaCK LARCH. Leaves short, less than I inch long; cones $\frac{1}{2}$ to $\frac{3}{4}$ inch long; scales few and rounded. Mainly in cool swamps.

2. Larix Europæ̀a, Michx. EURopeAn LARCH. Leaves and cones longer than in the preceding species; cones many-scaled. Introduced from Europe as an ornamental tree.

\section{THUัYA.}

(The ancient name.)

Trees or shrubs, with short, lance-shaped, or awl-shaped, or even blunt, leaves, which are borne opposite each other in 4 appressed rows. Flowers monœcious, both staminate and pistillate catkins terminal and quite small ; staminate catkins globose and pistillate catkins ovoid or oblong. The scales of the pistillate cones opposite, and each with 2 erect ovules (rarely $2-5$ ovules). Cones spreading or recurved; the 6-1o coriaceous scales also spreading when mature.

I. Thuya occidentàlis, L. (L., occidentalis, pertaining to the west; from occidere, to set.) ARBOR VIT Æ, WHITE CEDAR. Conical trees; the 2 lateral rows of leaves keeled and the 2 outer rows flat, giving the branchlets a 2-edged appearance. Mature cones from $\frac{1}{3}$ to $\frac{1}{2}$ inch long, with obtuse scales. Commonly planted as an ornamental tree.

\section{JUNÍPERUS. Juniper.}

(Name Celtic.)

Trees or shrubs, with opposite 4-ranked leaves, or leaves verticillate in threes; leaves small and lance- or awl-shaped. Flowers usually diœcious, but sometimes monœcious; catkins very small, the staminate oblong or ovoid, and the pistillate globose, consisting of a few fleshy scales, each bearing a single erect ovule, or rarely two ovules. Globose cones berrylike by a coalescence of the scales, and containing I-6 bony seeds.

I. Juniperus commùnis, L. (L., communis, common.) JUNIPER. Shrub or low tree. Leaves in whorls of 3 , linear, awl-shaped; aments axillary, cones berrylike.

2. Juniperus Virginiàna, L. RED CEDAR or SAVIN. From shrubs to tall trees. Leaves mostly opposite and of two forms, awl-shaped, or scalelike. Aments terminal. Heart wood red and fragrant. 


\section{Class II. - ANGIOSPERM $Æ$.}

Ovules inclosed in an ovary which is composed of one or more spore-bearing leaves known as carpels (see Botany, page 20r), the ripened ovary and contents constituting the fruit.

\section{Subclass I. MONOCOTYLEDONES.}

Embryo in the seed with but one cotyledon, the first leaves being alternate; stem showing no distinction into wood, pith, and bark, the vascular bundles being promiscuously distributed throughout the stem. Leaves mostly parallel-veined; parts of the flower usually in one or more whorls of threes or sixes.

\section{TYPHÀCE}

Marsh or water plants, with creeping rootstocks and flat, linear leaves, sheathing at the base. Flowers monœcious, in dense, terminal spikes. Parts of the perianth reduced to bristles. Ovary I to 2-celled with as many styles. Fruit nutlike.

\section{TỲPHA. Cat-tail Flag.}

(The old Greek name Typhe.)

Plants erect, growing 6 feet or more in height. Leaves erect and flat, sheathing a jointless stem. Rootstock creeping. Flowers in terminal, dense, cylindrical spikes, which are staminate above and pistillate below. Pistils stipulate, I-celled. Nutlets minute and long-stalked.

I. Typha latifòlia, L. (L., latus, broad; folium, leaf.) BROAD-LEAvEd CATTAlL. Stems from 4 to 8 feet tall; leaves $\frac{1}{4}$ to $I$ inch broad. Terminal spikes pistillate below and staminate above; staminate and pistillate portions in close proximity, and each about 3 to 6 inches long; pistillate portion I inch in diameter. In marshes and wet places.

\section{GRAMÍnE}

Nearly all annual or perennial herbaceous grasses; usually hollow internodes and solid nodes. Leaves alternate, 2-ranked, sheathing the stem at the base, the sheath split open longitudinally on the side opposite the leaf blade. Flowers in spikelets which are panicled or spiked. Spikelets composed of one or more flowers which may be perfect. 
monœcious, or diœcious, and of several 2-ranked bracts or glumes. Stamens commonly 3 , and styles usually 2 , but varying from I to 3 .
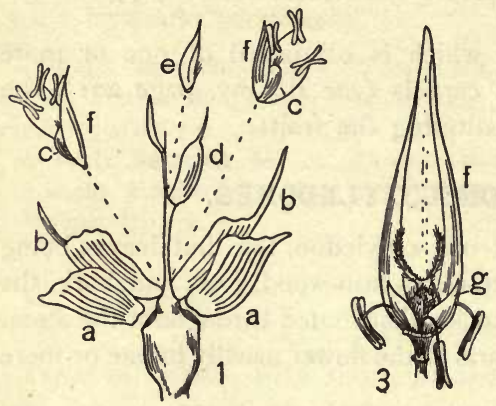

Ovary I-celled and I-ovuled. Fruit a seedlike grain. See Fig. 34I for a diagrammatic representation of the structure of the flower.

\section{PÒA. Meadow-Grass. Spear Grass.}

(Greek name for grass or fodder.)

Annuals or perennials, leaves flat or convolute. Spikelets flattened, ovate, or lance-ovate, 2-6-flowered, in more or less open panicles. Flowers usually perfect, rarely diœcious. The two lower glumes empty, I-3-nerved; the glumes next the flower longer, and usually with cobwebby hairs at the base, 5nerved; palets (see Fig. 34I) shorter than the glumes, and 2-nerved or 2-keeled.

Poa praténsis, L. (L., pratensis, growing in meadows, from pratum, a meadow.) KENTUCKY BLUE Grass. Smooth, slender stems springing from running rootstocks; sheaths sometimes longer than the internodes; ligules short and blunt; spikelets nearly sessile, $3-5$-flowered; upper glumes hairy on the margins and keel. Commonly planted for lawns, meadows, etc.

\section{TRÍPSACUM. Gama Grass. Sesame Grass.}

(Gr., tribo, to rub, in allusion to the polished spikes.)

Stems solid, stout and tall, from thick, creeping rootstocks. Leaves broad and flat. Monœcious spikelets in jointed unilateral spikes; staminate spikelets in the upper part of the spike, and the pistillate below; staminate spikelets in pairs, 2-flowered; pistillate spikelets with I perfect flower. 


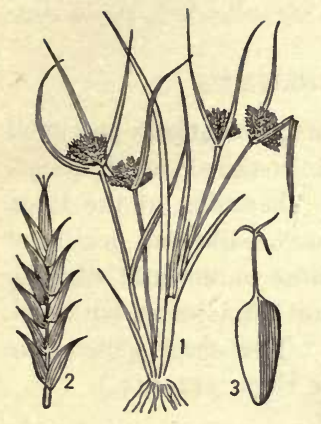

FIG. 342 .

Cyperus inflexus. I, the entire plant; 2 , a single spike; 3 , the pistil. After BRITTON and BRown.

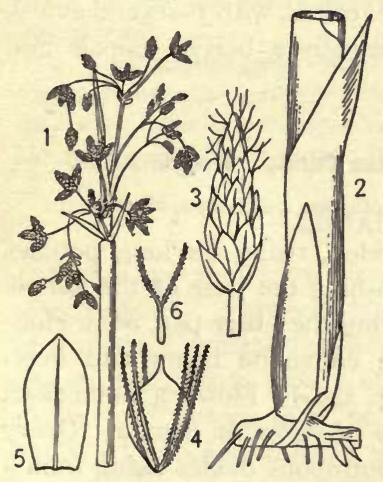

FIG. 344 .

Scirpus lacuistris. I, inflorescence; 2 , basal portion of the plant; 3 , a single spike; 4 , an achene; 5 , scale of a spike; 6, bifurcate style.-After BRITTON and BROWN.

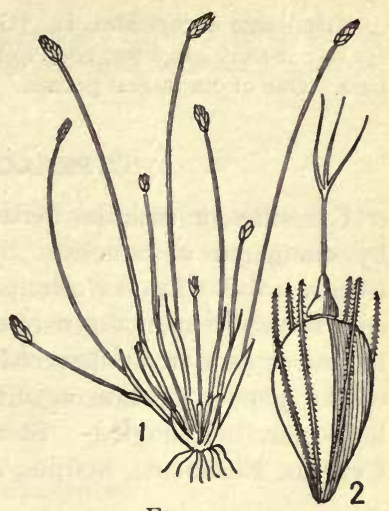

FIG. 343 .

Eleócharis dlbida. I, the entire plant ; 2, the pistil.-After BRITTON and BROWN.

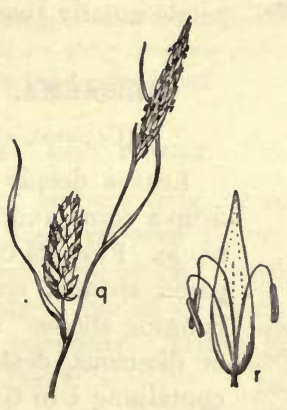

FIG. 345.

Càrex Mèadii. q, upper portion of a plant showing a staminate spike above and a pistillate spike below; $r$, a staminate flower; $s$, a pistillate flower. 
Tripsacum dactyloìdes, L. (Gr., daktylos, finger; eidos, resemblance.) GAMA GRASS. Stems 4 to 8 feet tall; spikes single or several, branching from a common base. One of our largest grasses. Moist soil.

\section{CYPERÀCE}

Grasslike or rushlike herbs with fibrous roots; sometimes perennial by elongated rootstocks. Stems 3-4-angled, rounded or flattened, usually solid. Leaves alternate and 3-ranked, sheathing at the base, and not split open down one side as in grasses. Flowers perfect or imperfect in I-many-flowered spikelets; I flower in the axil of each of the glumes or bracts. Style 2-3-cleft; fruit an acheme, flattened, lenticular, or 3-angled. Stamens usually 3. The chief genera are Cypèrus, Eleócharis, Scírpus, and Càrex. (See Figs. 342-345.)

\section{ARÀCE ÆE. ARUM FAMILY.}

Herbs with long-petioled, simple, or compound leaves, rising from a corm or tuberous rootstock; sap usually very pungent. Flowers monœcious or diœcious and densely crowded on a spadix which is usually surrounded by a spathe (see Fig. 346). Stamens 4-10 with short fila-

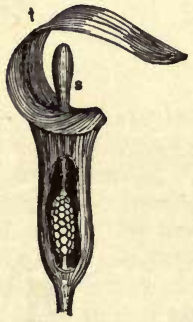

Fig. 346 .

Inflorescence of Arisæma. $t$, the spathe, cut open below and showing the flowers clustered at the base of the spadix, $s$.

ments ; ovary I-several-celled, with I-several ovules in each cell. Fruit usually a berry. Sepals and petals usually absent.

\section{ARISAÈMA. Indian Turnip. Dragon Arum.}

(Gr., aris, a kind of arum, and haima, blood.)

Leaves deeply divided, rising on long petioles from a corm, and sheathing the base of the simple scape. Flowers covering the lower part of an elongated spadix; spathe convolute below and overhanging above. (Fig. 346.) Flowers monœcious or diœcious, destitute of calyx or corolla. Ovary containing 5 or 6 orthotropous ovules rising from a basal placenta. Fruit a globose red berry.

I. Arisæma triphýllum, Torr. (L., triphyllum, 3-leaved, from Greek tri, three; phyllon, leaf.) INDIAN TURNIP. Jack-in-the-pulpit. Leaves 3 -foliate with ovate, entire segments. Spathe green with purple stripes, broad and overhanging at the summit. Rich woods.

2. Arisæma Dracóntium, Schott. (Gr., drakon, snake, or dragon.) GREeN 


\section{Monocotyledones.}

Dragon. Leaves pedately divided into $5^{-\mathrm{r}} 7$ segments. Spadix long and tapering, exceeding the greenish or whitish, narrow, convolute, and pointed spathe. Rich woods or low grounds.

\section{COMMELINÀCE AE. SPIDERWORT FAMILY.}

Stems herbaceous and jointed; leaves lanceolate, linear, or ovate, sheathing the stem at the base, parallel veined. Roots perennial, fibrous, or thickened. Flowers usually perfect, consisting of 3 persistent green sepals, and 3 blue, purple, or rose-colored, ephemeral petals, 6 stamens, and a single 2-3-celled superior ovary, containing 3 -several ovules, and surmounted by a single undivided style. Fruit a 2-3-celled capsule.

\section{TRADESCÁNTIA. Spiderwort.}

\section{(Tradescant, gardener to Charles I. of England.)}

Stems upright, nearly simple, and mucilaginous, bearing keeled leaves. Flowers crowded in terminal or axillary umbels; filaments bearded.

I. Tradescantia Virginica, L. COMMON SPIDÉRWORT. Leaves linear or linear-lanceolate, flat or channeled, sometimes I foot or more long; stems varying in height from about 8 inches to 3 feet; leaflike bracts subtending the inflorescence. Flowers of various shades of blue and purple, from $I$ to 2 inches broad. Rich ground.

2. Tradescantia ròsea, Vent. (L., roseus, rosy; from rosa, a rose.) Leaves narrowly linear and grasslike; stems erect, from 6 to 12 inches tall; bracts below the flowers short and scarious; umbels on long terminal peduncles; rose-colored corolla from $\frac{1}{2}$ to $\frac{2}{3}$ inch broad. In dry and sandy woods.

\section{LILIÀCE $\not$. LILY FAMILY.}

Herbs, perennial by means of bulbs, corms, or rootstocks ; rarely woody plants. Flowers regular and symmetrical ; perianth consisting of 6 distinct or nearly distinct segments, which (excepting in Trillium) are colored nearly alike; stamens 6, borne on or at the base of the perianth, I before each of its segments. Ovary superior, 3-celled. Fruit a loculicidal capsule or a berry.

Woody climbers.

Smilax I.

Not woody climbers.

Flowers umbellate on naked scapes, -

Having the odor of onions.

Allium II.

Not having the odor of onions.

NOTHOSCORDUM III.

Flowers in racemes or spikes on naked scapes, -

Divisions of the perianth separate nearly cr quite to the base.

Camassia IV.

Divisions of the perianth united into a tube below. Hyacinthus V. 
Flowers borne on stems which are leafy, at least near or at the base, -

Stems leafy only at the base; flower single and erect.

Stems leafy only at the base; flower single and nodding.

Stem with leaves close to the flower.

Stem simple and leafy throughout, -

From a scaly bulb.

From a rootstock; flowers in terminal racemes.

From a rootstock; flowers axillary.

TULIPA VI.

ERYTHRONIUM VII.

TRILLIUM VIII.

LiLium IX.

Smilacina X.

Polygonatum XI.

\section{SMillAX. Greenbrier.}

(An ancient Greek name.)

Climbing by tendrils from the bases of the petioles. Leaves simple, parallel ribbed, and netted veined. Flowers diœcious in axillary umbels. The greenish or yellowish perianth of 6 distinct, deciduous segments ; anthers apparently I-celled on linear filaments; ovules 1 or 2 in each of the 3 cells of the ovary. Fruit, a small berry.

I. Smilax rotundifòlia, L. (L., rotundas, round; folium, leaf.) СоммоN GrEenbrier. Stems and branches beset with sharp prickles. Leaves broadly ovate or round ovate, 2 to 3 inches long, 5-nerved, abruptly pointed at apex and cordate at base. Peduncles shorter or hardly longer than the petioles. Fruit, a blue-black berry. Woods and thickets.

2. Smilax Pseùdo-Chìna, L. (Gr., pseudos, false.) Stems and branches without prickles, or with very few. Leaves mostly ovate or ovate-oblong, sometimes lobed at the base, acute or cuspidate at the apex, 7-9-nerved. Peduncles 2-4 times the length of the petiole. Umbels 12-40-flowered. Berries black. Dry or sandy thickets.

\section{II. ÁLlIUM. Onion. Garlic.}

(Ancient Latin name of garlic.)

Leaves and scape rising from a coated bulb; leaves linear, lanceolate-oblong, or lanceolate. Flowers white, pink, purple, or greenish, in rather dense terminal umbels, which are subtended by scarious bracts. Plants with the odor of onions. Ovules, $1-2$ in each cell.

I. Allium mutábile, Michx. (L., mutabilis, changeable.) WILD ONION. Coats of the bulb fibrous reticulated; scape from I to 2 feet tall, pedicels nearly I inch long; divisions of the perianth thin. Moist soil.

2. Allium Nuttállii, S. Wats. (L. genitive, of Nuttall the ,botanist.) Bulb as in the above species; scape from 4 to 8 inches tall; pedicels from $\frac{1}{3}$ to $\frac{1}{2}$ inch long; perianth becoming rigid in the fruit. On prairies. 


\section{NOTHOSCÓRDUM.}

(Gr., nothos, false; skordion, garlic.)

Similar to Allium, but without the smell of onions. Flowers greenish yellow in rather loose umbels. Ovules, several in each cell of the ovary.

I. Nothoscordum striàtum, Kunth. (L., striatus, furrowed; from stria, a furrow.) Scape usually less than I foot high; leaves narrowly linear, thick, scarcely equaling the scape; bracts of the umbel 2, lanceolate, persistent; pedicels filiform, becoming I to 2 inches long. Flowers about $\frac{1}{2}$ inch long, with oblong-lanceolate, thin segments. Open woods and prairies.

\section{CAMÁSSIA.}

(From native Indian name, quamash or camass.)

Linear leaves and naked scape from a coated bulb. Flowers on jointed pedicels in bracted racemes. Divisions of the perianth parted almost or quite to the base, blue or purple. Ovary oblong or obovate, with a filiform style. Several seeds in each cell.

I. Camassia Fràseri, Torr. (L. genitive, of Fraser.) Wild HyacintH. Scapes from I to 2 feet tall. Bulb ovoid; leaves narrowly linear. Racemes 3 to 8 inches long. Flowers on filiform pedicels; parts of the perianth narrowly oblong, longer than the stamens; blue to almost white. In rich and moist ground.

\section{HYACINTHUS. Hyacinth.}

(Gr., hyakinthos.)

Perianth united into a tube below; stamens inserted at the throat, which is open and spreading. Flowers in spicate racemes, varying in color from white through various shades of blue and purple.

Here belong the cultivated varieties of hyacinth.

\section{TÙLIPA. Tulip. \\ (Per., dulband, a turban.)}

Stem I- or 2-leaved near the ground, bearing a large, erect flower; perianth bell-shaped, of 6 separate segments, which are broad and erect; stigmas 3 , sessile; ovary triangular; seeds many.

Here belong the various kinds of cultivated tulips. 


\section{ERYTHRònIUM. Dogtooth Violet. Easter Bell.}

(Gr., Erythronion, name of the European purple species; from erythros, red.)

Scape bearing a single nodding flower, 2-leaved near the ground. Divisions of the perianth lanceolate, spreading or recurved above. Stamens 6, hypogynous, anthers not versatile. Style elongated, thickened above, 3-lobed or cleft. Flowering very early in the spring; bulb cormlike.

I. Erythronium álbidum, Nutt. (L., albidus, whitish.) WhITE DoGrooth VIOLET. Leaves elliptical oblanceolate, very little or not at all spotted. Flowers white, bluish, or purplish; parts of the perianth oblong, tapering, recurved. Asexual propagation by offshoots from the base of the corm. In moist woods and thickets or on prairies.

2. Erythronium mesachòrium, Knerr. (Gr., mesos, middle; chora, land.) MidLAND DOGTOOTH VIOLET. Leaves narrowly oblong, not spotted, from $\frac{1}{4}$ to $\mathrm{I}$ inch wide. Perianth not recurved nor much spreading. No offshoots from the bulbs. On open prairies.

\section{TRÍLLIUM. Wake-robin.}

\section{(L., triplum, triple, all parts being in threes.)}

Unbranched herbs. Stem rising from a short rootstock and bearing near the summit 3 leaves in a whorl, which subtend the solitary sessile flower. The outer whorl of the perianth of 3 green sepals, inner whorl of 3 white, pink, purple, or greenish petals. Six hypogynous stamens with linear anthers. Ovary 3 -celled, styles 3 ; numerous ovules in each cell.

I. Trillium cérnuum, L. (L., cernuus, nodding.) NODDING WAKE-ROBIN. Leaves broadly ovate or rhombic, acuminate above, 2 to 7 inches long. Slender stems from 8 to 20 inches high. Peduncle scarcely $1 \frac{1}{4}$ inches long, recurved; petals recurved. In rich woods.

2. Trillium eréctum, L. (L., erectus, erect.) ILL-SCENTED WAKE-ROBIN. Stout stem from 8 to 16 inches high. Leaves sessile or short-petioled. Petals from to $x_{\frac{1}{2}}^{\frac{1}{2}}$ inches long, ovate or lanceolate. Peduncle $x_{\frac{1}{4}}$ to 4 inches long, erect or declined. Corolla spreading. In woods.

3. Trillium grandiflòrum, Salisb. (L., grandis, large; flos, floris, flower.) LARGE-FLOWERED WAKE-ROBIN. Similar to the above, but petals obovate or oblanceolate, from $I \frac{1}{1}$ to $2 \frac{1}{2}$ inches long, white or pink, exceeding the sepals. Peduncles erect or inclined. Rich woods. 


\section{Monocotyledones.}

\section{LÍLIUM. Lily.}

(The classical Latin name.)

Simple leafy stems rising from scaly bulbs. Large, showy flowers, erect or drooping. Perianth colored all alike, or nearly so. Anthers 6 and versatile. Ovary 3-celled with 2 rows of ovules in each cell. Style long, club-shaped above, stigma 3 -lobed.

r. Lilium umbellàtum, Pursh. (L., umbella, a little sunshade.) WESTERN RED LILY. I to 3 erect flowers which are 2 to 3 inches high. Leaves narrowly linear, nearly all alternate, or the upper leaves verticillate. Bulb of narrow, jointed, fleshy scales. Perianth red, yellow, or orange. In dry soil.

\section{SMILAcina. False Solomon's Seal.}

(Diminutive of smilax.)

Simple stems from creeping or thickened rootstocks. Leaves alternate. Flowers sometimes fragrant, in terminal racemes. Perianth 6-parted, white. Stamens 6, style short, stigma 3-lobed. Ovary 3-celled, 2 ovules in each cell. Berry 1 -2-seeded and globular. Flowers in terminal racemes.

I. Smilacina racemòsa, Desf. (L., racemosus, full of clusters.) WILD SPIKENARD. Stems from $I$ to 3 feet high, bearing numerous oblong lanceolate or oval, sessile or short-petioled leaves. Flowers on short pedicels in terminal racemose panicles; flowers about 1 inch broad, stamens exceeding the parts of the perianth. Berries aromatic, pale red, spotted with purple. Rootstock thick and fleshy. Moist thickets.

2. Smilacina stellàta, Desf. (L., stellatus, set with stars; from stella, star.) Stems scarcely I foot high, bearing 7-12 oblong-lanceolate leaves. Flowers in simple, few-flowered racemes; perianth segments longer than the stamens. Berries black or green striped with black. In moist soil.

\section{POLYGONÀTUM. Solomon's Seal.}

(The ancient name, from Gr., polys, many; gony, knee.)

Stems simple, usually somewhat curved, nearly erect, springing from a jointed rootstock. Leaves sessile and partly clasping. Flowers solitary, or from 2 to 10 on drooping axillary peduncles; perianth oblongcylindrical, or somewhat expanded near the apex, lobes 6 and short; stamens 6 , included, more or less adnate to the perianth. 2-6 ovules in each of the 3 cells of the ovary, style slender; stigma capitate, somewhat 3-lobed. 
I. Polygonatum biflòrum, Ell. (L., bis, twice; flos, floris, flower.) SMaLler Solomon's SEAL. Slender stems, from I to 3 feet high, bearing lanceolate, oval, or ovate leaves which are usually minutely pubescent beneath. Peduncles I-4, usually 2-flowered. Perianth from $\frac{1}{3}$ to $\frac{1}{2}$ inch long. Filaments roughened, inserted near the summit of the perianth. On wooded hillsides, or in woods and thickets generally.

2. Polygonatum gigantèum, Dietrich. (L., giganteus, gigantic.) GREAT Solomon's SEAL. Stout stems, from 2 to 7 feet high, bearing ovate to lanceolate leaves which are from 2 to 6 inches long; not pubescent beneath. Peduncles 2-8flowered; flowers from $\frac{1}{2}$ to $\frac{3}{4}$ of an inch long. Filaments smooth, inserted about the middle of the perianth tube. Along banks of streams or in moist woods.

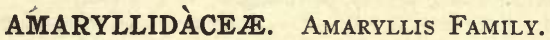

Herbaceous plants with scapes and flat linear leaves springing from a bulb, corm, or rootstock. Corolla 6-parted, adherent to the ovary; stamens 6 , inserted at the base of the perianth segments opposite the lobes. Ovary usually 3-celled, with numerous, rarely a few, ovules in each cell. Style filiform, entire, or 3-lobed or divided.

\section{HYPÓXIS. Star Grass.}

(Gr., hypoxys, subacid.)

Scape and linear, hairy leaves rising from a corm or solid bulb. Flowers few on a scape. The 6 parts of the perianth greenish outside, yellow within, separate nearly to the ovary, and withering on the pod. Stamens 6; filaments short; anthers sagittate or entire. Style short, stigmas 3 .

I. Hypoxis erécta, L. (L., erectus, erect.) Few-flowered scape from 3 to 8 inches high. Leaves narrowly linear, becoming longer than the scapes. Perianth segments spreading, bright yellow within, hairy and greenish without, and from to nearly $\frac{1}{2}$ inch long. In meadows and open woods.

\section{NARCÍSSUS.}

(The Latin name.)

Scape and leaves from a coated bulb. Tube of the perianth somewhat cylindrical, the 6 segments widely spreading ; a cup-shaped, funnelshaped, or saucer-shaped crown on the throat of the perianth; unequal stamens included in the crown.

Here belong the various varieties and species of Narcissus cultivated in gardens, and the daffodils, jonquils, and Chinese sacred lily. 


\section{GALÁNTHUS. Snowdrop.}

(Gr., gala, milk; anthos, flower. From the color of the flower.)

Scape from a coated bulb, bearing usually a single nodding flower. The 3 inner segments of the perianth shorter than the outer, and less spreading, notched at the apex. Anthers erect and not versatile.

I. Galanthus nivàlis, Linn. (L., nivalis, snowy.) Leaves 2, linear and pale; scape bearing a drooping white flower; inner perianth segments tipped with green. Flowering in early spring.

\section{IRIDÀCEA. IRIS FAMILY.}

Herbs with equitant 2-ranked leaves rising from corms, rootstocks, or tubers. The regular or irregular, mostly clustered flowers usually subtended by bracts. Perianth coherent to the ovary, the segments in an outer and an inner whorl of 3 each. Stamens 3, separate or monadelphous, inserted on the perianth opposite the outer whorl of segments. Ovary 3 -celled and 3 -angled or lobed; ovules numerous in each cell; style 3-lobed.

\section{I. İRIS. Flower-de-luce.}

(Ancient name of these flowers, from Gr., iris, rainbow.)

Erect or ascending equitant leaves from horizontal rootstocks. Flowers large and terminal. Tube of the perianth somewhat prolonged above the ovary, 6-cleft above, the 3 outer segments broad and spreading or reflexed, the inner 3 segments erect as a rule and usually narrower than the outer segments. The 3 stamens inserted at the base of the outer perianth segments; anthers linear or oblong. Ovary 3-celled; style petallike, united below with the tube of the perianth; overarching above and bearing a shelflike stigma below the apex.

I. Iris Germánica, L. CoMmon Flower-DE-LuCE. Flowers large and scentless. Outer divisions of the perianth bearded, deep violet, pendent, about 3 inches long; inner, obovate divisions lighter colored, and nearly as large.

2. Iris pùmila, L. (L., pumilus, dwarf.) DWARF GARDEN IRIS. Outer divisions of the perianth bearded; flowers few, violet or purple, close to the ground in early spring. Stem from 4 to 6 inches high. 


\section{SISYRÍNCHIUM. Blue-eyed Grass.}

(Gr., sisyrinchion, a bulbous plant.)

Grasslike plants with very short rootstock and fibrous roots. Leaves linear; stems flattened and 2-edged. Flowers umbellate from a pair of green bracts. Tube of the perianth short or none; segments of the perianth obovate or oblong and spreading. Ovary 3 -celled, with several ovules in each cell; branches of the style threadlike and alternating with the stamens. Stamens monadelphous.

r. Sisyrinchium angustifòlium, Mill. (L., angustus, narrow; folium, leaf.) Stem from 3 to $I_{4}$ inches tall, usually simple, erect. Linear leaves shorter than the stem; narrowly linear or nearly setaceous bracts subtending the umbel very unequal. Flowers white or delicate blue. Common in meadows.

2. Sisyrinchium ánceps, Cav. (L., anceps, two-headed.) Stems broadly 2-winged, from 6 to $r 8$ inches tall, terminating with two unequal branches. above, which are subtended by a grasslike leaf. Bracts subtending the umbels about equal. In meadows.

\section{ORCHIDÀCE ÆE. ORCHIS FAMILY.}

Perennial herbs with corms, tubers, or tuberous roots. Leaves sheathing and entire, alternate, and plainly parallel-nerved. Solitary, racemed or spiked flowers, often curiously irregular. Perianth in 2 whorls, each of 3 divisions; the 3 outer divisions or sepals more or less petallike; one of the inner whorl or petals differing from the others in size and shape, and termed the lip. Ovary inferior, I-celled, with numerous ovules on 3 parietal placentæ. I or 2 perfect stamens coherent with the style or fleshy stigma (see Fig. 289), forming what is here termed the column; each cell of the 2-celled anthers containing masses of waxy or powdery pollen, termed the pollinia when adherent in a definite mass.

\section{CYPRIPÈDIUM. Lady's Slipper.}

(Gr., Kypris, Venus; podion, a buskin, a low-cut woman's shoe.)

Stems or scapes leafy; leaves broad and many-nerved. Roots a tuft of fleshy fibers. Flowers few or solitary, large and drooping. Sepals spreading, or 2 united under the lip. The 2 lateral petals spreading and usually narrower than the sepals; the lip a large inflated sac. Column declined, having 2 fertile stamens laterally and a petaloid sterile stamen above. The broad terminal stigma obscurely 3 -lobed. 


\section{Dicotyledones.}

I. Cypripedium pubéscens, Willd. (L., pubescens, becoming downy.) LARGER YeLLow LADY'S SLIPPER. Leafy stems from I to 2 feet high. Leaves broadly oval or elliptical; both leaves and stems pubescent. Sepals elongate-lanceolate. Lip much inflated, from I to 2 inches long, pale yellow with purple lines. In bogs or woods.

\section{II. ÓRCHIS.}

(The ancient Greek name.)

Stems scapelike, from I- to 2-leaved at the base. Roots fleshyfibrous. Flowers in short terminal spikes. Sepals and petals similar. Anther I with divergent sacs; pollinia attached by means of a caudicle to a basal viscid disk, which is inclosed in a pouch.

I. Orchis spectábilis, L. (L., spectabilis, showy.) SHOwy Orchis. Stems 4-5-angled; 2 obovate leaves borne near the base, 3 to 6 inches long. Spikes $3-6$ flowered. Sepals and lateral petals connivent above, pinkish purple in color; lip whitish and obtusely spurred. In rich woods.

\section{Subclass 2. DICOTYLEDONES.}

Embryo in the seed with 2 cotyledons, stem differentiated into bark, wood, and pith, the vascular bundles being laid down in the form of a ring, and possessed of a cambium zone. Leaves mostly netted-veined. Parts of the flower usually in one or more whorls of fours or fives.

\section{JUGLANDÀCEÆ. Walnut Family.}

Trees. Leaves alternate and pinnately compound. Flowers monœcious; the staminate in aments, and the pistillate occurring singly or a few in a cluster. Staminate flowers of 3-many stamens and an irregular calyx; pistillate flowers of a single pistil and a 4-5-lobed calyx adherent to the ovary. Ovary incompletely 2-4-celled with a single ovule. Fruit a drupe with a fibrous or woody husk inclosing a bony nut. Embryo large, fleshy and oily, the crumpled cotyledons deeply 2-lobed.

\section{JÙGLANS. Walnut.}

\section{(L., Fovis glans, the nut of Jupiter.)}

Staminate flowers in solitary aments, produced from buds on stems of the previous year's growth; stamens 12-40; pistillate flowers borne singly or in groups at the end of the current season's growth, 4 small petals borne in the sinuses of the 4-toothed calyx. Drupe large and 
globose or ovoid; husk fibrous and indehiscent; the shell or endocarp rugose or sculptured.

I. Juglans cinèrea, L. (L., cinereus, ash-colored.) BUTTERnUT or WHite WALNUT. Trees, usually of moderate size and rather smooth gray bark; young shoots, etc., viscid-pubescent. Oblong lanceolate leaflets II-I9, downy beneath. Drupes oblong and pointed and clammy. Rich woods.

2. Juglans nigra, L. (L., niger, black.) BLACK WALnUT. Becoming large trees with rough bark; petioles and young shoots puberulent, becoming glabrous when older. Ovate-lanceolate leaflets 13-23. Fruit globose, not viscid. Rich woods.

\section{CÁRYA. Hickory.}

(From Gr., karya, the walnut.)

Staminate flowers of 3-10 stamens in clustered lateral aments; pistillate flowers in clusters of 2-5 on a terminal peduncle. Calyx 4toothed, petals wanting. Drupes subglobose, oblong, or ovoid; husk separating into 4 valves; nut bony, smooth, or angled.

I. Carya álba, Nutt. (L., albus, white.) SHEllbaRK or SHAGBARK HICKORY. Old bark falling off in broad strips. Leaflets 5 , or rarely 7 , the 3 upper lance-obovate and much larger than the lower. Opening terminal buds very conspicuous, the bracts enlarging and persisting until the flowers are fully developed. Staminate flowers on slender peduncles at the bases of shoots of the current season. Nut somewhat compressed and angled, and slightly mucronate at the apex. Shell rather thin. Husk splitting into 4 valves. Seed sweet. In rich soil.

2. Carya sulcàta, Nutt. (L., sulcatus, furrowed.) BIG SHELlbark. Bark separating in long strips. Leaflets usually 7-9, downy beneath. Nut from $I_{4}^{\frac{1}{4}}$ to 2 inches long, usually angular and prominently pointed at both ends. Seed sweet. Husk and shell thick. Bottom lands and moist woods.

3. Carya porcina, Nutt. (L., porcinus, pertaining to a hog.) PIGNUT. Sometimes becoming large trees; bark rough; foliage usually smooth, sometimes pubescent; leaflets $3-7$, infrequently 9 , oblong-lanceolate or obovate-lanceolate, acuminate, 3 to 6 inches long. Fruit obovoid or obovoid-oblong. Husk thin, incompletely or tardily dehiscent. Nut thin-shelled, angled, and pointed. Seed bitter. Uplands.

4. Carya olivæfórmis, Nutt. (L., oliva, olive; forma, form.) Pecannut. Becoming tall trees with rough bark. Shoots pubescent while young, but becoming glabrous with age. Leaflets II-I5, short-petioled, oblong-lanceolate, and somewhat falcate toward the apex. Staminate aments fascicled near the apex of the previous year's growth. Fruit from $I \frac{1}{2}$ to $2 \frac{1}{2}$ inches long, oblong-cylindrical; husk thin; nut thin-shelled and smooth; seed sweet. In moist soil and along streams. 


\section{SALICÀCE E. WilLow FAMILY.}

Shrubs or trees with light wood and alternate stipulate leaves. Flowers diœcious, both staminate and pistillate in aments, which expand before or with the leaves. Stamens I-many on the concave receptacle. Pistillate flowers of a single, I-celled ovary with numerous ovules on 2-4 parietal or basal placentæ. Seeds tufted with silken hairs.

\section{PóPUlus. Poplar.}

(The classical Latin name.)

Trees with rounded or angular twigs and resinous buds. Leaves usually long-petioled, from narrow to broad. Staminate aments dense and pendulous; pistillate aments less dense, often racemelike. Staminate flowers of 4-60 stamens; stigmas 2-4, often large. Scales of the aments more or less fringed. Flowers appearing before the leaves.

I. Populus álba, L. (L., albus, white.) White PoplaR. Young branches and under surface of leaves white tomentose. Bark smooth and light gray. Leaves truncate-ovate to nearly orbicular, with acute apices, irregularly dentate or 3-5lobed. Producing suckers from adventitious buds on the roots. Often planted for shade.

2. Populus monilífera, Ait. (L., monile, necklace; ferre, to bear.) COTTONwoon. A large tree with grayish green bark which becomes roughened with age. Leaves smooth and shining, broadly deltoid-ovate, acuminate at the apex and crenulate on the margins; petioles flattened and about as long as the blade. Stamens as many as 60 ; pistillate flowers in long, pendulous aments. Along streams and in moist soil.

\section{SÀLIX. Willow.}

(The classical Latin name.)

Shrubs or trees. Leaves entire, usually long and pointed, with short petioles; branches very slender. Staminate flowers of 1-10, usually 2 stamens. Bracts of the aments entire; flowers with small glands. Buds with a single scale and lining membrane.

I. Salix nìgra, Marsh. (L., niger, black.) BLACK Willow. Stamens 3-7 with pubescent filaments; capsule ovoid and about twice as long as the pedicel; leaves short-petioled, narrowly lanceolate, and green on both sides. Stipules nearly cordate, conspicuous, Along banks of lakes and streams. 
2. Salix amygdaloídes, Anders. (Gr., amygdale, an almond; eidos, form.) PEACH-LEAVED Willow. Stamens more than 2 ; with pubescent filaments; capsule acutely and narrowly ovoid, about as long as the pedicel; leaves slenderpetioled, broadly lanceolate with long acuminate apex; dark green above and paler and somewhat glaucous beneath. Stipules fugacious. Along streams, etc.

3. Salix lùcida, Muhl. (L., lucidus, bright.) SHINING or Glossy Willow. Stamens 5, the filaments pubescent near their bases. Capsules longer than the pedicels and narrowly ovoid. Leaves ovate to lanceolate, long acuminate, serrulate on the border, green and shining on both surfaces. Stipules glandular and nearly cordate to oblong.

4. Salix longifölia, Mubl. (L., longus, long; folium, leaf.) SANDBAR or RIVERBANK WILlow. Stamens 2 with pubescent filaments. Capsules ovoid or conical, glabrous or silken. Leaves linear-lanceolate or linear-oblong, acuminate, and sparingly denticulate. Stipules small or none. Generally a much-branched shrub. Occurring in thickets along lakes and water courses.

5. Salix Missouriénsis, Bebb. Missouri Willow. Stamens 2 with glabrous filaments. Glabrous capsules narrowly ovoid, and 3 or 4 times longer than the pedicels. Leaves lanceolate to oblanceolate, acuminate, finely serrate, with minute glands on the teeth, green above and pale or glaucous beneath. Stipules about $\frac{1}{2}$ inch long. Becoming tall trees with thin gray bark. Along river banks.

\section{CUPULİFERE. OAK FAMILY.}

Trees or shrubs with monœcious flowers, the staminate flowers in aments or clustered; the pistillate flowers either solitary or clustered or in catkins. Fruit a I-celled and I-seeded nut, with or without an involucre. Ovary 2-7-celled with $1-2$ ovules in each cell, but becoming reduced in number in the fruit.

\section{BÉTULA. Birch.}

(The ancient Latin name.)

Trees or shrubs. Flowers of both kinds in aments, expanding before or with the leaves. Staminate flowers in clusters of threes in the axil of each bract of the ament, each flower consisting of two 2-parted filaments bearing $I$ anther sac on each fork. Pistillate flowers from $I$ to 3 in the axil of each bract, without a perianth. Ovary 2-celled and styles 2. Fruit becoming a small, compressed, laterally winged nut. Leaves thin and dentate or serrate.

I. Betula papyrífera, Marsh. (Gr., papyros, papyrus rush; L., ferre, to bear.) PAPER or CANOE BIRCH. Trees with chalky-white bark. Leaves ovate and finely dentate, smooth and green above, and glandular and pubescent on the veins beneath. 
Bark on trunk and old branches pealing off in thin layers. Fruiting aments peduncled. Nut narrower than the wings. In rich woodland and along streams.

2. Betula occidentàlis, Hook. (L., occidentalis, western.) WESTERN RED BIRCH. Trees with smooth brown or greenish brown bark. Leaves from ovate to nearly orbicular, sharply serrate; smooth on both sides or only slightly pubescent on the veins beneath: Nut narrower than the wings. Fruiting aments peduncled.

3. Betula nigra, L. (L., niger, black.) RIVER or RED BIRCH. Slender trees with reddish brown or greenish brown bark which peels in thin layers. Rhombic ovate leaves which are dark green and smooth above, and pale and smooth or somewhat tomentose beneath. Obovate nut broader than the wings. Pistillate aments peduncled. Along streams, etc.

\section{CARPINUS. Hornbeam or Ironwood.}

(The ancient Latin name.)

Trees or shrubs with smooth gray bark, and leaves with lateral veins from the midrib nearly parallel and straight. Inflorescence expanding before the leaves. Staminate aments sessile at the ends of short branches; I staminate flower of 3-12 stamens in the axil of each bract of the ament; short filaments 2-cleft, each branch bearing an anther sac. Two pistillate flowers in the axil of each bract of the small terminal pistillate aments. Ovary 2-celled; stigmas 2, sessile, or nearly so. Fruit a small, ovate, nerved nut, subtended by a persistent foliaceous bractlet.

r. Carpinus Caroliniàna, Walt. AMerican HoRnbeam or Blue or WATER BEECH. Small tree with slender gray twigs. Leaves ovate-oblong, acuminate, very sharply doubly serrate, somewhat falcate or inequilateral, green on both sides, and nearly smooth; bractlet subtending the nutlet 3 -lobed and halberd-shaped. Along streams and in moist woods.

\section{III. ÓSTRYA. Hop Hornbeam or Ironwood.}

(Greek name for hardwood tree.)

Slender trees with brownish bark and furrowed trunks. Leaves ovate or oblong-ovate, somewhat inequilateral, slightly pubescent above and beneath, petioles very short. Staminate flowers, of several stamens, occurring singly in the axil of each bract; filaments forked, each fork bearing an anther sac. Two pistillate flowers in the axil of each bract of the short pistillate ament; a tubular bractlet surrounding each flower and becoming bladdery in fruit and completely inclosing the small nutlet. Ovary incompletely 2 -celled, with 2 ovules; stigmas 2, long and linear. Border of the adherent calyx short and bearded. 
I. Ostrya Virginica, Willd. AMERICAN HOP HORNBEAM. Leaves sharply doubly serrate; tubular bractlets bristly hairy at the base. Nutlets flattened and shining. In rich woods.

\section{CÓRYLUS. Hazelnut or Filbert.}

(The classical Latin name.)

Shrubs or small trees, with broad, thin leaves serrulate or incised. Staminate flowers of about 4 stamens, solitary, in the axil of each bract of the staminate aments, which terminate the shoots of the previous year; filaments 2-forked, each fork bearing an anther sac. Pistillate flowers clustered at the end of short branches of the current season, each flower consisting of a calyx adnate to an incompletely 2-celled ovary, which is surmounted by a short style and 2 slender stigmas. Fruit, a large bony nut, inclosed in 2 foliaceous or coriaceous bractlets, which are closely adherent and often grown together at the lacerated margins.

r. Corylus Americàna, Walt. HAZELnut. Shrubs with ovate or broadly oval leaves, glabrous above and tomentose beneath. Bractlets compressed beyond the apex of the nut, and laciniate along the margins. In thickets.

2. Corylus rostràta, Ait. (L., rostratus, beaked.) BEAKED HAZELnUt. Shrubs with nearly glabrous ovate or narrowly oval leaves, with margins incised-

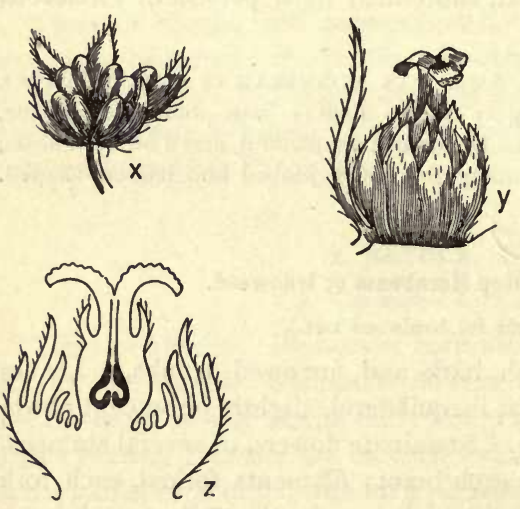

FIG. 347 .

Diagrams of the flowers of an oak. $x$, staminate flower; $y$, pistillate flower; $z$, longitudinal diagram of a pistillate flower. - After STRASBURGER. serrate and serrulate. Bractlets prolonged beyond the nut into a long, tubular, laciniate beak. In thickets.

V. QUÉRCUS. Oak.

(The classical Latin name.)

Trees or shrubs. Flowers appearing before or with the leaves. Staminate flowers of a 2-8-parted or lobed calyx and 3-12 stamens. Pistillate flowers solitary or somewhat clustered, subtended by a scaly involucre, which becomes a hard cup in the fruit. Ovary nearly 3 -celled, with 2 ovules in each cell, becoming usually a I-celled and I-seeded acorn or nut. . (Fig. 347.) 


\section{Dicotyledones.}

I. Quercus rùbra, L. (L., ruber, red.) RED OAK. Leaves oval or obovate, obes usually triangular and bristle-tipped. Acorns about I inch long, and about 2-4 times as long as the flat or saucer-shaped cup. In rich or poor soils.

2. Quercus álba, L. (L., albus, white.) WHite OAK. Leaves obovate, green above and pale or somewhat glaucus beneath, divided into $3-9$ oblong lobes, which are often toothed at the apices, but not bristle-pointed. Acorns ovoidoblong, becoming sometimes I inch long and from 3 to 4 times as long as the shallow cup. In various soils.

3. Quercus macrocárpa, Michx. (Gr., makros, long; karpos, fruit.) BUR OAK. Leaves obovate or oblong-obovate, variously lobed, pinnatifid or crenate, bright green above and grayish tomentulose beneath. Acorn from to $I_{\frac{1}{2}}$ inches long, about half immersed in, or scarcely exceeding, the cup, whose uppermost bracts are extended in the form of a fringe. In rich soil.

4. Quercus Prinus, L. (Gr., prinos, the evergreen oak.) Rock CHestnuT OAK. Leaves obovate, oblong, or oblong-lanceolate, and coarsely crenate, dark green and smooth above, grayish tomentulose beneath. Acorns I to $I \frac{1}{4}$ inches long, and from 2 to 3 times as long as the tuberculate cup. In dry soil or on rocky banks.

\section{URTICÁCE e. Nettle Family.}

Trees, shrubs, or herbs with monœcious, diœcious, or sometimes perfect flowers, and leaves with stipules. The perfect flowers with a regular, inferior calyx. Ovary superior, 1-celled, rarely 2-celled. Stamens as many as the calyx lobes, and opposite them, or fewer.

\section{I. ÚLMUS. Elm.}

(The classical Latin name.)

Trees with 2-ranked, simple, serrate, straight-veined leaves, with perfect or polygamous flowers in lateral clusters, expanding before the leaves. Calyx 4-9-lobed and campanulate. Flowers in clusters on twigs of the preceding season; cells of the ovary $1-2$, each with a single ovule; the 2 styles diverging, and stigmatic along the inner edge; fruit a I-seeded samara, winged all around.

I. Ulmus Americàna, L. AMERICAN or White Elm. Leaves oval or obovate, only slightly roughened above, 2-4 inches long. Branches without corky wings (as in the case of those of the winged elm, Ulmus alata). Samara ovateoval, nearly $\frac{1}{2}$ inch long, ciliate on the margins of the reticulate-veined wing. Along streams or in rich and moist soil.

2. Ulmus fúlva, Michx. (L., fulvus, tawny.) SLIPPERY or RED ElM. Leaves from ovate to obovate, much roughened above, doubly serrate, 4 to 8 inches long. Branches without corky wings, inner bark very mucilaginous: Samara from $\frac{1}{2}$ to of an inch long, without ciliate borders. In rich soil. 


\section{CÉLTIS. Hackberry.}

(Pliny's name for an African lotus.)

Trees or shrubs, bearing polygamous or monœcious, rarely diœcious, flowers in the axils of leaves on shoots of the current season. Staminate flowers in clusters, and the pistillate usually solitary, but sometimes in clusters of 2 or 3. Sepals distinct or calyx 4-6-parted. Ovary I-celled, with a single ovule. Fruit an ovoid or globose drupe.

I. Celtis occidentàlis, L. (L., occidentalis, western.) HACKBERRY. Leaves ovate or ovate-lanceolate, often inequilateral, serrate, usually thin. The globose drupes purple, black, or orange when mature. In woods and along river banks.

\section{MòRUS. Mulberry.}

(The classical Latin name.)

Trees or shrubs with milky sap. Flowers monœcious or diœcious, the staminate and pistillate flowers on separate spikes, the pistillate spikes becoming juicy, aggregate fruits. Stamens 4; perianth 4-parted, persisting in the pistillate flowers and becoming fleshy, and inclosing the ovary, in the fruit.

I. Morus rùbra, L. (L., ruber, red.) RED MULBERRY. Leaves ovate to orbicular, rough above and pubescent beneath. Pistillate spikes $I$ to $I \frac{1}{2}$ inches long; staminate spikes longer. Fruit dark purple when ripe. In rich woods.

\section{SANTALÀCE}

Herbs or shrubs. Leaves entire, opposite or alternate, from oval to lanceolate. Flowers perfect, monœcious or diœcious; inflorescence various. Calyx campanulate, 3-6-lobed, adnate to the ovary below. Stamens of the same number as the calyx lobes and opposite them. Ovules 2-4 and pendulous from the top of the I-celled ovary. Fruit a I-seeded drupe or nut.

\section{COMÁNDRA. Bastard Toadflax.}

(Gr., kome, hair, and andres, for stamens, in allusion to hairs on calyx lobes attached to the stamens.)

Erect perennial herbs, sometimes growing parasitically on the roots of other plants. Stamens 5 , rarely 4 , inserted at the base of the lobes of the campanulate or urn-shaped calyx, and between the lobes of a 


\section{Dicotyledones.}

fleshy disk; anthers connected to the middle of the calyx lobes by tufts of hairs. Globose fruit surmounted by the persistent calyx.

I. Comandra umbellàta, Nutt. (L., umbella, a little sunshade.) BASTARD TOADFLAX. Stems slender and branched, from 6 to 18 inches tall. Leaves sessile, oblong to oblong-lanceolate, somewhat acute at both ends, from $\frac{1}{2}$ to $\mathrm{r}$ inch and more long. Flowers in corymbose clusters near the summit of the plant. Fruit globose. Dry grounds.

2. Comandra pállida, A. DC. (L., pallidus, pale.) Pale Comandra. Leaves linear or linear-lanceolate; flowers corymbose-clustered. Fruit ovoidoblong. In dry soil.

\section{POLYGONÀCEÆ. BuckwheAt FAMily.}

Shrubs or trees, herbs, and twining vines; stems tumid at the nodes and usually sheathed by the united stipules or ocreæ. Flowers small, regular, and perfect, monœcious, diœcious, or polygamous ; inflorescence various. Stamens 4-12, inserted on the base of the 3-6-lobed calyx. Sepals sometimes petaloid, petals none. The superior ovary I-celled with a single erect or pendulous ovule. Fruit usually a 3 -angled achene.

\section{RÙMEX. Dock or Sorrel.}

(The ancient Latin name.)

Annual or perennial herbs with grooved and usually branched stems of various habits. Leaves entire, or undulate, flat or crisped; ocreæ cylindrical and fugacious. Inflorescence of simple or compound racemes. Calyx 6-parted, the inner 3 parts usually developing into wings in the fruit. Stamens 6, style 3-parted. Flowers greenish, small, crowded in panicled racemes.

I. Rumex altíssimus, Wood. (L., superlative of altus, high.) TALL or PEACHLEAved Dock. Perennial. Stems simple or little branched, 2 to 4 feet tall. Leaves ovate-lanceolate to lanceolate, from 2 to to inches long. Flowers densely whorled in open panicles. The 3 inner sepals forming the wings usually I-tubercled. Pedicels about the length of the wings, and jointed below the middle. Moist soil.

2. Rumex críspus, L. (L., crispus, curled.) CURLED Dock. Perennial. Stem from I to $3^{\frac{1}{2}}$ feet tall, simple or branched above. Leaves lanceolate, long-petioled, 3 to 12 inches long, crisped and wavy-margined. Flowers in loose whorls; pedicels in fruit $\mathrm{I}_{2}^{\frac{1}{2}-2}$ times as long as the wing sepals. Wings with a tubercle. In waste grounds.

3. Rumex hastátulus, Baldw. (L., hastatus, armed with a spear.) HALBERDLEAVED SORREL. Stems simple, I to 2 feet high from a running rootstock. Leaves halberd-shaped, $\mathrm{I} \frac{1}{2}$ to 3 inches long. In pastures and waste places. 


\section{NYCTAGINÀCEAE. FOUR-O'CLOCK FAMILY.}

Herbs outside the tropics. Leaves simple and entire. Flowers perfect; calyx corollalike and inferior. Ovary I-celled and I-ovuled. Calyx persisting in fruit and forming a nutlike pericarp. Stems swollen at the joints; an involucre below the flowers.

\section{OXÝBAPHUS.}

(Gr., oxybaphon, a vinegar saucer, from the shape of the involucre.)

Herbs with thick perennial roots and opposite leaves. Flowers in clusters of 3-5 above a 5-lobed involucre which enlarges and becomes membranous in the fruit. Stamens $3-5$, mostly 3. Style filiform.

r. Oxybaphus nyctagineus, Sweet. (Gr. nyx, night; L. ending agin-.) HEART-LEAVED UMBRELLA-WORT. Stems usually slender and angled or 4 -sided, from I to 3 feet tall. Leaves ovate, acuminate at the apex and cordate to truncate at the base; all but the uppermost ones with petioles. Perianth red; stamens and style exserted; involucre shorter than the flowers. Fruit oblong and very pubescent. In dry soil.

\section{PORTUlacáce e. Purslane Family.}

Usually succulent herbs. Sepals mostly 2 , seldom 5 ; petals 4 or 5 . Stamens usually of the same number as the petals, sometimes more numerous. Ovary superior, I-celled, style 2-8-cleft or divided; ovules 2 or many. Capsules opening by 3 valves, or circumscissile.

\section{CLAYTÓNIA.}

(Named from Dr. John Clayton, early botanist.)

Perennial herbs with simple stems rising from a bulb, and bearing a pair of opposite leaves. Stamens 5 , adhering to the base of the petals. Pod 3-valved and 3-6-seeded. Flowers in a loose raceme in early spring; petals rose color, with veins of a darker color.

I. Claytonia Virgínica, L. SPRING BeAUTY. Leaves 3 to 7 inches long, linear-lanceolate. In moist open woods.

2. Claytonia Caroliniàna, Michx. Carolina Spring Beauty. Leaves I to 2 inches long, ovate-lanceolate or spatulate oblong. 


\section{CARYOPHYLLÁCE ze. Pink Family.}

Annual or perennial herbaceous plants with opposite, entire leaves, and usually swollen nodes. Flowers perfect or rarely diœcious. Sepals 4 or 5 , separate or united below into a tube. Petals of the same number as the sepals, or wanting. Stamens twice as many as the sepals or fewer. Ovary I-celled or sometimes 3-5-celled, containing few to many ovules on a central placenta; styles $2-5$, rarely united. Fruit a dehiscent capsule, or an achene or utricle.

\section{SILÈnE. Catchfly or Campion.}

(Gr., sialon, saliva, from viscid exudation of some species.)

Sepals united below into a more or less tubular campanulate or inflated tube, and 5-toothed or cleft above. Petals 5, narrow and clawed. Stamens Io. Styles usually 3. Ovary I-celled or incompletely 2-4-celled. Pod dehiscing by 6 or 3 apical teeth. Seeds spiny or tubercled.

I. Silene Virgínica, L. FIRE PINK. A slender, perennial, erect herb, with flowers in terminal cymes, crimson, from I to $I_{\frac{1}{2}}^{\frac{1}{2}}$ inches broad; petals spreading. Calyx campanulate, nearly I inch long, enlarging in fruit. Lower leaves spatulate to oblanceolate, upper leaves lanceolate, opposite. Open woods.

\section{CERÁSTIUM. Chickweed.}

(Gr., keras, a horn.)

Sepals distinct or united only at the base, 5, seldom 4. Petals of the same number as the sepals, rarely absent, emarginate or bifid at the summit. Stamens Io, seldom less. Capsule cylindrical, and dehiscing by 8 -10 teeth. Pubescent annual or perennial herbs, bearing dichotomous cymes of white flowers.

I. Cerastium viscòsum, L. (L., viscosus, full of bird lime.) MousE-EAR CHICKwEED. Viscid-pubescent, erect, or spreading stems. Upper leaves ovate or obovate, lower spatulate. Petals shorter than the sepals. Flowers glomerate with pedicels not longer than the sepals. Annuals. In grassy places.

2. Cerastium brachýpodum, Robinson. (Gr., brachys, short; pous, podas, foot.) SHORT-STALKED CHICKWEED. Viscid-pubescent annuals, 3 to Io inches tall. Lower leaves oblanceolate or spatulate, upper linear to lanceolate. Petals longer than the sepals. Pedicels shorter or but little longer than the calyx. In dry soil.

3. Cerastium arvénse, L. (L., arvum, a plowed field.) FIELD CHICKWEed. Tufted, erect, or ascending perennials; lower leaves linear-oblong, upper linear to lanceolate. Petals obcordate, longer than the sepals. Flowers cymose, from $\frac{1}{2}$ to of an inch broad. Styles 5 . In dry or rocky places. 


\section{STELLÀRIA. Chickweed or Starwort.}

$$
\text { (L., stella, star.) }
$$

Herbs, mostly tufted and diffuse. Sepals distinct or only slightly united at the base, 4 or 5 . The 4-5 petals deeply 2-cleft. Stamens 8-10, rarely fewer. Pod ovoid; styles $3-5$; valves twice the number of the styles. Flowers white, solitary or cymose.

I. Stellaria mèdia, Smith. (L., medius, medium.) Common ChickweEd. Tufted, branched annuals, decumbent or ascending, 4 to $I 6$ inches long; stems with I or 2 longitudinal, pubescent lines; leaves ovate to oval. Flowers small, on slender pedicels, in leafy cymes, or solitary in the axils of the leaves. Petals 2-parted, shorter than the calyx; stamens 2-Io. The ovoid capsule longer than the calyx. Common in moist meadows, woods, and waste places.

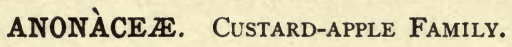

Shrubs or trees, with entire alternate leaves. Sepals 3, and petals about 6 in 2 whorls; stamens many with adnate, extrorse anthers; carpels several, distinct or coherent, usually becoming fleshy in fruit.

\section{Asímina. Papaw.}

(From the Indian name, Assimin.)

Shrubs or small trees. Flowers axillary and nodding. The 3 sepals ovate; the 3 outer petals larger than the 3 inner. Stamens and carpels 3-15. Fruit an oblong, fleshy berry. Seeds large and flat.

I. Asimina tríloba, Dunal. (Gr., tri, three; lobos, lobe.) North AMERICAN PAPAW. Small trees, with obovate-lanceolate leaves. Petals veiny and of a deep reddish purple. Flowers appearing with the leaves. Fruit large, fleshy, sweet, and edible. In rich woods.

\section{RANUNCULÀCE Æ. CROWfoot Family.}

Annual or perennial herbs or climbing shrubs. Sepals often petallike, 3-15; petals of about the same number and sometimes wanting. Stamens numerous; carpels numerous or solitary, I-celled, and Imany-ovuled. Fruit an achene, follicle, or berry. Flowers regular or irregular.

Leaves ternately or biternately compound.

Flowers regular.

Pistils 2 or more.

ISOPYRUM I.

Pistil r.

ACTEA II.

Flowers irregular. 


\section{Dicotyledones.}

Leaves simple, variously lobed or divided.

Flowers irregular.

Delphinium IV.

Flowers regular.

With basal and involucral leaves only.

Involucre more or less remote from the flowers.

Involucre close to the flowers and resembling a calyx.

With basal leaves only.

With both basal and stem leaves; involucre none.

Anemone V. Hepatica Vi. Myosurus VII. Ranunculus VIII.

\section{ISOPỲRUM.}

(From ancient Gr., isopyron.)

Slender herbs, with ternately decompound leaves and white, solitary, or panicied flowers. Five deciduous, petallike sepals; petals wanting in our species. Carpels $2-6$ or more, forming a head of follicles in fruit.

I. Isopyrum biternàtum, T. \& G. (L., bis, twice; terni, three each.) FALSE RUE ANEMONE. Slender, paniculately branched above, from fibrous or sometimes tuberiferous roots. Lower leaves long-petioled, biternate, the leaflets lobed or divided. Stamens many, the filaments thickened above. Pistils commonly 4, spreading in fruit. In moist woods or on moist, shady banks.

\section{ACT झ̀ेA. Baneberry or Cohosh.}

(Gr., aktea, name of the elder.)

Tall, erect, perennial herbs, with regular, small, racemose flowers and large, ternately compound leaves. Sepals petallike, 3-5; petals small, 3-10, rather narrow and clawed. Stamens numerous; ovary 1 with many ovules; fruit a rather large berry.

I. Actæa rùbra, Willd. (L., ruber, red.) RED BANEBERRY. From I to 2. feet high. Leaflets toothed or cleft. Racemes ovate, pedicels slender, berries red. In rich woods.

2. Actæa álba, Mill. (L., albus, white). White BaneberRy. Similar to the preceding in habit. Racemes oblong. Fruiting pedicels thick. Berries white. In rich woods.

\section{AQUILĖGIA. Columbine.}

(L., aquilla, eagle, with reference to the spurs of the petals.)

Erect, perennial herbs, with showy flowers and ternately or biternately compound leaves. Sepals 5 , regular, of the same color as the petals ; petals 5 , extending backward into hollow spurs, concave and spreading in front. Stamens numerous ; carpels 5 and many-ovuled. 
I. Aquilegia Canadénsis, L. WILD Columbine. Erect and branching, from I to 2 feet high. Flowers about 2 inches long, usually scarlet and yellow, nodding so that the straight spur points upward. In woods or on shaded, rocky banks.

\section{DELPHÍNIUM. Larkspur.}

(From the Greek name, delphinion.)

Erect, branching annuals or perennials, with palmately lobed or divided leaves, and irregular, showy, racemose, or paniculate flowers. Sepals 5, petallike, the upper one prolonged backward into a spur. Petals, 4, irregular, the upper pair sending spurs into the spur of the sepal, the lower petals small, sometimes wanting. Carpels I-5, forming many-seeded pods in fruit.

I. Delphinium azùreum, Michx. (L., azureus, sky-blue.) Stems I to 2 feet high, slender and somewhat pubescent. Flowers blue to white, about I inch long, with upward-curving spur. Leaves deeply 3-5-parted, the divisions again deeply cleft into linear segments. Racemes short and strict; follicles erect. In open grounds.

2. Delphinium tricórne, Michx. (L., tricornis, 3-horned.) DWARF LARKSPUR. Stout, 6 inches to 3 feet high. Leaves 5-7-parted, the divisions again lobed or cleft. Racemes 4 to 7 inches long, loosely or compactly several to manyflowered. Flowers I inch or more long, spur slightly curved. Follicles widely spreading. In open grounds.

\section{ANEMÒNE. Windflower.}

(The old Greek and Latin name, from Gr., anemos, wind.)

Perennial herbs, with both radical and stem leaves, those of the stem forming an involucre near or remote from the flower; the radical leaves variously lobed, divided, or dissected. Sepals 4-20, petallike; petals none. Fruit an achene, pointed or tailed.

I. Anemone Caroliniàna, Walt. Carolina Anemone. Simple stem from a tuberous root; becoming 4 to ro inches high. Basal leaves usually 3 -divided, the divisions variously lobed or cleft. Involucre of 3 wedge-shaped divisions, which are again 3 -cleft. Flowers from $\frac{3}{4}$ to $1 \frac{1}{2}$ inches broad. Sepals 6-20, linear oblong, purplish or whitish. In open meadows.

2. Anemone Pennsylvánica, L. Pennsylvania Anemone. From $I$ to 2 feet high; somewhat hairy, particularly on the under side of leaves. Basal leaves 3-5parted, and the divisions variously cleft; long-petioled. The leaves of both primary and secondary involucres sessile. Peduncle branched above the primary involucre, the branches bearing 2-leaved involucres at the middle. Flowers from $\frac{1}{2}$ to $I$ inch or more broad; sepals white. Achenes merely pubescent or smooth. In low grounds or in woods. 


\section{Dicotyledones.}

3. Anemone quinquefòlia, L. (L., quinque, five; folium, leaf.) WINDFLowER. (Anemone Nemerosa in Gray's "Manual.") 4 to 9 inches high from horizontal rootstocks. Basal leaves long-petioled, appearing later than the flowering stem. 5-parted, the divisions dentate. Involucre leaves 3-5-parted, on slender petioles. Solitary flower about I inch broad; sepals $4-9$, white or purplish. Achenes pubescent, tipped with the hooked style. Margins of woods.

4. Anemone pàtens, L. (L., patens, exposed.) Var. Nuttalliana, Gray. PASQUE FLOWER. Perennial herbs rising from a thick rootstock, from 6 to I6 inches high, silky-villous. Basal leaves much divided into linear lobes, on slender petioles. Involucre leaves a short distance below the flowers, erect, and forming a shallow cup, divided into linear segments above. Light bluish purple sepals ovate-oblong. Achenes with long, persistent, plumose styles. In dry prairies.

\section{HEPÁTICA. Liverleaf.}

(Gr., hepatikos, from hepar, liver; from supposed resemblance of leaves to the liver.)

Low perennial herbs with long-petioled, 3-lobed, basal leaves, persisting through the winter. Flowers rather large, solitary, on slender scapes, white or purple. Sepals petallike. Involucre of 3 sessile leaves close under the flower and resembling a calyx.

I. Hepática tríloba, Chaix. (Gr., tri, three; lobos, lobe.) Round-LOBED LIVERLEAF. Lobes of the leaves and of the involucre rounded or obtuse. Flowers blue, purple, or white on villous scapes. In woods.

2. Hepatica acùtiloba, DC. (L., acutus, sharp; Gr., lobos, lobe.) SHARPLOBED LIVERLEAF. Lobes of the leaves and of the involucre acute. In other respects closely resembling the preceding species. In woods.

\section{MYOSÙRUS. Mousetail.}

$$
\text { (Gr., mys, mouse; oura, tail.) }
$$

Small annuals with linear basal leaves and I-flowered scapes. Sepals usually 5 with a basal spur. Petals 5 , small, with a slender claw which is nectariferous at the summit. Stamens 5-25. Pistils many on a slender axis which becomes greatly elongated in fruit.

I. Myosurus mínimus, L. (L., minimus, least.) Mousetall. From I to 6 inches high; scapes at length longer than the leaves. The receptacle becoming an inch or more in length. In moist soil.

\section{RANÚNCULUS. Crowfoot or Buttercup.}

(Pliny's name for these plants, meaning a little frog, since many occur where frogs abound.)

Annual or perennial herbs, usually with both basal and stem leaves, without involucres and with both sepals and petals, flowers usually yel- 
low, sometimes white or red. Sepals 5 and deciduous; petals of the same or greater number, with a nectariferous pit and a scale at the base of the blade. Flowers solitary or corymed. Achenes capitate or spicate, tipped with the persisting style, numerous.

I. Ranunculus abortivus, L. (L., abortivus, abortive.) SMALL-FLOWERED CRowfoot. From 6 inches to 2 feet high. Basal leaves usually ovate-reniform. crenate, long-petioled. Stem leaves sessile and divided into oblong or cuneate lobes. Flowers yellow; petals shorter than or equaling the calyx; style short; achenes smooth, with a minute beak. In the moist ground of woods and banks of streams.

2. Ranunculus micránthus, Nutt. (Gr., mikros, small; anthos, flower.) Rock Crowfoot. Similar to the above, but basal leaves ovate or orbicular, usually 3-lobed; plant hairy; roots tuberous. In rich woods.

3. Ranunculus recurvàtus, Poir. (L., recurvatus, curved backward.) HookeD Crowfoot. From 6 inches to 2 feet high; leaves broad, deeply 3-parted or lobed, the lobes variously toothed, long-petioled. Plant usually hairy. Flowers yellow, from $\frac{1}{3}$ to nearly $\frac{1}{2}$ inch broad. Achenes tipped with a prominent, recurved, hooked beak. In woods.

4. Ranunculus septentrionàlis, Poir. (L., septentrionalis, northern.) SWAMP or MARSH BUTTERCUP. A branching plant from $I$ to 3 feet high, smooth or only slightly pubescent. Roots fibrous. Leaves long-petioled, 3 -divided, the divisions stalked and cleft or lobed and cuneate at the base. Flowers yellow, an inch or more in diameter. Petals obovate, and twice the length of the sepals. Achenes with an awl-shaped beak, flat and broadly margined. In low and moist ground.

5. Ranunculus híspidus, Michx. (L., hispidus, bristly.) HisPID BUTTERCuP. Stems 8 inches to 2 feet tall, ascending or spreading. Densely hairy while young, but becoming less so with age. Roots of thickened fibers. Leaves 3-5-divided, the divisions ovate, oblong, or obovate. Flowers from $\frac{1}{2}$ to $I_{2}^{\frac{1}{2}}$ inches broad, yellow. Achenes narrowly margined and tipped with an awl-shaped beak. In dry woods and thickets.

6. Ranunculus fasciculàris, Muhl. (L., fasciculus, a small bundle.) EARLY or TUFTED BUTTERCUP. Tufted plants from 6 to I2 inches tall. Leaves petioled, 3-5-divided, the divisions stalked and with oblong or linear lobes. Flowers yellow, about I inch broad. Achenes slightly margined with awl-shaped beak. Somewhat pubescent. Root fibers thickened. On hills or in woods.

\section{BERBERIDÀCE ÆE. BARBERRY FAMILY.}

Herbs or shrubs. Both sepals and petals imbricated in the bud and usually in two whorls each, with 3 , rarely 2-4, segments in each whorl. Stamens of the same number as the petals and opposite them. Ovary superior, I-celled: Anthers opening by uplifted valves, excepting in the genus Podophyllum. 


\section{BÉRBERIS. Barberry.}

(From Berberys, Arabic name for the fruit.)

Shrubs with yellow wood; leaves often spiny. Flowers usually terminal, solitary or racemed. Bractlets subtending the 6 sepals. The 6 petals obovate, with 2 glandular-spots (nectaries) above the claw. The 6 stamens sensitive and closing around the pistil when shocked. Fruit a I-seeded berry.

I. Berberis vulgàris, L. (L., vulgaris, common.) EUROPEAN BARBERRY. A smooth shrub, from 6 to 8 feet high. Twigs ash-colored, leaves simple, alternate or fascicled, obovate or spatulate, bristly serrate. Inflorescence a many-flowered drooping raceme. Petals not notched. Berries oblong. In thickets.

2. Berberis Canadénsis, Mill. American BARberRy. Shrubs from I to 6 feet high, with dark brown twigs. Leaves obovate or spatulate, with spreading teeth. Racemes few-flowered. Petals emarginate or notched at the apex. Berries from oval to nearly globose.

\section{CAULOPHÝllum. Blue Cohosh.}

(Gr., kaulos, stem; phyllon, leaf, the stem seeming to form a stalk for the large sessile leaf.)

Herbs from thickened rootstocks. Stem simple, bearing a large ternately compound leaf, and terminated by a raceme or panicle of greenish yellow or purplish flowers. Sepals 6, subtended by 3-4 bractlets. Petals 6, thick and somewhat hooded. Ovary finally bursting and withering away, leaving the 2 spherical, drupelike seeds naked on their seed stalks.

r. Caulophyllum thalictroides; Michx. (Gr., thalictron, meadow rue; eidos, form.) BLUE COHOSH. From I to 3 feet high. Leaflets $2-3$-lobed; divisions of the leaf long-petioled. Flowers greenish purple. In rich woods.

\section{PODOPHÝLLUM. Mandrake or May Apple.}

$$
\text { (Gr., pous, foot; phyllon, leaf.) }
$$

Erect, perennial herbs from horizontal, poisonous rootstocks. Leaves large, peltate, and palmately lobed. Flowers solitary ; white, sometimes pink. Sepals 6, petaloid and fugacious. Petals 6-9, exceeding the sepals. Stamens as many or twice as many as the petals, the linear anthers dehiscing longitudinally. Ovary usually $\mathrm{I}$, and ovules in many rows on the large parietal placenta. Fruit, a fleshy berry.

I. Podophyllum peltàtum, L. (L.,peltatus, armed with a small shield.) MANDRAKE or MAY APPLE. From I to $I_{2}^{\frac{1}{2}}$ feet high. Flowerless stems terminated by 
a large, round, peltate, 7-9-lobed leaf. Flowering stems usually bearing 2 large lobed leaves, with petioles fixed near one side. Nodding white flowers, sometimes nearly 2 inches broad, springing from the fork of the leaves. Fruit edible, but foliage and roots poisonous. In rich woods.

\section{MENISPERMÀCE ÆE. MOONSEED FAMILY.}

Herbaceous or woody climbing plants, with alternate leaves, and small diœcious flowers, usually in panicles. Carpels usually 3-6, sometimes more, superior, 1 -ovuled. Sepals 4-12; petals 6 in 2 rows, sometimes fewer or wanting. Stamens nearly of the same number as the petals. Fruit a I-seeded drupe.

\section{CALYCOCÁRPUM. Cupseed.}

$$
\text { (Gr., kalyx, cup; karpos, fruit.) }
$$

A high climber, with long-petioled, palmately lobed leaves. Flowers greenish in narrow, drooping panicles. Sepals 6, petallike; petals none. Stamens about 12 ; pistils 3 , with laciniate stigmas. Droop oval, and flattened or hollowed on one side.

I. Calycocarpum Lyòni, Nutt. (Latin genitive of proper name.) CUPSEED. Leaves 5 to 8 inches long, palmately $5-7$-lobed. Diœcious flowers in long, loose panicles. Flowers about $\frac{1}{8}$ inch broad. Drupe about I inch long and black when ripe. In rich woods.

\section{MENISPÉRMUM. Moonseed.}

\section{(Gr., mene, moon; sperma, seed.)}

A tall climber. Leaves in our species on slender petioles, broadly cordate-ovate, entire, or 3-7-angled or lobed. Sepals 4-8 in 2 rows, exceeding the 6-8 petals. Flowers diœcious. Stamens 12-24 in the staminate flowers; pistils $2-4$ in the pistillate flowers, on a short, common receptacle. Ovary incurved after flowering. Fruit globular.

I. Menispermum Canadénse, L. MoonseED. Flowers small and white. Leaves 4 to 8 inches wide, peltate near the base. Drupe $\frac{1}{4}$ to $\frac{1}{8}$ inch in diameter; the clusters of fruit resembling small grapes. In woods.

\section{LAURÀCE Æ. LAUREL FAMILY.}

Aromatic trees or shrubs, with mostly alternate, simple leaves, frequently marked with pellucid dots. Petals none; sepals 4-6, colored. Stamens inserted on the calyx in several whorls; anthers opening by 2 or 4 uplifted valves. Ovary superior, I-celled and I-ovuled. Fruit, a $\mathrm{I}$-seeded drupe or berry. 


\section{Dicotyledones.}

\section{SÁSSAFRAS.}

(Sp., sasafras, used as a popular name by early French settlers.)

Trees, with aromatic bark and mucilaginous leaves and twigs. Leaves entire or 1-3-lobed. Flowers diœcious, yellow, in umbelled, clustered racemes, unfolding with the leaves. Calyx 6-parted and spreading. Staminate flowers of 9 stamens in 3 rows. Pistillate flowers of an ovoid ovary and 6 abortive stamens. Fruit, an oblongglobose, blue drupe.

I. Sassafras officinàle, Nees. (L., officina, a shop.) SASSAFRAS or AgUE TREE. Becoming a large tree with rough bark. Leaves oval and entire, or I-3-lobed, petioles about $I$ inch long. Drupe about $\frac{1}{2}$ inch long. In dry or sandy soil or in rich woods.

\section{PAPAVERÀCE Æ. POPPY FAMILY.}

Herbs, with milky or colored secretions, which exude when the plants are broken or deeply injured. Leaves mostly alternate and without stipules. Inflorescence various, but peduncles mostly I-flowered. Sepals 2-3, falling as the flower opens. Petals $4-6$ or more, deciduous. Stamens few to many, distinct and hypogynous. Ovary usually I-celled, many-ovuled. Fruit, mostly a dry, I-celled pod.

\section{ARGEMòne. Prickly Poppy.}

(Gr., argema, a disease of the eye. Name applied to a plant used as a remedy.)

Annual or biennial herbs, with yellow secretions and lobed and spinytoothed leaves. Flowers large and showy. Sepals 2-3, petals 4-6. Stamens many. Placentæ 4-6, many-ovuled; stigma 3-6-radiate, dilated. Capsule oblong, prickly, dehiscent at the apex.

I. Argemone álba, Lestib. (L., albus, white.) White Prickly POPPy. Stout and often tall, with pinnatifid or pinnately lobed leaves. Flowers white, sometimes 3 to 4 inches in diameter. Capsules $I$ to $I \frac{1}{2}$ inches long, dehiscent at the apex by valves. On dry, open prairies or plains.

\section{SANGUINÀRIA. Bloodroot.}

(Latin name, from sanguis, blood. Named from the red color of its secretion.)

Low perennials from horizontal rootstocks; secretions or latex red. Flower and leaf appearing together in early spring. Leaf rounded and palmate-lobed. Flower white, solitary on a naked scape. Sepals 2 
early deciduous; petals $8-12$, in 2-3 whorls. Stamens many. Placentæ 2. Capsule oblong, turgid, I-celled and 2-valved, dehiscing to the base.

I. Sanguinaria Canadensis, L. BLoodroot. Petioles 6 to $\mathrm{I}_{4}$ inches long. Flowers $I$ to $I \frac{1}{2}$ inch broad. Flowers at first overtopping the leaves, but finally exceeded by them. In open woods.

\section{STYLÓPHORUM. Celandine Poppy.}

(Gr., stylos, style; phero, to bear.)

Low perennial herbs from stout rootstocks, with both basal and stem leaves. Latex yellow. Leaves pinnatifid. Flowers yellow, clustered or solitary. Sepals 2, hairy; petals 4 ; stamens many. Placentæ 2-4. Capsule oblong or ovoid, hirsute, dehiscent to the base.

I. Stylophorum diphýllum, Nutt. (Gr., di, two; phyllon, leaf.) YELlow or CELANDine Poppy. Leaves pinnatifid, the 5-7 lobes sinuate. Upper stem leaves opposite. Plant becoming 12 to 18 inches high, and the basal leaves 4 to 10 inches long. Flowers terminal, 2-4, about I inch broad, deep yellow. In damp woods.

\section{FUMARIÀCEAE. FUMITORY FAMILY.}

Delicate, smooth, and succulent herbs, with compound, dissected leaves. Flowers irregular. Sepals 2 and inconspicuous, soon falling off; petals 4, somewhat united; stamens 6, diadelphous. Ovary I-celled and I-seeded, or several seeds on 2 parietal placentæ.

\section{DICÉNTRA. Dutchman's Breeches.}

$$
\text { (Gr., dis, twice; kentron, a spur.) }
$$

Corolla cordate or 2 -spurred at the base; the inner 2 petals coherent above, and crested or winged on the back. The 6 stamens, in 2 sets, placed opposite the outer petals, the filaments somewhat diadelphous. Placentæ 2 ; style slender; and stigma 2-4-lobed. Low perennials, with ternately compound and dissected leaves. Flowers racemose and nodding; pedicels bracted.

I. Dicentra cucullària, DC. (L. cucullus, a hood or cap.) Dutchman's BREECHES. Delicate, smooth herbs with ternately compound, finely dissected leaves. Racemes simple, 4-10-flowered; flowers white or whitish, nodding. Spurs divergent and inner petals minutely crested. In rich woods. 


\section{Dicotyledones.}

\section{CORÝDALIS.}

(Ancient Greek name for the crested lark.)

Only the upper outer petal spurred at the base. Pod with few to many crested seeds. The interior petals narrow and keeled at the back. Stamens 6 in 2 sets, opposite the outer petals. Placentæ 2 ; style persistent. Capsule linear or oblong, 2-valved. Flowers in racemes.

I. Corydalis flàvula, DC. (L., favus, golden yellow.) PALE CoRydalis. Flowers $\frac{1}{4}$ to $\frac{1}{d}$ inch long; spur short; pods drooping or spreading. Pedicels slender in the axils of conspicuous bracts; flowers pale yellow. Stems slender, diffuse or ascending. Lower leaves petioled, the upper nearly sessile, finely dissected. In woods or on rocky banks.

2. Corydalis micrántha, Gray. (Gr., mikros, small; anthos, flower.) SMALLFLOWERED CORYDALis. Similar to the above, but with pods ascending and short-petioled.

3. Corydalis aùrea, Willd. (L., aureus, golden.) Golden Corydalis. Flowers from $\frac{1}{4}$ to $\frac{3}{3}$ inch long, spur conspicuous, about half as long as the corolla. Corolla golden yellow. Pods spreading or pendulous, becoming swollen at intervals. Var. occidentalis, Englm., has the spur nearly as long as the body of the corolla. On rocky banks.

\section{CRUCÍfER Ae. MUSTARd FAMILy.}

Mostly herbs with acrid juice. Leaves alternate, and flowers in racemes or corymbs. Flowers cruciform. Sepals 4 ; petals 4, spreading in the form of a cross. Stamens 6 (sometimes fewer) and tetradynamous, 2 being usually shorter than the other 4 . Pistil superior, consisting of 2 carpels united to form a compound pistil with a thin partition connecting the 2 parietal placentæ. Fruit a silique or silicle, usually 2-celled, rarely I-celled.

FLOWERS, WHITE.

\section{SEEDS IN 2 ROWS IN EACH CELL.}

Pods more or less compressed parallel with the partition.

(a) Low-tufted herbs, often with stellate pubescence.

(b) Leaves lanceolate or linear; pods at length orbicular.

(c) Pods linear; seeds often wing-margined.

Draba XII.

Alyssum XIV.

ARABIS XIII.

Pods more or less compressed at right angles to the partition.

(a) Pods short, obcordate-triangular.

Capsella $\mathrm{X}$.

Pods terete or turgid.

(a) Pods elongated, often angled; valves I-3-nerved.

(b) Pods globular to oblong-linear; valves nerveless.

Sisymbrium II. NAsturtium IV.

II. SEEDS IN I ROW, OR SINGLE IN EACH CELL.

Pods more or less compressed parallel with the partition.

(a) Pods becoming orbicular; leaves oblong or linear; leafy throughout.

Aュyssum XIV. 
(b) Pods linear, flattened; valves mostly nerveless; leafy throughout.

(c) Stems leafless below, from horizontal, tuberous rootstocks.

Cardamine V.

(d) Pods broadly linear, nerveless; stems low and scapelike.

Dentaria Vi.

LEAVENWORTHIA VIII.

(e) Pods linear; valves mostly I-nerved; seeds usually winged or margined.

Pods more or less compressed at right angles to the partition.

ARABIS XIII.

(a) Pod short, flat, rounded, 2 -seeded.

Pods terete or turgid.

(b) Pods elongated, often angled; valves I-3-nerved.

LEPIDIUM I.

Sisymbrium II.

FLOWERS, YELLOW.

I. SEEDS IN 2 ROWS IN EACH CELL.

Pods more or less compressed parallel with the partition.

(a) Leaves pinnatifid; valves thin, finely veined and nerveless.

(b) Low-tufted herbs, often with stellate pubescence.

(c) Pods obovoid or pear-shaped; valves I-nerved.

Pods terete or turgid.

(a) Pods elongated, often angled; valves I-3-nerved.

(b) Pods globular to oblong-linear; valves nerveless.

(c) Pods globular-inflated; leaves simple with stellate hairs.

(d) Pods obovoid or pear-shaped; valves I-nerved.

Selenia VII.
Draba XII.
Camelina XI.

SisYmbrium II.

NASTURTIUM IV.

Lesquerella IX.

Camelina XI.

II. SEEDS IN I ROW, OR SINGLE IN EACH CELL.

Pods terete or turgid.

(a) Pods elongated, often angled; valves 1-3-nerved; style or beak short.

(b) Pods elongated, tipped with a stout beak; valves 1 -5-nerved; lower leaves pin. natifid, upper leaves dentate or entire.

Brassica III.

FLOWERS, PURPLE.

I. SEEDS IN 2 ROWS IN EACH CELL.

Pods more or less compressed parallel with the partition.

(a) Pods linear; seeds often wing-margined.

ARABIS XIII.

II. SEEDS IN I ROW IN EACH CELL.

Pods more or less compressed parallel with the partition.

(a) Pods linear and flattened; valves mostly nerveless; leafy throughout.

(b) Stems leafless below, from horizontal tuberous rootstocks.

Cardamine V.

(c) Pods broadly linear, nerveless; stems low and scapelike.

Dentaria VI.

Leavenworthin VIII.
(d) Pods linear, mostly I-nerved; seeds often wing-margined.

LEAVENWORTHIA VIII.

\section{LEPÍDIUM. Pepperwort or Peppergrass.}

(Gr., lepidion, a little scale, with reference to the little pods.)

Flowers white; pods short, the boat-shaped valves or carpels flattened contrary to the narrow partition; pod 2-seeded and very flat. Stamens sometimes fewer than 6 , and petals sometimes wanting. 


\section{Dicotyledones.}

r. Lepidium Virginicum, L. WILD PEPPERGRASS. Pods slightly winged above, orbicular or oval, about $\frac{1}{12}$ inch broad. Petals usually present. Basal leaves from spatulate to obovate, somewhat pinnatifid. Stem leaves sessile, lanceolate or linear, entire or dentate. Pedicels slender and spreading, from $\frac{1}{6}$ to $\frac{1}{4}$ inch long in fruit. Roadsides and fields.

\section{SISÝMBRIUM. Hedge Mustard.}

(Ancient Greek name for a plant of this family.)

Flowers mostly white or yellow. Seeds usually in only I row in each cell of the elongated terete or angled siliques, sometimes 2-rowed; valves I-3-nerved. Mostly tall and erect annuals and perennials. Leaves simple, entire, lobed, or pinnatifid.

r. Sisymbrium officinàle, Scop. (L., officina, a workshop.) HEDGE MUSTARD. Leaves runcinate-pinnatifid. Flowers yellow; pods linear, about $\frac{1}{2}$ inch long, appressed. In waste places.

2. Sisymbrium canéscens, Nutt. (L., canescens, becoming white.) TANSY MUSTARD. Leaves 2-pinnatifid, frequently hoary or downy. Flowers very small and yellowish. Pods oblong-club-shaped or oblong-linear, shorter than the nearly horizontal pedicels. Seeds in 2 rows in each cell. In waste places.

3. Sisymbrium Thaliàna, Gaud. (Gr., thaleia, blooming.) MOUSE-EAR CRESS. Leaves obovate or oblong, entire or barely toothed. Flowers white. Pods linear and somewhat 4 -sided, longer than the spreading pedicels. Slender and branching; about 9 inches tall. Old fields and waste places.

\section{BRÁSSICA.}

(Latin name for cabbage.)

Flowers showy and yellow in elongated racemes. Siliques sessile, elongated, terete or 4-sided, tipped with a more or less elongated beak; the convex valves $\mathbf{1 - 5}$-nerved. Seeds in I row in each cell. Erect, branching, herbaceous annuals, biennials, or perennials, with basal leaves pinnatifid and stem leaves dentate or nearly entire.

I. Brassica nìgra, Koch. (L., niger, black.) BLACK MUSTARD. Pods from $\frac{1}{2}$ to $\mathrm{I}$ inch long, slender, and appressed. Flowers bright yellow, from $\frac{1}{4}$ to nearly $\frac{1}{2}$ inch broad. From 2 to 7 feet high. Lower leaves slender-petioled, pinnatifid, with a large, terminal lobe, the lobes dentate; the upper leaves much smaller, entire, and lanceolate, or oblong. Brassica juncea, Cosson., has slender, erect pods, from I to 2 inches long, on slender pedicels, and runcinate-pinnatifid lower leaves. Brassica sinapistrum, Boiss., has spreading or ascending pods from $\frac{1}{2}$ to $\frac{3}{3}$ inch long, somewhat constricted between the seeds, on stout pedicels not more than $\frac{1}{4}$ inch long in fruit. In fields and waste places.

2. Brassica campéstris, Linn. (L. campestris, pertaining to a level field.) RUTA-BAGA or SWEDISH TURNIP. Flowers creamy yellow; roots tuberous. In cultivated grounds. 


\section{NASTÚRTIUM. Water Cress.}

(L., nasus tortus, a wry nose, in allusion to the pungent qualities of the genu.)

Flowers yellow or white. Pods short or elongated, from globular to oblong-linear, terete or nearly so; valves nerveless. Leaves mostly pinnate or pinnatifid. Seeds in most cases in 2 rows in each cell. Embracing aquatic and marsh species.

I. Nasturtium officinàle, R. Br. (L., officina, workshop.) TRUE WATER CREss. Petals white, twice as long as the sepals; pods linear, from $\frac{1}{2}$ to $\frac{2}{3}$ inch long; leaves with 3-II odd-pinnate leaflets. Aquatic plants, rooting at the nodes. In brooks and ditches.

2. Nasturtium sinuàtum, Nutt. (L., sinuatus, curved or wavy.) SPREAdiNG YELLOW CRESS. Flowers yellow, about $\frac{1}{6}$ inch broad. Pods linear-oblong, from $\frac{1}{3}$ to $\frac{3}{4}$ inch long, on slender pedicels, somewhat elongated. Low diffuse perennials, with lanceolate, oblanceolate, or oblong leaves, pinnatifid into linear or oblong lobes, or merely sinuate-dentate, from 2 to 3 inches long and usually less than an inch in breadth. In moist ground.

3. Nasturtium palústre, DC. (L., palus, a swamp.) MARSH CREsS. Flowers yellow, from $\frac{1}{6}$ to $\frac{1}{3}$ inch broad. Pods spreading or curved, about $\frac{1}{4}$ inch long, on pedicels of nearly the same length; style quite short. Erect, branching annuals or biennials. Lower leaves deeply pinnatifid, lanceolate, or oblanceolate, petioled; upper leaves sessile or nearly so, dentate or lobed. In wet places or shallow water.

\section{CARdÁmine. Bitter Cress.}

(Greek name of a cress.)

Flowers white or purple, in racemes or corymbs. Pods linearelongate, flattened parallel with the partition, usually erect; valves hardly or not at all nerved, dehiscing elastically at maturity. Seeds in I row in each cell, flattened and marginless.

I. Cardamine Pennsylvánica, Muhl. Pennsylvania Bitter Cress. Erect, stout, or slender stems, 8 inches to 3 feet tall, leafy throughout. Basal leaves 2 to 6 inches long, pinnately divided into $4^{-8}$ pairs of mostly narrow segments, the terminal segment obovate, oval, or nearly orbicular. Flowers white. Pods narrowly linear, rather more or less than I inch long. In swamps and wet places.

2. Cardamine bulbòsa, B. S. P. (L., bulbosus, full of bulbs.) Bulbous CresS. Erect stems from a tuberous base, simple or sparingly branched; 6 inches to $I_{\frac{1}{2}}$ feet tall. Basal leaves long-petioled, entire, oval to orbicular, sometimes cordate. Stem leaves mostly sessile, oblong or lanceolate, dentate or entire. Flowers white; petals much longer than the calyx. The linear-lanceolate pods about $I$ inch long. In wet meadows and springs. 


\section{DENTÀRIA. Toothwort or Pepperroot.}

(L., dens, a tooth.)

Flowers white, rose-colored, or purple, in corymbose clusters, petals much exceeding the calyx. Pods linear and flattened, parallel with the partition, dehiscing from the base. Seeds in I row in each cell. Perennial herbs from fleshy, horizontal rootstocks. Stems leafless below, 2-4-leaved above. Leaves 3-divided, or palmately laciniate, petioled.

I. Dentaria laciniàta, Muhl. (L., lacinia, a flap.) CuT-LeAved ToothWORT or PEPPERROOT. Basal leaves, when present, similar to the stem leaves, which are 3-parted, the divisions lanceolate or oblong, lobed or cleft. Leaves petioled, 2 to 5 inches broad; stem leaves close together, usually 3. Flowers rather more than $\frac{1}{2}$ inch broad, white or pinkish. In moist woods.

2. Dentaria diphýlla, Michx. (Gr., di, two; phyllon, leaf.) Two-LEAveD Too'rHwort. Basal leaves long-petioled, 4 to 5 inches broad, ternate, the divisions broadly ovate, dentate, or lobed; the two stem leaves similar to the basal leaves, and opposite or nearly so. Flowers white. In woods and meadows.

\section{SELÈNIA.}

(Gr., selene, the moon; from fancied resemblance of the pods.)

Flowers yellow. Pods flattened parallel to the broad partition, oblong, and narrowed at both ends. Valves thin, finely veined, and nerveless. Styles long and slender. Seeds in two rows in each cell. Low annuals with pinnatifid leaves, and leafy-bracted racemes.

I. Selenia aurea, Nutt. (L., aureus, golden yellow.) Stems 2 to 8 inches high. Basal leaves $I$ to 2 inches long, once or twice pinnatifid; stem leaves smaller but similar. Pedicels about $\frac{1}{2}$ inch long in fruit. Pod often more than $\frac{1}{2}$ inch long and $\frac{1}{6}$ to inch broad. In open places.

\section{LEAVENWÒRTHIA.}

(Named from M. C. Leavenworth.)

Upper part of corolla white or purple, yellow toward the base. Pods broadly oblong-linear, flattened parallel to the partition, $\frac{1}{2}$ to $\mathrm{I}$ inch and more long. Valves nerveless, finely reticulate-veined. Seeds in I row in each cell. Low annuals with scapelike stems, and lyrate-pinnatifid basal leaves. Flowers terminal, solitary, or few.

I. Leavenworthia Michaùxii, Torr. (L., genitive of proper name.) Stems tufted, 3 to 6 inches high; basal leaves $I$ to 4 inches long; stem leaves few or wanting. Petals wedge-shaped, about twice the length of the calyx. Pods oblong or linear. Leavenworthia torulòsa, Gray, is similar to the above, but with pods constricted between the seeds. In open dry places. 


\section{LESQUERÉLLA.}

(Named for Leo. Lesquereux.)

Flowers yellow. Pod globular-inflated, nerved from the apex to the middle, seeds few to several in 2 rows. Low annuals or herbaceous perennials, having simple leaves beset with stellate pubescence.

I. Lesquerella globòsa, Watson. (L., globus, a sphere.) Sparingly branched annuals or biennials, 6 to 20 inches high, beset with fine, stellate pubescence. Basal leaves I to $I \frac{1}{2}$ inches long, oblong-obovate, obtuse. Stem leaves smaller, linear or oblong, sessile, entire, or margins slightly undulate. Pods nearly globular. In open places.

2. Lesquerella Ludoviciàna, Watson. Biennials or perennials, 6 to 18 inches high, densely stellate-pubescent throughout. Leaves linear to oblanceolate, blunt and entire, lower leaves 2 to 3 inches long. Flowers yellow. Pedicels nearly $\mathrm{I}$ inch long, spreading or recurved in fruit. Prairies.

3. Lesquerella grácilis, Watson. (L., gracilis, slender.) Slender, sparingly pubescent annuals, much branched, I to 2 feet tall. Leaves linear to oblanceolate. Pods globose and glabrous. Prairies.

\section{CAPSÉlla. Shepherd's Purse.}

(Latin diminutive of capsa, box.)

Flowers white. Pod short, obcordate-triangular, flattened contrary to the narrow partition. Seeds numerous in 2 rows in each cell. Annuals.

Capsella Bursa-pastòris, Mœnch. (L., meaning shepherd's purse.) Annuals, 6 to 20 inches high. Basal leaves clusteret and more or less pinnatifid and toothed. Stem leaves much smaller, few, often dentate and auriculate. Flowers small in an elongate raceme. Pods on slender pedicels, erect or spreading. Common in waste places.

\section{CAMÉLINA. False Flax.}

(Gr., chamai, dwarf; linon, flax.)

Flowers yellow and small. Pods obovoid or pear-shaped, only slightly flattened parallel with the partition; valves I-nerved. Seeds several in 2 rows in each cell; style slender. Erect annuals with entire or toothed and pinnatifid leaves.

I. Camelina sativa, Crantz. (L., sativus, sown or planted.) Gold-of-PLEASURE or FALSE FLAX. I to 2 feet high. Leaves lanceolate, the upper clasping by a sagittate base, mostly entire. Pedicels slender and spreading or ascending. In fields and waste places. 


\section{DRÀBA. Whitlow Grass.}

Low tufted herbs, often with stellate pubescence. Flowers white or yellow; pods elliptic, oblong, or linear, flattened parellel with the partition. Seeds several in 2 rows in each cell. Stems scapose or leafy.

I. Draba vérna, L. (L., verna, a native.) Vernal Whitlow Grass. Flowers white; petals deeply 2-cleft. I to 5 inches high; flowering stems leafless. Leaves tufted at the base, oblong or spatulate-oblanceolate, entire or only dentate, beset with stiff, stellate hairs. Pods oblong or oval, smooth, shorter than the pedicels. In fields and sandy waste places.

2. Draba Caroliniàna, Walt. CARolina Whitlow Grass. Flowers white; petals entire. Pods inear, longer than the ascending pedicels. Flowering stems I to 5 inches high. Leaves obovate and entire, clustered at the base, or only a short distance up the stem, beset with stellate pubescence.

3. Draba cuneifòlia, Nutt. (L., cuneus, wedge; folium, leaf.) Flowers white; petals emarginate; pods oblong-linear, minutely hairy, longer than the horizontal pedicels. 4 to 8 inches high, branching and leafy below; leaves obovate, cuneate, or the lowest spatulate, dentate toward the summit. In fields and grassy places.

4. Draba brachycàrpa, Nutt. (Gr., brachys, short; karpos, fruit.) SHORTFRUITED WHITLOW GRASS. Flowers yellow; the oblong pods $\frac{1}{12}$ to $\frac{1}{6}$ inch long. Basal leaves $\frac{1}{3}$ to $\frac{1}{2}$ inch long, ovate or obovate, stem leaves oblong and entire. Dry hills and fields.

\section{XIII. ÁRABIS. Rock Cress.}

(Named from Arabia.)

Flowers white or purple. Pods linear, elongated, and flattened parallel with the partition; valves mostly I-nerved. Seeds in I or 2 rows in each cell, usually margined or winged. Leaves seldom divided.

I. Arabis Ludoviciàna, Meyer. Stems ascending froin $\frac{1}{2}$ to I foot high. Stem leaves pinnatifid, oblong, and narrow. Flowers very small and white. Pods linear, spreading, nearly $I$ inch long; seeds as broad as the pod, and winged. In open places.

2. Arabis dentàta, T. \& G. ToOTheD Rock CRess. Petals greenish white, hardly exceeding the calyx; pods narrowly linear, sometimes exceeding $I$ inch in length. Seeds oblong, in I row in each cell. Stems sparingly branched, I to 2 feet high. Basal leaves obovate and dentate, on margined petioles; stem leaves oblong or oblanceolate, dentate, sessile, base auricled and clasping.

\section{ALÝSSUM.}

(Gr., $a$, without or depriving; lyssa, madness. Greek name of a plant supposed to have remedial value.)

Flowers white (or sometimes yellow). Pods orbicular, flattened at the margins parallel with the partition; seeds only 1 or 2 in each cell. 
x. Alyssum maritimum, L. (L., maritimus, relating to the sea.) SwEET Alyssum. White, honey-scented flowers. Stems spreading; leaves lanceolate or linear, entire, green, or slightly hoary. Rounded pods with a single seed in each cell. Cultivated.

\section{SAXIFRAGÀCE Æ. SAXIFRAGE FAMILY.}

Herbs or shrubs with opposite or alternate, exstipulate leaves. Flowers perfect or polygamo-diœcious. Calyx mostly 5-lobed or parted, usually persistent and more or less adnate to the ovary or free from it. Petals 4-5. Stamens sometimes twice as many as the petals (sometimes more numerous), when of the same number alternate with them, perigynous or epigynous. Carpels I-several, mostly 2, united or free. Styles as many as the carpels or cells of the ovary, or united into I. Fruit a capsule, follicle, or berry; seeds mostly many.

\section{SAXÍFRAGA. Saxifrage.}

(L., saxum, a rock; frangere, to break.)

Perennial herbs. Calyx 5-lobed, free from or adnate to the base of the ovary. Petals 5 and perigynous. Stamens 10, inserted with the petals. Ovary 2-lobed and 2-celled. Capsule 2-beaked. Seeds numerous.

I. Saxifraga Pennsylvánica, L. Swamp Saxifrage. Stout, I to 3 or more feet high. Leaves 4 to Io inches long and sometimes 3 inches wide, varying from oval to oblanceolate, narrowing at the base into a short petiole, clustered at the base. Stem scapose, bearing flowers in large, oblong, open panicles. Calyx reflexed. Petals longer than the calyx, greenish. Follicles divergent when mature. On wet banks or in bogs.

2. Saxifrage Virginiénsis, Michx. EARLy SAXIFrage. Scapes 4 to 12 inches high, viscid-pubescent. Leaves obovate-spatulate, narrowing into a petiole, crenate or dentate, $x$ to 3 inches long, or longer. Flowers clustered in cymes, the inflorescence becoming a loose panicle. Flowers white, to to $\frac{1}{4}$ inch broad. Calyx lobes erect, shorter than the petals. Carpels nearly separate and becoming widely divergent in fruit. Dry hillsides and rocky woodlands.

\section{PHILADÉLPHUS. Mock Orange or Syringa.}

(Gr., philos, loving; adelphos, brother. No obvious reason for the name.)

Shrubs with opposite, petioled, exstipulate leaves. Flowers large, white or cream-colored, terminal or axillary. Calyx tube coherent with the ovary, 4-5-lobed. Petals 4-5, rounded or obovate. Stamens 20-40. Ovary 3-5-celled; styles 3-5, distinct or united. Capsule top-shaped, 


\section{Dicotyledones.}

dehiscing loculicidally. The common name syringa is the proper generic name for the lilac.

I. Philadelphus coronàrius, L. (L., coronarius, pertaining to a wreath.) GARDEN SYRINGA or MOCK ORANGE. A shrub 8 to Io feet high. Leaves 2 to 4 inches long, elliptic or ovate-elliptic, pubescent beneath, denticulate. Flowers racemose at the ends of the branches, an inch or more broad, creamy white and fragrant. Cultivated; escaped from gardens in some localities.

\section{RIBES. Gooseberry and Currant.}

$$
\text { (Ar., ribes, gooseberry.) }
$$

Shrubs with aiternate and often fascicled, lobed leaves. Calyx 5lobed, the tube coherent with the ovary. Petals 5 and small, inserted at the throat of the calyx. Stamens 5 , alternate with the petals. Ovary I-celled, with 2 parietal placentæ. Styles 2, distinct or united. Fruit a pulpy, globose, or ovoid berry, bearing the remains of the calyx at its summit.

I. Ribes Cynósbati, L. (Gr., kynosbatos, the dog-thorn.) WILD GooseberRY or DogBerRy. Flowers I-3. Calyx tube ovoid-campanulate, green. Berry beset with awl-shaped prickles. In rocky woods.

2. Ribes setòsum, Lindl. (L., setosus, bristly.) BRISTLY GooseberRy. Flowers I-4, calyx tubular and white. Stem with numerous prickles. Fruit glabrous or only sparingly prickly. In thickets and along lake shores.

3. Ribes grácile, Michx. (L., gracilis, slender.) MisSouri GoosererRY. Flowers about 3, white or greenish, drooping. Lobes of the calyx longer than the tube. Stamens much exserted. Berry reddish purple. In rocky or dry soil.

4. Ribes oxycanthoides, L. (Gr., oxys, sharp; akanthos, spine; eidos, resemblance.) HAWTHORN or NORTHERN GOOSEBERRY. Flowers I-3 on short pedicels, greenish purple or white. Stamens short, not exserted. Stems scarcely prickly. Fruit reddish purple when ripe, smooth. In low grounds or damp woods.

5. Ribes flóridum, L'Her. (L., foridus, flowery.) WILd BlaCk CURRANT. Leaves somewhat pubescent and resinous-dotted beneath. Flowers many in pendulous racemes, greenish white. Calyx tube cylindric. Fruit smooth, black, and globose-ovoid when ripe. In woods.

6. Ribes rùbrum, L. (L., ruber, red.) RED CURRANT. Without prickles. Leaves 3-5-lobed and serrate. Flowers in loose, pendulous racemes, greenish or purplish. Calyx tube campanulate. Stamens short. Fruit red and smooth.

7. Ribes cèreum, Dougl. (L., cereus, waxy.) WHITE-FLOWERED or SQUAW CURRANT. Flowers sessile or on short pedicels in short racemes, from the same buds as the rounded, reniform leaves, whitish or greenish white. Calyx tube tubular and glandular. Fruit red and insipid.

8. Ribes aúreum, Pursh. (L., aureus, golden yellow.) Golden, Buffalo, 
or MISSOURI. CURRANT. Flowers several in leafy-bracted racemes, yellow, spicyscented, $\frac{1}{2}$ to $r$ inch long. Calyx tube cylindric, about 3 times as long as the lobes. Fruit smooth, yellow, becoining black. Along streams.

\section{ROSÀCE \&. Rose FAmily.}

Herbs, trees, or shrubs with alternate, mostly stipulate, leaves. Flowers regular; sepals 5 , often subtended by as many sepal-like bractlets; petals 5, apparently inserted on the calyx; stamens usually indefinite, apparently inserted on the calyx (expanded border of the base of the receptacle). Pistils I-many, distinct or united. Some of our most beautiful flowers and best fruits belong to this family.

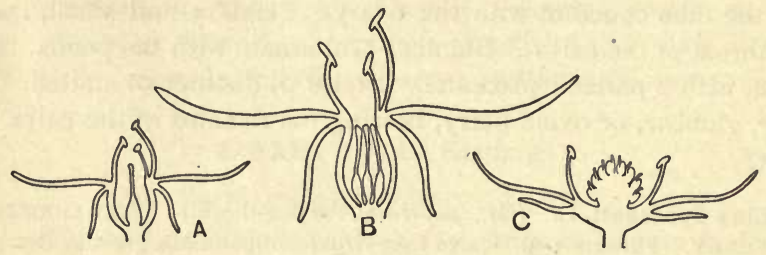

FIG. 348 .

Longitudinal diagrams of type flowers of the Rosaceæ. $A$, plum; $B$, rose; $C$, strawberry.

Ovary superior or half superior.

Ripened pistil a drupe or drupelet.

Fruit consisting of a single pistil.

Prunus I.

Fruit consisting of several pistils cohering over an elongated receptacle. RuBUS IV. Ripened pistil a few- to several-seeded pod.

Pistils 5-8; pods not inflated.

SPIREA II.

Pistils $1-5$; pods inflated.

Physocarpus III.

Ripened pistil an achene.

Carpels distinct and numerous on a convex receptacle, which becomes fleshy and edible in fruit.

Fragaria V.

Carpels distinct on a dry receptacle; styles not lengthening in fruit; bracts conspicuous at the sinuses of the calyx.

Potentilla Vi.

Carpels 2-6 on a short receptacle; styles not lengthening in fruit; bracts at the sinuses of the calyx minute or wanting.

WaLDSTEINIA VII.

Carpels numerous on a dry conical or cylindrical receptacle; style persisting as a hairy Ovary inferior. or jointed tail to the achene.

Geum VIII.

Pistils several, inclosed in an urn-shaped receptacle.

Rosa IX.

Pistil single, compound, its cells as many as the styles (2-5).

Fruit a pome; ovary 5 -celled, its carpels 2-seeded.

Pyrus X.

Fruit a small, berrylike pome; ovary becoming ro-celled, its carpels 2-seeded.

Fruit a small, drupelike pome with $\mathrm{r}-5$ bony stones.

Amelanchier XI. Crategus XII. 


\section{Dicotyledones.}

\section{PRÙNUS. Plum, Cherry, Peach.}

(The ancient I atin name.)

Trees or shrubs, with leaves mostly simple and serrate. Flowers perfect; lobes of calyx and corolla 5 ; ovary superior and free from the so-called calyx tube at maturity. Stamens I5-20; pistil I, with 2 pendulous ovules. Fruit a fleshy drupe with a hard stone.

I. Prunus Americàna, Marsh. Wild Yellow or RED Plum. Flowers in lateral umbels, white, appearing before the leaves, about I inch broad. Calyx lobes entire and pubescent within. Leaves ovate or obovate-acuminate, nearly glabrous when mature. Branches somewhat thorny. Fruit red or yellow, globose, little or no bloom; stone slightly flattened. Shrubs or small trees. River banks and woods.

2. Prunus Wàtsoni, Sargent. (Latin genitive of proper name.) SAND Plum. A somewhat spiny shrub, 6 to to feet high. Flowers in numerous lateral fascicles, about $\frac{1}{2}$ inch in diameter. Leaves ovate to ovate-lanceolate, finely serrulate, shining above. Petals oblong-obovate and short-clawed. Globose fruit about $\$$ inch in diameter, no bloom, flesh yellow. In sandy soil.

3. Prunus Chicàsa, Michx. (Latinized form of Chickasaw, Indian name.) Chickasaw Plum. A small tree with somewhat thorny branches. Flowers in lateral umbels, expanding shortly before or with the leaves. Leaves lanceolate or oblong-lanceolate, serrulate, glabrous when mature. Drupe red, $\frac{1}{2}$ to inch in diameter; bloom scant; skin thin; stone ovoid, hardly flattened. In dry soil.

4. Prunus Bésseyi, Bailey. (Latin genitive of proper name.) WESTERN SAND CHERRY. A shrub, I to 4 feet high. Flowers in lateral umbels, expanding with the leaves, $\frac{1}{3}$ to nearly $\frac{1}{2}$ inch in diameter. Leaves mostly elliptic or oblongelliptic. Stipules of young shoots often longer than the short petioles. Fruit black, mottled, or yellow, $\frac{1}{2}$ to $\frac{2}{2}$ inch in diameter, astringent. Branches often spreading and prostrate. Prairies.

5. Prunus Pérsica, Sieb. \& Lucc. (L., persicus, persian.) PEACH. Small trees. Leaves thin, lanceolate, serrate. Fruit large and edible; stone thick-walled, somewhat compressed, deeply wrinkled. Flowers pink, about $\frac{3}{4}$ inch in diameter; borne in clusters.

6. Prunus Pennsylvánica, L. f. Wild Red Cherry. Small tree. Flowers on long pedicels, many in a corymbose cluster. Leaves oval or lanceolate on slender petioles, shining on both sides, serrulate, unfolding with the flowers. Fruit small and globose, with thin skin and sour flesh, light red. Stone globular. In rocky woods.

7. Prunus Virginiàna, L. Choke Cherry. A shrub 2 to to feet high. Flowers in racemes, terminating shoots of the season. Leaves obovate or broadly oval, sharply serrulate with slender teeth. Drupe red or nearly black, about $\frac{1}{\frac{1}{3}}$ inch in diameter, very astringent. Stone globular. Along river banks. 
8. Prunus serotina, Ehrh. (L., serotinus, late ripe.) Wild BlACK Cherry. Large tree. Flowers in racemes, terminating leafy branches. Leaves thick, oval to oval-lanceolate. Drupe dark purple or black, about $\frac{1}{3}$ inch in diameter, somewhat astringent, but sweetish and pleasant. In woods.

\section{SPIRł̀A. Meadowsweet.}

(Gr., speirao, to twist; from the spiral pods of some species.)

Shrubs or perennial herbs, with simple, pinnatifid, or pinnate leaves and white or rose-colored flowers in corymbs and panicles. Calyx 5-cleft, short, and campanulate. Petals 5, inserted on the calyx. Stamens 10-60. Pods 5-8, not inflated, few to several-seeded.

I. Spiræa corymbòsa, Raf. (Gr., korymbos, a cluster.) CORymbed SPIRÆA. Shrub, I to 3 feet high. Leaves oval to orbicular, unequally and coarsely serrate from some distance above the base, thick. Flowers in terminal corymbs, white, about $\frac{1}{6}$ inch broad. Pods glabrous. Mountains and rocky places.

2. Spiræa salicifòlia, L. (L., salix, willow; folium, leaf.) WILLOW-LEAVED or Common Meadowsweet. An erect shrub, 2 to 4 feet high. Leaves oval, obovate, or oblanceolate, sharply serrate above the middle; nearly glabrous throughout. Flowers in dense terminal panicles. Flowers white or tinged with pink, about $\frac{1}{6}$ to $\frac{1}{4}$ inch broad. In swamps or moist grounds.

3. Spiræa lobàta, Jacq. (Gr., lobos, a lobe.) Queen of THE PRAIRIE. Perennial herb, 2 to 8 feet tall. Leaves interruptedly $3-7$-foliate; leaflets $3-5$-lobed or parted and unequally serrate or incised; terminal leaflet 7-9-parted; the lower leaves sometimes 3 feet long. Stipules persistent and serrate. Flowers pink or purple, fragrant, borne in a panicle on a long, naked peduncle. Pods 5-8 I-2-seeded. Moist ground and prairies.

4. Spiræa Arúncus, L. (L., aruncus, beard of a goat.) GoAT's BEARD. Smooth, tall, perennial herb with 2-3-pinnate, large leaves on long petioles; leaflets ovate to lanceolate, sharply doubly serrate. Flowers small, whitish, and diœcious in panicled, slender spikes. Pods $3-5$, several-seeded, pedicels reflexed in fruit. In rich woods.

\section{PHYSOCÁRPUS. Nine-bark.}

$$
\text { (Gr., physa, a bladder; karpos, fruit.) }
$$

Branching shrubs with palmately lobed leaves, and white flowers in umbel-like corymbs. Carpels I-5, inflated; stamens 30-40. In other respects like Spiraa.

I. Physocarpus opulifòlius, Maxim. (L., opulus, a kind of maple; folium, leaf.) NiNE-BARK. Shrub, 3 to to feet high with recurved branches, the bark peeling off in thin strips. Leaves petioled, ovate-orbicular, 3 -lobed, serrate, I to 2 inches long, or longer on young shoots. Corymbs terminal, peduncled, nearly 


\section{Dicotyledones.}

spherical, many-flowered, 1 to 2 inches across. Flowers white or purplish. Pods purplish and conspicuous. River banks and rocky places.

\section{RÙBUS. Bramble. Raspberry. Blackberry.}

(The Roman name, allied to L., ruber, red.)

Herbs, shrubs, or trailing vines. Calyx without bractlets, 5-parted. Petals 5 ; stamens numerous. Carpels several, seldom few, on a convex or elongate receptacle, ripening into drupelets and forming an aggregate fruit. Flowers usually white, sometimes pink or purple, and fruit edible.

I. Rubus strigòsus, Michx. (L., strigosus, lean or thin.) WILD RED RASPBERRY. Biennial shrubby stems, 3 to 6 feet high, densely covered with weak, glandular bristles, or hooked prickles on the older stems. Leaves 3-5-foliate, leaflets ovate or ovate-oblong. Inflorescence both terminal and axillary. Flowers white, $\frac{1}{3}$ to $\frac{1}{2}$ inch broad; petals and sepals about equal, both spreading. Fruit light red, elongate-hemispheric. Hills and thickets.

2. Rubus occidentàlis, L. (L., occidentalis, western.) BLACK RASPBERRY. THImblebERRY. Stems canelike and recurved, sometimes as much as 12 feet long, decidedly glaucous, and sparingly beset with small, hooked prickles. Leaves mostly 3 -foliate, serrate, and somewhat incised, white-pubescent beneath. Inflorescence usually terminal, compact-corymbose. Fruit purple black, hemispheric.

3. Rubus triflòrus, Richardson. (L., tri, three; flos, floris, flower.) DwARF RASPBERRY. Stems 6 to 18 inches long, trailing or ascending, somewhat pubescent, and without prickles. Leaves pedately or pinnately 3 -foliate, sometimes 5 -foliate. Flowers I-3 on slender, glandular-pubescent peduncles; sepals reflexed. Fruit red purple. Swamps or wooded hillsides.

4. Rubus híspidus, L. (L., hispidus, bristly.) RunNing Swamp BLaCKBERRY. Stems slender and creeping, slightly woody, beset with weak bristles; erect or ascending branches, 4 to 12 inches long, with few or no prickles. Leaves of 3 , rarely 5 obovate, obtuse, unevenly serrate leaflets. Flowers racemose and axillary or terminal, $\frac{1}{2}$ to $\frac{2}{3}$ inch in diameter. Fruit composed of a few drupelets, small, black, and sour, remaining on the receptacle. In low woods or swamps.

5. Rubus triviàlis, Michx. (L., trivialis, common.) Low-BUSH BlACKBERRY. Stems several feet long, trailing or procumbent, bristly and prickly. Leaves mostly 3 -foliate, coriacious and evergreen, nearly or quite glabrous. Peduncles prickly, I-3-flowered. Flowers about I inch broad. Sepals reflexed, much shorter than the petals. Fruit black, sometimes I inch long, pleasant, remaining on the receptacle. In sandy soil.

6. Rubus Canadénsis, L. Low-RUNNING BLACKBERRY or DEWBERRY. Stems shrubby, becoming several feet long, and trailing, naked or with scattered prickles. Leaves $3-7$-foliate, leaflets ovate to ovate-lanceolate. Flowers few, terminal, and racemose or solitary; peduncles leafy. Fruit delicious, sometimes I inch long, remaining on the receptacle. In dry soil. 


\section{FRAGÀRIA. Strawberry.}

(L., fraga, strawberry.)

Acaulescent, perennial herbs, propagating by runners. Leaves 3-foliate, basal, and tufted, on long petioles with a sheathing membranous stipule. Flowers on erect, naked scapes, corymbose or racemose, polygamo-diœcious. Sepals 5-bracteolate, persistent, deeply 5-lobed. Petals 5, obovate, clawed, white. Stamens numerous. Carpels numerous, on an elongated receptacle which becomes fleshy and edible in fruit; carpels becoming dry achenes.

I. Fragaria Virginiàna, Duchesne. Virginia or Scarlet Strawberry. Leaflets thick, broadly oval or obovate; petioles 2 to 6 inches long; inclined to be villous-pubescent with spreading or appressed hairs. Fruit ovoid, red, the achenes imbedded in pits. Scape shorter than the leaves. In fields or woodlands.

2. Fragaria vésca, L. (L., vescus, small or thin.) European WOOD STRAWBERRY. Leaflets thick, broadly oval or ovate, usually not so villous as the preceding. Scapes longer than the leaves, and the fruit lifted above them. Fruit hemispheric or conic, red, achenes not imbedded in pits. Fields and rocky places.

\section{POTENTílla. Cinquefoil or Five-finger.}

(L., potens, powerful, from reputed medicinal value of one of the species.)

Herbs, rarely shrubs. Leaves digitately or pinnately compound, alternate, stipulate. Flowers perfect, cymose or solitary. Calyx usually 5-lobed, subtended by as many bractlets. Petals mostly 5, often emarginate, yellow, white, or purple. Stamens usually many, sometimes 5-Io. Carpels numerous, on a dry receptacle which is often hairy.

I. Potentilla argùta, Pursh. (L., argutus, sharp, pungent.) TALL or GlandULAR CINQueforl. Flowers white, cymose. Stout and erect, I to 4 feet high. Basal leaves with 7-II leaflets, long-petioled. Stem leaves shorter with fewer leaflets. Leaflets cut-serrate. Flowers white, about $\frac{1}{2}$ inch broad, in terminal cymes. Plant glandular-pubescent.

2. Potentilla argéntea, L. (L., argenteus, silvery.) SILvery or HOARY CINQUEFOIL. Flowers yellow, cymose. Stems ascending, tufted, 4 to 12 inches long, white from woolly pubescence. Leaves digitately 5 -foliate, the divisions lanciniate beyond the middle, green above, white beneath. In dry soil.

3. Potentilla Norvégica, L. Rough Cinqueforl. Flowers yellow in terminal cymes. Erect and stout annuals or biennials with rough pubescence, 6 inches to 2 feet or more high. Leaves 3 -foliate, the lower petioled, upper stem leaves nearly or quite sessile. Leaflets obovate to oblong-lanceolate. Styles glandularthickened at the base. In dry soil.

4. Potentilla leucocàrpa, Rydberg. (Gr., leukos, white; karpos, fruit.) DIF- 
FUSE CINQUEFOIL. Flowers yellow in loose, leafy cymes. Diffuse, rather weak annual, 6 inches to 3 feet high. Leaves, all but the uppermost, 3 -foliate and petioled. Leaflets thin, oblong, incisely serrate. Styles thickened below. In damp soil.

5. Potentilla Anserina, L. (L., anserinus, pertaining to geese.) SILverWEED. Flowers yellow, solitary and axillary. Herbaceous and tufted, spreading by slender runners. Leaves pinnate; leaflets 7-25, oblong to obovate, serrate, white-pubescent beneath. Style filiform. River banks, lake borders, etc.

6. Potentilla Canadénsis, L. Common Cinquefoll or Five-Finger. Flowers yellow, solitary and axillary. Stems tufted, and spreading by slender runners. Leaves digitately 5 -foliate, sometimes $3-4$-foliate, petioled. In dry soil.

\section{WALDSTEINIA.}

\section{(Named for Francis von Waldstein.)}

Perennial herbs resembling the strawberry, but with yellow flowers and 2-6 carpels inserted on a short receptacle. Flowers corymbose on bracted scapes. Petals conspicuous and stamens numerous.

I. Waldsteinia fragarioides, Tratt. (L., fraga, strawberries; Gr., eidos, resemblance.) BARREN OR DRY STRAWBerRy. Leaves on long petioles, tufted, mostly 3 -foliate; leaflets obovate-cuneate, dentate, crenate, or incised. Scapes corymbosely 3-8-flowered; pedicels slender and sometimes drooping. Wooded hillsides.

\section{GËUM. Avens.}

(Ancient Latin name.)

Perennial herbs, with pinnatifid or odd pinnate, stipulate leaves; basal leaves clustered, stem leaves smaller. Calyx somewhat campanulate, 5 -lobed, usually with 5 bractlets at the sinuses. Petals 5 , exceeding the calyx. Stamens many, inserted on the disk below the calyx. Carpels many, on an elevated, dry receptacle. Styles persisting in the form of hairy, naked, or jointed tails to the achenes.

I. Geum rivàle, L. (L., rivalis, belonging to a brook.) PURPLE or WATER Avens. Flowers purple and nodding, calyx lobes erect or spreading. Erect, I to 3 feet high, pubescent. Basal leaves lyrately, interruptedly pinnate; stem leaves 3-lobed or 3-pinnate. Achenes very pubescent; style jointed, and plumose below. In wet meadows and swamps.

2. Geum ciliàtum, Pursh. (L., cilium, an eyelash.) LONG-PLUmed PURPLE AvENS. Flowers light purple; styles very long and plumose throughout. Scapose, pubescent herbs, 6 to 18 inches tall. Scapes 3-8-flowered. Basal leaves tufted, pinnate, leaflets very numerous and cut-toothed. In rocky soil.

3. Geum álbum, Gmel. (L., albus, white.) White Avens. Flowers white, less or more than $\frac{1}{2}$ inch broad. Plants softly pubescent or nearly glabrous, $I^{\frac{1}{2}}$ to 
$2 \frac{1}{2}$ feet high. Basal leaves 3 -foliate or pinnately divided, the terminal lobe larger and broadly ovate. Stem leaves 3-5-lobed or divided, nearly or quite sessile. Receptacle densely bristly, and styles glabrous, or pubescent below. In shady places.

4. Geum Virginiànum, L. RoUGH Avens. Resembling the preceding species, but stouter and bristly pubescent. Flowers creamy white. Receptacle glabrous or merely downy. Low grounds and borders of woods.

5. Geum macrophýllum, Willd. (Gr., makros, large; phyllon, leaf.) LARGELEAVED Avens. Flowers yellow. Basal leaves lyrate-pinnate, the terminal lobe much exceeding the others. Lateral lobes $3-6$, with smaller lobes interspersed. Upper leaves of 2-4 leaflets. Receptacle glabrous; style slender and jointed, pubescent below. Stems erect and bristly-pubescent.

\section{RòsA. Rose.}

\section{(L., rosa, rose.)}

Erect or climbing, generally prickly, shrubs. Flowers showy, red, pink, or white, rarely yellow. Lobes of the calyx usually 5 ; petals 5 ; stamens many, all borne around the margin of an urn-shaped receptacle, in which are inclosed numerous carpels, arising from the base. Fruit berrylike, consisting of the thickened, hollow receptacle and inclosed carpels.

I. Rosa setígera, Michx. (L., seta, bristle; gerere, to bear.) ClimBING or PRAIRIE ROSE. Stems climbing, becoming several feet long, beset with stout, scattered prickles. Leaflets commonly 3, sometimes 5. Stipules very narrow. Styles cohering in a column. Fruit globular and somewhat glandular. Prairies and thickets.

2. Rosa blánda, Ait. (L., blandus, of a smooth tongue, agreeable.) SмоoтH or MEADOW RosE. Erect, 2 to 4 feet tall, almost destitute of prickles. Leaflets 5 7; stipules rather broad. Styles separate, fruit globose or pyriform, nearly or quite glabrous, tipped by the persistent, long, erect, or spreading sepals. In moist and rocky places.

3. Rosa Arkansàna, Porter. ARkansas Rose. Erect, I to 2 feet high, the stems beset with slender bristles. Leaflets 7-II. Lanceolate sepals persistent, spreading, or reflexed. Fruit globose and glabrous. Prairies.

4. Rosa Woodsii, Lindl. (Latin genitive of proper name.) Woods' RosE. I to 3 feet high. Straight spines on the stems, at least below. Leaflets $5-9$. Acuminate, lanceolate sepals erect on the globose fruit. Usually with spines just below the stipules. Prairies.

5. Rosa hùmilis, Marsh. (L., humilis, low.) Low or PASTURE Rose. From $\frac{1}{2}$ to 6 feet high, bushy. Leaflets mostly 5 , sometimes 7 ; straight spines below the stipules. Flowers solitary or few together. Sepals deciduous, spreading, commonly lobed. In dry and rocky soil. 


\title{
Dicotyledones.
}

\section{PỲRUS. Pear and Apple.}

\author{
(L., pirus, a pear tree.)
}

Trees or shrubs, with conspicuous flowers in corymbed cymes. Receptacle (in this instance commonly called the calyx tube) urnshaped, fleshy, and adherent to the carpels. Sepals, or lobes of the calyx, 5 ; petals 5 ; stamens numerous. Styles $2-5$; carpels $2-5$, their walls of cartilaginous texture, ovules 2 in each cavity. Fruit, a pome or berrylike.

r. Pyrus commùris, L. (L., communis, common.) Common PeAR. Bark smooth, branches apt to have somewhat thorny spurs. Leaves ovate with small teeth. Flowers pure white. Fruit tapering toward the base; flesh containing grit cells. Native of Europe and Asia.

2. Pyrus Màlus, L. (L., malum, an apple.) Common APple. Trees with spreading branches. Leaves broadly ovate or oval, rounded, or subcordate at the base. Flowers pink or white; calyx tomentose. Fruit $I_{2}^{\frac{1}{2}}$ to 3 inches in diameter. Native of Europe and Western Asia.

3. Pyrus coronària, L. (L., coronarius, pertaining to a wreath or crown.) AmERICAN CRAB APPLE. A small tree. Leaves ovate to triangular-ovate, sharply serrate, and frequently somewhat lobed, rounded or somewhat cordate at the base. Flowers rose-colored and very fragrant. Styles woolly and united below. Fruit very acid, greenish yellow, fragrant. In thickets.

4. Pyrus angustifòlia, Ait. (L., angustus, narrow ; folium, leaf.) NARRowLEAVED CRAB APPLE. A small tree. Leaves oval to oblong-lanceolate, commonly ovate-lanceolate and narrowed at the base, dentate or entire. Flowers pink and fragrant. In thickets.

5. Pyrus Ioénsis, Bailey. (Latinized form, meaning pertaining to Iowa.) Western Crab APPLE. Resembling Pyrus coronaria, but the leaves are whitepubescent on the lower surface, oval or ovate, usually narrowed at the base. Fruit, dull green with small light dots. In thickets.

6. Pyrus Japónica, Thunb. (Latinized form, signifying relating to Japan.) JAPAN QUINCE. A cultivated, thorny, and much-branched shrub from Japan. Flowers scarlet red, produced in great abundance before the leaves. Leaves oval or wedge-oblong. Fruit hard and green; speckled.

\section{AMELÁNCHIER. Juneberry. Service Berry. Shad Bush.}

(Savoy name of the medlar.)

Shrubs or small trees, with solitary or racemose white flowers and simple, petioled, serrate leaves. Calyx campanulate and more or less adnate to the ovary, with 5 narrow, reflexed, persistent lobes. Styles 2-5, cavities of the ovary becoming twice as many, with I ovule in each cavity. Pome small and berrylike, 4-ro-celled. 
I. Amelanchier Canadénsis, T. \& G. Shad Bush or Service Berry. A tree, seldom more than 25 feet high, with ovate or ovate-lanceolate leaves, acute or acuminate at the apex, and rounded or cordate at the base. Flowers in spreading or drooping racemes, pedicels long and slender. Bracts and stipules long, silkyciliate. The sweet pome globose, red or purple. In dry woodlands.

2. Amelanchier rotundifolia, Rœm. (L., rotundus, round; folium, leaf.) ROUND-LEAVED JUNEBERRY. Similar to the above, but with leaves ovate to orbicular, and more or less rounded at both ends. In woods and thickets.

3. Amelanchier alnif́lia, Nutt. (L., alnus, alder; folium, leaf.) NORTHWESTERN JUNE or SERvice BERry. Shrub 3 to 8 feet high. Leaves elliptic to orbicular, serrate above the middle. Flowers in short, dense racemes. A bloom on the purple, globose pome. In dry soil.

\section{CRATAÈGUS. Hawthorn or White Thorn.}

(Gr. kratos, strength, referring to the toughness of the wood.)

Thorny shrubs or small trees. White or pink flowers in terminal, corymbose clusters. Leaves simple and often lobed. Receptacle (socalled calyx tube) cup-shaped, adherent to the I-5 carpels. Sepals or calyx lobes 5 ; petals 5 ; stamens numerous. Pome small and drupelike with I-5 I-seeded stones.

I. Cratægus Crús-gálli, L. (L., crus, leg; galli, genitive of gallus, a cock.) COKSPUR THORN. Shrub or small tree, with obovate or oblanceolate, serrate leaves, glabrous, shining above and dull beneath. Stems with slender thorns, which are 2 to 4 inches long. Fruit globular and red. In thickets.

2. Cratægus coccínea, L. (L., coccineus, of a scarlet color.) SCARLET THORN or HAW or RED HAW. Shrub or small tree, with stout spines $I \frac{1}{2}$ to 2 inches long. Leaves broadly ovate or orbicular, truncate or subcordate at the base, sharply incised and serrate, with glandular-tipped teeth. Glandular pubescence on the calyx and pedicels. Red, globular fruit about $\frac{1}{2}$ inch in diameter, sometimes more. In thickets.

3. Cratægus móllis, Scheele. (L., mollis, soft.) RED-FRUITED THORN or HAw. Similar to Cratagus coccinea, but with leaves sometimes 5 inches long, usually very pubescent beneath, and hairy fruit, sometimes I inch in diameter. In thickets.

4. Cratægus flàva, Ait. (L., favis, golden yellow.) SUMmer or Yellow HAW. Small and often quite thorny tree. Leaves obovate, often obtuse and glandular-dentate at the apex, narrowed at the base, at first pubescent on both sides. Fruit globose to pyriform, yellow, red, or greenish. In sandy thickets.

5. Cratægus Oxyacántha, L. ENGLISH HaWTHORN. Shrubs or trees, with stout and frequent thorns. Leaves generally broadly ovate or obovate, sharply 3-7-lobed, broadly cuneate at the base, I to 2 inches long. Flowers sometimes more than $I$ to 2 inches in diameter, white or pink. Fruit, deep red, globose or ovoid. Roadsides and thickets. 


\section{LEGUMINÒS E. PUlse FAMily.}

Herbs, trees, or shrubs, with papilionaceous or more or less irregular flowers, and alternate, stipulate, pinnately or palmately compound leaves. Stamens usually Io, monadelphous, diadelphous, or sometimes distinct. Ovary superior, of a single carpel, and becoming a legume in fruit.

Herbs.

Stamens distinct.

BAptisia I.

Stamens diadelphous, or sometimes all united near the base.

Leaves palmately 3 -foliate.

Leaves odd-pinnate.

Leaves abruptly pinnate.

Trifolium II.

Astragalus Vi.

Vicia VII.

Herbs or sometimes shrubs.

Stamens distinct or united only at the base.

Leaves bipinnately compound; stamens 8-r2.

Leaves bipinnately compound; stamens ro-5.

Stamens monadelphous or diadelphous.

Schrankia XI.

Desmanthus XII.

Psoralea III.

Trees or shrubs.

Stamens distinct or united only at the base.

Leaves once or twice pinnately compound.

Trees without thorns.

Trees with conspicuous thorns.

Leaves simple.

Stamens monadelphous; leaves odd-pinnate.

Stamens diadelphous; leaves odd-pinnate.

Gymnocladus IX.

Gleditschia X.

Cercis VIII.

Amorpha IV.

Robinia V.

\section{BAPTísIA. False Indigo.}

(Gr., baptisis, dipping or dyeing.)

Erect, perennial herbs, with palmately 3 -foliate or rarely simple leaves. Flowers yellow, white, or blue, truly papilionaceous, borne in racemes. Stamens 10 and distinct; ovary stipitate, and pod inflated. Standard about equaling the wings and keel, its sides reflexed.

I. Baptisia tinctòria, R. Br. (L., tinctorius, pertaining to dyeing.) WILD INDIGo. Erect, glabrous, 2 to 4 feet high. Leaves 3 -foliate, the leaflets obovate or oblanceolate, petioles short. Flowers yellow. Pods raised on a stipe longer than the calyx, and tipped with the awl-shaped style. In dry soil.

2. Baptisia leucophaèa, Nutt. (Gr., leukos, white; phaios, gray.) LARGEBRACTED WILD INDIGO. About I foot high, with divergent branches, pubescent throughout. Leaves 3 -foliate, sessile, or short-petioled; leaflets spatulate or oblanceolate, with ovate or lanceolate, persistent stipules. Flowers white or cream color, about I inch long, borne in a many-flowered raceme, which sometimes becomes I foot long. Pods hoary, pointed at both ends. On prairies. 


\section{TRIFÒLIUM. Clover or Trefoil.}

(L., tri, three; folium, leaf.)

Herbs, with mostly slender branches and 3 -foliate leaves, the leaflets denticulate. Flowers pink, purple, white, or yellow, papilionaceous. Stamens I0, diadelphous, or sometimes all united near the base. Flowers in heads or spikes, and pods straight and membranaceous.

I. Trifolium praténse, L. (L., pratensis, growing in a meadow.) RED or MEADOW Clover. Flowers red purple, sessile, in globose or ovoid heads, the heads nearly or quite sessile. Somewhat pubescent, branching, perennial herbs. Leaflets short-stalked from the same point, often dark-spotted near the middle. In fields and meadows.

2. Trifolium rèpens, L. (L., repens, creeping.) White Clover. Creeping, mostly glabrous perennials, rooting at the nodes. Leaves rising on long petioles; leaflets obovate, emarginate-denticulate. Flowers white, in globose heads borne on long peduncles; flowers of the head raised on pedicels. In fields and open places.

\section{PSORÀLEA.}

(Gr., psoraleos, scurfy, alluding to glandular dots of the leaves.)

Herbs or shrubs, with $1-5$-foliate, glandular-dotted leaves. Flowers bluish purple or white, borne mostly in spikes or racemes. Calyx 5-cleft, the lower lobe longest. Stamens diadelphous, sometimes monadelphous. Ovary I-ovuled; the short pod ovoid and indehiscent.

I. Psoralea tenuiflòra, Pursh. (L., tenuis, slender, small; flos, floris, flower.) FEW-Flowered Psoralea. Erect and slender, 2 to 4 feet high, hoary with an appressed pubescence. Leaves short-petioled, digitately $3-5$-foliate, mostly oblongoval or elliptic or obovate, sometimes mucronate at the apex. Peduncles slender and much exceeding the leaves, loosely 6-14-flowered. Corolla purplish; corolla about twice the length of the calyx. Prairies.

2. Psoralea argophýlla, Pursh. (Gr., argos, white; phyllon, leaf.) SilverLEAF PSORALEA. Silvery pubescent with white appressed hairs. I to 3 feet tall. Petioles about equaling the leaves. Leaflets $3-5$, digitate, oblong-oval to oblongobovate. Inflorescence interrupted-spicate, peduncles much exceeding the leaves. Flowers deep blue or purplish, about $\frac{1}{8}$ inch long. Calyx lobes lanceolate, very densely pubescent. Pod ovate, with a straight beak. On prairies.

3. Psoralea floribúnda, Nutt. (L., flos, floris, flower + adjectival ending, signifying plenty.) MANY-FLowered PSORALEA. Stems much branched, I to 4 feet high, hoary, not glandular. Leaves mostly $3-5$-foliate, sometimes 7 -foliate. Leaflets oblong, canescent beneath and glandular on both surfaces. Peduncles 2 to 7 inches long, usually many-flowered. Lobes of the calyx triangular-acute. Prairies.

4. Psoralea esculénta, Pursh. (L., esculentus, edible.) Pomme Blanche. 


\section{Dicotyledones.}

PRAIRIE APPLE or TURNIP. 4 to 18 inches high from a tuberous root. Densely hairy all over. Leaves digitately 5 -foliate. Leaflets mostly obovate or obovateoblong. Peduncles often longer than the petioles, bearing dense, oblong, spikelike racemes. Calyx nearly equaling the bluish corolla. Prairies.

\section{AMÓRPHA. False Indigo.}

(Gr., amorphos, deformed, from the absence of 4 petals.)

Shrubs, with odd-pinnate, glandular-punctate leaves. Flowers mostly violet or purple. Corolla consisting of the standard alone, the wings and keel wanting. Stamens Io, monadelphous below, anthers all alike. Pod I-2-seeded.

I. Amorpha fruticòsa, L. (L., fruticosus, shrubby.) FALSE INDIGo. A rather tall shrub. Leaflets II-2I, elliptic to oblong. Flowers dense in solitary or clustered spicate racemes. Standard violet-purple. River banks and hillsides.

2. Amorpha microphýlla, Pursh. (Gr., mikros, small; phyllon, leaf.) FrAGRANT FALSE INDIGO. A bushy shrub, scarcely exceeding I foot in height, nearly glabrous. Standard purplish. Flowers fragrant; racemes mostly solitary. Prairies.

\section{ROBÍNIA. Locust Tree.}

(Named for John Robin, herbalist to Henry IV. of France.)

Trees or shrubs, with odd-pinnate leaves and racemes of showy flowers. Stipules often spiny. Calyx 5 -toothed, the 2 upper teeth somewhat united. Standard broad and reflexed. Stamens diadelphous. Ovary several-ovuled. Pods linear and flat, becoming margined on the seed-bearing edge.

I. Robinia Pseudacàcia, L. (Gr.,pseudes, false + acacia.) COMmon Locust TREE or FALSE ACACIA. Becoming a large tree. Leaflets 9-19; stalked, ovate or oval. Stipules often spiny. Flowers white and fragrant in loose, drooping racemes. Twigs, petioles, and pods glabrous. Extensively planted.

\section{ASTRÁGALUS. Milk Vetch.}

(Ancient Greek name of a leguminous plant.)

Chiefly perennial herbs, with odd-pinnate leaves and flowers in racemes. Stamens diadelphous with the anthers all alike. Calyx tubular, the teeth nearly equal. Standard narrow and the keel of the corolla blunt. Pod somewhat turgid, the sutures often projecting so as to divide the cavity into two.

I. Astragalus caryocárpus, Ker. (Gr., karyon, a nut; karpos, fruit.) Ground PLUM. Flowers violet-purple, about inch long, in short racemes. Pods globose 
or oval, sessile, short-pointed, glabrous, fleshy, 2-celled, sometimes I inch in diameter. Branches 6 to $I_{5}$ inches long, ascending or decumbent. Leaflets $I_{5-25}$. mostly oblong-elliptic. Peduncles equaling or shorter than the leaves. Plant covered with a pale, minute, appressed pubescence. Prairies.

2. Astragalus Mexicànus, A. DC. LARger Ground Plum. Corolla cream color, purplish at the tip, about I inch long. Globose, glabrous pods sometimes exceeding I inch in length, pointless, 2-celled. Pubescence somewhat spreading. Leaflets I7-33, oblong to obovate. Prairies.

3. Astragalus gracilis, Nutt. (L., gracilis, slender.) Slender MiLK VetCH. Flowers purple, $\frac{1}{4}$ inch or less in length, in slender, spikelike racemes. Pendent pods I-celled, concave on the back, at first hoary, but becoming glabrous. Plants erect and slender, I to 2 feet high, finely pubescent. Leaflets II-2I, distant and narrowly linear. Prairies.

\section{VícIA. Vetch or Tare.}

(The classical Latin name.)

Herbaceous vines, climbing or trailing. Leaves evenly pinnate and tendril-bearing. Stipules mostly half-sagittate. Calyx teeth 5, about equal, or the 2 upper shorter. Standard obovate or oblong, emarginate; wings adhering to the middle of the keel. Stamens more or less diadelphous, 9 and I. Style slender, hairy at the summit.

I. Vicia Americàna, Muhl. American Vetch or PEA Vine. Flowers bluish purple, somewhat less than I inch long, $3-9$ in a loose-flowered raceme. Mostly glabrous perennial, 2 to 3 feet long. Leaflets $8-\mathbf{I} 4$, elliptic or ovate-oblong, conspicuously veined. Pod glabrous, 4-7-seeded. In moist soil.

2. Vicia lineàris, Green. (L., linearis, linear.) Flowers and fruit as in Vicia Americana, but the leaflets are narrowly linear, and the branches are weak and often zigzag. In dry soil.

\section{CÉRCIS. Redbud or Judas Tree.}

(Ancient name of the Oriental Judas tree.)

Shrubs or small trees, with red purple flowers in umbellike clusters along last year's twigs or older branches, appearing just before the leaves. Leaves simple, broad, and heart-shaped. Standard in the bud inclosed by the wings. Stamens 10 and distinct. Pods oblong and flat.

I. Cercis Canadénsis, L. REDBUD. A small tree, often planted for ornamental purposes. In rich soil. 


\section{GYMNócLADUS. Kentucky Coffee Tree.}

(Gr., gymnos, naked ; klados, branch, alluding to the somewhat naked branches.)

Trees with bipinnate leaves and regular, diœcious, or polygamous, whitish flowers in terminal racemes. Calyx tubular, 5-lobed; petals usually 5, oblong or oval, inserted at the summit of the calyx tube; stamens Io, distinct, shorter than the petals. Pod oblong, 2-valved, thick and leathery, pulpy between the seeds.

I. Gymnocladus Canadénsis, Lam. Ken'rucky Coffee Tree. Becoming a large tree with rough bark. The bipinnate leaves quite large, of $7-15$ leaflets, the leaflets mostly ovate, acuminate, I to 3 inches long. Racemes many-flowered. Pods 5 to Io inches long. Rich woods.

\section{GLEDÍTSCHIA. Honey Locust.}

(Named for J. G. Gleditsch, botanist.)

Becoming large, thorny trees. Leaves evenly once or twice pinnate. Flowers small, greenish, in slender, axillary racemes, polygamous. Calyx campanulate, 3-5-cleft; petals 3-5, inserted on the summit of the calyx tube; stamens 6-10, distinct. Pod flat, I-many-seeded.

I. Gleditschia triacánthos, L. (Gr., tri, three; akantha, thorn.) THREeTHORNED ACACIA or HONEY LOCUST. Stems often armed with numerous stout, simple, or branching thorns. Leaflets oval to oblong-lanceolate, about $\mathrm{x}$ inch long. Pod I foot or more long, shining and twisted, many-seeded; a sweet pulp between the seeds. Woods and pastures.

\section{SCHRÁNKIA. Sensitive Brier.}

(Named for F. P. Schrank, botanist.)

Perennial herbs or shrubs, prostrate or procumbent, with bipinnate leaves, which are somewhat sensitive, the leaflets small and numerous. Flowers perfect or polygamous, borne in axillary, peduncled heads or spikes. Calyx 5-toothed, minute; petals united to the middle, 5-cleft above; corolla pink or purple. Stamens 8-12, distinct or united at the base. Plant beset with recurved prickles. Pods spiny, linear, acute, or acuminate.

I. Schrankia uncinàta, Willd. (L., uncinatus, barbed.) SENSITIve BRIER. Herbaceous perennial, branches decumbent, 2 to 4 feet long, very prickly. Stems grooved and angled. Pinnæ $4^{-8}$ pairs. Leaflets obliquely elliptic, 8-15 pairs, prominent elevated veins beneath. Heads very dense, globose. Flowers pink. In dry soil. 


\section{DESMÁNTHUS.}

(Gr., desma, a band; anthos, flower.)

Perennial herbs or shrubs, with bipinnate leaves and small, regular, greenish, or whitish flowers in peduncled, axillary heads or spikes, perfect or polygamous. Calyx campanulate, 5-toothed. Petals 5 and distinct or slightly coherent below. Stamens 10 or 5 , distinct, exserted. Pod flat, several-seeded.

r. Desmanthus brachýlobus, Benth. (Gr., brachys, short; lobos, lobe.) Stems I to 3 feet high, ascending or erect, nearly or quite glabrous. Pinnæ 6-15 pairs. Leaflets $20-30$ pairs. Stamens 5. Pods curved, oblong, or lanceolate, in globose heads. Prairies and river banks.

\section{GERANIÀCE Æ. Geranium FAMily.}

Chiefly herbs, with perfect and mostly symmetrical flowers. Parts of the flower usually in 5's. Stamens commonly as many or twice as many as the sepals, often 5 long and 5 short. Ovary 5 -lobed and 5-celled. Axis of the dry fruit persisting.

\section{GERÀNIUMM. Cranesbill.}

(Gr., geranos, a crane, from fancied resemblance of the long carpels to a beak of the crane.)

Herbs with palmately lobed, parted, or divided leaves, and flowers on axillary I-few-flowered peduncles. Stamens Io, 5 long and 5 short. Sepals and petals 5 , imbricated in the bud. Ovary 5 -lobed and 5-celled, beaked by the compound style; ovules 2 in each cavity. Carpels breaking away from the central axis in dehiscence.

I. Geranium maculàtum, L. (L., maculatus, spotted.) WILD or SPOTTED Cranesbill. Perennials with a thick rootstock. I to 2 feet high, branching above; pubescent, with more or less spreading hairs. Basal leaves long-petioled, deeply 3-5-parted. Stem leaves shorter, but similar. Petals $\frac{1}{2}$ inch long, light purple, bearded on the claw. Sepals hairy and awn-pointed. Carpels pubescent. Woods.

2. Geranium Caroliniànum, L. CARolina Cranesbill. Annuals, 6 to I5 inches high, branched from the base, diffuse, loosely pubescent. Leaves 5-9parted, the divisions cleft into somewhat linear lobes. Flowers whitish or pale rose, in compact clusters; peduncles and pedicels short and hairy. Beak of the hispid-pubescent ovary nearly I inch long. In barren soil and waste places. 


\section{Dicotyledones.}

\section{II. óxALIS. Wood Sorrel.}

(Gr., oxys, sour, relating to the sour juice.)

Herbs with sour juice; leaves radical or alternate, mostly of 3 obcordate leaflets. Sepals 5 and persistent; petals 5 , somewhat united at the base. Stamens I0, alternately longer and shorter, mostly monadelphous at the base. Ovary 5-lobed and 5-celled, the carpels dehiscing on the back. Styles 5 and distinct. Seeds 2 or more in each cell. Flowers often dimorphous or trimorphous (see Botany, page 176).

I. Oxalis violàcea, L. (L., violaceus, violet-colored.) VIOLET WOoD SoRREL. Perennial, acaulescent herbs from a scaly bulb, 4 to 9 inches high. Scapes umbellately several-flowered, mostly exceeding the leaves. Petals rose-violet, rarely white. Open woods or rocky places.

2. Oxalis strícta, L. (L., strictus, close, tight.) UPRIGHT YELLOW WOOD SoRREL. Annual or perennial. Commonly branching at the base, the branches spreading, about 6 inches long. Stems leafy to the top; flowers yellow. In woods and fields.

\section{RUTÀCE⿸广. RUE FAMILY.}

Mostly trees or shrubs. Leaves usually compound. Flowers in our species diœcious or polygamous. Sepals and petals 3-5; stamens as many or twice as many as the sepals. Pistils $2-5$, distinct, or as many carpels united to form a compound ovary. Fruit usually a capsule. Plants secrete a pungent and acrid volatile oil; the foliage dotted with pellucid glands.

\section{XANThÓXYLUM. Prickly Ash.}

(Gr., xanthos, yellow; xylon, wood.)

Trees or shrubs. Leaves alternate, odd-pinnately compound. The small whitish or greenish flowers diœcious, cymose, axillary, or terminal. Sepals and petals 4-5. Stamens 4 or 5. Pistils 2-5, united only by their styles. Stems, and often the petioles, prickly. Pods fleshy, 2-valved, I-2-seeded.

I. Xanthoxylum Americànum, Mill. Prickly AsH or Toothache TreE. Shrub with yellowish green flowers appearing before the leaves. Flowers in sessile, axillary, umbellate clusters. Leaves odd-pinnate, leaflets ovate, 5-II. Bark, leaves, and pods very pungent-aromatic. In woods and thickets. 


\section{EUPHORBIÀCEE. SPURGE FAMILY.}

Herbs, sometimes shrubs or trees, with milky secretions. Flowers monœcious or diœcious. Flowers mostly apetalous, and sometimes
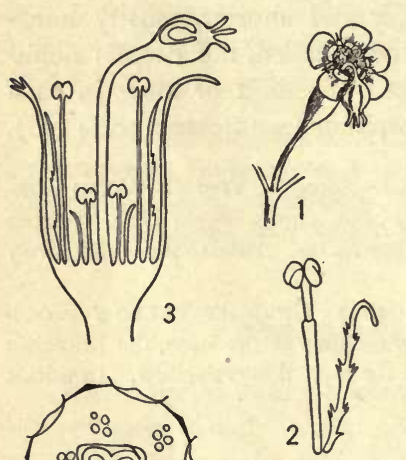
much reduced and subtended by an involucre, which resembles a calyx. Stamens few to many, filaments sometimes united. Ovary usually 3-celled, with $\mathbf{I}-2$ ovules in each cavity. Fruit usually a 3-lobed capsule, dehiscing elastically when mature. (Fig. 349.)

\section{EUPHÓRBIA. Spurge.}

(Named for Euphorbus, physician to King Juba.)

Flowers without a calyx and clustered in a cup-shaped, calyxlike involucre, the cluster easily mistaken by the beginner for a single flower. The flowers of two kinds within the involucre, many staminate flowers consisting of a single stamen, and a single pistillate flower consisting of a single 3-lobed pistil protruding above the staminate flowers. Styles 3 and stigmas 6.

I. Euphorbia sérpens, H. B. K. (L., serpens, creeping.) ROUND-LEAVED SPREADING SPURGE. Annuals, branching from the base, the branches prostrate, slender, 2 to 12 inches long. Leaves orbicular, ovate, or oval, often less than $\frac{1}{4}$ inch long, less than twice as long as broad. Stipules triangular and membranaceous. In open places.

2. Euphorbia corollàta, L. (L., corolla, a little crown.) Flowering SPURGE. Perennials from stout rootstocks, I to 3 feet tall, umbellately branched above. Leaves ovate, lanceolate, or linear, only the uppermost opposite or whorled. Involucre with showy white appendages appearing like petals. In dry soil.

3. Euphorbia marginàta, Pursh. (L., marginatus, provided with a border.) 


\section{Dicotyledones.}

White-MARGINED SPURGE. Erect annuals with stout stems from I to 3 feet high. Bracts of the involucre white-margined and petallike. The uppermost leaves opposite or whorled and with conspicuous white, petallike margins. In dry soil.

\section{ANACARDIÀCEZE. SUMAC FAMILY.}

Shrubs or small trees, with acrid, resinous, or milky secretions, mostly alternate leaves, and perfect or polygamo-diœcious flowers. Calyx 3-7-cleft, most frequently 5-cleft, and petals when present of the same number. Stamens usually as many or twice as many as the petals. Ovary I-celled and I-ovuled. Styles I-3. Fruit generally a small drupe. Sometimes poisonous.

\section{RHÚS. Sumac.}

(The old Greek and Latin name.)

Shrubs or trees, with alternate, odd-pinnate, 3-foliate, or simple leaves. Flowers mostly polygamous in axillary or terminal panicles. Calyx 5-parted, and petals 5. Stamens 5 , inserted between the lobes of a flattened disk at the base of the calyx. Fruit generally a small, dry drupe.

I. Rhus, trilobàta, Nutt. (Gr., tri, three; lobos, lobe.) ILL-SCENTEd SumaC or SKunk Bush. Shrub 2 to 6 feet high, mostly glabrous. Leaves 3 -foliate, the leaflets sessile or nearly so, $\frac{1}{2}$ to I inch long, few lobed or incised toward the summit. Flowers yellow green, in clustered spikes, appearing before the leaves. Unpleasantly scented. Rocky hillsides.

2. Rhus aromática, Ait. (Gr., aromatikos, pertaining to spice.) (R. Canadensis, Marsh.) Fragrant or SweEt-Scented SumaC. Similar to the preceding species, but the leaflets 2 to 4 inches long and pleasantly aromatic, and crenate or dentate above the middle. Rocky hillsides.

3. Rhus rádicans, L. (L., radicans, having roots.) ( $R$. Toxicodendron in Gray's "Manual.") PoISON IVy or PoISON OAK. Woody; climbing trees, etc., by means of adventitious rootlets. Sometimes shrubby and not climbing. Leaves 3 -foliate, leaflets $\mathrm{I}$ to 4 inches long, the terminal longer stalked than the lateral leaflets. Flowers green in loose, axillary panicles. In thickets and low grounds.

4. Rhus hírta, Sudw. (L., hirtus, hairy.) StaG-HoRn Sumac. Large shrub or small tree. Leaves pinnate, with II-3I leaflets. Leaves and twigs with a velvety pubescence. In dry and rocky soil.

5. Rhus glàbra, L. (L., glaber, bald.) Sмоoth or Scarlet Sumac. Shrub or small tree. Leaflets II-3I. Foliage and twigs glabrous and somewhat glaucous. In dry soil. 


\section{SAPINDÀCE F. SOAPBERRY FAMILY.}

Trees or shrubs, with simple or compound leaves. Flowers mostly unsymmetrical and often irregular. Sepals and petals 4-5. Stamens 5-10, inserted in a fleshy disk. Ovary 2-3-lobed, with as many cells. Ovules $\mathbf{I}-2$ in each cell.

\section{I. ÀCER. Maple.}

(Latin name of the maple.)

Mostly trees, with palmately lobed, opposite leaves and small, polygamo-diœcious flowers. Calyx usually 5 -lobed or parted; petals of the same number or wanting. Stamens 3-12. The 2-celled ovary with a pair of ovules in each cell. Styles 2, stigmatic along their inner surfaces. Fruit, 2 diverging, long-winged samaras, joined together at their bases.

I. Acer dasycárpum, Ehrh. (Gr., dasys, dense or thick; karpos, fruit.) SiLVER, SOFT, or WHITE MAPLE. Becoming large trees. Leaves (white and somewhat pubescent beneath) deeply 5 -lobed, 4 to 6 inches long, the lobes irregularly dentate. Flowers greenish or reddish, in dense, sessile, lateral clusters, appearing before the leaves. Petals none. Ovary woolly when young. Along streams,

2. Acer saccharìnum, Wang. (Gr., sakcharon, cane or palm sugar.) SUGAR or ROCK MAPLE. Large trees, whose sap yields most of the maple sugar of commerce. Leaves $3-7$-lobed, with rounded sinuses, pale beneath and dark green above. Flowers in lateral or terminal corymbs on long, slender, drooping, hairy pedicels, appearing with the leaves. In rich woods.

3. Acer Negúndo, L. (New Latin for a native name.) Box Elder or AsHLEAVED MAPLE. (Negundo aceroides, Mœnch, in Gray's "Manual.") Trees with pinnately $3-5$-foliate leaves. Flowers diœcious, appearing shortly before the leaves, greenish in drooping clusters. Along streams.

\section{STAPHYLÈA. Bladder Nut.}

$$
\text { (Gr., staphyle, a cluster.) }
$$

Upright shrubs, with opposite, 3 -foliate, or pinnate leaves and panicles or racemes of white flowers terminating branchlets of the current season. Lobes of the 5-parted calyx erect and whitish; petals 5, inserted on the margin of a thick disk at the base of the calyx. Stamens 5 , alternating with the petals. Carpels 3, united along their inner faces, and forming in fruit a 3 -lobed and 3-celled pod, membranous and inflated. 


\section{Dicotyledones.}

I. Staphylea trifolia, L. (L., tri, three; folium, leaf.) AMERICAN BLADDER NUT. Branching, 6 to $I_{5}$ feet high. Leaflets mostly 3 , ovate-acuminate. Flowers white, campanulate. Carpels in fruit separate at the summit and dehiscing along their inner margins. In moist woods and thickets.

\section{AÉSCULUS. Horse-chestnut or Buckeye.}

(Latin name of an oak tree.)

Trees or shrubs. Leaves opposite, palmately 3-9-foliate. Flowers in terminal panicles. Calyx unequally 5 -lobed or cleft. Petals 4-5, unequal. Stamens usually 7 , filaments slender and often unequal. Ovary 3-celled with 2 ovules in each cell. Style slender. Fruit, a leathery 3-celled and 3-seeded capsule; sometimes 2 seeds become abortive. Seeds large with thick and shining coat; scar of the seed large and pale.

I. EEsculus glàbra, Willd. (L., glaber, without hair.) FETID or OHIO BuckEYE. Trees. Leaflets $5-7$, mostly $5 ; 3$ to 6 inches long. Flowers pale yellow, in loose, pubescent panicles. Woods and river banks.

2. Fesculus argùta, Buckl. (L., argutus, fiery.) SHRUbBY or WESTERN Buckeye. Shrub, 3 to to feet high. Leaflets $7-9$, narrow, 3 to 4 inches long. Flowers yellow with reddish center, in dense panicles. Along streams and in moist woods and thickets.

\section{RHAMNÀCE ÆE. BuCKTHORN FAMILY.}

Shrubs or small trees, sometimes with thorny branches. Leaves simple and alternate. Flowers small and sometimes apetalous, often polygamous, and in some instances diœcious. The limb of the obconic or cylindric calyx tube 4-5-toothed. Petals 4-5, inserted on the calyx. Stamens of the same number as the petals and inserted with and opposite them. Petals more or less concave or hooded in the bud. Ovary 2-5-celled, with I ovule in each cavity. Stigmas 2-5. Fruit, a drupe or capsule, often 3 -celled.

\section{RHÁMNUS. Buckthorn.}

(The ancient Greek name.)

Shrubs or small trees, with flowers in axillary clusters, greenish, polygamous, and diœcious. Calyx somewhat urn-shaped, lined with a fleshy disk below, its limb 4-5-toothed. Petals 4-5, or none, emarginate and hooded, short-clawed. Ovary 3-4-celled and free from the disk. Drupe berrylike, containing 2-4 separate nutlets. 
I. Rhamnus lanceolàta, Pursh. (L., lanceolatus, armed with a little lance.) LANCE-LEAYED BUCKTHORN. A tall shrub with thornless branches. Leaves ovate-lanceolate, short-petioled, minutely serrulate. Greenish flowers in groups of 2 or 3 in the axils of the leaves. Drupe containing 2 grooved nutlets. In dry soil.

2. Rhamnus alnifolia, L'Her. (L., alnus, alder; folium, leaf.) ALDER-LEAVED BUCKTHORN or DWARF ALDER. A small shrub with thornless branches. Leaves oval to elliptic, serrated. Flowers greenish, 2 or 3 together in the axils, diœcious, without petals, appearing with the leaves. In swamps.

3. Rhamnus Caroliniàna, Walt. CAROLina BUCKThorn. Thornless shrub or small tree. Leaves broadly oblong or oblong-elliptic. Flowers several together in axillary, peduncled umbels. Petals present. Drupe globose and sweet, containing 3 seeds. In swamps and along rivers.

\section{CEANÒthuS. New Jersey Tea or Redwood.}

(Gr., keanothos, a kind of thistle.)

Shrubs, with white, blue, or yellow flowers in axillary or mainly terminal, clustered umbels. Calyx mostly hemispheric and 5-lobed. Petals 5, spreading, incurved, and clawed. Ovary adnate to the disk at the base of the calyx, 3-lobed. Style short and 3-cleft. Fruit 3lobed, and separating at maturity into 3 nutlets.

I. Ceanothus Americànus, L. NEW Jersey TEA or Redroot. Stems ascending or erect, generally several together from a deep reddish root. Leaves ovate to ovate-lanceolate, finely pubescent, particularly beneath. Flowers white. In dry, open woods.

2. Ceanothus ovàtus, Desf. (L., ovatus, egg-shaped.) SMALLER REDRoot. Similar to the preceding species, but the leaves are oblong or oval-lanceolate, and nearly glabrous. On prairies and in rocky places.

\section{VITÀCE Æ. GRAPE FAMILY.}

Woody vines trailing or climbing mostly by tendrils. Leaves mostly palmately lobed, dentate, or compound. Flowers small and greenish, polygamous or diœcious. Petals 4-5, hypogynous or perigynous, falling away without expanding. Limb of the calyx mostly obsolete or 4-5-lobed. Stamens of the same number as the petals and opposite them. The single ovary often immersed in a fleshy disk, 2-6-celled, with I-2 ovules in each cavity. Fruit, a I-6-celled, but commonly 2-celled berry. Stigma slightly 2 -lobed, on a short style or sessile. 


\section{VìTIS. Grape.}

(The Latin name.)

Plants climbing high by coiling tendrils. Leaves simple, rounded, variously sharply-incised, and lobed. Flowers polygamo-diœcious, sometimes perfect, borne in a compound thyrse. Petals falling off without expanding. Five nectariferous glands alternating with the stamens. Fruit, a pulpy, edible berry.

I. Vitis æstivàlis, Michx. (L., astivalis, pertaining to summer.) SUMmer or SMALL GRAPE. Terete branches climbing high. Leaves large, dentate, or 3-5lobed; young leaves and branches quite woolly. Tendrils and flower clusters not present opposite each third leaf. Berries sour, but edible, with a bloom. In thickets.

2. Vitis cinèrea, Englm. (L., cinereus, ashy.) Downy GraPE. Climbing. Branches angled. Leaves entire or only slightly 3-lobed, dentate; pubescence whitish or grayish, especially pronounced on the under side of leaf. Berries without bloom, black, edible, somewhat sour.

3. Vitis ripària, Michx. (L., riparius, relating to a river bank.) RIVERSIDE or SWEET-SCENTED GRAPE. Climbing or trailing. Branches only slightly angled or rounded. Plant glabrous throughout. Leaves shining, mostly sharply 3-7lobed; stipules $\frac{1}{6}$ to $\frac{1}{4}$ inch long, persisting until the fruit is formed. Berries bluish black, with a bloom, sweetish, approaching $\frac{1}{3}$ inch in diameter. Along rocky stream banks or near water.

4. Vitis cordifòlia, Michx. (L., cor, cordis, heart; folium. leaf.) FROST or Chicken Grape. Climbing. Branches round or only slightly angled, Leaves shining above, only slightly pubescent beneath, acuminate, very coarsely serrate, sometimes slightly 3 -lobed, cordate at the base; stipules small. Berries black and shining, ripening after frost. In moist thickets and along streams.

\section{AMPELÓPSIS. Virginia Creeper.}

(Gr., ampelos, a vine; opsis, appearance.)

Mostly climbing, woody vines. Leaves digitately 3-5, mostly 5foliate. Leaflets oblong-lanceolate, sparingly serrate above. Tendrils with clinging, suckerlike disks at their tips.

I. Ampelopsis quinquefolia. (L., quinque, five; folium, leaf.) VIRGINIA Creeper, FAlSE GRAPE, or AMERICAN IVy. Climbing high on trees or walls. Clinging sometimes by rootlets as well as by tendrils. Flowers in panicles, which are spreading in fruit. Berries bluish. In woods and thickets. Commonly planted for covering walls. 


\section{TILIÀCEX. LINDEN FAMILY.}

Mostly trees or shrubs. Leaves simple and generally alternate, with small, deciduous stipules. Flowers axillary or terminal, cymose or paniculate. Sepals mostly 5 , valvate. Petals of the same number as the sepals, sometimes less or wanting, mostly valvate. Stamens many, 5-1O-adelphous. Ovary 2-10-celled. Styles lobed or entire. Peduncles springing from a leaflike expansion.

\section{TÍLIA. Linden or Basswood.}

(The classical Latin name.)

Trees, with serrate, cordate, somewhat inequilateral leaves, and white or cream-colored flowers. Sepals and petals 5. Stamens many, cohering in 5 sets (5-adelphous). Ovary 5 -celled with 2 ovules in each cell. Style I ; stigma 5-toothed. Fruit, I-2-seeded, drupaceous.

I. Tilia Americàna, L. Basswoon or AMERICAN Linden. A large tree. Leaves 2 to 5 inches wide, smooth on both sides. Flowers fragrant and much visited by bees for nectar. Along river bottoms.

2. Tilia heterophýlla, Vent. WhITE BASSWOOD. Leaves whitened beneath with a downy pubescence. In mountainous, wooded districts.

\section{MALVÀCE $\not$. MALLow Family.}

Herbs or shrubs, with alternate leaves having small, deciduous stipules. Flowers mostly perfect, often showy. Sepals mostly 5 and valvate, somewhat united at the base. Petals of the same number and convolute in the bud. Stamens many, united by their filaments around the pistil, and adherent to the bases of the petals. Anthers I-celled. Ovary of several cells, entire or lobed. Styles united below, but separate above and usually projecting beyond the stamens.

\section{MÁLVA. Mallow.}

(The Latin name.)

Herbs. Calyx with a 3-leaved involucre at the base. Stamen-column bearing anthers only at the summit. The numerous styles stigmatic along the inner side. Fruit flattened and circular, of several beakless I-seeded carpels.

I. Malva rotundif lia, L. (L., rotundus, round; folium, leaf.) RounDLEAVED or RUNNing MAllow. Procumbent annual or biennial. Leaves round- 
reniform, crenate, with 5-9-rounded, shallow lobes. Flowers bluish white, clustered in the axils, $\frac{1}{3}$ to $\frac{1}{2}$ inch broad. Petals about twice the length of the calyx lobes. Waysides and cultivated grounds.

\section{CALLÍRRHOË. \\ (Gr., kalos, beautiful; rheo, flower.)}

Herbs, with lobed or divided leaves and showy flowers. Bracts of the involucre when present 1-3. Calyx 5-parted; petals truncated at their apices, as many as the lobes of the calyx. Stamen column antherbearing at the summit. Carpels about 10-20, united in a circle, each I-seeded, beaked at the apex.

I. Callirrhoe alcæoides, Gray. (Gr., alkea, wild mallow ; eidos, form.) LiGHT POPPY MALlow. Erect perennial, 8 to 20 inches high from a thickened root. Basal leaves triangular and lobed; stem leaves digitately divided. Bracts of the involucre none. Flowers pink or white, about I inch broad. Carpels pubescent. In dry soil.

2. Callirrhoe digitàta, Nutt. (L., digitatus, having fingers.) FRINGED POPPY Mallow. Similar in habit to the preceding species. Flowers $I \frac{1}{2}$ to 2 inches broad; petals fimbriate along their upper margin; red purple to white. Carpels hardly pubescent. In dry soil.

3. Callirrhoe involucràta, Gray. (L., involucrum, a wrapper.) PURPLE POPPY MALlow. Perennials, I to 2 feet long, procumbent or ascending from a deep root. Leaves cordate-orbicular, palmately lobed or incised. Bracts of the involucre 3. Peduncle slender and I-flowered. Flowers red purple, I to $2 \frac{1}{2}$ inches broad. Carpels rugose-reticulate. In dry soil.

\section{VIOLÁCE ze. VIOLET FAMily.}

Herbs, with irregular, I-spurred corolla and adnate anthers conniving over the ovary. Ovary I-celled with 3 parietal placentæ. Sepals 5 and petals 5. Leaves stipulate; flowers nodding. Style club-shaped with a I -sided stigma.

\section{I. vìolA. Violet or Heart's-ease.}

(The classical Latin name.)

Herbs, with basal leaves commonly clustered. Flowers usually scapose and solitary, nodding, often of two kinds, open and showy, and cleistogamous and inconspicuous beneath the leaves. Sepals more or less auricled. Lower petal spurred at the base, and the two lower anthers with spurlike nectaries. Capsule dehiscing into 3 valves. 
Acaulescent from a rootstock.

Flowers various shades of violet and purple to almost white.

Lateral petals bearded.

(a) Outer leaves crenate-dentate, inner leaves variously palmately lobed.

Viola palmata I.

(b) Leaves pedately parted into linear, obtuse lobes.

Viol.a PEDATIFIDA II.

(c) Leaves reniform to ovate-wedge-shaped, cordate at the base; margins crenatedentate; plants glabrous.

Viola cucullata III.

(d) Leaves ovate to orbicular-ovate; peduncles and other parts of the plant villous.

Viola soraria IV.

Lateral petals beardless.

(a) Leaves pedately parted or divided.

Viola pedata V.

(b) Leaves ovate to orbicular, cordate at the base, crenate-margined, sweet-scented. Cultivated.

(c) Leaves lanceolate to linear-lanceolate.

VIOLA ODORATA VI. Caulescent.

Flowers yellow.

(a) Leaves reniform to broadly ovate; plant villous or pubescent.

Viola pubescens VIII.

(b) Leaves reniform to ovate, only slightly pubescent; stems slender or decumbent.

VIOLA SCABRIUSCULA IX.

Flowers various shades of blue or violet to white, rarely yellowish.

(a) Leaves ovate to nearly orbicular, acuminate or acute; stipules ovate to lanceolate and entire.

Viola Canadensis X.

(b) Leaves as above, but stipules dentate, pinnatifid, or fimbriate.

VIOLA STRIATA XI.

(c) Leaves varying from nearly orbicular to oblong-ovate or oblong-elliptic; stipules large and leaflike, pinnatifid or lyrate.

VIOLA TRICOLOR XII.

(d) Leaves, etc., similar to the above, but the whole plant smaller and more slender.

VIOLA TENELLA XIII.

I. Viola palmàta, L. (L., palmatus, hand-shaped.) EARLY BluE Violet. Acaulescent, leaves and flowers arising from a rootstock which is scaly and thick. Outer and lower leaves crenate-dentate, inner leaves variously palmately lobed. Flowers blue of different shades, sometimes nearly white. Lateral petals bearded; styles beardless. Mostly in woods.

2. Viola pedatifida, Don. (L., pes, pedis, the foot; findo, fidi, to divide.) PRAIRIE VIOLET. Acaulescent. Leaves and flowers from a short, scaly rootstock. Leaves pedately parted into linear, obtuse lobes. Flowers bright blue; lateral petals bearded. On prairies.

3. Viola cucullàta, Ait. (L., cucullus, a hood.) MEADOw or Hooden VIOLET. Acaulescent from a thick, scaly rootstock. Leaves varying from reniform to ovatewedge-shaped, cordate at the base, crenate-dentate. Flowers blue, varying to white ; lateral petals bearded. Plants glabrous, or only slightly pubescent when young. Common in various habitats.

4. Viola soròria, Willd. (L., sororius, sisterly.) WOOLLY BLUE VIOLET. Acaulescent from short and thick rootstock. 'Leaves mostly ovate or orbicularovate, pointed at the apex and cordate at the base. Leaves crenate-villous when young, but becoming less so with age; ascending. Flowers blue; petals more or less bearded. Peduncles villous. Mostly in shady, dry soil. 
5. Viola pedàta, L. (L., pedatus, furnished with feet.) BIRD'S-FOOT VIOLET. Acaulescent from a thick, short rootstock. Leaves pedately parted or divided. Flowers fragrant, blue to violet, opening widely; petals beardless. Stigma beardless and beakless. On hillsides or in sandy or gravelly soil.

6. Viola odoràta, L. (L., odoratus, fragrant.) SWEET VIOLET. -A native of Europe, cultivated in gardens, and running wild in some localities. Spreading by stolons, which take root at the nodes. Leaves and flowers rising from a thick rootstock. Leaves ovate to orbicular, cordate at the base, crenate on the margins. Flowers mostly blue, varying to white; petals beardless; fragrant.

7. Viola lanceolàta, L. (L., lanceolatus, arıned with a little lance.) LANCELEAVED VIOLET. Acaulescent; spreading by stolons, with root at the nodes. Leaves and flowers from a slender rootstock. Leaves lanceolate to linearlanceolate, gradually tapering to a petiole, barely crenate. Flowers white, the lower and lateral petals purplish-veined, beardless. Along streams and in moist meadows.

8. Viola pubéscens, Ait. (L., pubescens, hairy.) HAIRY Yellow VIOLET. Caulescent, villous, or pubescent. Basal leaves long-petioled, withering early. Upper leaves short-petioled. Leaves reniform to broadly ovate, finely crenatedentate. Flowers yellow, purple-veined; spur short. In woods.

9. Viola scabrisúcula, Schwein. (L., diminutive adjective from scaber, rough.) SMOOTHISH YELLOW VIOLET. Resembling the preceding species, but less pubescent, and stems slender and sometimes decumbent; basal leaves usually persisting through the period of blossoming. In woods and thickets.

ro. Viola Canadénsis, L. CaNADA Violet. Caulescent. Stems tufted and leafy throughout. Leaves ovate, sometimes nearly orbicular, glabrous, finely crenate, acuminate, or acute. Stipules entire, ovate to lanceolate. Flowers pale violet to white, purple-veined. In woods of hills or mountains.

II. Viola striàta, Ait. (L., striatus, furrowed.) PALE or STRIPED VIOLET. Similar in general aspect to the preceding species, but the stipules are dentate, pinnatifid, or fimbriate; petals cream-colored, light blue, or white, much veined. In moist woods or thickets.

12. Viola tricolor, L. (L., tri, three; color, color.) PANSY or HEART'S-EASE. A native of Europe, cultivated in gardens, and sometimes running wild. Rather stout annuals with leafy stems, and large, leaflike, pinnatifid, or lyrate stipules. Flowers large, especially in cultivation, variously yellow, purple, blue, violet, and white.

13. Viola tenélla, Muhl. (L., tenellus, somewhat tender.) Field Pansy. A native annual resembling the preceding species, but the plants are more slender, stipules smaller, and flowers smaller. In woods and fields.

\section{ONAGRÀCEA. Evening Primrose Family.}

Annuals or perennials, mostly herbaceous. Flowers axillary, spicate, or racemose. Ovary inferior, the so-called calyx tube often prolonged far beyond its summit. Calyx usually 4-lobed, sometimes 2-6-lobed. 
Petals usually 4, sometimes 2-9, convolute in the bud. Stamens as many or twice as many as the petals, and inserted with them on the tube of the calyx. Ovary usually 4-celled; stigmas 2-4-lobed or capitate. Pollen often bound together by cobwebby threads.

\section{CENOTHÈra. Evening Primrose.}

(Old name for a species of Epilobium.)

Calyx tube prolonged beyond the ovary. Petals and lobes of the calyx 4. Lobes of the calyx reflexed. Stamens 8, the anthers mostly versatile. Ovary elongated and 4-celled. Pollen cobwebby. Flowers white, yellow, or rose-color.

I. Enothera biénnis, L. (L., bi, twice; annus, year.) Common Evening PRIMROSE. Erect and mostly stout annuals or biennials, I to 5 feet high, more or less pubescent. Leaves lanceolate, acute, or acuminate at the apex, narrowed toward the base, sessile or short-petioled, $\mathrm{I}$ to 6 inches long, repand-denticulate. Flowers opening in the evening, bright yellow, $\mathrm{I}$ to 2 inches broad. Calyx tube I to $2 \frac{1}{2}$ inches long; reflexed lobes of the calyx cohering at their tips.

2. Enothera speciòsa, Nutt. (L., speciosus, showy.) Showy Primrose. Erect or more or less decumbent perennials, from 6 inches to 3 feet high. Leaves lanceolate to linear-lanceolate, sessile or short-petioled, repand-denticulate or sinuatepinnatifid, 2 to 3 inches long. Flowers usually few, $I^{\frac{1}{2}}$ to $3 \frac{1}{2}$ inches broad, white to pale pink. Tube of the calyx rather longer than the ovary. Capsule strongly 8-ribbed. Plant pubescent. Prairies.

3. Enothera Missouriénsis, Sims. Missouri Evening Primrose. Decumbent perennial with short, silken pubescence. Leaves rather thick, oval to linear or oblong-lanceolate, narrowing to a slender petiole, 2 to 6 inches long, remotely denticulate or entire. Flowers axillary, yellow, 3 to 6 inches broad, very striking. Calyx tube 2 to 6 inches, much exceeding the ovary. Capsules very broadly winged. Crests of limestone hills.

\section{UMBELlíferae. Parsley or Carrot Family.}

Herbs, mostly with hollow, ribbed stems and compound or decompound leaves which clasp the stem at the base. Flowers small in simple or compound umbels; in the latter case the ultimate umbels are called umbellets; the whorl of bracts usually subtending the general umbel is called the involucre, while that subtending the umbellet is termed the involucel. Ovary entirely inferior, 2-celled and 2-ovuled; the limb of the calyx surmounting the ovary either wanting or reduced to a mere 5-toothed border. Styles 2 and filiform, their bases frequently thickened, forming a stylopodium. Petals and stamens 5 , inserted on a 


\section{Dicotyledones.}

disk crowning the ovary. Fruit consisting of 2 seedlike carpels, each of which bears 5 primary ribs, and often 4 intermediate ones. Longitudinal oil tubes commonly occur in the tissue of the carpels between the ribs; these are best seen in cross sections of the carpels.

\section{HERACLÈUM. Cow Parsnip.}

(Named for Herakles, Greek form of Hercules.)

Tall, stout, and often pubescent perennials, with large, ternately compound leaves and broad, compound umbels of white flowers. Involucre of the general umbel deciduous or none; bracts of the involucels numerous and linear. Calyx teeth obsolete or wanting. Petals obcordate, the outer commonly larger and 2-cleft. Stylopodium or disklike expansion at the base of the style common in this family, thick and conic. Fruit broadly oval, obovate, or orbicular, flattened dorsally, and broadly winged on the sides. Ribs filiform with a single oil tube in each interval between the ribs extending only halfway down the fruit, as seen in cross sections.

I. Heracleum lanàtum, Michx. (L., lanatus, woolly.) Cow Parsnip. Stems 4 to 8 feet high, stout, ribbed, and woolly. Leaflets broad, irregularly lobed and cut-toothed, pubescent beneath. In moist ground.
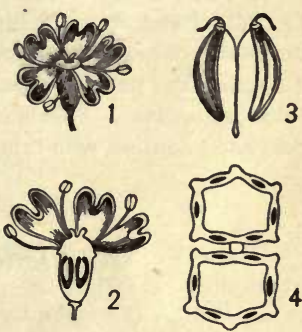

FIG. 349.

Diagrams of Carum Carvi : I, a single flower; 2 , longitudinal diagram of a flower; 3 , ripened fruit; 4 , cross-section of a fruit, showing oil ducts in black. - AFter Wossidlo.

\section{PASTINÀCA. Parsnip.}

(The Latin name, from pastus, food.)

Mostly biennial, tall, branching, and glabrous herbs. Leaves pinnately compound. Flowers yellow in compound umbels; involucre and involucels usually wanting. Calyx teeth obsolete. Stylopodium depressed. Fruit flattened dorsally, winged on the margins, and with filiform ribs on the back; a single oil tube in each interval.

I. Pastinaca sativa, L. (L., sativus, that is sown or planted.) WILD PARSNIP. Stems 2 to 5 feet high from a fleshy, conic root. Lower leaves about $I_{\frac{1}{2}}^{2}$ feet long, petioled, pinnately compound; upper leaves much smaller; leaflets cut-toothed. Roadsides and waste places. 


\section{PEUCÉdANum. Parsley.}

(The old Greek name.)

Perennial herbs, nearly or quite acaulescent, from thickened roots. Leaves mostly bipinnate or finely dissected. Flowers white or yellow in compound umbels. General involucre wanting, but involucels of several bracts. Calyx teeth mostly obsolete. Fruit orbicular, oval, or oblong, flattened dorsally, and winged on the margins. Dorsal and intermediate ribs filiform; $\mathbf{I}-4$ oil tubes in the intervals.

I. Peucedanum fœniculàceum, Nutt. (L., fœniculum, fennel.) FENNELLEAVEd PARsLey. Peduncles 4 to ro inches high, overtopping the leaves, tomentose or nearly smooth. Leaves twice or thrice pinnate, the segments finely dissected, the petioles sheathing at the base. Bractlets of the involucels united below, tomentose. Flowers yellow. Umbels unequally 3-12-rayed. Fruit broadly oval and glabrous, with thin lateral wings. Prairies.

\section{SANÍCULA. Sanicle or Black Snakeroot.}

(From low Latin, sanicula, diminutive of sanus, healthy.)

Rather tall, glabrous, perennial herbs, with few palmately lobed or parted leaves, the basal leaves long-petioled; flowers greenish or yellowish in irregular or compound umbels, capitate in the umbellets. Involucre foliaceous; involucels of few leaves. Fruit globular, not ribbed, and thickly covered with hooked prickles, each with 5 oil tubes.

I. Sanicula Marylàndica, L. Black SNAKeroot or SANicle. Usually unbranched, from $I \frac{1}{2}$ to 4 feet high. Leaves 3-7-parted, the divisions obovate to oblanceolate, irregularly serrate and dentate. Leaves of the general involucre 3-cleft; involucel leaves few and small. Umbels 2-4-rayed. Flowers both perfect and staminate, the staminate in separate heads. Petals greenish white, scarcely exceeding the calyx. Fruit ovoid and beset with stout bristles; the styles recurved, longer than the bristles. In rich woods.

2. Sanicula Canadénsis, L. SHORT-STYLEd SNAKEROoT. Staminate flowers never in separate heads, and styles shorter than the prickles on the carpels. Leaves 3-5-divided, petioled, the divisions sharply serrate. In dry woodlands.

3. Sanicula gregària, Bicknell. (L., gregarius, belonging to a herd or flock.) Clustered SNAKeroot. Stems usually clustered, and yellow petals much surpassing the calyx. I to 3 feet tall. Leaves 5 -divided, the divisions lanceolate to obovate-cuneate. In moist woods and thickets. 


\section{Dicotyledones.}

\section{CHÆROPHÝLLUM. Chervil.}

(Gr., chairo, to gladden; phyllon, leaf. From agreeable odor of the leaves.)

Annuals, growing mostly in moist soil. Leaves ternately decompound with pinnatifid leaflets. Flowers white, few in the umbellets; umbels few-rayed. Involucre usually none; involucels of numerous small bracts. Calyx teeth obsolete; petals reflexed at the apex. Carpels more or less 5 -angled. Fruit oblong to linear-oblong. Ribs slender and obtuse; oil tubes solitary in the intervals.

I. Chærophyllum procúmbens, Crantz. (L., procumbens, falling forward.) SPREADING CHERviL. Stems slender, branched, mostly spreading, more or less pubescent, 6 to 18 inches high. Umbels 2-6-rayed. Flowers few in the umbellets. Fruit linear-oblong and glabrous. In moist ground.

\section{OSMORRHìzA. Sweet Cicely.}

(Gr., osme, a scent; rhiza, root.)

Perennial herbs from fleshy, clustered, aromatic roots. Leaves ternately decompound. Flowers white in few-rayed umbels. Involucre and involucels wanting or of few bracts. Calyx teeth obsolete; petals incurved at the apex. Fruit oblong-linear, short-beaked, attenuate at the base, usually quite bristly along the equal ribs. Oil tubes obsolete or none.

I. Osmorrhiza brevístylis, DC. (L., brevis, short; stylus, stem or point.) SHORT-STYLED or WOolly SWEeT Cicely. Erect, rather stout, becoming branched above, $1 \frac{1}{2}$ to 3 feet high, villous pubescent. Lower leaves large, longpetioled, sometimes I foot across. Umbellets on long peduncles, 2-6-flowered. Style and stylopodium about $\frac{1}{24}$ inch long. In woods.

2. Osmorrhiza longistylis, DC. (L., longus, long; stylus, stem or point.) LONGER-STYLED or SMOOTHER SWEET Cicely. Similar to the preceding species, but only slightly prbescent or glabrous, and style and stylopodium about $\frac{1}{12}$ inch long. In woods.

\section{ERIGÈNIA. Harbinger of Spring.}

( $\mathrm{Gr}$., erigeneia, born in the spring.)

Low, glabrous, nearly acaulescent plants rising from a deep tuber. Leaves ternately decompound, generally only I or ${ }^{2}$. Flowers white in small umbels. Calyx teeth obsolete; petals flat and entire. Fruit nearly orbicular, incurved at top and bottom; carpels nearly kidneyshaped, each 5 -ribbed, and I-3 small oil tubes in each interval. 
I. Erigenia bulbòsa, Nutt. (L., bulbosus, full of bulbs.) HARBINGER oF SPRING. Stem 3 to 9 inches high with a leaf subtending the general umbel. Petioles sheathing at the base. Pedicels very short in flower. Fruit about $\frac{x}{12}$ inch tall, broader than tall.

\section{CORNÀCE E. DOGWOOD FAMILY.}

Shrubs or trees, usually with entire leaves. Flowers perfect, polygamous, or diœcious, in cymes or heads. Ovary inferior. Calyx teeth 4-5 or wanting. Petals 4-5, sometimes none, inserted at the base of an epigynous disk. Stamens inserted with the petals, and of the same number or more numerous. Ovary I-2-celled with a single ovule in each cavity. Style I. Fruit a I-2-celled, I-2-seeded drupe.

\section{CóRNuS. Cornel or Dogwood.}

\section{(L., corn $u$, horn, from the hardness of the wood.)}

Shrubs or trees, with mostly opposite or verticillate leaves, and small white, greenish, or purple flowers in cymes or involucrate heads. Calyx 4-toothed; petals 4; stamens 4 . Ovary 2-celled with I ovule in each cavity. Stone of the drupe 2-celled and 2-seeded.

I. Cornus flòrida, L. (L., floridus, flowery.) Flowering Dogwood. A shrub or small tree, with mostly ovate or oval, petioled leaves. Flowers greenish yellow, in heads; bracts of the involucre $I_{2}^{\frac{1}{2}}$ to $2 \frac{1}{2}$ inches long, white or pinkish, emarginate, strongly veined. Fruit ovoid and scarlet, crowned by the persistent calyx. In woods.

2. Cornus circinàta, L'Her. (L., circinatus, rounded.) Round-LEAVED CORNEL or DOGWOoD. Shrub, 3 to to feet high. Branches greenish and warty. Leaves orbicular or very broadly ovate, woolly beneath. Flowers in flat cymes; fruit blue. In shady and rocky places.

3. Cornus serícea, L. (L., sericeus, silken.) SILKy CORNEL or KinNikinNIK. A shrub, 3 to Io feet high, with purplish, pubescent twigs. Leaves narrowly ovate or elliptical, downy beneath. Calyx teeth lanceolate. Cymes flat. Fruit pale blue. Along streams and in damp woods.

4. Cornus asperifolia, Michx. (L., asper, rough; folium, leaf.) RougHLEAVED CoRnel or Dogwood. Shrub, 3 to $I_{5}$ feet high; branches brownish and rough pubescent. Leaves ovate or elliptic, acuminate, downy beneath and rough pubescent above. Calyx teeth minute; fruit white. In dry soil or exposed hillsides.

\section{ERICÀCE}

Shrubs or perennial herbs. Ovary superior. Calyx and corolla more or less 4-5-parted or cleft. Stamens usually as many or twice as many as the lobes of the corolla. Anthers usually opening by terminal chinks or pores. Style I; ovary 3-ro-celled. 


\section{Dicotyledones.}

\section{EPIGAÈ. Ground Laurel or Trailing Arbutus.}

(Gr., epi, upon; ge, the earth. From the trailing habit.)

Prostrate, more or less hairy, branching shrubs, with evergreen leaves. Flowers clustered at the end of the branches, white or pink, fragrant. Sepals 5, oblong. Corolla salver-formed, mostly 5-lobed. Stamens 10, attached to the base of the corolla and about as long as its tube. Ovary ovoid, hirsute. Style columnar; stigma 5-lobed.

I. Epigæa rèpens, L. (L., repens, trailing.) TRAlling ARBUTUS. MaYFLOWER. GROUND LAUREL. Leaves oval, oblong-ovate, or nearly orbicular, thick. Branches 6 to 15 inches long. Flowers appearing very early in the spring, exhaling a spicy fragrance. In sandy woods or rocky soil, especially under evergreen trees.

\section{GAYLUSSÀCIA. Tangleberry or Huckleberry.}

(Named for Gay-Lussac, chemist.)

Shrubs, with alternate, entire leaves, sometimes serrated. Flowers in lateral, bracted racemes, small and white or pink. Corolla tube urnshaped or campanulate, with 5-lobed limb. Calyx tube short, the limb with 5 short lobes or teeth. Stamens 1o, included. Anthers tapering upward and opening at the summit. Ovary Io-celled. Fruit a berrylike drupe with ro seedlike nutlets.

I. Gaylussacia fronđòsa, T. \& G. (L., frondosus, leafy.) BLUE TANGLE or TANGLEBERry. Erect shrubs, 2 to 4 feet high. Leaves obovate-oblong, blunt, under surface pale and glaucous, and resinous. Flowers in loose racemes, greenish pink, round-campanulate. Fruit globose, dark blue, with a bloom, sweet and edible. In moist woods and thickets.

2. Gaylussacia resinòsa, T. \& G. (L., resinosus, full of resin or gum.) BLACK or HIGH-BUSH HUCKLEBerRy. Shrub, I to 3 feet high. Branches numerous, erect or ascending, and rigid, somewhat pubescent. Leaves mostly oval or oblong-ovate, when young thickly covered with resinous globules, green on both sides. Inflorescence a r-sided raceme. Bracts shorter than the pedicels and deciduous. Fruit black, without bloom, sweet. Rocky woods and thickets, and in swamps.

\section{VACcínIUM. Blueberry, Bilberry, Whortleberry, or Cranberry.}

(The old Latin name.)

Shrubs, with alternate and often coriaceous leaves. Flowers small, white, pink, or red, urn-shaped or campanulate, in terminal or lateral racemes, or sometimes solitary. Calyx tube globose and adnate to the ovary, the persistent limb 4-5-toothed or lobed. Stamens 8-10, the 
anthers becoming tubular above, sometimes awned on the back, opening by terminal pores. Ovary 4-5-celled, or 8-10-celled by false partitions. Fruit a many-seeded berry.

I. Vaccinium corymbòsum, L. (Gr., korymbos, a cluster.) HIGH-BUSH or TAll BlUeBerRY. Shrub, 6 to 15 feet high. Leaves mostly oblong-ovate or elliptical. Flowers tubular, urn-shaped, appearing with the leaves, in short racemes. Calyx 5-lobed; corolla 5-toothed. Stamens Io. Berries blue, with a bloom, pleasantly acid. In swamps and low thickets.

2. Vaccinium Canadénse, Richards. Canada BlueberRy. Low-branching shrub, 6 inches to 2 feet high. Leaves narrowly oval to elliptic-lanceolate, pubescent at least beneath. Branches downy. Flowers oblong-campanulate, greenish white, appearing with the leaves. Berries mostly blue, with a bloom. Swamps and moist woods.

3. Vaccinium Pennsylvánicum, Lam. DWARF or LOW-BUSH BlueberRy. Similar in general aspect to the preceding species, but branches and leaves nearly or quite glabrous, the branches green and warty. Corolla white or pinkish, slightly constricted at the throat. Berries blue, with a bloom, sweet. Dry hills or dry, sandy soil:

\section{PRIMULÀCE Æ̇. Primrose FAmily.}

Herbs, with simple leaves, which are mostly opposite or verticillate, but sometimes alternate. Calyx, with rare exceptions, free from the ovary, mostly 5-parted. Corolla gamopetalous, the limb usually 5-cleft or lobed. Stamens as many as the lobes of the corolla and opposite them, inserted on the tube of the corolla or at its base. Ovary I-celled, with a free central placenta bearing many ovules.

\section{PRÍmula. Primrose or Cowslip.}

(Latin diminutive of primus, first, alluding to early flowering.)

Perennial herbs, with leaves clustered at the base, and flowers often borne in umbels at the end of a scape. Corolla salver-shaped, the tube often enlarging above the insertion of the stamens, 5 -lobed, the lobes mostly notched or obcordate. The five stamens not exceeding the tube of the corolla. Ovary superior, oblong, ovoid, or globose ; style filiform, and stigma capitate, dehiscing at the apex into 5 valves or io teeth.

I. Primula Sinénsis, Sabine. (Latinized form, relating to China.) CHINESE PrimRose. Cultivated in greenhouses, etc. Flowers showy, white, purple, or pink. Lobes of the corolla sometimes cut-fringed. Plant downy; leaves variously cut or crisped. Calyx inflated.

2. Primula grandiflòra, Lam. (L., grandis, large; flos, floris, flower.) TRUE Prim Rose. Cultivated from Europe. Sulphur-yellow flowers rising on slender pedicels from the axils of basal leaves, no proper scape being developed. Corolla flat. Leaves somewhat hairy on their under sides. 


\section{ANDRÓSACE. Starfoil.}

(Greek name of a polyp supposed to be a plant.)

Low annual or sometimes perennial herbs, with small, tufted basal leaves, and small white or pink flowers borne in scapose, involucrate umbels. Calyx 5-lobed or cleft, persistent. Corolla salver- or funnelshaped, contracted at the throat, the tube shorter than the calyx. Stamens 5, included. Ovary globular or turbinate. Capsule 5-valved.

I. Androsace occidentàlis, Pursh. (L., occidentalis, western.) WESTERN STARFolL. Small, nearly or quite glabrous annual. Scapes I to 3 inches long, mostly clustered, erect or ascending from fibrous roots. The basal leaves and leaves of the involucre oblong-ovate. Corolla white, shorter than the calyx. Pedicels slender. In dry soil.

\section{ANAGÁllis. Pimpernel.}

(The old Greek name, possibly from ana, again; agallo, to delight in.)

Diffuse or erect annual or perennial, with mostly opposite or verticillate sessile or short-petioled leaves, and white, blue, red, or pink flowers on axillary peduncles. Calyx and corolla 5-parted; corolla rotate, longer than the calyx. Stamens 5 , inserted at the base of the corolla, more or less pubescent, distinct or united into a ring at the base. Ovary globose with many ovules. Capsule circumscissile.

I. Anagallis arvènsis, L. (L., arvensis, belonging to the fields.) СоммоN PIMPERNEL. Usually much branched annual. Leaves ovate and sessile, shorter than the peduncles. Slender peduncles recurved in fruit. Lobes of the corolla fringed with teeth or glands. Flowers scarlet, sometimes white, opening only in bright weather. In waste places.

\section{CENTÚNCULUS. Chaffweed.}

(Derivation uncertain.)

Low, glabrous annuals, with small, alternate, and entire leaves and minute, solitary, axillary flowers. Calyx 4-5-parted; corolla 4-5-cleft, with urn-shaped tube and spreading lobes, shorter than the calyx. Stamens 4-5, filaments short, inserted on the throat of the corolla. Capsule globose, containing many seeds, circumscissile.

r. Centunculus mínimus, L. (L., minimus, least.) CHAFFweEd or FALSE PIMPERNEL. I to 6 inches high. Leaves spatulate or obovate, short-petioled. Flowers minute, pink, nearly sessile in the axils of the leaves, mostly 4-parted. In moist soil. 


\section{DOdecàtheon. American Cowslip.}

(Gr., dodeka, twelve; theoi, gods. Pliny's name for the primrose, supposed to be under the care of the gods.)

Glabrous perennial herbs, with entire or merely repand basal leaves, and flowers in involucrate umbel terminating a scape. Calyx 5-parted with lobes at first reflexed. Corolla 5 -parted with reflexed lobes, the tube short and thickened at the throat. Stamens 5, monadelphous, connivent into a cone, inserted on the throat of the corolla. Ovary ovoid or nearly globose, containing numerous ovules.

I. Dodecatheon Mèadia, L. American Cowslip or Shooting Star. Perennial, with short rootstock and fibrous roots. Leaves mostly oblanceolate, narrowing into a petiole, entire or sometimes toothed. Scape 8 inches to 2 feet high. Corolla pink, purple, or white. Often cultivated. Rich woods and prairies.

\section{EBENÀCE ÆE. EBony Family.}

Trees or shrubs, with alternate, exstipulate, entire leaves and polygamous, regular flowers. Calyx 3-7-lobed, free from the 3-12-celled ovary. Corolla gamopetalous, 3-7-lobed. Stamens inserted on the tube of the corolla, and 2 to 4 times as many as its lobes. Fruit a berry containing I or more seeds, with bony testa.

\section{DIOSPỲROS. Date Plum or Persimmon.}

(Gr., dios, of Jove; pyros, grain.)

Trees or shrubs, with simple leaves and lateral, solitary, or clustered, diœciously polygamous flowers. Corolla urn-shaped, 4-6-lobed. Calyx 4-6-lobed. Stamens 8-20 in the sterile flowers, fewer and imperfect, or even wanting, in the pistillate flowers. Ovary globose or ovoid; styles 2-6. Berry large and pulpy, containing 4-12 hard and flat seeds.

I. Diospyros Virginiàna, L. Common Persimmon. Tree, with hard and dark bark. Leaves thickish, ovate-oblong to oval, nearly or quite glabrous. Calyx 4-parted. Corolla mostly 4-lobed, greenish yellow, thickish, campanulate, or somewhat urn-shaped; sterile flowers smaller than the fertile. Fruit very astringent when green, becoming reddish yellow, and sweetening after exposure to frost. Woods and old fields.

\section{OLEÀCE Æ̇. OLIVE FAMILY.}

Trees or shrubs, with mostly opposite, pinnate, or simple exstipulate leaves, and flowers in panicles, cymes, or fascicles. Calyx 4-cleft or obsolete; corolla 4-cleft, or 4-petalous, or sometimes wanting. Stamens 


\section{Dicotyledones.}

2-4, mostly 2 , inserted on the corolla. Ovary superior, 2-celled, and with 2 , or at most a few, ovules in each cavity. Fruit a berry, drupe, samara, or capsule.

\section{SYRÍNGA. Lilac.}

(Gr., syrinx $x$, a pipe, possibly relating to the narrow tube of the corolla.)

Shrubs, with simple, entire, opposite leaves and dense panicles of gamopetalous flowers. Calyx mostly 4 -toothed. Corolla salver-formed with a 4-lobed limb. Stamens 2, inserted near the summit of the corolla tube. Ovary 2 -celled with 2 ovules in each cell. Style elongate; stigma 2-cleft. Fruit a narrowly oblong capsule. Natives of the Old World, cultivated for ornament.

I. Syringa vulgàris, L. (L., vulgaris, common.) Common Lilac. A shrub, common in gardens. Leaves ovate, somewhat cordate at base, acuminate at the apex, green and smooth on both sides. Flowers lilac or pale violet, in compact, terminal panicles or thyrses appearing soon after the leaves, fragrant. A white variety also occurs.

2. Syringa Pérsica, L. Persian lilac. Base of leaves narrower and somewhat tapering; leaves nearly lance-ovate.

\section{FRÁXINUUS. Ash.}

(The classical Latin name.)

Trees, with opposite, odd-pinnate leaves and polygamous or diœcious flowers in dense panicles or racemes in the axils of last year's leaves. Calyx small, 4-cleft, or toothed, or entire, sometimes wanting. Petals 2-4, or wanting. Stamens usually 2, sometimes 3-4. Fruit a flat samara, winged all around or only at the apex.

I. Fraxinus Americàna, L. White ASH. A tall tree. Leaflets 5-9, mostly 7 , petioled, commonly ovate to ovate-lanceolate, entire or denticulate, dark green above and paler or sometimes pubescent beneath. Flowers diœcious, rarely monœcious. Fruit nearly cylindrical, about half as long as the wing, which springs from its summit. In rich woods.

2. Fraxinus quadrangulàta, Michx. (L., quadrus, square; angulatus, angled.) BLUE ASH. A large tree, with angular twigs and 7-II chiefly lanceolate leaflets, which are short-stalked, green on both sides, and denticulate or finely serrate. Flowers diœcious. Fruit narrowly oblong, winged on the sides as well as apex; wing often notched. In woods.

\section{ASCLEPIADÀCE ÆE. MilkweEd FAMily.}

Perennial herbs or shrubs, usually exuding latex or milk when wounded. Flowers perfect and mostly in umbels. Ovary of 2 carpels, which are united only at the summit with a fleshy, stigmatic disk. 
Numerous ovules on a parietal placenta in each carpel. Anthers more or less coherent and forming a closed tube around the carpels. Pollen coherent in rather horny masses, called pollinia, the adjacent pollinia of contiguous anthers being joined together by an inverted $V$-shaped, horny excretion from the stigmatic disk (see Fig. 108). Nectar receptacles occurring as a 5 -lobed or parted crown or corona.

\section{ASCLÈPIAS. Milkweed or Silkweed.}

(Gr., asklepios, god of medicine.)

Herbs, usually with opposite or verticillate leaves. Each nectar receptaele bearing an incurved horn within. Calyx usually small, divided into 5 segments; the 5 corolla segments reflexed when open. Anthers tipped with a scale and winged along the sides. Seeds hairy-tufted in all but I species (see Fig. 129).

I. Asclepias tuberòsa, L. (L., tuberosus, full of humps or lumps.) BUTTERFLY WEED or PLEURISY ROOT. Stems erect or ascending, hirsute-pubescent, milky secretions not exuding when the stems are broken. Leaves alternate, lanceolate-oblong. Flowers orange-colored in terminal cymose umbels. In dry fields.

2. Asclepias decúmbens, L. (L., decumbens, falling down.) Decumbent BUTTERFLY WEED. Somewhat similar to the preceding species. Leaves elliptic to oblong. Stems at first decumbent, but erect nearer the apex. In dry fields.

3. Asclepias Cornùti, Decaisne. (L., cornutus, horned.) Common MilkWEED or SILKWEED. Stems erect and stout, 3 to 5 feet high, finely pubescent. Leaves oval-oblong, pubescent beneath. Flowers greenish white with purplish tinge, in dense umbels. Nectar receptacles exceeding the anthers, and incurved horns. Follicles 3 to 5 inches long, tomentose and beset with soft spinose processes. In rich ground.

\section{ASCLEPIODÒRA.}

(Gr., asklepios, god of medicine; doron, gift.)

Similar to Asclepias, but without horns from the nectar receptacles, and with corolla lobes ascending or spreading.

I. Asclepiodora víridis, Gray. (L., viridis, green.) GREEN MILKWEED. Stems about I foot high, nearly or quite glabrous. Leaves ovate-oblong to lanceolate, short petioled, alternate. Flowers green with a purplish crown. In dry soil.

\section{CONVOLVUlÀCE Æ̇. MORning GLoRy Family.}

Mostly twining or trailing herbs, sometimes with milky secretions. Leaves alternate. Flowers axillary, solitary, or cymose, regular and perfect. Calyx 5-parted or divided. Corolla gamopetalous, generally 
with an elongated tube, the limb 5-angled, lobed, or entire. Stamens 5, alternate with the lobes of the corolla, inserted low down in the tube. Ovary superior, 2-3-celled, with 2 erect ovules in each cavity; or sometimes false partitions seem to double the number of cavities, with apparently I ovule in each cell. Fruit, a globular 2-4-valved capsule.

\section{IPOMOÈA. Morning Glory. \\ (Ips, a worm; homoios, like.)}

Mostly twining or trailing annuals or perennials, with showy, axillary, solitary, or cymose flowers. Sepals 5, sometimes unequal. Corolla salver-shaped to funnel-form, or sometimes nearly campanulate, 5-angled or lobed. Style simple, terminated by $1-3$-capitate or globose stigmas. Stamens and style often included. Capsule globular, 4-6-seeded and 2-4-valved.

I. Ipomœa panduràta, Meyer. (L., pandura, a sort of fiddle.) WILD PotATo VINE or MAN-OF-THE-EARTH. Nearly smooth perennial, trailing, barely climbing, from a massive root. Leaves broadly cordate, or sometimes angular, 3-lobed or fiddle-shaped. Peduncles I-5-flowered, elongating in fruit. Sepals oblong, obtuse, or acute. Corolla funnel-form, 2 to 3 inches long, white with purple stripes in the throat; limb 5 -lobed. In dry soil.

2. Ipomœa leptophýlla, Torr. (Gr., leptos, thin; phyllọn, leaf.) BUSH MORNING GLORY. Smooth perennial, with erect, ascending, or reclining stem, 2 to 4 feet long from an enormous root. Much branched. Leaves 2 to 5 inches long, linear, acute at the apex, on short petioles. Peduncles I-4-flowered, nearly erect, shorter than the leaves. Sepals broadly ovate. Corolla about 3 inches long, funnelform, scarcely-lobed, pink or purple. Capsule ovoid, 2-celled, nearly I inch long. In dry soil of western plains.

\section{II. convólvulus. Bindweed.}

(L., convolvere, to twine.)

Mostly perennials, with twining, trailing, or erect stems from slender rootstocks. Leaves mostly cordate or sagittate. Calyx sometimes with a pair of bracts at its base. Corolla funnel-form to campanulate, solitary or clustered in the axils of the leaves, white, purple, or pink. Stamens included in the tube of the corolla. Style slender; stigmas ${ }^{\circ}$, linear, awl-shaped, or ovoid. Ovary 1-2-celled and 4-ovuled.

I. Convolvulus sèpium, L. (Gr., sepion, bone of cuttle-fish.) HEDGE or GREAT BINDWEED. Stems 3 to Io feet long, trailing or twining. Leaves triangularhastate on slender petioles. Flowers funnel-form on slender peduncles, white or tinged with pink. Two large bracts at the base of the calyx. Stigmas oblong. In fields and waste places, preferring moist soil. 
2. Convolvulus rèpens, L. (L., repens, trailing.) TRAILING BINDWEed. I to 3 feet long, trailing or twining, pubescent or tomentose. Leaves sagittate with basal lobes óbtuse or rounded, sometimes cordate. Peduncles I-flowered. Flowers white. Stigmas oblong. Calyx 2-bracted. In dry fields.

3. Convolvulus spithamaèus, L. UPRIGHT BINDWEED. Erect or ascending, 6 to $I 2$ inches high, somewhat pubescent. Leaves mostly oblong-oval and shortpetioled. Flowers white, solitary on long peduncles. Calyx subtended by 2 large oval bracts. Stigmas thick, oblong. In dry, sandy, or rocky soil.

4. Convolvulus arvénsis, L. (L., arvensis, belonging to the fields.) SMALL BINDWEED. Stems slender, trailing or decumbent, I to $2 \frac{1}{2}$ feet long, nearly or quite glabrous. Leaves sagittate at the base and somewhat acute at the apex. Peduncles I-4-flowered, but commonly 2-flowered. Corolla short-funnel-form, white or pink. Peduncles, and usually the pedicels, bracted. Calyx not bracted. Stigmas linear. In fields and waste places.

\section{POLEMONIÀCE E. Phlox Family.}

Herbs. Flowers in corymbose or paniculate clusters, perfect and mostly regular. Calyx 5-cleft, tubular or campanulate. Corolla 5-lobed, tubular, campanulate, or rotate. Stamens 5 , inserted on the tube of the corolla and alternate with its lobes. Ovary superior, 3-celled; style 3-lobed. Capsule few- to many-seeded, 3-valved.

\section{PHLOX.}

(Gr., phlox, flame, an ancient name for Lychnis.)

Perennial or annual herbs, with opposite, entire leaves, and flowers borne in cymose, mostly bracted, clusters. Calyx tubular or tubularcampanulate, becoming distended and rupturing by the ripening capsule. Corolla salver-form with broad, spreading lobes. Stamens included, inserted at different heights on the corolla tube. Ovules I-4 in each of the 3 cavities of the ovary.

I. Phlox maculàta, L. (L., maculatus, stained or spotted.) WILD SweE'T William. Stems erect, $I \frac{1}{2}$ to 5 feet high. Sometimes puberulent, and often flecked with purple. Leaves, excepting the uppermost, opposite, ovate to ovatelanceolate, 2 to 5 inches long. Flowers short-pediceled, borne in elongated, leafy panicles. Calyx teeth lanceolate. Flowers mostly pink or purple.

2. Phlox glabérrima, L. (L., glaberrimus, very smooth.) Smooth Phlox. Stems I to 3 feet high, smooth and slender. Leaves linear-lanceolate, or linear below, $1 \frac{1}{2}$ to 4 inches long. Flowers in cymes grouped in a corymbose cluster. Calyx teeth lanceolate-awl-shaped. Corolla mostly pink, with obovate lobes longer than the tube. Prairies and open woods.

3. Phlox pilósa, L. (L., pilosus, downy.) Downy Phlox. Stems slender, I to 2 feet high. Plant downy or hairy, sometimes glandular. Leaves $I$ to 4 inches 


\section{Dicotyledones.}

long, lanceolate or linear. Flowers in corymbose-cymes. Calyx teeth awl-shaped. Corolla pink, purple, or white with obovate lobes and somewhat pubescent tubes. In dry soil.

4. Phlox divaricàta, L. (L., divaricatus, spread out or apart.) WiLD BLUE PHLOX. Stems decumbent at the base, spreading, viscid-pubescent, 9 to $r 8$ inches high. Leaves lanceolate to ovate-lanceolate, about $I_{\frac{1}{2}}^{\frac{1}{2}}$ inches long, Flowers in loosely-flowered cymules, blue or lilac, slightly fragrant. Calyx teeth awl-shaped. Lobes of the corolla obcordate, emarginate, or entire, slightly longer than the tube. In damp woods.

5. Phlox subulàta, L. (L., subula, an awl.) Ground or Moss Pink. Stems diffuse, matted, branches 2 to 6 inches long. Leaves mostly linear-awl-shaped or linear-lanceolate, from $\frac{x}{8}$ to nearly I inch long, ciliate, often fascicled at the nodes and widely spreading. Calyx teeth somewhat awl-shaped. Corolla pink, purple, or white, its lobes shorter than the tube. On rocky or sandy hills or banks.

\section{HYDROPHYLLÀCE}

Herbs, with leaves mostly alternate. Corolla gamopetalous, 5-lobed or parted, salver-form to campanulate or rotate. Calyx 5-cleft or divided. Stamens 5 , inserted on the tube of the corolla or at its base, alternate with its lobes. Ovary superior, I-celled with 2 parietal placentæ, or 2-celled by the ingrowth and coalescence of the placentæ. Styles 2, sometimes partly united. Fruit, a 2-valved, 4-many-seeded capsule. Flowers in cymes, racemes, or spikes, sometimes solitary.

\section{HYDROPHÝLLUM. Waterleaf.}

(Gr., hydor, water; phyllon, leaf, application not evident.)

Perennial or biennial herbs. Leaves lobed or pinnately divided or parted. Flowers white, blue, or purple, in cymes. Calyx deeply 5-parted, the divisions lanceolate or awl-shaped. Corolla campanulate, 5-cleft, with a linear, grooved appendage extending down the tube opposite each lobe. Stamens 5, exserted. Filaments bearded. Ovary I-celled, the fleshy placentæ nearly filling the cavity, each bearing 2 ovules. Capsule $\mathrm{r}-4$-seeded.

I. Hydrophyllum Virgínicum, L. Virginia Waterleaf. Perennial from scaly rootstock. Nearly or quite glabrous. Stem slender, ascending or erect, I to 3 feet long. Leaves with 5-7 ovate-lanceolate or oblong, pointed, pinnate divisions, the divisions cut-toothed. Peduncles longer than the petioles of the upper leaves. Calyx lobes narrowly linear, ciliate, the sinuses not appendaged. Flowers about $\mathrm{I}$ inch long, purplish or white. In woods.

2. Hydrophyllum Canadénse, L. BROAd-Leaved WATERleaf. Perennial from a scaly rootstock. Stems slender, nearly or quite glabrous, I to $2 \frac{1}{2}$ feet high. 
Leaves rounded, cordate at the base, palmately 5-7-lobed, the lobes pointed and unequally toothed, somewhat pubescent. Lower leaves long-petioled and very broad, sometimes nearly I foot in diameter; upper leaves smaller and shorterpetioled. Flowers in cymes, purplish or white. Calyx lobes linear-awl-shaped, smoothish, sometimes with minute appendages in the sinuses. Corolla shortcampanulate. In woods.

3. Hydrophyllum appendiculàtum, Michx. (L., appendicula, a small appendage.) APPENDAged WAterleaf. Rough and hairy biennial. Stems slender, I to 2 feet long. Lower leaves long-petioled and pinnately incised or divided, the lobes dentate or incised. Upper leaves smaller, rounded, palmately 5 -lobed, the lobes pointed and irregularly toothed. Calyx with a reflexed appendage in each sinus. In damp woods.

\section{ELLÍSIA.}

(Named for John Ellis, naturalist.)

Slender branching annuals, with pinnately lobed or divided leaves and small white or bluish flowers, solitary or racemed. Calyx spreading, 5-lobed or parted, enlarging in fruit. Corolla campanulate or cylindric, hardly, or not at all, exceeding the calyx ; 5 minute appendages within the tube. Stamens included. Ovary I-celled; placentæ as in Hydrophyllum, each 2-4-ovuled.

I. Ellisia Nyctèlea, L. (Gr., nyktelios, nightly.) Somewhat hairy, 6 to I2 inches high. Leaves ovate-oblong in outline, the lobes mostly oblong and cuttoothed. Corolla whitish. Peduncles opposite the leaves, I-flowered. In shady and damp situations.

\section{BORAGINÀCE $\mathbb{E}$. BORAGE FAMILY.}

Mostly rough, hairy herbs, but sometimes shrubs and trees, with alternate, entire leaves and perfect flowers borne in cymes, racemes, or spikes, which are often scorpioid. Calyx 5-lobed, cleft, or parted. Corolla gamopetalous, mostly 5-lobed. Stamens 5, inserted on the tube of the corolla and alternate with its lobes. Ovary superior, mostly deeply 4-lobed. Style undivided or 2-cleft. Fruit appearing like 4 I-seeded nutlets.

\section{CYNOGLÓSSUM. Hound's Tongue.}

(Gr., kyon, dog; glossa, tongue.)

Coarse herbs, with long-petioled basal leaves and mostly sessile upper leaves. Flowers in panicled, somewhat scorpioid racemes. Calyx 5-parted. Corolla funnel-form, its tube about equaling the calyx, and the throat closed by obtuse scales opposite the lobes. Stamens included. Nutlets beset with short, barbed prickles. 


\section{Dicotyledones.}

I. Cynoglossum officinàle, L. (L., officina, a workshop.) CoMmON Hound's TONGUE. $I^{\frac{1}{2}}$ to 3 feet high. Soft, hairy biennial. Upper leaves lanceolate and sessile; lower leaves broadly lanceolate, tapering into a long petiole. Flowers in simple or branched racemes, which elongate in fruit. Calyx lobes ovate-lanceolate. Corolla reddish purple, sometimes white, about $\frac{1}{2}$ inch in diameter. Nutlets flattened on their upper face. In pastures and waste places.

\section{LITHOSPÉRMUM. Gromwell or Puccoon.}

(Gr., lithos, stone; sperma, seed, from the hardness of the seeds.)

Hairy or rough annual, biennial, or perennial, with alternate, entire, sessile leaves. Roots thick and often reddish. Flowers in leafy-bracted racemes or spikes, or solitary. Calyx 5-cleft or parted, with narrow lobes. Corolla salver-form or funnel-form, sometimes crested or pubescent in the throat. Stamens 5, included, inserted on the tube of the corolla and alternate with its lobes. Nutlets 4 or less, white and shining or brown and wrinkled.

I. Lithospermum canéscens, Lehm. (L., canescens, becoming hoary.) HOARY Puccoon. Perennial, pubescent, and somewhat hoary, 6 to $x 8$ inches high. Leaves oblong or ovate-oblong, blunt, downy beneath and roughish above. Flowers in short, leafy racemes. Segments of the calyx linear-lanceolate, shorter than the tube of the corolla. Corolla orange-yellow with rounded, entire lobes, crested in the throat. Nutlets white, smooth, and shining. On prairies or in open woods.

2. Lithospermum angustifólium, Michx. (L., angustus, narrow ; folium, leaf.) NARRow-leaved PucCoOn. Pubescent and rough perennial from a deep root, 6 to 18 inches high. Leaves linear and acute. Flowers in terminal, leafy racemes. The early flowers more showy and with longer tubes than the later. Corolla of the early flowers about I inch long, bright yellow, the tube much longer than the segments of the calyx, the lobes erose-denticulate, and the throat crested. Pedicels of the later, cleistogamous flowers recurved in fruit. Nutlets white and shining, often punctate. On prairies or in dry soil.

3. Lithospermum arvénse, L. (L., aroum, a plowed field.) CORN GromwELL. Rough, somewhat hoary. Leaves lanceolate to linear. Flowers whitish; corolla scarcely longer than the calyx; throat naked. In waste soil.

\section{ONOSMÒDIUM. False Gromwell.}

(Named from resemblance to genus Onosma.)

Stout-bristly or rough-pubescent perennial herb, with alternate, entire, prominently veined leaves and white, greenish, or yellowish flowers in leafy-bracted scorpioid racemes or spikes. Calyx with 5 narrow segments. Corolla tubular or tubular-funnel-form with 5 erect lobes. 
Stamens 5, included, inserted on the tube of the corolla. Style filiform, long-exserted. Ovary 4-parted, but producing only I-2 white, shining nutlets.

I. Onosmodium Caroliniànum, DC. Shaggy FALSE Gromwell. I to 3 feet high, beset with rough, spreading hairs. Leaves lanceolate or ovate-lanceolate, acute. Corolla pubescent outside, yellowish white. On prairies or in dry fields.

2. Onosmodium mólle, Michx. (L., mollis, soft.) SofT-hairy Fal.SE GromWELL. I to 2 feet high, pale, clothed with short, soft hairs. Leaves ovate-lanceolate, acute. On prairies.

3. Onosmodium Virginiànum, DC. Virginia False Gromwell. $I$ to 2 feet high. Clothed with short, appressed, bristly hairs. Leaves oblong to oblonglanceolate, blunt, oblanceolate below, narrowing to a petiole. Corolla yellowish white. On hillsides or banks or in dry thickets.

\section{VERBENÀCE ÆE. VERVAIN FAMILY.}

Herbs or shrubs, with mostly opposite or verticillate leaves. Flowers somewhat 2-lipped, or regular, in spikes, racemes, cymes, or panicles. Calyx 4-5-lobed or cleft. Corolla mostly with cylindric tube and 2lipped or 4-5-lobed and regular. Stamens 4, didynamous, or as many as the lobes of the corolla, sometimes only 2, inserted on the tube of the corolla. Ovary superior, mostly 2-4-celled, but not 4-lobed. Style simple; stigmas $\mathbf{I}-2$. Fruit separating at maturity into 2-4 nutlets.

\section{VERBÈNA.}

(Latin name for any sacred herb.)

Herbs, with opposite leaves and flowers in terminal, simple, or panicled spikes. Calyx tubular, 5-angled, or toothed. Corolla salver-form or funnel-form, with spreading, 5-lobed limb. Stamens 4, didynamous, or rarely 2, included. Ovary 4-celled with I ovule in each cavity. Fruit splitting into 4 nutlets.

I. Verbena Aublètia, L. LARGe-Flowered Verbena. Perennial, I foot high or less, soft pubescent or smoothish. Leaves ovate to ovate-oblong in outline, incisely lobed or toothed, or 3-cleft, the lobes dentate. Inflorescence capitate, but becoming spicate. Bracts of the inflorescence shorter than, or hardly equaling, the calyx. Calyx teeth slender, awl-shaped. Corolla reddish purple or lilac, rarely white, about I inch long, and the limb $\frac{1}{2}$ inch or more in diameter. On prairies or in open woods.

2. Verbena bipinnatifida, Nutt. (L., bi, twice; pinnatus, feathered; findere, to cut.) Small-flowered Verbena. Perennials, producing suckers, erect, 6 to 


\section{Dicotyledones.}

I8 inches high. Leaves I-2-pinnatifid into oblong or linear divisions. Spikes solitary at the ends of the branches. Bracts of the inflorescence mostly exceeding the calyx. Corolla bluish purple or lilac, limb of the corolla less than $\frac{1}{2}$ inch broad. On open prairies.

3. Verbena bractedsa, Michx. (L., bractea, a thin plate.) LARGE-BRACTED VERvaIN. Perennial. Stems 4-sided; much branched from the base, the branches decumbent or ascending, 6 to $I_{5}$ inches long. Leaves wedge-lanceolate in outline, short-petioled, cut-pinnatifid, or 3-cleft. Spikes dense, becoming 4 to 6 inches long, with conspicuous bracts longer than the purple flowers. Corolla about $\frac{1}{4}$ inch long. On prairies and in waste places.

\section{LABIAT Æ. Mint FAMily.}

Aromatic herbs or shrubs, with opposite leaves and usually 4-sided stems. Flowers chiefly in cymose clusters, which are often aggregated into spikes or racemes. Calyx mostly 5-toothed or lobed, persistent. Corolla gamopetalous, mostly 2-lipped, the limb 4-5-lobed. Stamens mostly 4 and didynamous, inserted on the tube of the corolla, sometimes only 2. Ovary superior and deeply 4-lobed, with I ovule in each lobe. Fruit, 4 seedlike nutlets at the bottom of the persistent calyx.

\section{SCUTELLÀRIA. Skullcap.}

(L., scutella, a dish, alluding to calyx in fruit.)

Bitter annual or perennial herbs. Flowers in spikelike racemes, blue or violet. Calyx 2-lipped, the upper lip with a prominent protuberance. Corolla 2-lipped, its tube long, ascending, and somewhat curved, dilated in the throat; the upper lip arched and the lower spreading or bent downward. Stamens 4, all anther-bearing, didynamous, the lower anthers but I-celled. Ovary 4-parted; style unequally 2-cleft. Lips of the calyx closed in fruit.

I. Scutellaria serràta, Andrews. (L., serrātus, notched like a saw.) SHowy SkULLCAP. Slender perennial, I to 2 feet high, nearly glabrous. Leaves ovate to ovate-oblong, coarsely serrate or dentate. Flowers in terminal loose racemes. Corolla blue, about I inch long, very minutely pubescent, the upper lip somewhat shorter than the lower. In woods.

2. Scutellaria pilòsa, Michx. (L., pilosus, hairy.) Hairy Skullcap. Perennial, clothed with spreading hairs. Leaves ovate to oval or oblong, crenate or coarsely serrate, I to 3 inches long, rather remote, the lower long-petioled. Racemes short. Corolla blue, about $\frac{1}{2}$ inch long, the lower lip a little shorter than the upper. In dry woods or thickets.

3. Scutellaria Wrightii, Gray. (Latin genitive of proper name.) Resinous Skullcar. Perennial from a woody root, 6 to to inches high. Minutely hairy 
and generally resiniferous. Leaves ovate to spatulate-oblong, entire, obtuse, from $\frac{1}{4}$ to $\frac{1}{2}$ inch long. Flowers solitary in the axils of the upper leaves. Corolla $\frac{1}{2}$ to $\frac{3}{8}$ inch long, violet to nearly white, pubescent. On western plains.

4. Scutellaria párvula, Michx. (L., parvulus, very small.) Small SkuldCAP. Perennial from slender, tuberiferous rootstocks. Erect or ascending, 3 to I2 inches high. Minutely downy. Lower leaves round-ovate, upper lance-ovate. Flowers solitary in the axils of the upper leaves. Corolla $\frac{1}{6}$ to $\frac{1}{3}$ inch long, violet, pubescent. In moist, sandy soil.

5. Scutellaria campéstris, Britton. (L., campestris, belonging to the field.) PraIRIE SkUllcap. Perennial from tuberous-thickened rootstocks. More or less spreading. Leaves ovate, rounded or truncate at the base, often dentate. Minutely pubescent. Flowers violet or purple. Listed by Gray as var. mollis of the preceding species. In dry, sandy soil.

6. Scutellaria nervosa, Pursh. (L., nervosus, full of nerves.) VeINEd SkullCAP. Perennial from slender stolons. Slender, 8 inches to 2 feet high. Lower leaves rounded to ovate, coarsely serrate or dentate, upper leaves ovate-lanceolate and entire. Flowers solitary in the axils of the upper leaves. Leaves prominently nerved beneath. Corolla bluish, about $\frac{1}{3}$ inch long. Lower lip longer than the concave upper one. In moist thickets or woods.

\section{NÉPETA. Cat Mint.}

(The Latin name.)

Erect or creeping herbs. Flowers in verticillate clusters. Calyx tubular, obliquely 5 -toothed. Corolla 2 -lipped, the upper lip erect, 2-cleft, or notched, somewhat concave ; the lower lip 3-cleft and spreading. Stamens 4, ascending close to the upper lip, didynamous, the lower pair shorter. Anthers approximate in pairs.

I. Nepeta Glechoma, Bentham. (Gr., glechon, pennyroyal.) Ground IVy. GiLL-over-THE-Ground. Perennial, pubescent, creeping and trailing. Leaves round-kidney-shaped, green both sides, petioled, crenate. Corolla light blue, 2 or 3 times longer than the calyx. In damp or shady places.

\section{LÀmium. Dead Nettle.}

(Gr., laimos, throat, alluding to the ringent corolla.)

Mostly diffuse annual or perennial herbs. Leaves commonly heartshaped in general outline, and crenate, dentate, or entire. Flowers verticillate in axillary or terminal clusters. Calyx tubular-campanulate, with 5 equal or unequal teeth, and about 5 -nerved. Tube of the corolla longer than the calyx, dilated in the throat, 2-lipped; the upper lip concave and generally entire, narrowed at the base; the lower lip spreading, 3-lobed, the middle lobe emarginate. Stamens 4, didynamous, close under the upper lip, approximate in pairs, the anterior pair longer. 
I. Lamium amplexicaùle, L. (L., amplexus, an encircling; caulis, stock or stem.) Greater Henbit or Henbit DeAd NetTle. Slender, ascending or decumbent annual or biennial, 6 to 18 inches long. Leaves nearly orbicular, coarsely crenate-toothed, the lower petioled, the upper sessile and clasping. Flowers purple, few and small in terminal and axillary clusters. Calyx nearly as long as the slender tube of the corolla. Upper lip of the corolla pubescent and the lower lip spotted. In fields and waste places.

\section{SÁlVIA. Sage.}

(The Latin name, from salvus, safe, alluding to the healing properties.)

Mostly herbs, with clustered and generally showy flowers. Calyx 2-lipped, the upper lip 3-toothed or entire, the lower 2-cleft or toothed. Corolla deeply 2-lipped, the upper lip mostly entire, straight or curved, concave; the lower lip spreading or pendent, 3-lobed. Stamens 2, the filaments short, surmounted by long filiform connectives which bear a perfect anther-sac at their upper ends, and only a rudimentary anthersac or none at all at their lower ends (see p. 188, Fig. 105). Style 2-cleft. Nutlets smooth.

I. Salvia lyràta, L. (Gr., lyra, lyre.) LyRe-Leaved SAge. Perennial or biennial, more or less pubescent or hirsute, I to 3 feet high. Basal leaves petioled, tufted, often lyre-shaped or sinuate-pinnatifid. Stem leaves sessile, narrower, often entire, and seldom more than a single pair. Flowers in loose whorls forming an interrupted raceme. Calyx campanulate, the teeth of the lower lip longer than those of the upper. Corolla blue purple, pubescent, about I inch long, upper lip short and straight, smaller than the lower lip. Both anther-cells pollen-bearing. In woods, thickets, and meadows.

2. Salvia lanceolàta, Willd. (L., lanceolatus, provided with a little spear.) LANCE-LEAvED SAGE. Erect or diffuse annual, 6 to 18 inches tall, branched and very leafy. Leaves lanceolate to linear-oblong, narrowing to a petiole, sparingly serrate or entire. Flowers opposite or in interrupted, slender, spikelike racemes. Upper lip of the calyx entire, the lower 2-cleft. Corolla blue, about $\frac{1}{3}$ inch long, the lower lip about twice as long as the upper. Lower end of the connective not anther-bearing, dilated. On our western plains.

\section{SOLANÀCE E. NightShadE FAMILY.}

Mostly herbs, sometimes vines or shrubs, with leaves usually alternate and without stipules. Calyx gamosepalous, mostly 5-lobed. Corolla gamopetalous, salver-form or tubular to campanulate or rotate. Stamens generally 5 , inserted on the tube of the corolla and alternate with its lobes. Corolla generally plaited in the bud. Ovary superior, 
entire, 2-celled, rarely 3-5-celled. Ovules numerous on axillary placentæ which often project prominently into the cell. Often rank-scented and poisonous.

\section{SOLÀNUM. Nightshade.}

(Low Latin name for nightshade.)

Herbs or shrubs. Flowers mostly cymose, umbellate, or racemose, yellow, white, bluish, or purplish. Calyx campanulate or rotate, 5toothed or cleft. Corolla rotate, 5-lobed or angled. Stamens inserted on the throat of the corolla, the anthers connate or connivant, oblong, each cell opening by a terminal pore, or by a longitudinal slit. Ovary and berry generally 2 -celled.

I. Solanum Carolinénse, L. HoRse NetTle or APPLE of Sodom. Perennial, green, beset with 4-8-rayed stellate hairs, and armed with straight, yellow prickles. Leaves oblong-ovate, sinuate-toothed or pinnatifid. Flowers racemose, violet or white. Lobes of the calyx lanceolate or ovate, acuminate, about half the length of the corolla. Berries orange-yellow, about $\frac{a}{3}$ inch in diameter. In fields or waste places.

2. Solanum rostràtum, Dunal. (L., rostratus, beaked.) SAND BUR or TEXAS THistLE. Annual, densely armed with awl-shaped prickles and beset with 5-8rayed stellate hairs. Leaves deeply lobed or pinnatifid. Calyx very prickly, inclosing the berry. Corolla yellow, about I inch broad. One anther much exceeding the others in length and diameter. On our western prairies and in waste places.

3. Solanum Dulcamàra, L. (L., dulcis, sweet; amarus, bitter.) BITTERSWEET or NightShaDE. Perennial with climbing or rambling stems, woody below. Leaves ovate or heart-shaped, the upper leaves often halberd-shaped, or with 2 leaflets or more at the base. Corolla deeply 5-cleft, violet-purple, with greenish spots at the base of the lobes. Berries red, oval or globose. In thickets or moist places.

\section{LÝcIUM. Matrimony Vine.}

(Named from the country of Lycia.)

Low shrubs, or woody trailing or climbing plants, generally spiny. Leaves small, alternate, entire, often clustered on lateral spurs. Flowers solitary or clustered, purple, greenish, or white. Calyx 3-5-toothed or lobed, persisting, but not enlarging. Corolla mostly funnel-form or salver-form, 5-lobed. Stamens 5, the anthers dehiscing longitudinally. Style filiform, stigma capitate. Ovary and small, globose berry 2-celled.

I. Lycium vulgàre, Dunal. (L., vulgaris, common.) COMMON MATRIMONY VINE or BOX-THORN. Often somewhat spiny. Stems lithe, trailing, climbing, or recurved. Leaves oblong-spatulate or lanceolate. Flowers axillary, solitary or few-clustered on slender pedicels. Corolla funnel-form, purplish to greenish. Berry orange-red, oval. In thickets or waste places. 


\section{SCROPHULARIÀCE ÆE. FIGWORT FAMILY.}

Mostly herbs. Flowers with a 2-lipped or otherwise irregular gamopetalous corolla and 2,4 , or 5 , often didynamous stamens inserted on the corolla tube. Calyx generally $4-5$-toothed or cleft. Ovary superior, 2-celled, entire or sometimes 2-lobed, ovules several to many on axillary placentæ. Style simple and slender, entire or 2-lobed.

\section{PENTSTÈmon. Beardtongue.}

(Gr., pente, five, stemon, stamen, the fifth stamen being present, though sterile.)

Perennial herbs, branching from the base, with leaves usually opposite or verticillate, the upper sessile and often clasping. Flowers showy, white, purplish, or red, racemose or paniculate. Calyx 5-parted. Corolla mostly tubular-inflated, 2-lipped, the upper lip 2-lobed, under lip 3-lobed. Stamens 5, 4 anther-bearing and didynamous, and I sterile, either naked or bearded. Style filiform and stigma terminal. Capsule oblong to globose, containing many seeds.

I. Pentstemon pubéscens, Soland. (L., pubescens, hairy.) HAiRy BEARDTONGUE. Stems slender and pubescent, I to 3 feet high. Leaves varying from ovate and petioled below to lanceolate and sessile above. Inflorescence loose, glandular-pubescent. Beard at the base of the lower lip nearly closing the throat of the purplish to whitish corolla. Sterile filament bearded for about half its length. In dry woods or rocky grounds.

2. Pentstemon Digitàlis, Nutt. (L., digitalis, finger-shaped.) FoxGlove BEARDTONGUE. Stem 2 to 5 feet high. Glabrous below, but inflorescence glandular-pubescent. Lower leaves oblong-oval, narrowing below. Upper leaves ovatelanceolate to lanceolate, sessile, and somewhat clasping. Corolla white, slightly 2-lipped, open in the throat, I inch or more long. Upper part of sterile filament bearded. In fields and thickets.

3. Pentstemon grácilis, Nutt. (L., gracilis, slender.) SLENDER BEARDTONGUE. 6 to 18 inches tall, glabrous below, but the lax inflorescence glandularpubescent. Lower leaves linear-oblong to spatulate. Upper leaves mostly linear-lanceolate. Corolla purple or whitish, $\frac{8}{4}$ to $x$ inch long, tubular-funnel-form, open in the throat. Sterile filament bearded above for about $\frac{1}{2}$ of its length. On moist prairies.

4. Pentstemon Cobaèa, Nutt. (From proper name, Cobó, a Spanish botanist.) Cobea Beardtongue. Stems stout, $\mathrm{I}$ to 2 feet high, soft-pubescent beneath and glandular-pubescent above. Leaves ovate to oblong-ovate or ovate-lanceolate, often sharply dentate, the upper clasping. Corolla sometimes 2 inches long, much inflated above the middle, whitish or purplish, its lobes.about equal, rounded and spreading. Sterile filament bearded. On dry prairies. 


\section{VERÓNICA. Speedwell.}

(Possibly named for St. Veronica.)

Chiefly herbs, with opposite or sometimes alternate or verticillate leaves. Flowers usually small, inflorescence racemose, spicate, or solitary. Calyx mostly 4-parted, sometimes 3- or 5-parted. Corolla rotate or salver-form, generally with a 4-parted border, the upper lobe commonly broader than the others. Stamens 2, exserted and divergent, inserted on each side of the upper lobe of the corolla. Capsule flattened, notched or obtuse at the apex, 2-celled, few- to many-seeded. Style and stigma simple.

I. Veronica officinàlis, L. (L., officina, workshop.) COMmON SPEEDwelL. Pubescent. Stems ascending or prostrate, rooting at the base, 3 to ro inches long. Leaves mostly obovate, serrate, short-petioled. Racemes spikelike and manyflowered. Flowers pale blue, about inch broad. Capsule much flattened and broadly notched. Perennial. In dry fields and open woods.

2. Veronica serpyllifolia, L. (L., serpyllum, wild thyme; folium, leaf.) Thyme-Leaved Speedwell. Perennial. Glabrous or softly pubescent. Decumbent at the base, much branched, the branches ascending or erect, 2 to Io inches high. Leaves mostly petioled, oblong, oval or ovate, crenate, those of the inflorescence lanceolate. Inflorescence a terminal, spicate, loose-flowered raceme. Corolla pale blue with dark stripes, or white, about $\frac{f}{6}$ inch broad. Capsule broader than long, obcordate, or emarginate. Roadsides, fields, and thickets.

3. Veronica peregrìna, L. (L., peregrinus, foreign, or exotic.) PURSLANE SPEEDWELL or NECKWEED. Nearly smooth or glandular-puberulent annual. Erect or ascending, 3 to $I 2$ inches high. Lowest leaves oval-oblong, short-petioled, or sessile, toothed, thickish. Upper leaves mostly oblong or spatulate, longer than the solitary flowers in their axils. Corolla minute, whitish. Capsule obcordate, nearly orbicular, shorter than the calyx. In moist waste and cultivated grounds.

4. Veronica arvénsis, L. (L., arvum, a field.) CORN or WALl SPEEdWEll. Slender, pubescent annual, simple or diffusely branched, 3 to ro inches tall. Lowest leaves petioled, ovate, and crenate. Upper leaves ovate to lanceolate, frequently alternate and entire with a minute blue or nearly white flower on a short pedicel in the axil of each. Capsule minute, broadly obovate or obcordate. In cultivated grounds, fields, woods, or waste places.

\section{CASTILlèra. Painted Cup.}

(Named for Castillejo, Spanish botanist.)

Herbs. Parasitic on the roots of other plants. Leaves alternate, entire or cut-lobed. Flowers red, purple, white, or yellow, in leafy bracted spikes, the bracts being often expanded and more brightly colored than the flowers. Calyx tubular, somewhat compressed laterally, 
cleft on the upper and sometimes on the lower side. Corolla 2-lipped, the upper lip elongated, compressed laterally, sometimes curved and keeled; the lower lip short and 3-lobed. Stamens 4, didynamous, inclosed in the upper lip of the corolla. Capsule many-seeded; style filiform, and stigma entire or 2-lobed.

I. Castilleia coccínea, Spreng. (L., coccineus, of a scarlet color.) SCARLET PAINTED CUP or INDIAN PAINT-BRUSH. Slender annual or biennial, I to 2 feet high, villous-pubescent. Basal leaves tufted, obovate or oblong, mostly entire. Stem leaves 3-5-cleft into linear or lanceolate segments. Bracts of the inflorescence brilliant scarlet, or rarely yellow. Corolla greenish yellow, its tube included within the 2-cleft calyx. In low meadows and moist thickets.

2. Castilleia sessiliflòra, Pursh. (L., sessilis, low-growing; flos, floris, flower.) Downy PainTed Cup. Densely leaved perennial, 6 to I5 inches tall, clothed with a fine cinereous pubescence. Lower leaves commonly linear and entire, the upper deeply cleft into narrow, entire, or cleft lobes. Bracts of the inflorescence not brilliantly colored, green. Lobes of the calyx linear-lanceolate. Corolla yellowish, nearly 2 inches long, its lower lip narrowly lobed. On prairies.

\section{PEDICULÀRIS. Lousewort.}

(L., pediculus, louse; application not evident.)

Herbs, with pinnately-lobed, cleft, or divided leaves, mostly lanceolate or linear in general outline. Flowers in terminal spikes or spicate racemes. Calyx tubular, variously cleft or toothed. Corolla 2-lipped, with cylindric tube; the upper lip laterally flattened, curved, and sometimes beaked; lower lip erect, with 3 spreading lobes. Stamens 4, didynamous, within the upper lip; anther-sacs equal. Capsule ovate or lanceolate, generally oblique, several-seeded.

I. Pedicularis Canadénsis, L. WoOD Betony or Lousewort. Hairy perennial with generally clustered stems 6 to 18 inches high. Lower leaves oblong, slender-petioled, pinnately-lobed or parted, the lobes variously toothed. Flowers in short spikes which become elongated in fruit. Calyx cleft on the lower side. Corolla usually greenish yellow or purplish, about $\frac{3}{4}$ inch long; upper lip arched, with 2 minute teeth near the apex. Capsule lanceolate and flat. On banks and in thickets.

\section{LENTIBULARIÀCE ÆE. BLADDERWORT FAMILY.}

Herbs, growing in water or in wet places, either floating or rooted. Flowers irregular, solitary or racemose, borne on erect scapes. Calyx 2-lipped. Corolla 2-lipped and personate, the upper lip usually erect and concave; lower lip 3-lobed, spreading, forming a nectariferous spur below. Stamens 2, with confluent anther-sacs. Ovary superior, 
I-celled, with numerous ovules on a free, central placenta. Stigma I-2lipped, either sessile or borne on a short style. Capsules bursting irregularly or dehiscing by valves.

\section{UTRICULÀRIA. Bladderwort.}

\section{(L., utriculus, a little bladder.)}

Herbs, either floating free or rooted, the aquatic species having finely dissected leaves bearing numerous small bladders which are bristled at the orifice. Flowers few or solitary on slender scapes, prominently personate. (See Fig. 73, p. 145.)

I. Utricularia vulgàris, L. (L., vulgaris, common.) GREATER BLADDERWORT or HOODED WATER MILFOIL. Floating free, the branches becoming sometimes I foot long. Scapes 3 to $\mathrm{I}_{4}$ inches high with few or no scales. Divisions of the leaves capillary, bearing numerous bladders. Racemes 3-20-flowered. Corolla yellow, about $\frac{1}{2}$ inch broad, the spur conic and somewhat curved, shorter than the slightly 3 -lobed lower lip. Pedicels recurved in fruit. In ponds and slow streams.

2. Utricularia minor, L. (L., minor, smaller.) LESSER BLADDERWORT. Floating free. Leaves scattered, dichotomously branched into a few setaceous divisions. Bladders among the leaves, few and often none. Scapes slender, 3 to 7 inches high, bearing I-Io flowers. Corolla pale yellow, $\frac{1}{4}$ inch broad or less. Spur very much reduced, or hardly apparent. Pedicels recurved in fruit. In bogs and shallow ponds.

\section{OROBANCHÀCEA. BROOM RAPE FAMILY.}

Root parasites, from nearly white to brownish or purplish, with leaves reduced to scales, and irregular, perfect flowers, which are sessile in spikes or peduncled and solitary in the axils of scales. Calyx gamosepalous, 4-5-toothed or cleft, sometimes divided on one or both sides. Corolla gamopetalous, more or less 2-lipped, the upper lip 2lobed or entire, lower lip 3-lobed; throat of the tubular corolla ringent. Stamens 4, didynamous, alternate with the lobes of the corolla and inserted on its tube. Ovary superior and I-celled, with numerous ovules on 2-4-parietal placentæ. Capsule I-celled and 2-valved.

\section{APHÝLLON. Naked Broomrape.}

(Gr., a, without; phyllon, leaf.)

Brownish or whitish herbs. Scapes naked. Flowers purplish or whitish on long, glandular-pubescent peduncles. Calyx campanulate, about equally 5-cleft. Corolla somewhat 2-lipped, the upper lip 2-lobed and the lower 3 -lobed. Stamens included. The 4 placentæ equidistant or grouped in pairs. 
x. Aphyllon uniflorum, Gray. (L., unus, one; flos, floris, flower.) ONEFLOWERED BROOMRAPE or CANCER ROOT. Stem hardly appearing above the ground, and sending up I-flowered, scapelike peduncles, 3 to 8 inches high. Calyx less than $\frac{1}{2}$ the length of the corolla, its divisions awl-shaped. Corolla about I inch long, white to violet, its lobes obovate and short, and tube slightly curved. Placentæ equidistant. In damp woods, on the roots of various plants.

2. Aphyllon fasciculàtum, Gray. (L., fasciculus, a small bundle.) ClusTERED BROOMRAPE or CANCER ROOT. Stems 2 to 4 inches high, glandularpubescent, bearing 3 to $I_{5}$ I-flowered peduncles. Corolla purplish yellow, plainly 2-lipped. In sandy soil, parasitizing the roots of various plants.

\section{CONÓPHOLIS. Squaw Root or Cancer Root.}

\section{(Gr., konos, cone; pholis, scale.)}

Light brown, scaly herb, parasitic on the roots of trees. Flowers in dense, scaly spikes. Calyx irregularly 5-toothed, split down the lower side, subtended by 2 bractlets. Corolla 2-lipped, its tube slightly curved; upper lip nearly erect, concave, notched at the summit; lower lip shorter, 3-lobed and spreading. Stamens exserted; anthers pubescent. The 4 placentæ about equidistant.

I. Conopholis Americàna, Wallr. SQUAW ROOT. Stems usually clustered, 3 to Io inches high, light brown, covered with overlapping scales, lower scales much shorter than the upper. Flowers about $\frac{1}{2}$ inch long; corolla pale yellow. Among fallen leaves in rich woods.

\section{BignONiàce E. Bignonia or Trumpet Creeper Family.}

Shrubs or trees or woody vines. Leaves generally opposite. Flowers large, clustered, more or less irregular. Calyx 2-lipped, 5-cleft, or entire. Corolla gamopetalous, more or less funnel-form, tubular, or campanulate, sometimes 2-lipped. Anther-bearing stamens, 2 or 4 and didynamous, alternate with the lobes of the corolla and inserted on its tube. Ovary superior, often 2-celled by ingrowth of the placentæ or projections from them; many-ovuled. Fruit, a dry capsule. Seeds flat and winged.

\section{BIGNònIA. Cross Vine or Tendriled Trumpet Flower.}

\section{(Named for Abbé Bignon.)}

Tall, woody climber, with compound leaves ending in a tendril. Calyx short, somewhat 5 -toothed or undulate. Corolla gamopetalous, inflated above the calyx, 5-lobed, somewhat 2 -lipped. Perfect stamens 4, didynamous, included. Capsule linear, flattened parallel with the partition. Seeds transversely winged. 
I. Bignonia capreolàta, L. (L., capreolus, small tendril.) Cross Vine. Leaves long-petioled, 2-foliate, ending with a tendril; leaflets oblong-ovate. Corolla 2 inches long, orange-red, puberulent without, yellow on the inside. Pod about 6 inches long.

\section{CATÁlPA. Catalpa or Indian Bean or Candle Tree.}

(The aboriginal Indian name.)

Trees or sometimes shrubs, with mostly opposite, simple, petioled, ovate, or cordate leaves and showy white or mottled flowers. Tube of the corolla much swollen, its limb 5-lobed and 2-lipped. Perfect stamens 2, or sometimes 4. Capsule cylindric, long and slender, 2-celled. Seeds flat, with lateral wings fringed on the border.

I. Catalpa Bignonioídes, Walt. (From proper name Bignon, and Gr., eidos, form.) Catalpa. Tree with thin bark. Leaves mostly broadly ovate and entire, acute or acuminate, strong scented. Corolla much spotted within, the lower lobe entire. Often planted for shade tree. In woods in the Gulf States.

2. Catalpa speciòsa, Warder. (L., speciosus, showy.) CATALPA. Tree with thick and rough bark. Leaves much as in the last species, but without strong scent. Corolla little mottled within, the lower lobe emarginate. In rich woods. Often planted.

\section{ACANTHÀCEZE. ACANTHUS FAMILY.}

Mostly herbs, with opposite, simple leaves and regular, or only slightly irregular, perfect flowers. Calyx 4-5-parted or cleft, persistent. The gamopetalous corolla 5-lobed, nearly regular, or somewhat 2-lipped. Perfect stamens 4, and didynamous, or only 2. Ovary superior, 2-celled, with 2-10 ovules in each cavity. Seeds borne on curved projections from the placentæ. Capsule 2-celled, opening elastically by 2 valves on drying.

\section{RUÉLLIA.}

(Named for John Ruelle, herbalist.)

Perennial herbs or shrubs, with entire, or rarely dentate, leaves and large, solitary or clustered flowers in the axils of the leaves, or terminal. Calyx 5-cleft or parted. Corolla funnel-form or salver-form, with large, spreading border. Stamens 4. Ovules 3-10 in each cavity of the ovary. Style recurved toward the apex.

I. Ruellia strèpens, L. (L., strepens, murmuring ; application not evident.) Sмоотн RUelis. Smooth or slightly pubescent perennial, I to 4 feet high. Leaves oblong-ovate or oval on short petioles. Flowers solitary or clustered in the axils. Divisions of the calyx linear-lanceolate. Corolla blue, about $I_{2}^{\frac{1}{2}}$ to 2 
inches long, the diameter of the corolla about equaling the tube. Capsule longer than or equaling the calyx. In rich soil or dry woods.

2. Ruellia ciliòsa, Pursh. (L., cilium, an eye-lash.) Hairy Ruellia. Rather stout, I to $2 \frac{1}{2}$ feet high, beset with soft, whitish hairs. Leaves oval or ovateoblong, sessile or short-petioled. Flowers solitary or clustered in the axils. Corolla blue to violet-purple, its tube $I_{\frac{1}{2}}^{\frac{1}{2}}$ to 2 inches long. Capsule shorter than the calyx. In dry soil.

\section{DIANTHËRA. Water Willow.}

(Gr., dis, double; anthera, anther, alluding to the separated anther cells.)

Mostly perennial herbs, growing in wet places. Leaves opposite and entire. Flowers very irregular, purplish, in axillary, peduncled spikés or heads. Calyx 5-parted; corolla 2-lipped, the upper lip erect, concave, 2-toothed; lower lip spreading and 3-cleft. Stamens 2, inserted on the throat of the corolla. Each cavity of the ovary containing 2 ovules.

I. Dianthera Americàna, L. Dense-flowered WAter Willow. Erect, smooth perennial, I to 2 feet high, with lanceolate or linear-lanceolate leaves. Flowers violet to nearly white, in short, long-peduncled spikes. Tube of the corolla shorter than the lips. In water and wet places.

\section{PLANTAGINÀCE Á. Plantain Family.}

Chiefly acaulescent or short-stemmed annuals or perennials. Leaves mainly basal, with prominent parallel ribs. Flowers chiefly in spikes or heads on long scapes. Calyx 4-parted. Corolla 4-lobed, membranaceous. Stamens mostly 4 , inserted on the tube of the corolla and alternate with its lobes. Ovary superior, I-2-celled, or falsely 3-4celled, with I-several ovules in each cavity.

\section{PLANTÀgo. Plantain.}

(The Latin name.)

Short-stemmed or acaulescent herbs, with mostly prominently ribbed leaves and greenish or purplish flowers in spikes on slender scapes. Calyx of 4 membranous-margined sepals, persistent. Corolla salverform or rotate, 4-parted, withering on the pod. Stamens mostly 4, sometimes 2, exserted. Ovary generally 2-celled, with I or more ovules in each cell. Capsule with circumscissile dehiscence.

I. Plantago màjor, L. (L., major, larger.) Common or Greater PlanTAIN. Perennial. Scapes sometimes becoming 2 feet tall, longer than the leaves. Spikes dense, linear-cylindric. Withered corolla not closing over the capsule in fruit. Leaves on long petioles, mostly ovate, with 3 to II ribs, which remain free 
to the base. Flowers proterogynous, stamens 4. Capsule about twice the length of the calyx. Seeds, several in the capsule. In yards and waste places.

2. Plantago lanceolàta, L. (L., lanceolatus, armed with a little lance.) RiBGRASS or ENGLISH PLANTAIN. Somewhat hairy perennial or biennial, with oblonglanceolate leaves tapering to a petiole; ribs of the leaves $3-5$, free to the base. Scapes exceeding the leaves, becoming sometimes 2 feet tall. Spikes at first short and dense, becoming later cylindric. Sepals with green midrib, and scarious on the margins. Capsule 2-seeded, somewhat longer than the calyx. In fields and waste places.

3. Plantago cordàta, Lam. (L., cordatus, heart-shaped.) HEART-LEAvED or WAter Plantain. Glabrous perennial. Leaves ovate to orbicular, pinnately veined, cordate or abruptly narrowed at the base, long-petioled. Scapes exceeding the leaves; spikes becoming loosely flowered. Bracts fleshy. Capsule 2-4-seeded. Found in swampy places and along streams.

4. Plantago Púrshii, R. \& S. (Latin genitive of proper name.) PuRsh's Plantain. (Plantago Patagonica, var. gnaphalioides, in Gray's "Manual.") Woolly or hairy annual, with slender scapes and linear leaves. Spikes dense, cylindric, and very woolly; bracts equaling or only slightly exceeding the flowers. Capsule 2-seeded, but little exceeding the calyx. On dry prairies.

5. Plantago aristàta, Michx. (L., aristatus, having ears.) LARGE-BRACTED PlantaIN. Villous or glabrate annual, with linear leaves and dense, cylindric, pubescent, but not woolly spikes. Bracts of the inflorescence linear, ascending, many times exceeding the flowers. On dry plains or prairies.

\section{RUBIÀCEÆ. MADDER FAMILY.}

Herbs or shrubs, with leaves opposite or verticillate, often connected by intermediate stipules. Flowers perfect, but sometimes dimorphous. Calyx coherent with the ovary. Corolla gamopetalous, 4-5-lobed. Stamens as many as the lobes of the corolla and inserted on its tube, alternate with the lobes. Ovary 2-4-celled, with ovules solitary or many in each cavity. Fruit various.

\section{HOUSTÒNIA. Bluets.}

(Named for Dr. William Houston, English botanist.)

Mostly tufted, erect or spreading herbs, with opposite and entire, sometimes ciliate leaves, and commonly dimorphous, blue, purple, or white flowers. Calyx 4-lobed, corolla funnel-form or salver-form, 4-lobed. Stamens 4. Ovary 2-celled, with several ovules in each cell. Style slender; stigmas 2.

I. Houstonia cœrùlea, L. (L., coruleus, azure.) BLUETs or INNOCENCE. Smooth, with erect and slender stems sparingly branched from the base, 3 to 7 inches high. Lower leaves spatulate or oblanceolate; upper leaves oblong-elliptic, 
about $\frac{1}{8}$ inch long. Peduncles slender, $x$ inch or more long. Corolla light blue to nearly white, with yellow center, $\frac{1}{2}$ to $\frac{1}{2}$ inch broad. Perennial by slender rootstock. In grassy or moist places.

2. Houstonia minima, Beck. (L., minimus, smallest.) LEAST BLUETS. Diffuse or spreading, and generally rough, annual, I to $2 \frac{1}{2}$ inches high. Lower leaves spatulate or ovate, upper leaves oblong-elliptic to nearly linear. Peduncles I inch or less long. Calyx lobes rather broad and much exceeding the capsule. Corolla violet to purple. In dry soil.

3. Houstonia purpùrea, L. (L., purpureus, purple.) LARGE Houstonia. Erect and stout, mostly glabrous perennial, 4 to 18 inches high. Leaves ovate or ovate-lanceolate, sometimes 2 inches long. Flowers in terminal cymes or cymose clusters. Corolla funnel-form, lilac or purple. Calyx lobes longer than the globular pod. In woodlands or open places.

4. Houstonia ciliolàta, Torr. (L., cilium, an eye-lash.) FrINGed Houstonia. Tufted, erect perennial, 4 to 7 inches tall. Leaves oblanceolate to obovate, ciliatefringed. Corolla purple or lilac. On rocky banks.

5. Houstonia angustifòlia, Michx. (L., angustus, narrow; folium, leaf.) NARROW-LEAVED Houstonia, Stiff, erect, glabrous perennial, rising I to 2 feet from a deep root. Leaves mostly linear, often in fascicled clusters. Flowers on short pedicels in dense, terminal, cymose clusters. Corolla purplish to white, its lobes bearded within. Capsule compressed-obovoid, nearly as long as the calyx lobes. In open places.

\section{Mítchella. Partridge Berry.}

(Named for Dr. John Mitchell, botanist.)

Creeping herbs, with opposite, evergreen leaves and terminal, dimorphous flowers in pairs, with united ovaries. Calyx usually 4 -toothed. Corolla funnel-form and 4-lobed. Stamens 4, inserted on the throat of the corolla alternate with its lobes. Ovary 4-celled, with I ovule in each cavity. Style exserted; stigmas 4 . Flowers white or tinged with purple, fragrant. Fruit, 2 united, scarlet, edible drupes.

I. Mitchella rèpens, L. (L., repens, creeping or trailing.) PARTRIDGEBERRY or TWIN-BERRY. Stems slender and rooting at the nodes. Leaves petioled, rounded-ovate, sometimes variegated with white lines. Flowers white and sessile. Corolla about $\frac{1}{2}$ inch long. Fruit persisting through the winter. At the bases of trees in dry woods.

\section{GÀLIUM. Bedstraw or Cleavers.}

(Gr., gala, milk, which some species have the property of curdling.)

Slender herbs, with square stems and whorled leaves, and small flowers in axillary or terminal cymose clusters. Tube of the calyx somewhat globose, its teeth minute or none. Corolla rotate, mostly 4-parted. 
Stamens 4, or sometimes 3, exserted. Ovary 2-celled with I ovule in each cavity. Fruit separating into 2 I-seeded carpels.

I. Galium Aparine, L. (Gr., aparine, goose-grass.) CleAvers or GooseGRASS. Weak annual; the stem becoming 2 to 5 feet long, beset with backwardgrowing bristles. Leaves lanceolate or oblanceolate, I to 3 inches long, about 8 in a whorl, rough on margins and midrib. Flowers white, $\mathrm{I}-3$ in the axils. In shaded grounds, or habitat various.

2. Galium circaèzans, Michx. WILD LIQUORICF. Somewhat pubescent perennial, I to 2 feet high. Leaves ovate to oval-lanceolate, 4 in a whorl. Peduncles forked; corolla greenish, hairy outside. In dry woods.

\section{CAPRIFOLIÀCEE. HONEYSUCKLE FAMILY.}

Shrubs and woody vines, or sometimes herbs, with opposite, exstipulate leaves. Calyx 3-5-toothed or lobed, its tube coherent with the ovary. Corolla gamopetalous, rotate to tubular. Stamens 5 , or sometimes 4 , inserted on the tube of the corolla alternate with its lobes. Ovary 1-6-celled. Fruit a berry, drupe, or capsule.

\section{SAMBÙCUS. Elder.}

(The Latin name.)

Shrubs, with pinnate leaves and serrate or laciniate leaflets, and small, white flowers in dense, compound cymes. Calyx tube 3-5-toothed or lobed. Corolla somewhat campanulate, 3-5-lobed. Stamens 5, inserted on the base of the corolla. Ovary 3-5-celled. Stigmas 3 . Fruit a small, berrylike, juicy drupe.

I. Sambucus Canadénsis, L. Common Elder. Nearly glabrous, 3 to to feet high. Younger stems with large, white pith. Leaflets 5-II, ovate or oval, serrate, acuminate. Cymes broad and flat. Fruit purple to black. Usually in moist soil.

2. Sambucus racemòsa, L. (L., racemosus, full of clusters.) RED-BERRIED ELDER. 2 to 12 feet high, with commonly pubescent twigs and leaves, and warty bark. Leaflets $5-7$, ovate-lanceolate, sharply serrate. Cymes somewhat pyramidal. Fruit bright red. In rocky places.

\section{VIBÚRNUM.}

(The Latin name.)

Shrubs or trees, with simple leaves and mostly white flowers in compound cymes. Calyx 5-toothed. Corolla deeply 5 -lobed, spreading. Stamens 5 , inserted on the tube of the corolla, exserted. Ovary in- 
ferior, I-3-celled, with a single ovule in each cell. Style short; stigmas I-3. Fruit a I-celled, I-seeded drupe.

r. Viburnum Ópulus, L. (L., opulus, a kind of maple.) Cranberry-Tree or High-BUSh CRANBERry. Shrub, becoming sometimes I2 feet high. Branches upright and smooth. Leaves deeply 3-lobed toward the apex, the lobes dentate. Marginal flowers much larger than the others. Drupe globose, red, acid. In low ground along streams.

2. Viburnum pubéscens, Pursh. DownY-Leaved ARrow-Wood. Low branching shrub, 2 to 5 feet high. Leaves ovate, coarsely serrate, nearly sessile, acute, soft-pubescent beneath. All the flowers perfect. Drupes oval and nearly black. In rocky woods,

3. Viburnum prunifölium, L. (L., prunus, plum; folium, leaf.) BLACK HAW, STAG-BUSH, or SLOE. Shrub or small tree, with ovate or oval, finely serrate leaves, I to 3 inches long, obtuse at the apex. Cymes compound and sessile. Petioles hardly or not at all margined. Fruit ripening in the fall, oval, bluish black, sweet. In dry or moist soil.

4. Viburnum Lentàgo, L. NANNY-BERRY, SHEEP-BER RY, or SweEt VibURNUM. Shrub or small tree with ovate, finely serrate leaves, acuminate at the apex, 2 to 4 inches long; petioles long and often margined. Drupes oval, bluish black, with a bloom. Cymes compound and sessile. Along banks of streams and in woods.

\section{TRIÓSTEUM.}

(Abbreviation of triostesspermum, from Gr., tri, three; osteon, bone; sperma, seed; alluding to the three bony seeds.)

Perennial herbs, with opposite leaves much narrowed below the middle, sessile and connate around the stem. Flowers axillary, sessile, solitary or clustered. Calyx with 5 linear-lanceolate lobes, its tube ovoid. Corolla but little longer than the calyx, 5 -lobed, its tube campanulate or tubular, swollen at the base. Stamens 5 , inserted on the corolla tube. Ovary generally 3-celled with I ovule in each cavity. Style slender; stigma 3-5-lobed. Fruit an orange or red drupe, containing bony nutlets.

I. Triosteum perfoliàtum, L. (L., per, through; folium, leaf.) FEvER-worT or Horse-Gentian. Stems stout, 2 to 4 feet high, beset with soft hairs. Leaves oval, abruptly constricted below the middle, 4 to 9 inches long. Flowers brownish purple, clustered in the axils of the leaves. Drupes orange-color. In rich soil.

2. Triosteum angustifolium, L. (L., angustus, narrow; folium, leaf.) YELLOW or NARROW-LEAVED HORSE-GENTIAN. Stems more slender than in the preceding species, bristly hairy, I to 3 feet tall. Leaves lanceolate, tapering below the middle, 3 to 5 inches long. Flowers pale greenish yellow, usually solitary in the axils. In shady situations. 


\section{LONÍCERA. Honeysuckle or Woodbine.}

(Latinized form of Lonitzer, German herbalist.)

Erect or climbing shrubs, with opposite, entire, sometimes perfoliate leaves. Flowers in clusters, or sometimes solitary. Calyx nearly globular, slightly 5 -toothed. Corolla tubular or funnel-form, more or less irregularly 5-lobed. Stamens 5, inserted on the tube of the corolla. Ovary 2-3-celled, with numerous ovules in each cavity. Style slender and stigma capitate; berry fleshy.

I. Lonicera Sullivántii, Gray. (Latin genitive of proper name.) SUllIVANT'S HONEYSUCKLE. Smooth and glaucus, 3 to 6 feet long. Leaves oval or obovate, glaucus, and often pubescent beneath. Corolla pale yellow, hairy within, its tube about $\frac{1}{2}$ inch long. Filaments nearly glabrous. In woods.

2. Lonicera sempervirens, L. (L., semper, always; virens, growing green.) TRUMPET or CoRAL Honeysuckle. Climbing high. Leaves oval, the uppermost connate-perfoliate, dark green above, glaucus beneath. Flowers in whorls in terminal, interrupted spikes. Corolla scarlet outside, yellow inside, and sometimes yellow throughout, from I to $I_{\frac{1}{2}}$ inches long, trumpet-shaped. Berries scarlet. Common in cultivation. In copses, or habitat various.

\section{VALERIANÀCE E. VALERIAN FAMILy.}

Herbs, with opposite, exstipulate leaves, and small, more or less irregular flowers in forked or panicled cymes. Calyx tube adherent to the ovary, its limb nearly or quite wanting, often becoming prominent in the fruit. Corolla tubular or funnel-form, mostly 5-lobed, and sometimes irregular. Stamens $\mathbf{I}-4$, inserted on the tube of the corolla alternate with its lobes, mostly exserted. Ovary inferior, I-3-celled; I cell containing a single ovule and the others empty. Style slender; stigmas 1-3. Fruit dry and indehiscent.

\section{VALERIÀNA. Valerian.}

(Latin name of unknown origin.)

Perennial, mostly tall herbs with thickened, strong-scented roots, and paniculate, cymose flowers. Limb of the calyx divided into several plumose bristles which are inrolled in the flower, but straighten out and become conspicuous in the fruit. The funnel-form or tubular corolla gibbous near the base and nearly regularly 5-lobed above. Stamens mostly 3. Fruit I-celled and seedlike.

I. Valeriana pauciflora, Michx. (L., paucus, few ; fos, floris, flower.) LARGE- 


\section{Dicotyledones.}

Flowered VAlerian. Stem smooth, erect or ascending, I to 3 feet high. Base leaves cordate or ovate, stem leaves with $3-7$ ovate leaflets. Divisions of the panicled cyme few-flowered. Corolla pale pink, its tube $\frac{1}{2}$ inch or more long. In moist soil.

2. Valeriana édulis, Nutt. (L., edulis, edible.) Edible Valerian. Smooth or finely pubescent, erect, I to 4 feet high from a deep, fusiform root. Basal leaves spatulate to lanceolate. Stem leaves pinnately 3-7-parted into linear or lanceolate segments. Inflorescence an elongate, interrupted panicle. Flowers small, yellowish white, nearly diœecious. On moist prairies.

\section{VALERIANÉLLA. Corn Salad or Lamb-lettuce.}

(Name a diminutive of Valerian.)

Annuals or biennials, mostly glabrous, and dichotomously branched. Leaves tender and succulent, those of the base tufted, entire; the stem leaves often dentate. Limb of the calyx obsolete or short and toothed. Corolla funnel-form or tubular, equally or unequally 5 -lobed, small, and white or whitish. Stamens 3. Style slightly 3-lobed. Fruit 3-celled, 2 cells empty and the third containing a single seed. Inflorescence a corymbed or panicled cyme.

r. Valerianella chenopodifòlia, DC. (Gr., chen, goose; pou, foot; folium, leaf.) Goose-FOOT CORN SALAD. Smooth, I to 2 feet high. Lower leaves broadly spatulate, somewhat repand, stem leaves oblong-oval to lanceolate. Cymes $\frac{1}{2}$ inch or more broad. Flowers very small, white; fruit triangular-pyramidal in form. In moist soil.

2. Valerianella radiàta, Dufr. (L., radiatus, provided with spokes or rays.) BEAKED CORN SALAD. Nearly or quite glabrous, 6 to 18 inches high. Lower leaves spatulate or oblong-oval, entire; upper leaves oblong-elliptic to lanceolate, often dentate. Cymes about $\frac{1}{2}$ inch broad or less. Flowers white and very small. Fruit tetragonal, broadly grooved, oblong or ovoid, mostly downy pubescent. In moist soil.

3. Valerianella stenocàrpa, Krok. (Gr. stenos, narrow; karpos, fruit.) NARROW-CELled CORN SALAD. Similar in general aspect to the preceding species, but the fruit commonly glabrous, sometimes downy-pubescent, and the grooves narrow. In moist soil.

\section{CAMPANULÀCE Æ. Bellflower Family.}

Herbs, mostly with a milky juice, alternate, exstipulate leaves, and solitary or scattered flowers. Calyx tube adherent to the ovary, its limb mostly 5 -lobed or parted. Corolla gamopetalous, 5 -lobed, its tube sometimes parted down one side. Stamens 5 , inserted with the corolla and alternate with its lobes. Ovary generally 2-5-celled with axillary plaçentæ; or sometimes I-celled with 2 parietal placentæ. Style simple 
with a tuft of hairs above; stigma generally 2-5-lobed. Fruit a capsule or berry with many small seeds.

\section{SPECULÀRIA. Venus's Looking-glass.}

(L., specularia, a window glass made of talc; from bluish color of the flowers.)

Low annuals, with alternate leaves and axillary blue or purplish flowers; the earlier flowers small and cleistogamous. Calyx 5-, or sometimes 3-4-lobed. Corolla wheel-shaped, 5-lobed. Stamens 5, with membranous, hairy filaments. Ovary mostly 3-celled; sometimes 2- or 4-celled. Stigma usually 3-lobed. Capsule opening by lateral valves.

I. Specularia perfoliàta, A. DC. (L., per, through; folium, leaf.) Somewhat pubescent, 6 to 20 inches tall. Leaves rounded, crenate-dentate, cordate and clasping at the base. Flowers solitary, or few together in the axils, sessile. The upper and older flowers with an expanded and conspicuous corolla, blue or violet. In open grounds or dry woods.

2. Specularia leptocàrpa, Gray. (Gr. leptos, small ; karpos, fruit.) Simple or branched, 6 to ${ }_{5} 5$ inches high. Leaves lanceolate, with mostly solitary sessile flowers in the axils. Calyx lobes 4-5, awl-shaped, but in the earlier flowers only 3 . Corolla of the earlier flowers rudimentary, of the later flowers rotate, $\frac{1}{2}$ inch or more broad. Capsule nearly cylindrical. In dry soil.

\section{COMPÓSIT Ze. CoMposite FAMILY.}

Flowers in a compact head, borne on an enlarged common receptacle, the head having the appearance of a double flower. Many bracts subtend the head, forming an involucre somewhat simulating a calyx. Limb of the calyx (termed pappus in this family), rising from near the summit of the I-celled ovary in the form of bristles, awns, scales, or teeth, or in the form of a cup, or sometimes entirely absent. Corolla strap-shaped, or tubular, mostly 5-lobed. Stamens 5, rarely 4, inserted on the tube of the corolla; anthers united into a tube around the style. Ovary I-celled and I-seeded; style 2-cleft. Fruit a dry, I-seeded achene. Heads which are composed of strap-shaped (ligulate) corollas throughout, or only at the margin, are termed radiate, flowers with ligulate corollas being termed ray flowers. Heads without ray flowers are termed discoid. The family is divided into two series or suborders: I. Tubuliflora, in which the corollas are all tubular; or ligulate only at the margin of the head, where they are pistillate, or neutral. II. Liguliflorce, in which the corollas are ligulate in all flowers of the head, and all of the flowers are perfect. Frequently bracts, in this family called chaff, 


\section{Dicotyledones.}

occur on the receptacle among the flowers. The receptacle is said to be naked when these are absent.

$A$. Corollas all tubular, or ligulate and pistillate, or neutral only, at the margin of the head. Flowers of the head all tubular; diœcious.

Antennaria II.

Disk flowers tubular; ray flowers ligulate.

Ray flowers white, pink, or purple, rarely yellow.

- Pappus of capillary bristles.

Ray flowers numerous and pistillate; bracts of the involucre little imbricated; receptacle naked.

ERIGERON I.

Pappus a minute crown or wanting.

(a) Ray flowers pistillate or neutral; bracts of the involucre imbricated in several rows; receptacle convex and chaffy near the summit; heads on long, terminal peduncles.

Anthemis III.

(b) Ray flowers pistillate; bracts of the involucre imbricated in few rows, appressed; receptacle chaffy; heads small in corymbose clusters.

Achillea IV.

(c) Ray flowers pistillate; disk flowers with flattened tubes; involucral scales imbricated in several rows; receptacle without chaff; heads large on long peduncles.

Chrysanthemum V.

Ray flowers yellow.

Pappus of capillary bristles; disk flowers as well as ray flowers, when present, yellow.

Senecio VI.

$B$. Corollas all ligulate, and all flowers of the head perfect.

Heads borne singly on a scape.

(a) Leaves tufted at the base, entire.

(b) Leaves tufted at the base, pinnatifid or runcinate.

Troximon VII.

Heads borne on long, bracted peduncles, sometimes scapose.

TARAXACUM VIII. margins.

Pyrrhopappus IX.

Sonchus X.

\section{Tubuliflore.}

\section{ERÍGERON. Fleabane.}

(Gr., eri, early; geron, old man, from the hoariness of some of the early spring species.)

Heads radiate on the margin. Ray flowers white, pinkish, or purple; disk flowers yellow. Ray flowers numerous and pistillate. Involucre hemispheric or campanulate, its bracts narrow and little, or not at all, imbricated. Receptacle naked, nearly flat. Pappus a row of capillary bristles with smaller ones interspersed. Achenes flattened, often pubescent and 2-nerved.

I. Erigeron ánnuus, Pers. (L., annuus, annual.) SweEt Scabious. Slightly pubescent annuals, I to 4 feet high, corymbosely branched above. Lower leaves petioled, ovate, or ovate-lanceolate, mostly coarsely dentate; the upper leaves lanceolate or ovate-lanceolate, sessile or short-petioled, sharply dentate. 40-70 linear, white or purplish, rays. Pappus doubled by an outer row of slender scales; the inner row of capillary bristles often wanting in the ray flowers. In fields and waste places. 
2. Erigeron strigòsus, Muhl. (L., strigosus, thin or narrow.) DAISY FLEABANE. Resembling the preceding species, but smaller, and the lower leaves oblong or spatulate, and tapering into a slender petiole; upper leaves linear-oblong or linear-lanceolate. Clothed with an appressed pubescence. Rays white, and twice the length of the puberulent involucre. In fields.

3. Erigeron bellidifòlius, Muhl. (L., bellis, bellidis, the ox-eye daisy; folium, leaf.) RoBin's Plantain. Hairy perennial, producing stolons or offsets at the base. Basal leaves tufted, obovate, or spatulate; upper leaves few and distant, anceolate-oblong, partly clasping. Heads $I$ to $I \frac{1}{2}$ inches broad on slender peduncles. Rays about 5o, violet or purple. Pappus simple. Moist banks and copses.

4. Erigeron Philadélphicus, L. COMMON FleAbane. Hairy or nearly glabrous perennial, forming stolons or offsets at the base. Stems slender, I to 3 feet high. Lower leaves obovate or spatulate, dentate, tapering to a short petiole. Upper leaves oblong or lanceolate, clasping at the base. Rays Ioo or more, light rose-purple. Pappus simple. In fields and woods.

\section{ANTENNÀRIA. Everlasting.}

(Named from resemblance of the pappus to the antennæ of some insects.)

Flowers of the head many, diœcious, all tubular. Corolla of the staminate flowers truncate and minutely dentate, the anthers caudate, pappus scant, club-shaped, barbed, or smooth above. Pistillate flowers with slender, tubular corolla and copious, capillary pappus, united beneath to form a ring. Involucre white or colored, and dry, the scales numerous and imbricated. Receptacle without chaff, and convex or flat. Perennial herbs clothed with a white wool. Leaves entire.

I. Antennaria plantaginifòlia, Richards. (L., plantago, plantain ; folium, leaf.) Plantain Leaf or MOUSE-EAR Everlasting. 3 to 18 inches high, spreading by stolons. Young leaves softly woolly, becoming green above and hoary beneath. Basal leaves petioled, obovate, or broadly spatulate, 3-nerved. Stem leaves lanceolate, appressed. Heads in corymbose clusters. Scales of the involucre white or greenish white, imbricated in about 3 rows. In dry soil or open woods.

\section{III. ÁNTHIMIS. Chamomile.}

(The ancient Greek name of the chamomile.)

Heads radiate on the margins; ray flowers white or yellow, either pistillate or neutral. Disk flowers yellow, perfect. Involucre hemispheric, with bracts imbricated in several rows. Receptacle convex and chaffy, at least near the summit. Pappus a minute crown or wanting. Achenes oblong and ribbed. Strong-scented annual or perennial herbs, with alternate, pinnatifid, or dissected leaves and heads usually borne on long terminal peduncles. 


\section{Dicotyledones.}

I. Anthemis Cótula, L. (Gr., kotyle, a small measure or cup.) MAyweed or Dog FENNEL. Strong-scented and pungent annual, branched, I to 2 feet high. Leaves finely I-3 pinnately dissected. Rays white, Io-18, neutral or with an abortive pistil. Pappus none. Receptacle with bristly chaff near the summit only. By roadsides or in fields and waste places.

\section{ACHILLÈA. Yarrow.}

(Named for Achilles, who is supposed to have discovered its virtues.)

Heads small, with both radiate and tubular flowers, in corymbose clusters at the ends of the branches. Ray flowers white, few and fertile. Disk flowers yellow. Bracts of the involucre imbricated in a few rows, appressed. Receptacle chaffy, convex, or flattish. Pappus none. Achenes flattened and somewhat margined. Perennial herbs, with serrate, pinnatifid, or finely dissected, alternate leaves.

I. Achillea Millefollium, L. (L., mille, thousand; folium, leaf.) СоммоN YARROW or MiLfoIL. Perennial, from a horizontal rootstock, becoming I to 2 feet high. Leaves twice pinnatifid into slender segments. Flowers in a compound, flat-topped corymb. Heads small and numerous; ray flowers 4-5, mostly white, sometimes pink or purple; bracts of the involucre oblong, acute. Habitat various.

\section{CHRYSÁNTHEMUM. Ox-eye Daisy.}

(Gr., chrysanthemon, golden flower.)

Heads composed of both tubular and ray flowers; the rays fertile, white, rose-colored, or yellow. Disk flowers with flattened tubes, perfect. Scales of the involucre imbricated in several rows. Receptacle flat or convex, without chaff. Biennial, perennial, or annual herbs, with mostly large heads borne on long peduncles.

I. Chrysanthemum Leucánthemum, L. (Gro, leukos, white; anthemon, flower.) OX-EYE or White Daisy. Perennial, I to 3 feet high. Branches terminated by a single large head on a long peduncle. Rays white, 20-30. Pappus none. Basal leaves petioled, spatulate, incised. Upper leaves spatulate to linear, cut-toothed, clasping at the base. Scales of the involucre with scarious and brown margins. In pastures, fields, and waste places.

\section{SENĖCIO. Groundsel.}

(L., senex, old $m$ ?n, from the hoariness of some species.)

Heads usually consisting of both disk and ray flowers. Ray flowers, when present, pistillate; disk flowers perfect and fertile. Both disk and ray flowers usually yellow. Pappus of numerous fine capillary bristles. 
Involucre of I row of bracts, subtended by a few bractlets. Receptacle flat and naked. Annual or perennial herbs, with solitary or corymbed heads. Achenes mostly cylindrical, 5-Io-ribbed, downy.

I. Senecio lobàtus, Pers. (Gr., lobos, lobe.) BUTterweed or CREsS-LEAved GrouNDSEL. Glabrous or only slightly woolly annual, I to 3 feet high. Leaves pinnately divided, the lower ones petioled. Heads rather more than $\frac{1}{2}$ inch broad, numerous in terminal corymbs. Rays 6-Io, conspicuous. Involucre nearly cylindric, usually with no smaller outer bracts. In wet grounds.

2. Senecio aùreus, L. (L., aureus, golden yellow.) GoLDEN RAGwORT or SQUAW WEED. Nearly or quite glabrous perennial, I to $2 \frac{1}{2}$ feet high. Basal leaves long-petioled, rounded or nearly heart-shaped, crenate. Stem leaves varying from lyrate below to lanceolate above, and more or less pinnatifid, sessile, and clasping. Heads $\frac{2}{3}$ of an inch or more broad, borne on slender peduncles in an open corymb. Rays 8-I2, yellow. This species is quite variable. Var. obovatus, T. \& G., has basal leaves round-obovate, the earliest being almost sessile and tufted. Var. Balsamite, T. \& G., has spatulate, oblong, or lanceolate basal leaves, lyrate, pinnatifid upper leaves, and heads small and numerous. In moist, open ground.

\section{Liguliflore.}

\section{TRÓXIMON.}

Perennial or annual herbs, with leaves tufted at the base, linear or lanceolate and entire. Heads of ligulate flowers large, yellow or rarely purple, borne singly at the end of a naked or sometimes bracted scape. Scales of the involucre imbricated in 2-3 rows. Receptacle flat, naked, or pitted. Achenes beaked, or sometimes beakless, Io-ribbed. Pappus of many rigid capillary bristles.

I. Troximon cuspidàtum, Pursh. (L., cuspidatus, pointed.) Scape I foot high. Leaves lanceolate, elongate, and tapering, entire, woolly on the margins. Achenes beakless. Plains.

2. Troximon glaùcum, Nutt. (Gr., glaukos, bluish gray.) Scape $I$ to 2 feet high. Leaves oblong or lanceolate, entire, or sometimes dentate or pinnatifid. Heads $I$ to 2 inches wide. Achenes prominently beaked. Plains.

\section{TARÁXACUM. Dandelion.}

(Gr., tarasso, to disquiet, in allusion to medicinal properties.)

Perennial or biennial herbs from a thickened tap-root. Heads large, many-flowered, solitary on the summit of a slender, hollow scape, which exudes a milky secretion when broken. Scales of the involucre in 2 rows, the outer scales short, reflexed with age, those of the inner row 


\section{Dicotyledones.}

linear and erect. Achenes 4-5-ribbed, prolonged into a beak bearing a copious, soft, capillary pappus. Flowers yellow.

r. Taraxacum officinàle, Weber. (L., officina, workshop.) Common DANDEL.iON. Leaves pinnatifid or runcinate, clustered near the ground. Heads crowded with many golden yellow flowers. Beak bearing the pappus becoming very elongate and filiform in fruit. In pastures, fields, and yards.

\section{PYRRHOPÁPPUS. False Dandelion.}

(Gr., pyrrhos, reddish or flame-colored; pappos, pappus.)

Mostly perennial herbs, with the general characteristics of the dandelion. Large heads of yellow flowers borne on long and usually bracted peduncles. Pappus of a reddish color, surrounded at the base by a soft, hairy ring, and borne above the achene on an elongate beak.

I. Pyrrhopappus Caroliniànus, DC. Stems branching, $x$ to 2 feet high. Basal leaves oblong or lanceolate, entire, toothed, or pinnatifid. Upper leaves nearly lanceolate and partly clasping. Outer bracts of the involucre awl-shaped and spreading, inner bracts erect and indurated at the apex. Beak much longer than the achene. In dry fields.

2. Pyrrhopappus scapòsus. (Gr., skapos, a staff.) Rough FALSE DANDELion. Perennial from tuberous thickened roots. No stem leaves present. Basal leaves deeply pinnatifid. Scape often naked, or with a small basal leaf. On prairies.

\section{SóNCHuS. Sow Thistle.}

(The ancient Greek name.)

Coarse annual or perennial herbs, exuding a milky secretion when wounded. Leaves mostly lobed or pinnatifid, with prickly, toothed margins. Heads of yellow flowers in corymbose or paniculate clusters. Bracts of the involucre imbricated in several rows, becoming shorter toward the outside. Achenes somewhat flattened, 10-20-ribbed. Pappus of many soft and fine bristles, not borne on an elongated beak.

I. Sonchus oleràceus, L. (L., oleraceus, pertaining to vegetables.) CомmoN Sow THistle or Hare's LeTtuce. Annual, $x$ to 5 feet high. Basal leaves petioled, lyrate-pinnatifid in outline. Upper leaves sessile and clasping by an auricled or sagittate base, margins with mucronate teeth. Achenes striate and transversely wrinkled. Flowers pale yellow. In fields and waste places.

2. Sonchus ásper, Vill. (L., asper, rough.) SPINY-LEAVEd Sow THISTLE. Similar in general aspect to the preceding species. Lower leaves petioled, obovate, or spatulate. Upper leaves sessile and clasping, with rounded basal lobes; the margins more rigidly spiny-toothed than in the preceding species. Achenes flattened and margined, 3 -nerved on each side. In fields and waste places. 



\section{INDEX TO FLORA.}

Acanthacex, 108.

Acanthus family, 108.

Acer, 74 .

A chillea, 119.

Actra, 39.

Esculus, 75.

Ague tree, 45.

Allium, 20.

Alyssum, 53, 54.

Amaryliidaceæ, 24.

A melanchier, 63.

A merican Black Larch, 14 .

American cowslip, 90.

American ivy, 77.

American pea vine, 68.

A morpha, 67.

Ampelopsis, 77.

A nacardiaceæ, 73.

A nagailis, 89 .

Androsace, 89.

Anemone, 40.

Angiospermæ, 15.

Anonaceæ, 38.

Antennaria, 118.

A nthemis, 118, 119.

A phylion, 106, 107.

A pple, 63.

Apple of Sodom, 102.

Aquilegia, 39.

Arabis, 53.

Araceæ, 18.

A rbor vitæ, 14.

Argemone, 45.

Arisæma, 18; inflorescence of, 18.

Arrow-wood, 113.

A rum family, 18.

Asciepiadaceæ, 91.

Asclepias, 92.

A scleplodora, 92.

Ash, 91.

Asimina, 38.

Astragalus, 67, 68.

Avens, 61, 62 .

Baneberry, 39.

Baptisia, 65.
Barberry, 43.

Barberry family, 42.

Barren strawberry, 61 .

Basswood, 78.

Bastard toadflax, 34, 35.

Beardtongue, 103.

Bedstraw, 111.

Bellflower famiiy, 115 .

Berberidaceæ, 42.

Berberis, 43.

Betula, 30 .

Bignonia, 107, 108.

Bignoniaceæ, 107.

Bignonia family, 107.

Biiberry, 87.

Bindweed, 93, 94.

Birch, 30, 31.

Bitter cress, 50 .

Bittersweet, 102.

Blackberry, 59.

Black haw, 113.

Black mustard, 49.

Black snakeroot, 84.

Bladder nut, 74, 75.

Bladderwort, 106.

Bladderwort family, 105.

Bloodroot, 45, 46.

Blueberry, 87, 88.

Blue birch, 81.

Blue cohosh, 43.

Blue-eyed grass, 26.

Blue grass, 16.

Bluets, 110, 111.

Borage family, 96 .

Boraginaceæ, 96.

Box elder, 74.

Box-thorn, 102.

Bramble, 59.

Brassica, 49.

Broom-rape family, 106.

Bryophyta, 5.

Buckeye, 75.

Buckthorn, 75, 76.

Buckthorn family, 75 .

Buckwheat family, 35 .

Bulbous cress, 50 .

Buttercup, 41, 42.
Butterfly weed, 92.

Butternut, 28.

Butterweed, 120.

Callirrhoe, 79.

Calycocarpum, 44.

Camassia, 21.

Camelina, 52.

Campanulaceæ, 115.

Campion, 37.

Cancer root, 107.

Candle tree, 108.

Caprifoliaceæ, 112.

Capsella, 52.

Cardamine, 50.

Carex, 17.

Carpinus, 31.

Carrot family, 82.

Carya, 28.

Caryophyllaceæ, 37.

Castillela, 104, 105.

Catalpa, 108.

Catchfly, 37.

Cat mint, 100.

Cat-tail, 15.

Caulophyllum, 43.

Ceanothus, 76.

Cedar, white, 14 ; red, 14.

Celandine poppy, 46.

Celtis, 34 .

Centunculus, 89 .

Cerastium, 37.

Cercis, 63.

Chærophyllum, 85.

Chaffweed, 89.

Chamomile, 118.

Cherry, 57, 58.

Chervil, 85.

Chickweed, 37, 38.

Chinese primrose, 88 .

Chrysanthemum, 119.

Cinquefoil, 60, 61.

Claytonia, 36.

Cleavers, 111, 112.

Clover, 66.

Cohosh, 39.

Columbine, 39, 40. 
Comandra, 34, 35.

Commelinaceæ, 19.

Compositæ, 116.

Composite family, 116 .

Conifere, 12.

Conopholls, 107.

Convolvulaceæ, 92.

Convolvulus, 93, 94.

Cornaceæ, 86.

Cornel, 86.

Corn salad, 115.

Cornus, 86.

Corydalis, 47.

Corylus, 32.

Cottonwood, 29.

Cow parsnip, 83.

Cowslip, 88.

Crab apple, 63.

Cranberry, 87.

Cranberry tree, 113.

Cranesbill, 70.

Cratægus, 64.

Cress, 49, 50.

Cross vine, 107, 108.

Crowfoot, 41, 42.

Crowfoot family, 38.

Cruciferæ, 47.

Cupseed, 44.

Cupuliferæ, 30.

Currant, 55, 56.

Custard-apple family, 38 .

Cynoglossum, 96, 97.

Cyperaceæ, 18.

Cyperus, 17.

Cypripedium, 26.

Daisy, 119.

Dandelion, 120.

Date plum, 90.

Dead nettle, 100, 101.

Delphinium, 40.

Dentaria, 51.

Desmanthus, 70.

Dewberry, 59.

Dianthera, 109.

Dicentra, 46.

Dicotyledones, 27.

Diospyros, 90.

Dock, 35.

Dodecatheon, 90.

Dog-fennel, 119.

Dogtooth violet, 22.

Dogwood, 86.

Dogwood family, 86 .

Draba, 53.

Dragon arum, 18.

Dry strawberry, 61 .
Dutchman's breeches, 46. Dwarf alder, 76.

Easter bell, 22.

Ebenaceæ, 90.

Ebony family, 90 .

Elder, 112.

Eleocharis, 17.

Ellisia, 96.

Elm, 33.

Epigæa, 87.

Ericaceæ, 86.

Erigenia, 85, 86.

Erigeron, 117, 118.

Erythronium, 22.

Euphorbia, 72.

Euphorbiaceæ, 72.

Evening primrose, 82.

Evening primrose family, 81 .

False acacia, 67.

False dandelion, 121.

False flax, 52.

False grape, 77.

False gromwell, 97, 98.

False indigo, 65, 67.

False pimpernel, 89.

False rue anemone, 39 .

False Solomon's seal, 23, 24.

Fever-wort, 113.

Figwort family, 103.

Filbert, 32.

Fire pink, 37.

Five-finger, 60, 61 .

Fleabane, 117, 118.

Flower-de-luce, 25.

Four o'clock family, 36 .

Foxglove beard tongue, 103.

Fragaria, 60.

Fraxinus, 91.

Fumarlaceæ, 46.

Fumitory family, 46.

Galanthus, 25.

Galium, 111, 112.

Gama grass, 16.

Garlic, 20.

Gaylussacia, 87.

Geraniaceæ, 70.

Geranium, 70.

Geranium family, 70 .

Geum, 61, 62.

Gill-over-the-ground, 100.

Gleditschia, 69.

Goat's beard, 58 .

Golden ragwort, 120.
Gold-of-pleasure, 52.

Gooseberry, 55.

Goosegrass, 112.

Gramineæ, 15.

Grape family, 76 .

Grape, 77.

Grass family, 15.

Grase flower, dlagram of, 16.

Greater henbit, 101.

Greenbrier, 20.

Green dragon, 18.

Green milkweed, 92.

Gromwell, 97.

Ground ivy, 100.

Ground laurel, 87.

Ground pink, 95.

Ground plum, 67, 68 .

Groundsel, 119.

Gymnocladus, 69.

Gymnospermæ, 12.

Hackberry, 34.

Hackmatack, 14.

Harbinger of spring, 85, 86.

Hare's lettuce, 122.

Haw, 64.

Hawthorn, 64.

Hazelnut, 32.

Heart's-ease, 79, 81.

Heath family, 86 .

Hedge mustard, 49.

Henbit, 101.

Hepatica, 41.

Heracleum, 83.

Hickory, 28.

Honey locust, 69.

Honeysuckle, 114.

Honeysuckle family, 112.

Hop hornbeam, 31, 32.

IIornbeam, 81 .

Horse-chestnut, 75.

Horse-gentian, 113.

Horse nettle, 102.

Hound's tongue, 96, 97.

Houstonia, 110, 111.

Huckleberry, 87.

Hydrophyllace, 95.

Hydrophyllum, 95, 96.

Hypoxis, 24.

Indian bean, 108.

Indian paint-brush, 105.

Indian turnip, 18.

Innocence, 110, 111.

Ipomœa, 93.

Iridaceæ, 25.

İris, 25. 


\section{Index to Flora.}

Ironwood, 31.

Isopyrum, 39.

Judas tree, 68.

Juglandaceæ, 27.

Juglans, 27.

Juneberry, 63, 64.

Juniper, 14.

Juniperus, 14.

Kentucky blue grass, 16.

Kentucky coffee tree, 69.

Key to families, 7-11.

Kinnikinnik, 86.

Labiatæ, 99.

Lady's slipper, 26, 27.

Lamb-lettuce, 115.

Lamium, 100, 101.

Larch, 14.

Larix, 13, 14.

Larkspur, 40.

Lauraceæ, 44.

Leaven worthia, 51 .

Leguminosæ, 65 .

Lentibulariaceæ, 105.

Lepidium, 48.

Lesquerella, 52.

Lilac, 91.

Liliaceæ, 19.

Lilium, 23.

Lily, 23.

Lily family, 19 .

Linden, 78 .

Linden family, 78.

Lithospermum, 97.

Liverleaf, 41.

Locust, 67.

Lonicera, 114.

Lousewort, 105.

Lycium, 102.

Madder family, 110.

Mallow, 78 .

Mallow family, 78 .

Malva, 78.

Malvaceæ, $7 \mathrm{~s}$.

Mandrake, 43.

Man-of-the-earth, 93.

Maple, 74.

Marsh cress, 50.

Matrimony vine, 102.

Mayapple, 43.

Mayflower, s7.

Mayweed, 119.

Meadow-grass, 16.

Meadowsweet, 58.
Menispermaceæ, 44.

Menispermum, 44.

Milfoil, 119.

Milk vetch, 68.

Milkweed, 92.

Milkweed family, 91 .

Mint family, 99.

Mitchella, 111.

Mock orange, 54, 55.

Monocotyledones, 15.

Moonseed, 44.

Morning glory, 93.

Morning glory family, 92 .

Morus, 34 .

Moss pink, 95.

Mouse-ear cress, 49.

Mouse-ear everlasting, 118.

Mousetail, 41.

Mulberry, 34 .

Mustard family, 47.

Myosurus, 41.

Nanny-berry, 113.

Narcissus, 24.

Nasturtium, 50.

Neckweed, 104.

Nepeta, 100.

New Jersey tea, 76.

Nightshade, 102.

Nightshade family, 101.

Nine-bark, 58.

Nothoscordum, 21.

Nyctaginaceæ, 36.

Oak, 32,33 ; diagram of flowers of, 32 .

Oak family, 30 .

(Enothera, 82.

Oleaceæ, 90.

Olive fanily, 90.

Onagraceæ, 81.

Onion, 20.

Onosmorlium, 97.

Orchidaceæ,"26.

Orchis, 27.

Orchis family, 26.

Orobanchaceæ, 106.

Osmorrhiza, 85.

Ostrya, 31.

Oxalis, 71.

Ox-eye daisy, 119.

Oxybaphus, 36.

Painted cup, 104, 105.

Pansy, 81.

Papaveraceæ, 45.

Papaw, 38.
Parsley, 84.

Parsley family, 82 .

Parsnip, 83.

Partridge berry, 111.

Pastinaca, 83.

Peach, 57, 58.

Pear, 63.

Pecan nut, 28.

Pedicularis, 105.

Pentstemon, 108.

Peppergrass, 48, 49.

Pepperwort, 48, 51.

Persimmon, 90.

Peucedanum, 84.

Philadelphus, 54, 55.

Phlox, 94.

Phlox family, 94.

Physoearpus, 58.

Pignut, 28.

Pimpernel, 89.

Pine family, 12.

Pine, white, yellow, spruce, pitch, 13.

Pink, 37, 95.

Pink family, 37.

Pinus, 13.

Plantaginaceæ, 109 .

Plantago, 109, 110.

Plantain, 109, 110.

Plantain family, 109.

Plantaln-leaf everlasting, 118.

Pleurisy root, 92.

Plum, 57.

Poa, 16.

Podophyllum, 43.

Poison ivy, 73.

Poison oak, 73.

Polemoniaceæ, 94.

Polygonaceæ, 35.

Polygonatum, 23, 24.

Pomme blanche, 66 .

Poplar, 29.

Poppy family, 45.

Poppy mallow, 79 .

Populus, 29.

Portulacaceæ, 36 .

Potentilla, 60.

Prairie apple, 67.

Prairie turnip, 67.

Prickly ash, 71.

Prickly poppy, 45.

Primrose, 88.

Primula, 88.

Primulaceæ, 88.

Psoralea, 66.

Pteridophyta, 6.

Puccoon, 97. 
Pulse family, 65 .

Pyrrhopappus, 121.

Pyrus, 63.

Queen of the prairie, 58.

Quercus, 32, 33.

Ranunculaceæ, 38.

Ranunculus, 41.

Raspberry, 59.

Redbud, 68.

Redroot, 76.

Rhamnaceæ, 75.

Rhamnus, 75, 76.

Rhus, 73.

Ribes, 55.

Robinia, 67.

Robin's plantain, 118.

Rock cress, 53.

Rosa, 62.

Rosacex, 56; diagrams of Hlowers of, 56 .

Rose, 62.

Rubiaceæ, 110.

Rubus, 59.

Rue family, 71 .

Ruellia, 108, 109.

Rumex, 35.

Ruta-baga, 49.

Rutaceæ, 71.

Sage, 101.

Salicacex, 29.

Salix, 29, 30 .

Salvia, 101.

Sambucus, 112.

Sandalwood family, 34 .

Sand-bur, 102.

Sanguinaria, 45, 46.

Sanicle, 84.

Sanicula, 84 .

Santalacer, 34.

Sapindaceæ, 74 .

Sassafras, 45 .

Savin, 14.

Saxafraga, 54.

Saxafragaceæ, 54.

Saxafrage, 54 .

Saxafrage family, 54 .

Schrankia, 69 .

Scirpus, 17.

Scrophulariaceæ, 103.

Scutellaria, 99.

Sedge family, 18 .

Selenia, 51.

Senecio, 119.

Sensitive brier, 69.
Service berry, 63, 64.

Sesame grass, 16.

Shad bush, 63, 64 .

Sheep-berry, 113.

Shepherd's purse, 52.

Shooting star, 90.

Silene, 37.

Silkweed, 92.

Sisymbrium, 49.

Sisyrinchium, 26.

Skullcap, 99, 100.

Skunk bush, 73.

Sloe, 113.

Smilacina, 23.

Smilax, 20.

Snakeroot, 84.

Soapberry family, 74 .

Solanacer, 101.

Solanum, 102.

Sonchus, 121.

Sorrel, 35.

Sow thistle, 121.

Spear grass, 16.

Specularia, 116.

Speedwell, 104.

Spermatophyta, 6, 12 .

Spiderwort, 19.

Spiderwort family, 19.

Spiræa, 58.

Spreading yellow cress, 50 .

spring beauty, 86 .

Spurge, 72, 73.

Spurge family, 72.

Squaw root, 107.

Squaw weed, 120.

Stag-bush, 113.

Staphylea, 74.

Starfoil, 89.

Star grass, 24.

Starwort, 38.

Strawberry, 60.

Stellaria, 38.

Stylophorum, 46.

Sumac, 73.

Sumac family, 73.

Swedish turnip, 49.

Sweet alyssum, 54 .

Sweet cicely, 85 .

Sweet viburnum, 113.

Sweet William, 94.

Synopsis of main groups of vegetable kingdom, 5 .

Syringa, 54, 55, 91.

Tamarack, 14.

Tangleberry, 87.

Tansy mustard, 49.
Taraxacum, 120.

Tare, 68.

Tendriled trumpet creeper, 107.

Texas thistle, 102.

Thallophyta, 5 .

Three-thorned acacia, 69.

Thuya, 14.

Tilia, 78.

Tiliaceæ, 78.

Toothache tree, 71 .

Toothwort, 51.

Tradescantia, 19.

Trailing arbutus, 87.

Trefoil, 66.

Trifolium, 66.

Trillium, 22.

Triosteum, 113.

Tripsacum, 16, 18.

Troximon, 120.

Trumpet creeper family, 107.

Tulip, 21.

Tulipa, 21.

Twin-berry, 111.

Typha, 15.

Typhaceæ, 15 .

Ulmus, 33.

Umbelliferæ, 82.

Umbrella-wort, 36 .

Urticaceæ, 33.

Utricularia, 106.

Vaccinium, 87, 88.

Valeriana, 114.

Valerianacex, 114.

Valerianella, 115.

Valerian family, 114.

Venus's looking-glass, 116.

Verbena, 98, 99.

Verbenacex, 98.

Veronica, 104.

Vervain, 99.

Vervain family, 98 .

Vetch, 68.

Viburnum, 112, 113.

Vicia, 68.

Viola, $79,80,81$.

Violaceæ, 79.

Violet, $79,80,81$.

Violet family, 79 .

Virginia creeper, 77.

Vitaceæ, 76.

Vitis, 77.

Wake-robin, 22.

Waldsteinia, 61 . 


\section{Index to Flora.}

Walnut, 27, 28.

Walnut family, 27 .

Water birch, 31.

Water cress, 50.

Waterleaf, 95, 96.

Waterleaf family, 95 .

Water milfoil, 106.

Water willow, 109.

White daisy, 119.
White thorn, 64 .

Whitlow grass, 53.

Whortleberry, 87.

Wild hyacinth, 21.

Wild indigo, 65 .

Wild liquorice, 112.

Wild potato vine, 93 .

Wild spikenard, 23.

Willow, 29, 30.
Willow family, 29.

Windflower, 40, 41.

Wood betony, 105 .

Woodbine, 114.

Wood sorrel, 71.

Xanthoxylum, 71.

Yarrow, 119.

Yellow poppy, 46. 


THIS BOOK IS DUE ON THE LAST DATE STAMPED BELOW

AN INITIAL FINE OF 25 CENTS WILL BE ASSESSED FOR FAILURE TO RETURN THIS BOOK ON THE DATE DUE. THE PENALTY WILL INCREASE TO 50 CENTS ON THE FOURTH DAY AND TO \$1.00 ON THE SEVENTH DAY OVERDUE.

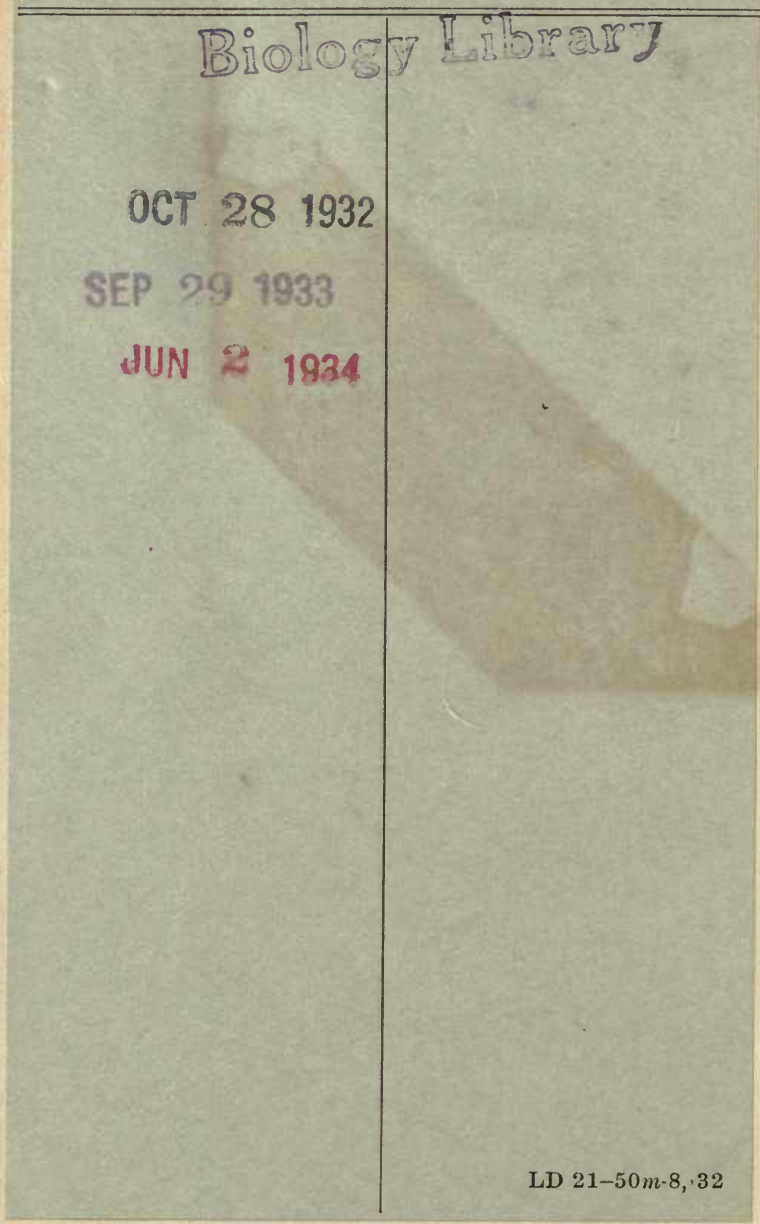




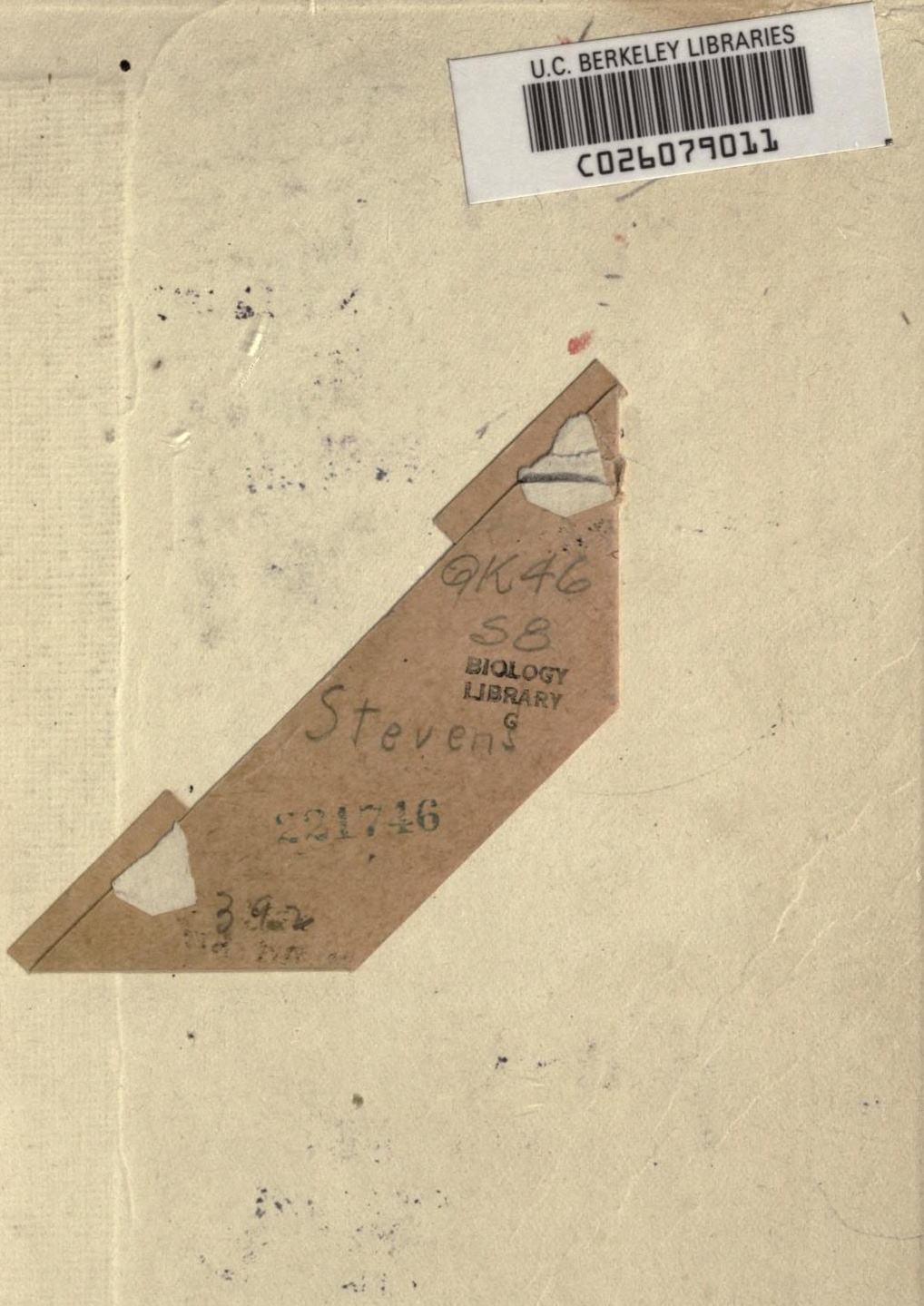


H.

\section{to}

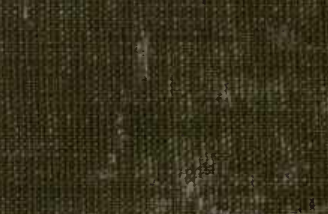

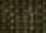

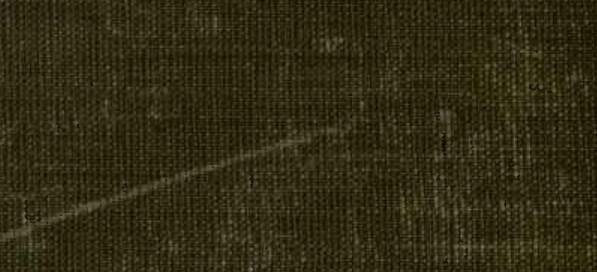

H 70) H. 